\title{
Design and Synthesis of Simplified Analogues of Pateamine A
}

\author{
by \\ Hemi Cumming
}

A thesis submitted to Victoria University of Wellington for the degree of Doctor of Philosophy in Chemistry

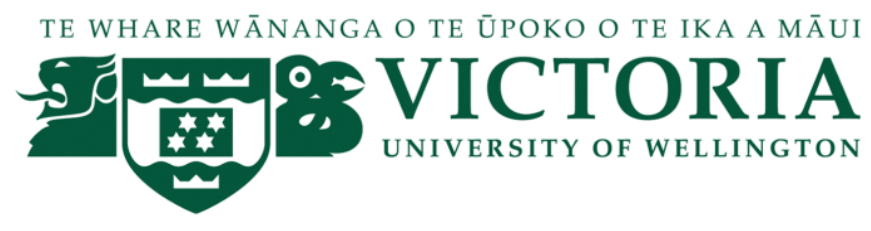

School of Chemical and Physical Sciences 

"Ko te manu e kai ana i te miro, nōna te ngahere. Ko te manu e kai ana i te mātauranga, nōna te ao."

\author{
"The bird that partakes of the miro berry reigns in the forest.
}

The bird that partakes of the power of knowledge has access to the world." 


\title{
Dedicated to mum and dad
}

(Bruce and Kathryn Cumming)

\author{
Everything I have and will achieve, \\ is because of you both.
}




\section{Abstract}

Pateamine A (14) is a natural product that was extracted from a marine sponge off the coast of the South Island of New Zealand. It exhibits potent biological activity, mediated by a number of protein targets. The most sensitive of these towards pateamine are the eIF4A isoforms, which have roles in translation of RNA into proteins and in nonsensemediated decay. The inhibition of these enzymes may be beneficial in the treatment of cancer or certain types of genetic diseases. Unfortunately, the naturally available supply of pateamine is very limited and its total synthesis is complex. This provides an imperative for the design of a synthetic strategy that would allow the preparation of simplified analogues of pateamine to gain further insight into the necessary features for activity and selectivity of the eIF4A isoforms.

Based on the principles of pharmacophore modification, chemical synthesis and the structure-activity relationships (SARs) reported by Romo and co-workers, a simplified analogue of pateamine, 107, was targeted that lacked a number of pendant methyl groups and contained a triazole in place of the thiazole.
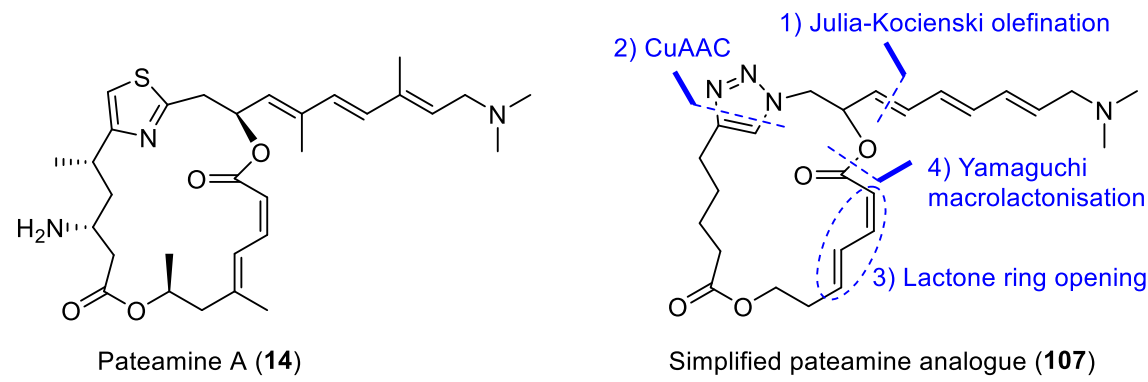

Synthesis of the target analogue 107 was achieved through preparation of four fragments, followed by an investigation of suitable coupling reactions and the optimal order of connectivity. This included the preparation of two macrocycles that lacked the trienecontaining sidechain, and of simplified model substrates that allowed investigation of two olefination reactions (namely, the Wittig and Julia-Kocienski reactions) for the attachment of the sidechain fragment. 
After substantial optimisation of the fragment preparation and connectivity, the complete synthesis of the target pateamine analogue 107 was achieved. The synthesis features: 1) a Julia Kocienski olefination between a highly functionalised three-carbon sulfone and a conjugated aldehyde to attach the sidechain; 2) copper-catalysed azide-alkyne cycloaddition ( $\mathrm{CuAAC}$ ) reaction to form the triazole; 3$)$ ring opening of a $\delta$-substituted $\alpha, \beta$-unsaturated lactone to access the Z,E-dienoate moiety; and 4) Yamaguchi macrolactonisation. This synthesis represents a convergent strategy with 11 steps in the longest linear sequence, which utilises easily accessible starting materials (i.e. furan (or cisbutenediol), epichlorohydrin, $\varepsilon$-caprolactone and 1,3-propanediol) and reagents. The approach is also broadly applicable to the preparation of a range of analogue variants.

The simplified analogue (107) was found to have significantly lower activity, in comparison to pateamine A (14), in a growth inhibitory assay. Presuming this loss of bioactivity is at least partially caused by the incorporation of the triazole (in place of the thiazole), this raises an interesting question as to the role of the thiazole moiety in the bioactivity of pateamine A.

The adaptation of the synthetic strategy devised in this thesis to the preparation of future analogues will enable study of the mechanism of action of pateamine and related compounds, and probe the requirements for effective binding to the eIF4A isoforms. 


\section{Acknowledgements}

Hei timata ake ra, he tika me tukuna te mihi tuatahi ki te timatanga o te ao, à, kia Io. Whai ake ra kia Ranginui rāua ko Papatuanuku. Ki aku tīpuna, ki aku whānau kua wehea ki te pō, he mihi pumou kia koutou. Ki aku kaumātua, John Moffatt me Peter raua ko Florence Cumming, na koutou i whakatau te tūāpapa mōku, no reira kei te mihi. Ka tangi tonu te ngakau mo koutou. Heoi ano, kia tatou e ora ake nei, e tutuki i o tatou wawata, kia ora hoki ra.

My PhD journey has been filled with challenges and has tested me in every way possible. Thus, it is with great satisfaction that I have conquered this lofty mountain and am able to thank those who enabled me to do so.

To my supervisors, Paul Teesdale-Spittle and Joanne Harvey. You have both been nothing short of amazing throughout these past few years, providing me with encouragement, patience, insight and guidance. Paul, your immense knowledge and humble nature is truly inspiring; and Joanne, your eye for detail and enthusiasm has been invaluable to my progress.

I was lucky enough to get financial support through my time of study, so many thanks for: the Victoria PhD scholarship, Nga Pae o te Maramatanga Travel Grant (allowing me to attend PacifiChem conference in Hawaii), the Curtis-Gordon Scholarship, the Âwhina Award, the PVC Māori grant and the Victoria PhD Submission Scholarship whose funding support was vital to my completion and for that, I am deeply apprecative.

My experience as part of the School of Chemical and Physical Sciences (SCPS) has been a real pleasure, with very competent, supportive and friendly staff. These include the Heads of the School, John Spencer and Uli Zuelicke, both of whom were very supportive of me. With special thanks to Ian Vorster for all his help and dedication to keeping the NMR machines going and running my samples on the mass spectrometer. Also, thanks to Jing Jing Wang for her time running samples on the mass spectrometer.

Furthermore, I want to acknowledge with much appreciation other members of the faculty that contributed to this project: Peter Northcote, for purification advice and the useful discussions around pateamine; Jono Singh and Helen Woolner, for running samples on the 600 NMR; Matthias Lein and Thomas Bevan, for all their help with molecular calculations on Gaussian; Dr Jessica Field and Richard Little, from the School of Biological Sciences (SBS), for growth inhibitory assay measurements and performing protein adduct experiments, respectively.

I am really thankful for all the members of our lab group during my PhD: Russell Hewitt, Febly Tho, Xuyu (Jonny) Lu, Sam Ting, Peter Moore, Mark Bartlett, Dylan Davies, Scott Riordan, Thomas Bevan, Claire Turner, Kalpani Somaranthe, Stephen Tat and Jing Jing Wang, for all the great discussions and making the daily grind in the lab much more enjoyable. 
I want to thank Liz Richardson who has been a steadfast supporter and provided me with many opportunities to grow through Te Rōpū Āwhina.

Thanks to Pauline Harris, for all the de-stressing tea breaks from the lab and your friendship and guidance though all the trying moments over the last few years. Ka nui te mihi.

Thanks to all my friends who have supported me and kept me going through the good and the bad during my PhD. In particular, I want to thank Dan and Teresa Chick, Royna Fifield and Annie Fay for all the support, fun and encouragement. Also, thanks to my flatties, Arama, Māui and Toma, who picked me up after long strenuous days and welcomed me home to the sweet smell of boiled salmon heads and a good kōrero and laugh.

To ALL my whānau, particularly: Mum, Dad, Nana (Grace Moffatt), Mathew \& Sacha, Ani \& Leland and Hohepa, who have patiently supported me to complete this long and stressful journey. I am truly blessed to have you all in my life. None of this would have been possible without the âroha and support that all of you have given me, both prior to and during my PhD journey. I am and always will be, eternally grateful.

Nō reira, mai i te hohonutanga o tāku ngākau, ka tuku nga mihi āroha ki a koutou katoa. 


\section{Table of Contents}

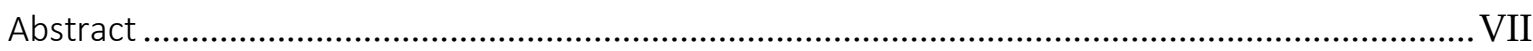

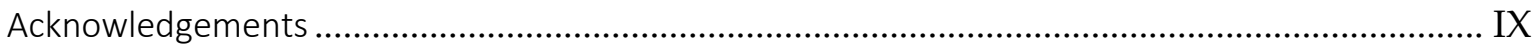

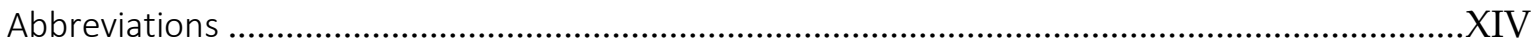

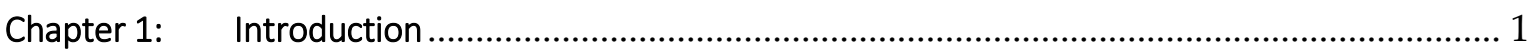

$1.1 \quad$ Natural Products and Analogues in Drug Discovery ............................................... 1

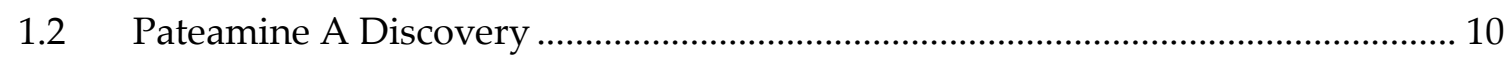

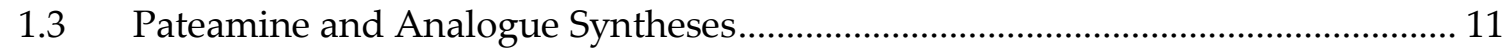

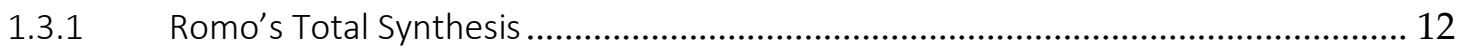

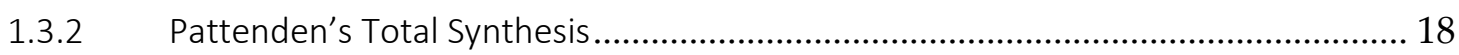

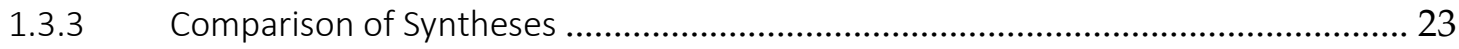

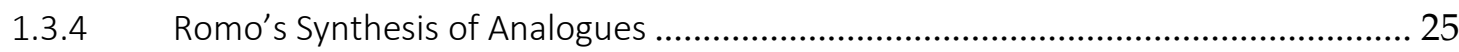

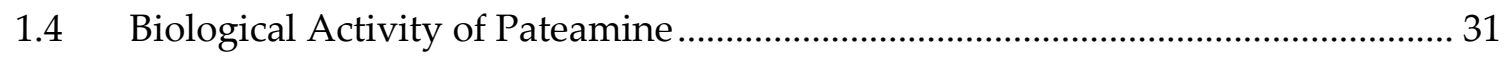

1.4.1 Discovery of Biological Target.................................................................................. 31

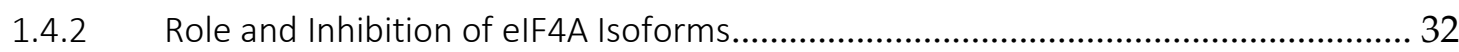

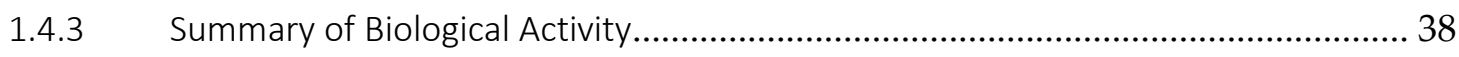

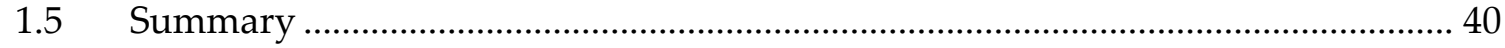

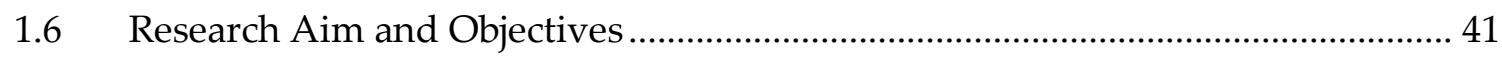

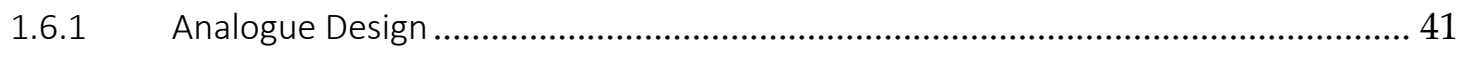

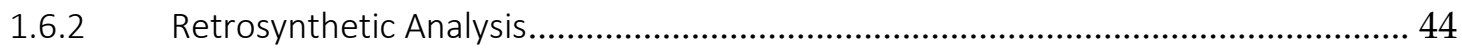

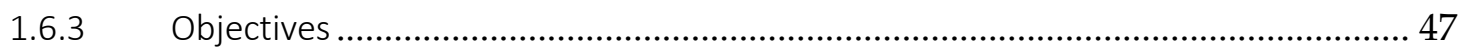

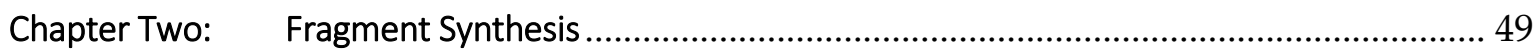

2.1 Synthesis of C17-C23 (Diene) Fragment .................................................................. 49

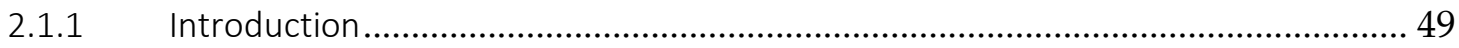

2.1.2 Strategy One: Reductive Furan Ring Opening Route ............................................ 54

2.1.3 Strategy Two: $\alpha, \beta$-Unsaturated $\delta$-Lactone Ring-Opening Elimination Route............ 59

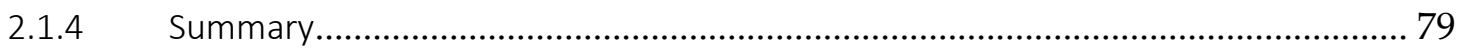

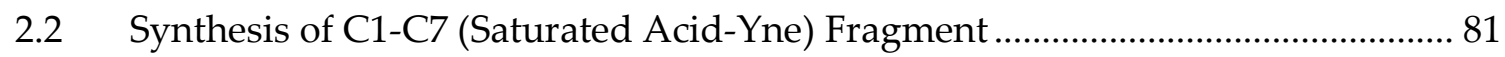

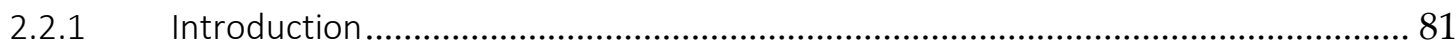

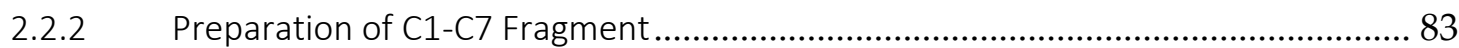


2.2.3 Summary .85

2.3 Synthesis of C8-C10 (Tri-Substituted Propane) Fragment.......................................... 86

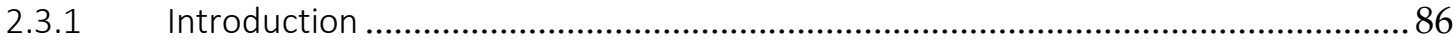

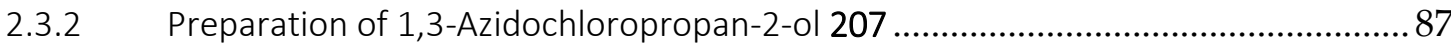

2.3.3 Preparation of 1,3-Azido(phenyltetrazole)propan-2-ol 208....................................... 89

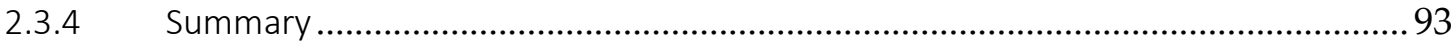

2.4 Synthesis of C11-C16 (Conjugated Side Chain) Fragment ......................................93

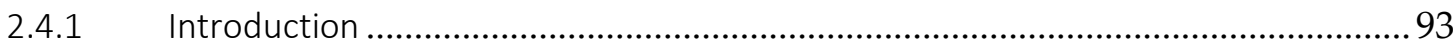

2.4.2 Preparation of C11-C16 Fragment 213 …………............................................... 95

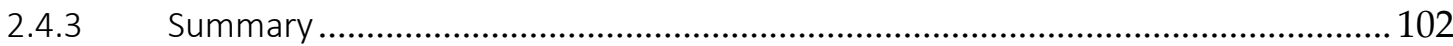

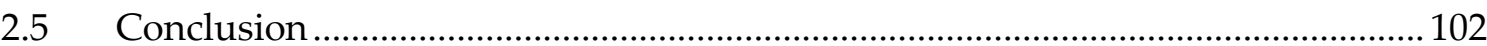

Chapter Three: Fragment Coupling and the Synthesis of Pateamine Analogue ........................ 105

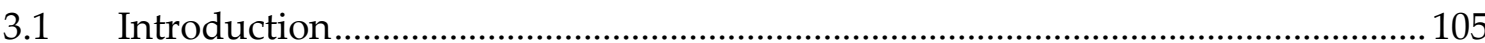

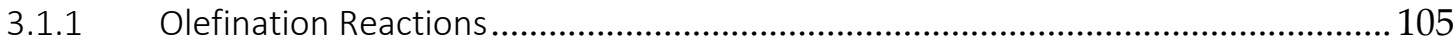

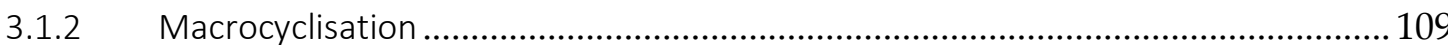

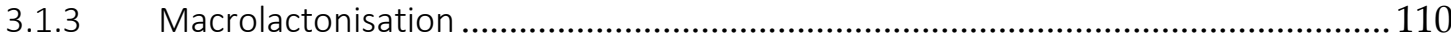

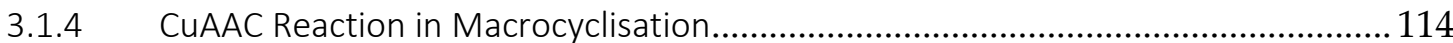

3.2 Fragment Coupling to Form a Sidechain Truncated Macrocycle ......................... 117

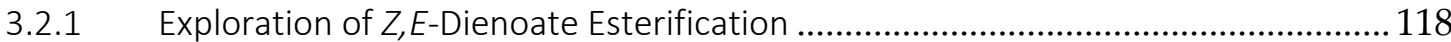

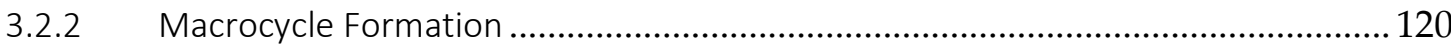

3.3 Model Studies for Side Chain Attachment............................................................... 124

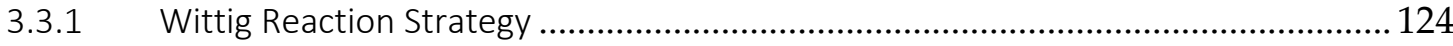

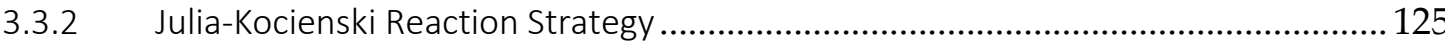

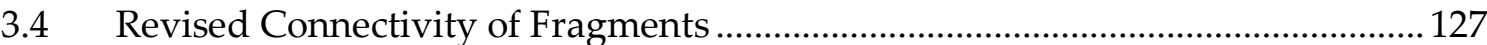

3.4.1 Preparation of Triene Sidechain Fragment …...................................................... 127

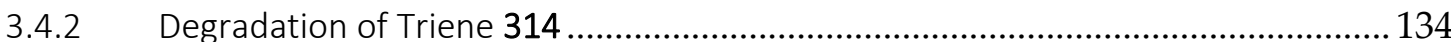

3.5 Completing Synthesis of Analogue 107................................................................ 139

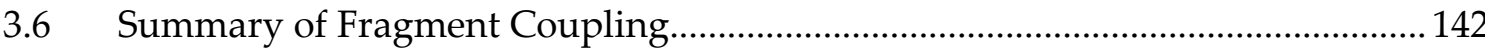

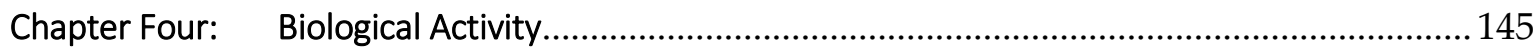

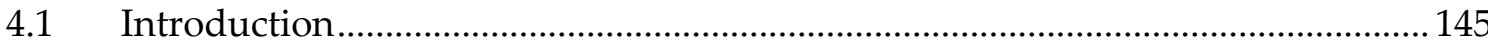

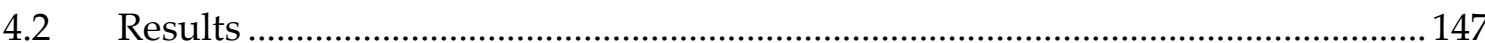




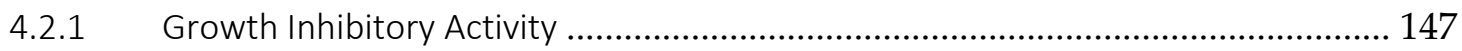

4.2.2 Searching for elF4A/Macrocycle Adducts ........................................................... 148

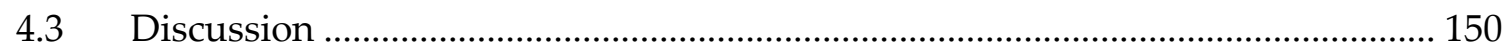

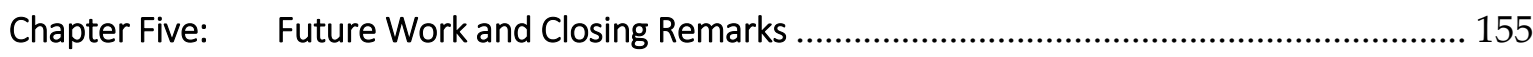

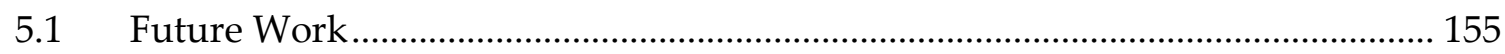

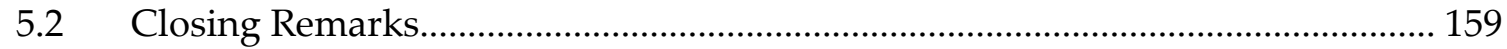

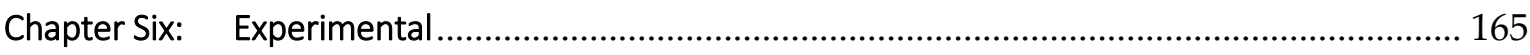

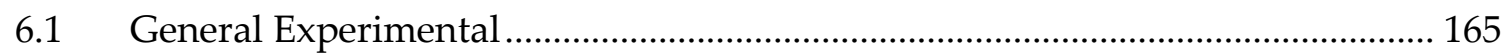

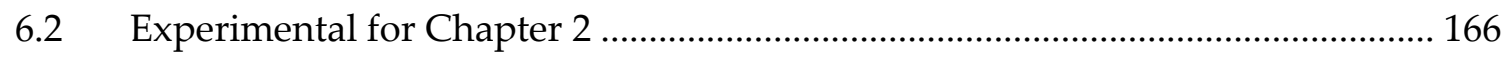

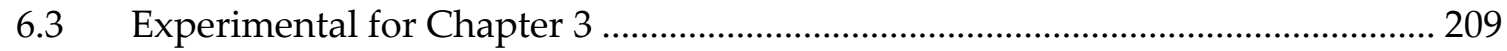

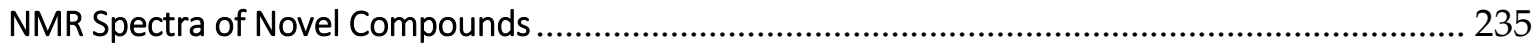

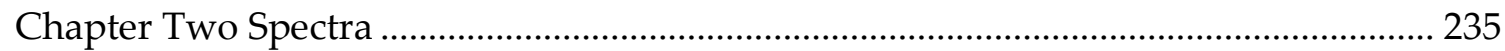

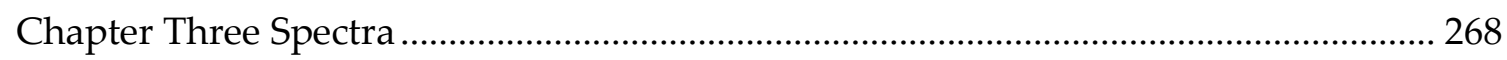

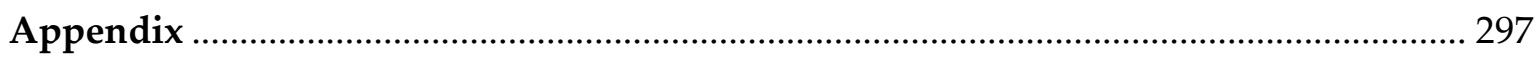

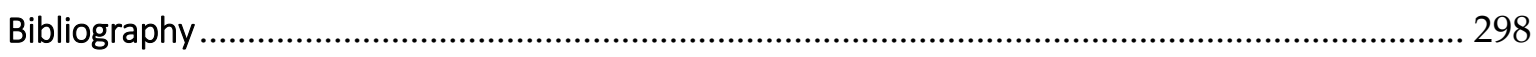




\section{Abbreviations}

18-crown-6 1,4,7,10,13,16-Hexaoxacyclooctadecane

2,6-lut 2,6-Lutidine

4A 4 Angstrom

4-PP 4-Pyrrolidinopyridine

Ac Acetyl

a.k.a Also known as

aq. Aqueous

BAIB Bis(acetoxy)iodobenzene

Bn Benzyl

Boc tert-Butyloxycarbonyl

BRSM Based on recovered starting material

CAN Ceric ammonium nitrate

cat. Catalytic

cf. confer ("compare to")

CSA Camphor-10-sulfonic acid

Cy Cyclohexyl

DCE 1,2-Dichloroethane

DCM Dichloromethane

DDQ 2,3-Dichloro-5,6-dicyano-1,4-benzoquinone

DEAD Diethyl azodicarboxylate

DIAD Diisopropyl azodicarboxylate

DIBAL-H Diisobutylaluminium hydride

DIPEA Diisopropylethylamine

DMAP 4-(N,N-Dimethylamino)pyridine

DMF $\quad N, N$-Dimethylformamide

DMSO Dimethylsulfoxide

EDCI $\quad N$-(3-Dimethylaminopropyl)- $N^{\prime}$-ethylcarbodiimide hydrochloride

e.g. exempli gratia ("for example")

Et Ethyl

EtOAc Ethyl acetate 


\begin{tabular}{|c|c|}
\hline gem- & Geminal \\
\hline $\mathrm{h}$ & Hour(s) \\
\hline $\mathrm{IC}_{50}$ & Inhibitory concentration for $50 \%$ effect \\
\hline i.e. & id est ("that is") \\
\hline imid. & Imidazole \\
\hline IR & Infrared \\
\hline KHMDS & Potassium hexamethyldisilazide \\
\hline LDA & Lithium diisopropylamide \\
\hline LiHMDS & Lithium bis(trimethylsilyl)amide \\
\hline M & Molar \\
\hline $\mathrm{Me}$ & Methyl \\
\hline MOM & Methoxymethyl \\
\hline NBS & $N$-Bromosuccinimide \\
\hline NMD & Nonsense-mediated decay \\
\hline NMR & Nuclear magnetic resonance \\
\hline -OTf & Trifluoromethanesulfonate (triflate) \\
\hline$-\mathrm{OTr}$ & Triphenylmethyl ether (trityl) \\
\hline $\mathrm{Pg}$ & Unspecified protecting group \\
\hline $\mathrm{Ph}$ & Phenyl \\
\hline PMB & para-Methoxybenzyl \\
\hline PMP & para-Methoxyphenyl \\
\hline $\operatorname{Pr}$ & Propyl \\
\hline PPTS & Pyridinium para-toluenesulfonate \\
\hline PTSA & para-Toluenesulfonic acid \\
\hline PT & Phenyl tetrazole \\
\hline pyr. & Pyridine \\
\hline quant. & Quantitative \\
\hline $\mathrm{R}$ & Unspecified Substituent \\
\hline $\mathrm{RCM}$ & Ring closing metathesis \\
\hline Rf & Retardation factor \\
\hline $\mathrm{rt}$ & Room temperature \\
\hline SM & Starting material \\
\hline TBA & Tetra- $n$-butylammonium \\
\hline
\end{tabular}


TBAF Tetra- $n$-butylammonium fluoride

TBAI Tetra- $n$-butylammonium iodide

TBS tert-Butyldimethylsilyl

TCBC 2,4,6-Trichlorobenzoyl chloride

TcBoc Trichloro-tert-butoxy carbamate

TEMPO 2,2,6,6-Tetramethyl-1-piperidinyloxy

THF Tetrahydrofuran

Ts para-Toluenesulfonate (tosyl)

UV Ultraviolet 


\section{Chapter 1: Introduction}

This thesis presents work on the design, synthesis and activity of simplified analogues of the marine natural product, pateamine A. This chapter begins by introducing the value of naturally sourced compounds in the discovery of medicinally beneficial therapeutic agents. Techniques and methods to design natural product analogues that have improved pharmacological properties or simplified syntheses are presented. Previous total syntheses of pateamine A will be discussed and comparisons of their approaches are examined. This is followed by an analysis of the findings to date for the biological activity of pateamine A and its analogues. Finally, the research objectives of this thesis will be presented, along with a retrosynthetic analyses of target analogues.

\subsection{Natural Products and Analogues in Drug Discovery}

As our understanding of the biochemical mechanisms of diseases increases, so too does our desire to discover new compounds to modulate these processes and ultimately provide therapeutic agents. The natural world is rich in diverse organisms that inhabit a wide range of environments. These organisms have evolved and adapted to their unique environments, producing a wide range of structurally and functionally diverse chemicals to aid in their survival. ${ }^{1}$ As a result, naturally produced chemicals from plants, microorganisms and animals represent valuable sources for leads in drug discovery. ${ }^{2}$ In addition, "natural products" by definition, are biosynthesised by their host, and therefore, it is reasonable to presume that these compounds would generally exhibit biocompatibility in other organisms (e.g. humans). Consequently, natural products are likely to have good ADME (absorption, distribution, metabolism and excretion) properties, which defines a drug's performance and pharmacological activity on the body as a whole. 
Despite the many advantages that they possess in drug discovery, natural products started to fall out of favour with big pharmaceutical companies in the last few decades due to: diminishing returns, high rediscovery rates, technology shifts, and problems in supply and screening. ${ }^{3}$ Consequently, a trend towards new technologies became popular that allowed for the random synthesis of millions of different chemical species that could be purified and screened for their biological activity. Use of combinatorial chemistry and high-throughput screening (HTS) in this way has the advantage of mass production and screening of many compounds in a relatively short time period compared with natural product isolation and total synthesis. The number of potential combinations of organic molecules that are of potential interest in drug discovery is in excess of $10^{60,4}$ based on combinations that follow Lipinski's "rule of $5^{\prime \prime} .5$ However, combinational chemistry and HTS has thus far failed to lead to high outputs of novel drugs. A scan of the small molecule drugs that were approved by the FDA over the last 30 years (1980-2010), shows that about two-thirds are naturally derived or naturally inspired (Figure 1.1). ${ }^{2}$ As a result, a resurgence in natural product-informed drug discovery is occurring. ${ }^{6}$

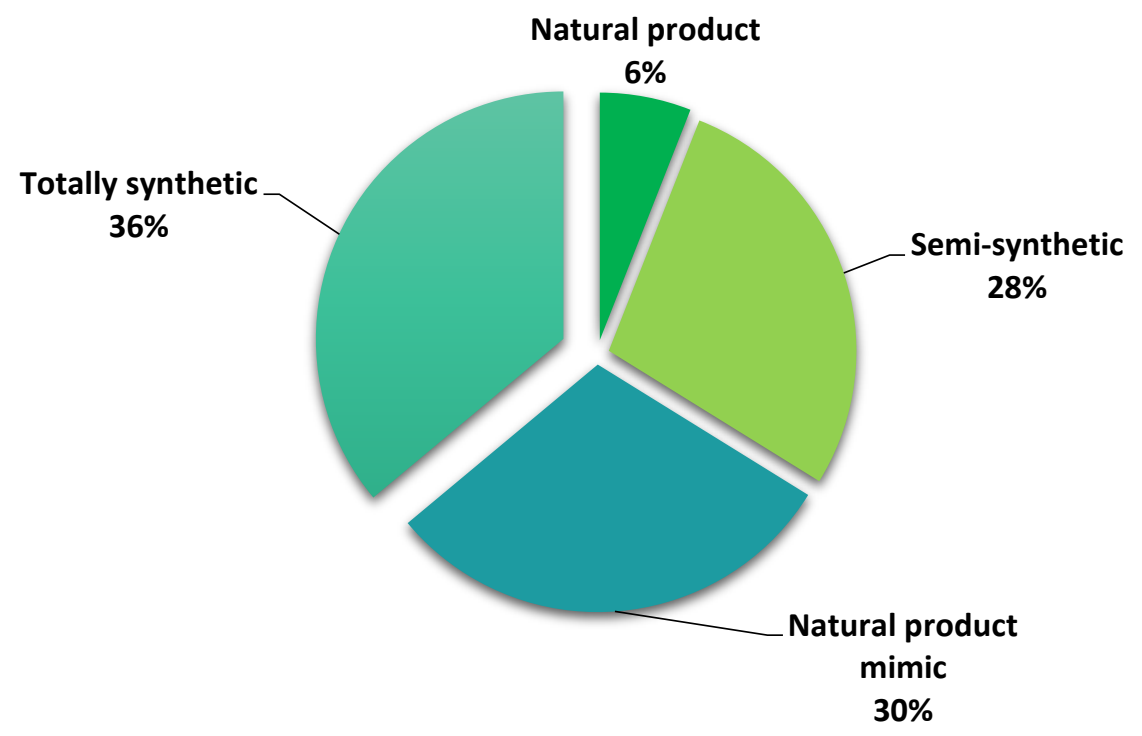

Figure 1.1 Source of small-molecule approved drugs between 1980 and 2010. (Modified from Newman, 2012) $)^{2}$

\footnotetext{
'Lipinski's "rule of 5" states that a molecule displays favourable pharmacokinetic properties in terms of absorption and distribution if at least two of the following four criteria are met: (1) Molecular weight less than $500 \mathrm{Da}$, (2) An octanol-water partition coefficient $(\log P)$ not greater than 5, (3) Not more than 10 hydrogen bond accepters, and (4) Not more than 5 hydrogen bond donors.
} 
Figure 1.1 shows that natural products have proven to be a rich source of compounds for drug discovery. However, these compounds are usually not evolved to perform the pharmacological function that is desired in humans, and therefore, an "analogue" of the natural product may be desirable. In medicinal chemistry, an analogue is a chemical that has some structural or pharmacological relationship to a known drug candidate. The preparation of analogues of a compound, either by total synthesis or by derivatising a natural product, is motivated by the desire to make improvements on its pharmacological activity, or alternatively, to simplify its chemical structure, thus facilitating large-scale synthesis.

The value of total synthetic strategies for the preparation of natural product analogues lies in their ability to access chemical structures that cannot be obtained from derivatisation of the natural product. A good example, is the work done by Danishefsky's group on analogues of epothilone B (EpoB). ${ }^{7}$ Originally extracted from the soil-dwelling grampositive bacterium Sorangium cellulosum, EpoB stabilises microtubules with a similar mode of action to the successful anticancer drug paclitaxel. However, in vivo studies revealed that EpoB is highly toxic in mice, even at subtherapeutic dosages. ${ }^{8}$ Through small perturbations of the epothilone framework, a series of analogues were synthesised that provided pharmacological improvements that could not otherwise be accessed from the natural product.

Figure 1.2 illustrates in a general sense how relatively small chemical modifications, accessed from de novo synthesis, can affect pharmacological properties. Thus, changing the epoxide of $\mathrm{EpoB}$ to an alkene (dEpoB) resulted in significant decrease in its toxicity. Dehydration at the C9 and C10 carbons (9,10-dehydro-dEpoB) further improved potency and biological stability. Furthermore, substitution of the methyl group for a trifluoromethyl group (fludelone) was found to further decrease the toxicity and broaden the therapeutic index. Substitution of the thiazole heterocycle for a isoxazole moiety (isofludelone) further increased efficacy and stability. All these analogues are currently at different stages of clinical trials as anticancer agents. 


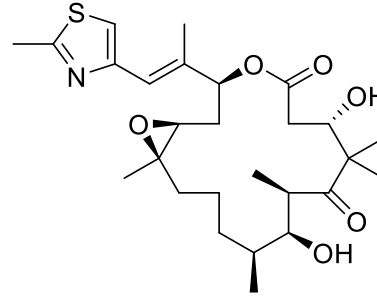

Epothilone B

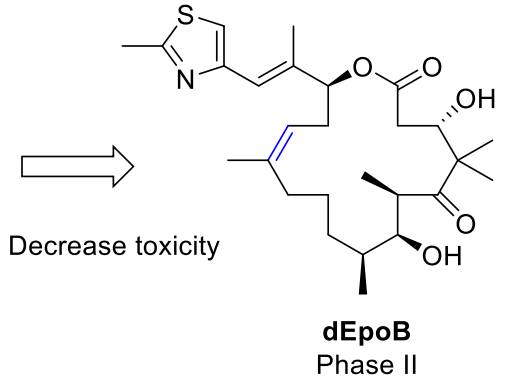

Phase II

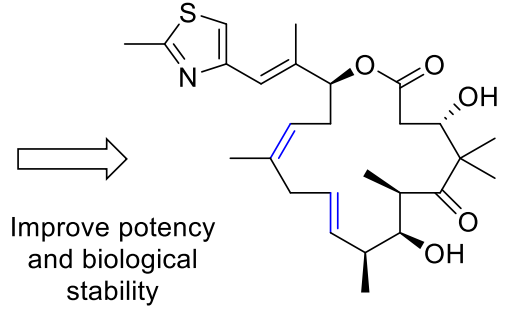

9,10-dehydro-dEpoB Phase I

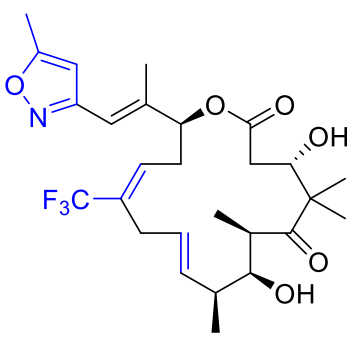

Iso-Fludelone (KOS-1803) Preclinical

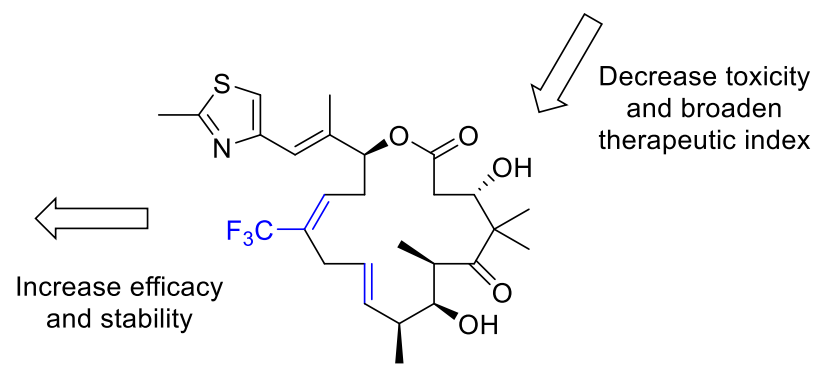

Fludelone Preclinical

Figure 1.2 Development of epothilone B analogues. ${ }^{7}$

FR901464 (1) provides another example of a natural product that, via total synthesis, has had analogues prepred that improve on its pharmacology. It was isolated from a culture broth of Pseudomonas bacteria, and found to have anti-tumour activity against a range of cancer cell lines with $\mathrm{IC}_{50}$ values of $0.6-3.4 \mathrm{nM} \cdot{ }^{9}$ It was observed that the $\beta$-epoxy hemiketal moiety was labile under physiological conditions, with a half-life of 4 hours in buffer at $\mathrm{pH} 7.4$ and $37^{\circ} \mathrm{C}$ (Figure $1.3 \mathrm{~b}$ )..$^{10}$ In an attempt to avoid this degradation, an analogue was designed and synthesised where the hydroxyl group of the hemiketal was substituted with a methyl group, hence preventing its ability to ring open, and this analogue was named meayamycin (2) (Figure 1.3a). As expected, meayamycin was significantly more stable than its natural counterpart with a half-life of 48 hours in buffer at $\mathrm{pH} 7.4$ and $37^{\circ} \mathrm{C}$ (Figure 1.3b)..$^{10}$ Interestingly, it was found that meayamycin was significantly more potent towards a breast cancer cell line (MCF-7) with an $\mathrm{IC}_{50}$ value of $10.2 \mathrm{pM}$ ( $c f$. IC 50 value of $1.1 \mathrm{nM}$ for FR901464). 
(a)
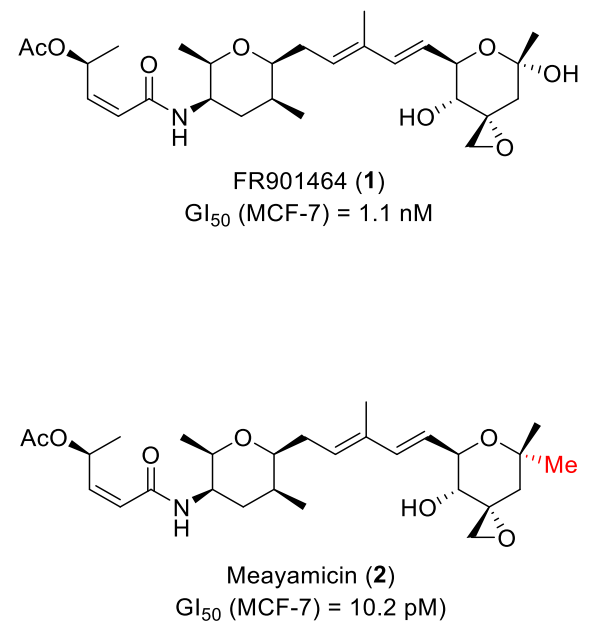

(b)

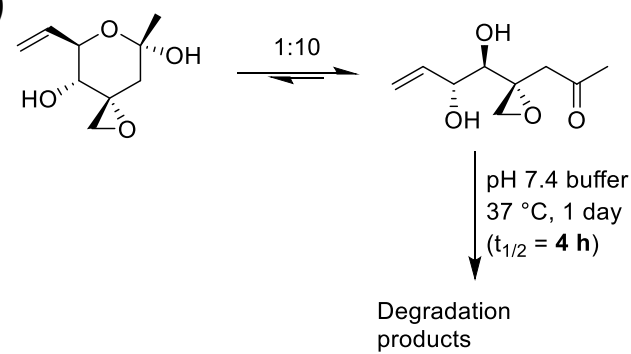

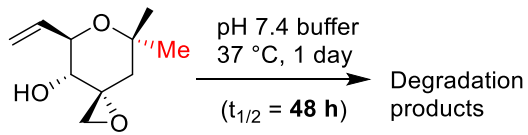

Figure 1.3 (a) FR901464 and meayamycin, with growth inhibition of MCF-7 cells in brackets, and (b) degradation studies of the $\beta$-epoxy hemiketal and gem-dimethyl moieties.

These examples highlight the value of synthetic analogues of biologically active natural products and how strategically modified structures can be accessed that improve the pharmacological properties of the natural product.

"Functional oriented synthesis" (FOS) is a synthetic strategy that aims to reduce the necessary synthetic steps to provide a functional chemical analogue. ${ }^{11}$ This is achieved by simplifying the structure relative to the natural product but maintaining the important pharmacophores (features necessary for target binding). This is exemplified by a set of three protein kinase $\mathrm{C}(\mathrm{PKC})$ modulators, namely phorbol esters, and simple functional analogues 1,2-diacyl-sn-glycerols and a designed agonist (Figure 1.4). Despite their drastically different chemical structures, all three of these compounds exhibit good binding to target PKC proteins. What they have in common is a set of hydrogen bond donors and acceptors that are a similar distance from each other that perform the function of binding into the active site of PKC proteins. ${ }^{11}$ 


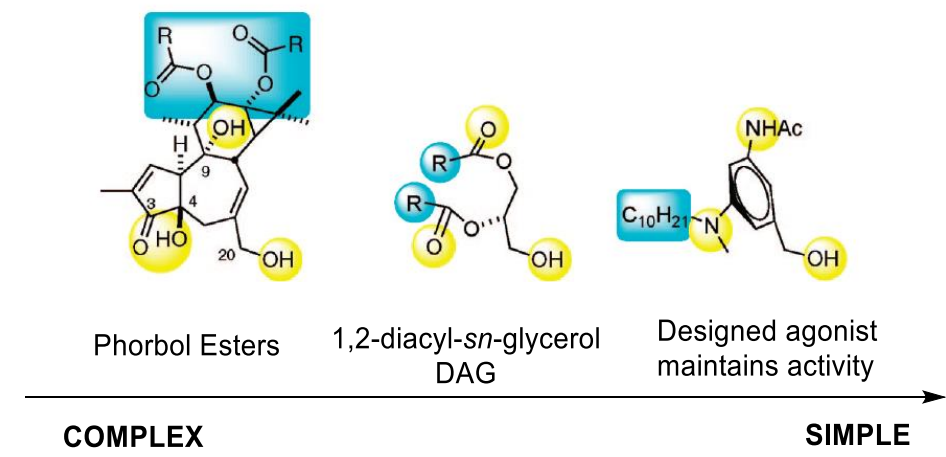

Figure 1.4 PKC modulators with key pharmacophoric moieties (yellow) and lipophilic regions (blue). ${ }^{11}$

As another example of preparing simplified analogues, bryostatin 1 (3), is a natural product produced by a bacterial symbiont of the marine bryozoan Bugula neritina. Original extracts of the marine species were found to have anti-cancer activity, and since then a unique portfolio of biological activities has been found for bryostatin 1, including modulation of apoptotic function ${ }^{12}$, reversal of multidrug resistance, ${ }^{13}$ stimulation of the immune system ${ }^{14}$ and induction of synaptogenesis (implicated in neurodegenerative disorders $) .15$

Unfortunately, bryostatin 1's scarcity in its natural source (18 grams from 14 tons of $B$. neritina, i.e. $0.00014 \%$ ), its relatively complex structure, and consequently, its lengthy synthesis (46 total steps), ${ }^{16}$ means that it is environmentally and economically impractical to obtain significant amounts of pure bryostatin 1 from its natural source or by total synthesis. Hence, a simpler structure was sought. Based on a FOS approach Wender et al. hypothesised that there was a 'spacer domain' which was necessary to control the correct orientation of the 'recognition domain' which directly interacted with the protein (Figure 1.5). ${ }^{17}$ Based on this hypothesis a range of analogues were designed and synthesised with the aim of maintaining these features. In this way, a bryostatin analogue (4) was prepared which reduced the number of steps (29 longest linear steps, cf. 46 for byostatin 1, 3) while maintaining (and enhancing) its inhibition of protein kinase C (PKC) isozymes. ${ }^{18}$ Here it can be seen that the 'spacer domain' has been stripped back in complexity to its structural skeleton, whilst the functionality of the 'recognition domain' has been maintained (Figure 1.5). Since activity is maintained, it would be plausible to suggest that the extra complexity in the 'spacer domain' is a biosynthetic accident. If readily available 
biosynthetic machinery and precursors produce a sufficiently active molecule, there is no evolutionary drive or necessity to produce a more simplified structure.

Although, it could be argued that this 'excess' complexity may indeed serve a function in its 'natural' bioactivity, for the purpose of synthesising a medicinally beneficial compound for humans, simplification allows access to the potential clinical use of these chemical structures.
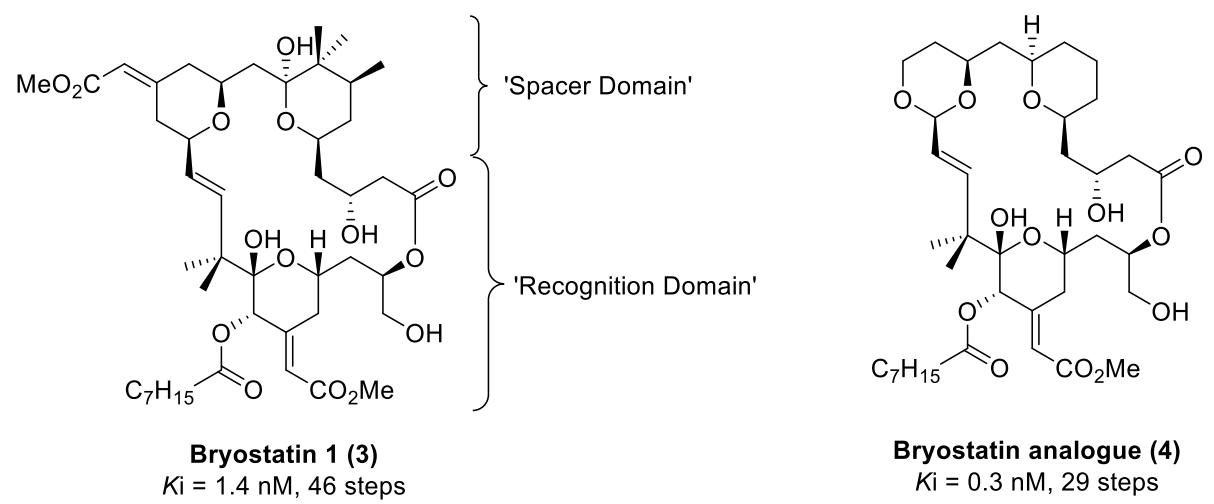

Figure 1.5 PKC modulators with key pharmacophoric atoms (yellow) and lipophilic regions (blue). ${ }^{11}$

Determining rational modifications that should be made to achieve the desired effect is a complex task, as it requires detailed knowledge of how the compound interacts with its biological target. In addition, such simplifications may change the interaction with other biomolecules leading to altered target selectivity or metabolism.

Complex natural products typically contain many stereocentres, and incorporating these stereocentres can sometimes complicate synthesis, and require expensive reagents, catalysts or starting materials. Thus, in the interest of a more cost- and step-economical synthetic route it would be desirable to exclude functionality that engenders chirality whenever this is not essential for bioactivity. For example, an analogue of laulimalide (5) (a marine sponge extract with microtubule-stabilising activity) was synthesised that lacked the C11 methyl group (6), thus removing a chiral centre, which maintained biological activity against a melanoma cell line, MDA-MB-435 (Figure 1.6). ${ }^{19}$ The synthesis of the C9-C13 fragment in laulimalide began with the relatively expensive chiral starting material, (R)-citronellic acid, and was converted in three steps to chiral aldehyde $7 .{ }^{20,21} \mathrm{In}$ 
contrast, the synthesis of the simplified C9-C13 fragment in 11-des-methyl-laulimalide began with $\delta$-valerolactone (over 15-times cheaper*), which was converted in two steps to achiral aldehyde $8 .^{19}$

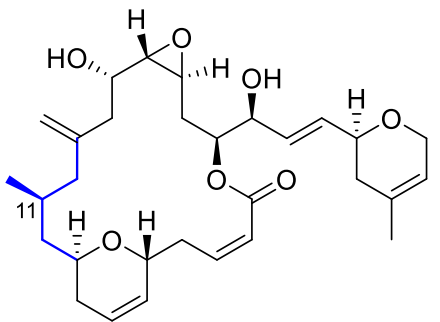

Laulimalide (5) $\mathrm{IC}_{50}(\mathrm{MDA}-\mathrm{MB}-435)=5.7 \mathrm{nM}$<smiles>Ic1ccccc1</smiles><smiles>CC(=O)CC(C)CC=O</smiles>

1) Esterification

2) i. $\mathrm{PhSeSePh}, \mathrm{H}_{2} \mathrm{O}_{2}$

ii. ${ }^{\mathrm{B}} \mathrm{BuOOH}$

3) $\mathrm{O}_{3}, \mathrm{MeOH}$ then $\mathrm{Me}_{2} \mathrm{~S}$<smiles>CC(C)=CCC[C@H](C)CC(=O)O</smiles>

(R)-citronellic acid

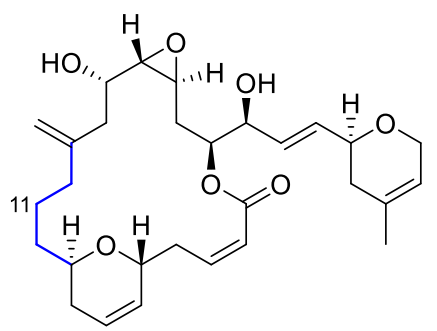

11-des-Methyl Laulimalide (6) $\mathrm{IC}_{50}(\mathrm{MDA}-\mathrm{MB}-435)=49.0 \mathrm{nM}$

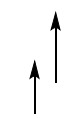<smiles>CC(=O)CCCC=O</smiles>

8 1) $\mathrm{MeOH}, \mathrm{H}^{+}$ 2) Oxidation<smiles>O=C1CCCCO1</smiles>
delta-valerolactone

Figure 1.6 Comparison of the cytotoxicity (against MDA-MB-435 cancer cells) and the preparation of the C9-C13 fragments of laulimalide and 11-des-methyl laulimalide.21,19

Methyl groups can sometimes be a logical choice when determining structural features to 'crop' for a complex target compound, as they are likely to contribute only weakly to overall protein binding, if at all, via van der Waals interactions, with dissociation energy < $4 \mathrm{~kJ} / \mathrm{mol}$. In comparison, hydrogen bonds through functional groups such as alcohols and carbonyls have dissociation energies of typically $5-21 \mathrm{~kJ} / \mathrm{mol} .{ }^{22}$ 
Additional examples of natural products that have been synthesised that lack a methyl group, include analogues of the natural products (-)-dictyostatin (9) and (+)discodermolide (10) (Figure 1.7). 16-des-methyl dictyostatin (11), ${ }^{23}$ and both the 16-desmethyl (12) ${ }^{23}$ and 14-des-methyl (13) ${ }^{24}$ analogues of discodermolide exhibit very similar biological activity to the natural products.

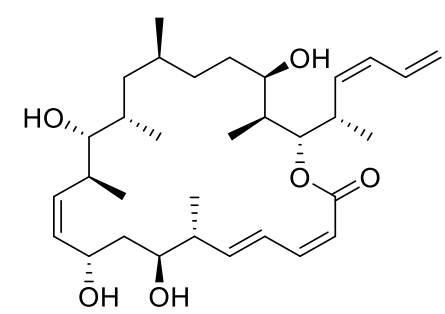

(-)-Dictyostatin (9) $\mathrm{IC}_{50}(1 \mathrm{~A} 9)=0.69 \mathrm{nM}$<smiles>C=C/C=C\[C@H](C)C(OC(N)=O)C(C)C(O)C(C)C/C(C)=C\C(C)[C@@H](O)[C@@H](C)/C=C\[C@H](O)C[C@H]1OC(=O)[C@H](C)[C@@H](O)[C@H]1C</smiles>

(+)-Discodermolide (10) $\mathrm{IC}_{50}(\mathrm{MG63})=6 \mathrm{nM}$ $\mathrm{IC}_{50}(\mathrm{MCF}-7)=28 \mathrm{nM}$

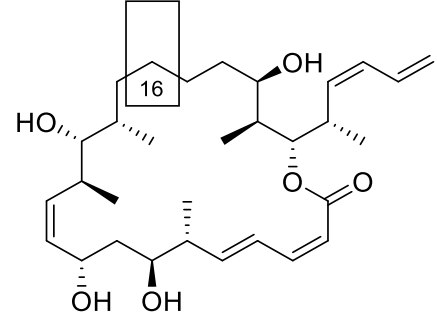

(-)-16-des-Methyl Dictyostatin (11) $\mathrm{IC}_{50}(1 \mathrm{~A} 9)=0.41 \mathrm{nM}$

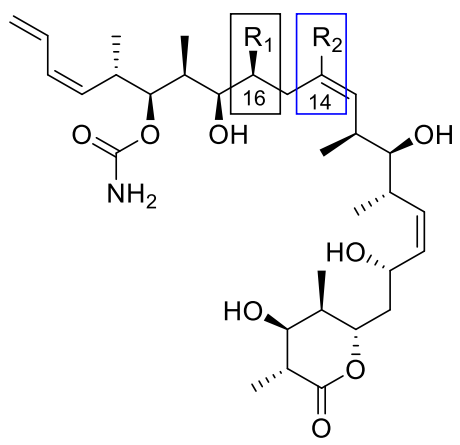

(+)-14/16-des-Methyl Discodermolide $R_{1}=H, R_{2}=M e: I C_{50}(M G 63)=10 \mathrm{nM}$ (12) $\mathrm{R}_{1}=\mathrm{Me}, \mathrm{R}_{2}=\mathrm{H}: \mathrm{IC}_{50}$ (MCF-7) $=46 \mathrm{nM}$ (13)

Figure 1.7 Comparison of the bioactivity of natural products and synthesised des-methyl analogues.

In summary, natural products are good sources of chemically and structurally diverse chemicals that are invaluable for drug discovery. Analogues of natural products allow us to fine-tune the biological activity of the original natural product to improve its potency or pharmacology. Simplification of complex structures makes preparation more practical and economical, and can often be achieved with limited loss of activity. Examples of desmethyl analogues that maintain good biological activity have been described. 


\subsection{Pateamine A Discovery}

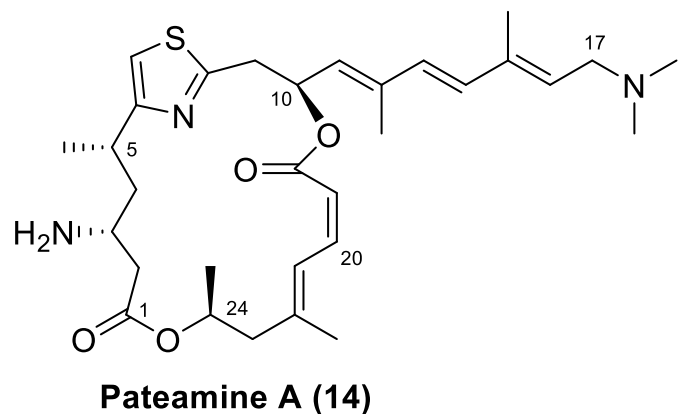

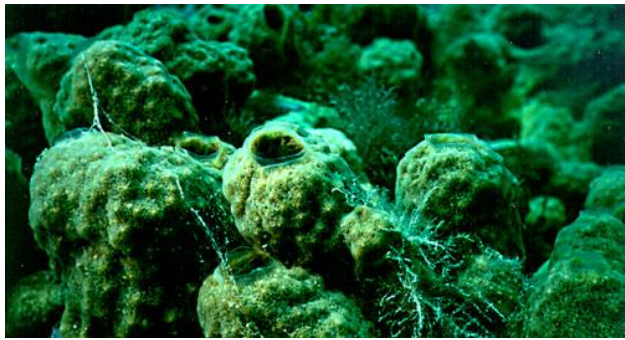

Mycale hentscheli

Figure 1.8 Structure of pateamine A and a photo of the marine sponge it was extracted from (Mycale hentscheli). ${ }^{+}$

Pateamine A (14) is a secondary metabolite that was isolated from the marine sponge Mycale hentscheli by Northcote et al. and its structure and preliminary bioactivity reported in 1991.25 The sponge is endemic to New Zealand waters and has been found as far south as Stewart Island/Rakiura and as far north as Kerikeri. However, pateamine A has only been extracted from the sponges found in the waters of Fiordland on the west coast of the South Island and, in much smaller amounts, in the Marlborough Sounds. ${ }^{26}$

The original isolation was directed by toxicity of sponge extracts against P388 leukemia cell lines, leading to the discovery that pateamine A was a potent cytotoxin to these fast growing cells, with an $\mathrm{IC}_{50}$ of $0.27 \mathrm{nM}$. In contrast, pateamine A was shown to have little toxicity against a BSC-1 cell line under static growth, with an IC 50 of $540 \mathrm{nM} \cdot{ }^{25}$ Consequently, this selectivity towards cancer cells has made pateamine an attractive candidate as an anti-cancer agent. Pateamine A was also shown to be a potent anti-viral and anti-fungal agent (see Section 1.4 for a more comprehensive discussion of biological activity).

It is also worth noting that other variants with very slight differences were extracted in smaller amounts, designated pateamine B and C (Figure 1.9). However, there was not sufficient material to unambiguously confirm these structures. ${ }^{27}$ The name 'pateamine' will be used for the remainder of this thesis to represent pateamine A. 


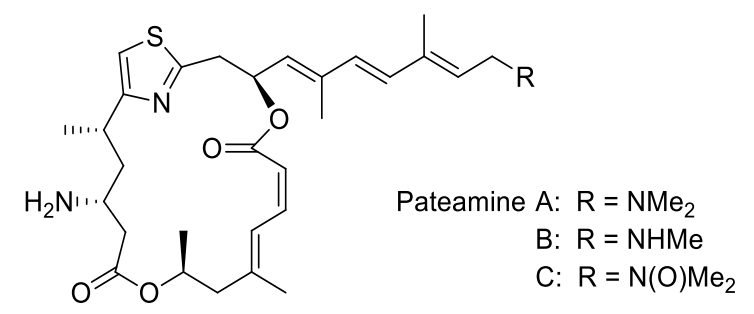

Figure 1.9 Structures of pateamine A, and proposed for pateamine B and C.

Two other families of biologically active compounds with potent cytotoxicity have also been extracted from the same marine sponge, namely the pelorusides and the mycalamides (Figure 1.10). Peloruside A was found to be a microtubule stabiliser with potency and mode of action similar to paclitaxel and the epothilones. ${ }^{26}$ The mycalamides are potent inhibitors of protein synthesis and were found to cause apoptosis. ${ }^{28}$
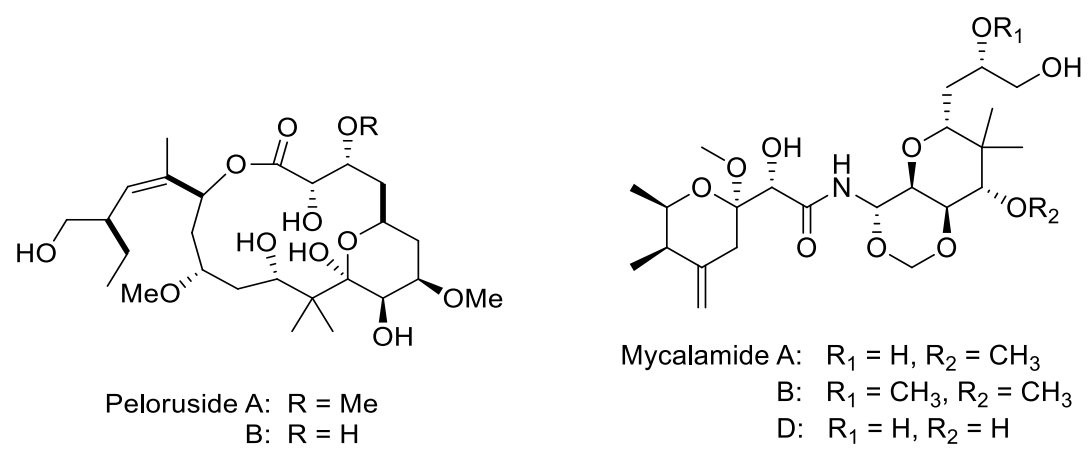

Figure 1.10 Structures of selected pelorusides and the mycalamides.

\subsection{Pateamine and Analogue Syntheses}

The high potency of pateamine and its selective activity towards cancer cell lines resulted in international interest. ${ }^{29}$ However, since pateamine could not be easily obtained from its natural source in adequate amounts for clinical testing, chemical synthesis was required to further examine its potential as a drug candidate. 
In 1998, seven years after pateamine was first reported, its total synthesis was reported by Romo's group at Texas A\&M University, USA. ${ }^{30}$ This was followed by a second total synthesis by Pattenden's group, reported in 2000, at the University of Nottingham, United Kingdom.

\subsubsection{Romo's Total Synthesis}

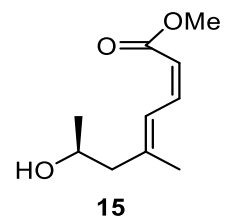

The first reported synthetic work on pateamine (14) was by Rzasa and Romo in 1995, when the synthesis of the C18-C24 section, 15, of the natural product was disclosed. ${ }^{31}$ Their first attempt, as outlined in Scheme 1.1 (blue arrows), utilised a Negishi tandem carboalumination-cross coupling as the key step to couple alkyne $\mathbf{1 7}$ and a (Z)bromoacrylate 18. However, this resulted in the undesired isomerisation to the more energetically favoured $(E, E)$-diene 19. To avoid isomerisation of the diene, a strategy involving a Sonogashira coupling was employed between propargyl alcohol and the vinyl iodide 20, derived from alkyne 17 by zirconium catalysed carboalumination. The resultant alcohol 21 was subjected to a two-step oxidation to afford carboxylic acid 22 and treatment with diazomethane to install the methyl ester. Desilylation with HF and reduction of the alkyne with Lindlars catalyst provided Z,E-dienoic ester 15. Preparation of Mosher's esters derived from 15 confirmed the stereochemical assignment at C24 of pateamine. 


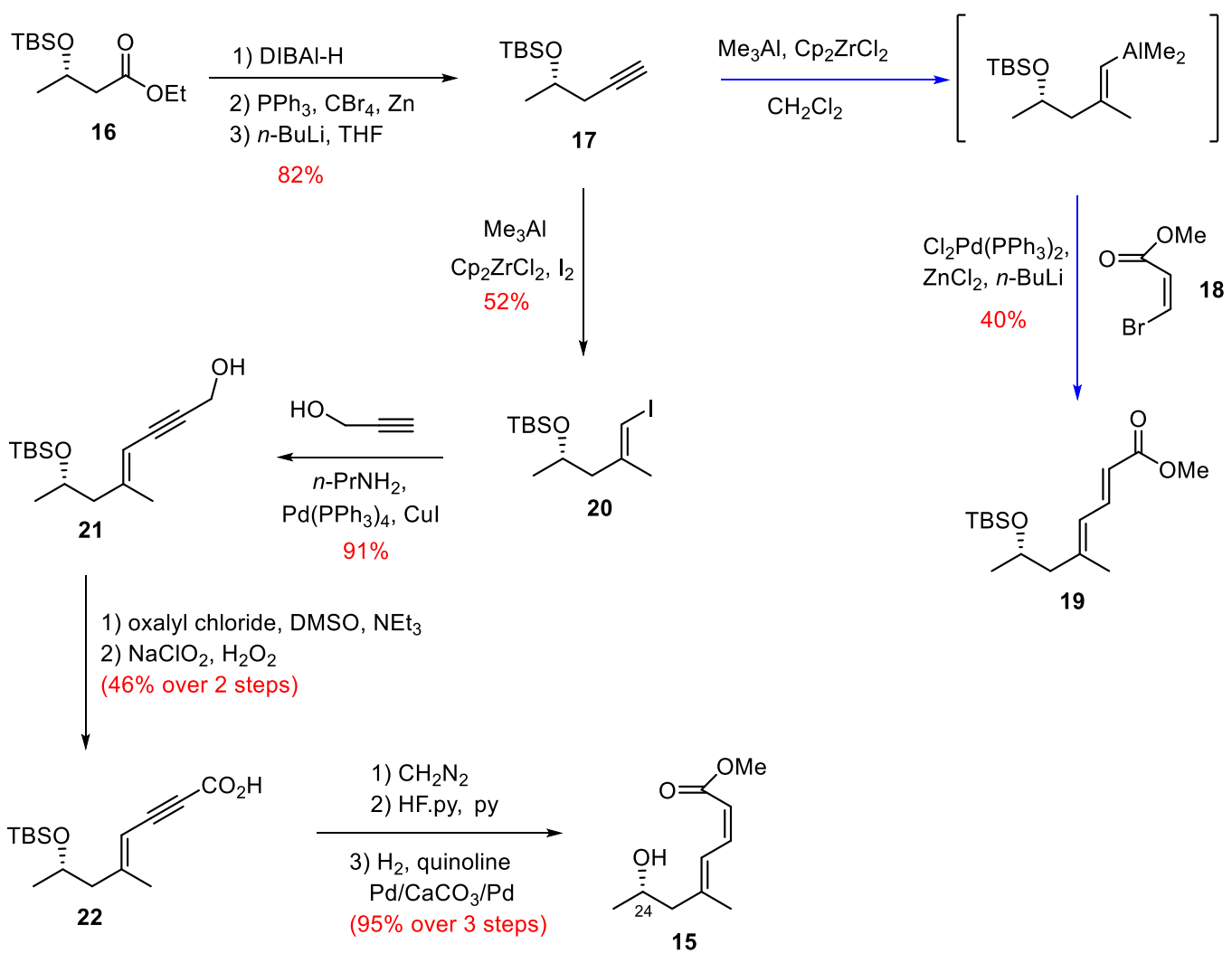

Scheme 1.1 Preparation of C18-C24 fragment of pateamine.

With the C18-C24 section of pateamine synthesised, the Romo group continued on with the synthesis, and in 1998 reported the first total synthesis. ${ }^{30}$ Their strategy involved the synthesis of three major fragments $(\mathbf{2 2}, 23$ and 24$)$ which could be connected to afford the full structure of the natural product (Figure 1.11).

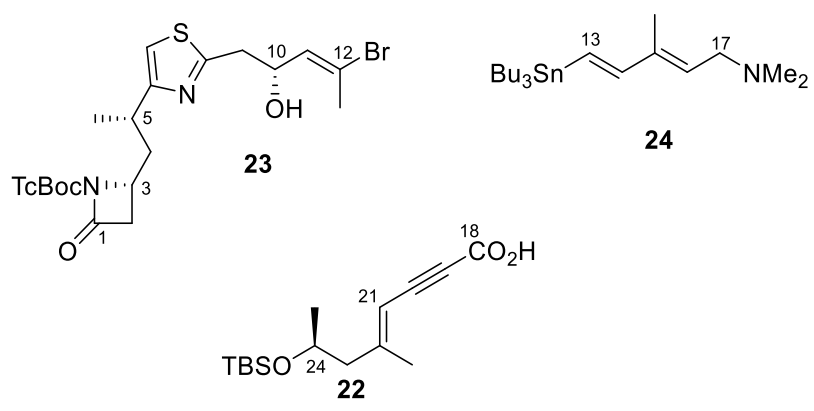

Figure 1.11 Major fragments in Romo's total synthesis. 
The C18-C24 fragment was synthesised as described for the hydroxydienoate 15. The preparation of the ester (S)-16 was prepared using a modified Noyori hydrogenation of ethyl acetoacetate (Scheme 1.2), which could be performed on a larger scale compared with the yeast reduction method previously used, and gave better yields and enantiomeric purity ( $94 \%$ ee vs $84-90 \%$ ee).

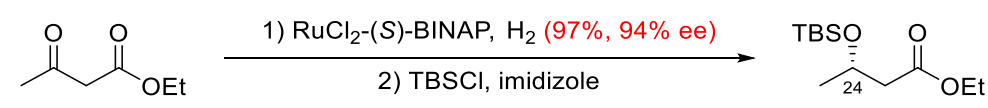

16

Scheme 1.2 Setting stereochemistry at C24 in the preparation of compound 3.

The hydrogenation of the alkyne 22 with Lindlar's catalyst (vide supra, Scheme 1.1) to give the $Z, E$-diene was performed during the final stages of the total synthesis to avoid the potential for isomerisation to the $E, E$-stereoisomer.

\section{Fragment C1-C12 (23)}

The synthesis of the C1-C12 fragment began with a Nagao aldol reaction between the $\mathrm{N}$ acetylthiazolidinethione 25 and the known aldehyde 26, via the presumed complex 27 (Scheme 1.3). This reaction produced the stereochemistry at the C10 position (natural product numbering) in a highly stereoselective fashion (>19:1 dr). Although this gives the non-natural configuration, it is inverted during the Mitsunobu coupling with fragment 22. Silyl protection of the alcohol and transamidation with gaseous ammonia gave amide $\mathbf{3 0}$. Conversion to thioamide 31 was achieved with Belleau's reagent. Initial attempts with Lawesson's reagent resulted in the formation of a volatile nitrile and elimination. Formation of the thiazole 32 was achieved using the modified Hantzsch conditions reported by Aguilar and Meyers. ${ }^{32}$ Reduction of the thiazole ester 32 to the aldehyde 33 with DIBAL-H was followed by a Horner-Wadsworth-Emmons coupling with the oxazolidinone-containing phosphonate 34 to afford compound 35 . Treatment with an 
excess of MeMgBr and an equivalent amount of CuBr.DMS according to the conditions of Hruby et al., ${ }^{33}$ led to the methylated product 37 in reasonably good diastereoselectivity (6.4:1 dr). This selectivity is proposed to occur with initial formation of a $\mathrm{Cu}(\mathrm{I}) \pi$-olefin $\mathrm{Mg}$ (II)-complex and addition of the methyl group from the opposite side to the phenyl group of the auxiliary (36). Transamidation of the amide auxiliary with $\mathrm{N}, \mathrm{O}-$ dimethylhydroxylamine hydrochloride and trimethyl aluminium to give the Weinreb amide 38 was followed by reduction with DIBAL-H to provide aldehyde 39. A crystalline by-product (40) was obtained during the formation of the Weinreb amide which was used to verify the stereochemistry at $\mathrm{C} 5$ and $\mathrm{C} 10$ (pateamine numbering) by $\mathrm{X}$-ray crystallography.

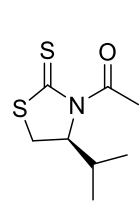

25
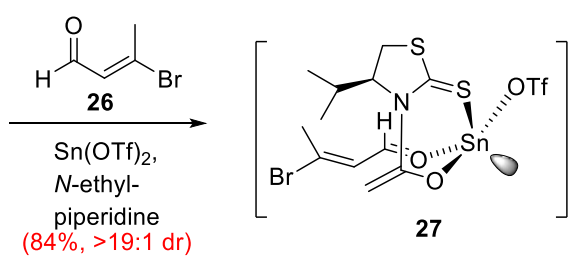

27

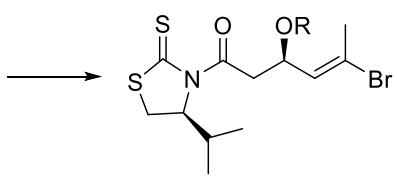

TIPSOTf 28: $\mathrm{R}=\mathrm{H}$

29: $R=$ TIPS $(99 \%)$
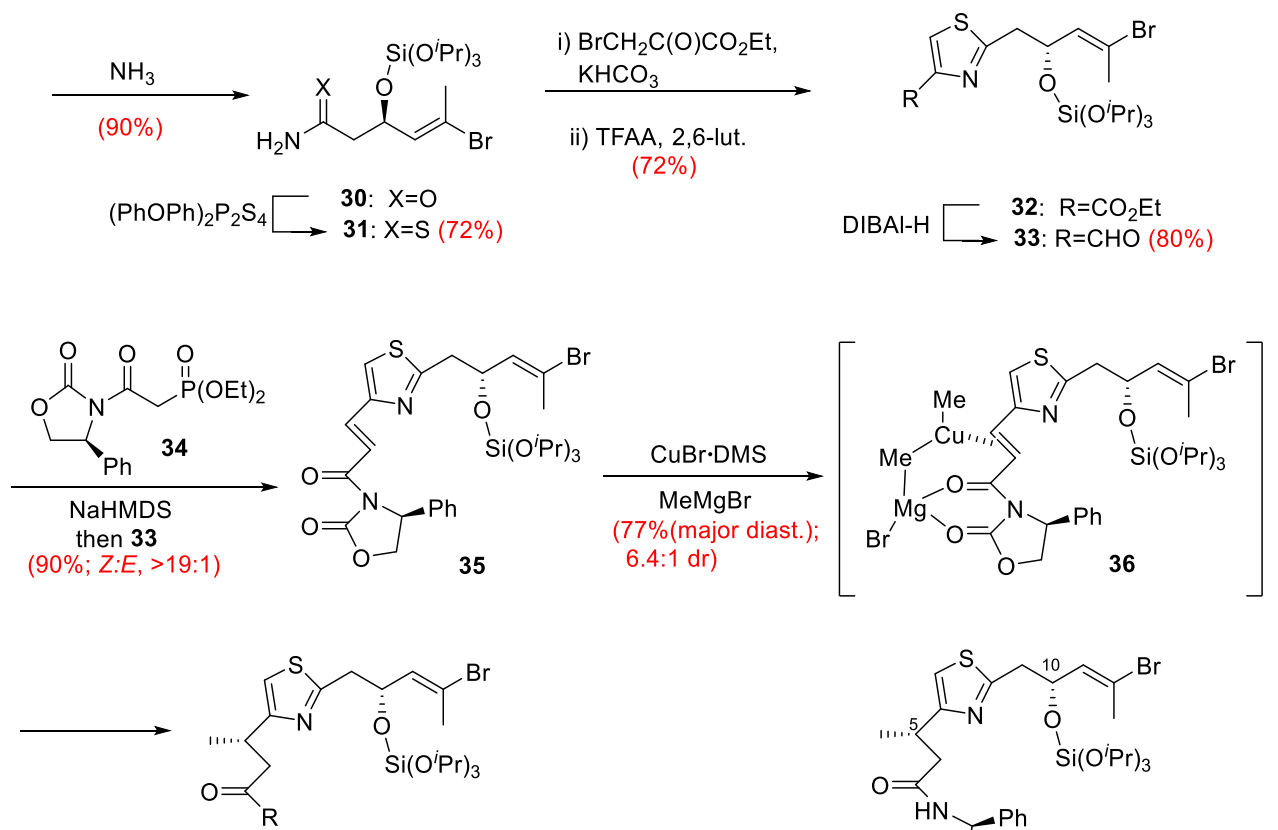

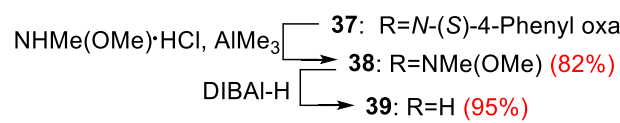

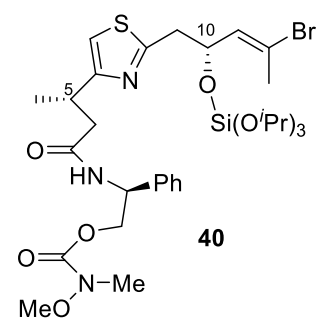

Scheme 1.3 Setting stereochemistry at C5 and C10 in the preparation of compound 39. 
Aldehyde 39 was reacted with $N$-acetylthiazolidinethione 25 in a second Nagao acetate aldol reaction providing 41 with good selectivity (>19:1 dr) for the product with inverse stereochemistry (relative to pateamine) at C3 (Scheme 1.4). The auxiliary was then removed by transamidation to the $N$-benzyloxy amide 42 followed by the Miller intramolecular Mitsunobu reaction to provide the $\beta$-lactam 43 with inversion at C3 to give the correct stereochemistry. The benzoyloxy group on the $\beta$-lactam nitrogen (43) was then removed with $\mathrm{SmI}_{2}$ in the presence of $\mathrm{H}_{2} \mathrm{O}$ and subsequent reprotection with a TcBoc group using 1,1-dimethyl-2,2,2-trichloroethyl chloroformate and DMAP to provide 45. This swapping of the tert-butyl carbamate for the benzoyloxy group was expected to allow a more compatible deprotection step with late intermediates. Deprotection of the silyl ether at C10 with TBAF afforded completed major section 23.
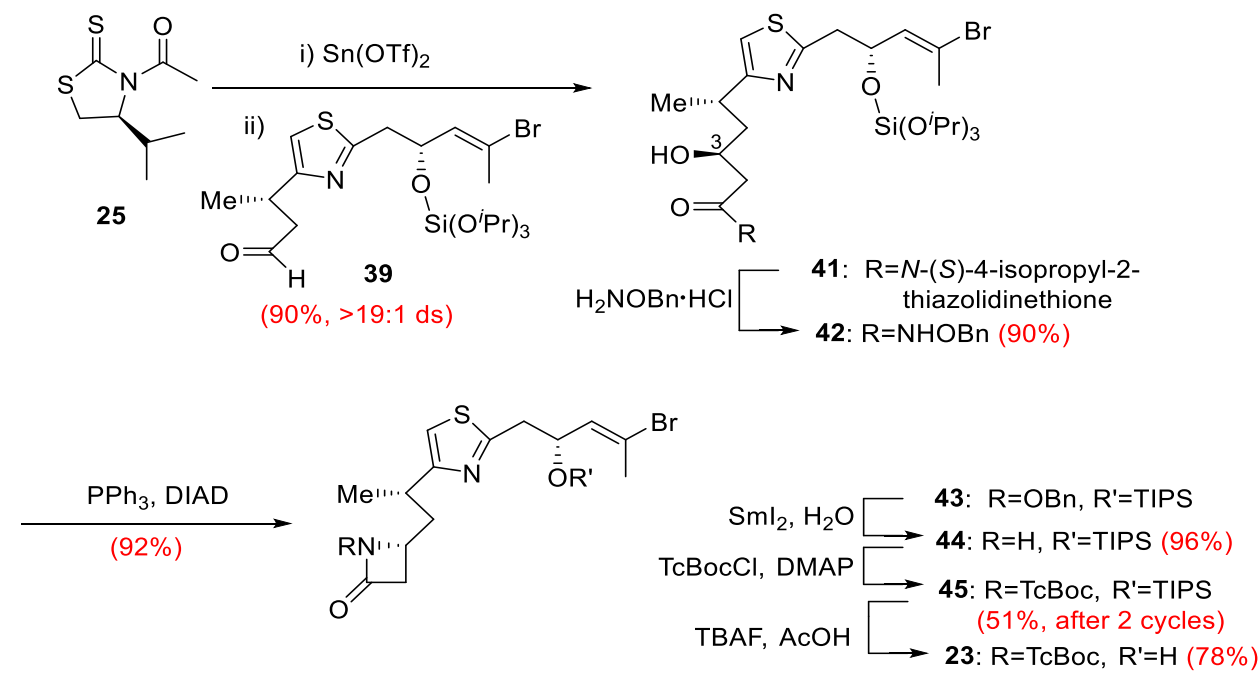

Scheme 1.4 Stereoselective installation of C3 in the preparation of major fragment 23.

\section{Fragment $113-17$ (24)}

The synthesis of the dienyl amino stannane 24 was accomplished in two steps from the known enyne alcohol 46 (Scheme 1.5). The substitution of the alcohol for dimethylamine was achieved in a one-pot reaction by tosylation of the alcohol followed by bubbling dimethylamine through the reaction mixture to provide the amine 47. Stannylcupration 
and quenching at low temperature according to the method of Aksela and Oehlschlager ${ }^{34}$ provided the desired dienyl stannane $\mathbf{2 4}$.

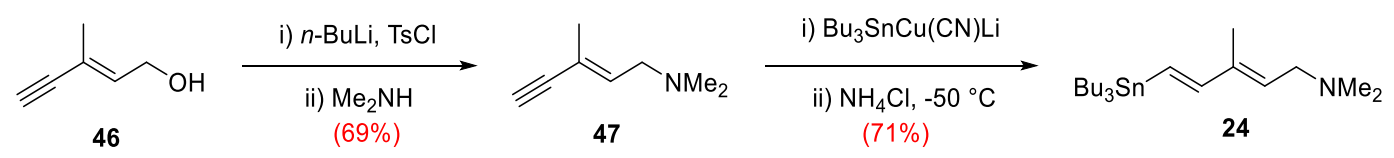

Scheme 1.5 Preparation of sidechain fragment 24.

\section{Coupling the fragments}

Coupling the fragments $\mathbf{2 3}$ and $\mathbf{2 2}$ was achieved with a Mitstunobu reaction (Scheme 1.6), which inverted the stereochemistry at C10 to give the correct (S)-configuration 48 . Desilylation with HF-pyridine was followed by $\beta$-lactam-opening macrolactonisation using a modification of Palomo's conditions ${ }^{35}$ to provide the 19-membered dilactone macrocycle 50. The alkyne was then reduced with Lindlar's catalyst to provide the E,Zdiene 51. The dienyl stannane 24 was attached via a Stille cross coupling, and macrolactone 52 was subsequently treated with a $\mathrm{Cd}-\mathrm{Pb}$ couple to remove the $\mathrm{TcBoc}$ group and provide pateamine $\mathbf{1 4}$. 

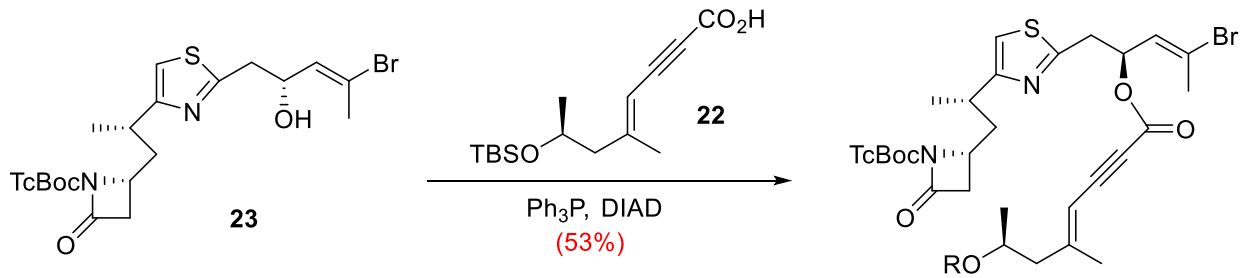

$\begin{array}{ll}H F \cdot p y & \text { 48: } \mathrm{R}=\mathrm{TBS} \\ & \text { 49: } \mathrm{R}=\mathrm{H}(78 \%)\end{array}$
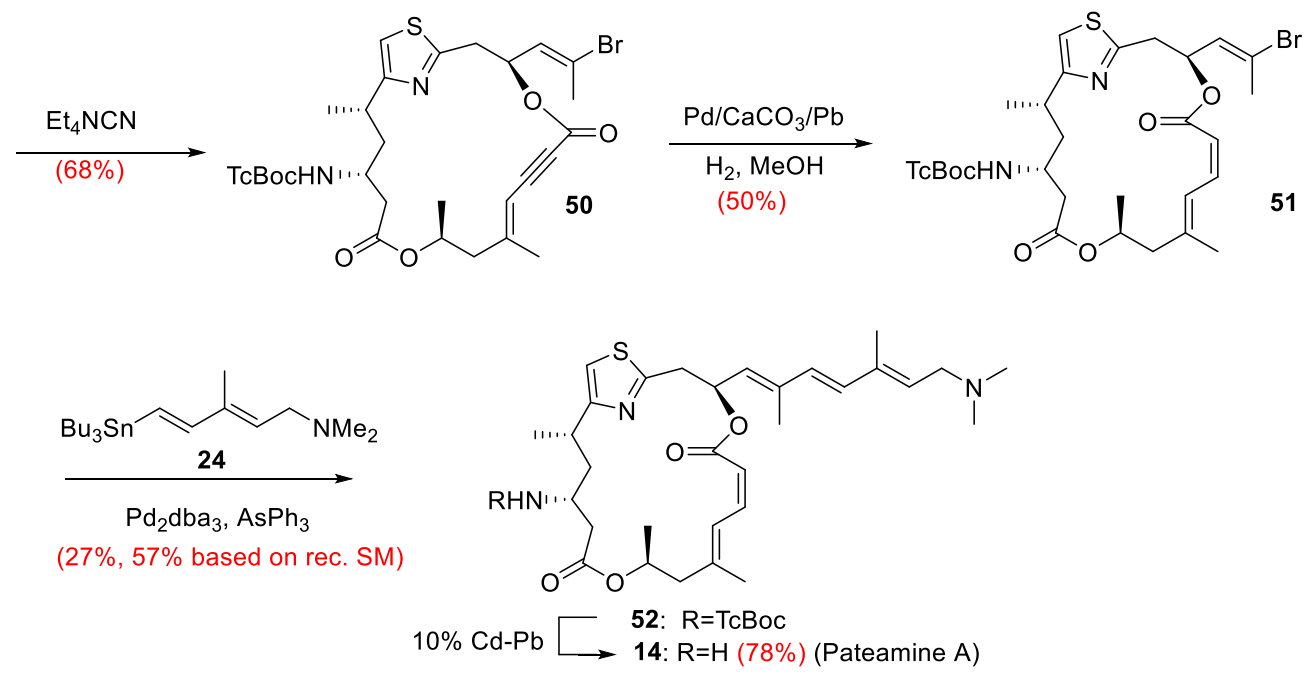

Scheme 1.6 Coupling of major fragments 22,23 and 24 to complete the synthesis of pateamine.

\subsubsection{Pattenden's Total Synthesis}

Concurrently to the Romo group working on the synthesis of pateamine, the Pattenden group at the University of Nottingham was also pursuing the same target. In 1996 they reported the synthesis of the 19-membered bis-lactone core ${ }^{36}$ (Figure 1.12) and in 2000 their total synthesis of pateamine was published. ${ }^{37}$

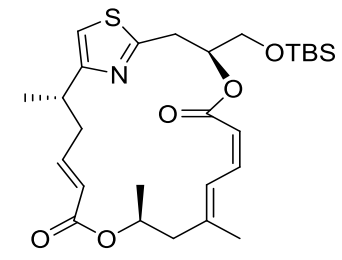

Figure 1.12 Bis-lactone core synthesised by Pattenden et al..$^{36}$ 
The Pattenden group's total synthetic strategy for pateamine involved the initial formation of the bis-lactone core, by the coupling of major fragment 53 with fragments $\mathbf{5 4}$ and 55 (Figure 1.13). This was followed by extension of the conjugated chain with fragment 56 followed by 57 .
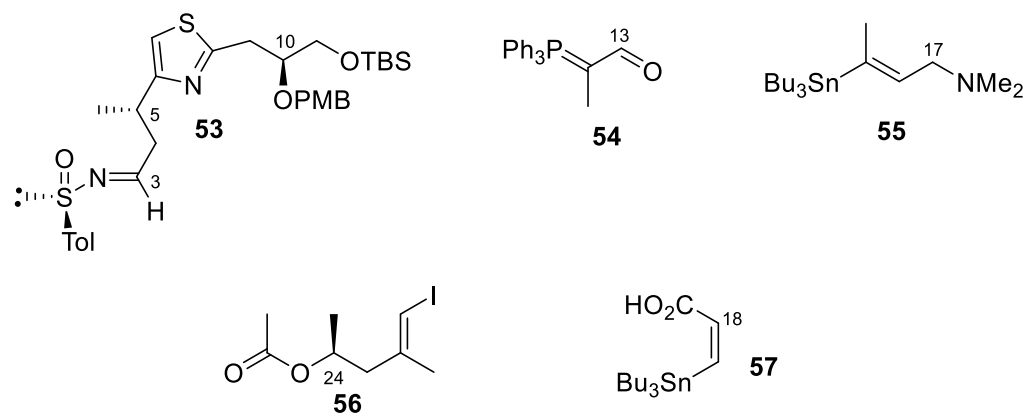

Figure 1.13 Major fragments in Pattenden's total synthesis.

\section{Fragment C3-C11 (53)}

The synthesis of fragment C3-C11 (53) was achieved by the coupling of compounds 64 and 69. Synthesis of compound 64 began with dimethyl L-malate 58 (which provided the stereocentre at C10) (Scheme 1.7). Selective reduction of the $\alpha$-methyl ester to alcohol 59 using borane-dimethyl sulfide complex and sodium borohydride, followed by sequential selective protections to give $\mathbf{6 0}$ then $\mathbf{6 1}$. Saponification of ester $\mathbf{6 1}$ provided carboxylic acid 62 which was subsequently transformed into the amide 63 via mixed anhydride formation and nucleophilic attack with ammonium hydroxide. Treatment with Lawesson's reagent provided the thioamide 64 . 

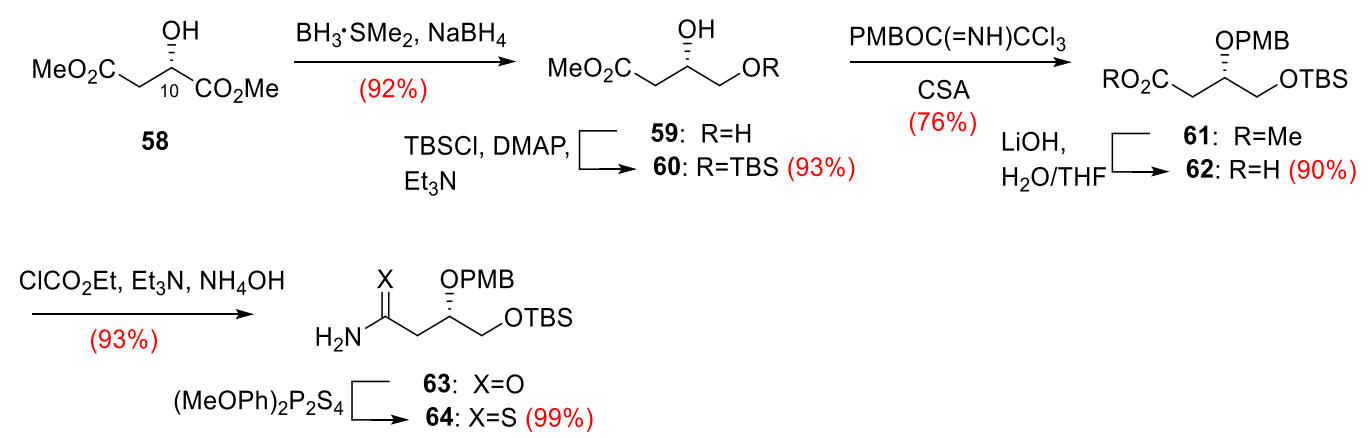

Scheme 1.7 Introduction of stereochemistry at C10 in the preparation of compound $\mathbf{6 4 .}$

Synthesis of compound 69 started with methyl (2S)-3-hydroxy-2-methylpropionate 65 (which set the stereochemistry at C5) that was silylated to give 66 followed by treatment with $\mathrm{N}, \mathrm{O}$-dimethylhydroxylamine hydrochloride and trimethyl aluminium to give the Weinreb amide 67 (Scheme 1.8). This was then converted to the methyl ketone 68 with methylmagnesium bromide, followed by kinetically favoured bromination at the less hindered $\alpha$-position with bromine in the presence of a bulky base at low temperature to provide the $\alpha$-bromo ketone 69 .

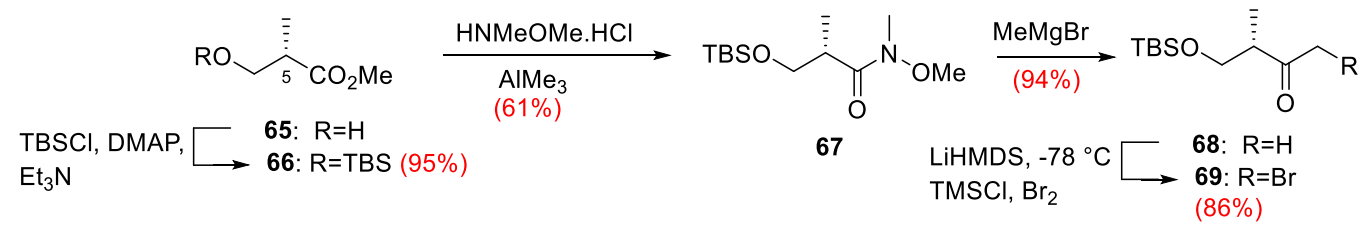

Scheme 1.8 Introduction of stereochemistry at C5 in the preparation of compound 69.

Compounds 64 and 69 were coupled using a modified Hantzsch reaction to provide the thiazole 70 (Scheme 1.9). Desilylation to afford alcohol 71 was followed by mesylation to give 72 and treatment with sodium cyanide to provide nitrile 73 . Reduction of the nitrile with DIBAL-H to afford the aldehyde 74 on hydrolytic work-up, was followed by the formation of the enantiopure sulfinimine 53 by treatment with $(R)$-tolylsulfinamide and $\operatorname{Ti}(\mathrm{OEt}) 4$. 


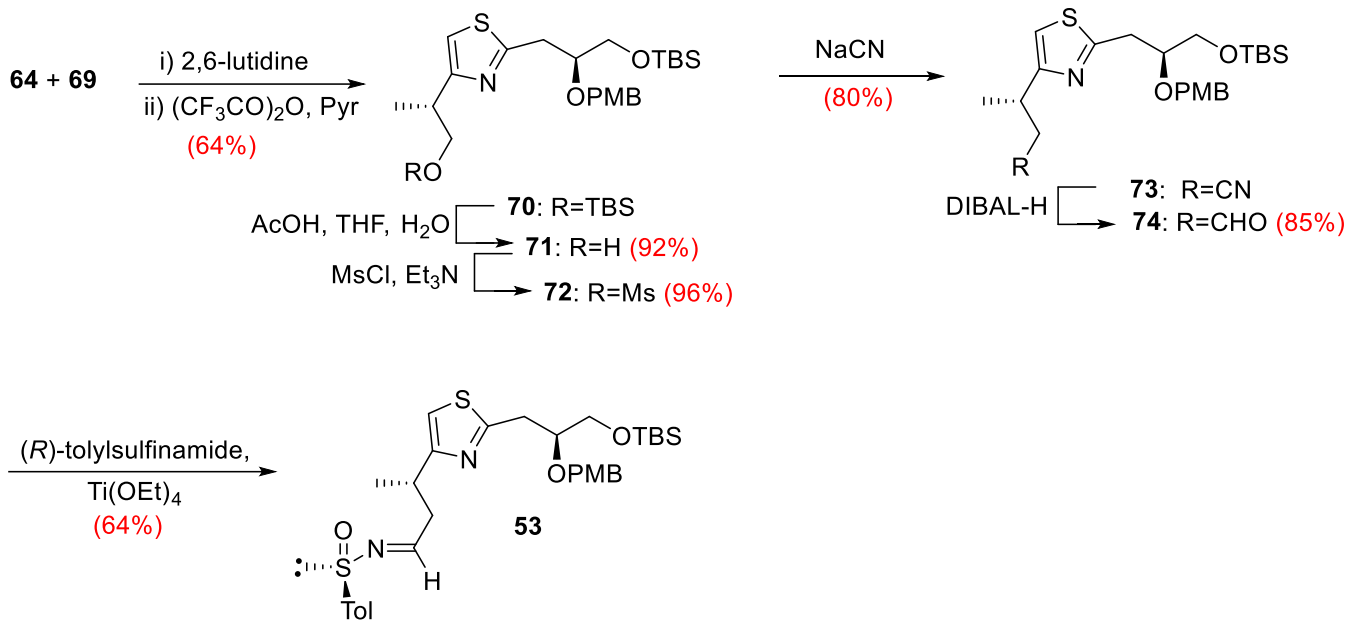

Scheme 1.9 Coupling of 64 and 69 in the preparation of major fragment 53.

\section{9-Membered bis-lactone core (80)}

The ester 56 (which was derived from (S)-propylene oxide to set the stereochemistry at C24) was coupled via its enolate to the sulfinimine 53 to provide compound 75 with approximately $90 \%$ diastereoselectivity for the $R$-configuration at C3 (Scheme 1.10). Removal of the $p$-toluenesulfinyl group with TFA-methanol gave amine 76, which was subsequently treated with 1,1-dimethyl-2,2,2-trichloroethyl chloroformate to provide the TcBoc protected amine 77. Deprotection of the PMB group with DDQ was followed by esterification, under Yamaguchi conditions, with (Z)-3-tri- $n$-butylstannylpropenoic acid 57 (derived from ethyl propiolate) to provide compound 79. Macrocyclisation was then achieved with a Stille coupling that provided the bis-lactone core $\mathbf{8 0}$ with complete preservation of the desired $E / Z$ diene stereochemistry. 

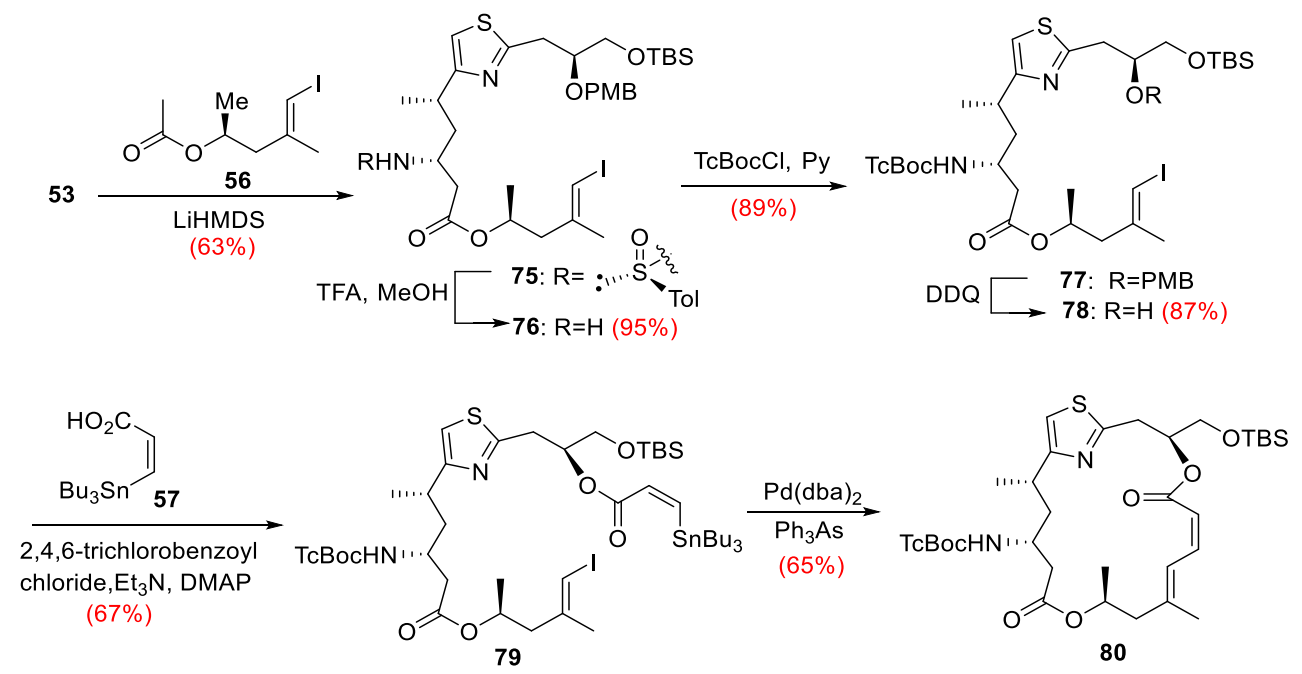

Scheme 1.10 Coupling of major fragments 53,56 and 57 to form macrocycle 80.

\section{Addition of conjugated chain to bis-lactone}

Desilylation of the bis-lactone 80 with TBAF and subsequent oxidation with pyridinebuffered Dess-Martin periodinane gave the aldehyde 82 (Scheme 1.11). A Wittig reaction between compound 82 and 2-(triphenylphosphoranylidene) propionaldehyde 54 gave the E-unsaturated aldehyde 83 which underwent a Takai reaction to provide the vinyl iodide 84. A Stille coupling of the dimethylamino-substituted vinyl stannane 55 with compound 86, followed by removal of the TcBoc group with a cadmium-lead couple provided pateamine (14). 


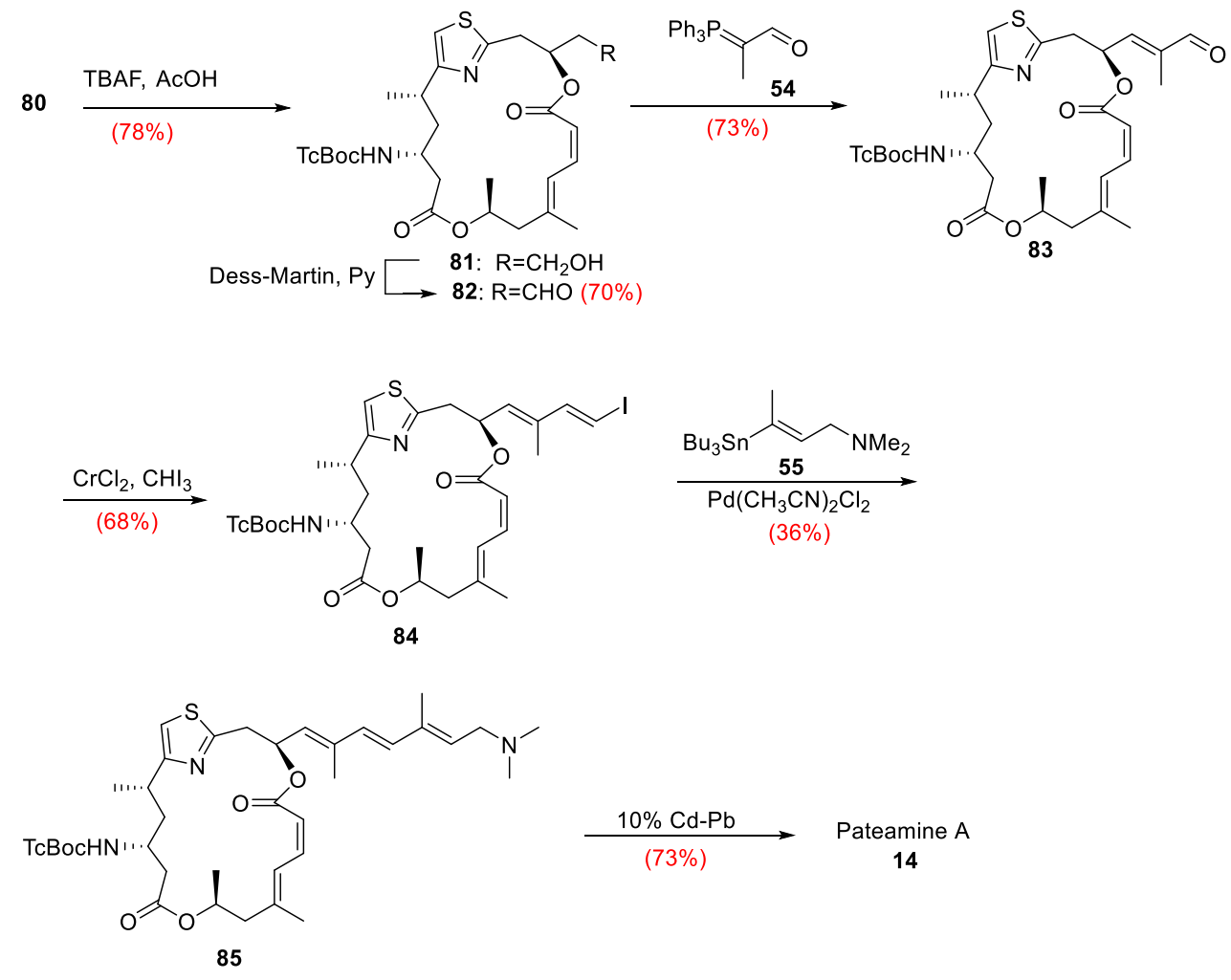

Scheme 1.11 Coupling sidechain fragments (54 and 55) to macrocycle 80 and completion of pateamine synthesis.

\subsubsection{Comparison of Syntheses}

Both Romo's and Pattenden's strategies involved the synthesis of the bis-lactone core followed by the addition of the conjugated chain. However, Pattenden's synthesis is more linear and involves the connection of smaller fragments (vide supra, Figure 1.13) compared with Romo's synthesis that involved the preparation then connection of three major fragments (vide supra, Figure 1.11). Figure 1.14 illustrates the comparison of the major connections involved in Romo's and Pattenden's syntheses. To form the macrocycle, Pattenden used a Stille coupling with a 65\% yield (vide supra, Scheme 1.10), and Romo obtained slightly better yields using a $\beta$-lactam macrolactonisation with $78 \%$ yield (vide supra, Scheme 1.6). 


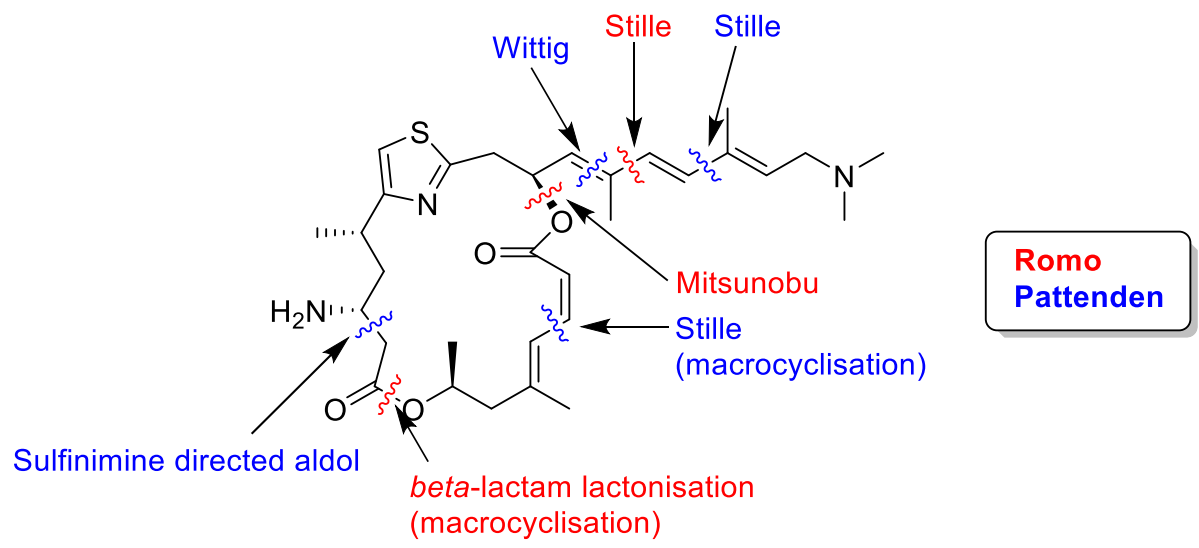

Figure 1.14 Comparison of major connections in Romo's and Pattenden's syntheses.

Pateamine contains four chiral centres (at C3, C5, C10 and C24), for which Pattenden's group used the chiral pool (commercially available chiral reactants) to obtain three of these chiral centres and one chiral auxiliary. In contrast, Romo's group used asymmetric reactions, three of which involved chiral auxiliaries and the other using a ruthenium complex with chiral ligands (Figure 1.15).

Pattenden's incorporation of stereochemistry from the chiral pool into his synthesis has the benefit of giving very good stereochemical purity to the final product. However, the chiral reactants used are fairly expensive (e.g. (S)-propylene oxide is \$NZ874 for 25 grams). $\ddagger$

Romo's incorporation of stereochemistry via chiral auxiliaries had the benefit of using cheaper chemicals, however, preparation of the auxiliaries made these reactions more involved. The effort required was decreased by utilising the same auxiliary in two of the chiral centres, C3 and C10. Romo obtained fairly good yields in his asymmetric reactions with $74-94 \%$ ee (Figure 1.15).

拉ice obtained from Sigma-Aldrich.com in NZ dollars (on $16^{\text {th }}$ September 2013) 


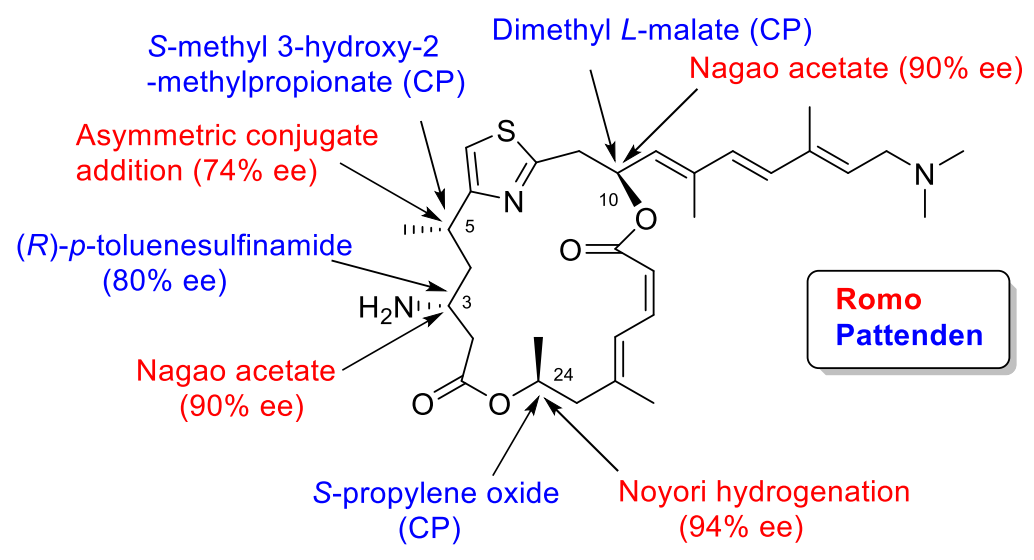

Figure 1.15 Source of chiral centres in Romo's and Pattenden's synthesis, showing obtained enantiomeric excess (ee). $\mathrm{CP}=$ chiral pool.

Additionally, it is noteworthy that both syntheses utilise many advanced starting materials, such as compounds 18, 25, 26, 34 and 46 (in Romo's synthesis) and compounds 54, 56 and 57 (in Pattenden's synthesis).

\subsubsection{Romo's Synthesis of Analogues}

In an effort to find simple active analogues and to determine some information about pateamine's structure-activity relationship with regard to its biological binding receptor, Romo's laboratory synthesised a range of pateamine analogues. These included the derivative devoid of the C3-amino and C5-methyl groups (DMDA-PatA) 86, substitution of the conjugated sidechain for various less-heavily unsaturated sidechains 87-89, and attachment of various sidechains to the enyne bis-lactone core 90-93 (Figure 1.16). ${ }^{38}$ 

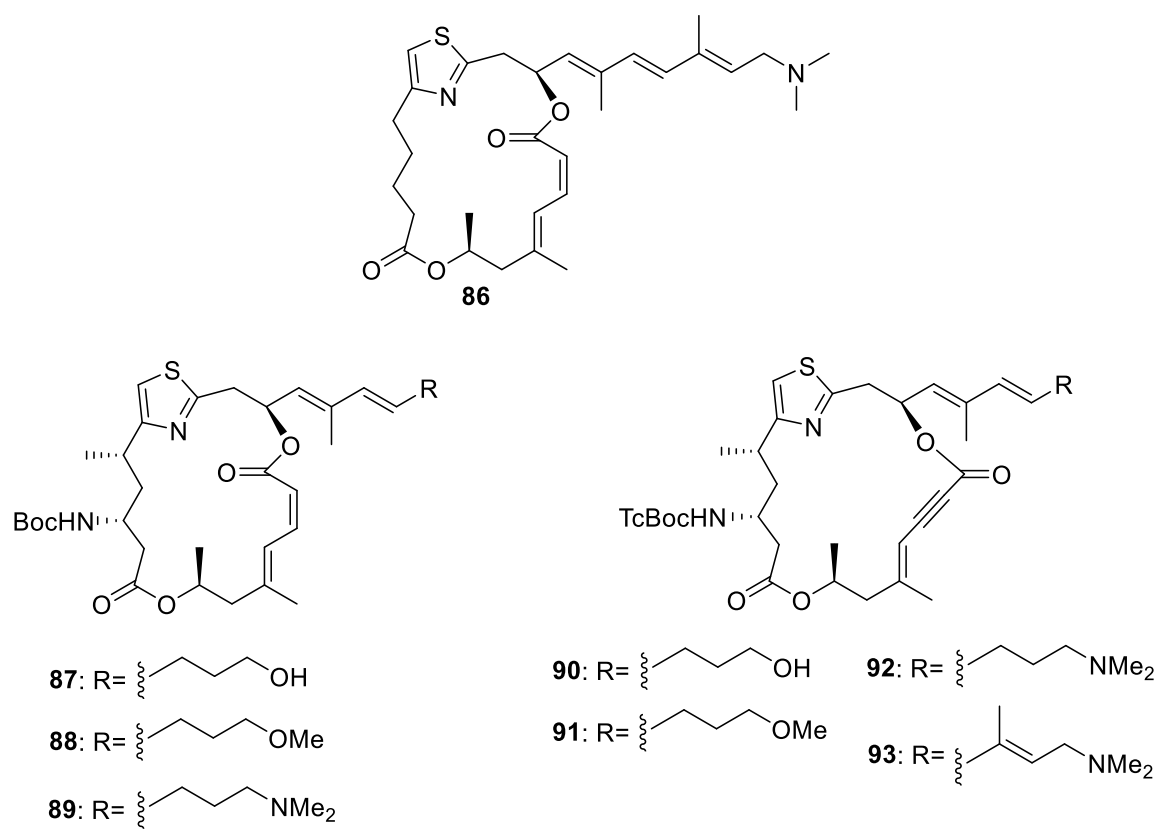

Figure 1.16 Pateamine analogues.

DMDA-PatA 86 was synthesised in a similar fashion to Romo's total pateamine synthesis (vide supra, Section 1.2.1) with two major differences. Firstly, in the preparation of the C1C12 fragment and secondly in the macrocylisation step, where a Yamaguchi lactonisation is used as opposed to the $\beta$-lactam lactonisation. The synthesis of DMDA-PatA began with 6-oxoheptanoic acid 94 which was esterified to give the trichloroethyl ester 95 and followed by bromination via the preformed TMS-enolate and treatment with NBS to provide the $\alpha$-bromo ketone 96 (Scheme 1.12). Hantzch thiazole coupling with thioamide 64 followed by desilylation provided the C1-C12 fragment 98. A Mitstunobu reaction between compound 98 and the more robust (than the previously described TBS enyne 22) TIPS protected fragment 99, provided compound 100. Desilylation with TBAF to give alcohol 101 and subsequent hydrolysis of trichloroethyl ester to give carboxylic acid 102 was followed with macrolactonisation using Yamaguchi's conditions to provide the bislactone core 103. Treatment of compound 103 with Lindlar's catalyst followed by Stille coupling with fragment 24 provided the complete DMDA-PatA 86. Overall, this provides a synthesis with 15 longest linear steps and 32 steps in total from commercially available chemicals. 

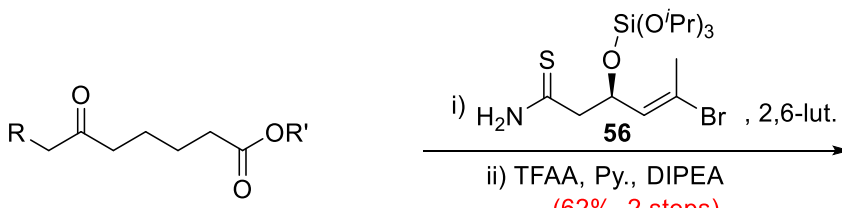

ii) TFAA, Py., DIPEA

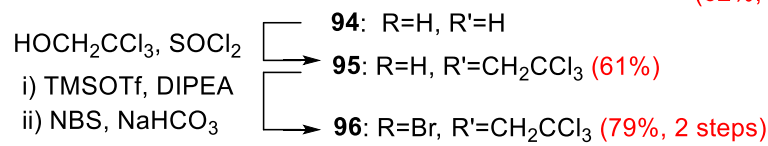<smiles>CC(Br)=CCc1nc(CCCCC(=O)OC(F)(F)Cl)cs1</smiles>

TBAF

97: $R=$ TIPS

98: $\mathrm{R}=\mathrm{H}(97 \%)$
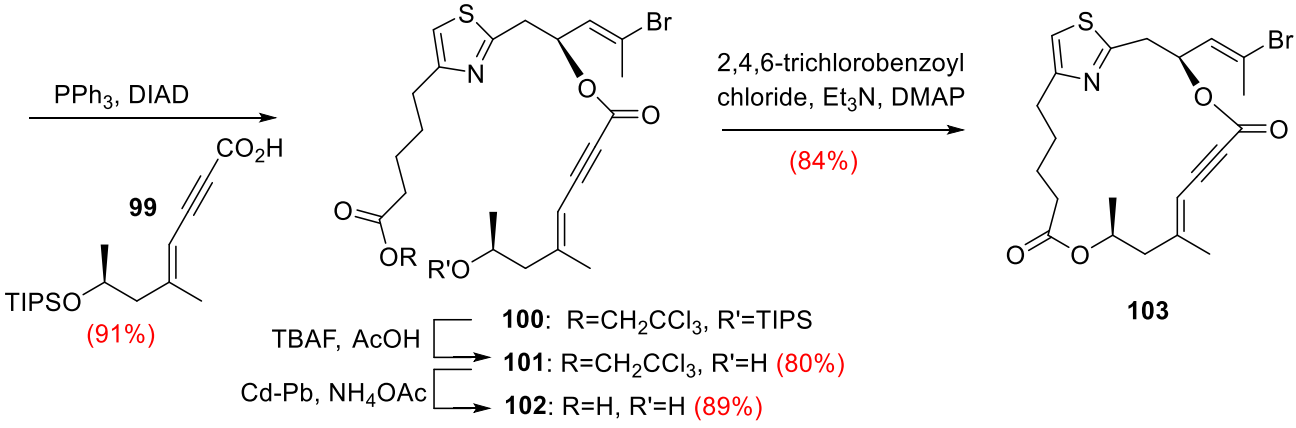

103

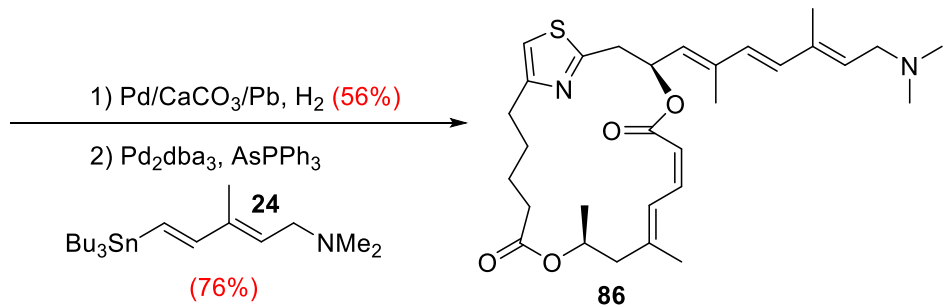

Scheme 1.12 Preparation of DMDA-PatA.

Synthesis of the pateamine analogues 87-93 again followed a similar scheme to Romo's total synthesis of pateamine. Previous biological studies had shown that the C3-Boc protecting group had only a minor effect on pateamine's activity (3-4 fold decrease) and improved its stability, therefore, it was decided to leave the Boc groups on the analogues for ease of handling. ${ }^{30}$ The synthesis of the partially saturated sidechains began with vinylstannane 104 (derived from 4-pentyn-1-ol), which was either methylated to provide the methyl ether 105, or substituted with dimethylamine to provide the amine 106 (Scheme 1.13). 


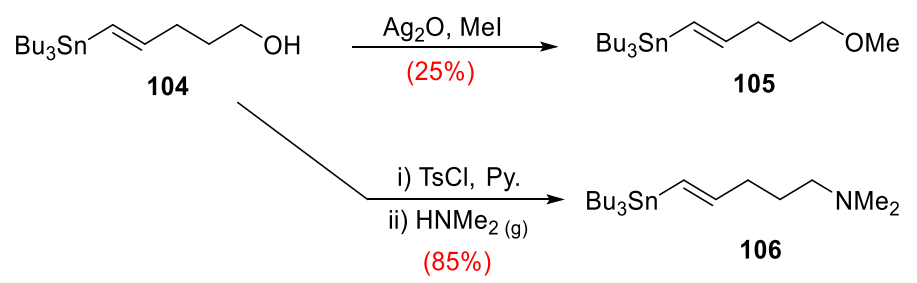

Scheme 1.13 Preparation of sidechains 105 and 106.

To complete the analogues 87-89, the bis-lactone core 107 (described in section 1.3.1, albeit protected with Boc instead of TcBoc) was reduced to the Z,E-diene with Lindlar's catalyst followed by attachment of vinylstannanes 104, 105 or 106 with Stille coupling to provide analogues 87, 88 and 89, respectively (Scheme 1.14). Enyne analogues 90, 91, 92 and 93 were synthesised by Stille coupling the vinylstananes 104, 105, 106 and 24, respectively, to the bis-lactone core 107.

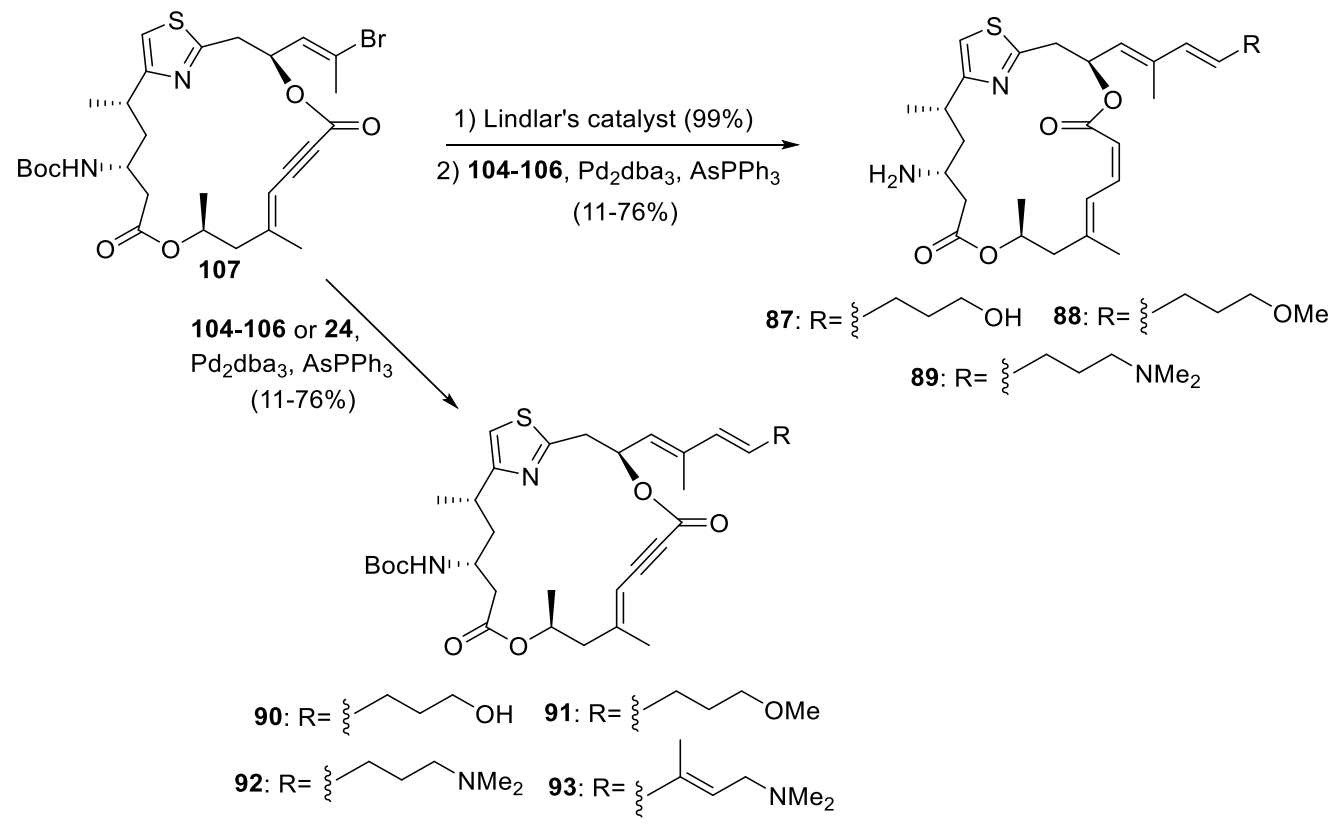

Scheme 1.14 Preparation of pateamine analogues.

Preliminary studies by a group at PharmaMar had shown potent activity by pateamine as an immunosuppressant. ${ }^{38}$ This led to the idea that pateamine was targeting an intracellular signaling pathway involved in T-cell activation. Romo et al. found that pateamine inhibited T-cell receptor-mediated IL-2 production ${ }^{30}$ and tested their 
synthesised pateamine and analogues in an assay that measured their ability to block the activation of an IL-2 promoter attached to a luciferase reporter gene. ${ }^{38}$ Interestingly, DMDA-PatA 86 showed somewhat higher activity in this assay compared with natural pateamine, with an IC 50 of $0.81 \mathrm{nM}$ (cf. pateamine with an IC 50 of $4.0 \mathrm{nM})$.

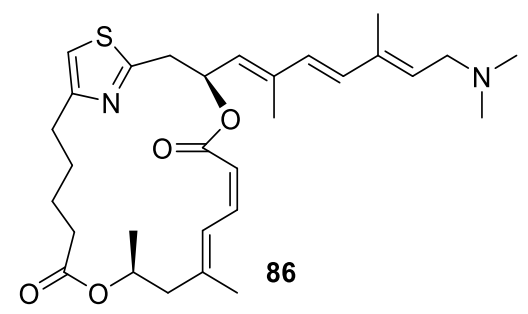

Figure 1.17 Simplified analogue of pateamine, DMDA-PatA (86).

The similar activity of DMDA-PatA and pateamine supported the hypothesis that pateamine has a 'scaffolding' domain that correctly orientates the 'binding' domain (Figure 1.18). This hypothesis was based on preliminary biological studies, structural analysis and simple molecular modelling studies of pateamine. ${ }^{38}$ In addition, biological assays of derivatised products of 'natural' pateamine supports the importance of flexibility of the 'scaffolding' domain for activity (see appendix). ${ }^{39}$

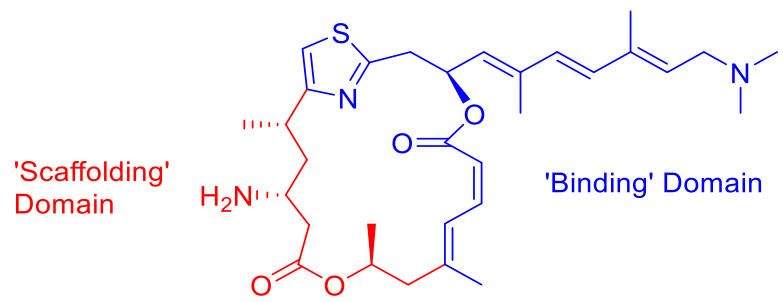

Figure 1.18 The hypothesised 'scaffolding' (red) and 'binding' (blue) domains of pateamine.

Table 1.1 summarises the inhibitory activity $\left(\mathrm{IC}_{50}\right)$ of the various pateamine analogues in the IL-2 assay. From the second column in Table 1.1, it appears that the diene containing analogues tend to have a downward trend in activity as the C10-C17 sidechain (in natural 
pateamine 14) is made more flexible (in 89) and then the amine substituted by the methyl ether (in 88) or the alcohol group (in 87).

When the alkyne containing variants were assayed (Table 1.1, column 3), no activity was observed with the conjugated amine chain (in 93) or the more flexible amine chain (in 92). What is interesting, however, is that when the amine was substituted for the methyl ether (in 91), high activity was observed ( $\mathrm{IC}_{50}$ of $55 \mathrm{nM}$ ). Removal of the methyl group to give the alcohol group (in 90) resulted in a 7-fold reduction in activity. This set of inconsistent results may suggest that the presence of the alkyne alters the mode of binding to the biological target, or that a different biological target is engaged.

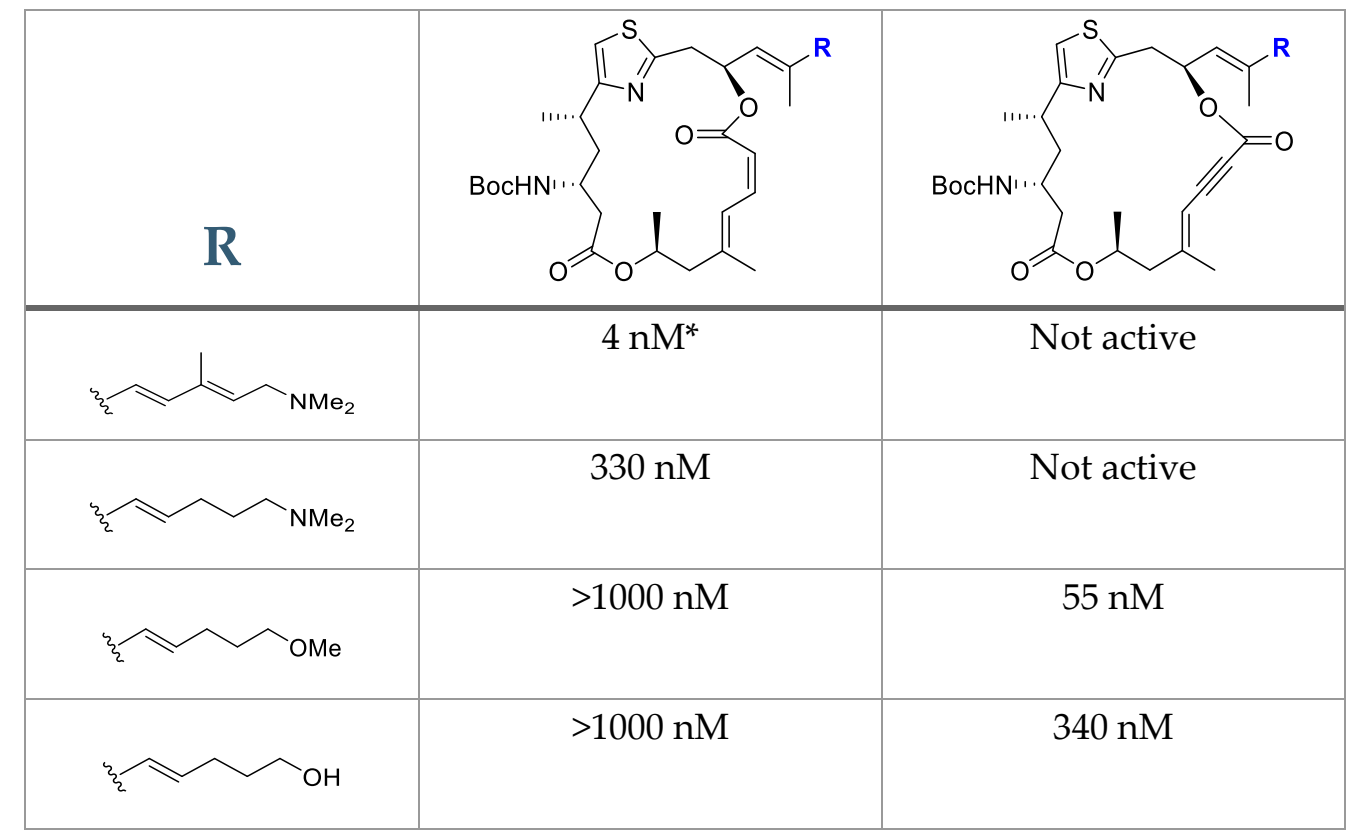

Table 1.1 IC 50 values for analogues from IL-2 reporter assay.

(*this value is for pateamine, i.e. without Boc group) 


\subsection{Biological Activity of Pateamine}

\subsubsection{Discovery of Biological Target}

Early biological studies had suggested that pateamine's target was a signalling pathway in $\mathrm{T}$ cell activation (as mentioned in section 1.3.2). However, it was later found that pateamine does not specifically target a signalling pathway, but targets a much more fundamental process, translation initiation.

In 2005 two research groups independently found that the biologically relevant target of pateamine was eukaryotic initiation factor 4A (eIF4A) family members (eIF4AI, -II and III). The first group comprising Professor Jerry Pelletier (at McGill University, Montreal) and co-workers (including at Victoria University of Wellington) discovered pateamine's target during a high-throughput screening campaign to identify inhibitors of eukaryotic protein synthesis. ${ }^{40}$ To narrow in on the specific target, an affinity matrix containing immobilised pateamine was loaded with total cell extracts from HL-60 cells. Proteins that were retained were then separated and visualised by SDS PAGE, and found to contain eIF4AI/II. Notably, the eIF4A proteins could only be eluted from the pateamine affinity matrix under forcing conditions, including heating in a thiol-containing loading buffer. ${ }^{40}$ This raised the possibility that there is a covalent interaction between pateamine and the eIF4As.

The second research group comprising Professors Daniel Romo and Jun Liu (Texas A\&M University, Texas and Johns Hopkins School of Medicine, Maryland, respectively) and coworkers continued their work on pateamine (described in previous sections) by coupling pateamine with biotin (Pat-B). ${ }^{41}$ Pat-B in conjunction with streptavidin-sepharose was used as an affinity binding assay with various cell lysates and eIF4AI was identified as having a strong affinity for Pat-B. 


\subsubsection{Role and Inhibition of elF4A Isoforms}

There are three isoforms of eIF4A, all of which have been found to bind pateamine and have their normal activity altered as a result of this binding. The eIF4A isoforms are members of the DEAD-box protein family of RNA helicases, defined by the presence of nine conserved motifs. Their RNA binding is stimulated by ATP, and their ATPase activity is dependent of eIF4A being bound to RNA. ${ }^{42}$ eIF4AI and eIF4AII are $90 \%$ identical in their amino acid sequences and have the same function. ${ }^{43}$ eIF4AIII on the other hand is approximately $65 \%$ identical to eIF4AI/II and is functionally different to eIF4AI/II. ${ }^{44}$

\section{D Structure of elF4AI/II and elF4AIII}

Although eIF4AIII is functionally different to eIF4AI/II and they have only $65 \%$ identical amino acid sequence, their overall stuctures are very similar. They both contain two domains that are connected by a flexible linker that are in an 'open' state when uncomplexed (Figure 1.19a), and in a 'closed' state when substrates ATP and RNA are bound (Figure 1.19b).

(a)

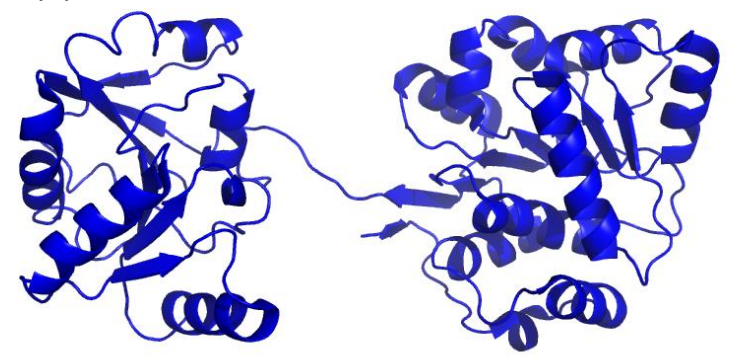

(b)

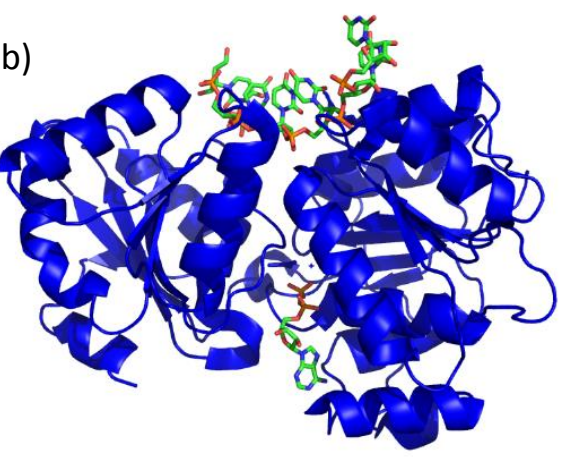

Figure 1.19 3D crystal structures of (a) eIF4A from yeast in 'open' state ${ }^{45}$ and (b) eIF4AIII containing bound RNA and ATP in its 'closed' state. ${ }^{46}$ (PDB 1FUU and 3EX7, respectively.)

Figure 1.20 illustrates the similarity of the three dimensional structures of eIF4AI/II and eIF4AIII, with superimposed crystal structures of eIF4AIII in its 'closed' state and separate 
$\mathrm{C}$ and $\mathrm{N}$ terminal domains of yeast eIF4A. This demonstrates that, although there is only $65 \%$ identical amino acid sequence the overall three-dimensional structures are almost matching. Although the protein fold is conserved, it is reasonable to suggest that the differences in the amino acid sequence of the isoforms may be exploited to allow the selective inhibition.

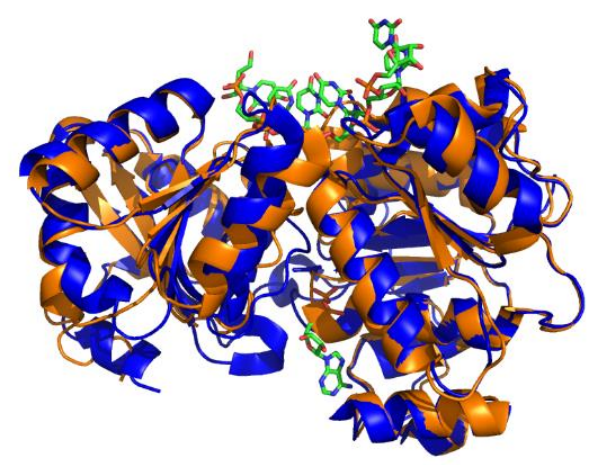

Figure 1.20 3D crystal structures of separate $\mathrm{C}$ and $\mathrm{N}$ terminal domains of yeast eIF4A (orange) overlaid onto eIF4AIII (blue) in its 'closed' state bound to RNA and ATP. (PDB 1FUK, 1QVA and 3EX7, respectively)

\section{elF4AI/II and Translation Initiation}

Translation is the process by which the mRNA trancript is decoded by a ribosome complex into proteins. This process can be broken down into three steps; initiation, elongation and termination. The initiation step of protein translation can be further simplified into four stages (refer to Figure 1.21): (a) the formation of the 435 pre-initiation complex, which contains the $40 S$ ribosomal subunit, initiator tRNA and other factors; (b) $48 \mathrm{~S}$ complex formation, which involves the mRNA bound eIF4F complex recruiting the $43 \mathrm{~S}$ complex; (c) $48 \mathrm{~S}$ complex moves along the mRNA until it recognises the start codon (AUG); and (d) recruitment of the $60 \mathrm{~S}$ ribosome subunit and start of translation of mRNA. 


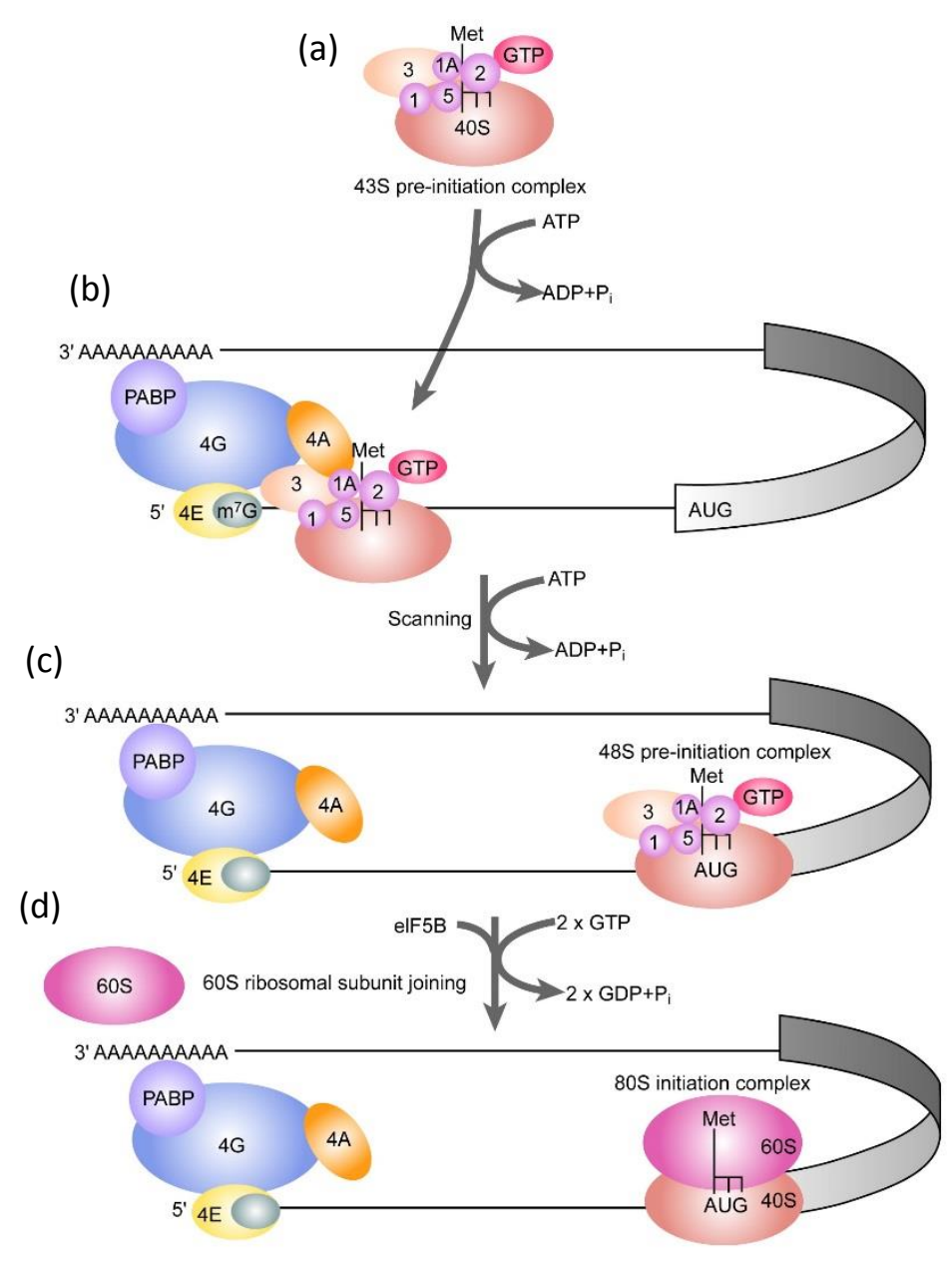

Figure 1.21 Translation initiation. (Reprinted with permission). ${ }^{29}$

Key: 4A, 4G and 4E represent eIF4AI/II, eIF4G and eIF4E, respectively. 40S and 60S represent the ribosome subunits. 1A, 1, 2, 3, 5, GTP and PABP represent other factors involved in translation initiation.

eIF4AI/II is part of the eIF4F complex, that also includes eIF4E, which binds to mRNA cap structure $\left(\mathrm{m}^{7} \mathrm{GpppN}\right.$, where $\mathrm{N}$ is any nucleotide) present at the $5^{\prime}$ end, and eIF4G, which is a scaffolding protein that interacts with eIF4E and eIF4AI/II. ${ }^{47}$ The putative role of eIF4AI/II is to unwind local mRNA secondary structure (the helicase activity of eIF4AI/II) to facilitate the binding of 435 pre-initiation complex (during step (b), Figure 1.21) and possibly to allow movement of the $48 \mathrm{~S}$ complex to scan for the start codon (during step (c), Figure 1.21).

eIF4AI/II exists in both the free form and complexed as part of eIF4F complex, and is thought to cycle between the two (Figure 1.22). ${ }^{43}$ As part of the eIF4F complex, eIF4AI/II is 
stabilised in its 'active' conformation with a 20-fold more efficient helicase activity over the free eIF4AI/II. ${ }^{48}$

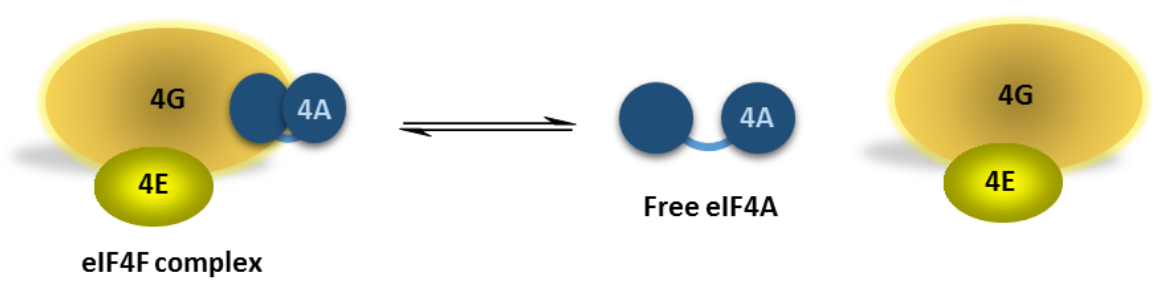

Figure 1.22 Reversible association of eIF4AI/II showing its free and complexed state. Key: See Figure 1.21.

Interestingly, the effect of pateamine on free eIF4AI/II is to increase both its RNA binding and RNA helicase activity, however, this inhibits interaction with eIF4G preventing the formation of the eIF4F complex that is essential for translation initiation (Figure 1.23). ${ }^{41}$

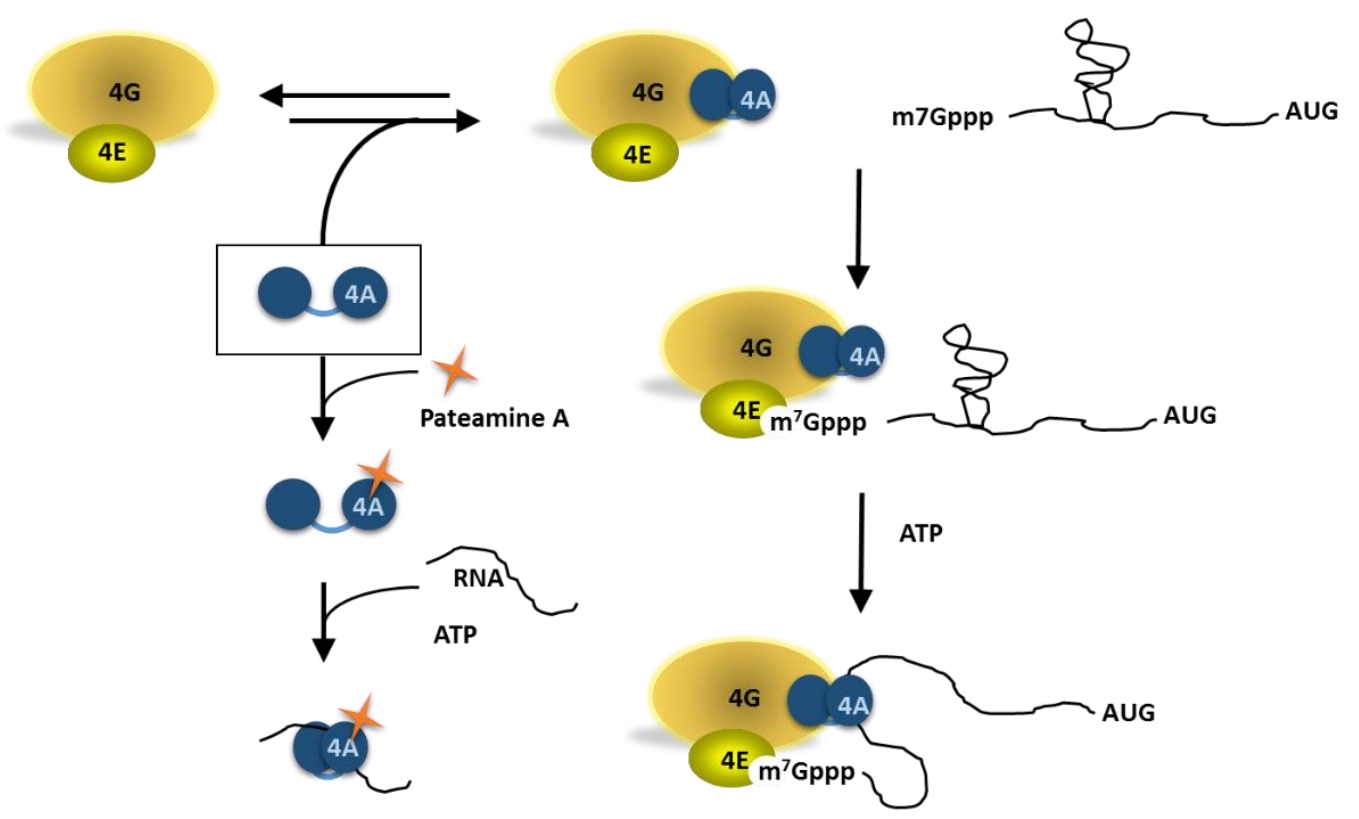

Figure 1.23 Proposed model of pateamine inhibition of translation initiation. ${ }^{49}$ Key: See Figure 1.21. 
eIF4AIII is part of the exon junction complex (EJC) core, which also includes MAGOHY14 hetero-dimer and Barentsz (Btz), and anchors EJC components to RNA (Figure 1.24). ${ }^{50}$

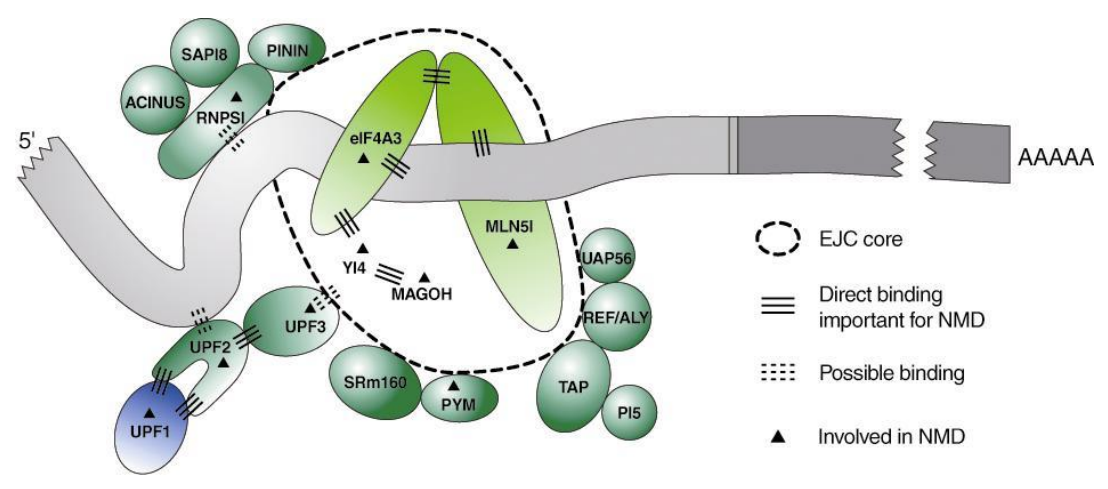

Figure 1.24 EJC components. (Reprinted with permission). ${ }^{50}$

NB: MLN51 is an alternative name for Btz.

The EJC assembles on an mRNA transcript in the nucleus during splicing. It is loaded in a sequence-independent manner at a fixed distance, 20-24 nucleotides upstream of the exon-exon junction. ${ }^{51}$ The putative roles of the EJC are to facilitate the transport of the mRNA through the nuclear membrane and in nonsense-mediated mRNA decay (NMD). ${ }^{52}$

NMD is a process that recognises a premature termination codon (PTC) in an mRNA sequence during the pioneer round of translation and signals the mRNA for degradation via the recruitment of other NMD factors (e.g. UPF1). ${ }^{50}$ NMD is considered to provide quality control for mRNA to prevent the production of non-functioning truncated proteins that are both energetically wasteful and potentially toxic to the cell. ${ }^{53}$

During the pioneer round of 'normal' translation, the ribosome travels along mRNA and dissociates the EJC from the mRNA construct (Figure 1.25b). The cap-binding protein $(\mathrm{CBC})$, which is attached to the $5^{\prime}$ end during transcription, is then replaced with eIF4E and 'bulk' translation begins. ${ }^{54}$ On the other hand, when an mRNA strand contains a PTC located at least 50-55 bases upstream of an EJC, NMD factors are recruited onto the EJC and ribosome complex, resulting in the dissociation of the ribosome complex and degradation of mRNA (Figure 1.25a)..$^{50}$ 
PTC-containing mRNA

(a)
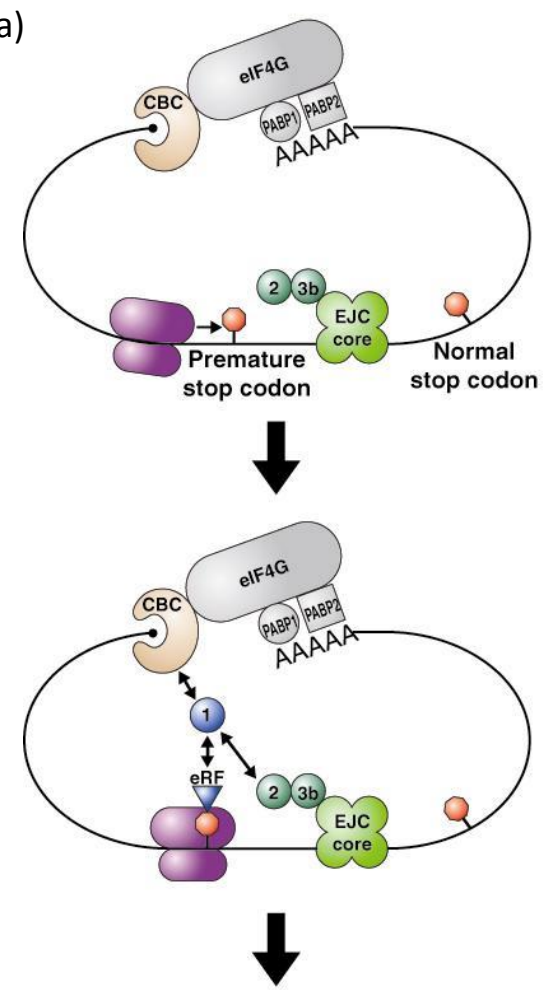

mRNA decay
Normal mRNA

(b)

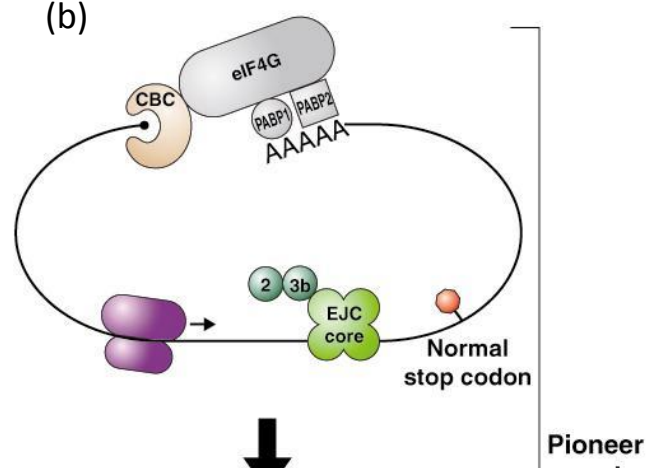

round

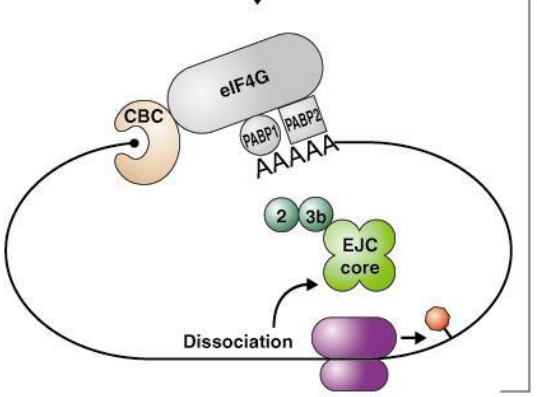

\section{$\downarrow$}

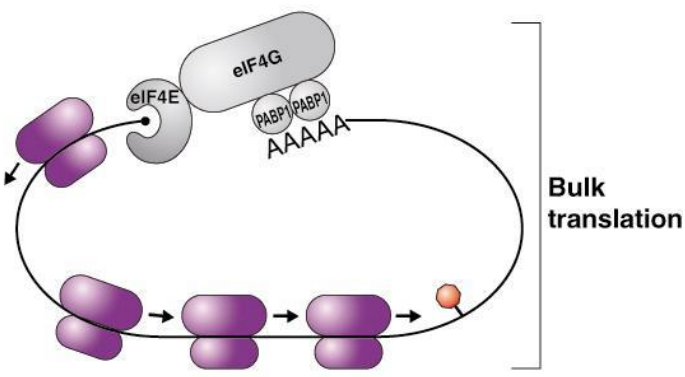

Figure 1.25 Simplified illustration of pioneer round of translation with (a) PTC present in mRNA (EJC model) and (b) in the absence of a PTC. (Reprinted with permission) ${ }^{50}$ Key: CBC, cap-binding complex; eRF, eukaryotic release factor; PABP1 and PABP2, poly(A) binding proteins 1 and 2, repectively; AAAAA, poly(A) tail of RNA; 1, 2 and $3 \mathrm{~b}$ represent UPF1, UPF2 and UPF3b, respectively.

Although many genetic and biochemical studies support the EJC model as the mechanism of NMD, there have been a few observations that are inconsistant. For example the S2 cells of fruit-flies ${ }^{55}$ and cells from Caenorhabditis elegans ${ }^{56}$ do not require introns or EJC proteins for NMD. Additionally, no NMD occured in S2 cells when an mRNA lacked the poly(A) tail or when poly(A) binding protein (PABP) was RNAi depleted, suggesting a role for PABP in NMD for that system. ${ }^{55}$ 
Due to the lack of understanding in this area, there is much value in synthesising compounds that can selectively target the EJC, to probe its importance in NMD.

\subsubsection{Summary of Biological Activity}

The biological targets of pateamine are the eIF4A isoforms. The eIF4AI/II isozymes and eIF4AIII are structurally very similar, ${ }^{57}$ but have different roles in the cell. ${ }^{44}$ The former are involved in protein translation initiation and the latter is putatively involved in mRNA quality control (i.e. mRNA nonsense-mediated decay (NMD)). Pateamine induces strong binding between eIF4A and its substrate RNA. With regards to eIF4AI/II, this prevents the formation of the eIF4F complex necessary for the ribosome-recruitment step of capdependent translation initiation. ${ }^{40}$ This results in the stalling of translation, and consequently, might be the cause of programmed cell death (apoptosis). ${ }^{28}$ Pateamine has also been demonstrated to have potent in vitro antifungal activity (against $C$. albicans and T. mentagrophytes), ${ }^{25}$ potent immunosuppressive activity through affecting T-cell receptormediated interleukin-2 (IL-2) production, ${ }^{30}$ induce stress granule formation by a novel pathway not involving eIF $2 \alpha,{ }^{58}$ and more recently, has been shown to prevent iNOSinduced muscle wasting and promote muscle fibre formation. ${ }^{59}$

Interest in pateamine (and the simplified active analogue DMDA-patA) as an anti-cancer agent is owed to its high potency and selectivity towards a range of cancer cell lines, as opposed to quiescent (non-proliferating) cell lines. This was demonstrated after pateamine's initial isolation with an IC 50 of $0.27 \mathrm{nM}$ against P388 leukemia cell line, in contrast to a BSC cell line under static growth with an $\mathrm{IC}_{50}$ of $540 \mathrm{nM} .^{25}$ In addition, a study with DMDA-PatA 86 demonstrated potent activity against 32 different human cancer cell lines, with IC 50 values of 5-8 nM, and a 1000-fold reduction in potency against a resting cell line (IMR-90 fibroblast cells) with an IC 50 of $8 \mu \mathrm{M} .{ }^{60}$ Furthermore, this latter study demonstrated that DMDA-patA retains almost full in vitro potency in Pglycoprotein-overexpressing cells, suggesting that DMDA-patA is not a substrate for Pglycoprotein-mediated drug efflux, a major known driver of multidrug resistance. ${ }^{60}$

Since translation initiation (i.e. function of eIF4AI/II) occurs prior to NMD (i.e. function of eIF4AIII) any inhibition of eIF4AI/II would disrupt NMD indirectly. Selectively inhibiting 
eIF4AIII over eIF4AI/II would inhibit NMD directly and may shed light on the proposed NMD mechanism (via the EJC model). Moreover, this may provide treatments for NMDrelated inherited conditions (e.g. some forms of cystic fibrosis and Duchenne muscular dystrophy).

At higher concentrations of pateamine, additional targets have been found to be affected, and these are summarised in Table 1.2.

\begin{tabular}{ll}
\hline Conc $(\mu \mathrm{M})$ & Effect \\
\hline $\mathbf{0 . 2}$ & IC50 Cap-dependent translation (4AI/II). ${ }^{40}$ \\
$\mathbf{0 . 5 - 1}$ & $\begin{array}{l}\text { eIF4AIII binding, NMD inhibition through stabilization of the interaction } \\
\text { between the EJC and mRNA. }{ }^{61}\end{array}$ \\
& IC50 DNA polymerase $\boldsymbol{\alpha}^{60}$ \\
$\mathbf{4}$ & $\sim 10 \mathrm{x}$ increase in eIF4AI/II ATPase activity. \\
& Significant increase in ATP and RNA binding to eIF4AI/II. ${ }^{40}$ \\
$\mathbf{1 0}$ & IC50 DNA polymerase $\boldsymbol{\gamma}^{60}$ \\
$\mathbf{2 0}$ & Equivalent suppression of microtubule formation to colchicine at $20 \mu \mathrm{M}^{62}$ \\
$\mathbf{1 3 0}$ & Mild increase in actin critical concentration. ${ }^{62}$ \\
$\mathbf{1 9 0}$ & IC50 initial rate of actin polymerisation. ${ }^{62}$ \\
$\mathbf{2 0 0}$ & Increase in actin critical concentration to $50 \%$ of control. ${ }^{62}$ \\
& Promotion of microtubule formation $\approx$ paclitaxel at \\
\hline
\end{tabular}

Table 1.2 Summary of biological targets and their IC 50 values.

Little is known about pateamine's mode of binding or the active site on its biological target. Analysis of 3-dimensional crystal structures reveals a pocket on eIF4A that contains a nucleophilic cysteine residue. We suspect that the dienoate of pateamine may act as a Michael acceptor to create a covalent cross-link with this cysteine residue causing the observed modulation of eIF4A activity. In addition, it is possible that the terminal amine on the sidechain of pateamine interacts with a phosphate group on the RNA bound to the target eIF4A protein. 


\subsection{Summary}

Natural products that have potent biological activity represent valuable candidates for drug discovery. ${ }^{2}$ The preparation of analogues of these natural products, allow us to 'finetune' the biological activity to improve their potency or pharmacology for use in humans, thus, improving their prospects as pharmaceutical agents.

Pateamine was extracted in small amounts from a marine sponge off the coast of the South Island of New Zealand. ${ }^{25}$ It exhibited potent and selective toxicity towards a cancer cell line, which prompted its total synthesis to allow further biological investigation. Two different total syntheses have been reported by Romo's and Pattenden's labs (in 1998 ${ }^{30}$ and $2000^{37}$, respectively), and the synthesis of analogues by Romo's lab (in 2004). ${ }^{38}$

Romo's group tested their pateamine analogues in an IL-2 reporter assay (immunosupressant activity) and obtained results that led them to hypothesise 'binding' and 'scaffolding' domains in pateamine that account for the activity. However, these results did not give a clear explanation with regards to the SAR. A year later (2005), the biological targets were independently determined by Pelletier's and Liu's labs and reported to be the eIF4A isoforms. These include the putative functionally identical isoforms eIF4AI/II (involved in translation initiation) and eIF4AIII (involved in NMD).

This reveals that the IL-2 reporter assay used in the analogue study (by Romo's lab) indirectly measured the real biological activity. Accordingly, one explanation for some of the observed discrepancies in the IL-2 reporter assay could be a consequence of switching in selectivity between the eIF4A isoforms and potentially other targets, caused by the chemical differences in the pateamine analogues. 


\subsection{Research Aim and Objectives}

The aim of this research is to design and synthesise analogues of pateamine for the purpose of selectively modulating the activity of eIF4AI/II or eIF4AIII, and as probes to determine the mode of binding.

There are two main facets of this project; firstly, to design simplified analogues that retain activity and will provide both information on key structural elements and a more practical synthesis than the natural product, and secondly, to design a range of analogues that can be tested for their ability to selectively inhibit either eIF4AI/II or eIF4AIII isoforms.

\subsubsection{Analogue Design}

With the aim of designing a compound that could mimic the activity of pateamine but is more easily synthesised, a simplified analogue was targeted (Figure 1.26). The features that were deemed important for activity, primarily based on Romo's analogue studies, were the structure and size of the macrocycle, the lactone functionalities, a heterocycle to scaffold the macrocycle, and the Z,E-dienoate, together with the conjugated triene sidechain with its terminal amine.
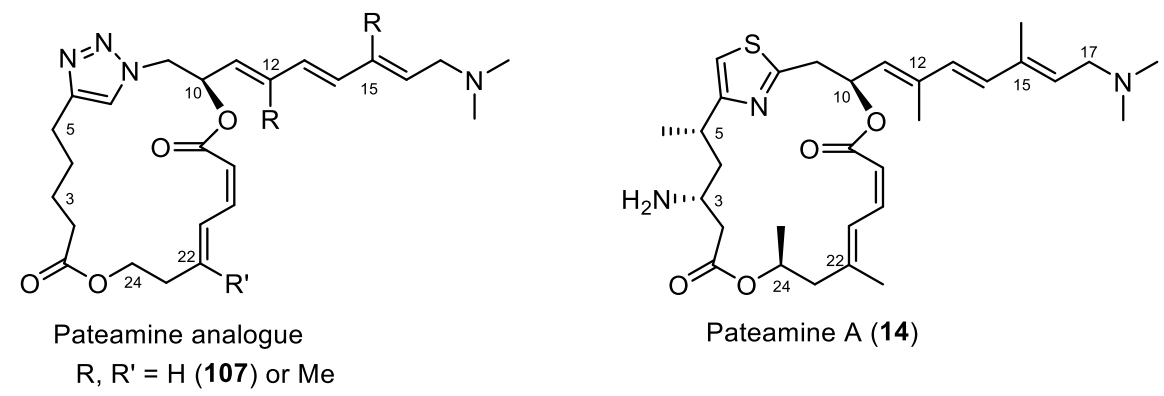

Figure 1.26 Comparison of pateamine and target analogues.

Des-methyl des-amino pateamine A (DMDA-PatA) 86 was shown to have comparable activity to the full natural product, ${ }^{38}$ and hence, we omitted the C3 and C5 substituents 
from the target analogue. The removal of the methyl group at C24 was desirable, as this removed a stereocenter. We proposed that methyl groups on C12, C15 and C22 do not play a major role in biological activity. Finally, we were interested in exploring how the use of an alternative five-membered heterocycle would affect the potency and selectivity of the pateamine analogue. We opted for a triazole, which was predicted to be synthetically easier to access (than the thiazole). Additionally, the copper catalysed azidealkyne cycloaddition reaction (CuAAC) may provide an effective method for macrocyclisation.

It was hoped that an alternative heterocycle would provide a slight electronic difference that might be of value in our desire to selectively target eIF4AIII over eIF4AI/II while retaining the conformation rigidity of the thiazole. We were encouraged by a study in search of HDAC1-selective inhibitors for anticancer agents, whereby an analogue of the marine natural product, largazole (108), was prepared that had a 4-methythiazoline replaced by a triazole (109) (Figure 1.27). ${ }^{63}$ Here, a significant effect in the selectivity was observed, indicating that such a modification can change selectivity between targets.
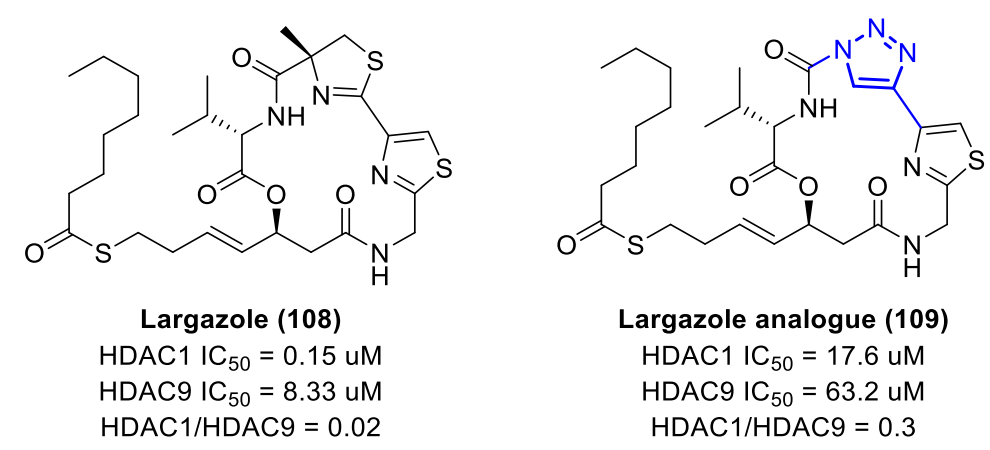

Figure 1.27 IC 50 values against HDAC1 and HDAC9 for largazole 108 and it's triazole analogue 109.63

Molecular modelling was carried out to assess the impact of the change from thiazole to triazole and accompanying removed methyl groups. A superimposed 'lowest energy conformation search' of DMDA-PatA 86 and a target analogue, 107, showed that the proposed simplifications did not cause a significant change in the overall conformation of our target (Figure 1.28). 


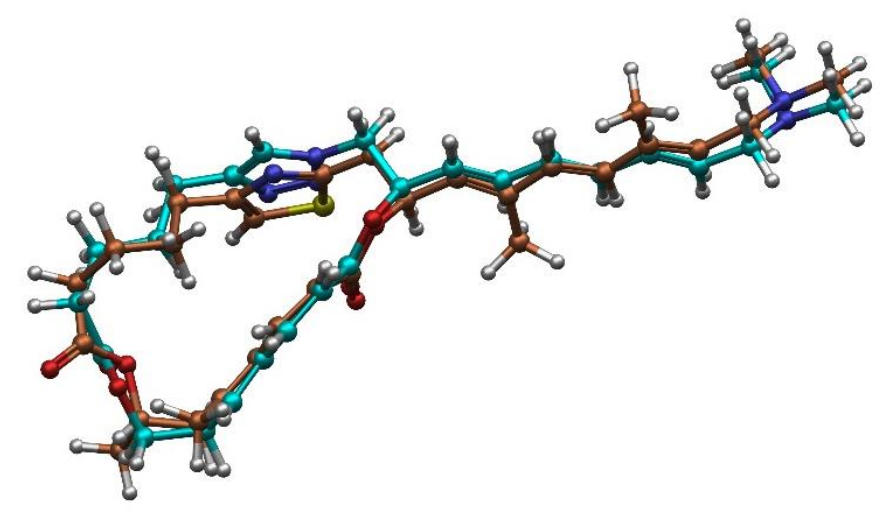

Figure 1.28 Superimposed structures of DMDA-pateamine $\mathbf{8 6}$ (copper) and simplified analogue 107 (cyan). $\$$

In addition, to determine the effect of the proposed changes of our target analogue on the overall charge distribution we calculated the electrostatic potential surface for both the target analogue 107 and DMDA PatA 86 (Figure 1.29). Here we can see that the sidechain is largely unaffected, but slight changes in the distribution of electrostatic potential is observed in the macrocycle. As expected, the effect of having a triazole (as in 107) instead of a thiazole (as in 86) resulted in significant differences in charge distribution (circled black in Figure 1.29).

$\S$ Lowest energy conformations were calculated using Maestro 9.0.109 software (Schrödinger, LLC), using Macromodel Monte Carlo conformation search with a force field (OPLS_2005) and a continuum solvent model to simulate water as solvent. The search used 30,000 iterations. 
(a)

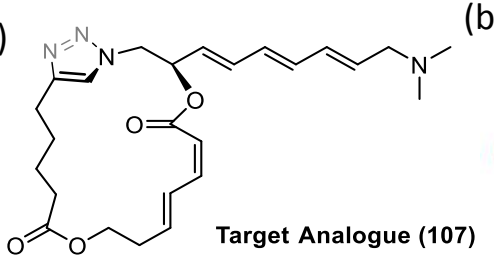

(b)

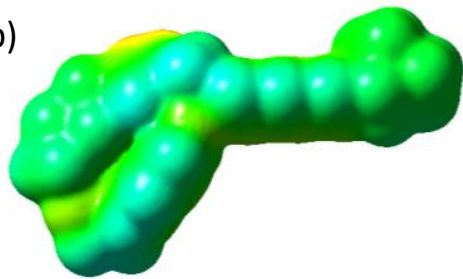

(c)

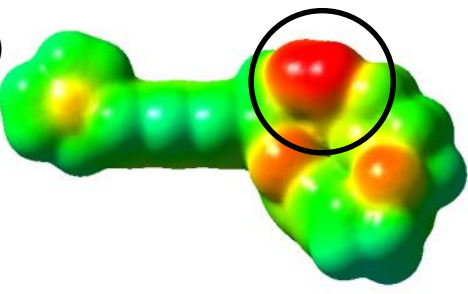

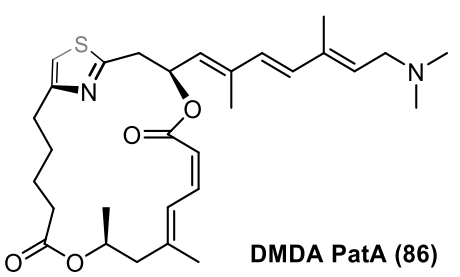
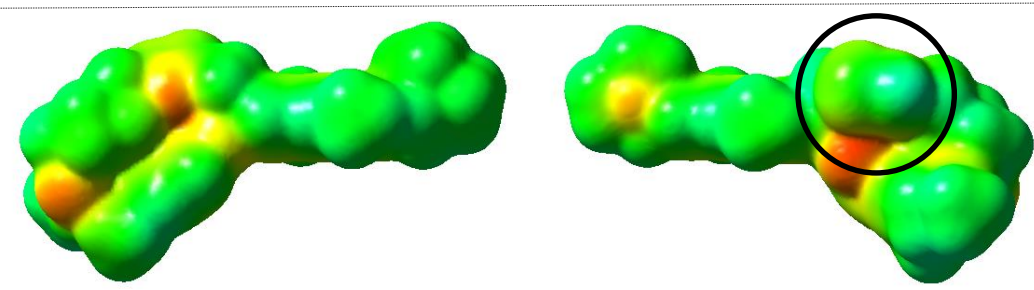

$-60 m \vee-v e$

$60 \mathrm{mV}$

Figure 1.29 Chemical structures, 'front' view (a), and calculated electrostatic potential surfaces, 'front' view (b) and 'back' view (c), of target analogue 107 and DMDA PatA 86.

Colour scale illustrates electrostatic potential, with values as shown on insert bar.

\subsubsection{Retrosynthetic Analysis}

The proposed synthesis of the pateamine analogues was designed to allow easy access to a range of variants. We envisioned a strategy that was convergent, involving the separate preparation of four fragments that could be coupled in different ways in the final steps of the synthesis. We wanted the synthetic strategy to be scalable, environmentally- and chemist-friendly. Thus, our choice of chemical methodology was influenced by the use of cheaper and less toxic starting materials and reagents.

Scheme 1.15 shows our first-generation broad retrosynthetic analysis of pateamine analogues. We envisioned that the conjugated sidechain (Fragment IV) could be coupled to macrocycle $\mathbf{1 1 0}$ as the final step using some form of olefination reaction, e.g. via a Wittig reaction (between a phosphonium ylide and an aldehyde) or a Julia-Kocienski reaction (between a heteroaromatic sulfone and an aldehyde). The macrocycle $\mathbf{1 1 0}$ would be constructed by coupling fragments I, II and III, using appropriate ester formation reactions and a copper(I)-catalysed azide-alkyne cycloaddition (CuAAC) reaction.

\footnotetext{
${ }^{* *}$ Electrostatic potential surfaces were calculated using Gaussian 09 software (and GaussView 5.0.9), performing a DFT (B3LYP) calculation with basis set 6-31G(d,p). Based on lowest energy conformation.
} 

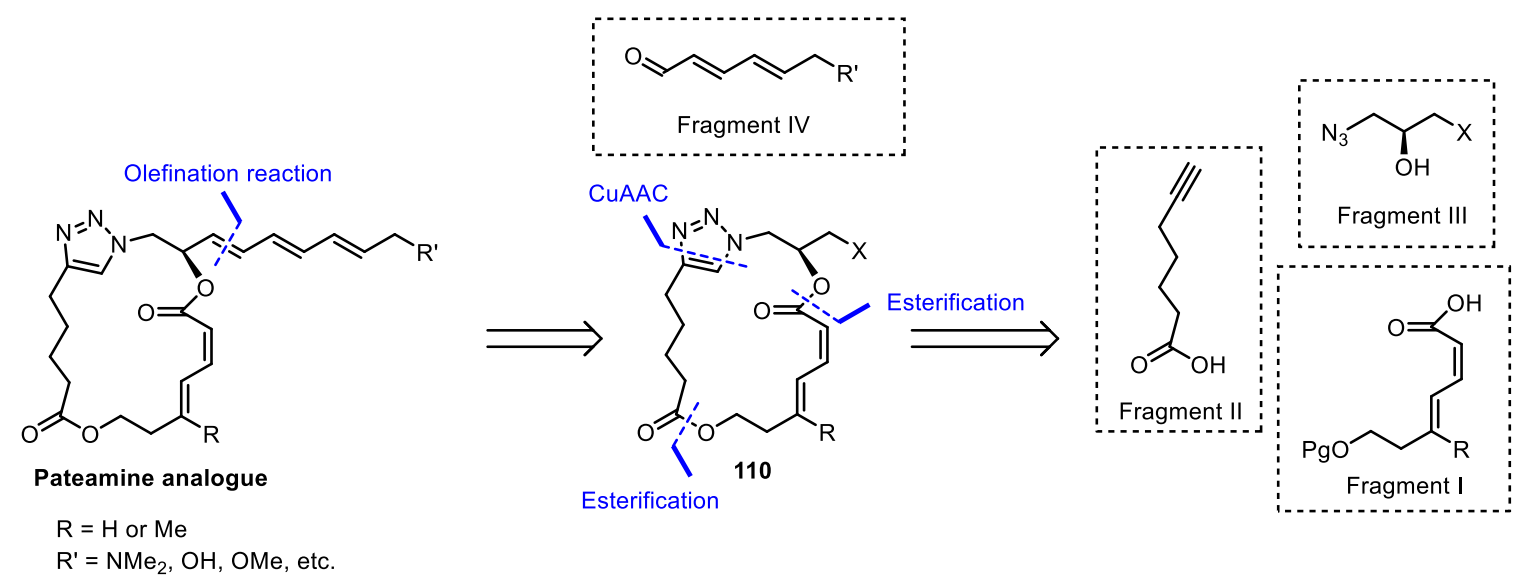

Scheme 1.15 First generation retrosynthetic analysis of pateamine analogues

There are multiple routes to combine three fragments into a macrocycle (i.e. the order in which they are coupled), so a strategy that limited the need for protecting groups was sought. Presuming that fragment I would be prepared as a free carboxylic acid (with a suitably protected alcohol), we initially plannned to couple this in the first instance to fragment III to allow differentiation from the carboxylic acid of fragment II in the subsequent steps (Scheme 1.16). After deprotection of the alcohol in fragment I to form compound 111, this would leave two alternative routes for coupling fragment II and macrocyclisation as the final macrocyclisation step, to be formed either as a macrolactonisation via 112 (blue retrosynthetic arrows) or using a CuAAC via $\mathbf{1 1 3}$ (red retrosynthetic arrows). Preparation of the four fragments will be discussed in the following chapter and the construction of the analogues in chapter three. 


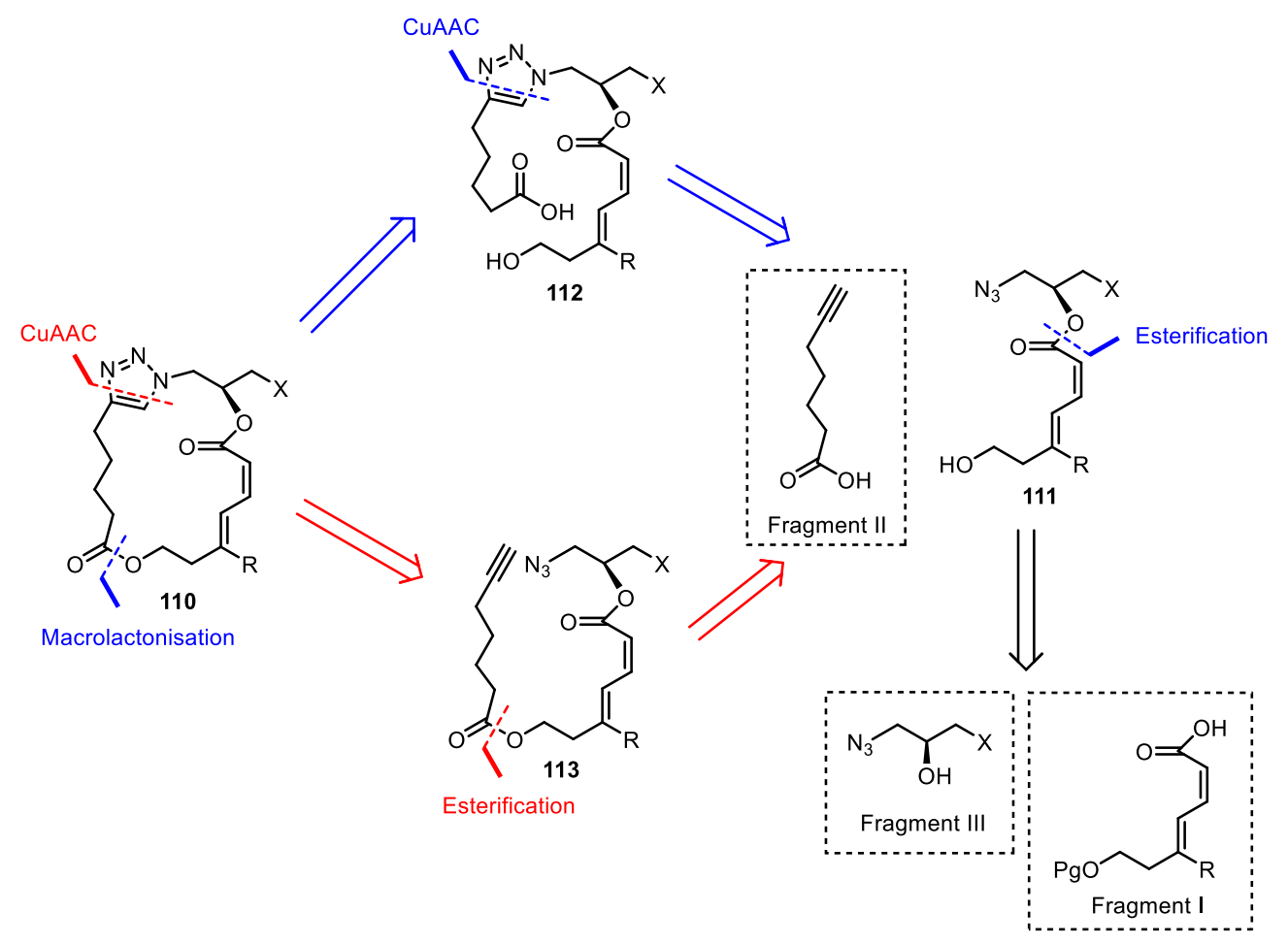

Scheme 1.16 Alternative macrocyclisation strategies. 


\subsubsection{Objectives}

The objectives of this project are listed as below:

- Prepare the four fragments for the target analogue.

- Establish appropriate reactions and reagents for fragment coupling.

- Explore a range of esterification/lactonisation conditions and evaluate the propensity for isomerisation at the Z,E-dienoate.

- Evaluate Wittig and Julia-Kocienski olefination reactions for installation of the sidechain using acyclic models.

- Establish a minimum protecting group strategy for analogue synthesis.

- Couple fragments to prepare simplified analogues.

- Determine the order of fragment coupling and the ideal site for the macrocyclisation.

- Attach sidechain fragment to a macrocycle to complete target analogue synthesis.

- Biological testing (to be performed by others)

- Cytotoxicity against a cancer cell line.

- Compare the binding/inhibition for individual eIF4A isoforms.

- Investigate mode and position of binding between pateamine analogue and eIF4A via mass spectrometry (inspecting for a possible covalent adduct).

- Use biological results to inform the design and synthesis of second generation analogues. 
48 


\section{Chapter Two: $\quad$ Fragment Synthesis}

As outlined in Section 1.6.2, the initial planned synthetic strategy involved the separate preparation of four fragments that would then be coupled together with appropriate reactions. This chapter describes the work towards the synthesis of these four fragments.

\subsection{Synthesis of C17-C23 (Diene) Fragment}

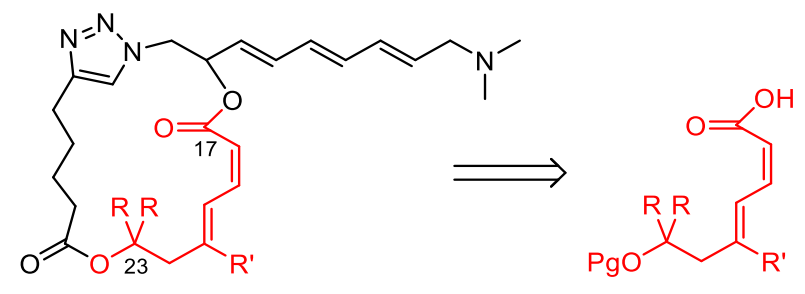

$\mathrm{R}$ or $\mathrm{R}^{\prime}=\mathrm{H}$ or $\mathrm{Me}$

$\mathrm{Pg}=$ protecting group

\subsubsection{Introduction}

The occurrence of diene $Z, E$-dienoate moieties in nature is reasonably rare, presumably due to the lower energy of its all-trans counterpart (i.e. the $E, E$-dieneoate). However, there are a handful of examples, which include; dictyostatin, zampanolide, stagonolide E, pateamine A, macrolactin A, and salarin C (Figure 2.1). All of these compounds possess potent biological activity, and all but stagonolide E (a terrestrial fungal extract) are sourced from the marine environment. 

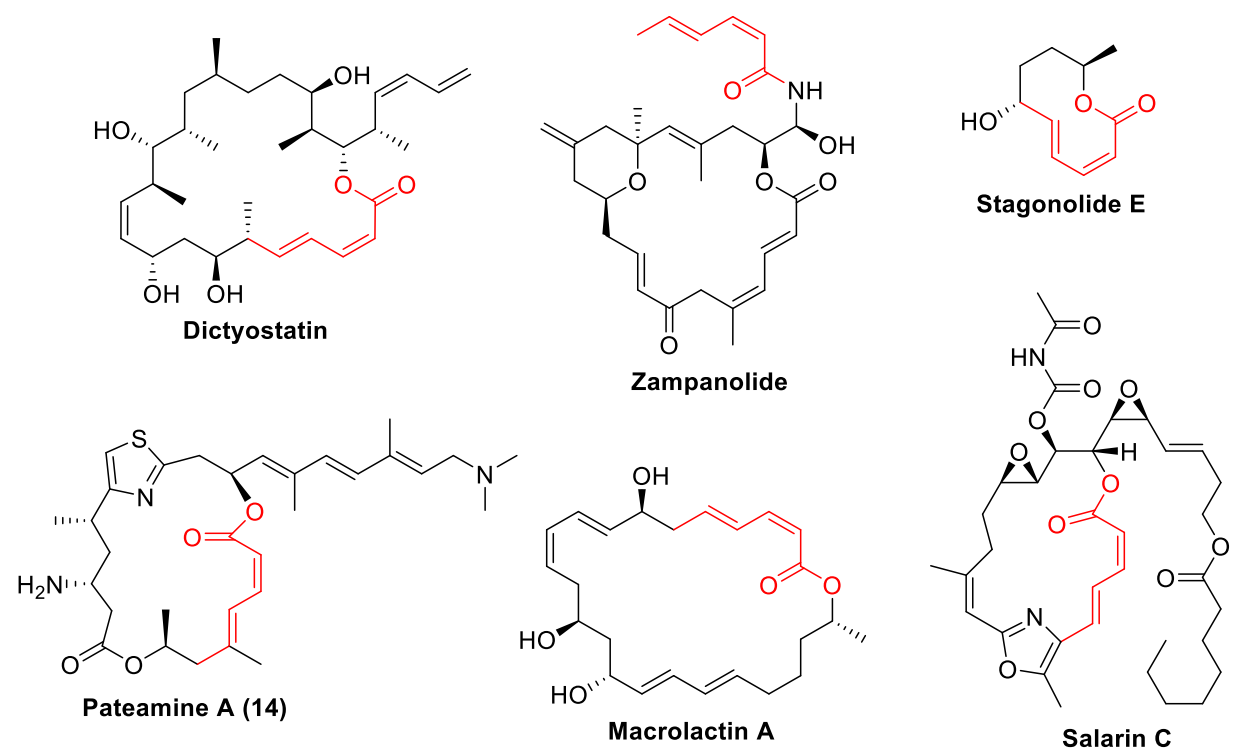

Figure 2.1 Examples of natural products containing Z,E-dienoates.

Given that these compounds exhibit potent biological activity, it is no surprise that much effort has been put towards synthesising them. A major consideration in the preparation of $Z, E$-dienoates is that the conjugation into the carbonyl makes them particularly susceptible to isomerisation to the more energetically stable $E, E$-dienes. Nevertheless, a range of synthetic methodologies have been successfully utilised to access $Z, E$-dienoates. These methods can be roughly classed as follows: (a) palladium or copper-catalysed Stille cross-coupling of a vinyl halide with a vinyl stannane; (b) Sonogashira cross-coupling between an $E$-vinyl iodide and an alkyne, followed by cis-selective reduction of the alkyne with Lindlar's catalyst; (c) Z-selective olefination reactions, and; (d) base-induced opening of an $\alpha, \beta$-unsaturated $\delta$-lactone.

The synthesis of macrolactin A utilised a palladium catalysed Stille cross-coupling reaction to couple the vinyl tributyltin 114 with (Z)-3-iodopropenoic acid 115, providing the key diene (Scheme 2.1a). ${ }^{64}$ Similarly, a copper-mediated Liebeskind-type Stille coupling of vinyl iodide 116 and Z-alkenyl stannane 117 used copper(I) thiophene-2carboxylate $(\mathrm{CuTC})^{65}$ as a catalyst in the synthesis of 10,11-dihydrodictyostatin (Scheme 2.1b).66 
(a)

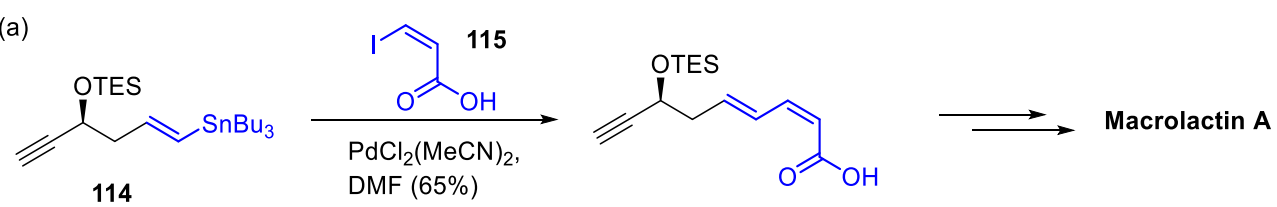

(b)
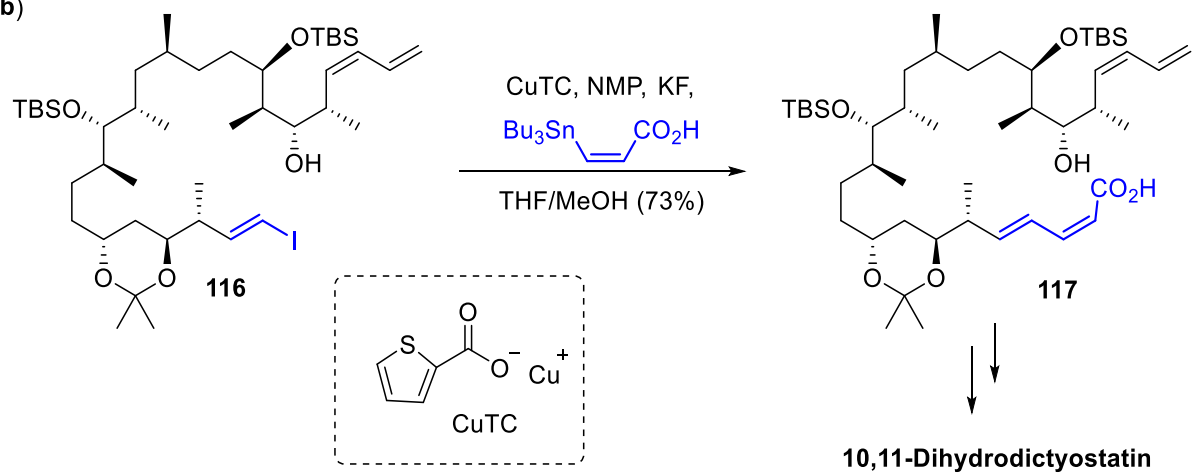

Scheme 2.1 Preparation of Z,E-dienoic acid moieties using (a) palladium ${ }^{65}$ or (b) copper ${ }^{66}$ cross coupling.

A method that prevents the risk of isomerisation is to use a Sonogashira palladium coupling of a vinylic halide with a terminal alkyne, whereby the alkyne can then be stereoselectively reduced to the cis alkene to provide $Z, E$-diene. The benefit of using this method is that the option of retaining the alkyne through subsequent reactions and reduced at a later stage is available, which prevents the potential for isomerisation of the cis alkene to the trans configuration en route to the final desired product. This approach was used in the synthesis of (-)-dictyostatin (Scheme 2.2), although in this case alkyne reduction immediately followed the Sonogashira coupling. ${ }^{67}$ 


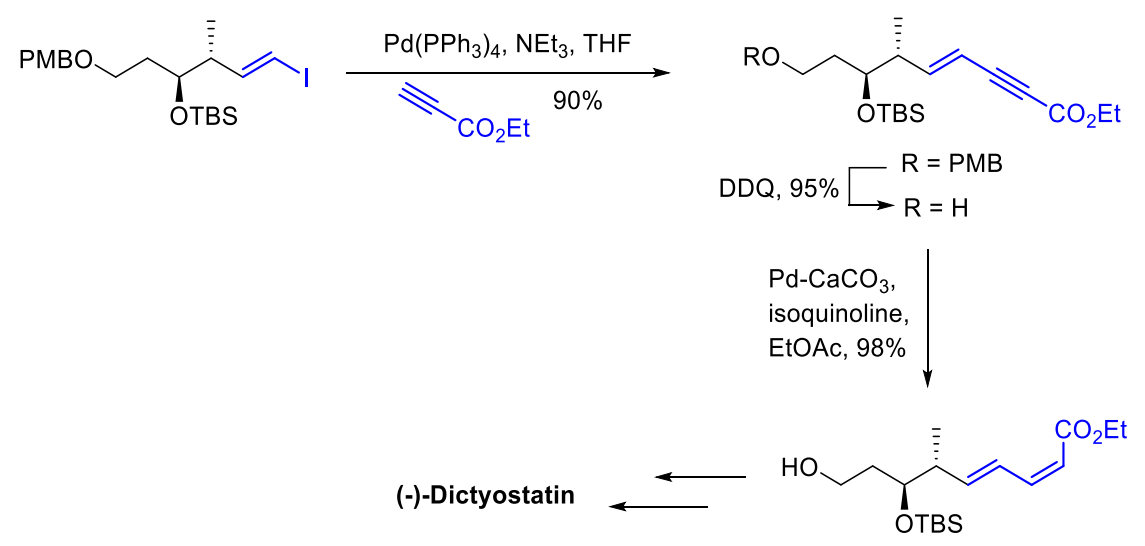

Scheme 2.2 Use of Sonogashira cross coupling and subsequent Lindlar reduction in the synthesis of (-)-dictyostatin. ${ }^{67}$

Typically, Horner-Wadsworth-Emmons (HWE) reactions of a stabilised phosphonate with an aldehyde predominantly gives the E-alkene. A modification of this which uses electron-withdrawing trifluoroethyl groups on the phosphonate (in combination with a strong base) provides predominantly Z-alkenes, known as the Still-Gennari modification. ${ }^{68}$ This method was used effectively in the synthesis of stagonolide $\mathrm{E}$ by reaction of bis(trifluoroethyl) phosphonate 118 with $\alpha, \beta$-unsaturated aldehyde 119 to provide Z,E-dienoate 120 with good stereoselectivity (95:5 Z,E:E,E) (Scheme 2.3). ${ }^{69}$
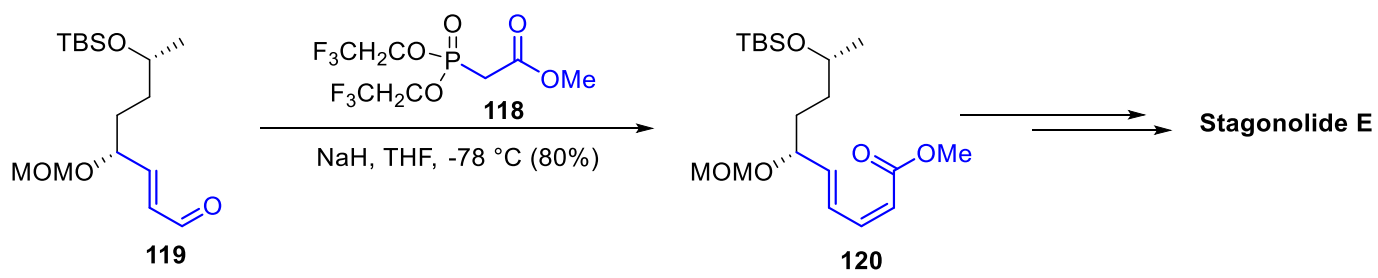

Scheme 2.3 Use of Still-Gennari reaction in the synthesis of stagonolide E. ${ }^{69}$

Another method for the preparation of $Z, E$-dienes, and one that has not been extensively utilised, is the base induced elimination of an $\alpha, \beta$-unsaturated $\delta$-lactone. This was first reported by Eisner et al. in 1953, and involved the treatment of parasorbic acid with sodium methoxide giving (2Z,4E)-hexadienoic acid 121 (Scheme 2.4a). ${ }^{70}$ This reaction was also utilised in the synthesis of dehydronerol (Scheme 2.4b). ${ }^{71}$ 
(a)

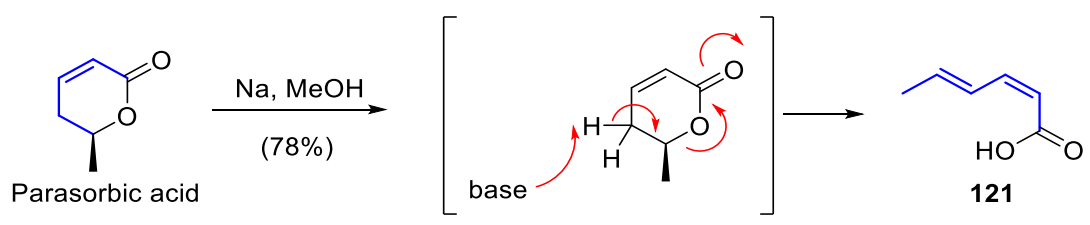

(b)

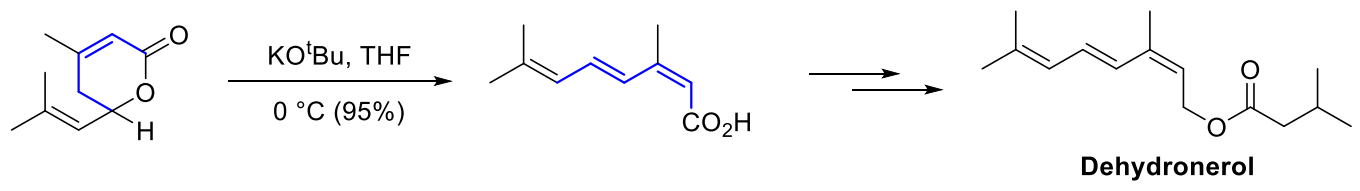

Scheme 2.4 Intramolecular elimination of $\alpha, \beta$-unsaturated lactones: (a) of parasorbic $\operatorname{acid}^{70}$ and (b) in the synthesis of dehydronerol. ${ }^{71}$

Another option for the synthesis of $Z, E$-dienoates would be to approach them from the corresponding 1-hydroxy-2,4-diene. One approach that is attractive because of its novelty, accessible starting materials and lack of application in target-driven synthesis, is the reductive opening of a substituted furan using Vitride ${ }^{\circledR}$ (sodium bis(2methoxyethoxy)aluminium hydride, a.k.a. Red-Al). This has been exemplified in the conversion of furan $\mathbf{1 2 2}$ to diene $\mathbf{1 2 3}$ (Scheme 2.5)..$^{72}$
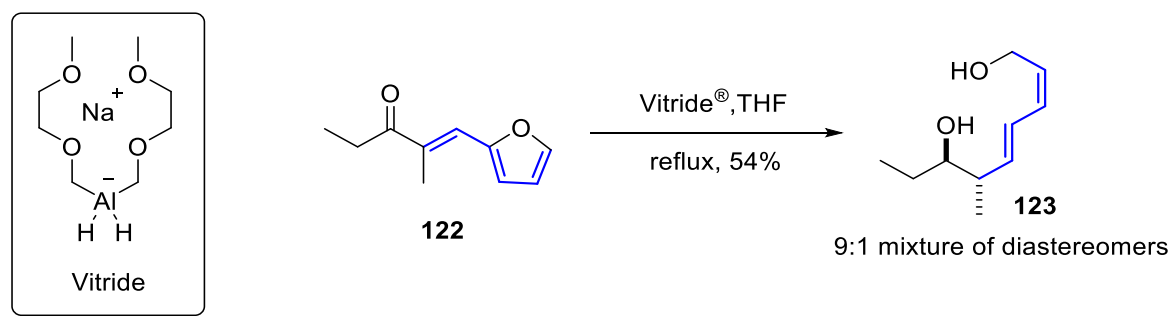

Scheme 2.5 Reductive opening of substituted furan. ${ }^{72}$

Gammill et al. proposed a mechanism that begins with aluminium coordination and reduction of the ketone 122 (Scheme 2.6). This then directs a hydride transfer resulting in ring opening and yielding the allene $\mathbf{1 2 5}$. This then undergoes an intramolecular collapse to the vinylogous aldolate intermediate 126. The aldehyde present in $\mathbf{1 2 6}$ undergoes hydride reduction to give bicyclic intermediate 127 , which on protonation provides diene product 123 . 


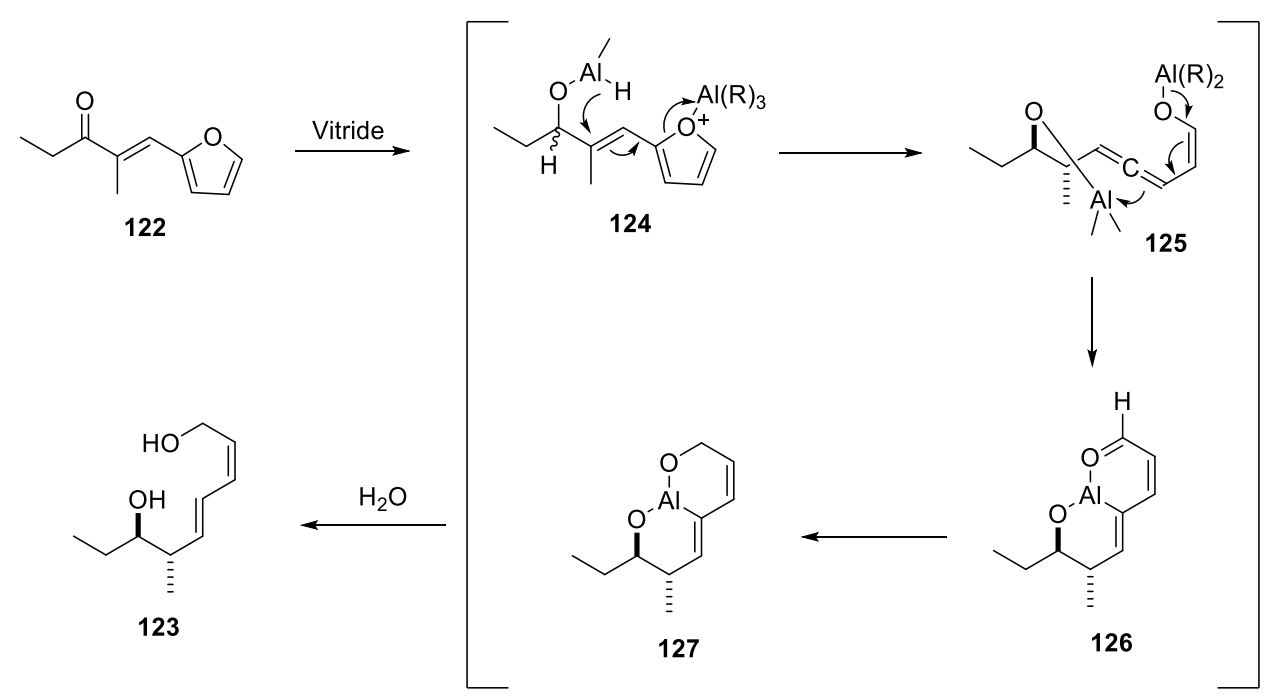

Scheme 2.6 Proposed mechanism for substituted furan reductive opening. ${ }^{72}$

\subsubsection{Strategy One: Reductive Furan Ring Opening Route}

Based on the transformation reported by Gamil and co-workers (vide supra, Scheme 2.5), it was proposed that the analogous substituted furan $\mathbf{1 2 8}$ could be utilised as a precursor to the $Z, E$-diene functionality for pateamine analogue synthesis (Scheme 2.7). The resultant diol 129 could then be selectively oxidised at the allylic alcohol with $\mathrm{MnO}_{2}$, and subsequently further oxidised to the acid $\mathbf{1 3 0}$.

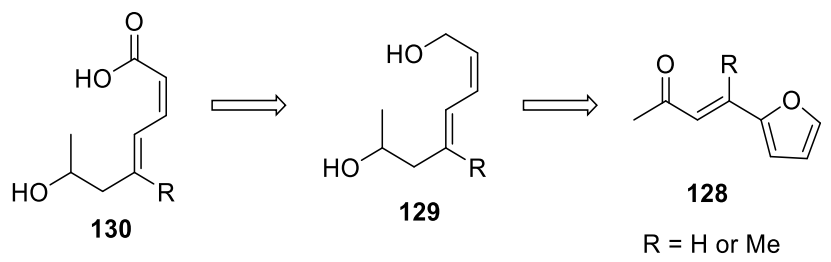

Scheme 2.7 Proposed retrosynthetic analysis of diene fragment utilising substituted furan reductive opening.

One concern was whether the Z-allylic alcohol would retain its alkene geometry upon treatment with $\mathrm{MnO}_{2}$ due to the tendency for isomerisation. However, the good cis- 
retention reported in the oxidation of (Z)-but-2-ene-1-ol with $\mathrm{MnO}_{2}$ to but-2-enal was encouraging (Scheme 2.8). ${ }^{73}$

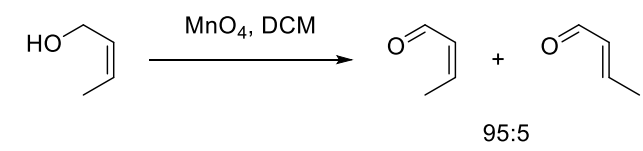

Scheme 2.8 Oxidation of an allylic alcohol with $\mathrm{MnO}_{2}$ with good retention of Zstereochemistry. ${ }^{73}$

The substituted furan 131 was prepared using two different methods from 2-furfural either: 1) by reacting with phosphorane 132 in a Wittig reaction; or 2) by treating with acetone in aqueous $\mathrm{NaOH}$ followed by acidification with $\mathrm{H}_{2} \mathrm{SO}_{4}$ in an aldol condensation reaction (Scheme 2.9). Both gave good yields and selectivity for the desired $E$-isomer.

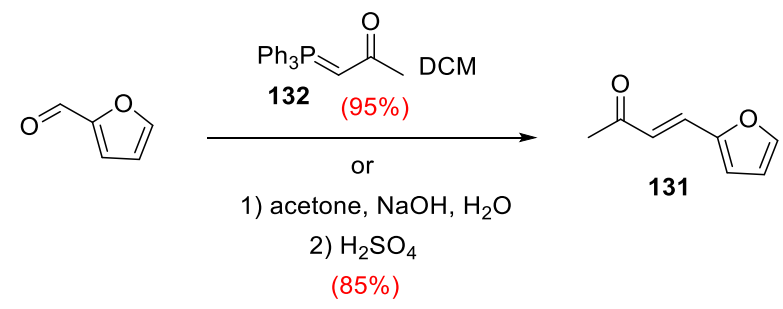

Scheme 2.9 Synthesis of substituted furan 131 via Wittig olefination or aldol condensation reaction.

Unfortunately, when the substituted furan 131 was treated with Vitride ${ }^{\circledR}$ in THF at reflux for two hours a mixture of products was obtained containing less than $5 \%$ of the desired product, based on ${ }^{1} \mathrm{H}$ NMR spectroscopy ${ }^{\dagger+}$, and all starting material consumed (Table 2.1, Entry 1). Varying temperature, reaction temperature and use of a different hydride source

${ }^{++}$This was based on analysis of the ${ }^{1} \mathrm{H}$ NMR and gCosy spectra from a crude mixture. With alkene peaks at $\left(500 \mathrm{MHz}, \mathrm{CDCl}_{3}\right): \delta 6.46-6.38(\mathrm{~m}, 1 \mathrm{H}), 6.10(\mathrm{t}, J=11.1 \mathrm{~Hz}, 1 \mathrm{H}), 5.82-5.72(\mathrm{~m}, 1 \mathrm{H}), 5.56$ $(\mathrm{td}, J=6.9,11.0 \mathrm{~Hz}, 1 \mathrm{H})$, which were consistent with ${ }^{1} \mathrm{H}$ NMR shifts of known compound, $(2 \mathrm{Z}, 4 E)$ deca-2,4-dien-1-ol. 
$\left(\mathrm{LiAlH}_{4}\right)$ provided mixtures of alkene containing compounds; unfortunately, no significant sign of the desired diol product 133 was observed (Entries 2-4).

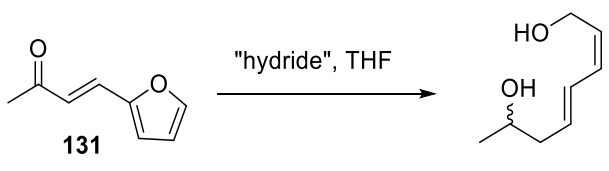

133

\begin{tabular}{cccc}
\hline Entry & $\begin{array}{c}\text { Hydride source } \\
\text { (equiv) }\end{array}$ & Conditions & NMR Yield \\
\hline $\mathbf{1}$ & Vitride $^{\circledR}(3)$ & 2 hours reflux & $<5 \%$ \\
$\mathbf{2}$ & Vitride $^{\circledR}(3)$ & 20 hours at rt & None \\
$\mathbf{3}$ & Vitride $^{\circledR}(3)$ & 20 hours at reflux & None \\
$\mathbf{4}$ & $\mathrm{LiAlH}_{4}(3)$ & 20 hours at rt & None \\
\hline
\end{tabular}

Table 2.1 Attempt at reductive opening of furanyl ketone 131.

With the failure to prepare the desired diol 133 from the furanyl ketone 131, attention was focused on a similar reductive opening of the furanyl alcohol 134. As well as providing an alternative substrate, we were motivated by the fact that this would allow the stereochemistry to be set at the $\mathrm{C} 2$ chiral centre using an asymmetric reduction of the ketone (e.g. CBS reduction). To test this, the racemic furanyl alcohol 134 was prepared by the reduction of the furanyl ketone 131 using sodium borohydride (Scheme 2.10). Disappointingly, treatment of the racemic alcohol with Vitride ${ }^{\circledR}$ in THF at a range of temperatures and varying reaction times, resulted in a mixture of alkene containing products with no significant sign of the desired diol product 133, similar to reactions with furanyl ketone 131.

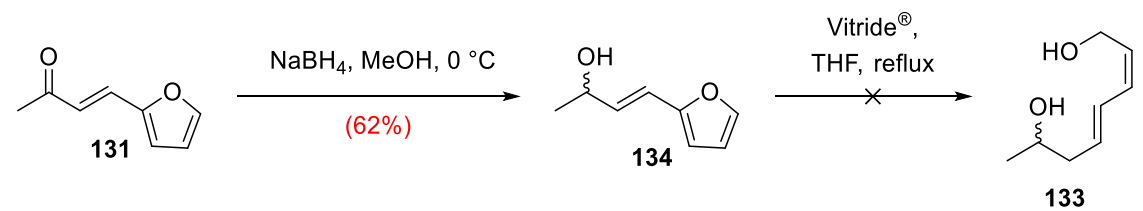

Scheme 2.10 Attempted reductive ring opening of furanyl alcohol 134. 
Exploring an alternative substituted furan was deemed appropriate, so the potentially more stable methylated compound 135 was prepared (Table 2.2). Taking the previously prepared phosphorane stabilised ylide 132 and reacting with 2-acetyl furan 136 led to no product (Entry 1). This was not highly surprising as stabilised ylides are typically not reactive towards ketones, although some success has been achieved using high temperature and pressure ${ }^{74}$ or with $\alpha$-oxo-ketones. ${ }^{75}$ Therefore, the more reactive HornerWadsworth-Emmons (HWE) reagent 137 was prepared. This was achieved by iodide substitution of chloroacetone under Finklestein conditions, followed by an Arbusov reaction with triethylphosphite, giving phosphonate 137 in $88 \%$ yield alongside a small amount (9\%) of the Perkow reaction phosphate product 138 (Scheme 2.11). ${ }^{76}$ Reaction of 2acetyl furan 136 with phosphonate 137 (pre-treated with $\mathrm{NaH}$ ) in DCM at room temperature, provided no product. Instead, recovered starting materials were obtained (Entry 2). Repeating the reaction in toluene at reflux provided the olefination product 138 in $9 \%$ yield (Entry 3). Using $\mathrm{KO} B \mathrm{Bu}$ as an alternative base in refluxing THF resulted in a very slight improvement in yield (12\% of 135) (Entry 4$)$.
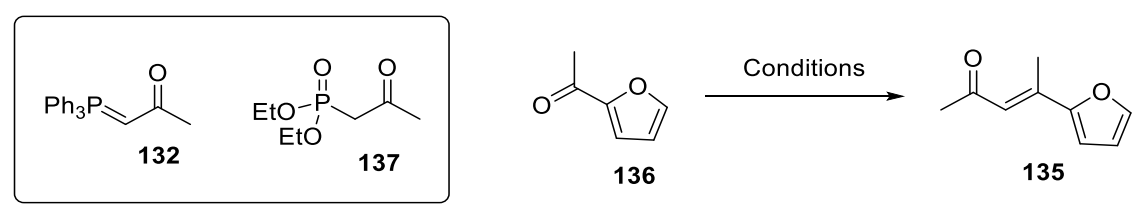

\begin{tabular}{cccc}
\hline Entry & Reagent (equiv*) & Conditions (equiv*) & Product \\
\hline $\mathbf{1}$ & Phosphorane $\mathbf{1 3 2}(1.2)$ & $\mathrm{DCM}, \mathrm{rt}, \mathbf{1 8 \mathrm { h }}$ & None \\
$\mathbf{2}$ & Phosphonate $\mathbf{1 3 7}(1.3)$ & $\mathrm{NaH}(1.3), \mathrm{DCM}, \mathrm{rt}, \mathbf{1 8} \mathrm{h}$ & None \\
$\mathbf{3}$ & Phosphonate $\mathbf{1 3 7}(1.3)$ & $\mathrm{NaH}(1.3)$, toluene, reflux, 18 h & $9 \%$ \\
$\mathbf{4}$ & Phosphonate $\mathbf{1 3 7}(1.3)$ & $\mathrm{KO}$ (Bu (1.3), THF, reflux & $12 \%$ \\
\hline
\end{tabular}

*Equivalents in relation to 2-acetyl furan.

Table 2.2 Synthesis of substituted furan 135. 


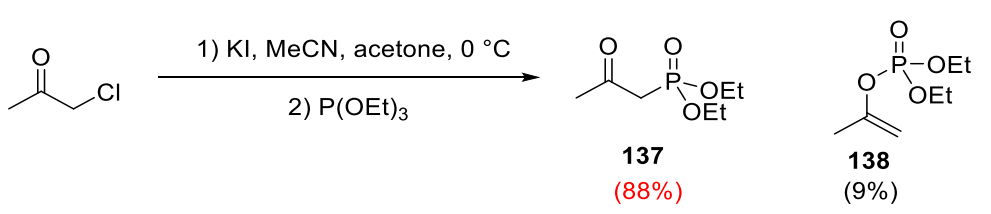

Scheme 2.11 Preparation of the HWE reagent 137.

It is interesting to note that only one stereoisomer was observed from the reaction of 2acetyl furan 136 and diethyl-(2-oxopropyl)phosphonate 137. Typically, HWE reactions with ketones give poor selectivity, so this result was surprising. Nevertheless, attempts were made to confirm the configuration of the alkene with NOE correlations. Irradiation of the $\mathrm{C} 3$ hydrogen afforded correlation to the $\mathrm{C} 1$ methyl protons, and irradiation of the hydrogens of the C5 methyl group caused enhancement of the signals of the C7 hydrogen on the furan (Figure 2.2). Both of these positive correlations would be possible in both configurations, so this did not solve the stereochemistry. However, no correlation was observed between the C3 hydrogen and the hydrogens of the C5 methyl group, which would support the designation as the E-isomer. However, the lack of an expected NOE correlation is not sufficient to unambiguously confirm the configuration.
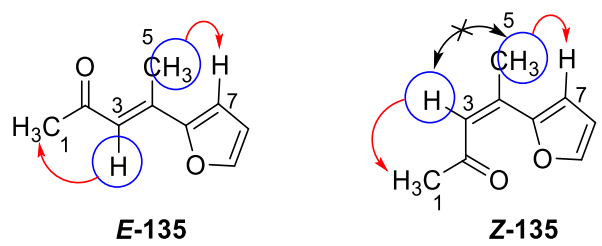

Figure 2.2 Attempt at determining alkene configuration using NOE correlations of $\mathbf{1 3 5 .}$

Proton irradiated (blue circle) with correlation observed (red arrow).

The inability to obtain sufficient $Z, E$-diene products from the reductive ring opening of substituted furans $\mathbf{1 3 1}$ or 134, as well as poor yields in the preparation of substituted furan 135, led us to abandon this approach. The use of base mediated $\delta$-lactone eliminative ring-opening to prepare the $Z, E$-diene fragment is described in the following section. 


\subsubsection{Strategy Two: $\alpha, \beta$-Unsaturated $\delta$-Lactone Ring-Opening Elimination Route}

\section{Preparation of $\delta$-methyl lactones}

As previously illustrated (Section 2.1.1), the base treatment of $\alpha, \beta$-unsaturated $\delta$-lactones containing a hydrogen at the $\gamma$-position provides $Z, E$-dieneoates as the sole stereoisomer..$^{77}$ However, the preparation of $Z, E$-dieneoates with a methyl group at the $\delta$ position had not been previously reported. We therefore set out to test the validity of this route by preparing model lactone 139 (Scheme 2.12). This was chosen due to the availability of the methyl ketone $\mathbf{1 4 0}$ starting material in the lab. Thus, allylation of methyl sec-butyl ketone using a Barbier reaction, followed by esterification of the resultant alcohol 141 with acryloyl chloride, provided diene 142 (Scheme 2.12). This was followed by ring closing metathesis (RCM) using Grubbs' second generation catalyst to give the lactone 139.

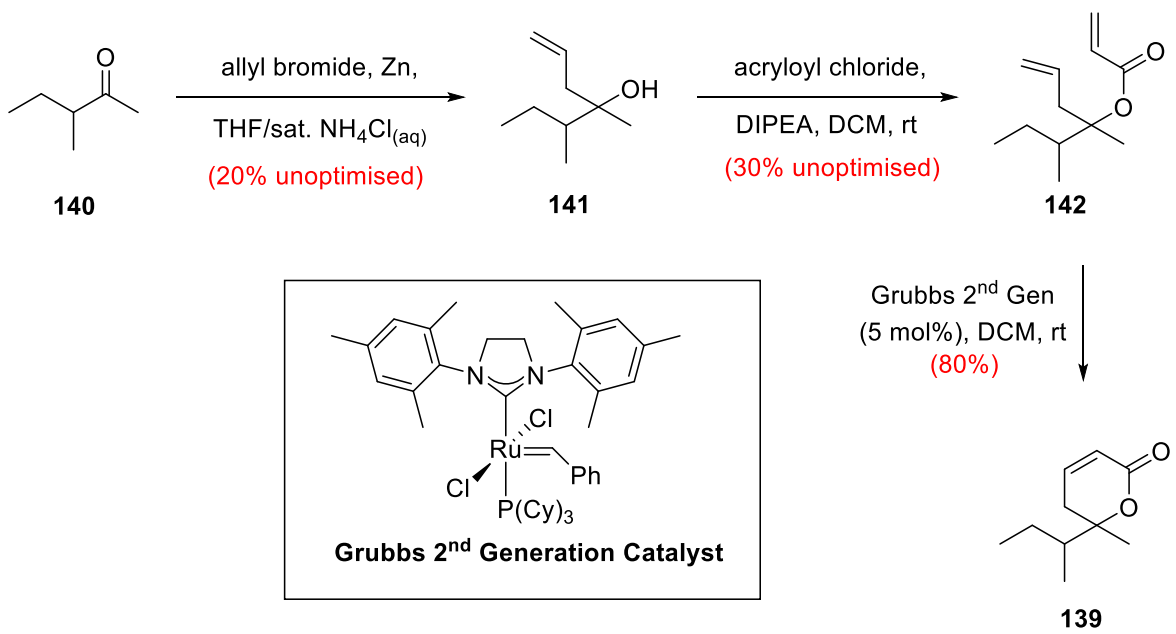

Scheme 2.12 Synthesis of model lactone 139.

Treatment of the $\delta$-lactone 139 with KHMDS in THF at $-78{ }^{\circ} \mathrm{C}$ provided the desired $Z, E$ diene 143 in 74\% yield (Table 2.3, Entry 1). Only the Z,E stereoisomer was observed, as confirmed using NOE correlations from the proton at $\mathrm{C} 3$ with the proton at $\mathrm{C} 2$ and $\mathrm{C} 5$ methyl protons. Using 3 equivalents of TBAF as the base at room temperature provided a 
similar result (Entry 2). Obtaining only one stereoisomer was significant as it suggested that the stereochemistry at the delta position of lactone $\mathbf{1 3 9}$ did not play a role in determining the stereoisomeric outcome of the $\gamma, \delta$-alkene of the diene 143. In addition, the fact that the reaction could be facilitated by $\mathrm{TBAF}$, a common reagent for silylether cleavage, allowed for a simultaneous silyl-deprotection/ring opening elimination at the later stages of the synthesis.

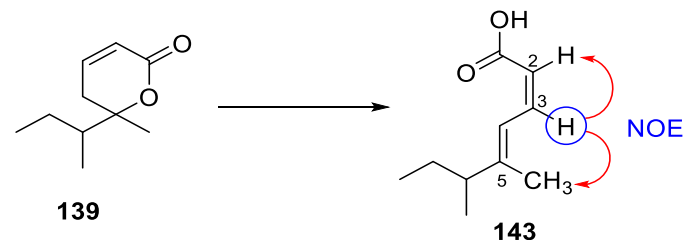

\begin{tabular}{ccccc}
\hline Entry & Base (equiv) & Conditions & Yield (ratio) & Product \\
\hline $\mathbf{1}$ & KHMDS (1.3) & THF, $-78^{\circ} \mathrm{C}, 2 \mathrm{~h}$ & $74 \%$ & Only Z,E-diene \\
$\mathbf{2}$ & $\mathrm{TBAF}(3)$ & $\mathrm{THF}, \mathrm{rt}, 2 \mathrm{~h}$ & $68 \%$ & Only Z,E-diene \\
\hline
\end{tabular}

Table 2.3 Base mediated ring-opening of model lactone 139. Proton irradiated (blue circle) with correlation observed (red arrow).

With the success of the model reaction providing the product with the desired $Z, E$-diene as the only stereoisomer, attention was directed towards the preparation of the pateamine diene fragment. The silyl protected lactone 144 was the first target, which was prepared by reaction of ethyl acetoacetate in a Barbier reaction that selectively allylated the ketone to give alcohol 145 (Scheme 2.13). This was then reduced with $\mathrm{LiAlH}_{4}$ at $0{ }^{\circ} \mathrm{C}$ to provide the diol 146. Selective silyl ether formation of the primary alcohol with the bulky TBS group gave compound 147. Treatment with acryloyl chloride gave diene 148, which was subjected to RCM with Grubbs $2^{\text {nd }}$ generation catalyst to provide lactone $\mathbf{1 4 4 .}$ 


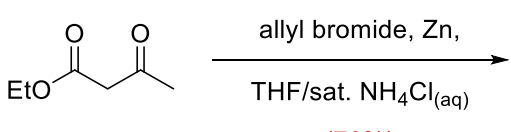

$(76 \%)$

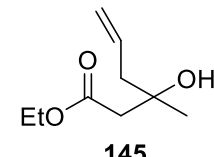

145

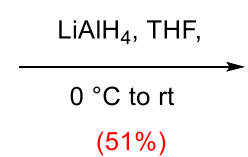

$(51 \%)$<smiles>C=CCC(C)(O)CO</smiles>

TBSCl, imid., DMF, $0{ }^{\circ} \mathrm{C}$ to $\mathrm{rt}$ $(88 \%)$

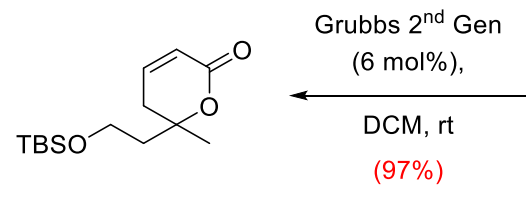

144
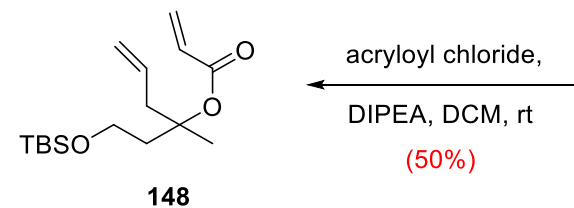

$(50 \%)$

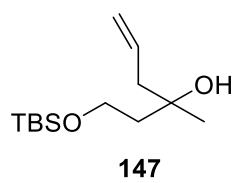

147

Scheme 2.13 Synthesis of TBS protected lactone 144.

Disappointingly, treatment of lactone 144 with a range of bases resulted in either no reaction or a mixture of many products (Table 2.4).

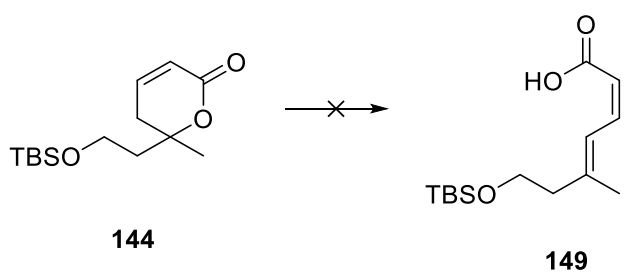

\begin{tabular}{|c|c|c|c|c|}
\hline Entry & Base (equiv) & Solvent & Temp & $\begin{array}{c}\text { Product } \\
\text { comments }\end{array}$ \\
\hline 1 & KHMDS (1.5) & THF & $-78^{\circ} \mathrm{C}$ & $\begin{array}{l}\text { Mixture with } \\
\text { some products }\end{array}$ \\
\hline 2 & KHMDS (1.5) & Diethylether & $-78^{\circ} \mathrm{C}$ & $\begin{array}{l}\text { Unidentifiable } \\
\text { mixture }\end{array}$ \\
\hline 3 & $\mathrm{NaH}$ (1.5) & Diethylether & $-78^{\circ} \mathrm{C}$ & $\begin{array}{c}\text { Significant } \mathrm{SM}+ \\
\text { mixture }\end{array}$ \\
\hline 4 & $\mathrm{NaO}^{\mathrm{t}} \mathrm{Bu}(1.5)$ & Diethylether & $-78^{\circ} \mathrm{C}$ & $\begin{array}{l}\text { Significant SM + } \\
\text { mixture }\end{array}$ \\
\hline 5 & KOtBu (1.3) & THF & $-78^{\circ} \mathrm{C}$ & $\begin{array}{l}\text { Unidentifiable } \\
\text { mixture }\end{array}$ \\
\hline 6 & KO'Bu (1.3) & $\begin{array}{c}\text { THF (18-crown-6, } \\
1.1 \text { equiv) }\end{array}$ & $\mathrm{rt}$ & $\begin{array}{l}\text { Mixture of diene } \\
\text { products }\end{array}$ \\
\hline
\end{tabular}

Table 2.4 Attempts at the base mediated ring-opening of TBS protected lactone 144 .

It was hypothesised that the bulk of the TBS group could be limiting reactivity or the lability of the silyl ether in the reaction conditions might be leading to complex mixtures. 
Therefore, a smaller non-silyl protecting group was chosen. The methoxymethyl ether seemed like an ideal choice, as it is non-bulky, stable to base, readily removed in acidic conditions and compatible with TBAF. A slightly modified approach was undertaken due to the previous route's reliance on the bulk of $\mathrm{TBSCl}$ to selectively protect the primary alcohol in the presence of a tertiary alcohol. Thus, MOM protection of 4-hydroxy-butan-2one using methoxymethyl chloride provided compound 150 (Scheme 2.14). Treatment of ketone 150 with allyl bromide and zinc in THF/saturated $\mathrm{NH}_{4} \mathrm{Cl}_{(\mathrm{aq})}$ provided tertiary alcohol 151, which was then reacted with acryloyl chloride to give diene 152. RCM with Grubbs' $2^{\text {nd }}$ generation catalyst provided lactone 153.

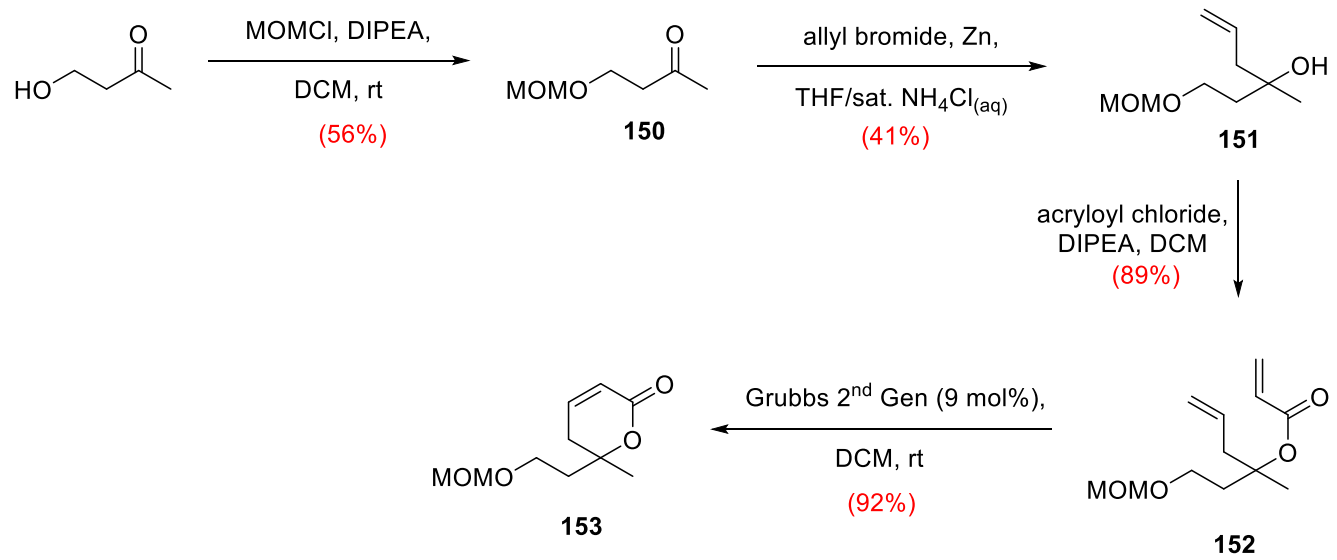

Scheme 2.14 Synthesis of MOM protected lactone 153.

Gratifyingly, when lactone 153 was treated with LiHMDS at $-78{ }^{\circ} \mathrm{C}$ the desired product was obtained (Table 2.5, Entry 1). Unfortunately, a significant amount of the undesired diastereoisomer $Z, Z$-dienoate was also made, resulting in a $Z, E: Z, Z$ ratio of $2.7: 1$. When the reaction was repeated at room temperature a $Z, E: Z, Z$ ratio of $1.7: 1$ was obtained (Entry 2). Use of TBAF as a base at room temperature provided similar results to the use of LiHMDS (Entry 3), suggesting that the stereoselectivity of the reaction is not significantly influenced by base. 


\begin{tabular}{|c|c|c|c|}
\hline Entry & Base (equiv) & Conditions & $Z, E: Z, Z$ ratio* \\
\hline 1 & LiHMDS (1.3) & $\mathrm{THF},-78^{\circ} \mathrm{C}$ to rt, $2 \mathrm{~h}$ & $2.7: 1$ \\
\hline 2 & LiHMDS (1.3) & THF, rt, 2 h & $1.7: 1$ \\
\hline 3 & TBAF (3) & THF, rt, $3 \mathrm{~h}$ & $1.6: 1$ \\
\hline
\end{tabular}

*Product isomeric ratio is based on ${ }^{1} \mathrm{H}$ NMR spectroscopy.

Table 2.5 Synthesis of MOM protected dienes Z,E-154 and Z,Z-154.

To distinguish the two diastereomers of the diene $\mathbf{1 5 4}$ it was necessary to obtain NOE correlations as the $1 \mathrm{D}{ }^{1} \mathrm{H}$ NMR signals were almost identical except for slight changes in shifts. Unfortunately, this required the differentiation of the proton shift at C3 for the two diastereomers, which was identical when the sample was run in $\mathrm{CDCl}_{3}$ as the solvent (Figure 2.3a). Satisfyingly, in deuterated benzene $\left(\mathrm{C}_{6} \mathrm{D}_{6}\right)$, the $\mathrm{C} 3$ proton became differentiated enough that NOE correlations could be obtained (Figure 2.3b). 


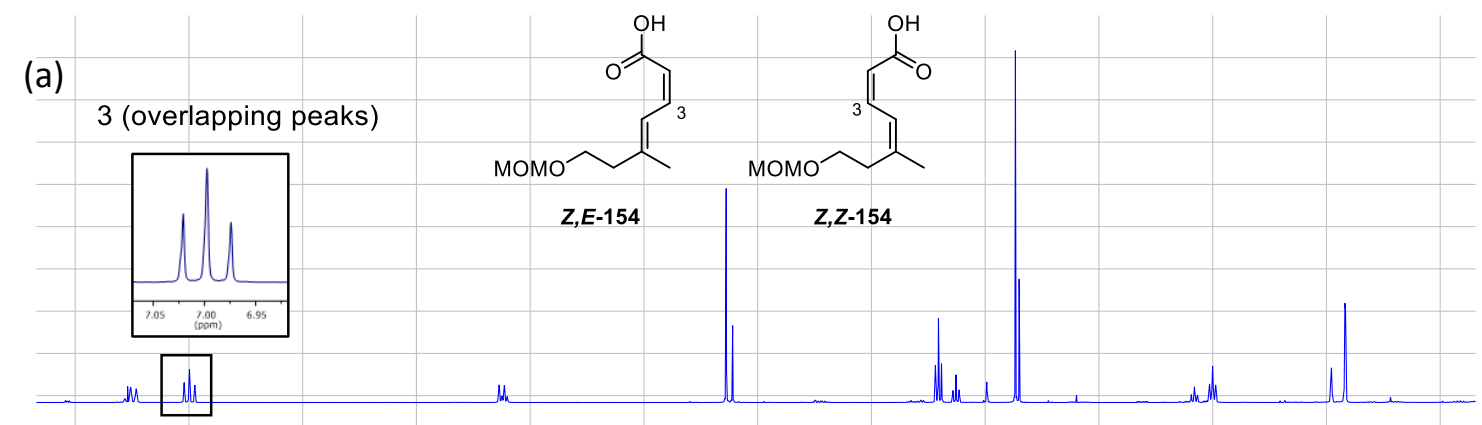

(b)

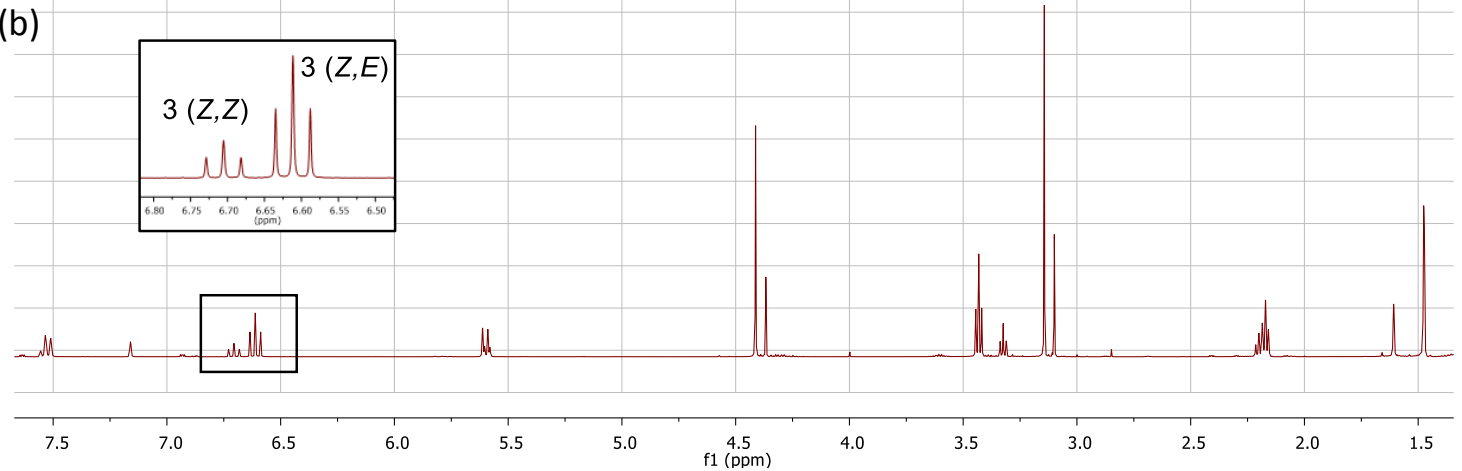

Figure $2.3{ }^{1} \mathrm{H}$ NMR of diene mixture run in $\mathrm{CDCl}_{3}\left(\mathrm{a}\right.$, blue) and $\mathrm{C}_{6} \mathrm{D}_{6}(\mathrm{~b}$, maroon) (with C3-proton shifts in black boxes).

Figure 2.4 shows the results of NOE measurements upon irradiation of the C3 proton of the two stereoisomers. Careful irradiation at $\delta 6.57$ (triplet, major component) resulted in NOE correlations with the C2 proton and C5-methyl protons (at 5.55 and 1.43 ppm, respectively), which confirms that it is the Z,E-diene 154 (Figure 2.4a). Conversely, careful irradiation of the signal at $\delta 6.66$ (triplet, minor component) resulted in NOE correlations with the C2 proton and the C6-allylic methylene protons (at 5.55 and $2.15 \mathrm{ppm}$, respectively), which confirms that it is the Z,Z-diene 154 (Figure 2.4b). 


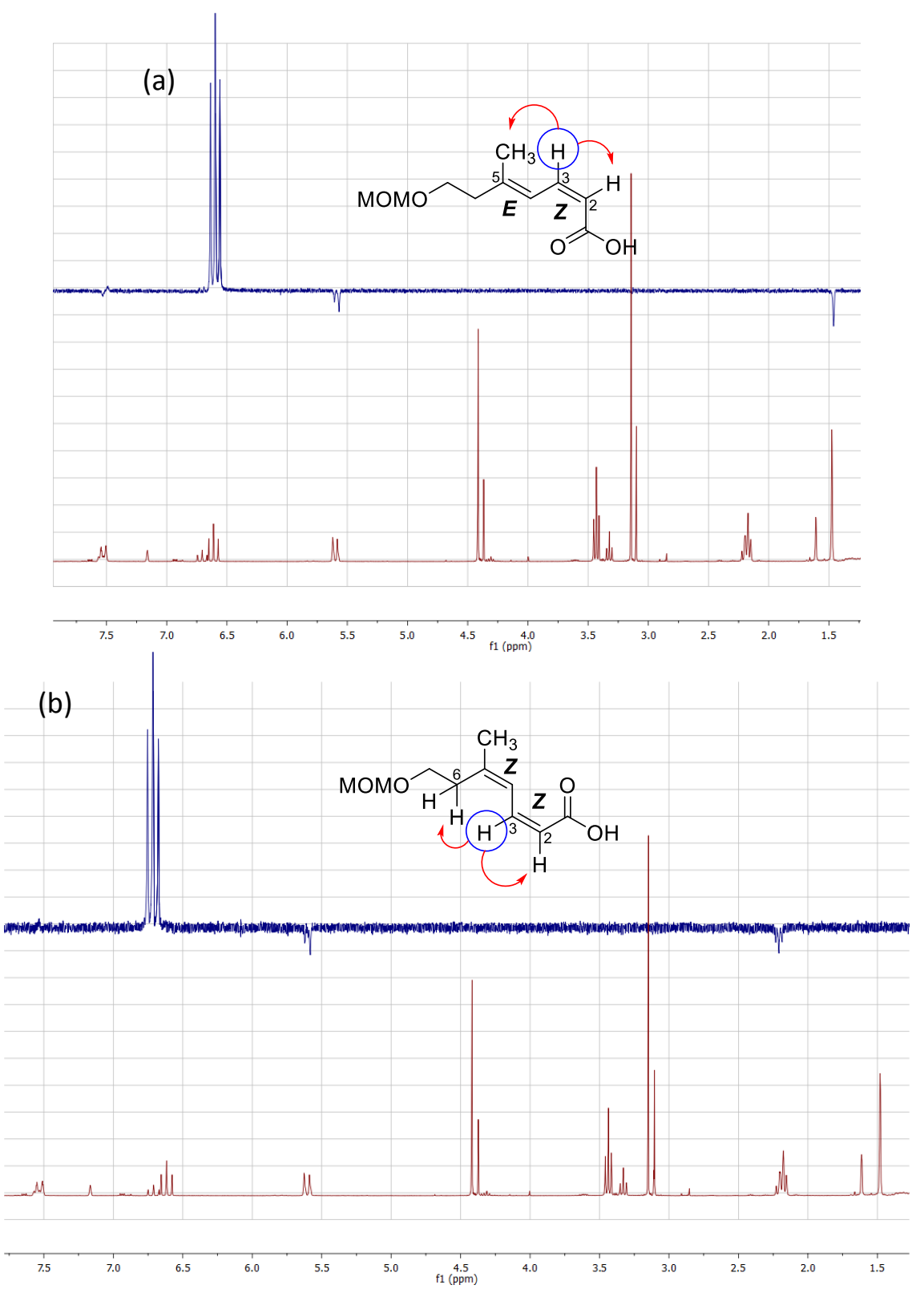

Figure 2.4 Comparison of NOE correlations (blue) and ${ }^{1} \mathrm{H}$ NMR spectrum (maroon), in $\mathrm{C}_{6} \mathrm{H}_{6}$, of diene mixture confirming configuration of $Z, E$-diene (154) as major isomer (a) and Z,Z-diene as minor isomer (b). Proton irradiated (blue circle) with correlation observed (red arrow).

Considering potential mechanisms for the elimination reaction, a concerted E2-type mechanism is plausible based on the reaction proceeding using a strong base (i.e. LiHMDS). However, it is conceivable that the reaction may proceed anywhere on the continuum of mechanistic possibilities (Figure 2.5). 


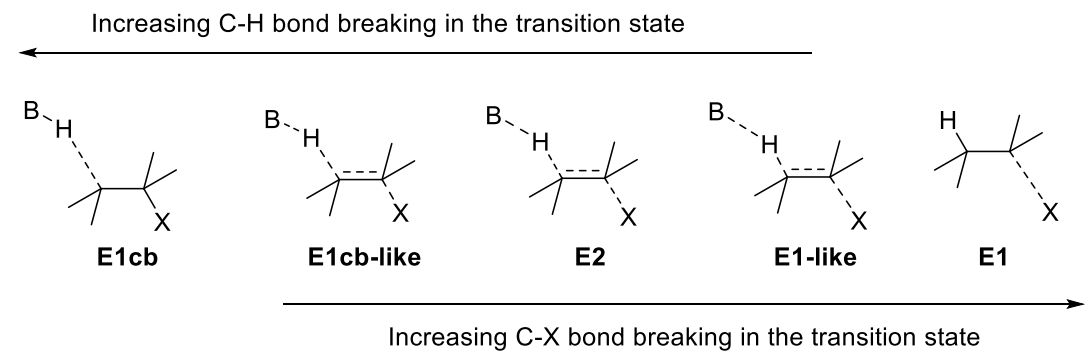

Figure 2.5 Continuum of mechanistic possibilities for $\beta$-elimination reactions. ${ }^{78}$

Nevertheless, presuming an elimination reaction that goes via a concerted route with syn elimination (i.e. E2-like mechanism), the deprotonation of two conformations may result in the different stereoisomers, one in preference to the other (Figure 2.6). In the transition state, $\mathrm{Hb}$ and the $\mathrm{C}-\mathrm{O}$ bond will be antiperiplanar. The relative orientations of the remaining hydrogen and the carbon of the lactone ring relative to the MOM-containing sidechain and methyl group will dictate the resulting geometries of the double bond. Thus, loss of $\mathrm{H}_{\mathrm{b}}$ in I will lead to the $Z, E$-diene product $Z, E$-154 and loss of the $\mathrm{H}_{\mathrm{a}}$ in II will lead to the Z,Z-diene product Z,Z-154.

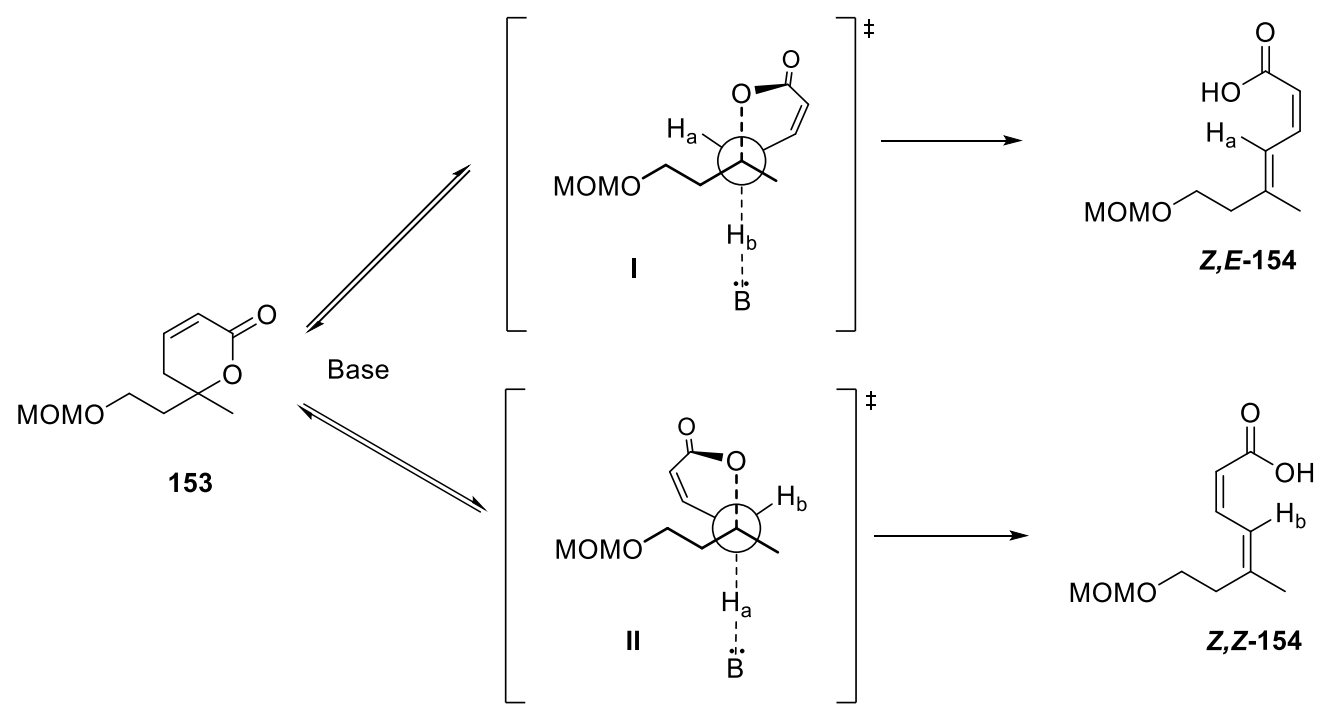

Figure 2.6 Proposed transition states en route to the two stereoisomer products, $Z, E-154$ and Z,Z-154. 
Consequently, it could be reasoned that increasing the steric bulk on the MOM-protected alcohol sidechain should improve the selectivity towards the formation of the $Z, E$-diene product. Prompted by the effect of branching in the reaction of model lactone 139 (vide supra, Table 2.3), it was decided that preparation of the gem-dimethyl lactone 155 would not only probe the factors that influence stereoselectivity, but would also provide an interesting alternative fragment for analogue synthesis. It was envisaged that the gemdimethyl groups would provide increased sterics and aid in the preference for the $Z, E$ configuration (Figure 2.7).

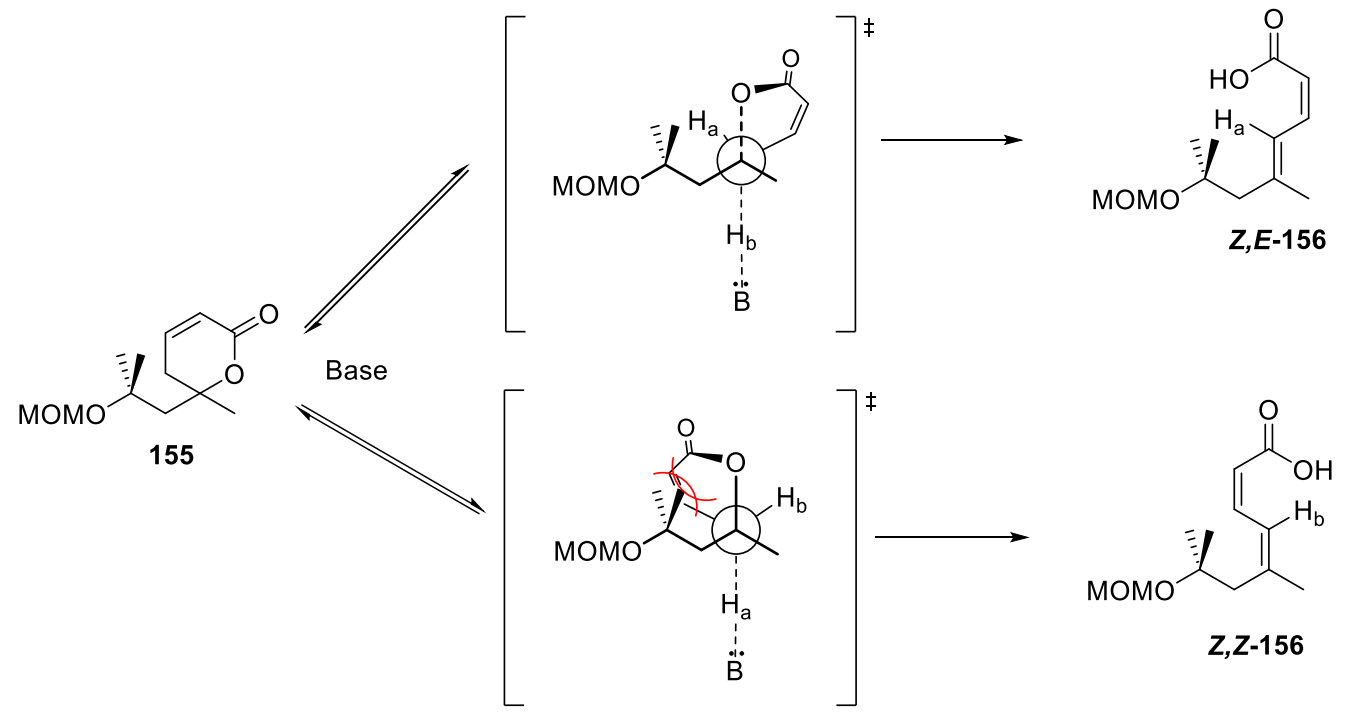

Figure 2.7 Proposed transition states en route to the two stereoisomer products, $Z, E-156$ and Z,Z-156.

The gem-dimethyl-substituted precursor 155 was synthesised using a similar reaction sequence as lactone 153 (vide supra, Scheme 2.14). Thus, MOM protection of 4-methyl-4hydroxypentan-2-one using methoxymethylchloride provided ketone 157. Treatment of ketone 157 with allylmagnesium bromide in THF provided alcohol 158, which was then reacted with acryloyl chloride to give diene 159. RCM with Grubbs' $2^{\text {nd }}$ generation catalyst provided lactone 155. 


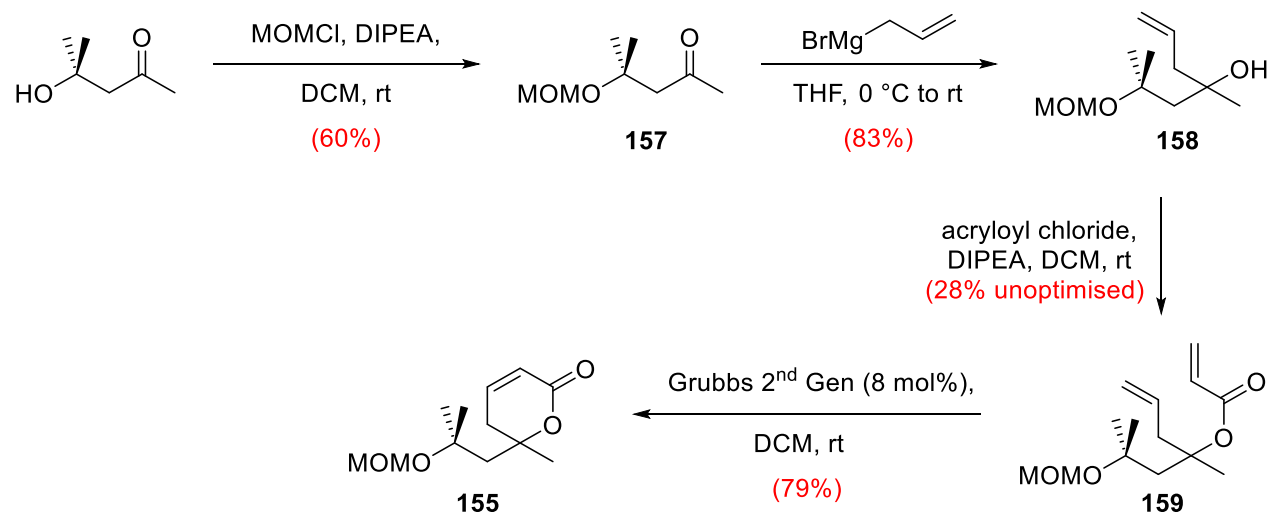

Scheme 2.15 Synthesis of gem-dimethyl MOM protected lactone 155.

Treatment of the gem-dimethyl lactone 155 with LiHMDS at $-78{ }^{\circ} \mathrm{C}$ resulted in the formation of two diastereomers in an almost equal ratio of $(1: 1.18)$ (Table 2.5, Entry 1). We were surprised to discover that the product in slight excess was in fact Z,Z-156. Therefore, not only had the introduction of the gem-dimethyl substituents worsened the ratio of the desired $Z, E$-stereoisomer, it had switched the reaction in preference (albeit slight) towards the undesired Z,Z-diene. Repeating the reaction at room temperature enhanced the ratio in favour of the undesired $Z, Z$-diene with a $Z, E: Z, Z$ ratio of $1: 1.32$ (Entry 2). Using TBAF as the base at room temperature provided a comparable result to that of LiHMDS (Entry 3).

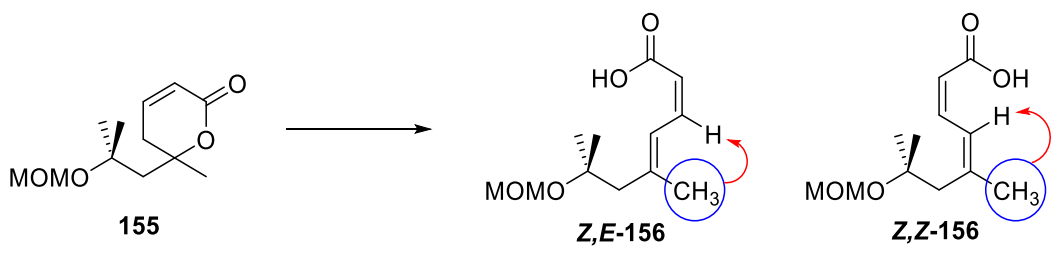

\begin{tabular}{cccc}
\hline Entry & Base (equiv) & Conditions & Z,E : Z,Z ratio* \\
\hline $\mathbf{1}$ & LiHMDS (1.3) & THF, $-78^{\circ} \mathrm{C}$ to rt, 3 h & $1: 1.18$ \\
$\mathbf{2}$ & LiHMDS (1.3) & $\mathrm{THF}, \mathrm{rt}, 2 \mathrm{~h}$ & $1: 1.32$ \\
$\mathbf{3}$ & $\mathrm{TBAF}(3)$ & $\mathrm{THF}, \mathrm{rt}, 3 \mathrm{~h}$ & $1: 1.35$ \\
\hline
\end{tabular}

*Ratio was based on ${ }^{1} \mathrm{H}$ NMR spectroscopy.

Table 2.5 Synthesis of MOM protected dienes Z,E-156 and Z,Z-156. NOE correlations used to determine alkene configuration are shown (irradiated proton in blue circle with red arrow pointing to correlating proton). 
It appears that increasing the bulk on the opposite side of the MOM-containing sidechain through the use of the gem-dimethyl lactone (155) increased the preference for the undesired Z,Z-diene compared to the linear sidechain (1:1.18 versus 2.7:1 Z,E:Z,Z at -78 ${ }^{\circ} \mathrm{C}$, respectively). Performing the reaction at higher temperature (i.e. at room temperature) in both cases consistantly resulted in an increased preference for the Z,Z-diene product. The use of TBAF as a base to facilitate the reaction gave comparable selectivity to LiHMDS, which expels the possibility that the stereoselectivity is influenced by the lithium cation interacting with both the carbonyl of the lactone and an oxygen in the MOM group. By comparison, the fact that the initial model reaction using lactone 139 gave solely the $Z, E$-diene would indicate that either the presence of the C6-methyl group, or the absence of oxygen functional groups prevented the undesired Z,Z-diene formation. This poses an interesting question as to what actually dictates the selectivity of the stereoisomers.

Since the transition state energies of the two pathways affect the relative rate of the two mechanistic pathways and hence, the isomeric ratios, the calculation of transition states could be instructive in understanding the selectivity. To do this, the lactones were modelled with a fluoride ion as a base in Gaussian and searched for a transition state energy pathway using either a Linear Synchronous Transit technique (using QST2) or optimising to a transition state (using Berny). 㧊 Two independent transition states were observed, i.e. proton abstraction and lactone ring opening (of deprotonated lactone). This suggests that the initial proton abstraction and lactone ring opening occur as independent steps, consistent with an E1cb-like mechanism (Scheme 2.16). Energies were obtained for starting material complex I (fluoride ion and lactone), the first transition state II (proton abstraction), the deprotonated intermediate III, the second transition state IV (lactone ring opening), and the product complex $\mathbf{V}$ (dienoic acid and hydrogen fluoride). Unfortunately, preliminary values obtained did not match what was observed experimentally and extensive examination of the calculations would be needed to obtain any useful information. Obtained values did, however, suggest a relatively low transition state energy for the proton abstraction, which suggests that the lactone ring opening step is the rate determining step.

¥‡Gaussian 09 software. DFT calculation performed using B3LYP, 6-31G(d,p) basis set. 


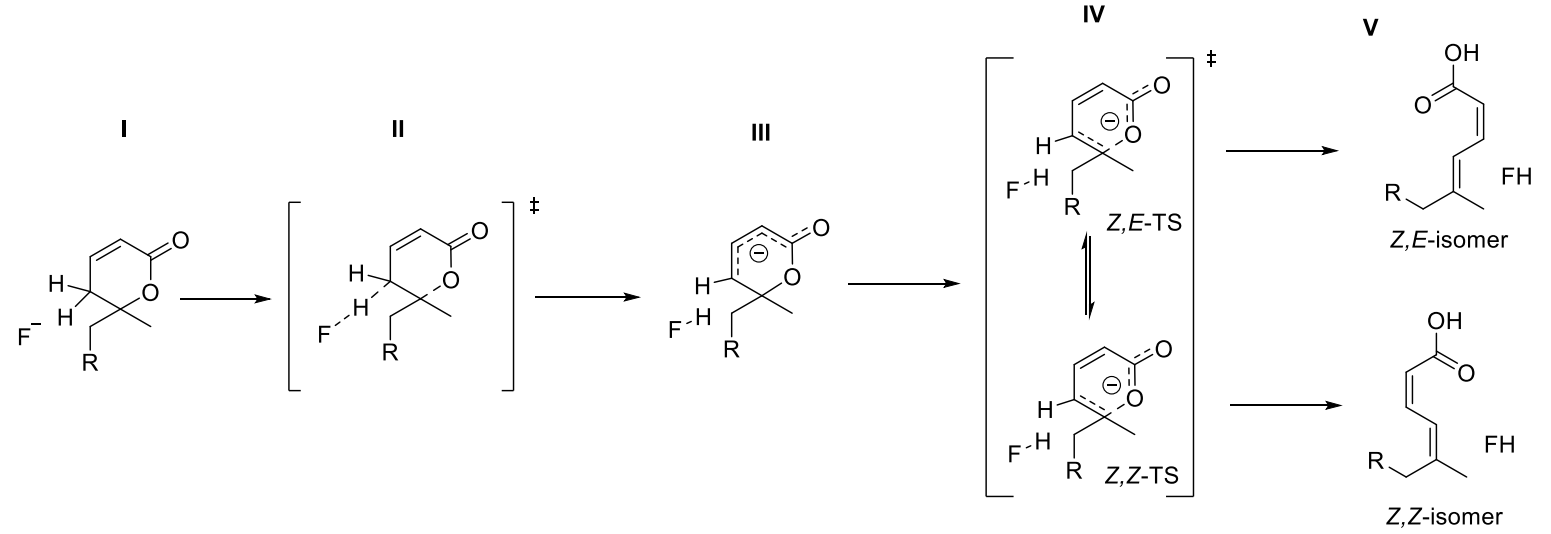

Scheme 2.16 Proposed mechanism of $\gamma$-methyl $\alpha, \beta$-unsaturated lactone ring-opening via an E1cb-like mechanism.

Preparation of another $\delta$-methyl lactone lacking an oxygen provided insight into the ring opening reaction (Figure 2.8). Thus, the outcome of the ring opening of lactone 160 should allow determination of whether the observed outcome of the initial model reaction of lactone 139 (Z,E-isomer only) was dictated by the presence of the methyl group at C6 (sterics), or the presence of the methoxymethyl functional group (electronics/complexation) as in lactone $\mathbf{1 5 3 .}$

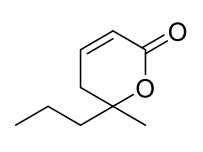

160

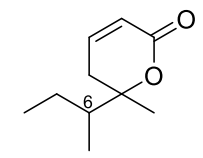

139

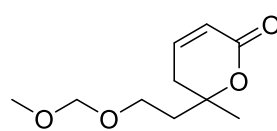

153

Figure 2.8 Comparison of lactone 160 with two previously prepared lactones 139 and 153.

Thus, lactone $\mathbf{1 6 0}$ was prepared in three steps from methyl propyl ketone (Scheme 2.17). A one-pot, two-step reaction with allylmagnesium bromide followed by acryloyl chloride provided diene 161. RCM with Grubbs' second generation catalyst then afforded the desired lactone $\mathbf{1 6 0 .}$ 


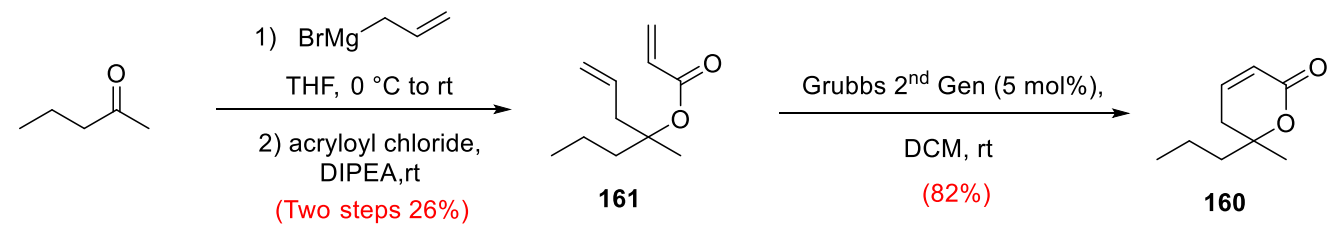

Scheme 2.17 Preparation of lactone 160.

Reaction of lactone 160 with LiHMDS at $-78{ }^{\circ} \mathrm{C}$ provided dienoic acid products, $Z, E-162$ and $Z, Z-162$ in a $1.9: 1$ ratio (Table 2.6, Entry 1 ). When the reaction was performed with TBAF at room temperature a $Z, E: Z, Z$ ratio of $1.7: 1$ was obtained (Entry 2). These results are comparable with those obtained using the MOM-containing substrate 156. Consequently, it would be reasonable to conclude that the MOM groups in substrates (153 and 155) does not significantly affect the diastereoselective outcome. In addition, this suggests that the presence of the C6 methyl group of lactone $\mathbf{1 3 9}$ prevents the formation of the undesired Z,Z-isomer.

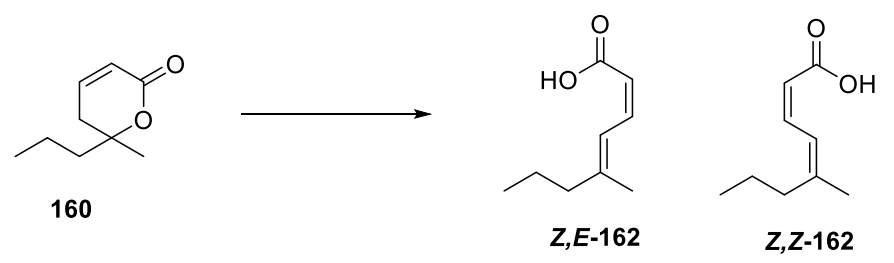

\begin{tabular}{cccc}
\hline Entry & Base (equiv) & Conditions & $Z, E: Z, Z$ ratio \\
\hline $\mathbf{1}$ & LiHMDS (1.3) & THF, $-78^{\circ} \mathrm{C}$ to $\mathrm{rt}, 2 \mathrm{~h}$ & $1.9: 1$ \\
$\mathbf{2}$ & $\mathrm{TBAF}(3)$ & $\mathrm{THF}, \mathrm{rt}, 3 \mathrm{~h}$ & $1.5: 1$ \\
\hline
\end{tabular}

${ }^{*}$ Ratio is based on ${ }^{1} \mathrm{H}$ NMR spectroscopy.

Table 2.6 Synthesis of dienes Z,E-162 and Z,Z-162.

Based on the experimental results (summarised in Table 2.7), a few preliminary conclusions can be made. As illustrated in Figure 2.9, alkyl substitution at C6 results in the $Z, E$-isomer as the sole product, branching at C7 increases selectivity for the Z,Z-isomer, and replacement of carbon at $\mathrm{C} 8$ for an oxygen has no significant effect on the selectivity of product. 
Base and Temperature

\begin{tabular}{|c|c|c|c|}
\hline Lactone & $\begin{array}{l}\text { LiHMDS (1.3 eq) } \\
-78{ }^{\circ} \mathrm{C}\end{array}$ & $\begin{array}{c}\text { LiHMDS (1.3 eq) } \\
20^{\circ} \mathrm{C}\end{array}$ & $\begin{array}{c}\text { TBAF (4 eq) } \\
20^{\circ} \mathrm{C}\end{array}$ \\
\hline & $1: 0^{*}$ & $1: 0$ & $1: 0$ \\
\hline & $2.7: 1$ & $1.7: 1$ & $1.6: 1$ \\
\hline & $1: 1.2$ & 1: 1.3 & $1: 1.4$ \\
\hline & $1.9: 1$ & - & $1.5: 1$ \\
\hline
\end{tabular}

${ }^{*}$ KHMDS was used as base.

Table 2.7 Summary of $Z, E: Z, Z$ ratios.

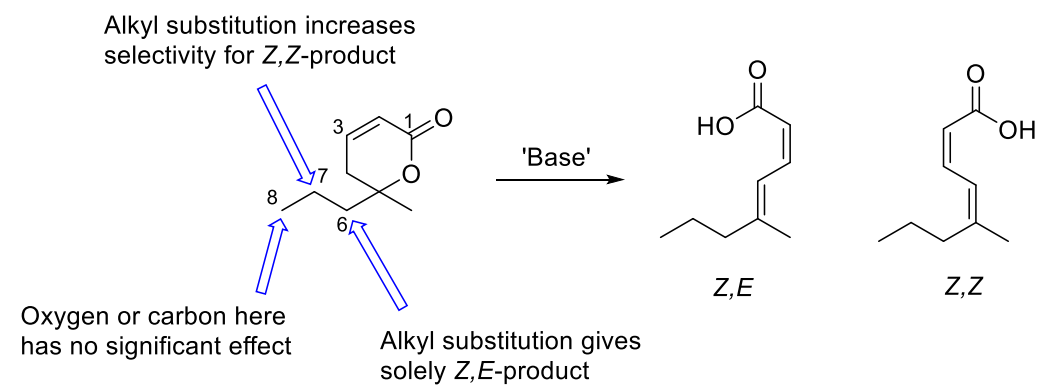

Figure 2.9 Summary of proposed apparent stereochemical effects on eliminative ring opening of $\delta$-methyl lactones.

\section{Preparation of unsaturated $\delta$-monosubstituted lactones}

In an effort to avoid the aforementioned streoselectivity issues with forming the $Z, E$-diene of pateamine, we decided to prepare a lactone lacking the $\delta$-methyl group. We chose to move to the use of a PMB-protecting group as opposed to the MOM-protecting group due to: the simiplicity of mono-protection (formed via a cyclic acetal); the extra mass to reduce volatility; and the added benefit of it being a chromophore to facilitate UV visualisation on the TLC plate. Thus, we heated a toluene solution of 1,3-propanediol in a Dean-Stark apparatus with para-anisaldehyde and catalytic para-toluene sulphonic acid (PTSA) to give the cyclic acetal 163 (Scheme 2.18). Treatment of the acetal 163 with DIBAL-H at -78 
${ }^{\circ} \mathrm{C}$ reductively cleaved the acetal to afford the mono-alcohol 164. Oxidation with a Swern oxidation provided aldehyde 165. The reaction with allylmagnesium bromide and acryloylation of the resultant alcohol in the same reaction pot afforded diene 166. Initially, ring closing metathesis using Grubbs' $2^{\text {nd }}$ generation catalyst provided lactone 167 in $40 \%$ yield. This was significantly lower than the Grubbs' catalysed lactone formation of the $\delta$ methyl lactones (79-97\%). It was hypothesised that the reduction in yield may have been caused by the lower substitution at the $\delta$-position, and therefore, the absence of a ThorpeIngold effect. ${ }^{79}$ For this reason, alternative approaches were investigated (see next section), however, later reactions with a different batch of Grubbs $2^{\text {nd }}$ generation catalyst improved the yield to $86 \%$.
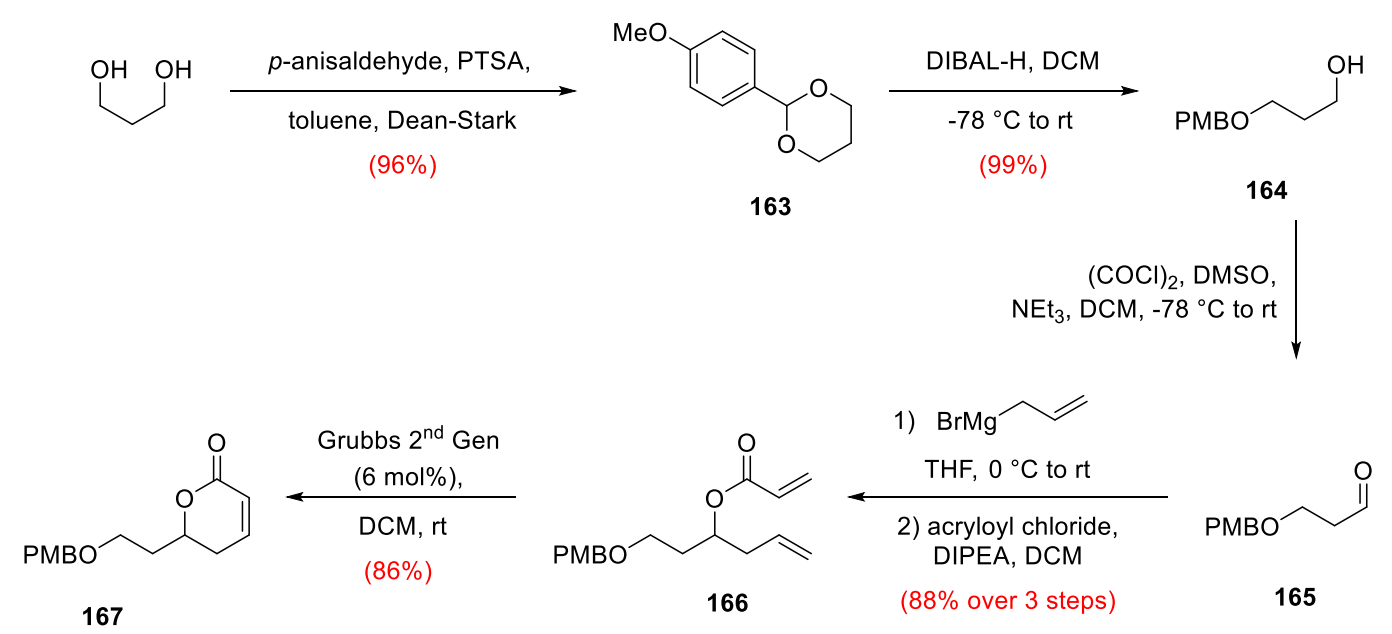

Scheme 2.18 Synthesis of lactone 167.

As hoped, the treatment of lactone 167 with TBAF at room temperature provided the $Z$,Edienoic acid 168 as the sole product in good yield (Scheme 2.19), confirming the lactone as a practical precursor to the $Z, E$-diene fragment for our pateamine analogue synthesis. 


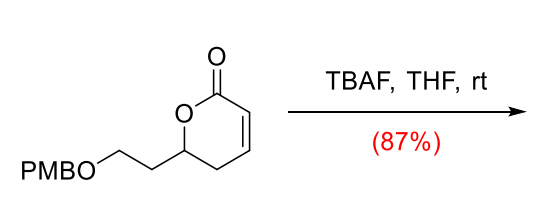

167

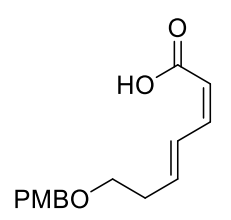

168

Scheme 2.19 Ring opening reaction of lactone 167.

Due to the initially low yields in the formation of $\delta$-lactone 167 , alternative methodologies were explored. Two approaches were considered, one used an RCM reaction on a more reactive substrate via a $\beta, \gamma$-unsaturated ester to an alternative lactone, the second avoided the use of an RCM reaction all together with an intramolecular condensation approach. These are described below.

\section{Preparation of $6, \gamma$-Unsaturated Lactone}

A recent paper by Schmidt and Kunz reported the preparation of $\beta, \gamma$-unsaturated lactones and their conversion to Z,E-dienoic acids (Scheme 2.20). ${ }^{77}$ The key improvement that this route provides over that utilising $\alpha, \beta$-unsaturated lactones is the improved reactivity of Grubbs $2^{\text {nd }}$ generation catalyst towards the more electron-rich $\beta, \gamma$-alkene of the starting material. Thus, good yields (46-96\%) are reported for the formation of a range of $\beta, \gamma$-unsaturated lactones by RCM using $1 \mathrm{~mol} \%$ catalyst loading. In addition, the higher acidity of the $\alpha$-hydrogens of the $\beta, \gamma$-unsaturated lactones, compared to the $\gamma$ hydrogens in the $\alpha, \beta$-unsaturated lactones, was presumed to improve the base-promoted lactone opening reaction. 


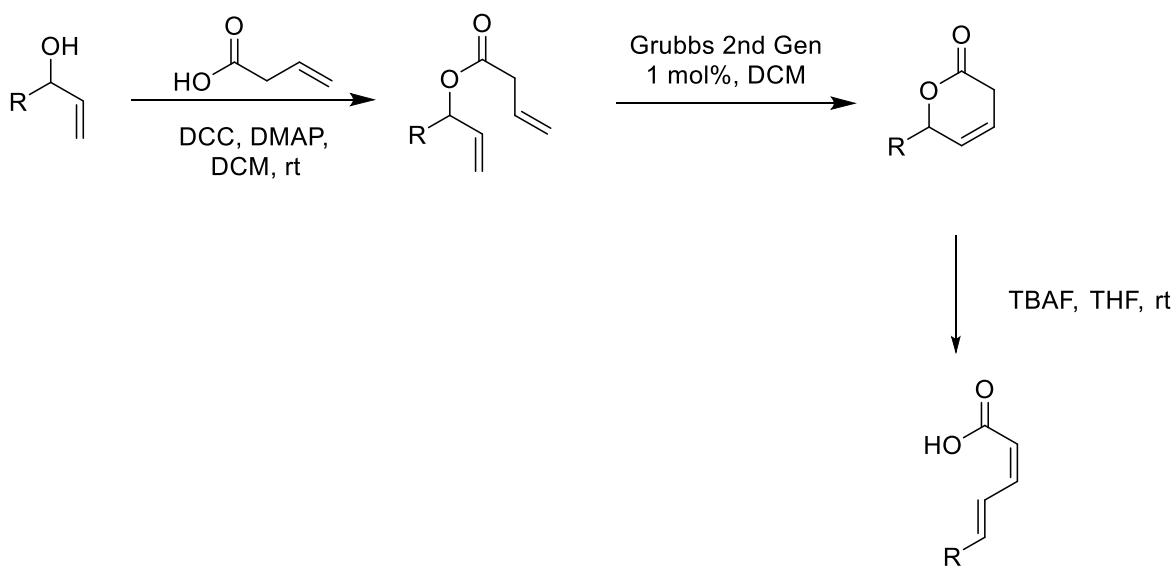

Scheme 2.20 General reaction for $Z, E$-dienoic acid formation via $\beta, \gamma$-unsaturated lactone. ${ }^{77}$

In order to explore the eliminative ring opening of a $\beta, \gamma$-unsaturated lactone in the pateamine analogue setting, the lactone 169 was prepared (Scheme 2.21). Thus, reaction of aldehyde 165 with vinylmagnesium bromide provided alcohol 170. Esterification with vinyl acetic acid, employing 1-ethyl-3-(3-dimethylaminopropyl)carbodiimide (EDCI) and 4-(N,N-dimethylamino)pyridine (DMAP) provided diene 171. Unfortunately this was accompanied with significant amounts of the conjugated dienes E-172 and Z-172. Separation of the isomers proved difficult, however, treatment of this mixture with Grubbs' $2^{\text {nd }}$ generation catalyst provided the $\beta, \gamma$-unsaturated lactone 169 which could then be purified from the other materialss. Due to performing the RCM reaction on an isomeric mixture (in which ratios were difficult to determine due to multiple overlapping peaks) it was not possible to quantify a yield from the reaction, however, significant lactone product was obtained using only $2 \mathrm{~mol} \%$ catalyst. Treatment of lactone 169 with TBAF in THF, provided the desired diene 168 in $44 \%$ yield. Nevertheless, based on the need to optimise the esterification reaction to minimise isomerisation of diene $\mathbf{1 7 1}$ and the low yield obtained from the lactone opening reaction this route was abandoned.

s\&Unreacted starting material, with no strong evidence for the presence of 5-membered lactone RCM product 173. 


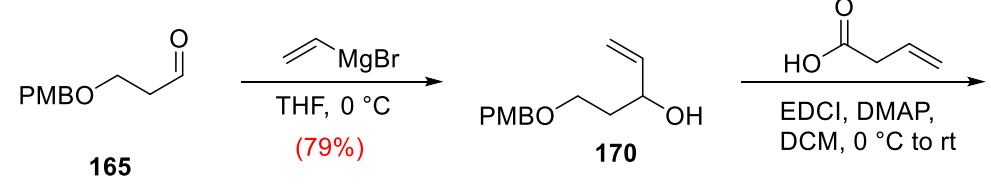<smiles>CC(C)(C)OCC/C=C/C=C/C(=O)O</smiles><smiles>CCCCC1C=CCC(=O)O1</smiles><smiles>CCCCCC1C=CC(=O)O1</smiles>

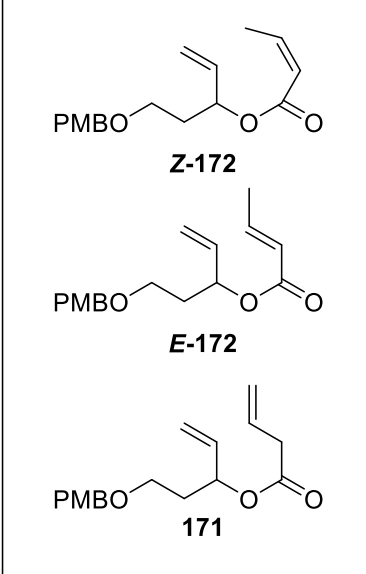

$(16 \%$, two steps
unoptimised) noptimised

Scheme 2.21 Preparation of $\mathbf{1 6 8}$ via $\beta, \gamma$-unsaturated lactone 169.

\section{Intramolecular condensation approach}

An alternative method that avoids the need for an RCM catalyst altogether, was used by Ciabatti and co-workers in their preparation of the Z,E-dienoic acid side chain $\mathbf{1 7 4}$ for ramolanin A2 (Scheme 2.22). ${ }^{80}$ Their synthesis commenced with Weiler dianion chemistry $^{81}$ of ethylacetoacetate and isovaleraldehyde 175 , to give the $\delta$-hydroxy $\beta$ ketoester 176. Reduction of the ketone with sodium borohydride provided the racemic diol 177. Refluxing in toluene with catalytic PTSA resulted in an intramolecular transesterification to give intermediate lactone 178, with subsequent condensation to furnish $\alpha, \beta$-unsaturated lactone 179. Treatment with TBAF provided the $Z, E$-dienoic acid 174. This method was attractive as it used much more readily available reagents, and reactions with easier work-up than the previous lactone formation strategy (i.e. acryloyl ester formation/RCM) which would facilitate easier scale-up. 


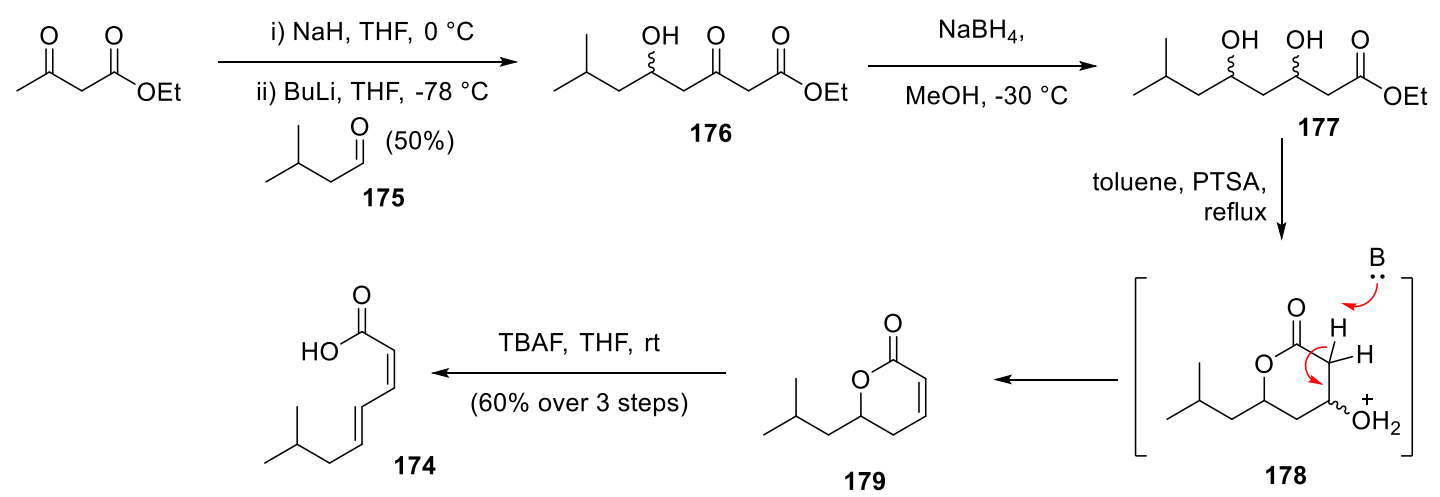

Scheme 2.22 Synthesis of sidechain for ramolanin A2.80

Consequently, we proposed that we could use the same methodology with previously synthesised aldehyde $\mathbf{1 6 5}$ to provide a starting material for this alternate route to the desired lactone 167 (Scheme 2.23).

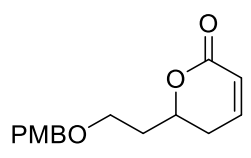

167

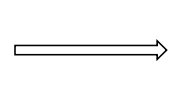

(1)

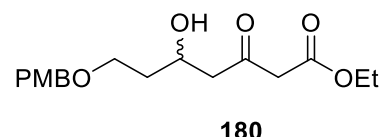

180

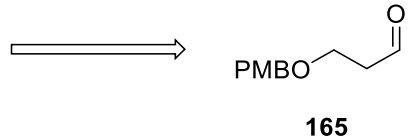

165

Scheme 2.23 Retrosynthetic analysis of alternate lactone preparation.

Starting with ethyl acetoacetate and forming the dianion using $\mathrm{NaH}$ then $n$-BuLi, allowed coupling with aldehyde 165, and gave the $\delta$-hydroxy $\beta$-ketoester 180 (Scheme 2.24). Reduction of the ketone with sodium borohydride provided diol 181 as a mixture of diastereomers. ${ }^{* * *}$ Overnight reflux in toluene and catalytic PTSA afforded only trace amounts of lactone 167, with a mixture of degraded and PMB cleaved products observed.

**** The success of the reduction reaction to afford 181, was characterised with ${ }^{1} \mathrm{H}$ NMR by observing the shift of a singlet at $3.50 \mathrm{ppm}$ ( $\alpha$-proton), with retention of peaks corresponding to the PMB and ethyl ester groups. 

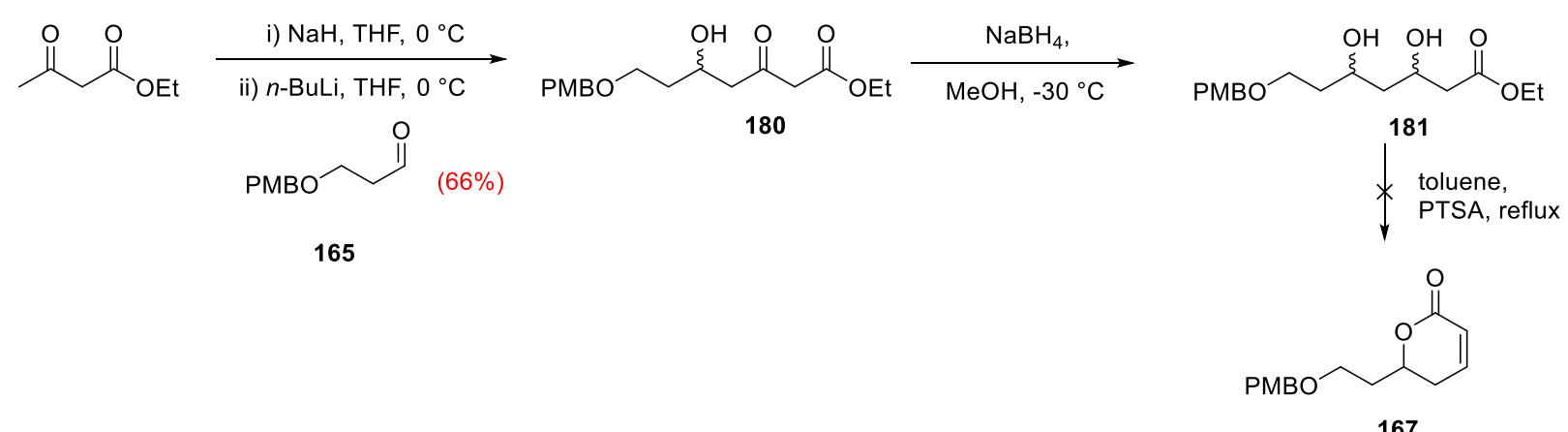

Scheme 2.24 Attempt at preparing lactone 167 using alternate method.

The failure in obtaining the desired lactone product 167 is almost certainly caused by the lability of the PMB ether in the reaction conditions (i.e. PTSA and heat) and, indeed, acidcatalysed cleavage is an established method for PMB ether deprotection (e.g. TFA in

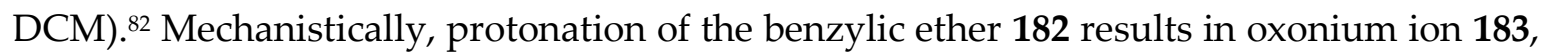
which fragments to the benzyl carbenium ion 184 and release of alcohol 185 (Scheme 2.25). The benzyl carbenium ion $\mathbf{1 8 4}$ then reacts with any nucleophile to afford the PMB adduct 186 .

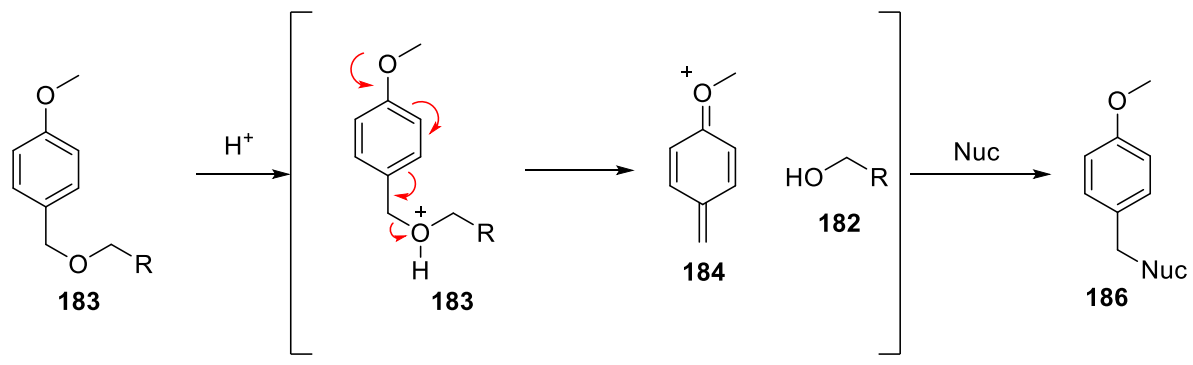

Scheme 2.25 Acid catalysed cleavage of PMB ether.

The lability of the PMB ether in strong acid urged us to examine whether the desired transformation (intramolecular transesterification/condensation) would occur using the buffered sulfonic acid, pyridinium para-toluene sulfonate (PPTS). In addition, the methyl ester was sought in an attempt to assist with reactivity, since methyl esters hydrolyse at a faster rate than ethyl esters..$^{83}$ Thus, starting with methyl acetoacetate and reacting the dianion with aldehyde 165 gave $\delta$-hydroxy $\beta$-ketoester 187 (Scheme 2.26). Reduction with sodium borohydride gave a mixture of stereoisomers of diol 188. Treatment of this 
mixture with catalytic PPTS in toluene at reflux overnight provided the desired lactone 167 in an uninspiring $20 \%$ isolated yield. The reaction products appear to be a mixture of unreacted starting material, PMB ether cleaved products, and stereoisomers of the intermediate lactone 189. In light of this lack of success, as well as the reasonable results subsequently obtained with the RCM approach, this route was not pursued.

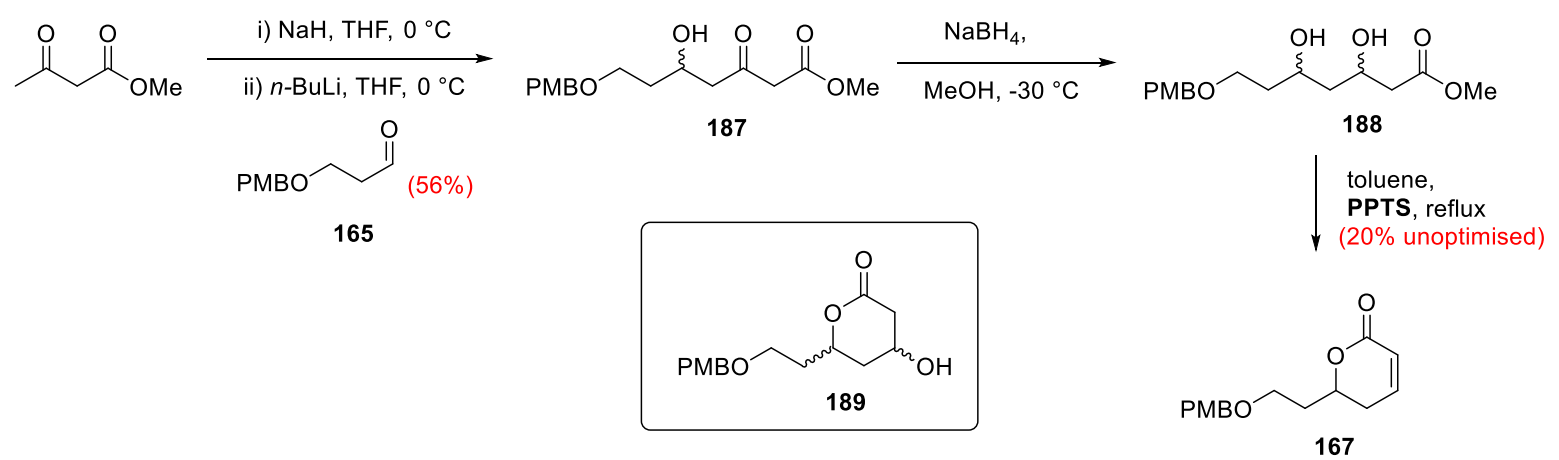

Scheme 2.26 Alternative preparation of lactone 167, using PPTS as transesterification catalyst.

\subsubsection{Summary}

In summary, a number of routes to $Z, E$-dienoates were investigated for the synthesis of fragment I of pateamine analogues. 2-Substituted furans were prepared and tested in a reductive ring opening. A range of conditions were attempted, however, we were unable to produce adequately pure products from this methodology and as a result this route was abandoned. $\alpha, \beta$-Unsaturated lactone 139 was synthesised as a model to test reaction conditions and diastereomeric outcome of base-induced ring opening. The reaction resulted in only one stereoisomer, the desired Z,E-dienoic acid 143. A TBS-protected desmethyl lactone 144 was found to be incompatible in this reaction with a range of bases. Fortunately, when the same lactone was prepared with MOM protection (153) instead of TBS, the product was obtained. However, unlike with the model lactone 139, a mixture of $Z, E$ and $E, E$ stereoisomers was obtained. Interestingly, a gem-dimethyl variant $\mathbf{1 5 5}$ resulted in an increased proportion of the undesired $Z, Z$ stereoisomer. The reactions done at room temperature and at $-78^{\circ} \mathrm{C}$, as well as the use of two distinctly different bases (TBAF and LiHMDS), were used in an attempt to understand the stereoselectivity. In 
addition, energy reaction profiles of the different reaction pathways that result in the different stereoisomers were calculated on Gaussian. Unfortunately, these calculations failed to explain the observed experimental selectivities, but suggests the reaction proceeds following an E1cb or E1cb-like mechanism.

Unable to obtain good stereoisomeric selectivity from $\alpha, \beta$-unsaturated lactones, the lactone lacking the methyl group at the $\delta$-position was prepared and, in doing so, successfully prepared Z,E-dienoic acid 167 as the sole product.

In an effort to improve the scalability of a lactone lacking the methyl group at the $\delta$ position, two alternative methods were explored. Firstly, $\beta, \gamma$-unsaturated lactone 169 was prepared to improve the RCM by Grubbs' $2^{\text {nd }}$ generation catalyst. However, issues with isomerisation in the esterification step, hindered the effectiveness of this strategy. Secondly, a PTSA catalysed tandem intramolecular transesterification/condensation reaction was attempted on diol 181. Unfortunately, the PMB ether was labile under these conditions.

In conclusion, lactones containing a methyl group at the $\delta$-position resulted in an undesirable mixture of diastereomers in the elimination ring opening. From our investigation, it would appear that the only solution to improving the selectivity in methylated variants, would be to incorporate the methyl group next to the lactone (i.e. with a vicinal relationship with the lactone ring). As this is not the most obvious analogue to seek, we opted to continue with des-methyl lactone 167 as the preferred analogue fragment. 


\subsection{Synthesis of C1-C7 (Saturated Acid-Yne) Fragment}

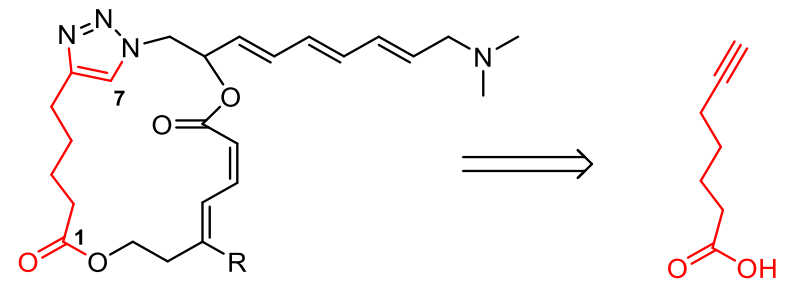

\subsubsection{Introduction}

The desire to access novel analogues that were more easily synthesised led us to target the triazole heterocycle in place of the thiazole that is present in pateamine. The triazole can be formed using the copper-catalysed azide alkyne cycloaddition (CuAAC) and thus, the target fragment for $\mathrm{C} 1-\mathrm{C} 7$ for our target analogue, 6-heptynoic acid 190, contains a terminal alkyne.

Terminal alkynes have been utilised in a diverse range of reactions in organic synthesis. ${ }^{84}$ The acidity of the terminal alkyne facilitates its use as a nucleophile with a variety of electrophiles, such as carbonyls, epoxides and alkylating agents. Terminal alkynes can also be chemoselectively activated via complexation with transition metals to perform a range of reactions, including olefin metathesis, ${ }^{85}$ Sonogashira cross coupling, ${ }^{86}$ and goldcatalysed cyclisations. ${ }^{87}$

Several methods have been utilised to prepare alkynes. Many of these methods make use of phosphorus chemistry such as thermal decomposition of $\alpha$-keto phosphoranes (Scheme 2.27a) ${ }^{88}$ or by use of phosphorus containing reagents that react with carbonyl compounds (e.g. via the Seyferth-Gilbert homologation reaction as in Scheme 2.27b). ${ }^{89}$ 
a)

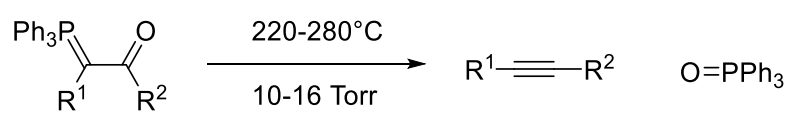

b)<smiles>[R]C#CCOc1ccc(C(C)(C)C)cc1</smiles>

Scheme 2.27 Examples of alkyne preparation using (a) thermal decomposition of an $\alpha$ keto phosphorane and (b) the Seyferth-Gilbert homologation reaction.

With regards to the formation of terminal alkynes, homologation reactions of carbonyl compounds are widely used due to the ease of preparation and versatility their substrates. ${ }^{90}$ Two reactions were chosen for exploration, namely the Corey-Fuchs method and the use of the Ohira-Bestmann reagent for the preparation of the required terminal alkyne. Both of these reactions achieve the same result, i.e. the one-carbon homologation and conversion of an aldehyde to a terminal alkyne. The Corey-Fuchs reaction involves the formation of a dibromomethyl phosphonium ylide 191 from carbon tetrabromide, triphenylphosphine and zinc powder, with reactivity similar to a Wittig reagent. The reaction with the aldehyde 192 forms a 1,1-dibromoalkenyl product 193 (Scheme 2.28). After isolation, the gem-dibromo alkene 193 is treated with two equivalents of $n$-BuLi, then quenched with aqueous ammonium chloride to afford the alkyne product 194.

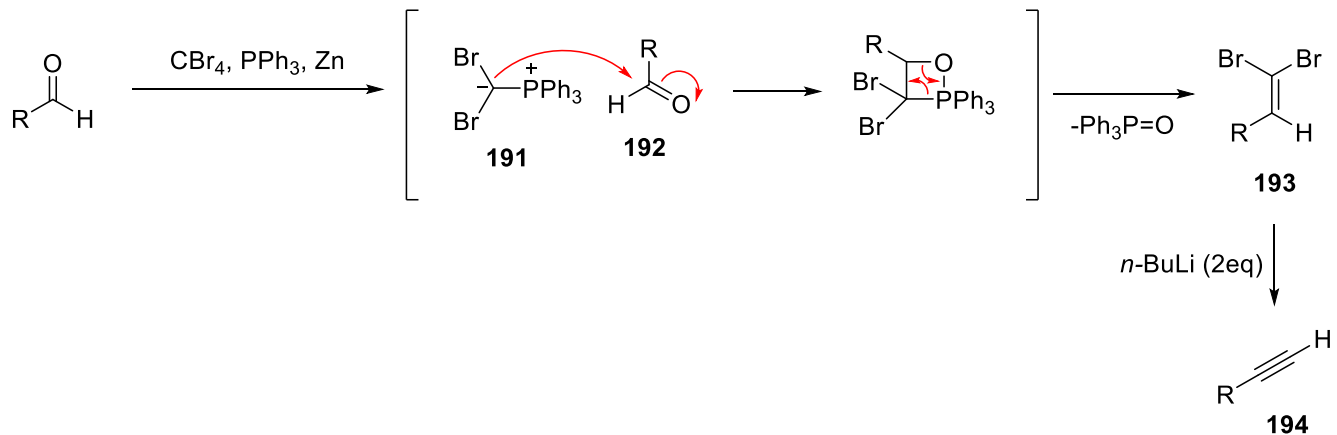

Scheme 2.28 The two-step Corey-Fuchs reaction.

Alternatively, the Ohira-Bestmann reagent 195 achieves the conversion in one step (Scheme 2.29). This reagent is a variant of the Seyferth-Gilbert reagent, with many 
advantages such as a greater substrate scope, milder conditions and stability to storage. ${ }^{90}$ Thus, the reaction of the Ohira-Bestmann reagent 195 with potassium carbonate in methanol forms the Seyferth-Gilbert phosphonate intermediate 196 in situ which then reacts with an aldehyde 197 to form phosphonate 198 (Scheme 2.29). Subsequent oxaphosphetane (199) formation and retro [2+2] cycloaddition provides diazoalkene 200. Liberation of $\mathrm{N}_{2}$ gas results in carbene formation with subsequent 1,2-migration of hydrogen providing the terminal alkyne 201.

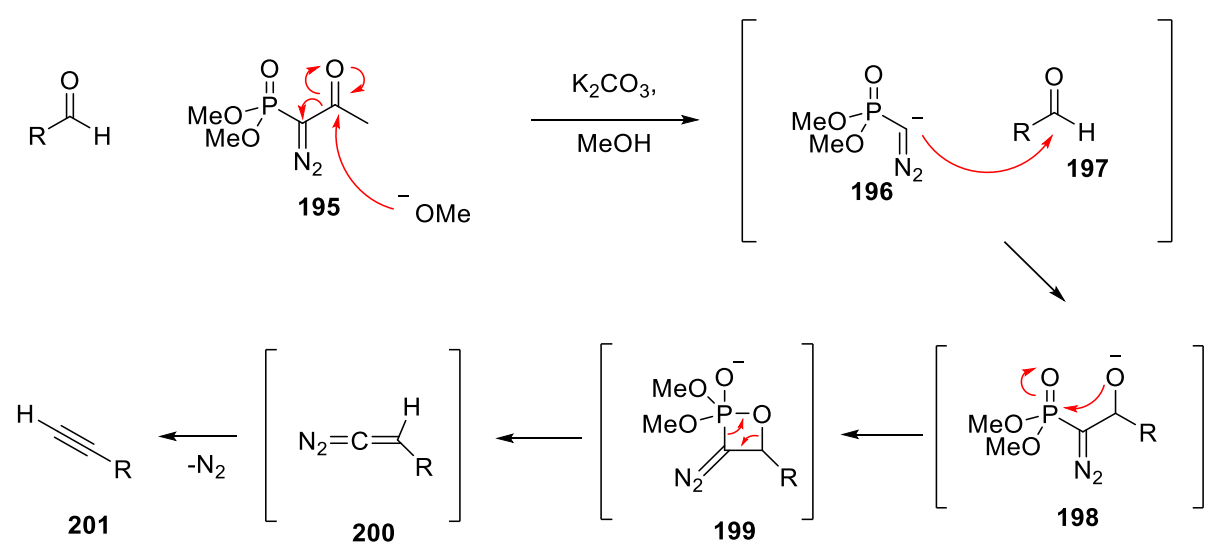

Scheme 2.29 Reaction mechanism for alkyne formation with Ohira-Bestmann reagent.

The following section describes the synthesis of heptynoic acid 190 utilising these two methodologies.

\subsubsection{Preparation of C1-C7 Fragment}

The preparation of fragment 190 started with the transesterification of $\varepsilon$-caprolactone with methanol to give the methyl ester 202 (Scheme 2.30). This was followed by oxidation of the hydroxyl group using catalytic TEMPO and BAIB as the co-oxidant to give aldehyde 203. This was then treated with carbon tetrabromide, zinc powder and triphenylphosphine to provide the vinyl gem-dibromo product 204, the first step of the Corey-Fuchs reaction. Treatment of dibromide 204 with two equivalents of n-BuLi resulted in a mixture of products due to nucleophilic substitution at the methyl ester. 
Treatment with the non-nucleophilic, but weaker base LiHMDS gave no reaction. It was then decided to first saponify the methyl ester 204 using $\mathrm{LiOH} / \mathrm{H}_{2} \mathrm{O}$ to provide the carboxylic acid 205. This removed the electrophilicity of the carbonyl group which was then treated with three equivalents of $n$-BuLi to provide the alkyne fragment 190.

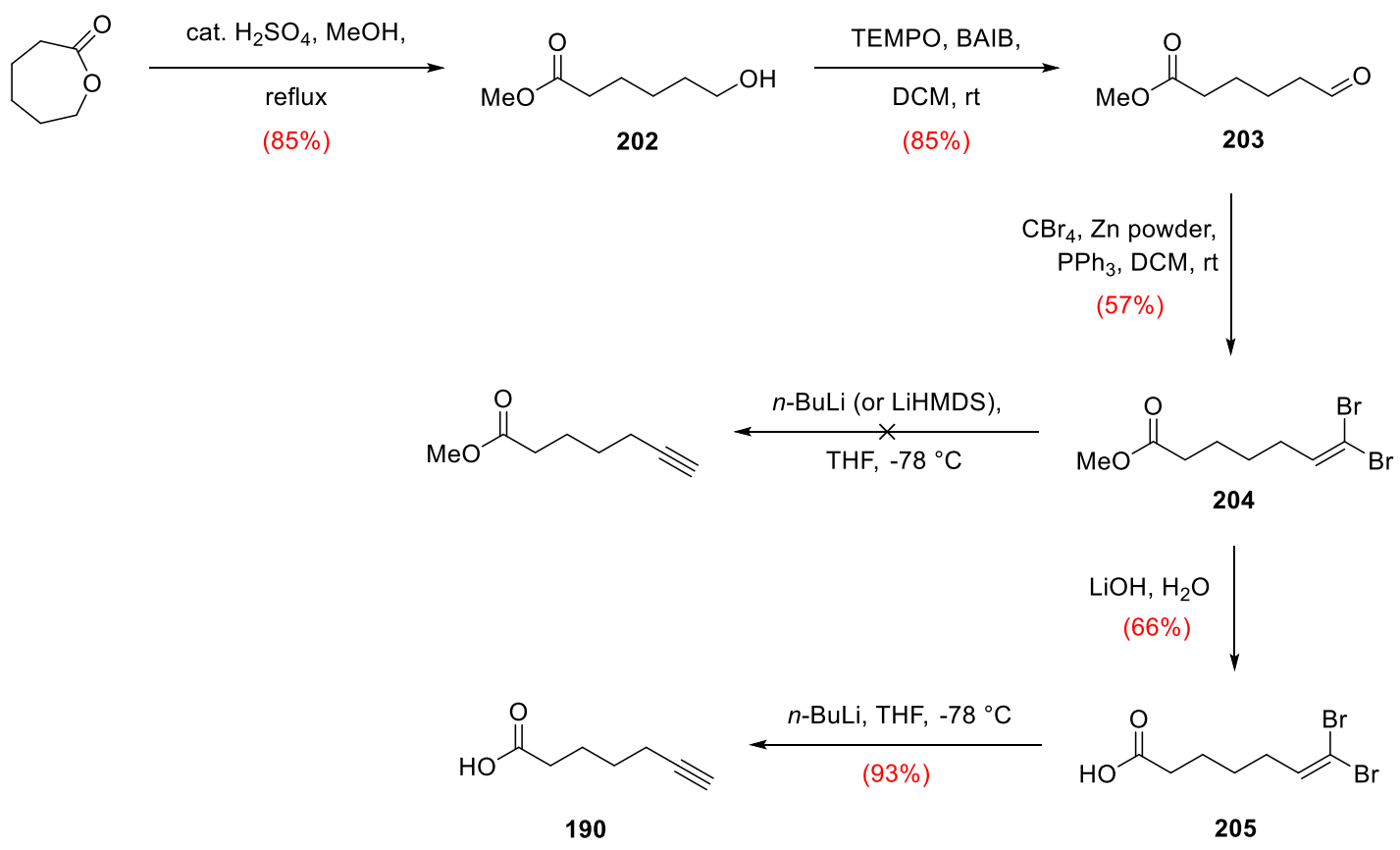

Scheme 2.30 Synthesis of 6-heptynoic acid 190 via Corey-Fuchs reaction.

A modified route based on the Seyferth-Gilbert method was envisaged to provide a more efficient route to 6-heptynoic acid 190. This first required the preparation of the OhiraBestman reagent 195, which was achieved in two steps, starting with formation of the previously described phosphonate $\mathbf{1 3 7}$ from chloroacetone and triethylphosphite in an Arbuzov reaction (Scheme 2.31). Treatment of phosphonate 137 with tosyl azide provided the desired Ohira-Bestmann reagent 195.

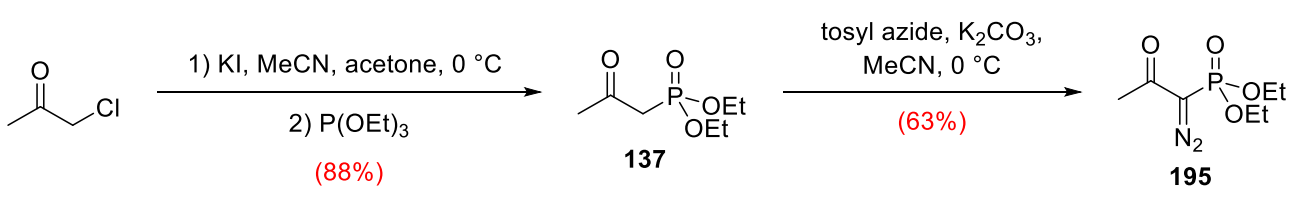

Scheme 2.31 Preparation of Ohira-Bestmann reagent 192. 
Aldehyde 203 was then treated with the Ohira-Bestmann reagent 195 to afford the alkyne 206 in 51\% yield and acid 190 in 40\% yield (Scheme 2.32). Saponification of the ester 206 product with $\mathrm{LiOH}$ in methanol provided the target 6-heptynoic acid 190 in 80\% yield. This gave $81 \%$ combined yield (over two steps) from the aldehyde 203 , in comparison to $34 \%$ (over three steps) for the same transformation using the Corey-Fuchs strategy. These strategies represent attractive alternatives to previously reported methods that use relatively expensive starting materials (e.g. 5-hexyn-1-ol, \$(NZ) 168 for 5 grams compared to $\varepsilon$-caprolactone, $\$(\mathrm{NZ}) 167$ for 500 grams). ${ }^{+++}$

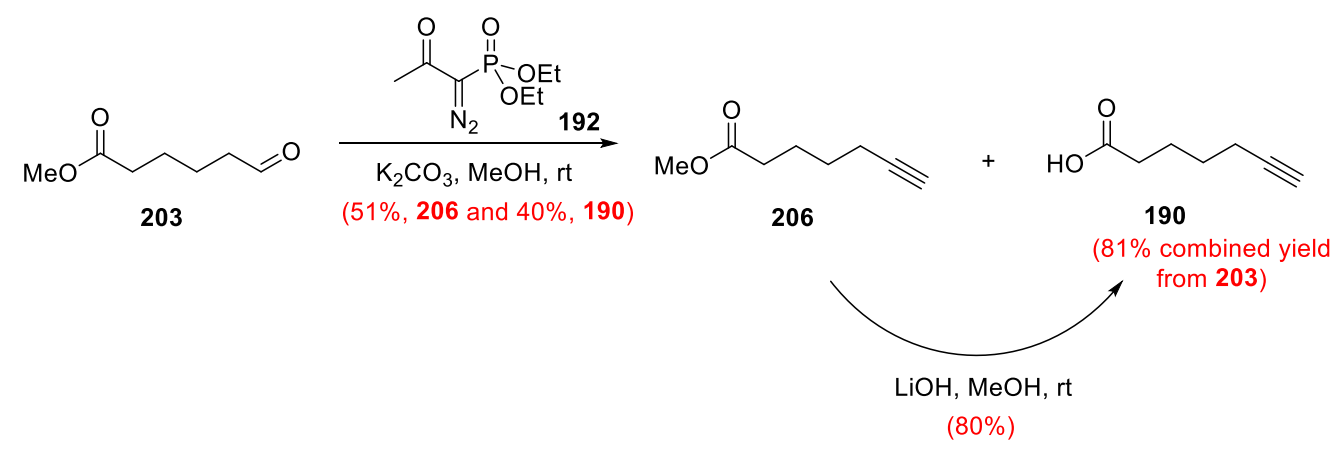

Scheme 2.32 Synthesis of 6-heptynoic acid (190) using Ohira-Bestmann reagent.

\subsubsection{Summary}

In summary, 6-heptynoic acid (190) (C1-C7 fragment) was efficiently prepared via two routes from $\varepsilon$-caprolactone. The first route utilised the two-step Corey-Fuchs reaction to access the terminal alkyne (vide supra, Scheme 2.30). Attempts to perform the second step of the Corey-Fuchs in the presence of the methyl ester 204 were unsuccessful. However, after saponification of the ester 204, the final step gave 6-heptynoic acid (190) in 25\% yield over 5 steps. Utilisation of the Ohira-Bestmann reagent provided a more efficient method for the preparation of 6-heptynoic acid (190) in 59\% yield over 4 steps.

t++ Prices taken from Sigma-Aldrich online catalogue (6 $6^{\text {th }}$ Aug 2013).

http://www.sigmaaldrich.com/new-zealand.html 


\subsection{Synthesis of C8-C10 (Tri-Substituted Propane) Fragment}

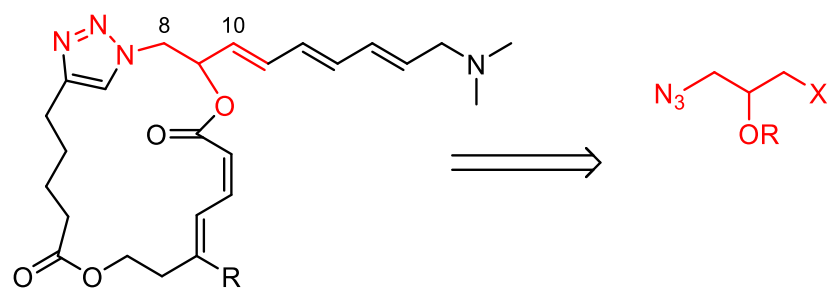

\subsubsection{Introduction}

The C8-C10 fragment is involved in two attachment points to the macrocycle of the proposed analogue (at the triazole and the unsaturated lactone) and links to the conjugated sidechain. Consequently, it requires three functional groups (an azide, an alcohol, and a group suitable for an olefination reaction) on a three-carbon chain, making it a functionally dense compound.

The epoxide opening of epichlorohydrin has been demonstrated with a range of nucleophiles using a variety of Lewis acids to provide trifunctional species, making this an appealing starting material. In addition, optically pure epichlorohydrin is commercially available and, therefore, the stereochemistry at the C9 carbon of the target analogue could be easily introduced. The classical protocol of opening epoxides with sodium azide and ammonium chloride requires a long reaction time (12-48 h) and often results in isomerisation and rearrangement of products. ${ }^{91}$ More effectively, the epoxide of epichlorohydrin has been reportedly opened with the following Lewis acids or additives: $\mathrm{CAN}{ }^{92} \mathrm{NaBF}_{4},{ }^{93} 4 \AA$ Ål sieves,,${ }^{94}$ Oxone ${ }^{\circledR},{ }^{95} \mathrm{Er}(\mathrm{OTf})_{3},{ }^{96}$ and (TBA) ${ }_{4} \mathrm{PFeW}_{11} \mathrm{O}_{39} \cdot 3 \mathrm{H}_{2} \mathrm{O},{ }^{97}$.

Two alternative olefination reactions were envisioned for coupling the conjugated sidechain to the C10 centre, namely E-selective Wittig or Julia-Kocienski reactions. Both would be tested and the results compared via the preparation of compounds $\mathbf{2 0 7}$ and 208 (for Wittig or Julia-Kocienski olefination, respectively). Both these routes relied on an epichlorohydrin derivative (Scheme 2.33). 


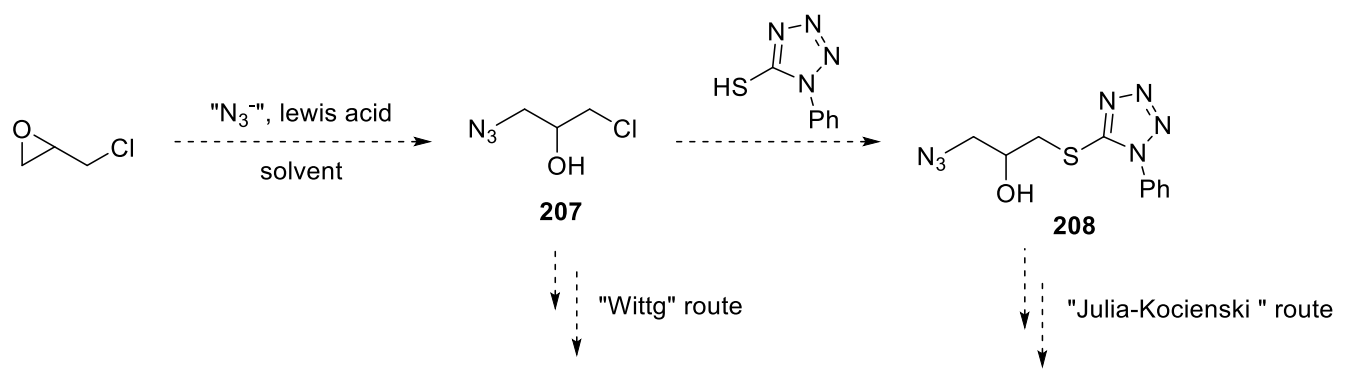

Scheme 2.33 Proposed C8-C10 fragments for the two alternative sidechain attachment reactions.

\subsection{2}

\section{Preparation of 1,3-Azidochloropropan-2-ol 207}

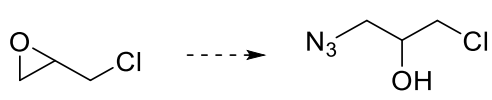

207

A range of reported conditions for opening the epoxide of epichlorohydrin with an azide species were tested. These included the use of CAN as a catalyst, ${ }^{92} \mathrm{NaBF}_{4}$ (modification from the reported use of $\left.\mathrm{LiBF}_{4}\right),{ }^{93} 4 \AA$ mol sieves (rt or heat) ${ }^{94}$ as additives and sodium azide impregnated on Amberlite XAD resin. ${ }^{98}$ Unfortunately, these reactions were found to be sluggish with very little conversion. Nonetheless, when $\mathrm{CeCl}_{3} .7 \mathrm{H}_{2} \mathrm{O}$ was used to facilitate the epoxide opening of epichlorohydrin (as reported by Sabitha and coworkers), ${ }^{99}$ significant conversion was observed in a test reaction, prompting further investigation of this route.

Following the conditions of Sabitha and co-workers, epoxide opening was carried out with sodium azide and $\mathrm{CeCl}_{3 .}{ }^{99}$ Good conversion was observed, but in addition to the desired product, a minor product was observed that was symmetrical based on the ${ }^{1} \mathrm{H}$ NMR signals attributed to it (Table 2.8, Entry 1). This compound was not separable from the product on silica gel chromatography, prompting the need to optimise the reaction conditions to increase yield whilst minimising this inseparable side product. Dropping the amount of $\mathrm{CeCl}_{3} .7 \mathrm{H}_{2} \mathrm{O}$ used resulted in a significant reduction in side product, however, it also resulted in a much lower yield (Entry 2). Whilst this side product was initially 
believed to be 1,3-diazidopropan-2-ol 210, investigation revealed it to be the unexpected 1,3-dichloropropan-2-ol 209, which was confirmed by comparison of the ${ }^{1} \mathrm{H}$ NMR spectrum with that of an authentic supply of this reagent. Increasing azide equivalents would suppress production of the undesired dichloro compound 209, but at the risk of producing the equally undesired 1,3-diazidopropan-2-ol 210. A comprehensive survey of the four variables (azide equivalents, catalyst loading, temperature and solvent) was challenging due to variability in the results of replicate experiments. It is plausible that this is a result of the reaction mixture not being fully homogeneous. Nevertheless, a range of general trends were obtained through trialling a range of conditions (Table 2.8). Reducing cerium chloride catalyst ( 0.5 to 0.3 equiv.), lowering temperature and increasing time $\left(80{ }^{\circ} \mathrm{C}\right.$, 3h to $\left.\mathrm{rt}, \mathrm{o} / \mathrm{n}\right)$ reduced diazido by-product 210 but lowered conversion (compare Entries 1 and 2 with 3, and also Entry 4 to 5). Reducing temperature reduced formation of the diazido by-product but lowered conversion (compare entries 2 with 3 and 4 with 5). Using 9:1 $\mathrm{MeCN}: \mathrm{H}_{2} \mathrm{O}$ resulted in significant dichloro by-product (Entry 1), most likely as a result of the limited solubility of $\mathrm{NaN}_{3}$ in this solvent mixture. Increasing the concentration of water (i.e. to $4: 1 \mathrm{MeCN}: \mathrm{H}_{2} \mathrm{O}$ ) drastically decreased the formation of dichloro by-product (compare Entry 1 with 2). Yet further increase, such as the use of 1:1 THF: $\mathrm{H}_{2} \mathrm{O}$ as a solvent mixture provided by-product ratios and yields that were comparable to the use of $4: 1 \mathrm{MeCN}_{\mathrm{H}} \mathrm{O}$ (compare Entries 5 to 6 , and 8 to 10). Interestingly, using $\mathrm{LiCl}$ in two equivalents as an additive instead of the $\mathrm{CeCl}_{3}$ catalyst, the diazido by-product was obtained as the sole product, albeit in moderate yield (Entry 9). Entry 11 shows the conditions judged to be optimal for the formation of 1,3azidochloropropan-2-ol 210. Although the yield was moderate (59\%), it provided the least amount of inseparable side-products. 


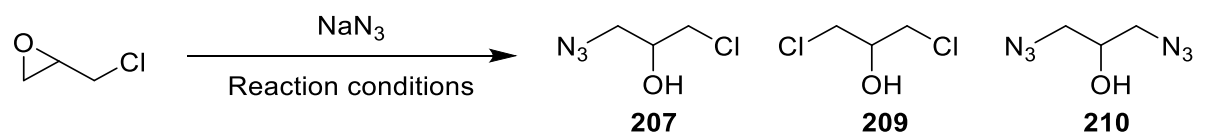

\begin{tabular}{ccccccc}
\hline Entry & $\mathbf{N a N}_{3}$ & $\mathbf{C e C l}_{3.7 \mathbf{H}_{2} \mathbf{O}}$ & Solvent & temp/time & $\begin{array}{c}\text { Ratio }^{\mathbf{b}} \\
\text { 207:209:210 }\end{array}$ & $\begin{array}{c}\text { Total Yield } \\
\text { (\%) }\end{array}$ \\
\hline $\mathbf{1}$ & 1.0 & 0.5 & $9: 1 \mathrm{MeCN}: \mathrm{H}_{2} \mathrm{O}$ & $80^{\circ} \mathrm{C}, 4 \mathrm{~h}$ & $1: 1.40: 0$ & 76 \\
$\mathbf{2}$ & 1.0 & 0.5 & $1: 1 \mathrm{MeCN}: \mathrm{H}_{2} \mathrm{O}$ & $80^{\circ} \mathrm{C}, 4 \mathrm{~h}$ & $1: 0.65: 0$ & 79 \\
$\mathbf{3}$ & 1.0 & 0.3 & $4: 1 \mathrm{MeCN}: \mathrm{H}_{2} \mathrm{O}$ & $\mathrm{rt}, \mathrm{o} / \mathrm{n}$ & $1: 0.16: 0$ & 47 \\
$\mathbf{4}$ & 0.5 & 0.3 & $4: 1 \mathrm{MeCN}: \mathrm{H}_{2} \mathrm{O}$ & $80^{\circ} \mathrm{C}, 4 \mathrm{~h}$ & $1: 1.6: 0$ & 89 \\
$\mathbf{5}$ & 0.5 & 0.3 & $4: 1 \mathrm{MeCN}: \mathrm{H}_{2} \mathrm{O}$ & $\mathrm{rt}, \mathrm{o} / \mathrm{n}$ & $1: 0.35: 0$ & 58 \\
$\mathbf{6}$ & 0.5 & 0.3 & $1: 1 \mathrm{THF}: \mathrm{H}_{2} \mathrm{O}$ & $\mathrm{rt}, \mathrm{o} / \mathrm{n}$ & $1: 0.21: 0$ & 71 \\
$\mathbf{7}$ & 2.5 & 0.3 & $1: 1 \mathrm{THF}: \mathrm{H}_{2} \mathrm{O}$ & $\mathrm{rt}, \mathrm{o} / \mathrm{n}$ & $1: 0: 0.58$ & 72 \\
$\mathbf{8}$ & $\mathbf{2}$ & 0.3 & $1: 1 \mathrm{THF}: \mathrm{H}_{2} \mathrm{O}$ & $\mathrm{rt}, \mathrm{o} / \mathrm{n}$ & $1: 0.12: 0$ & 88 \\
$\mathbf{9}$ & 2 & $\mathrm{LiCl}(2$ & $1: 1 \mathrm{THF}: \mathrm{H}_{2} \mathrm{O}$ & $\mathrm{rt}, \mathrm{o} / \mathrm{n}$ & $0: 0: 1$ & 38 \\
& & equiv) & & & & 96 \\
\hline $\mathbf{1 0}$ & 2 & 0.3 & $4: 1 \mathrm{MeCN}: \mathrm{H}_{2} \mathrm{O}$ & $\mathrm{rt}, \mathrm{o} / \mathrm{n}$ & $1: 0.23: 0.02$ & 59 \\
\hline $\mathbf{1 1}$ & 1.7 & 0.3 & $4: 1 \mathrm{MeCN}: \mathrm{H}_{2} \mathrm{O}$ & $\mathrm{rt}, \mathrm{o} / \mathrm{n}$ & $1: 0.04: 0$ & 56 \\
\hline
\end{tabular}

${ }^{\mathrm{a}} \mathrm{rt}=$ room temperature, $\mathrm{o} / \mathrm{n}=$ overnight (i.e. $18-20$ hours). ${ }^{\mathrm{b}}$ Determined by ${ }^{1} \mathrm{H} \mathrm{NMR}$ on reaction product mixture.

Table 2.8 Trialled conditions for the formation of 1,3-azidochloropropan-2-ol 207.

\subsubsection{Preparation of 1,3-Azido(phenyltetrazole)propan-2-ol 208}

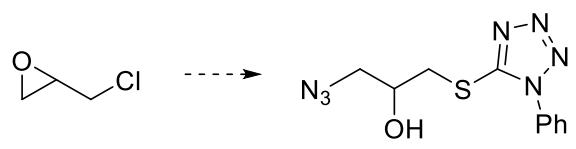

208

In order to prepare the thioether 208 necessary for a Julia-Kocienski reaction, phenyltetrazolethiol 211 was synthesised. This was efficiently prepared from phenyl isothiocyanate and sodium azide in water at reflux overnight to provide the thiol 211 in quantitative yield (Scheme 2.34). ${ }^{100}$ 


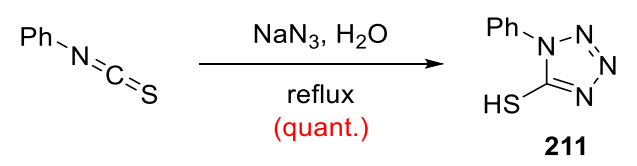

Scheme 2.34 Preparation of phenyltetrazolethiol 211.

The preparation of the thioether $\mathbf{2 0 8}$ was attempted by substitution of the alkyl chloride 207 with phenyltetrazolethiol 211 (Scheme 2.35). Treatment with an excess of a 'weak' base (NEt 3 ) or a 'strong' base $(\mathrm{NaH})$ at room temperature to facilitate deprotonation of the thiol resulted in no significant reaction, with recovery of alkyl chloride starting material. When the same reactions were repeated at refluxing THF a mixture of unidentifiable products were obtained.

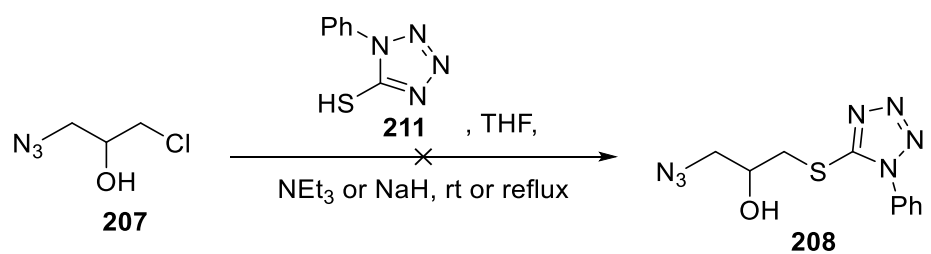

Scheme 2.35 Attempt at substituting alkyl chloride 207 with phenyltetrazolethiol 211.

The inability to effectively substitute the alkyl chloride to form the thioether 208 prompted us to go back a step and trial the use of thiol 211 to open the epoxide of epichlorohydrin. A literature search $¥ \neq \ddagger$ suggested that this exact reaction had not been reported. However, the use of phenyltetrazolethiol to open a non-halogenated epoxide using catalytic lithium bromide had been. ${ }^{101}$ Unfortunately, using $\mathrm{LiBr}$ (0.3 equivalents) resulted in a mixture of thiol and bromide substitutions due to reaction with the alkyl chloride (Scheme 2.36).

${ }^{\ddagger \neq \ddagger}$ Using SciFinder database; https://scifinder.cas.org (accessed March 2012) 


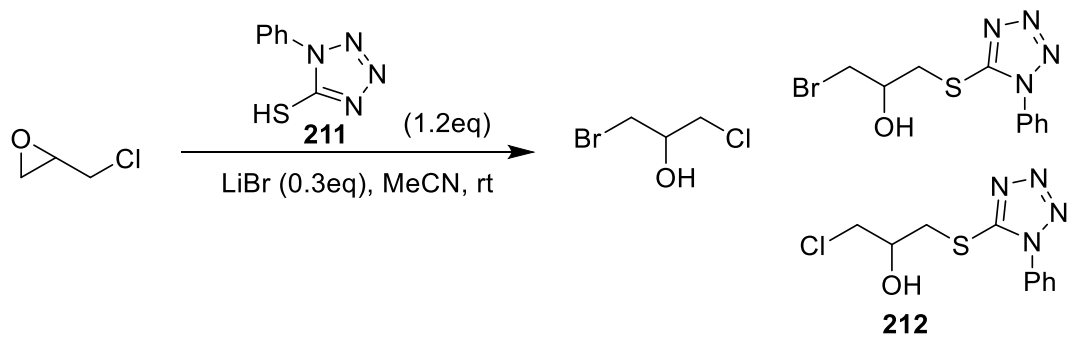

Scheme 2.36 Attempted epoxide opening with phenyltetrazolethiol 211 using LiBr.

A range of other reaction conditions were then screened for this transformation (Table 2.9). Cyanuric chloride had been previously reported as an efficient catalyst for epoxide opening with thiols, but after two days the reaction had not gone to completion (Entry 1). ${ }^{102}$ Similarly, using cerium chloride as a Lewis acid (0.3 equiv) gave incomplete conversion (Entry 2). ${ }^{99}$ Reaction in water, which has been reported with other thiols, ${ }^{103}$ gave good conversion (Entry 3). Satisfyingly, when the reaction was done neat, i.e. epichlorohydrin as the solvent, full conversion was observed in 20 hours (Entry 4). Given that no by-products were obtained for this reaction, performing the reaction under neat conditions provided a convenient method for conducting the reaction, as the excess epichlorohydrin could simply be removed under reduced pressure (b.p. $=115^{\circ} \mathrm{C}$ at $1 \mathrm{~atm}$ ) after the reaction was complete to provide pure thioether 209 in quantitative yields. 


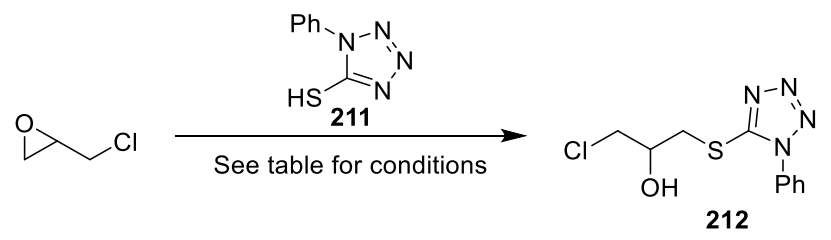

\begin{tabular}{cccccc}
\hline Entry & $\begin{array}{c}\text { Epoxide } \\
\text { (equiv) }\end{array}$ & $\begin{array}{c}\text { Thiol } \\
\text { (equiv) }\end{array}$ & $\begin{array}{c}\text { Conditions } \\
\text { (equiv) }\end{array}$ & $\begin{array}{c}\text { Time } \\
\text { (h) }\end{array}$ & Result \\
\hline $\mathbf{1}^{\mathbf{a}}$ & 3 & 1 & $\begin{array}{c}\text { cyanuric chloride }(0.1), \\
\mathrm{MeCN}, \mathrm{rt}\end{array}$ & 48 & $\begin{array}{c}\text { Modest } \\
\text { conversion }\end{array}$ \\
\hline $\mathbf{2}^{\mathbf{a}}$ & 3 & 1 & $\begin{array}{c}\mathrm{CeCl} 3.7 \mathrm{H}_{2} \mathrm{O}(0.3), \\
\mathrm{MeCN}: \mathrm{H}_{2} \mathrm{O}(4: 1), \mathrm{rt}\end{array}$ & 48 & $\begin{array}{c}\text { Modest } \\
\text { conversion } \\
\mathbf{3}^{\mathbf{a}}\end{array}$ \\
& 3 & 1 & 48 & $\begin{array}{c}\text { Good } \\
\text { conversion }\end{array}$ \\
$\mathbf{4}^{\mathbf{b}}$ & Excess & 1 & Neat, rt & 20 & Completion \\
\hline
\end{tabular}

${ }^{a}$ Reactions were conducted using phenyltetrathiol $211(20 \mathrm{mg}, 0.11 \mathrm{mmol})$, epichlorohydrin and additive in 4 $\mathrm{mL}$ solvent. The reactions were stirred at room temperature and analysed using TLC ( $\mathrm{R}_{\mathrm{f}}=0.43$ for

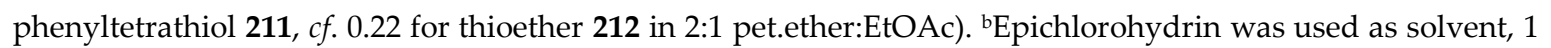
$\mathrm{mL}$.

Table 2.9 Reaction screening for epoxide opening of epichlorohydrin with phenyltetrathiol 211.

With an efficient and reliable method for opening the epoxide of epichlohydrin to provide the alkyl chloride $\mathbf{2 1 2}$, we were then able to substitute the alkyl chloride for the azide. As there was no concern of competitive reactivity, conditions that strongly favour nucleophilic substitutions could be applied. Thus, alkyl chloride $\mathbf{2 1 2}$ was reacted with an excess of sodium azide and catalytic tetra-n-butylammonium iodide (TBAI) in DMF at 80 ${ }^{\circ} \mathrm{C}$ overnight to provide the alkyl azide 208 in quantitative yield (Scheme 2.37).

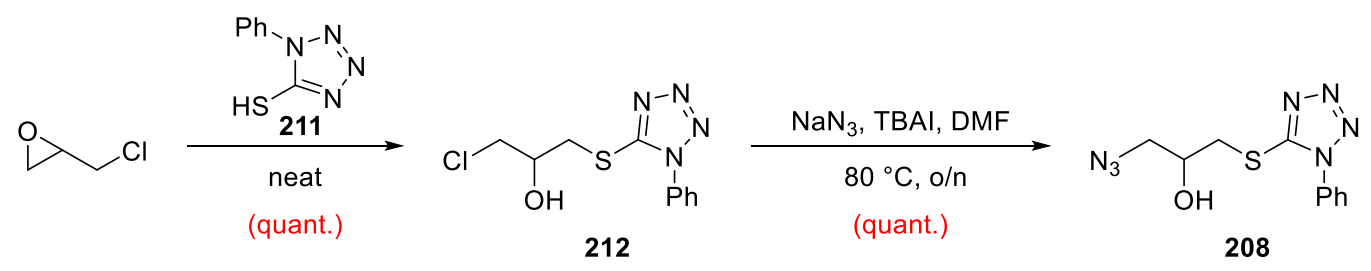

Scheme 2.37 Synthesis of thioether fragment 208 from epichlorohydrin. 


\subsubsection{Summary}

In summary, two trisubstituted propan-2-ol compounds (207 and 208) were prepared for testing as potential candidates for the $\mathrm{C} 8 \mathrm{-C} 10$ fragment. In the preparation of 207 , a range of reported procedures were screened for the epoxide opening of epichlorohydrin with an azide source. $\mathrm{CeCl}_{3} .7 \mathrm{H}_{2} \mathrm{O}$ was found to be effective at facilitating this reaction, however, competitive reactivity was observed. Attempts to purify the products by chromatographic separation from the by-products were unsuccessful, necessitating the need for optimising the reaction conditions to minimise by-products (vide supra, Table 2.8). Thioether 208 was efficiently prepared in two steps with quantitative yields (vide supra, Scheme 2.37).

\subsection{Synthesis of C11-C16 (Conjugated Side Chain) Fragment}
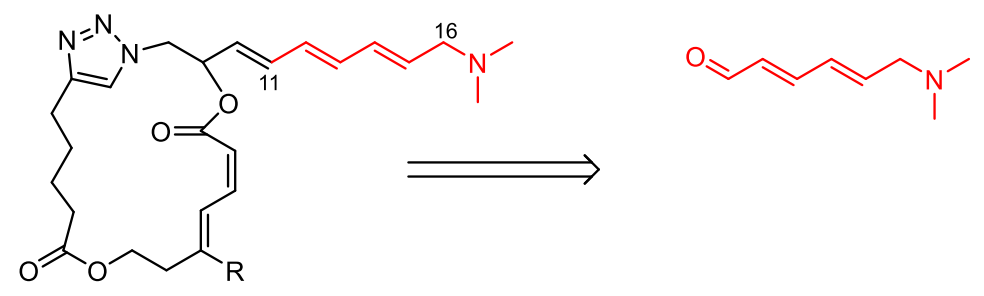

\subsubsection{Introduction}

Polyenes are present in a vast and diverse array of natural products, found in all forms of living organisms including bacteria, fungi, slime-moulds, plants, marine organisms and animals. ${ }^{104}$ Representative natural polyenes, which exhibit potent biological activity, are shown in Figure 2.10. The conjugation of double bonds provides structural rigidity in the carbon chain, and may also have additional function in the mechanism of bioactivity, such as electron affinity, radical formation, electron transfer or autoxidation. ${ }^{105}$ For example, amphotericin B, can undergo autoxidation with concomitant formation of free radicals. 
This has been proposed as an important mechanism for its antifungal activity, in addition to its ability to form cation selective channels. ${ }^{105}$

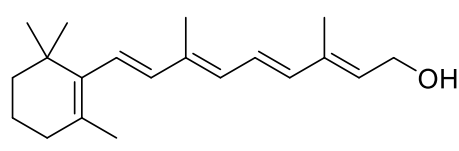

Retinol (vitamin A)
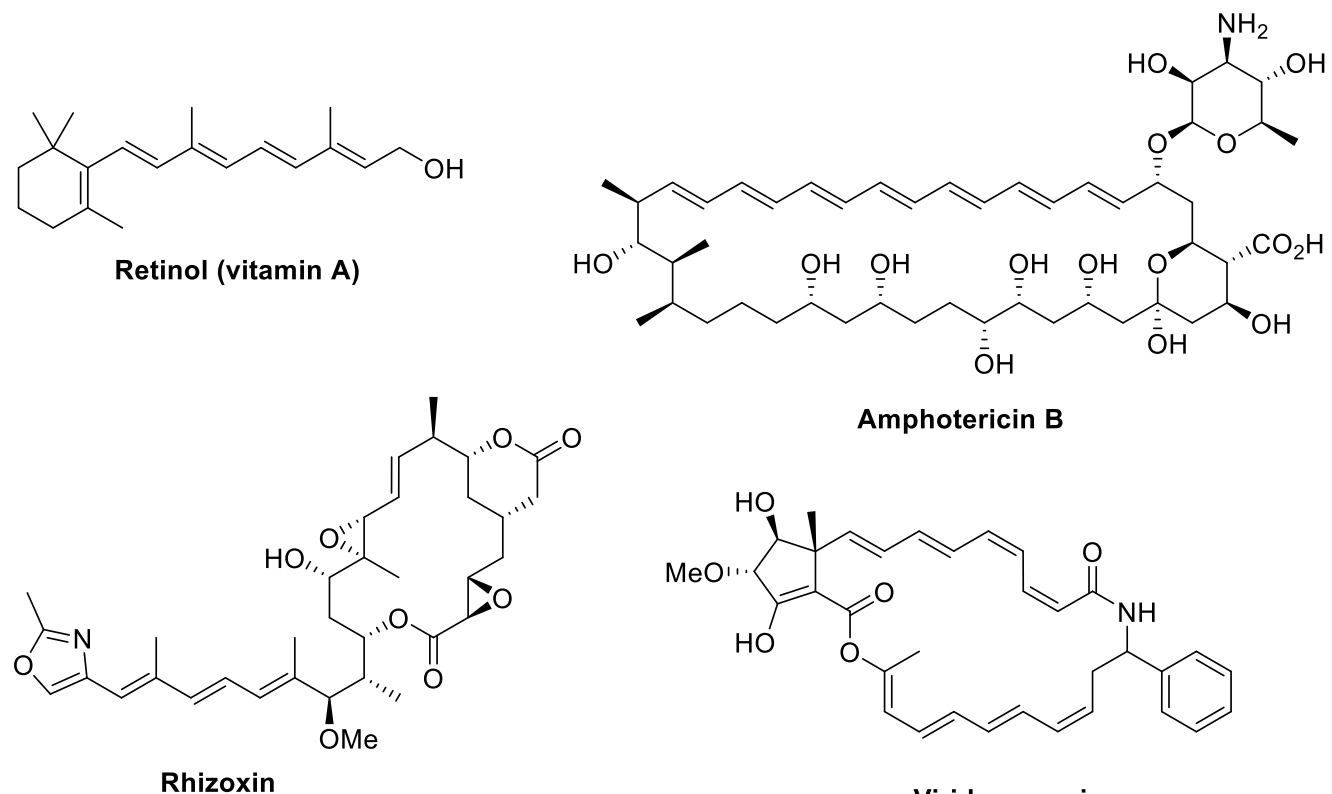

Amphotericin B

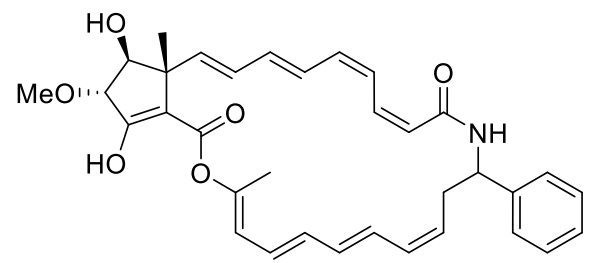

Viridenomycin

Figure 2.10 Examples of polyene containing biologically active natural products.

The general strategies to access polyenes synthetically, are summarised in Figure 2.11. Consideration of the synthetic functional group tolerance and stereoselectivity must be made when choosing synthetic methods in preparation of polyenes. In addition, polyenes in the all-trans configuration are generally more stable than those containing cis-alkenyl units; consequently, the latter are prone to isomerisation. 


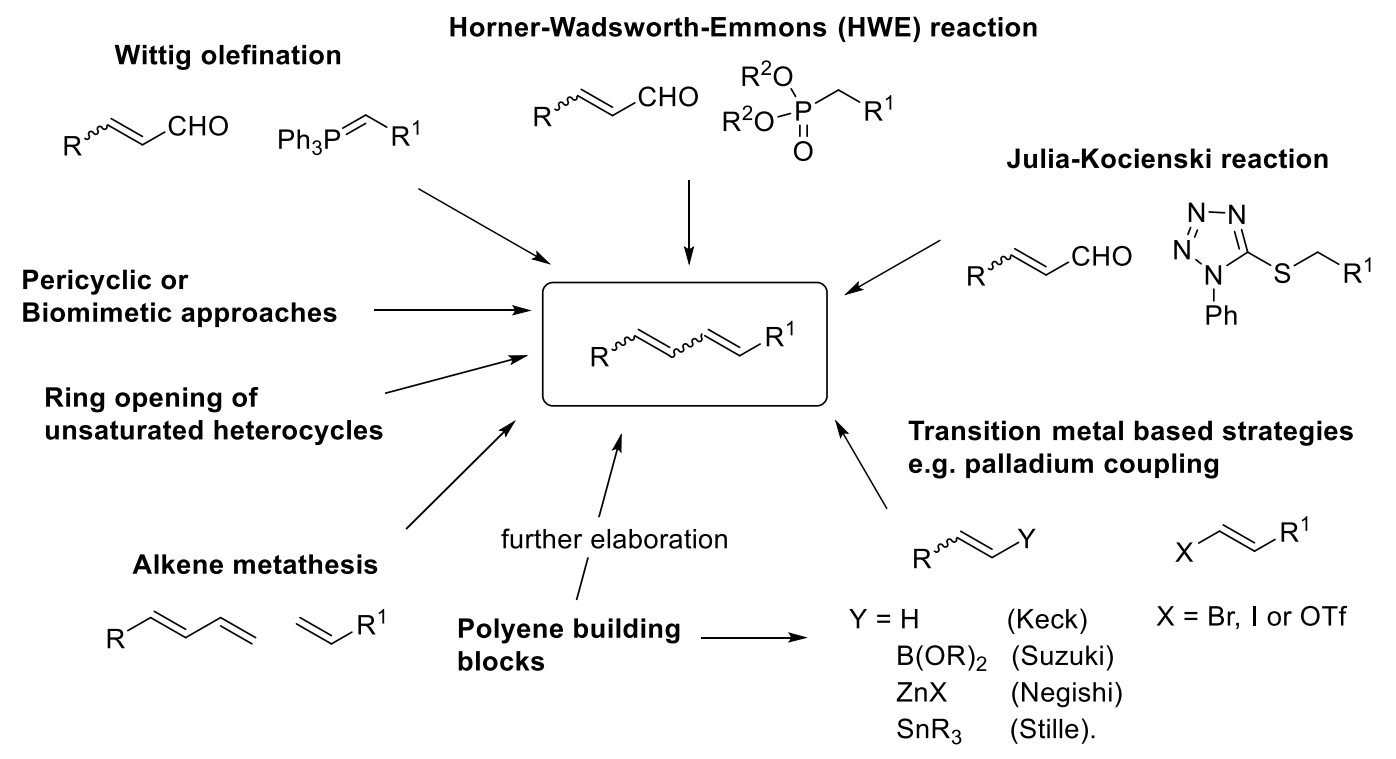

Figure 2.11 General strategies in the preparation of polyenes.

The triene-containing sidechain of pateamine has the all-E geometry. Scheme 2.38 shows the key retrosynthetic steps planned to prepare the side chain fragment 213 . The dimethylamine was envisioned to be introduced using either a reductive amination or an amine nucleophilic substitution. Wittig olefination and isomerisation of an $\alpha, \beta-$ unsaturated aldehyde 214 would provide the desired diene 215.

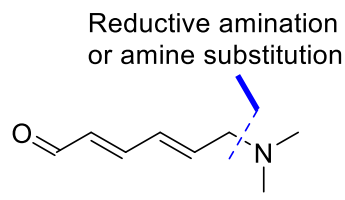

213
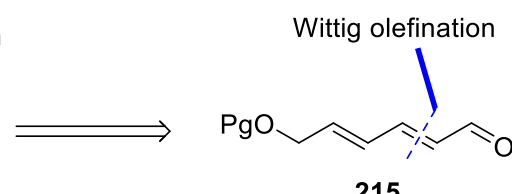

215

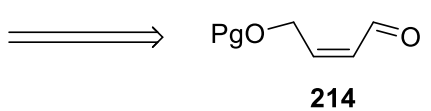

214

Scheme 2.38 Retrosynthetic analysis of fragment 213.

\subsubsection{Preparation of C11-C16 Fragment 213}

Our first attempt at the synthesis of the sidechain fragment 213 began with the monoprotection of cis-2-butene-1,4-diol to the para-methoxy benzyl (PMB) protected alcohol 216 (Scheme 2.39). This was achieved in a two-step process; firstly, formation of the PMP cyclic acetal 217 was achieved using p-anisaldehyde and heating in a Dean-Stark 
apparatus with catalytic para-toluenesulfonic acid (PTSA); and secondly, reduction of the acetal with DIBAL-H. Oxidation of alcohol 216 to aldehyde 218 was achieved using a Swern oxidation, initially at $-78{ }^{\circ} \mathrm{C}$ and warming to room temperature to allow isomerisation to the trans-alkene. Quenching the reaction without the warming phase was found to provide the cis-alkene as the major product. Wittig reaction of aldehyde $\mathbf{2 1 8}$ with the stabilised phosphorane ylide 219 provided ethyl ester 220, which was subsequently reduced to alcohol 221 with DIBAL-H.

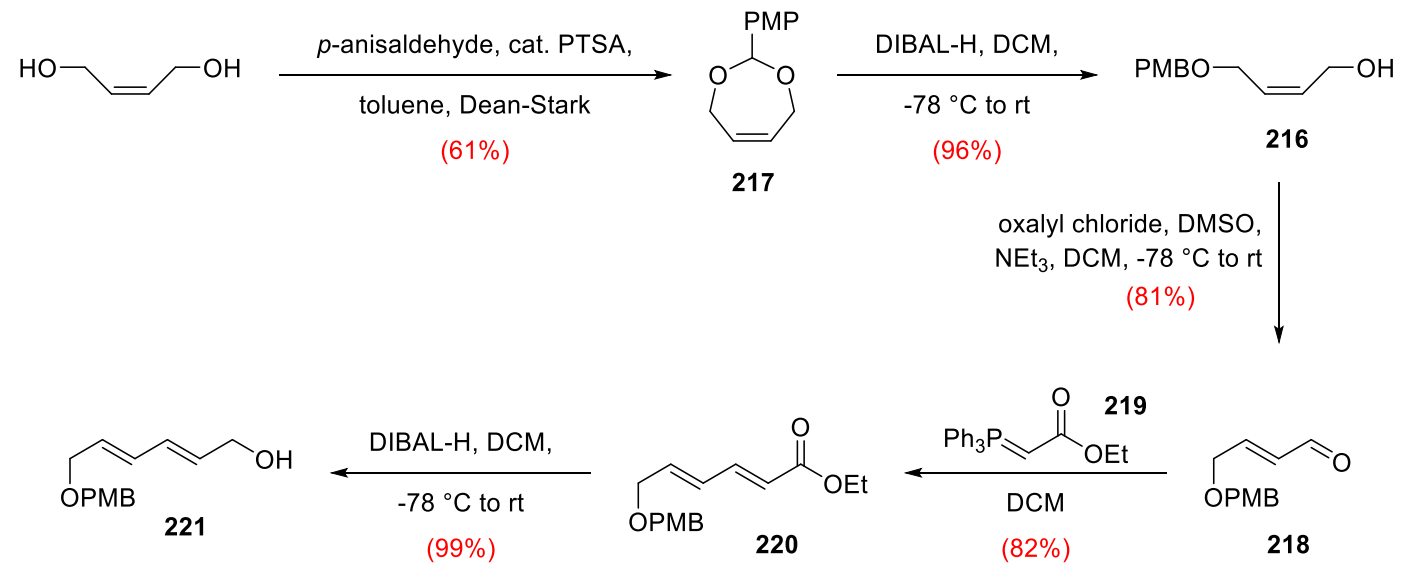

Scheme 2.39 Synthesis of PMB alcohol 221.

\section{Alcohol substitution on PMB protected diene}

With alcohol 221 in hand, it was necessary to substitute the hydroxyl group for the dimethylamine moiety. Thus, alcohol 221 was converted to the alkyl bromide 222 via an Appel reaction using carbon tetrabromide and triphenylphosphine (Scheme 2.40). The reaction of the alkyl bromide 222 with dimethylammonium hydrochloride and DIPEA resulted in a mixture of products. A significant amount of an undesired para-substituted product was observed, presumably formed from the cleavage of the PMB group. This byproduct could not be efficiently separated from the product 224 . To avoid the problems associated with PMB cleavage, we decided to repeat the reactions using a TBS protecting group. 


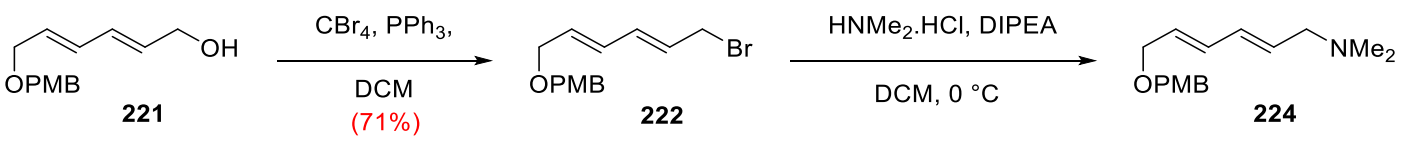

Scheme 2.40 Synthesis of dimethylamine 224 .

\section{Alcohol substitution on TBS protected diene}

TBS protected alcohol 225 was prepared in a similar manner as the PMB-protected alcohol 221 (Scheme 2.41). However, mono-protection of cis-butene diol was achieved in reasonable yield and just one step by reaction with $\mathrm{TBSCl}$ in an excess of the diol (cf. cyclic acetal formation followed by reduction to get PMB mono-protection). Alcohol 226 was then oxidised, the aldehyde 227 treated with the stabilised Wittig reagent and the resulting ester 228 reduced with DIBAL-H to provide TBS-protected alcohol 225.

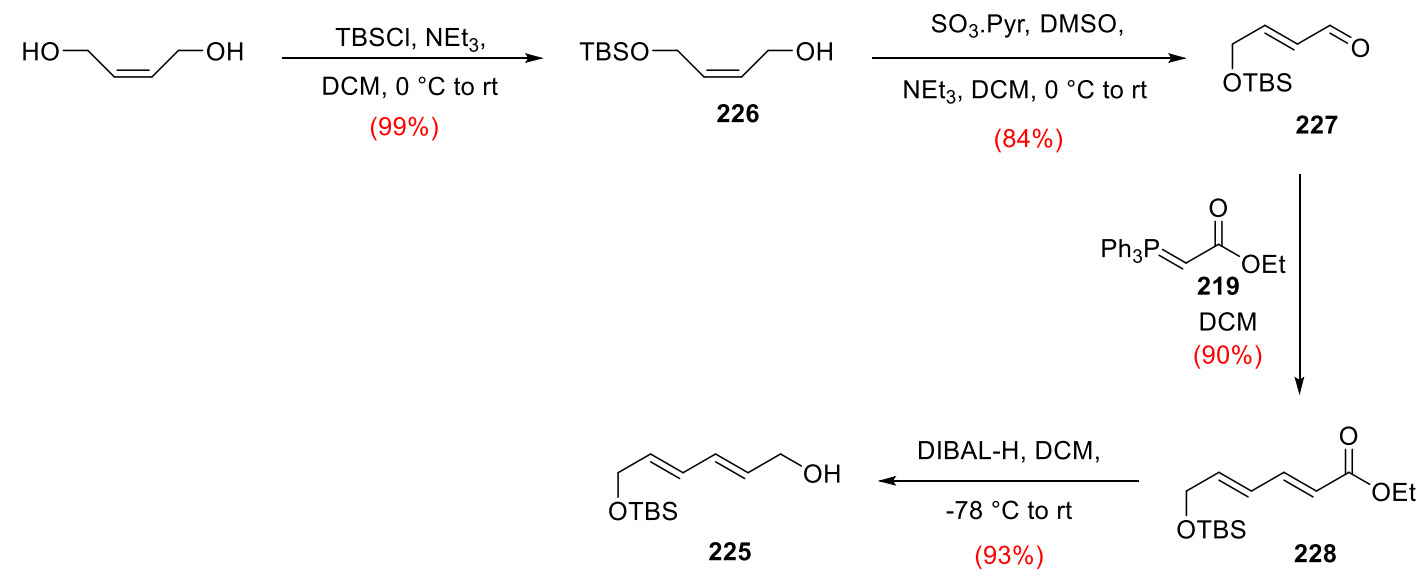

Scheme 2.41 Synthesis of TBS alcohol 228. 


\section{$S_{N} 2$ substitution (via alkyl bromide)}

With TBS-protected alcohol 225 in hand, we attempted to prepare the alkyl bromide 229. Unfortunately, the Appel reaction using $\mathrm{CBr}_{4} / \mathrm{PPh}_{3}$ resulted in poor yield and a mixture of by-products. We moved on to a variation of the Appel reaction that uses $\mathrm{PPh}_{3}$ with $\mathrm{N}$ bromosuccinimide (NBS). Thus, reaction of alcohol 225 with $\mathrm{PPh}_{3} / \mathrm{NBS}$ in $\mathrm{DCM}$ at $0{ }^{\circ} \mathrm{C}$ provided alkyl bromide 229 in 72\% yield (Table 2.10, Entry 1). A small amount of a pseudo-dimeric product was also observed, that is proposed\$\$s to be compound 230, formed via the reaction of alcohol 225 and alkyl bromide 229. In an attempt to eliminate the formation of the pseudo-dimeric product 230, the reaction was undertaken by first mixing $\mathrm{PPh}_{3}$ and NBS to pre-form the active reagent and adding the alcohol substrate last. Interestingly, this resulted in an increased formation of the dimeric product 230, in approximately a 1:1 ratio with desired product (Entry 2). Satisfyingly, reducing the temperature to $-30{ }^{\circ} \mathrm{C}$ and adding NBS last provided the alkyl bromide in $89 \%$ yield (Entry 3).

\begin{tabular}{|c|c|c|c|}
\hline 225 & 22 & & 230 \\
\hline Entry & Reagents & Temperature & Yield \\
\hline 1 & $\begin{array}{c}\mathrm{PPh}_{3}, \mathrm{DCM} \text {, } \\
\text { then NBS }\end{array}$ & $0^{\circ} \mathrm{C}$ & $72 \%$ \\
\hline 2 & $\begin{array}{c}\mathrm{PPh}_{3}, \mathrm{NBS}, \mathrm{DCM} \text {, } \\
\text { (preformed) } \\
\text { then } \mathbf{2 2 5}\end{array}$ & $0^{\circ} \mathrm{C}$ & $28 \%{ }^{*}$ \\
\hline 3 & $\begin{array}{c}\mathrm{PPh}_{3}, \mathrm{DCM} \text {, } \\
\text { then NBS }\end{array}$ & $-30^{\circ} \mathrm{C}$ & $89 \%$ \\
\hline
\end{tabular}

*1: 1 mixture of 229 and suspected pseudo-dimerised ether 230.

Table 2.10 Appel-like reaction on alcohol 225.

Reaction of the alkyl bromide 229 with 2 equivalents of dimethylammonium hydrochloride and DIPEA resulted in a very poor yield of amine 231, and a significant

$\$ \S \S$ Based on examination of ${ }^{1} \mathrm{H}$ NMR and ${ }^{13} \mathrm{C}$ NMR spectra. 
amount of the doubly substituted species 232 (Table 2.11, Entry 1). This was confirmed using HR-MS, and is likely formed from the reaction of unreacted starting material 229 with the desired dimethylamine 231. To counter the formation of the dimerised product 232, the quantity of dimethylammonium hydrochloride and DIPEA were increased, which resulted in a lower ratio of the by-product (Entries 2 and 3). However, even with 15 equivalents of dimethylammonium hydrochloride approximately $10 \%$ of by-product 232 was still formed (Entry 3). The poor yields obtained from this reaction are attributed to the tendency of the alkyl bromide to degrade during the reaction. It was also found that the alkyl bromide $\mathbf{2 2 9}$ had degraded significantly on storage at room temperature or, to a lesser extent, at $-18^{\circ} \mathrm{C}$.

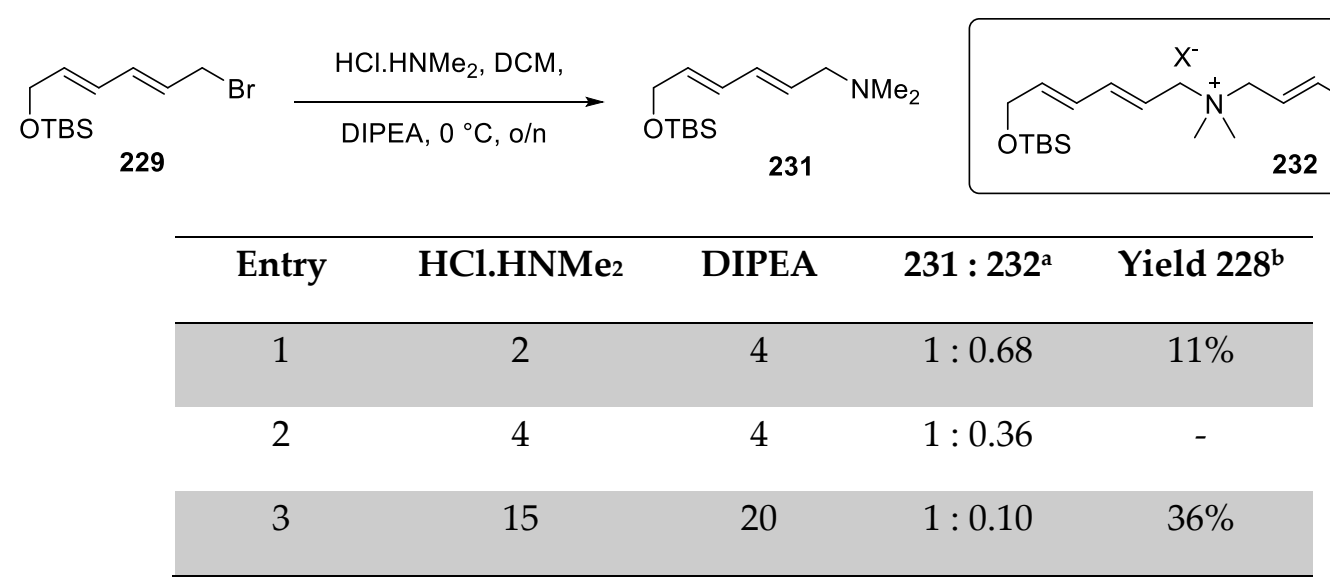

${ }^{a}$ Based on ${ }^{1} \mathrm{H}$ NMR of crude reaction product. ${ }^{b}$ Based on isolated yield.

Table 2.11 Substitution of alkyl bromide 229 with dimethylamine.

\section{Reductive amination}

The poor yield and the detrimental nucleophilicity of the diamine product 231 , resulting in dimer 232, prompted us to change tack and reductive amination of a conjugated dienecontaining aldehyde stood out as an attractive alternative that would avoid these issues. Accordingly, alcohol 225 was oxidised to aldehyde 233 using a Parikh-Doering oxidation (Scheme 2.42). Reductive amination was performed with an excess of the dimethylammonium hydrochloride salt, to form in situ the iminium ion 234, and subsequent reduction with tetramethylammonium triacetoxyborohydride to provide the 
dimethylamine 231. The first attempt was conducted using dichloromethane as a solvent, but this resulted in very poor yield, significant desilylation and the formation of bis(dimethylamino)methane 235. The bis-amine 235 is formed via the slow reaction of dimethylamine with dichloromethane (Scheme 2.43), which has been previously reported with other amines. ${ }^{106}$ Formation of bis-amine 235 increases $\mathrm{HCl}$ levels in the reaction mixture, which is presumably the cause of desilylation. We then moved to THF as the solvent, obtaining the desired amine 231 in a yield of $64 \%$, with the remainder being unreacted starting material. We had sufficient material to carry on so no further optimisation was performed on this substrate.

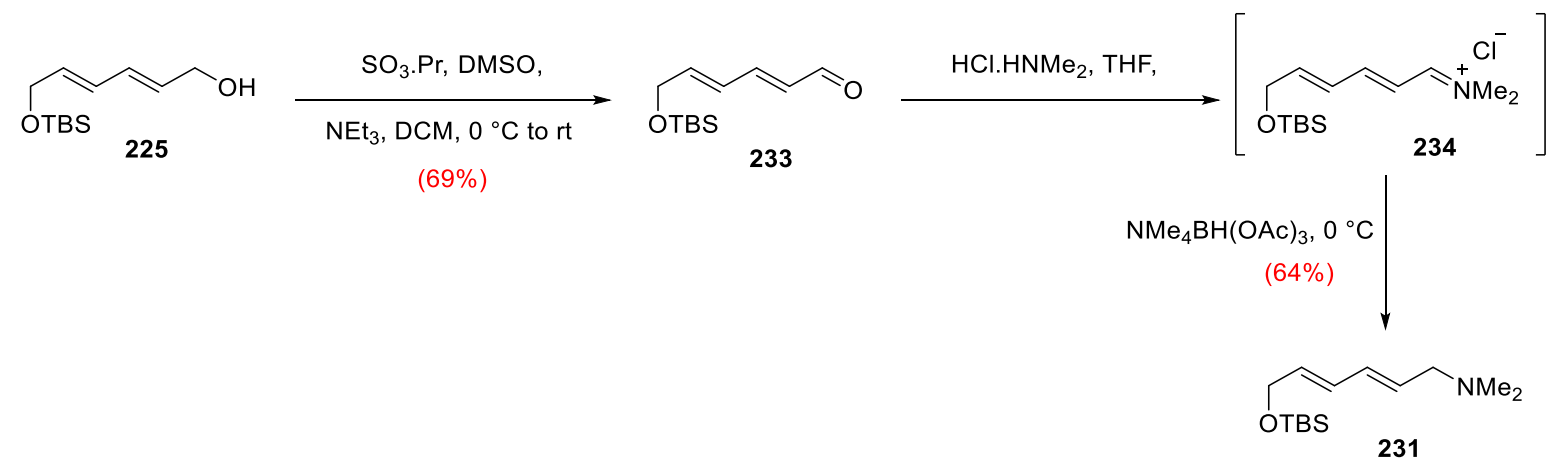

Scheme 2.42 Synthesis of dimethylamine 231 via reductive amination.

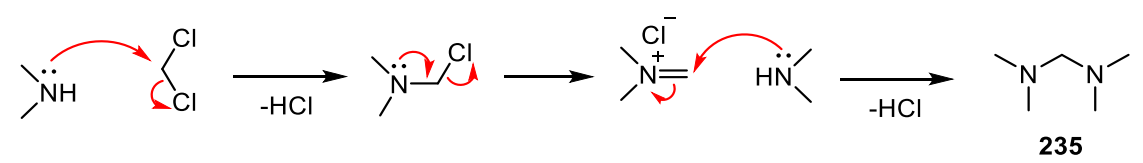

Scheme 2.43 Proposed mechanism for the reaction of dimethylamine with dichloromethane to give bis-amine 235 . $^{106}$

To complete the preparation of the desired aldehyde fragment 213, the compound 231 was desilylated using $1 \%$ concentrated $\mathrm{HCl}$ in methanol to give alcohol 236, followed by Parikh-Doering oxidation (Scheme 2.44). TBAF was also used for desilylation, which occurred with good conversion, but purification of the reaction mixture proved problematic due to the similar polarities of the tert-butylammonium salts and the amine product 236. 


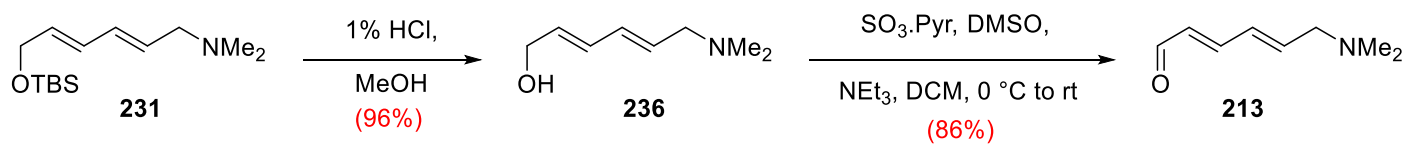

Scheme 2.44 Synthesis of aldehyde 213.

\section{Zincke aldehyde}

In the interest of preparing varied analogues and fragments that are more easily accessed, a conjugated sidechain was synthesised that was one carbon shorter. The shortened sidechain fragment 237, was conveniently prepared from the ring opening of a dinitrophenyl pyridinium salt 238. This process is based on reactions first reported by Zincke; consequently, the products are commonly referred to as Zincke aldehydes. ${ }^{107}$ Thus, reaction of pyridine and 1-chloro-2,4-dinitrobenzene 239 in acetone at reflux provided pyridinium salt 238 (Scheme 2.45). Opening of the pyridinium ring in 238 with two equivalents of dimethylamine (added as the hydrochloride salt) was followed by treatment with aqueous sodium hydroxide to hydrolyse the intermediate iminium $\mathbf{2 4 0}$ and afforded aldehyde 237.

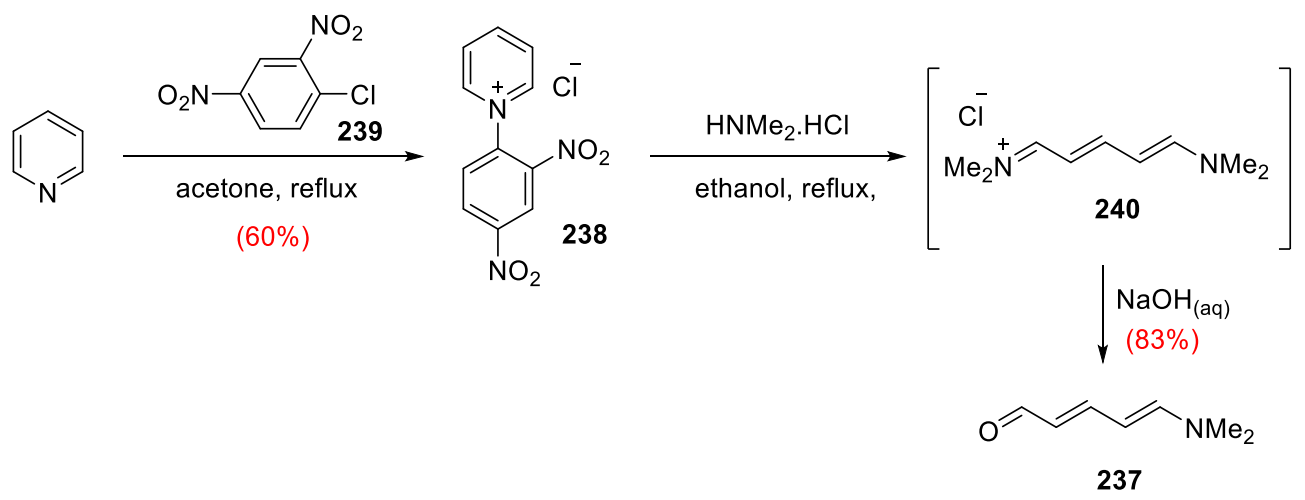

Scheme 2.45 Synthesis of Zincke aldehyde 237. 


\subsubsection{Summary}

In summary, the dimethylamine sidechain 213 was prepared in eight steps from cis-2butene-1,4-diol in 19\% isolated yield. The use of a PMB protecting group was found to be problematic due to its lability to subsequent reaction conditions, this was remedied by instead employing TBS protection. The activation of allylic alcohol 225 as a bromide and subsequent substitution with dimethylamine, were both accompanied with dimerisation issues. This prompted the use of a reductive amination between aldehyde 233 and dimethylamine, which resolved these issues.

\subsection{Conclusion}

In conclusion, four fragments of the target pateamine analogue were prepared (Figure 2.12). The C17-C23 fragment 167 was accessed via a RCM reaction and shown to provide, on treatment with base, the $Z, E$-dienoic acid as the sole stereoisomer in good yield. This method was favourable as the $\alpha, \beta$-unsaturated $\delta$-lactone acts as a 'masked' $Z, E$-dienoic acid, making its use in our synthesis attractive. Attempts to access the Z,E-dienoic acid with an extra methyl substituent at the $\delta$-position was hindered by the average diastereoselectivity for the Z,E-diene configuration over the Z,Z-diene.

The C1-C7 fragment 190 was efficiently accessed from $\varepsilon$-caprolactone, trialling both Corey-Fuchs and the use of the Ohira-Bestmann reagent as strategies to access the terminal alkyne. The use of the Ohira-Bestmann reagent provided the more efficient route, obtaining 190 in four steps and 59\% overall yield.

Two different C8-C10 fragments, 207 and 208, were prepared from epichlorohydrin. These were designed to allow the access to the Wittig or the Julia-Kocienski coupling olefination reactions, respectively.

The C11-C16 fragment 237 was accessed via a stabilised Wittig reaction. The amine proved troublesome to incorporate using standard $S_{\mathrm{N}} 2$ reaction strategies which was burdened 
with dimerization issues. The use of reductive amination provided a method which overcame these problems.

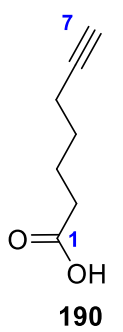

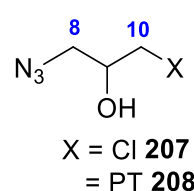

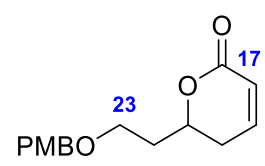

167

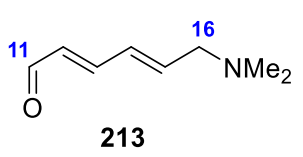

$$
P T=s^{3} \stackrel{P}{N}_{\substack{N-N \\ N}}^{N-N}
$$

Figure 2.12 Four fragments prepared of pateamine analogue target. 
104 


\section{Chapter Three: Fragment Coupling and the Synthesis of Pateamine Analogue}

\subsection{Introduction}

The four synthetic connections required to couple fragments described in the previous chapter are shown in Figure 3.1. These include two esterification reactions, a copper catalysed azide-alkyne cycloaddition (CuAAC) reaction to form the macrocycle, and an olefination reaction to attach the side chain.

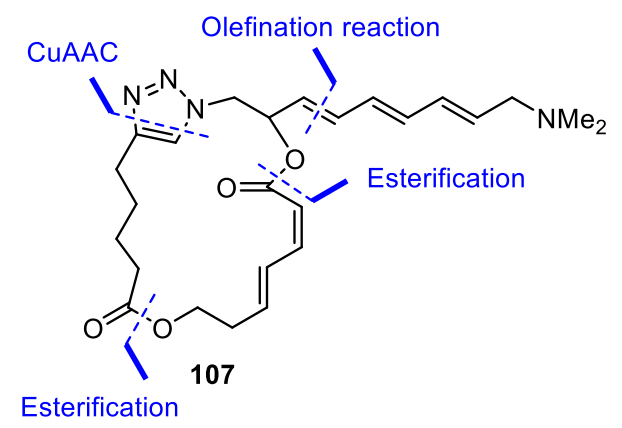

Figure 3.1 Major retrosynthetic disconnections.

\subsubsection{Olefination Reactions}

Two olefination reactions were chosen as candidates for the attachment of the side chain, namely the Wittig olefination and the Julia-Kocienski olefination. Standard Wittig conditions with unstabilised ylides typically provide Z-alkenes due to the preferred approach of the aldehyde and the phosphonium ylide to orientate the bulky groups ( $R$ and $\mathrm{R}^{\prime}$ ) away from each other in the transition state of the betaine formation (Scheme 3.1). After formation of the syn-oxaphosphetane intermediate 241 , the kinetically controlled Zalkene $\mathbf{2 4 2}$ is formed. Alternatively, the Schlosser modification of the Wittig reaction can be used to obtain the E-alkene. ${ }^{108}$ This is achieved by a multi-step one-pot reaction 
sequence that uses phenyllithium to facilitate the reaction of an aldehyde and phosphonium salt to form an $\alpha$-lithiated betaine (Scheme 3.1). The erythro intermediate 243 is again formed preferentially, but equilibrates in favour of the threo configuration to the extent of $\geq 99.5 \%$. ${ }^{109}$ Addition of one equivalent of tert-butanol (or $\mathrm{HCl}$ ) gives betaine 244 which is treated with potassium tert-butoxide to exchange lithium for potassium. This results in trans-oxaphosphetane formation, providing the $E$-alkene product 245.
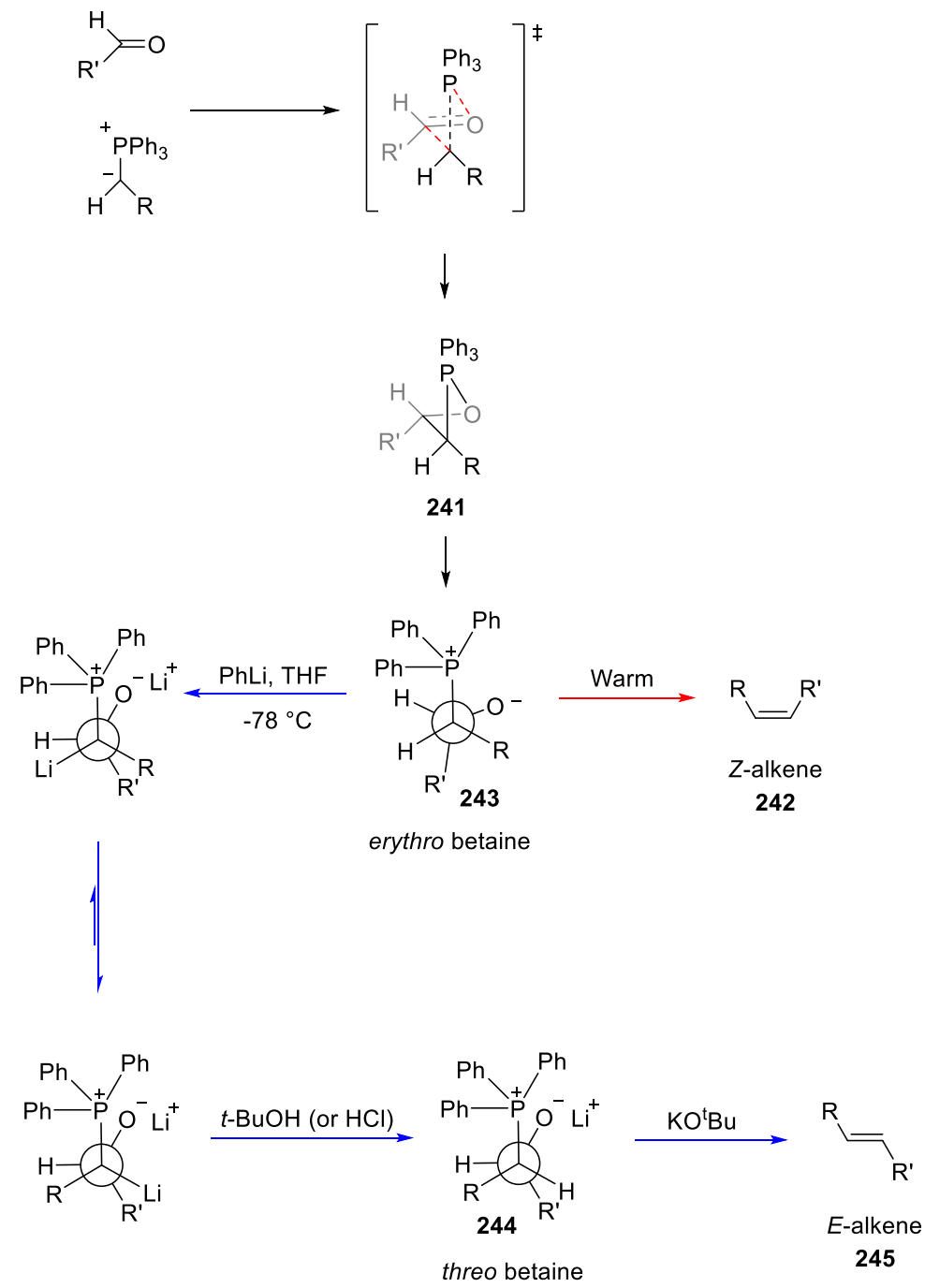

Scheme 3.1 Mechanism for the Wittig olefination to provide Z-alkene (standard conditions, red arrow) and the E-alkene (Schlosser's modification, blue arrows).

In addition, it is known that excellent E-selectivity can be obtained by using a trialkylphosphine instead of triphenylphosphine. ${ }^{110}$ The use of lithium salts and bulky 
groups on the phosphonium ion contribute to good E-selectivity. ${ }^{111}$ This is illustrated in Scheme 3.2, in a reaction towards an analogue of callystatin A, whereby tributylphosphonium salt $\mathbf{2 4 6}$ is deprotonated and reacted with aldehyde $\mathbf{2 4 7}$ to provide product 248 with high E-selectivity. ${ }^{112}$
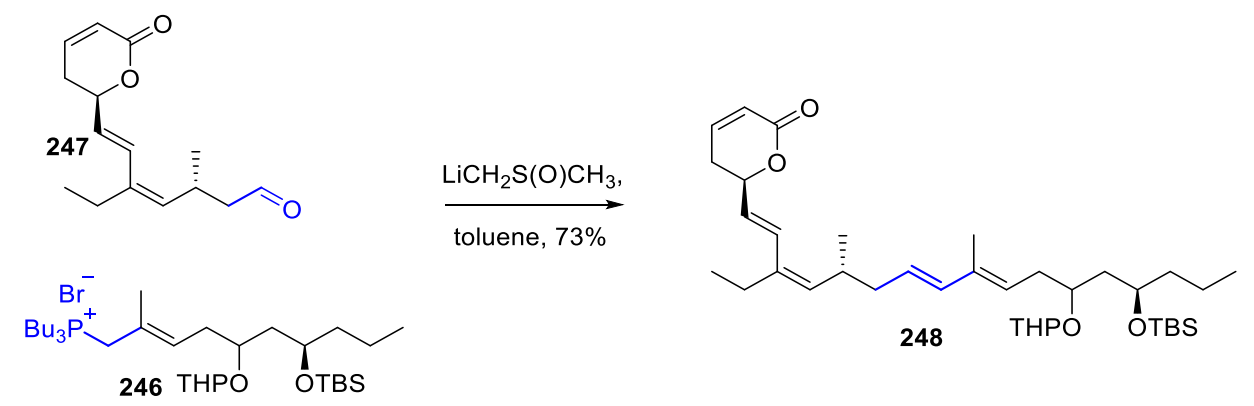

Scheme 3.2 E-Selective Wittig olefination reaction using a tributylphosphonium salt. ${ }^{112}$

On the other hand, the modified Julia-Kocienski reaction, in which an aldehyde reacts with a metallated 1-phenyl-1H-tetrazol-5-yl sulfone (PT) has emerged as a powerful approach to $E$-alkenes. ${ }^{113,114}$ The reaction begins with the treatment of the sulfone 249 with an appropriate metal base to give metallated sulfone 250 (Scheme 3.3). This then reacts quickly with an aldehyde to give the $\beta$-alkoxysulfone adduct 251, which is inherently unstable and experiences a facile Smiles rearrangement, via a putative spirocyclic intermediate 252 to give sulfinate salt 253. Spontaneous elimination of sulfur dioxide and lithium phenyltetrazole 254 from sulfinate salt 253 yields the alkene product 255 . 


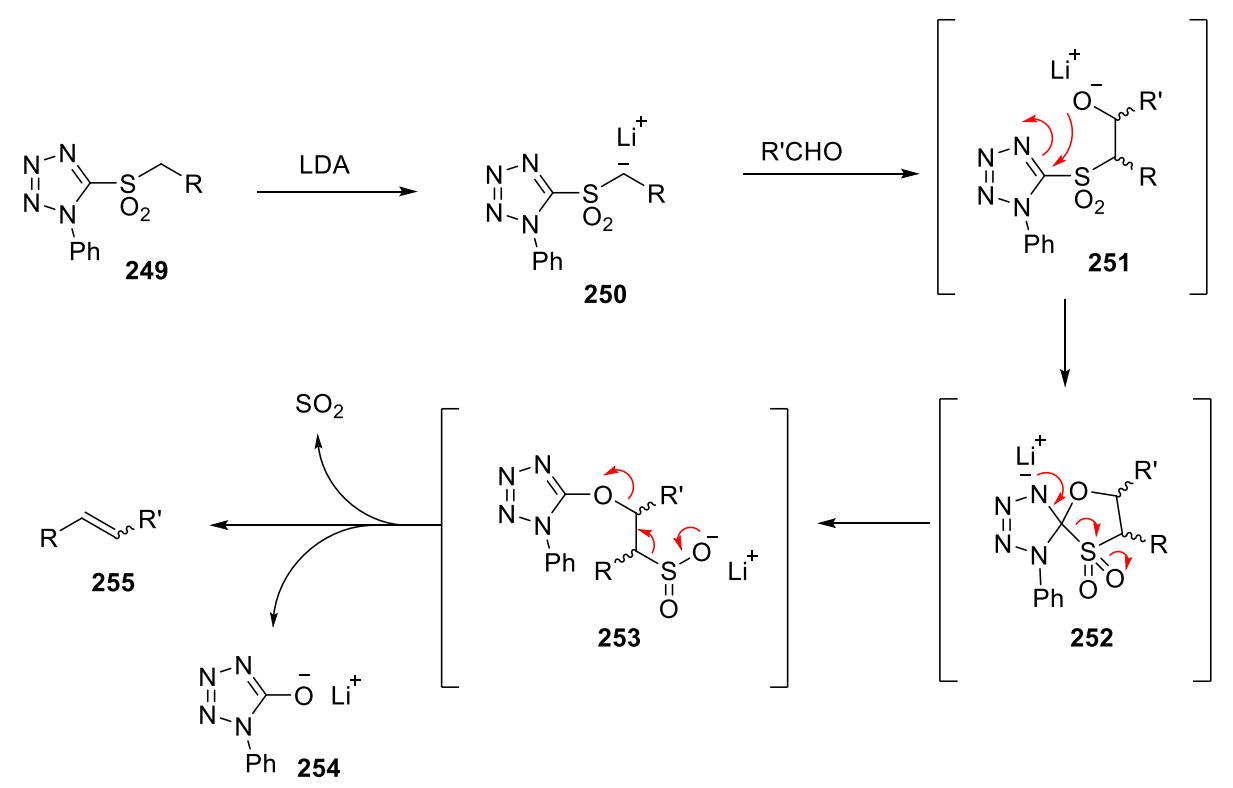

Scheme 3.3 Reaction mechanism of Julia-Kocienski olefination. ${ }^{113}$

The stereomeric outcome of the reaction is highly dependent on the base counter-cation and solvent that is used. In the reaction of PT-sulfone 256 and cyclohexanecarbaldehyde, using potassium as the base counter-cation provided the best E-selectivity in comparison to lithium and sodium (Table 3.1). In addition, E-selectivity improved as the reaction was done in increasingly polar solvent. ${ }^{115}$
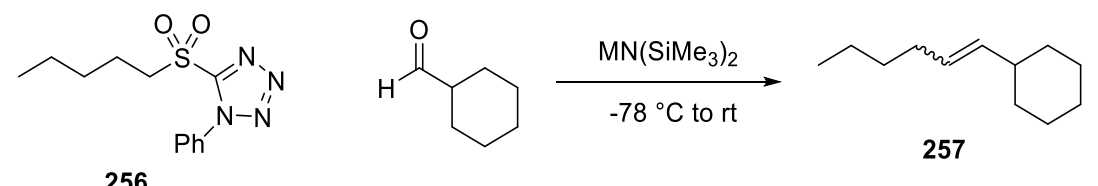

256

E:Z Ratio

\begin{tabular}{ccccc}
\hline $\mathbf{M}$ & toluene & $\mathbf{E t}_{\mathbf{2}} \mathbf{O}$ & THF & DME \\
\hline $\mathbf{L i}$ & $51: 49$ & $61: 39$ & $69: 31$ & $72: 28$ \\
$\mathbf{N a}$ & $65: 35$ & $65: 35$ & $73: 27$ & $89: 11$ \\
$\mathbf{K}$ & $77: 23$ & $89: 11$ & $97: 3$ & $99: 1$ \\
\hline
\end{tabular}

Table 3.1 Effect of solvent and base on E:Z ratio during coupling of PT-sulfone 256 and cyclohexanecarbaldehyde to form alkene 257.115 


\subsubsection{Macrocyclisation}

An intramolecular reaction of a linear chain that makes a cyclic compound (macrocycle) that is 12 or more atoms is known as macrocyclisation. Many biologically active natural products contain macrocycles and therefore much work has been put into this type of reactivity. ${ }^{116}$ The most common methods utilised to close large rings in target driven synthesis are palladium-catalysed cross coupling reactions, e.g. Stille reactions, macrolactonisation with an appropriate coupling reagent and ring closing metathesis (RCM) using, for example, Grubbs $2^{\text {nd }}$ generation catalyst.

The reactivity of a cyclisation reaction is determined by three key factors: the total energy of the system (enthalpy), loss of freedom (entropy), and the probability of end-to-end encounters. ${ }^{117}$ Activation energy reflects the strain energy of the transition state for the ring being formed, which arises from deviations of customary bond angles (Baeyer strain), imperfect staggering (Pitzer strain) and transannular strains due to repulsive interactions across the ring. In terms of entropy, the process of cyclisation results in a decrease in disorder due to the decrease in the freedom of the internal rotation of single bonds. This disorder increases with chain length. The probability of the chain terminals coming close enough to each other for the reaction to occur typically decreases as the chain gets longer.

Figure 3.2 shows the rate of reaction for the lactone formation from $\omega$-bromoalkanoic acids with increasing chain length. ${ }^{117}$ It can be seen here that for smaller ring lactones $(\mathrm{n}=$ 4-6) the enthalpic factor outweighs the entropic factor, as the probability of end-to-end encounters is high and ring strain of the transition state is low. As the ring size increases to medium sized rings $(n=8-12)$ strain energy increases due to lack of freedom to adopt ideal bond angles which, in combination with entropic factors, makes these ring sizes typically difficult to form. As the ring size increases to larger sized rings (13+) the ring strain decreases, but the probability of end-to-end encounters also decreases. 


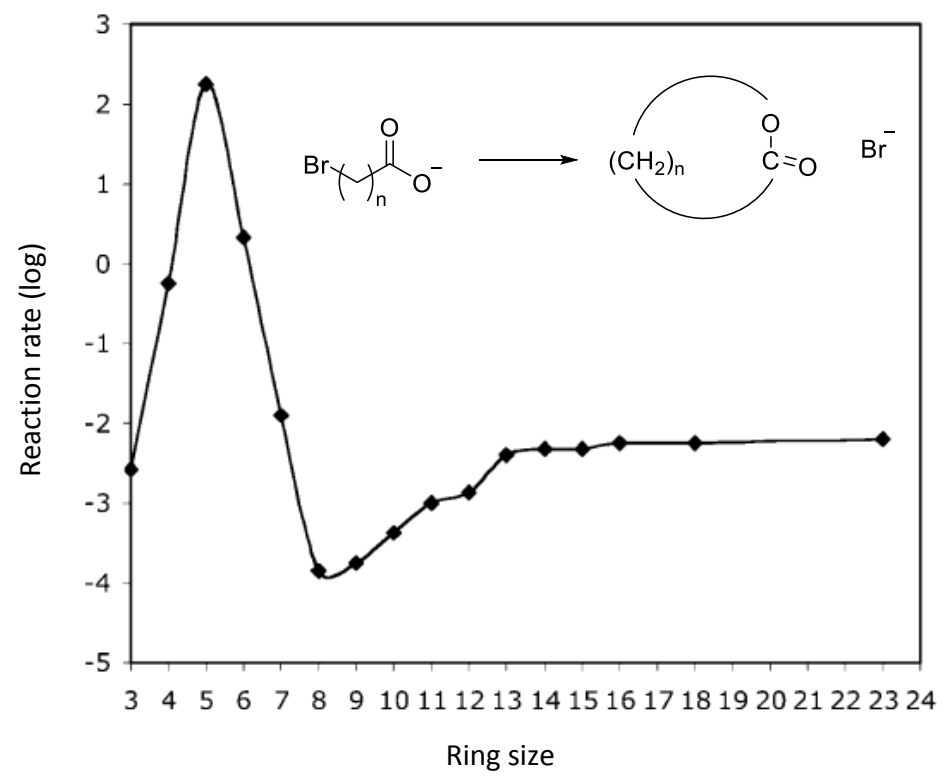

Figure 3.2 Reactivity profile of lactone formation. ${ }^{117}$

\subsubsection{Macrolactonisation}

Macrolactonisation of a seco acid is a fundamental reaction in organic synthesis. Many reagents and methods have been employed to achieve this coupling, most of which work by 'activating' either the carboxylic acid (Figure 3a) or the alcohol (Figure 3b), to overcome the energy barrier for reaction.

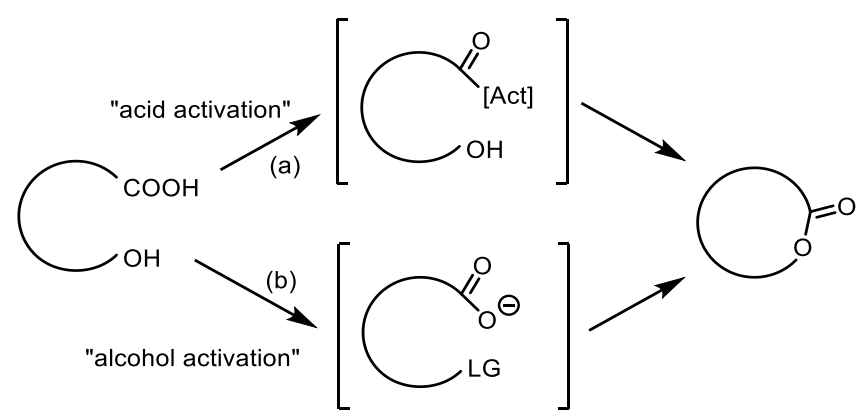

Figure 3.3 Schematic representation of macrolactonisation via acid or alcohol activation.

Figure 3.4 summarises some of the key macrolactonisation reactions and their mechanistic key steps that have been utilised in the synthesis of natural product macrocycles and their 
analogues. Each of the reactions exemplifies their respective 'class' of lactonisation reactions/reagents, of which many variations have been successfully used. ${ }^{118}$

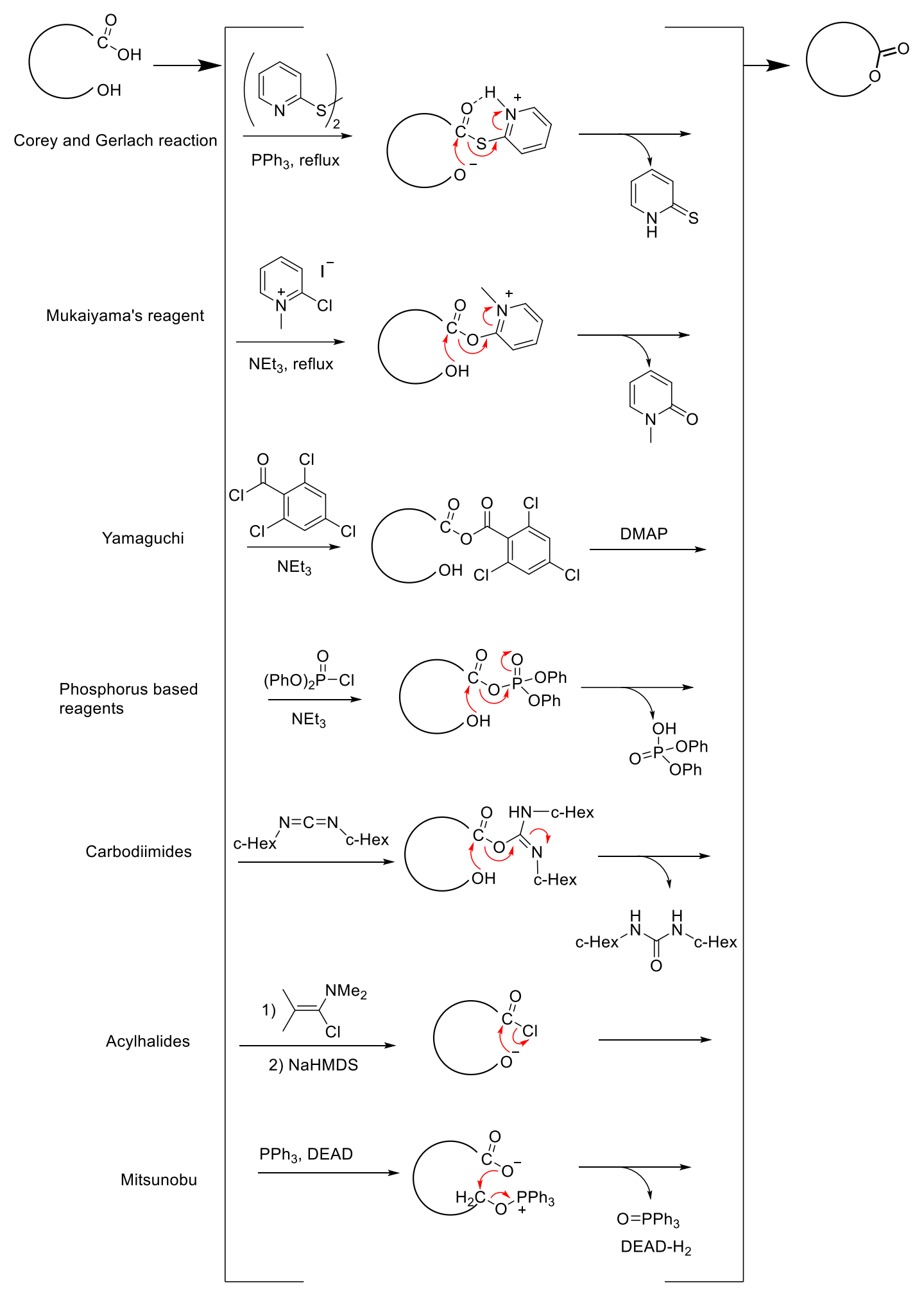

Figure 3.4 Summary of general macrolactonisation methods. 
Special care is required when coupling a Z,E-dienoic acid to an alcohol, owing to the fact that the $E, E$-isomer is thermodynamically more favoured, and therefore the $Z, E$-dienoic acid has a tendency to isomerise, as discussed in Section 2.1.1. For example, the coupling of alcohol 258 with dienoic acid 259 during the synthesis of varrucarin J (260) using DCC and 4-pyrrolidine pyridine (4-PP) resulted in significant isomerisation giving a 60:40 ratio of the Z,E : E,E product (Scheme 3.4). ${ }^{119}$ This was proposed to be caused by the 4-PP during the reaction.
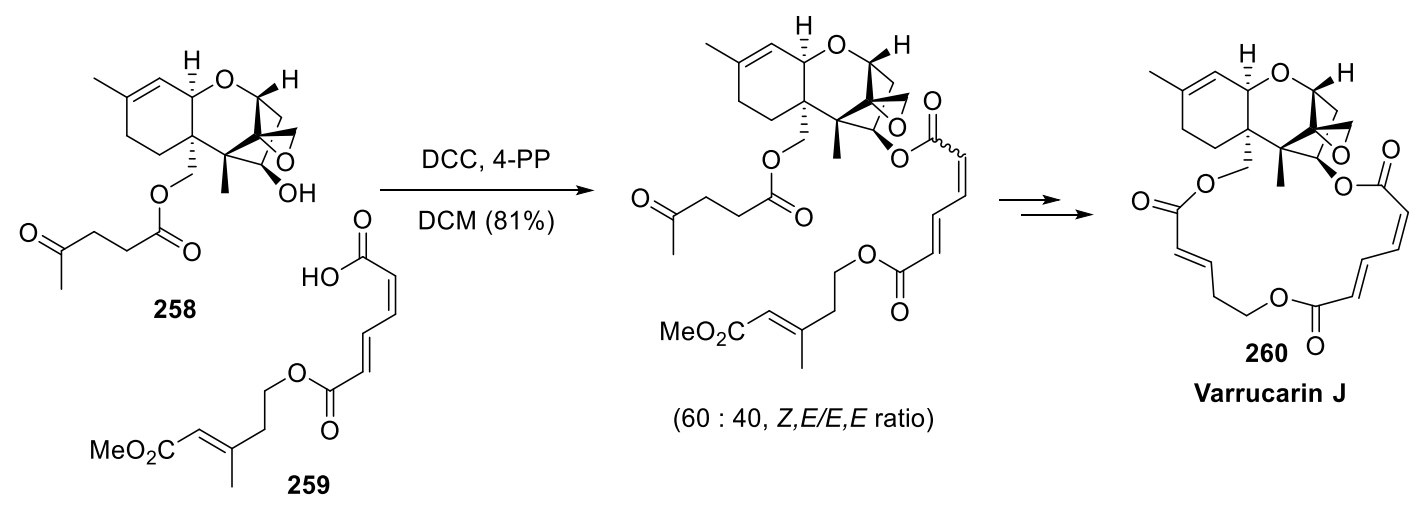

Scheme 3.4 Esterification reaction towards the synthesis of varrucarin J 260. ${ }^{119}$

In the synthesis of dictyostatin, an intermolecular esterification of alcohol 261 and dienoic acid 262 was attempted using 2-methyl-6-nitrobenzoic anhydride 263 (Shiina's reagent) which can sometimes suppress isomerisation. ${ }^{120}$ However, reaction with excess NEt 3 and DMAP gave a 58:42 ratio of the $Z, E$ to $E, E$ product 264 (Table 3.2, Entry 1). ${ }^{121}$ Attributing the isomerisation to the presence of DMAP, an esterification strategy was then used that was not reliant on an acylation catalyst. Thus, 1-chloro- $N, N$,2-trimethyl-1-propenylamine 265 (Ghosez's reagent) $^{122}$ was used as a mild and neutral chlorinating reagent to convert the carboxylic acid 262 into the acid chloride, followed by deprotonation of alcohol 261 with NaHMDS to facilitate esterification in a $75 \%$ yield with no observed isomerisation (Entry 2). 
<smiles>C=CC(=O)OC(CCC(C)CC(C)C(CC)C(C)C)C(C)C(C)O</smiles><smiles>C=CCC(O)CC(C)C=CC=CC(=O)O</smiles>
(see table)

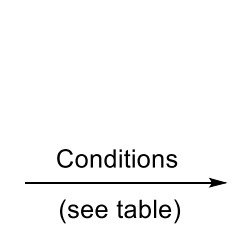

$$
\text { (1) }
$$

262
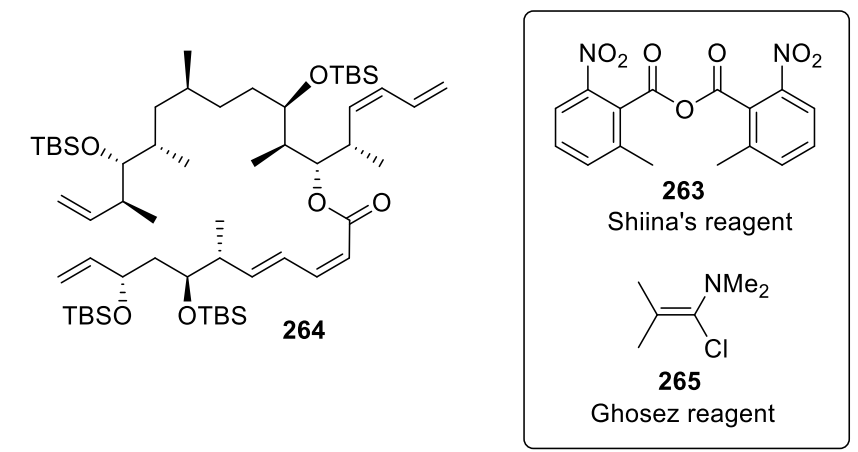

\begin{tabular}{clccc}
\hline Entry & \multicolumn{1}{c}{ Conditions } & Total yield & $\begin{array}{c}Z, E: \\
E, E\end{array}$ & Yield of $Z, E-264$ \\
\hline $\mathbf{1}$ & Shiina reagent, DMAP, NEt3 & $81 \%$ & $58: 42$ & $47 \%$ \\
$\mathbf{2}$ & $\begin{array}{l}\text { 1) Ghosez reagent } \\
\text { 2) NaHMDS }\end{array}$ & $75 \%$ & $100: 0$ & $75 \%$ \\
\hline
\end{tabular}

Table 3.2 Esterification of $Z, E$-dienoic acid 262 towards the synthesis of dictyostatin. ${ }^{121}$

Interestingly, in the synthesis of (+)-9-epi-dictyostatin, a Yamaguchi macrolactonisation was performed on seco acid 266 provided lactone 267 with only 5-10\% isomerisation (Scheme 3.5). ${ }^{123}$ This suggests that restriction of movement, as imposed during formation of a macrocycle, significantly reduces the isomerisation caused by the acylation catalyst DMAP.

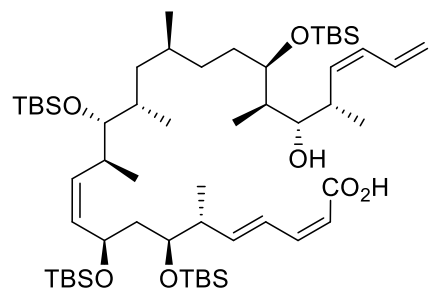

266

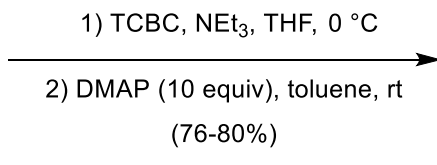

$(76-80 \%)$

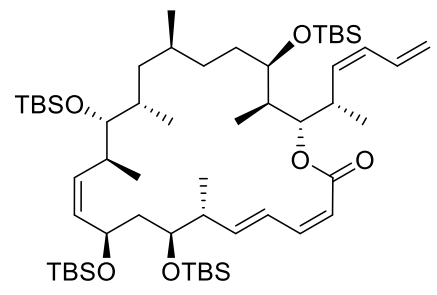

267

Scheme 3.5 Intramolecular Yamaguchi reaction of seco acid 266 to afford macrocycle 267 in the synthesis of (+)-9-epi-dictyostatin, accompanied by 5-10\% isomerisation to $2 E, 4 E-$ isomer of 267.123

An alternative strategy of ester formation was conducted in the synthesis of macrolactin A, using a Mitsunobu reaction between Z,E-dienoic acid 268 and alcohol 269 with no 
reported isomerisation (Scheme 3.6). ${ }^{64}$ Since the Mitsunobu reaction involves the activation of the alcohol functionality it is no surprise that diene isomerisation is not observed, which renders this an attractive esterification strategy to limit geometric isomerisation.

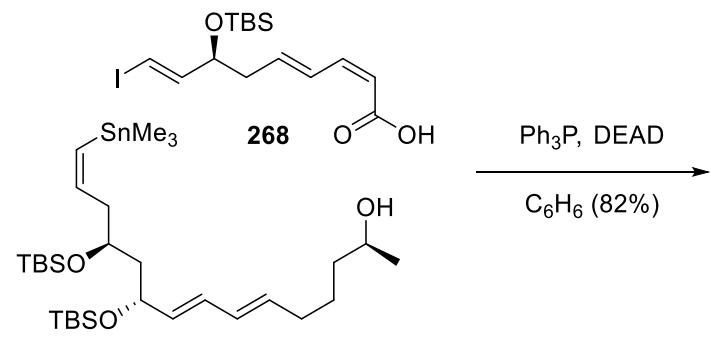

269

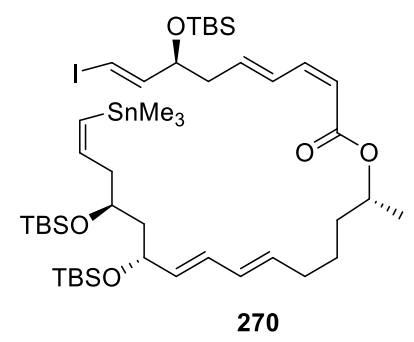

270

Scheme 3.6 Intermolecular Mitsunobu reaction between Z,E-dienoic acid 268 and alcohol 269 towards the synthesis of macrolactin A. ${ }^{64}$

\subsubsection{CuAAC Reaction in Macrocyclisation}

The formation of a triazole heterocycle is typically achieved using a copper catalysed azide-alkyne cycloaddition (CuAAC) reaction (Scheme 3.7). The CuAAC reaction involves the copper(I)-catalysed coupling of a terminal or internal alkyne and an alkyl azide to give the 1,2,3-triazole. It is a variant of the azide-alkyne Huisgen cycloaddition, which is a formal 1,3-dipolar cycloaddition that was first described by Michael ${ }^{124}$ and later investigated in detail by Huisgen and involves the uncatalysed formation of the 1,2,3triazole. ${ }^{125}$

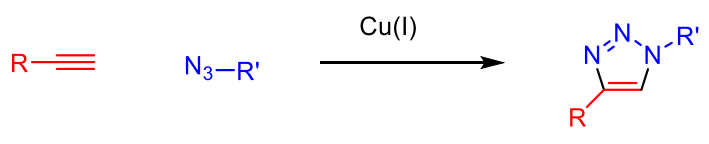

Scheme 3.7 Copper catalysed azide-alkyne cycloaddition (CuAAC) reaction. 
The CuAAC reaction is commonly referred to as a 'click' reaction due to it exhibiting most of the necessary features ${ }^{* * * *}$ for what is described as click chemistry. ${ }^{126}$ The CuAAC has found a variety of uses in medicinal chemistry, supramolecular chemistry, biological sciences ${ }^{127}$ and material sciences. ${ }^{128}$ This is due to its highly specific reactivity and the ability to perform reactions in either aqueous or organic solvents.

The mechanism of the CuAAC reaction involves the initial $\pi$-complexation of the copper(I) species with alkyne (A), thus lowering the $p$ Ka of the terminal alkyne proton and allowing transfer of $\mathrm{Cu}(\mathrm{I})$ onto the alkyne terminus (Scheme 3.8). The resultant copper-acetylide (B) then interacts with azide (C) forming an azido-moiety (D), which leads to a 6-membered intermediate (E). This breaks down into 5-membered ring (F), with subsequent release of the triazole product $(\mathrm{G})$ and regeneration of the catalytic copper(I) species.

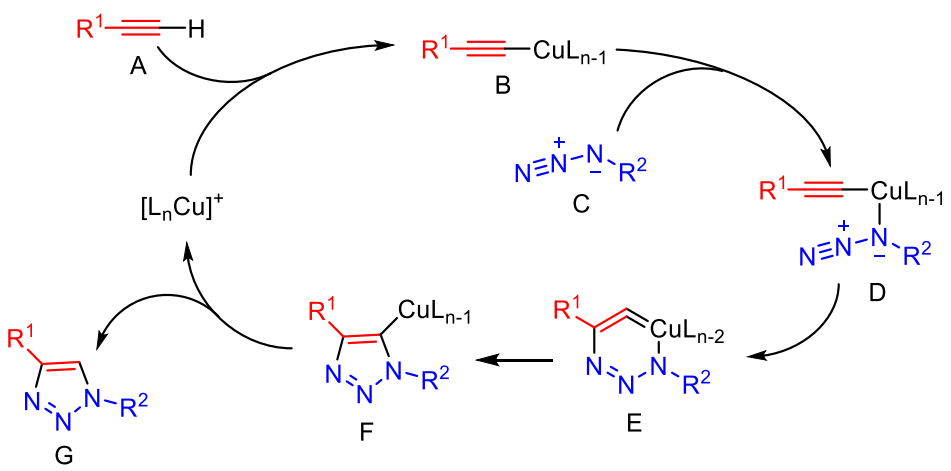

Scheme 3.8 Mechanism of CuAAC reaction. ${ }^{129}$

CuAAC can be used to generate macrolactones, however the outcome of these macrocyclisation reactions has been found to be highly structure dependent. From cyclisations of polypeptides it has been found that macrocycles containing 8 to 14 atoms are extremely difficult to achieve and mostly cyclic dimers and trimers are obtained (Figure 3.5)..$^{130}$

\footnotetext{
${ }^{* * * *}$ Criteria for the ideal 'click' reaction is: modular, wide in scope, high yielding, inoffensive by-products (that can be removed by non-chromatographic methods) and be stereospecific. The required process characteristics include simple reaction conditions (ideally, the process should be insensitive to oxygen and water), readily available starting materials and reagents, the use of no solvent or a solvent that is benign (such as water) or easily removed, and simple product isolation.
} 

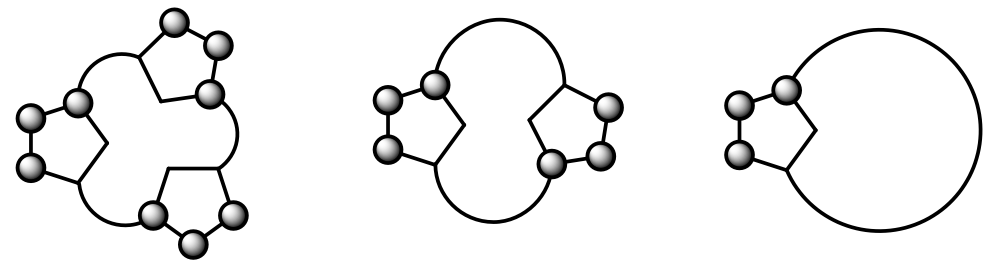

Polymers

Figure 3.5 Structural influence on the formation of macrocycles by CuAAC. ${ }^{130}$

The CuAAC-mediated cyclisation differs from that via macrolactonisation in the sense that three components come together in the transition state, i.e. the alkyne, the azide and the copper species. Furthermore, the copper(I) species is able to coordinate a second alkyne in the transition state. Consequently, if intermolecular reaction occurs, the probability of intramolecular formation is significantly increased due to the higher pseudoconcentration of reactive groups upon formation of the first triazole.

In the search for cyclic compounds capable of $\mathrm{Cu}^{\mathrm{II}}$ cation complexation, Allam and coworkers prepared triazole containing compound 271 (Scheme 3.9). ${ }^{131}$ Two strategies were attempted for the final stages of the synthesis, that involved either macrolactamisation (Path A) or CuAAC (Path B) as the final macrocyclisation step. In path A, intermolecular CuAAC reaction of alkyne $\mathbf{2 7 2}$ and azide $\mathbf{2 7 3}$ gave linear compound $\mathbf{2 7 4}$. This was then treated with a range of solvents at reflux, with no product formed and starting material recovered in all cases. On the other hand, in path B the intermolecular aminolysis of compounds 272 and 273 gave compound 275. Treatment of this linear compound with copper sulfate and sodium ascorbate in water resulted in two products, the desired 1,4triazole 271 monomer and the dimer 276, in a ratio of 80:20 and 84\% yield (Method A, Scheme 3.9). When the reaction was performed with $\mathrm{CuI}$ and diisopropylethylether in THF/acetonitrile (3:1) a similar result was obtained (Method B). Interestingly, by using similar conditions but in water (Method C) the dimer was obtained as the only product. This highlights that the different spatial constraints of these reactions (e.g. between lactamisation and $\mathrm{CuAAC}$ ) provide different favourability towards the success of macrocyclisation. 


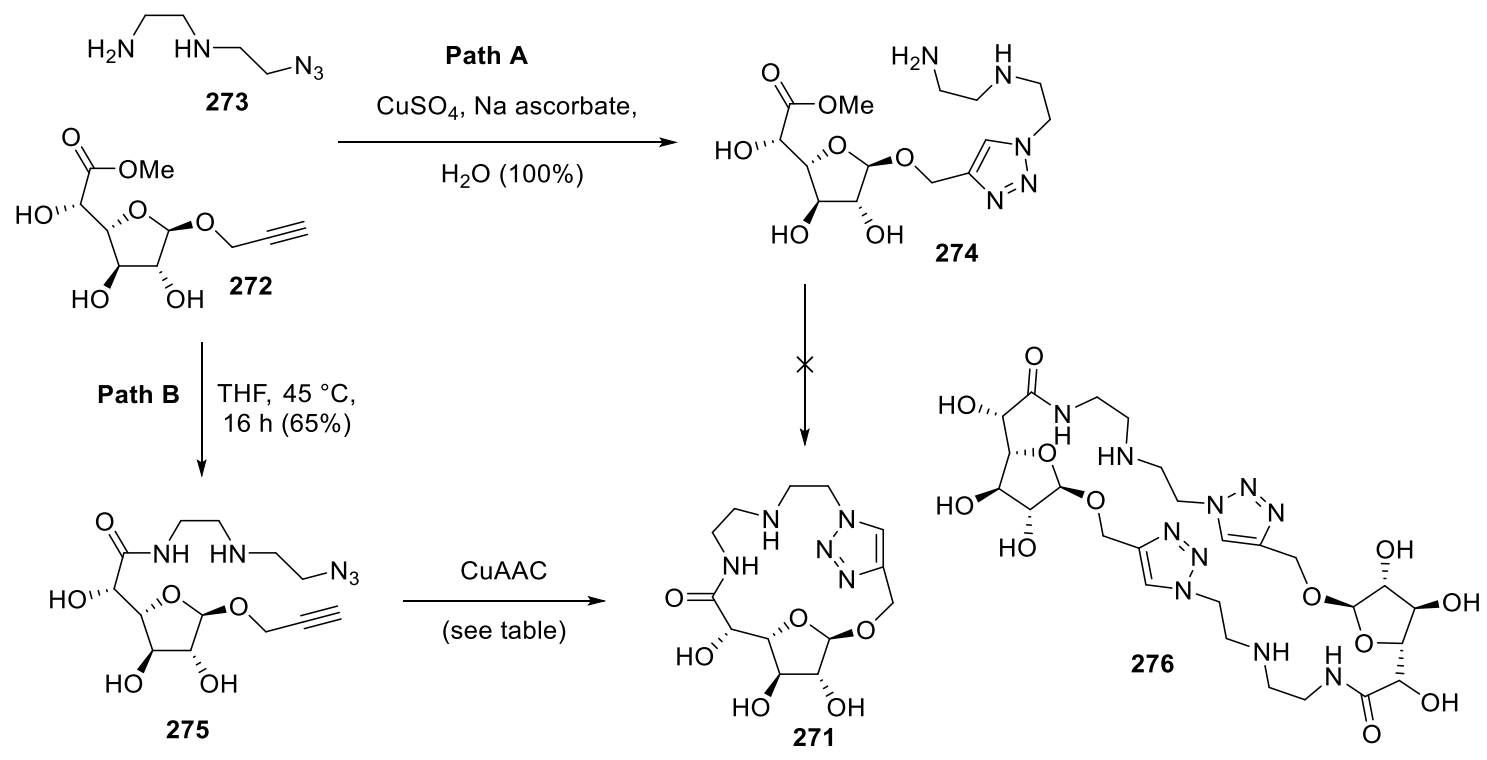

\begin{tabular}{cccc}
\hline Method & Conditions & Yield & Monomer : Dimer \\
\hline A & $\mathrm{CuSO}_{4}, \mathrm{Na}$ ascorbate, $\mathrm{H}_{2} \mathrm{O}, \mathrm{rt}$ & $84 \%$ & $80: 20$ \\
B & $\mathrm{CuI}, \mathrm{THF} / \mathrm{MeCN}, \mathrm{DIPEA}, \mathrm{rt}$ & $90 \%$ & $70: 30$ \\
C & $\mathrm{CuI}, \mathrm{H}_{2} \mathrm{O}, \mathrm{DIPEA}, \mathrm{rt}$ & $55 \%$ & $0: 100$ \\
\hline
\end{tabular}

Scheme 3.9 Use of CuAAC in cyclisation reaction. ${ }^{131}$

\subsection{Fragment Coupling to Form a Sidechain Truncated Macrocycle}

The combination of three fragments can be achieved via multiple orders of connectivity. This section outlines the work to determining a suitable order of connectivity and coupling reactions to provide a sidechain truncated macrocycle 277. In addition, this macrocycle is a valuable compound to seperately test the sidechain requirement for binding versus activity in eIF4A isoforms.

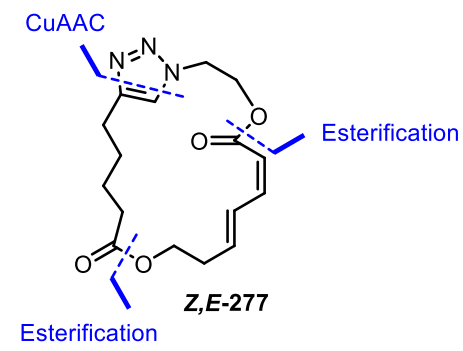

Figure 3.6 Sidechain truncated macrocycle Z,E-277. 


\subsubsection{Exploration of Z,E-Dienoate Esterification}

As discussed earlier (vide supra, Section 3.1.3), Z,E-dienoates have a tendency to isomerise to the E,E-stereoisomer during activation for esterification/lactonisation. Therefore, a suitable method to couple the $Z, E$-dienoic acid fragment in ester or lactone formation was sought. Zhu and co-workers' strategy to utilise Ghosez's reagent followed by NaHMDS (vide supra, Table 3.2), was successful in preventing isomerisation. However, preparation of the Ghosez reagent required the use of phosgene, which is highly toxic and volatile. It reacts with proteins in lungs resulting in suffocation and therefore we avoided this method. The Mitsunobu reaction seemed like an attractive choice as this did not involve activating the carboxylic acid and therefore limiting the risk of isomerisation.

Triphenylphosphine is a key reagent in the Mitsunobu reaction, but it also reacts with alkyl azides, as in the Staudinger reaction (Scheme 3.10). An excess of DIAD relative to the triphenylphosphine during pre-formation of reactive diazo-phosphonium species was used in an effort to avoid undesired side products. When this reaction was tested on alcohol 207 and cinnamic acid no product was observed, with significant recovery of starting materials and some unidentified side products (Scheme 3.11).

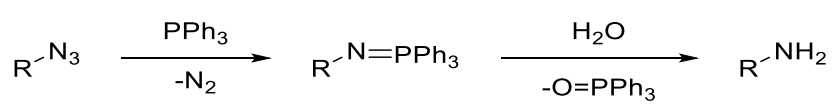

Scheme 3.10 Staudinger reaction.

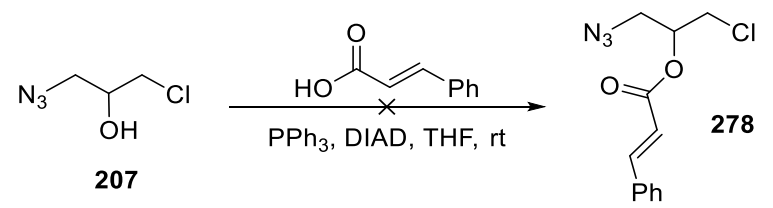

Scheme 3.11 Attempt at ester formation using Mitsunobu reaction.

We then tried EDCI as a coupling reagent with the use of catalytic DMAP (Scheme 3.12). The use of EDCI to perform the esterification reaction was appealing due to its simplicity (i.e. all reactants are added at the same time, typically performed at room temperature (or 
$0{ }^{\circ} \mathrm{C}$ ) and reaction is not particularly water sensitive). When the reaction was performed using alkyl chloride 207 or sulfide 208 at $0{ }^{\circ} \mathrm{C}$ or room temperature, good conversion of starting material to ester was obtained. Unfortunately, in all cases, complete isomerisation was observed, providing only the E,E-dienoate (279 and 280).
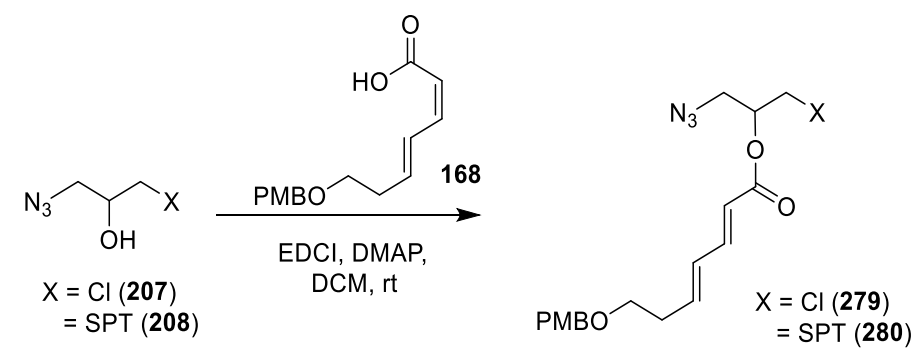

Scheme 3.12 Coupling of Z,E-dienoic acid 168 with EDCI.

Performing an intermolecular Yamaguchi reaction of Z,E-dienoic acid 168 and alkyl chloride 207 via 2,4,6-trichlorobenzoyl chloride (TCBC), resulted in a good yield and provided the desired product, Z,E-281 (Scheme 3.13), albeit accompanied by significant isomerisation with a 2:1 ratio of the $Z, E-281$ to $E, E-281$.

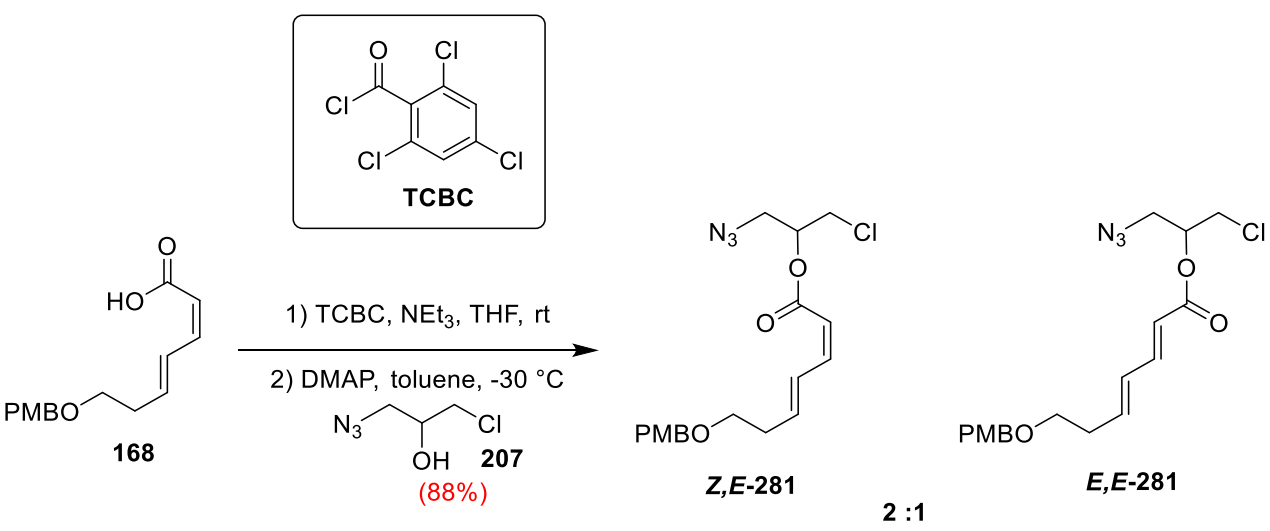

Scheme 3.13 Coupling of Z,E-dienoic acid 168 using Yamaguchi conditions.

From these reactions, the Yamaguchi esterification conditions gave the best results. Although the isomerisation was significant, it was satisfactory to test its utility in the 
formation of the macrocycle. It was also encouraging that the intramolecular Yamaguchi reaction that was performed by Gennari and co-workers on a Z,E-dienoic acid resulted in little (5-10\%) reported isomerisation (vide supra, Scheme 3.5), which suggested that the isomerisation is suppressed when performed intramoleculaly. ${ }^{123}$

\subsubsection{Macrocycle Formation}

We set out to test two routes of macrocyclisation utilising either the $\mathrm{CuAAC}$ reaction or the Yamaguchi macrolactonisation (Scheme 3.14). It was envisioned that compound 282, prepared from fragment 190 and 167, could be attached to 2-azidoethanol in two different orders of coupling, i.e. esterification followed by intramolecular CuAAC reaction (Scheme 3.14, blue route) or intermolecular $\mathrm{CuAAC}$ reaction followed by macrolactonisation (Scheme 3.14, red route). It is worth noting that this order of reactivity is possible due to the 'mild' reactivity of TBAF, which can function as a base to facilitate the lactone opening. This allows the $\delta$-lactone opening reaction to occur after coupling; in essence the lactone acts as a 'masked' $Z, E$-dienoic acid that can be revealed when necessary.

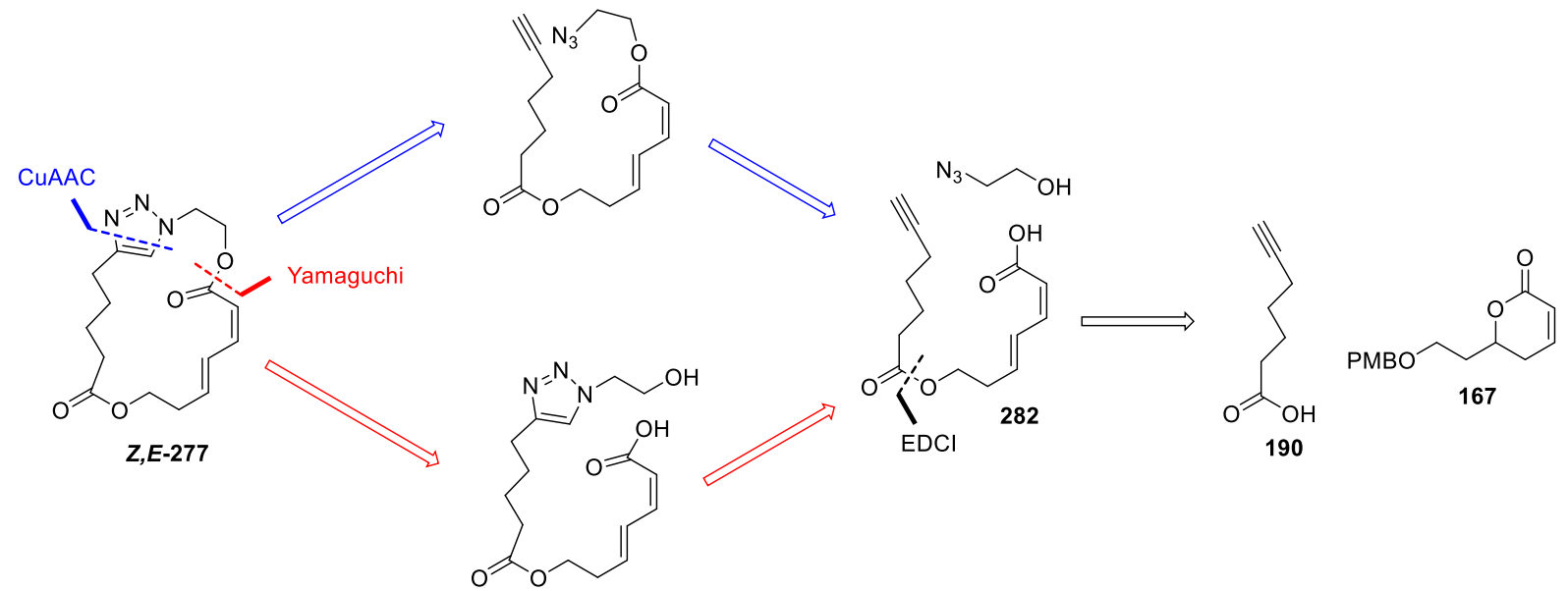

Scheme 3.14 Retrosynthetic analysis of macrocycle.

Preparation of Z,E-dienoic acid 282 was achieved by first cleavage of the PMB-ether of lactone 167 (from Section 2.1) using DDQ to provide alcohol 283 (Scheme 3.15). This was then coupled to C1-C7 fragment 190 (from Section 2.2) by esterification using EDCI with 
catalytic DMAP, providing compound 284. Lactone ring opening was then achieved by treatment with TBAF to give Z,E-dienoic acid $\mathbf{2 8 2}$ as the sole stereoisomer. 2-Azidoethanol was prepared from 2-chloroethanol by treatment with sodium azide and sodium iodide in refluxing water (Scheme 3.15, insert). ${ }^{132}$

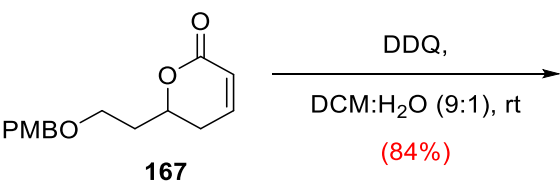

167

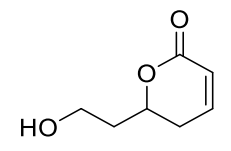

283

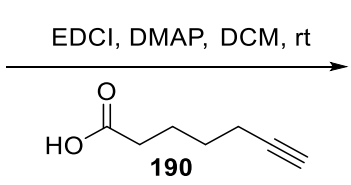

$(71 \%)$

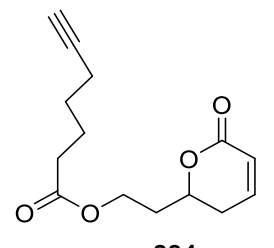

TBAF, THF, rt $(95 \%)$

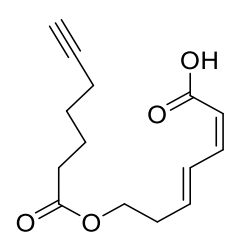

282

Scheme 3.15 Preparation of compound 282 and 2-azidoethanol (box insert).

With both dienoic acid $\mathbf{2 8 2}$ and 2-azidoethanol in hand, we were then able to test our two different routes for macrocyclisation. Firstly, the use of CuAAC as the macrocyclisation process was investigated. Thus, dienoic acid 282 was coupled to 2-azidoethanol using Yamaguchi esterification conditions, by reaction with TCBC and triethylamine at room temperature followed by dilution with DCM and addition of 2-azidoethanol and DMAP. Unfortunately, when the esterification step was performed at room temperature with a large excess of DMAP (20 equivalents), almost full isomerisation was observed with a 1:10 ratio of $\boldsymbol{Z}, \boldsymbol{E}-\mathbf{2 8 5}$ and $\boldsymbol{E}, \boldsymbol{E}-\mathbf{2 8 5}$ (Table 3.3, Entry 1). In an attempt to reduce isomerisation, the esterification step was performed at lower temperature $\left(-78^{\circ} \mathrm{C}\right.$ to room temperature $)$ and with catalytic DMAP (0.3 equivalence). This resulted in significantly less isomerisation, with a 2:1 ratio of $Z, E-285$ and $E, E-285$, but with a lower total yield (Entry 2). 


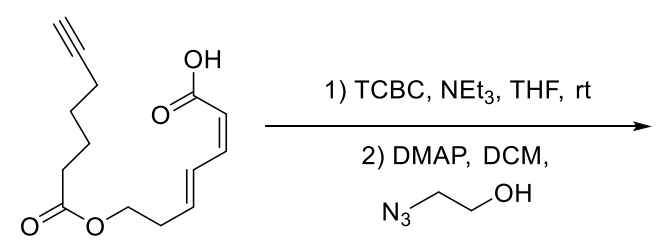

282

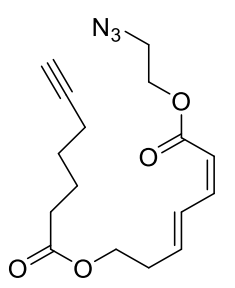

$Z, E-285$

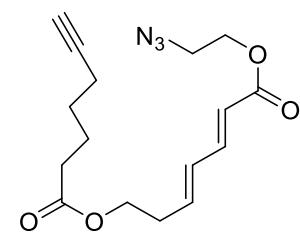

$E, E-285$

\begin{tabular}{ccccc}
\hline Entry & DMAP equiv & Temperature & Ratio $(Z, E: E, E)$ & Total yield \\
\hline $\mathbf{1}$ (G74) & 20 & rt & $1: 10$ & $71 \%$ \\
$\mathbf{2}$ (G76) & 0.3 & $-78^{\circ} \mathrm{C}$ to rt & $2: 1$ & $48 \%$ \\
\hline
\end{tabular}

Table 3.3 Yamaguchi esterification reaction of acid 282 and 2-azidoethanol.

The isomerisation occurring seen in the intermolecular esterification with Yamaguchi conditions was less than ideal. It did however, allow us to obtain macrocycle $E, E-277$ to compare its behaviour in biological systems with that of the $Z, E-277$. This was achieved by treating the acyclic $E, E-285$ with CuAAC conditions under high dilution to provide the macrocyclised product E,E-277 (Scheme 3.16). No dimerised products were observed by HR-MS.<smiles>C#CCCCC(=O)OCC/C=C\C=C\C(=O)OCCN</smiles>

$E, E-285$

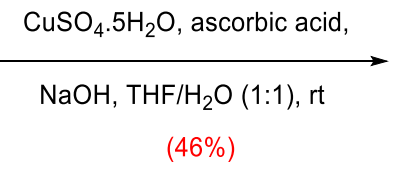

$(46 \%)$

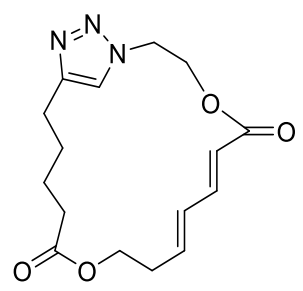

$E, E-277$

Scheme 3.16 Macrocyclisation with CuAAC to give $E, E-277$.

In studying the alternative route, Z,E-dienoic acid 282 was coupled to 2-azidoethanol using CuAAC conditions to provide seco acid 286 in 62\% yield (Scheme 3.17). The seco acid 286 was then subjected to Yamaguchi macrolactonisation conditions, through formation of the mixed anhydride with TCBC and triethylamine, followed by high dilution with DCM and addition of catalytic DMAP to the reaction mixture at $-78{ }^{\circ} \mathrm{C}$ and allowing the reaction to warm to room temperature (similar to the conditions that minimised isomerisation, $c f$. Table 3.3 , Entry 2) to provide macrocycle $Z, E-277$ in $22 \%$ 
yield. The Z,E- and E,E-macrocycles were clearly differentiated in their ${ }^{1} \mathrm{H}$ NMR spectra, by both the coupling of the unsaturated $\alpha$-proton $(J=11.2 \mathrm{~Hz}$ and $15.5 \mathrm{~Hz}$, respectively) and the characteristic signal shifts ${ }^{31}$ of the alkene protons (Figure 3.7).

Although the yield was poor from the Yamaguchi macrolactonisation (presumably due to incomplete conversion), it was satisfying that no isomerised product was observed. It was decided that this route was preferred as the isomerised products were not easily separated.

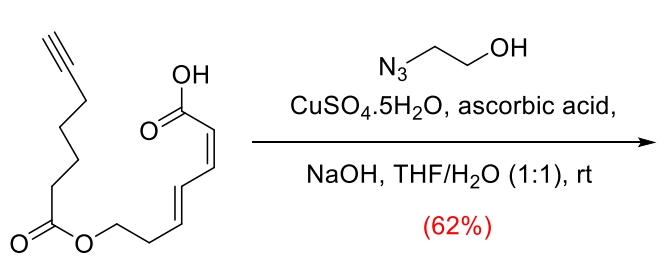

282<smiles>O=C(O)/C=C\C=C/COC(=O)CCCCc1cn(CCO)nn1</smiles>

286

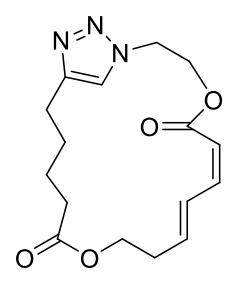

$Z, E-277$

Scheme 3.17 Preparation of the macrocycle $Z, E-277$ using macrolactonisation.
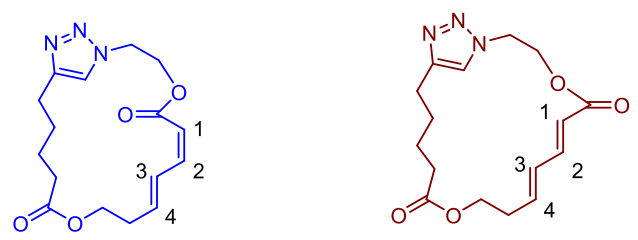

$$
\begin{gathered}
1 \\
(J=15.5 \mathrm{~Hz})
\end{gathered}
$$

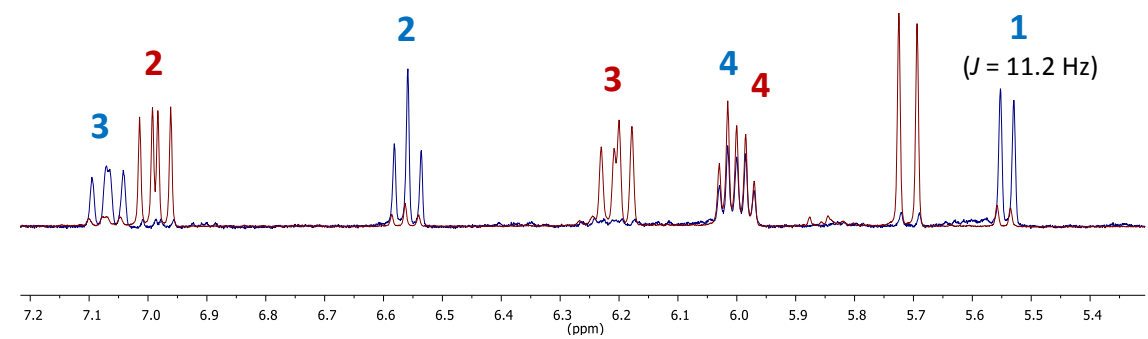

Figure 3.7 Comparison of the alkene region for the ${ }^{1} \mathrm{H}$ NMR spectra of $Z, E-277$ (in blue) and $E, E-277$ (in maroon). (Assigned using 2D NMR.) 


\subsection{Model Studies for Side Chain Attachment}

Having established methods for connecting the fragments to form the macrocycle, we then set out to test methods for attaching the conjugated sidechain. To save material, simplified acyclic models were prepared that would allow the validity of using either the Wittig or Julia-Kocienski olefination reactions to attach the sidechain.

\subsubsection{Wittig Reaction Strategy}

An acyclic model system was designed to test the validity of connecting the side chain via an alkyl chloride attached to the macrocycle (Scheme 3.18). This model was prepared by coupling alcohol 207 with cinnamic acid using EDCI to give ester 278. A copper-catalysed alkyne-azide cycloaddition (CuAAC) was then used to couple this to propargylic acetate, providing triazole 287 .

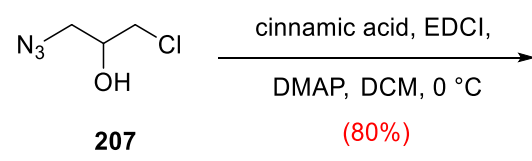

207

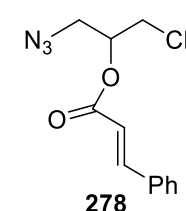

$278 \mathrm{Ph}$

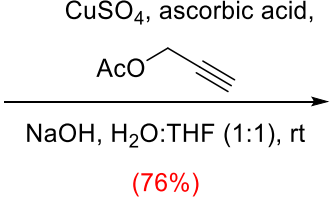

$(76 \%)$

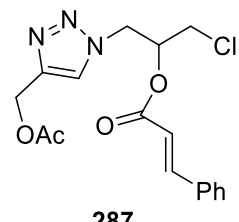

287

Scheme 3.18 Preparation of acyclic alkyl chloride model 287.

With the alkyl chloride $\mathbf{2 8 7}$ as a simplified model of the macrocycle, attempts were made to form the phosphonium salt 288. The reaction of 287 with triphenylphosphine and sodium iodide in acetonitrile at room temperature resulted in no reaction and recovery of starting material (Scheme 3.19). Repeating the reaction in DMF at higher temperature (120 $\left.{ }^{\circ} \mathrm{C}\right)$ resulted in degradation of substrate, with saponification of ester groups observed and no obvious evidence of forming any phosphonium salts. In the absence of success in this reaction, and the anticipated harsh conditions required for the E-selective Schlossermodified Wittig reaction, we decided to abandon this route and move on to testing the use of a Julia-Kocienski olefination reaction to attach the sidechain. 


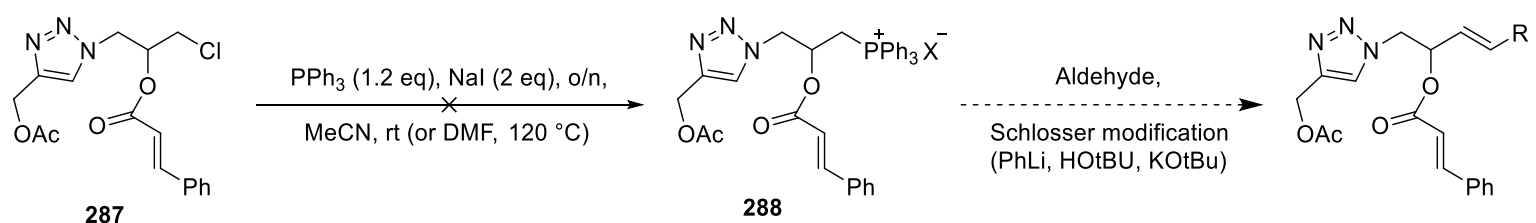

Scheme 3.19 Attempt at the formation of phosphonium salt 288 from alkyl chloride 287.

\subsubsection{Julia-Kocienski Reaction Strategy}

As formation of the phosphonium salt was unsuccessful from the acyclic model alkyl chloride, we turned our attention to the Julia-Kocienski olefination as the sidechain attachment method.

In addition to the more efficient access to the precursor thioether 208, in comparison to the alkyl chloride 207 (refer to Section 2.3), the Julia-Kocienski reaction was appealing in that milder reagents are used (i.e. KHMDS versus $\mathrm{PhLi}$ ) and good E-selectivity is usually obtained. ${ }^{115}$ Thus, an acyclic model of the macrocycle was prepared that would allow assessment of this method (Scheme 3.20). The thioether fragment 208 was esterified with cinnamic acid using EDCI to give the ester 289, followed by a CuAAC reaction with propargyl benzoate 290, providing the triazole 291. This thioether was oxidised with hydrogen peroxide and catalytic ammonium heptamolybdate to give the sulfone $292 .{ }^{133}$

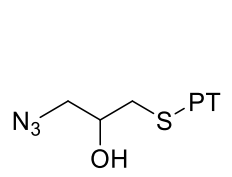

208

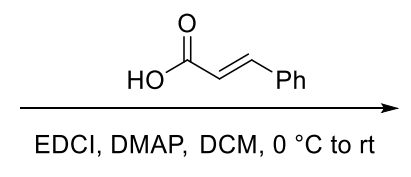

$(99 \%)$<smiles>NCC(CS[PH])OC(=O)C=Cc1ccccc1</smiles>

289

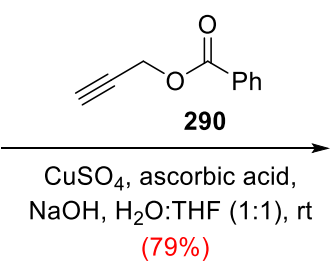

$(79 \%)$
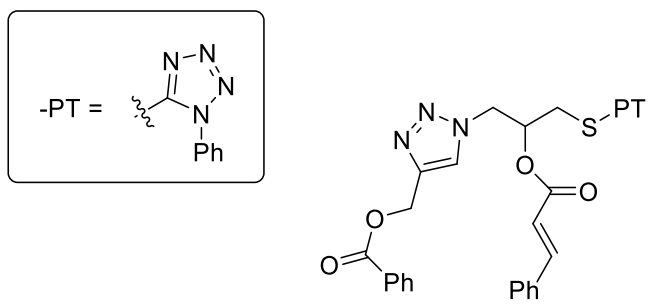

291

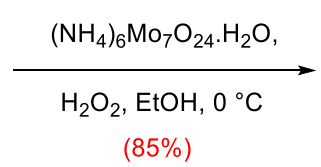

$(85 \%)$

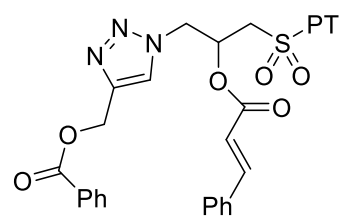

292

Scheme 3.20 Preparation of Julia-Kocienski precursor 292. 
With the sulfone model 292 in hand, we attempted a Julia-Kocienski olefination reaction with cinnamaldehyde using conditions that were optimised by Kocienski to provide high E-selectivity. ${ }^{134}$ Unfortunately, instead of the desired olefin product 293, deprotonation ( $\alpha$ to the sulfone) resulted in $\beta$-elimination of the ester giving the alkene product $\mathbf{2 9 4}^{+++t}$ (Scheme 3.21).<smiles>CCCS(=O)(=O)CC(Cn1cc(COC(=O)c2ccccc2)nn1)OC(=O)/C=C/c1ccccc1</smiles>

292

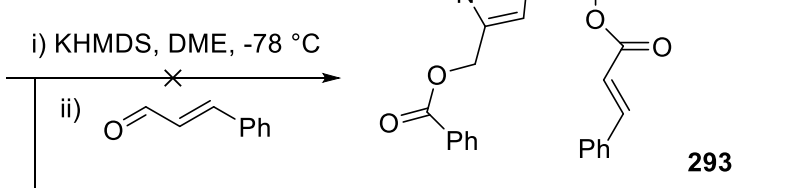<smiles>O=C(OCc1cn(C/C=C/S(=O)(=O)c2ccccc2)nn1)c1ccccc1</smiles>

Scheme 3.21 Attempted Julia-Kocienski reaction with acyclic model.

A scan through the literature revealed that Smith and co-workers, en route to the synthesis of (-)-callystatin, experienced a similar "facile $\beta$-elimination of sulfone (295) leading to decomposition" (Scheme 3.22). ${ }^{135}$ This pitfall led to a redesign of the synthetic plan.

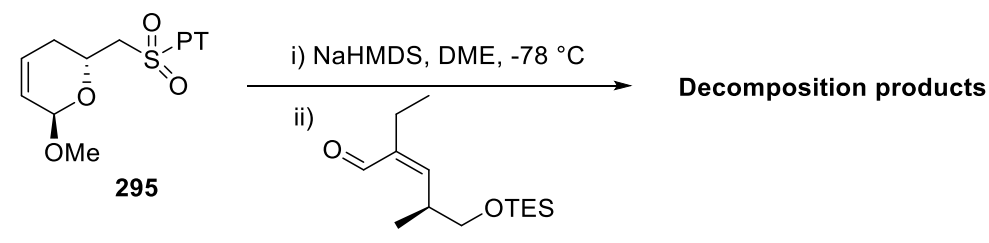

Scheme 3.22 Julia-Kocienski reaction in the synthesis of (-)-callystatin A. ${ }^{135}$

\footnotetext{
${ }^{++++}$Characterised using $1 \mathrm{D}$ and $2 \mathrm{D}{ }^{1} \mathrm{H}$ NMR $\left(500 \mathrm{MHz}, \mathrm{CDCl}_{3}\right)$, with signals corresponding to newly formed alkene at $\delta 5.17(\mathrm{~d}, J=7.8 \mathrm{~Hz})$ and $4.65(\mathrm{~d}, J=7.8 \mathrm{~Hz})$. HRMS-ESI $(\mathrm{m} / \mathrm{z}):[\mathrm{M}+\mathrm{H}]^{+}$calculated for $\mathrm{C}_{20} \mathrm{H}_{18} \mathrm{~N}_{7} \mathrm{O}_{4} \mathrm{~S}^{+}$, 452.1135; found 452.1137 .
} 


\subsection{Revised Connectivity of Fragments}

Based on the unsuccessful Wittig and Julia-Kocienski reactions on the acyclic models, it was clear that an alternative route was necessary. The $\beta$-elimination observed from the Julia-Kocienski reaction was a consequence of having the ester functionality in the $\beta$ position (C-9) to the sulfone (vide supra, Scheme 3.22). Thus, the new route involved performing the Julia-Kocienski reaction with the silylated compound 296 that is less prone to $\beta$-elimination and allow formation of triene 297 (Scheme 3.23). This could then be coupled to alkyne 284 via a CuAAC reaction. The desilylation and base-induced lactone ring opening was anticipated to be performed simultaneously, owing to the fact that both reactions are facilitated by TBAF. This enables a degree of step economy. ${ }^{11}$

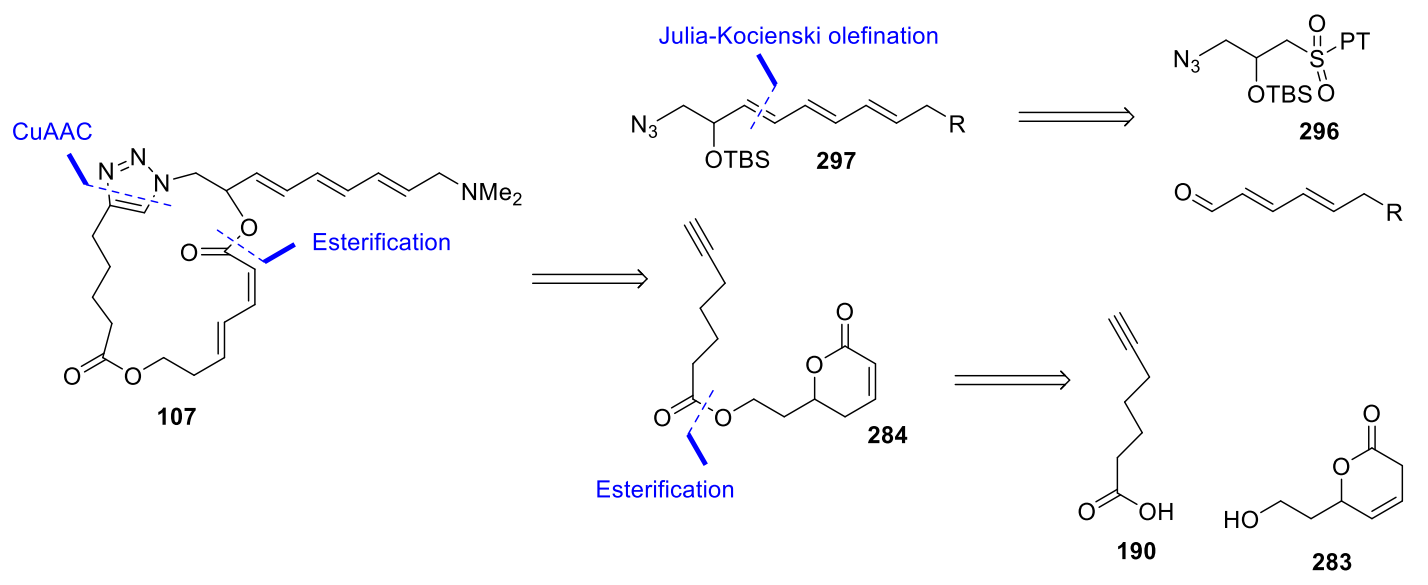

Scheme 3.23 Revised retrosynthetic analysis.

\subsubsection{Preparation of Triene Sidechain Fragment}

TBS-protected compound 296 was prepared by first silylating alcohol 208 using TBStriflate and imidazole. Oxidation of thioether 297 with hydrogen peroxide and catalytic ammonium heptamolybdate provided sulfone 296 (Scheme 3.24). ${ }^{133}$ 


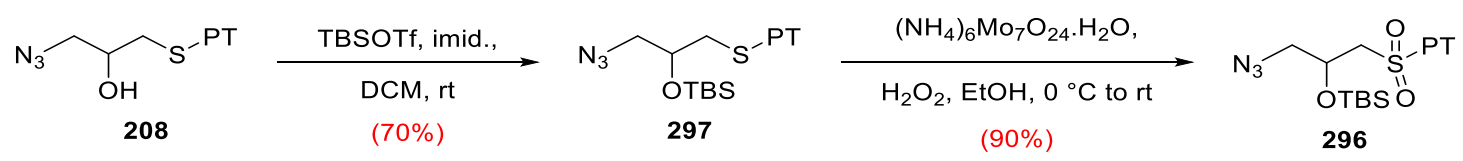

Scheme 3.24 Preparation of TBS-protected sulfone 296.

Sulfone 296 was then tested in reactions with a range of aldehydes by first pre-treating it with KHMDS at $-78^{\circ} \mathrm{C}$ followed by addition of the aldehyde (Table 3.4). The reaction with cinnamaldehyde provided the olefination diene product in a 10:1 (E:Z) mixture and $65 \%$ yield (Table 3.4, Entry 1). Disappointingly, when the same conditions were used with trans,trans-2,4-hexadienal, Zincke aldehyde $\mathbf{2 3 7}$ or the dimethyl amine sidechain fragment 213 (Entries 2-4, respectively), no desired olefination products were observed, and instead mixtures of unidentified products were obtained.
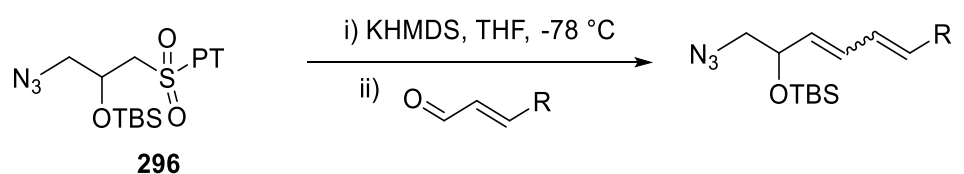

296

\begin{tabular}{ccc}
\hline Entry & R group & Result \\
\hline 1 & $-\mathrm{Ph}$ & Mixture of degradation products \\
2 & & Mixture of degradation products \\
3 & $\mathrm{NMe}_{2}(237)$ & Mixture of degradation products \\
\hline
\end{tabular}

Table 3.4 Julia-Kocienski reaction with a range of aldehydes.

Encouraged by the successful Julia-Kocienski reaction with cinnamaldehyde, other aldehydes were sought, that would allow access to a triene with suitable functional handles to allow progression of our pateamine analogue synthesis. Aldehyde 233, which was prepared en route to dimethyl amine 231, appeared to be a good candidate as this could then be further reacted to provide triene 299 (Scheme 3.25). Repeating the JuliaKocienski reaction conditions with aldehyde 233 , unfortunately resulted in the formation of a mixture of degradation products. 


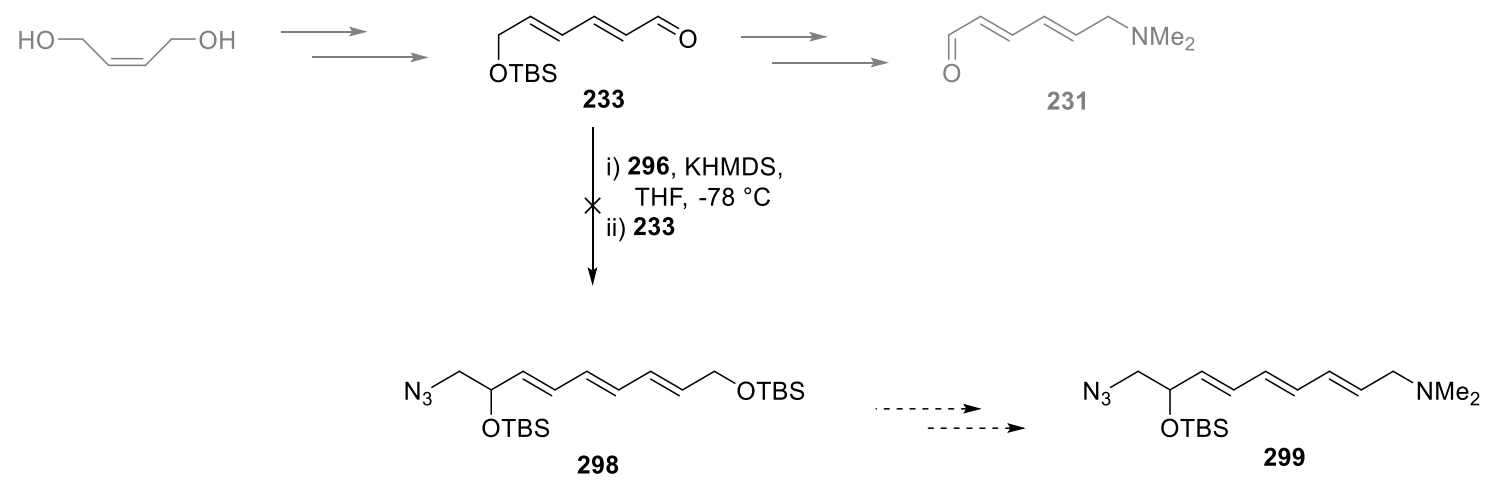

Scheme 3.25 Attempted Julia-Kocienski reaction on aldehyde 233 .

It was notable that the Julia-Kocienski reaction of sulfone 296 had provided the olefination product with cinnamaldehyde, but would give only degradation products with all other aldehydes that were trialled. It was recognised that a common feature of the unsuccessful Julia-Kocienski reaction substrates was the presence of an enolisable centre. It was then proposed that an aldehyde that was not able to enolise might be a better substrate in the Julia-Kocienski reaction. Thus, the ester $\mathbf{3 0 0}$ was targeted as a potential candidate. Aldehyde 301 was accessed from the previously prepared TBS protected alcohol 228, by treatment with $1 \% \mathrm{HCl}$ in ethanol to effect desilylation to alcohol 302, followed by oxidation using Parikh-Doering conditions (Scheme 3.26). Gratifyingly, reaction of aldehyde 301 with the TBS protected sulfone 296 provided the desired triene product 300 in a $10: 1(E: Z)$ mixture. 


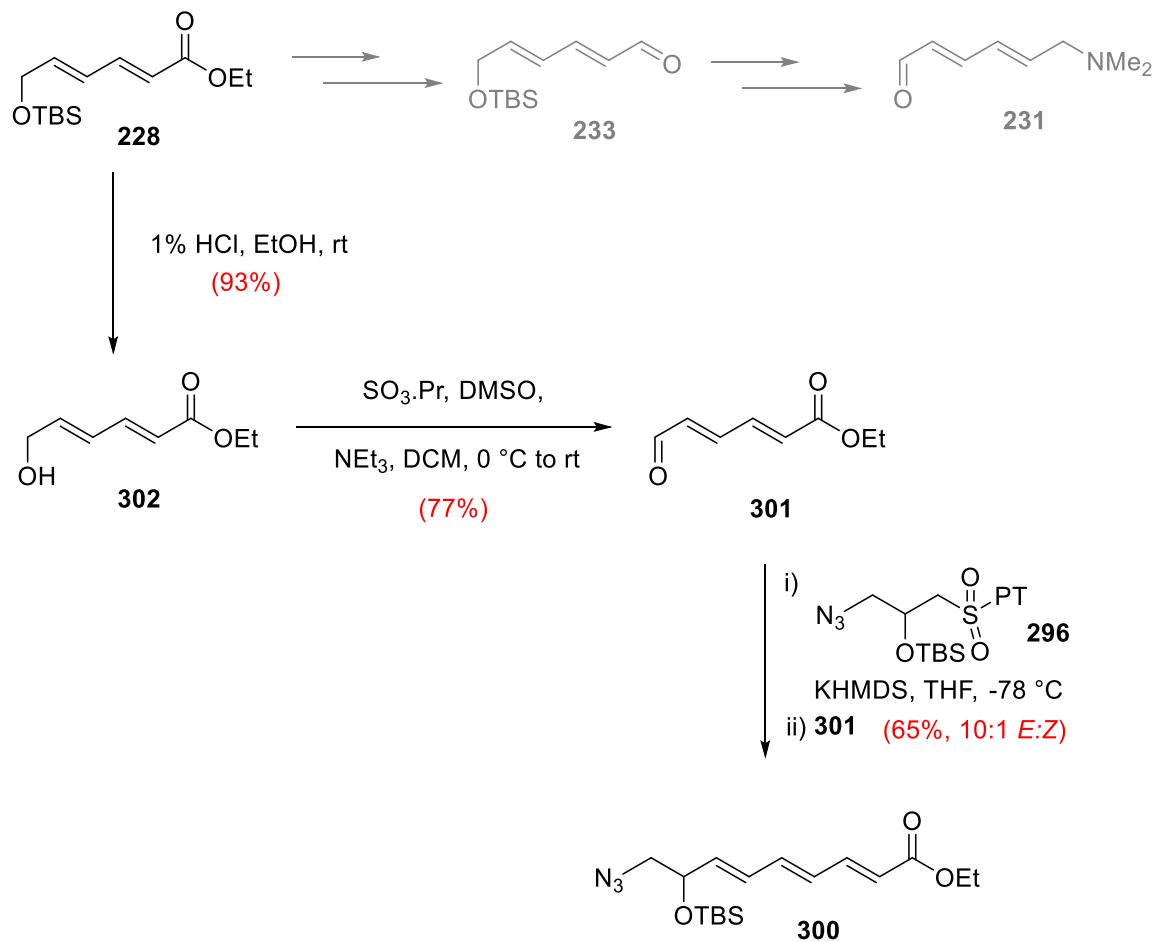

Scheme 3.26 Successful Julia-Kocienski reaction with aldehyde 301 to triene 300 .

It appears, based on the few examples assessed in this work, that having an enolisable diene on the aldehyde substrate of the Julia-Kocienski reaction is possibly the cause of the undesired reactivity. However, there are some published examples of such dienals that successfully couple in Julia-Kocienski reactions, for example the reaction of sulfone $\mathbf{3 0 3}$ with enolisable aldehyde 304 to give triene 305 , in the synthesis of (+)-thiazinotrienomycin E (Scheme 3.27).
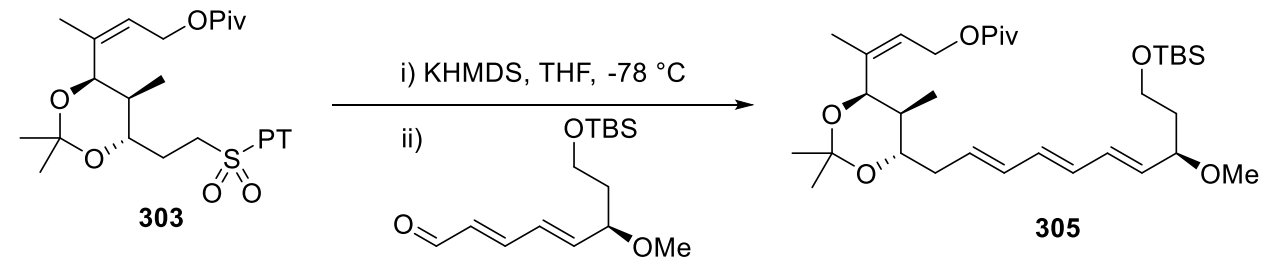

(85\%) 304

Scheme 3.27 Julia-Kocienski reaction of conjugated aldehyde in the synthesis of $(+)-$ thiazinotrienomycin E. ${ }^{133}$ 
With the success of the Julia-Kocienski reaction, a more convenient route to the new aldehyde substrate 301 was devised (Scheme 3.28). Thus, oxidative ring opening of furan with bromine and methanol followed by treatment with sodium bicarbonate provided fumaraldehyde bis(dimethyl acetal) 306. ${ }^{136}$ Mono-deprotection using water in acetone and catalysed with Amberlyst 15 (a solid supported sulfonic acid) gave aldehyde 307. Wittig olefination provided the ethyl ester 308, which was then treated with water in acetone, catalytic Amberlyst 15 and heating to $60{ }^{\circ} \mathrm{C}$ to afford the desired aldehyde 301 in $46 \%$ yield over 4 steps. ${ }^{137}$ This route had advantages over the previous route (vide supra, Section 2.4.2 and Scheme 3.26), in that it used cheaper starting materials and reagents and required fewer synthetic steps (e.g. by removing the need to use a silyl protecting group and oxidation reactions), making it much more scalable.

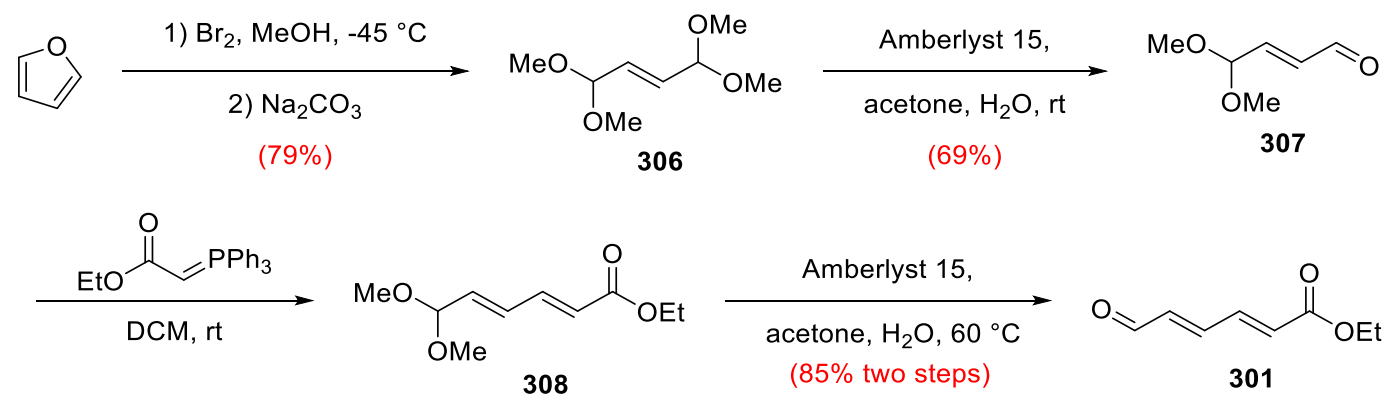

Scheme 3.28 More scalable synthesis of aldehyde 301.

With triene $\mathbf{3 0 0}$ in hand, we were able to access the desired trialkylamine derivative 299 (Scheme 3.29). This was achieved by reduction of ethyl ester 300 with DIBAL, which provided alcohol 309. Parikh-Doering oxidation afforded conjugated aldehyde 310, which was subsequently subjected to reductive amination using dimethylammonium hydrochloride and tetramethylammonium triacetoxyborohydride to give the dimethylamine-containing product 299. 


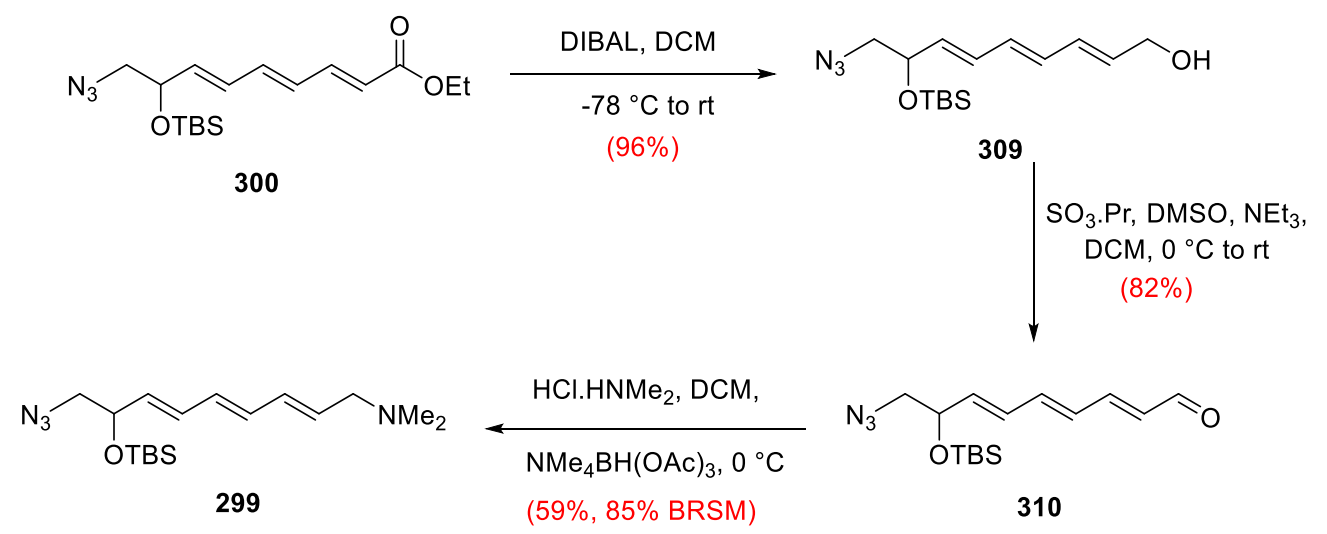

Scheme 3.29 Preparation of the completed amine-derivatised triene 299 .

From the previous model study (Section 3.3), the plan to attach a sidechain to a preformed macrocycle was not suitable. As a consequence the new strategy involved performing the Yamaguchi macrolactonisation in the presence of the dimethylamine functionality. With the observed nucleophilicity of the dimethylamine in the previous reaction that resulted in the formation of dimerised by-product (Table 2.11, Section 2.4), we were concerned about the compatibility of the dimethylamine functionality with the Yamaguchi conditions. Therefore, triene 299 was treated with 2,4,6-trichlorobenzoyl chloride (TCBC) and DIPEA in THF (Scheme 3.30), which indeed resulted in the formation of the quatinised amide 311. In the interests of avoiding the complications that formation of this salt might present in the macrolactonisation step, we chose to again review the synthetic strategy so that the inclusion of the dimethylamine (i.e. reductive amination) was the final synthetic step. This presented advantages in ultimately being able to explore a range of terminal amines, which would be preferential to introduce late in the synthesis.

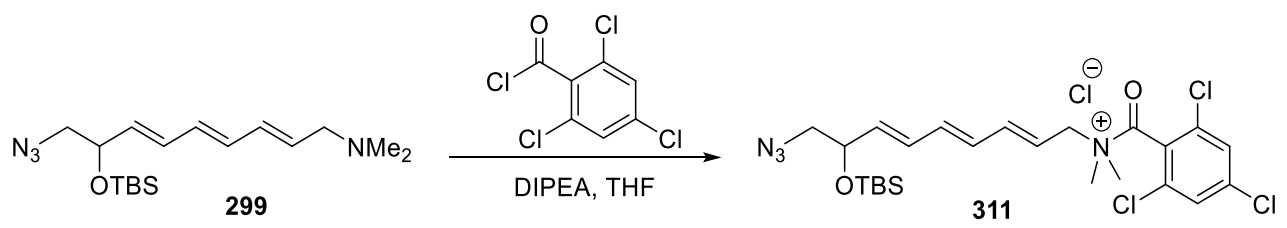

Scheme 3.30 Treatment of dimethyl amine 299 to Yamaguchi conditions. $¥ ¥ \neq$

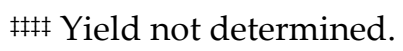


It seemed that the obvious way forward was to protect the triene alcohol 309 with a suitable protecting group, such as trityl 312 (Scheme 3.31). This could then be taken though steps to form macrocycle 313, followed by deprotection and completion of the pateamine analogue 107. The protecting group would need to be orthogonal to the coupling reactions (CuAAC and Yamaguchi reactions), TBAF treatment and ParikhDoering oxidation, and be removable afterwards. We felt that the trityl group best suited these conditions due to its stability to basic conditions and that it is readily cleaved in acidic treatment.
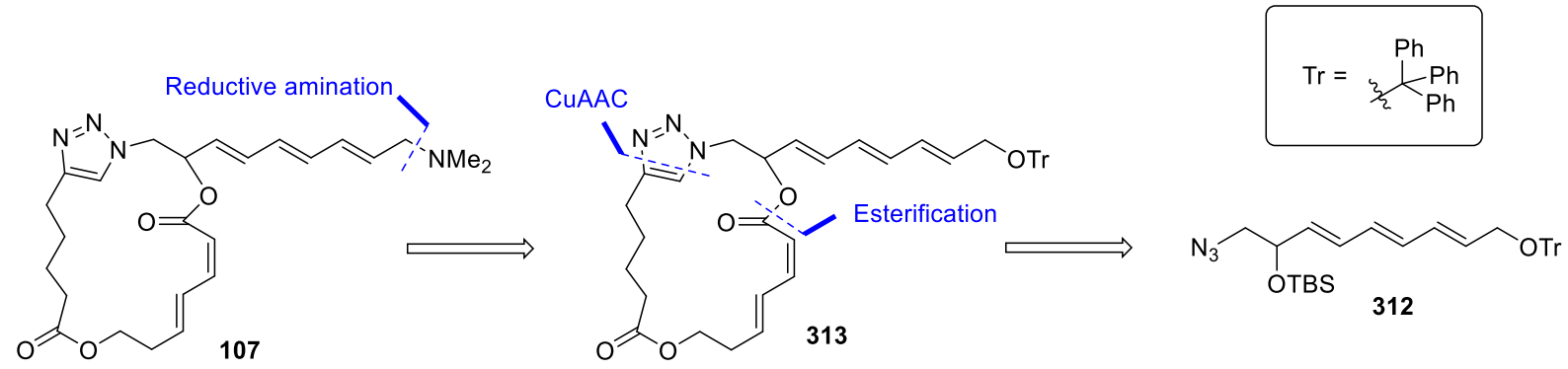

Scheme 3.31 Revised end game strategy to introduce amine as final step.

Thus, triene alcohol 309 was tritylated using trityl chloride $(\operatorname{TrCl})$ and triethylamine to give trityl-protected triene 312 (Scheme 3.32). Coupling this compound to fragment 284 with a CuAAC reaction then provided compound 314. However, it was noticed that degradation of the desired products was pronounced. In addition, the purified triene product 314 was found to degrade slowly on storage. ${ }^{1} \mathrm{H}$ NMR spectroscopy revealed significant loss of signals in the alkene region (5.5 - 6.5 ppm) while the rest of the molecule remained intact. The nature of the degraded product (or products) could not be determined by NMR techniques; however, HR-MS analysis showed that the major component had the mass of the product plus the mass of dioxygen (Figure 3.8). This suggested that the degraded product might be the result of oxidation via the addition of atmospheric oxygen. 


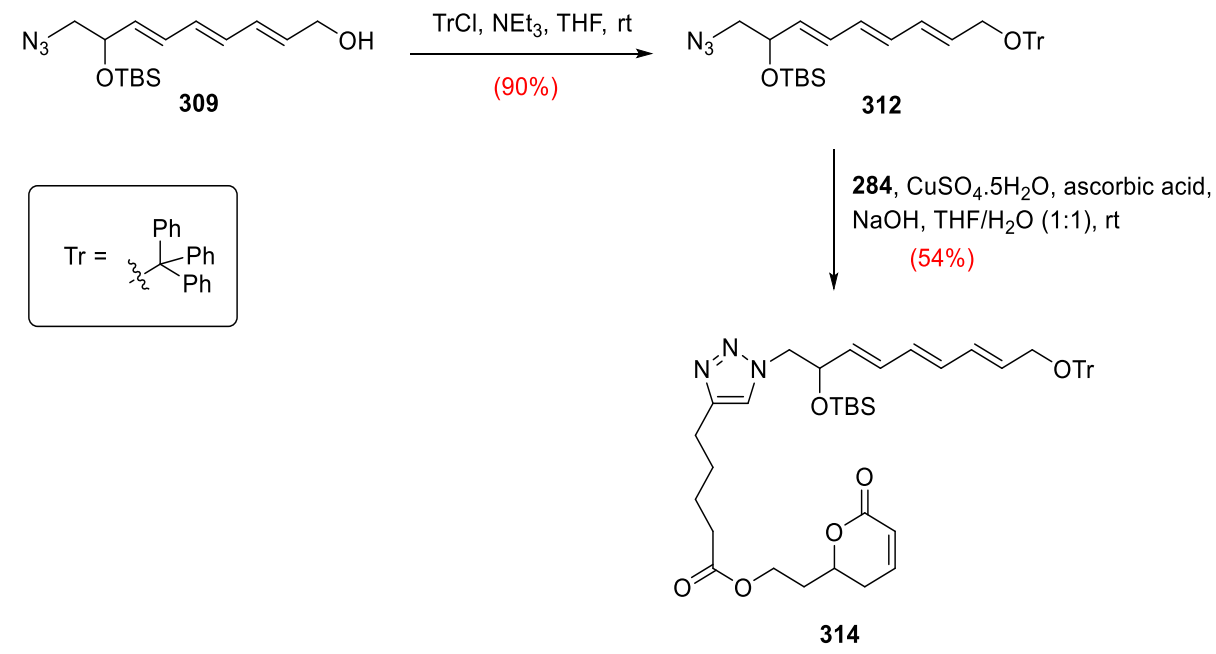

Scheme 3.32 Trityl protection and preparation of 314 .

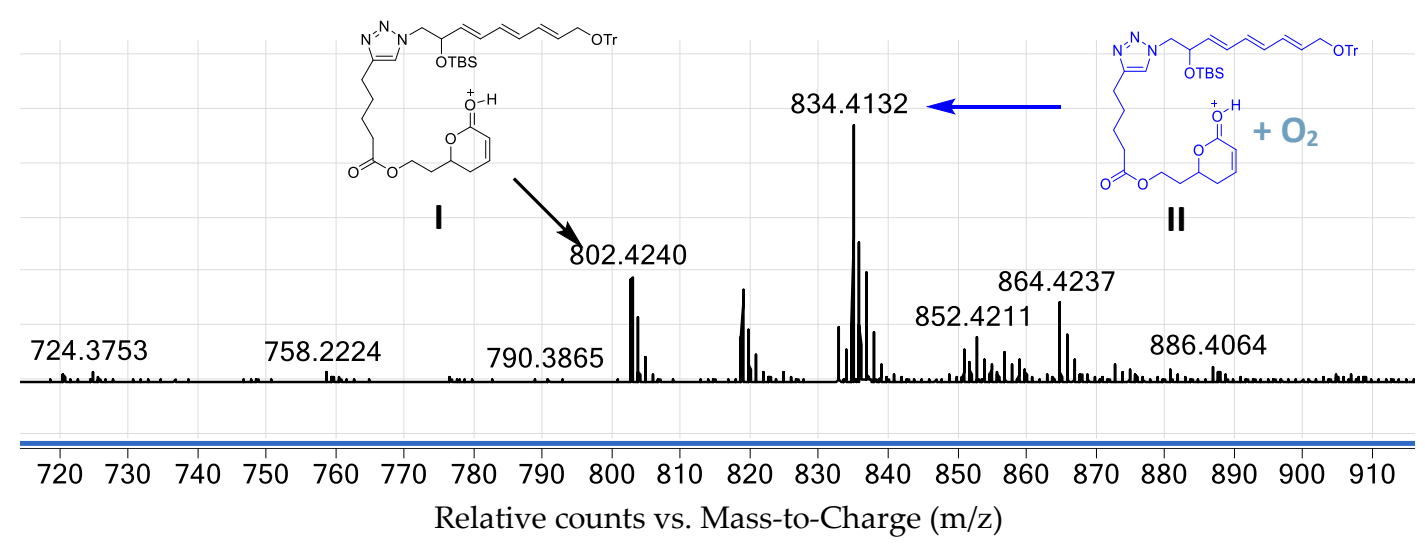

Figure 3.8 HR-MS of triene (314) degradation mixture.

Mass of charged compound I (in black, $\mathrm{m} / \mathrm{z}=802.4240$, calculated for $\mathrm{C}_{48} \mathrm{H}_{60} \mathrm{~N}_{3} \mathrm{O}_{6} \mathrm{Si}^{+}$, 802.4246) and II (in blue, $\mathrm{m} / \mathrm{z}=834.4132$, calculated for $\mathrm{C}_{48} \mathrm{H}_{60} \mathrm{~N}_{3} \mathrm{O}_{8} \mathrm{Si}^{+}, 834.4144$ )

\subsubsection{Degradation of Triene 314}

The addition of the exact mass of dioxygen indicated that degradation of $\mathbf{3 1 4}$ was caused by oxidation. The significant loss of peaks in the ${ }^{1} \mathrm{H}$ NMR spectrum in the alkene region indicated that the triene was the target of oxidation, and suggested that multiple oxygenated products were being formed. There were two potential mechanisms that we conceived could cause this oxygenation, namely the [4+2] cycloadition of singlet oxygen or autoxidation. 
The addition of singlet oxygen to double bonds is well established, for example in the synthesis of cyclic peroxide 315 from diene 316 (Scheme 3.33). ${ }^{138}$ However, this reaction requires the irradiation of a photo-sensitiser, such as rose bengal $\mathbf{3 1 7}$, to convert triplet oxygen into singlet oxygen.
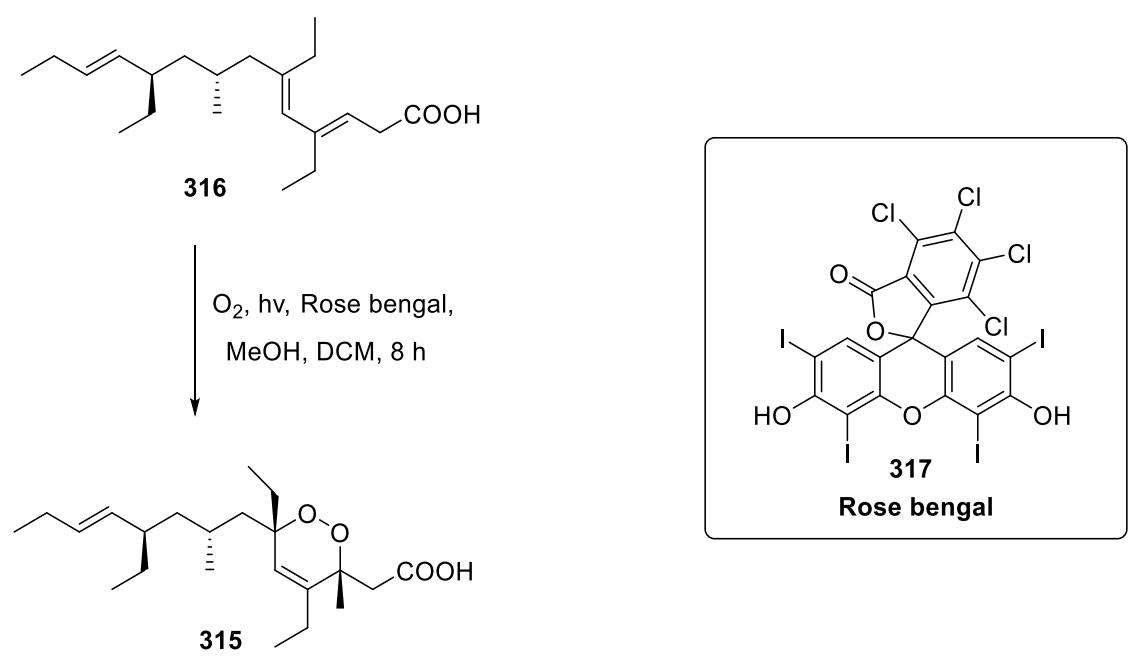

Scheme 3.33 Addition of singlet oxygen to diene $316 .{ }^{138}$

Based on the known addition of singlet oxygen to multiple bonds, it was conceived that cyclic peroxides 318 and 319 could be produced if singlet oxygen was present (Figure 3.9). However, it seems unlikely that the storage conditions (a closed system in the freezer or at room temperature), would give rise to the production of singlet oxygen. 


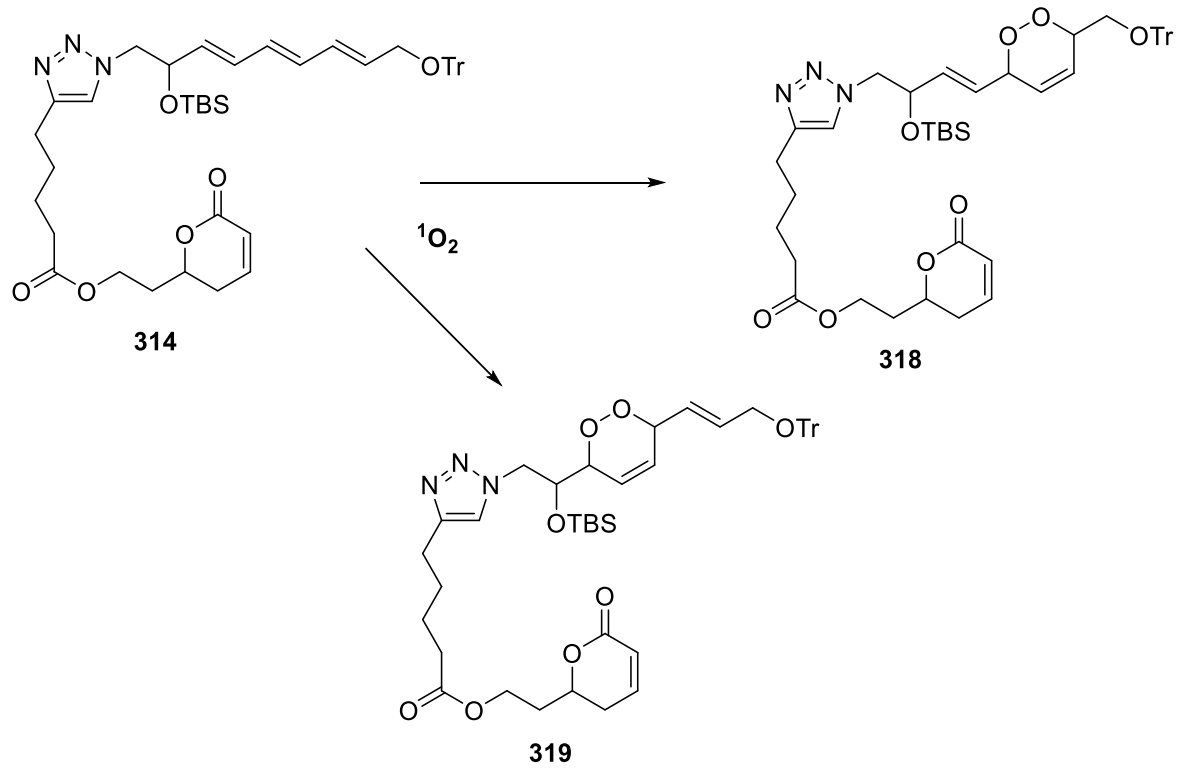

Figure 3.9 Potential degradation products via singlet dioxygen addition.

Alternatively, a mechanism via autoxidation may be the cause of the degradation as noted before with natural retinyl-polyenes such as $\beta$-carotene. ${ }^{139}$ The series of elementary reactions for the process of initiated autoxidation of polyenes has been described (Figure 3.10). ${ }^{139}$ Here $\mathrm{RH}$ represents the polyenic substrate that spontaneously undergoes thermal formation of carbon radical $R \cdot$, which reacts with molecular oxygen to form oxidation radical $\mathrm{RO}_{2}$. These then further react with other polyenic substrates $(\mathrm{RH})$ to propagate the process. The reaction of two oxygenated radical species $\left(\mathrm{RO}_{2} \cdot\right)$ eventually terminates the process to provide oxygenated non-radical products. 
Initiation

$\mathrm{RH} \longrightarrow \mathrm{R} \cdot$

\section{Propagation}

$$
\begin{aligned}
& \mathrm{R} \cdot+\mathrm{O}_{2} \rightleftarrows \mathrm{RO}_{2} \cdot \\
& \mathrm{RO}_{2} \cdot+\mathrm{RH} \longrightarrow \mathrm{R} \cdot(+ \text { products })
\end{aligned}
$$

\section{Termination}

$$
\mathrm{RO}_{2} \cdot+\mathrm{RO}_{2} \cdot \longrightarrow \text { non-radical products }
$$

Figure 3.10 Autoxidation of polyenes (Modified from Finkelshtein et al., 1996). ${ }^{139}$

On examination of other stored triene samples by ${ }^{1} \mathrm{H}$ NMR spectroscopy, namely compounds 309, 310 and 312, it was observed that there was significant degradation in all but aldehyde 310 (Figure 3.11).
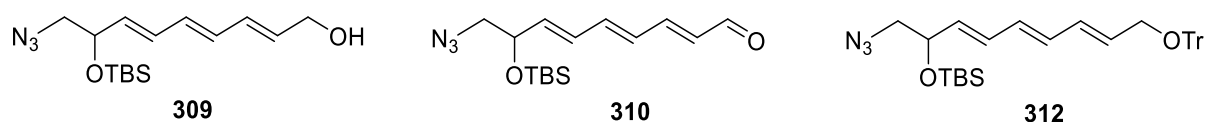

Figure 3.11 Triene samples examined for degradation.

This was initially surprising as aldehydes are typically susceptible to oxidation and therefore more prone to degradation, and with regards to autoxidation of saturated compounds this is certainly the case. ${ }^{140}$ However, polyenic aldehydes and ketones, instead, undergo much slower autoxidation than the corresponding hydrocarbons. There are two key steps in the autoxidation propagation, these are: hydrogen abstraction, and addition of peroxyls to the polyenic chain. ${ }^{139}$ It has been shown that the reactivity of compounds to peroxy radicals is directly related to the stabilisation energy of the radical formed, which increases with polyene length. Moreover, with high stabilisation energies of radical formation, the rate constants of allylic hydrogen abstraction are much greater than the rate constants of the addition of peroxyls. Thus, a polyene aldehyde lacking 
allylic hydrogens is much less reactive to autoxidation. Scheme 3.35 shows proposed mechanisms for the autoxidation of retinyl acetate via allylic hydrogen abstraction, and retinal via peroxyl addition. ${ }^{139}$
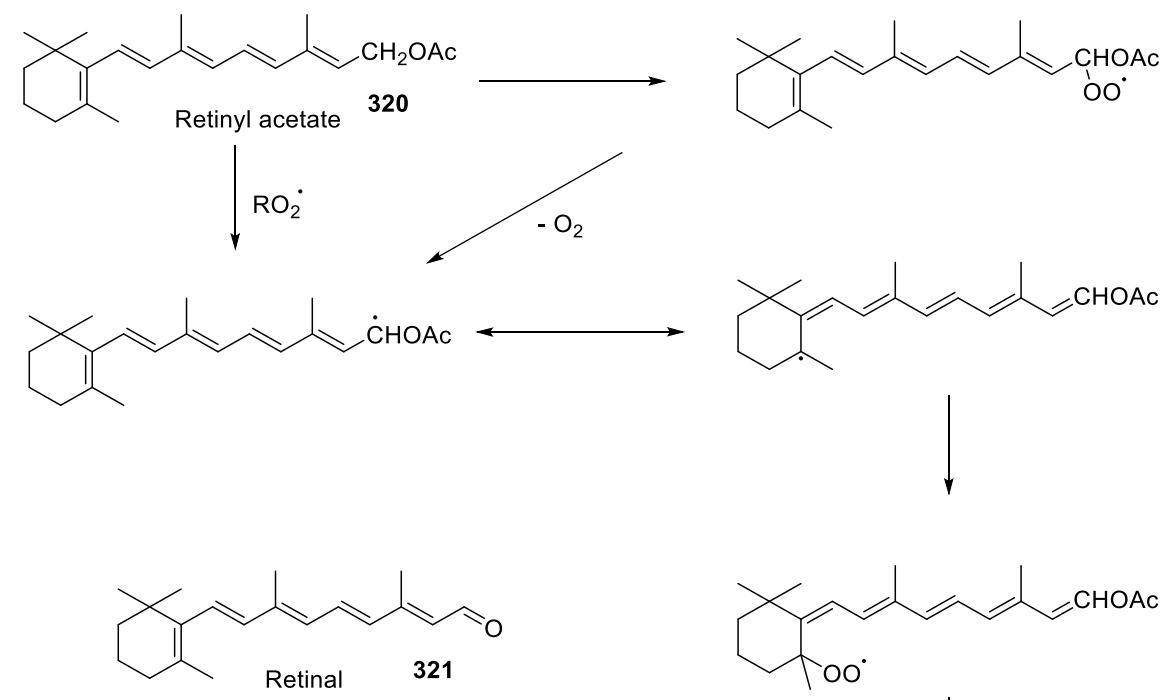

$\downarrow \mathrm{RO}_{2}$

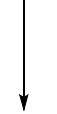<smiles>CC1=C(/C=C(C)/C=C/C=C/C(C)C(C=O)OC(C)C)C(C)(C)CCC1</smiles><smiles>CC(C)=CC=CC(C)C1C=CC2(C)OOC2(C)CCCC1(C)C</smiles>

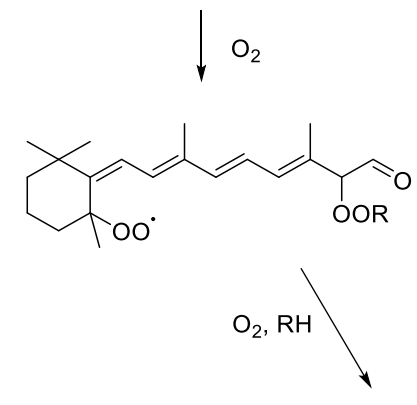
$\mathrm{O}_{2}$
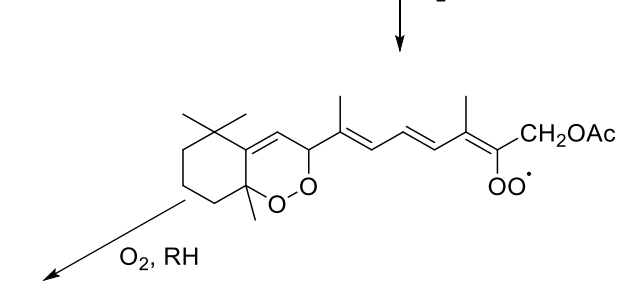

Oxidation Products

Scheme 3.35 Proposed autoxidation initial stage mechanism of retinyl acetate $\mathbf{3 2 0}$ (via hydrogen abstraction) and retinal 321 (via peroxyl addition). ${ }^{139}$

It was apparent that to limit the degradation of the triene sidechain it was necessary to avoid substrates that contained 'allylic' hydrogens at the triene terminus, thus we explored the potential of conducting the synthesis with the sidechain in an aldehyde form. 
This also had the advantage that no further transformations would be required ahead of final reductive amination.

\subsection{Completing Synthesis of Analogue 107}

The route was then modified to use the aldehyde $\mathbf{3 1 0}$ as a major fragment (Scheme 3.36). For this route to be viable, the conjugated aldehyde triene would have to be inert to reaction conditions and work-up for the CuAAC reaction, TBAF treatment and the Yamaguchi macrolactonisation.
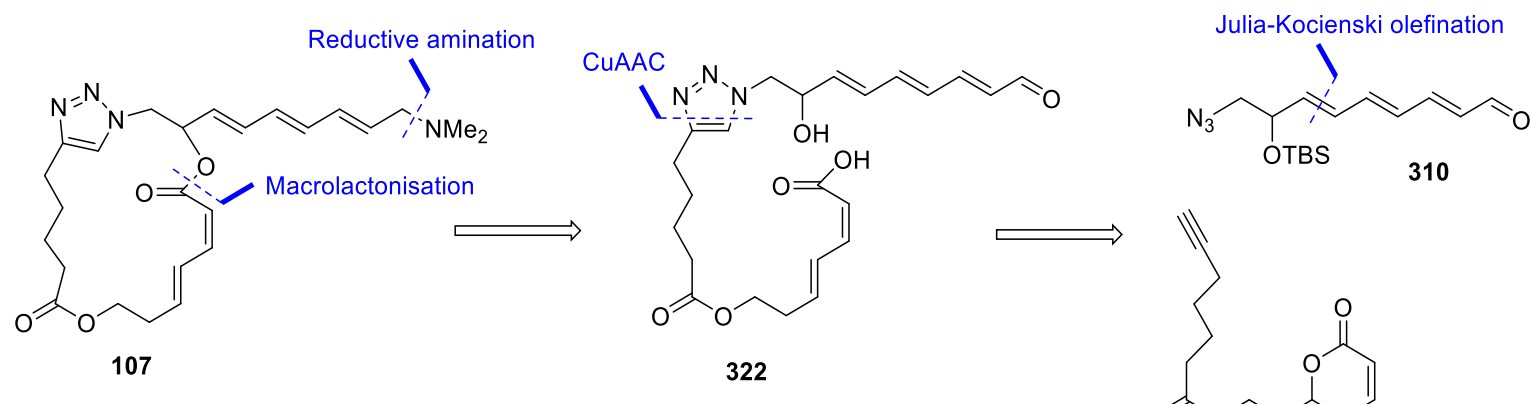

Scheme 3.36 Retrosynthetic analysis of aldehyde end-game.

Coupling alkyne 284 and the conjugated aldehyde 310 with a CuAAC reaction provided the triazole 323 (Scheme 3.37). Treatment of this compound (323) with excess TBAF in THF would allow the simultaneous silyl cleavage and the base induced lactone opening to afford the Z,E-diene seco acid 322. Unfortunately, significant degradation of the conjugated triene was observed due to these conditions, providing a 1:2 ratio of the desired product and a mixture of unidentified degraded products. 

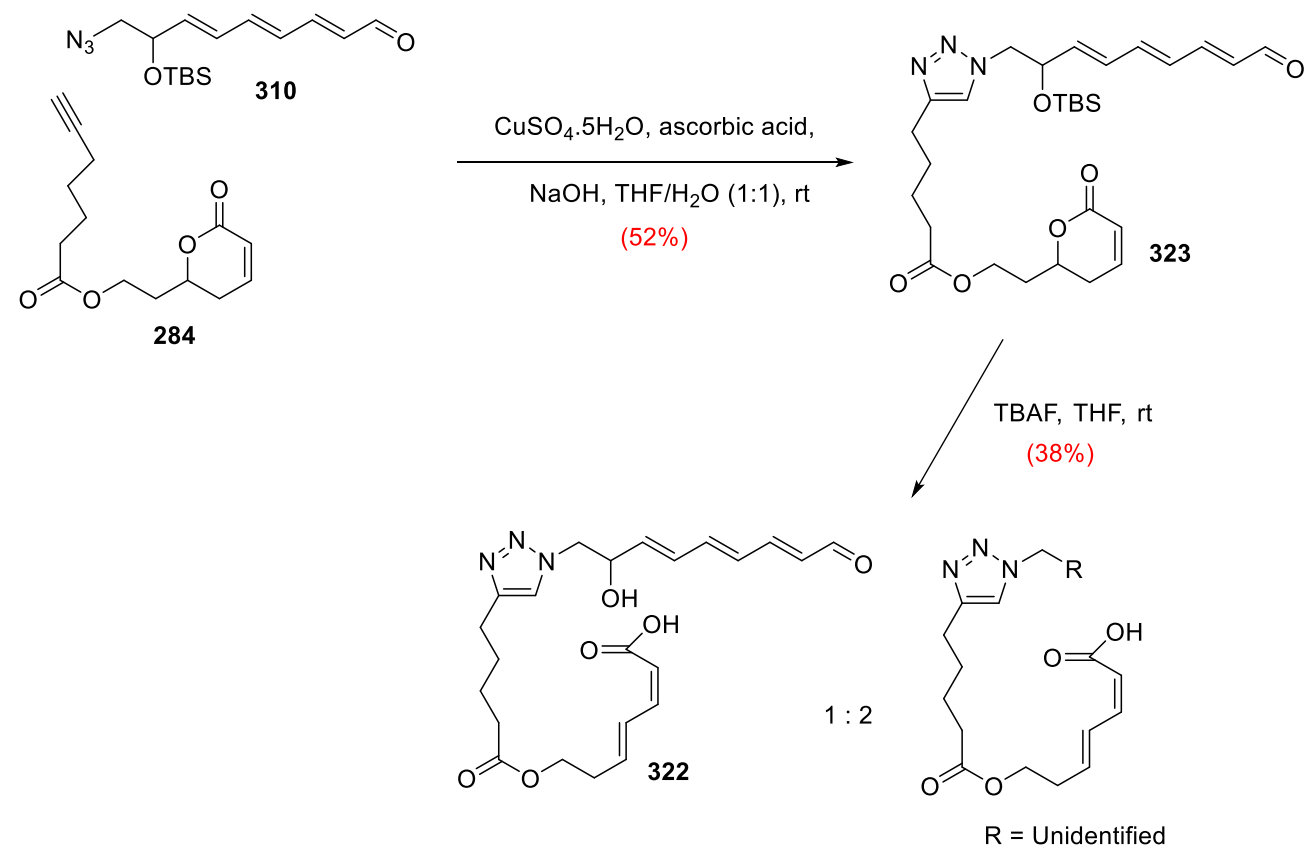

Scheme 3.37 Attempted one-pot lactone opening and silyl cleavage.

We then opted to perform the lactone opening and silyl cleavage on the separate fragments prior to coupling with the CuAAC reaction. Silyl deprotection of triene $\mathbf{3 1 0}$ with $2 \% \mathrm{HCl}$ in $\mathrm{MeOH}$ provided alcohol 324 (Scheme 3.38). Because of concerns about the potential for acid-catalysed elimination to the conjugated tetraene, we opted not to leave the reaction for too long ( 2 hours), after which time we got 22\% yield of alcohol 324 and $84 \%$ based on recovered starting material (BRSM). Lactone 284 was seperately treated with TBAF to facilitate lactone ring opening to $Z, E$-dienoic acid 282, which was subsequently coupled to triene 324 to provide seco acid 322. Yamaguchi esterification of seco acid 322 following conditions developed for the sidechain truncated macrocycle $Z, E$ 277 (vide supra, Section 3.2.2), afforded the macrocycle 325 with no observable geometric isomerisation. The low yield of this reaction is presumed to be a consequence of performing the reaction in the modified conditions found to prevent isomerisation (i.e. low temperature and catalytic DMAP). Further exploration of the reaction temperature and acylating catalyst loading would certainly improve the macrolactonisation yield. Reductive amination using dimethylammonium hydrochloride, sodium triacetoxyborohydride and triethylamine at $0{ }^{\circ} \mathrm{C}$ converted aldehyde 325 into the final target compound 107 in 56\% yield. Overall, this provides a synthesis with 11 longest 
linear steps and 23 steps in total from commercially available chemicals. $\$^{\$} \$$ In contrast, the total synthesis of the simplified des-methyl des-amino pateamine analogue 86 (see section 1.3.4) was achieved in 15 longest linear steps and 32 steps in total. ${ }^{38}$
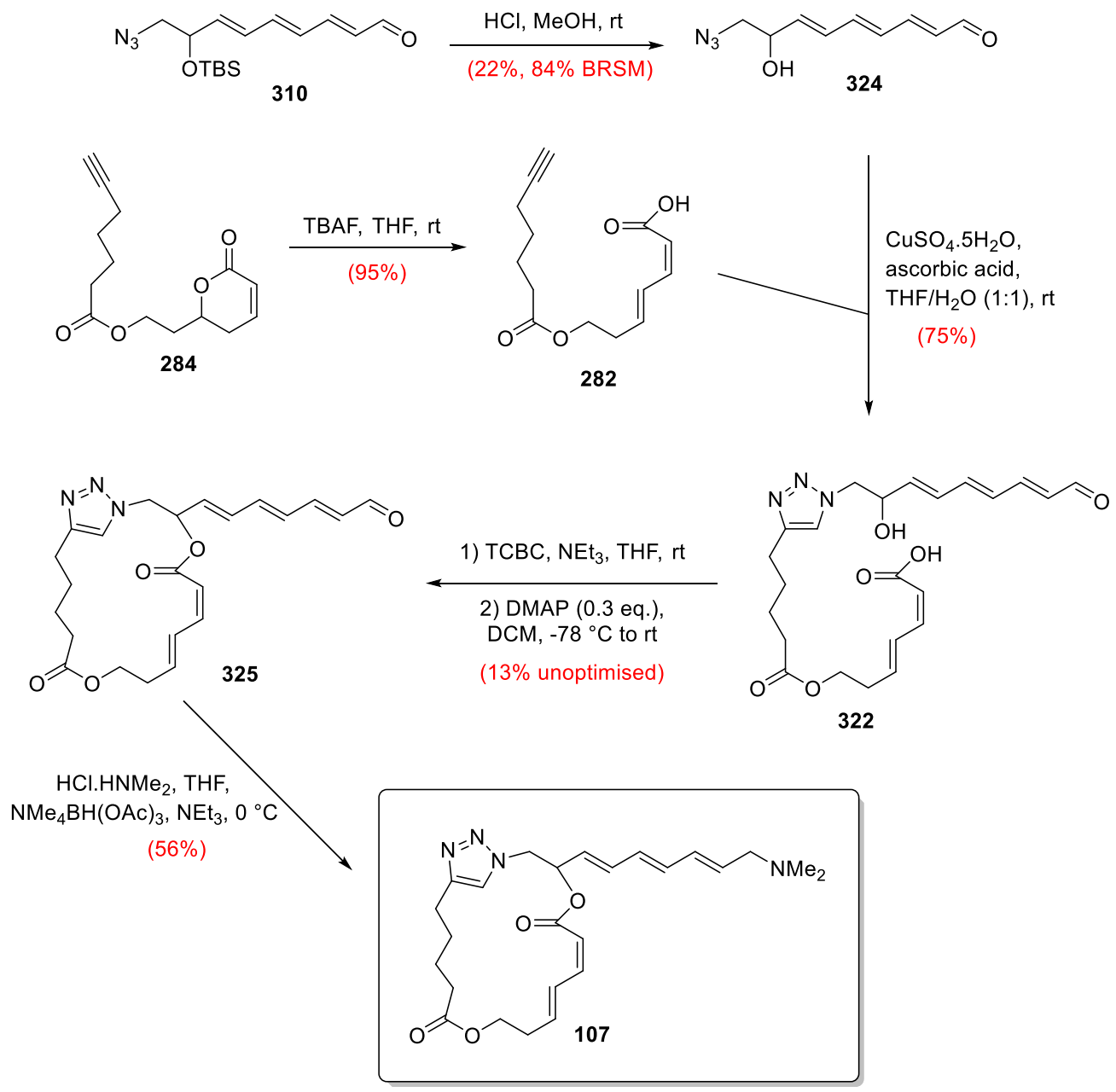

Scheme 3.38 Endgame of the synthesis of full pateamine analogue 107.

During the purification of pateamine analogue 107, we encountered the dimerised product $326^{* * * * * *}$ (Figure 3.12). It was not clear whether this was a product of the reductive

§s\& Taking into account that 6-heptynoic acid is commercially available.

***** Characterised by ${ }^{1} \mathrm{H}-\mathrm{NMR}$ and mass spectrometry. Proton spectrum was identical to that of 107, except the intergration of the peak corresponding to the methyl groups on the amine was halved. HRMS-ESI (m/z): [M] ${ }^{+}$calculated for $\mathrm{C}_{48} \mathrm{H}_{62} \mathrm{~N}_{7} \mathrm{O}_{8}{ }^{+}, 864.4654$; found 864.4618. $\mathbf{R}_{\mathbf{f}}$ : 0.67 (80:20:1 DCM:MeOH:saturated aqueous $\mathrm{NH}_{4} \mathrm{OH}$ ). 
amination, or produced during purification. A protonated amine could plausibly be substituted by a neighbouring un-protonated amine to provide this dimerised product, with loss of dimethylamine, as seen also in the Hofmann elimination. However, this type of reactivity had not been observed in any reductive amination reactions performed previously on other substrates. Nevertheless, the presence of this by-product proved difficult to separate from the product by silica gel chromatography, but fortunately use of reverse-phase HPLC (C18 column) allowed for clean separation.

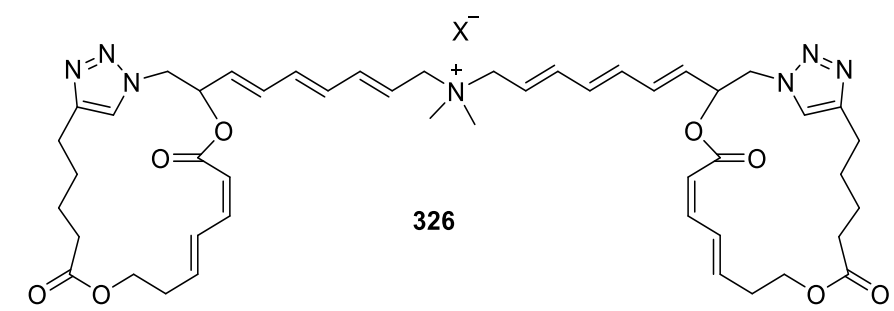

Figure 3.12 Dimerised side-product 326 observed during purification

It is also noteworthy that no sign of degradation of the triene sidechain of $\mathbf{1 0 7}$ was observed during purification and on storage on analysis of ${ }^{1} \mathrm{H}$ NMR spectrum and HRMS.

\subsection{Summary of Fragment Coupling}

Scheme 3.39 (fold out page) provides a summary of the successful route to the pateamine analogue 107, including fragment preparation, coupling and endgame. Many modifications of the original retrosynthetic analysis were made to the order of connectivity and reactions used.

The Yamaguchi reaction provided the most effective esterification method to minimise isomerisation of the $Z, E$-dienoic acid. Although significant isomerisation was observed in a model intermolecular reaction, performing the intramolecular Yamaguchi reaction (macrolactonisation) satisfyingly resulted in no observed isomerisation in the lactone product (Section 3.2).

\section{2}


Simplified model studies were conducted to test whether it was feasible to attach a conjugated sidechain to a macrocyclic species via a Wittig or Julia-Kocienski olefination reaction. However, we failed to form the phosphonium salt necessary for a Wittig reaction, and reaction of the sulfone 292 under Julia-Kocienski reaction conditions resulted in $\beta$-elimination (Section 3.3.2). This indicated that it was not viable to attach the sidechain to a formed macrocycle using these olefination reaction conditions, therefore, a new route that involved attachment of the sidechain prior to macrocyclisation was pursued (Section 3.4). Coupling sulfone 296 to an aldehyde-bearing sidechain in a JuliaKocienski reaction proved problematic, however persistence in testing different sidechains allowed us to find a suitable substrate (301). It appeared, based on the conjugated aldehyde sidechains tested, that having an enolisable proton was detrimental to the Julia-Kocienski reaction.

After preparing the dimethylamine substituted triene 299, it became evident that the nucleophilicity of the tertiary amine functionality renders itself incompatibile in the Yamaguchi macrolactonisation step suggesting that the amine functionality should be added as final step (Section 3.4.1). Thus, protection of the terminus of the sidechain as a trityl ether, which was envisioned could be cleaved after macrocyclisation allowing the completion of the synthesis. However, significant oxidative degradation products were encountered that we propose are caused by autoxidation.

Oxidative degradation of aldehyde $\mathbf{3 1 0}$ was not observed, prompting later-stage reactions to be performed with retention of the aldehyde functionality (Section 3.5). Reactivity of the sidechain of compound $\mathbf{3 2 3}$ with TBAF prevented the utility of TBAF to simultaneously facilitate silyl cleavage and lactone opening. Fortunately, the conjugated aldehyde remained intact during macrocycle formation, allowing the completion of the synthesis.

This synthesis represents an efficient strategy for the preparation of a simplified analogue to the marine natural product, pateamine A. The synthesis is characterised by utilisation of cheap starting materials, the avoidance of toxic chemicals (both to the environment and to the synthetic chemist) and minimal protection/deprotection steps. In addition, the convergent synthetic strategy allows easy access to range of alternative analogues. 
144 


\section{Chapter Four: Biological Activity}

\subsection{Introduction}

Pateamine is known to interact with a range of cellular components at different concentrations (see Section 1.4.3 for a summary of biological targets). Of these, eIF4AI/II and eIF4AIII were shown to be most sensitive.

It was found during the isolation of the eIF4A isoforms on pateamine affinity matrix that forcing conditions were necessary to remove the proteins from the resin. ${ }^{40}$ In particular, eIF4A was retained by the affinity matrix after extensive washing, including a high salt concentration wash that removed other proteins. To elute the eIF4A, the affinity matrix needed to be heated at $70{ }^{\circ} \mathrm{C}$ for 10 minutes in denaturing conditions with the thiolcontaining reducing agent dithiothreitol. This raised the possibility that there is a covalent interaction between pateamine and the eIF4As. It was hypothesised that this may be caused by the nucleophilic attack by a residue on eIF4A on the Michael acceptor (the $Z, E$-dienoate in the macrocycle) in pateamine.

A second hypothesis was that the tertiary amino group of the side chain will be protonated and form a strong, attractive electrostatic interaction with the target, potentially via a phosphate group on the backbone of the substrate RNA. Such an interaction may be essential for the role of pateamine in stabilising the interaction between the eIF4A proteins and RNA. Romo demonstrated that analogues with an oxygen-terminated side chain and a $Z, E$-dienoate had substantially reduced activity ${ }^{38}$. This reduction in activity might be due to a number of factors including: inability to bind due to a loss of a required electrostatic attraction; inability to bind due to the introduction of a potential electrostatic repulsion; no loss of binding, but a failure to stabilise the interaction between eIF4A and RNA.

Therefore, in addition to the full simplified pateamine analogue, analogues were synthesised that lacked the pateamine-like sidechain. These compounds were to be 
assessed to see whether they retained the ability to bind to eIF4A, in addition to their activity.

As previously mentioned (in Section 1.3.4), Romo's group hypothesised 'scaffolding' and 'binding' domains of pateamine A (see Section 1.3.4). The thiazole heterocycle sits within this proposed binding domain, however, there has been no experimental evidence that it has a role in binding to eIF4A. An alternative option is that it plays a role in structural integrity of the compound as a whole. To this end, the replacement of pateamine's thiazole with a triazole provides an opportunity to probe the effect an alternative 5membered heterocycle would have.

Modelling the lowest energy conformations of DMDA-PatA (86) and triazole-containing analogue 107 (see Section 1.6.1) suggested that the effect of a triazole in place of the thiazole would have minimal conformational implication. Therefore, the introduction of the triazole would represent an interesting chemical modification to both probe the role of the thiazole in binding to eIF4A and to investigate whether a switch in selectivity between different protein targets is obtained. 


\subsection{Results}

\subsubsection{Growth Inhibitory Activity}

To measure the growth inhibitory activity against cancer cells, the simplified analogue $\mathbf{1 0 7}$ and the precursor aldehyde 325 were assayed in an MTT assay against two cancer cell lines, namely HL-60 (human promyelocytic leukemia cells) and 1A9 (human ovarian carcinoma cells), by Dr Jessica Field (School of Biological Sciences, Victoria University of Wellington). The results are presented in Figure 4.1 and IC $_{50}$ values summarised below.

Aldehyde 325 IC50 values:

- $100 \mu \mathrm{M}(\mathrm{HL}-60)$

- $\quad>200 \mu \mathrm{M}(1 \mathrm{~A} 9)$

Full simplified analogue 107 IC50 values:

- $\quad 34.6 \mu \mathrm{M}(\mathrm{HL}-60)$

- $51.1 \mu \mathrm{M}(1 \mathrm{~A} 9)$.

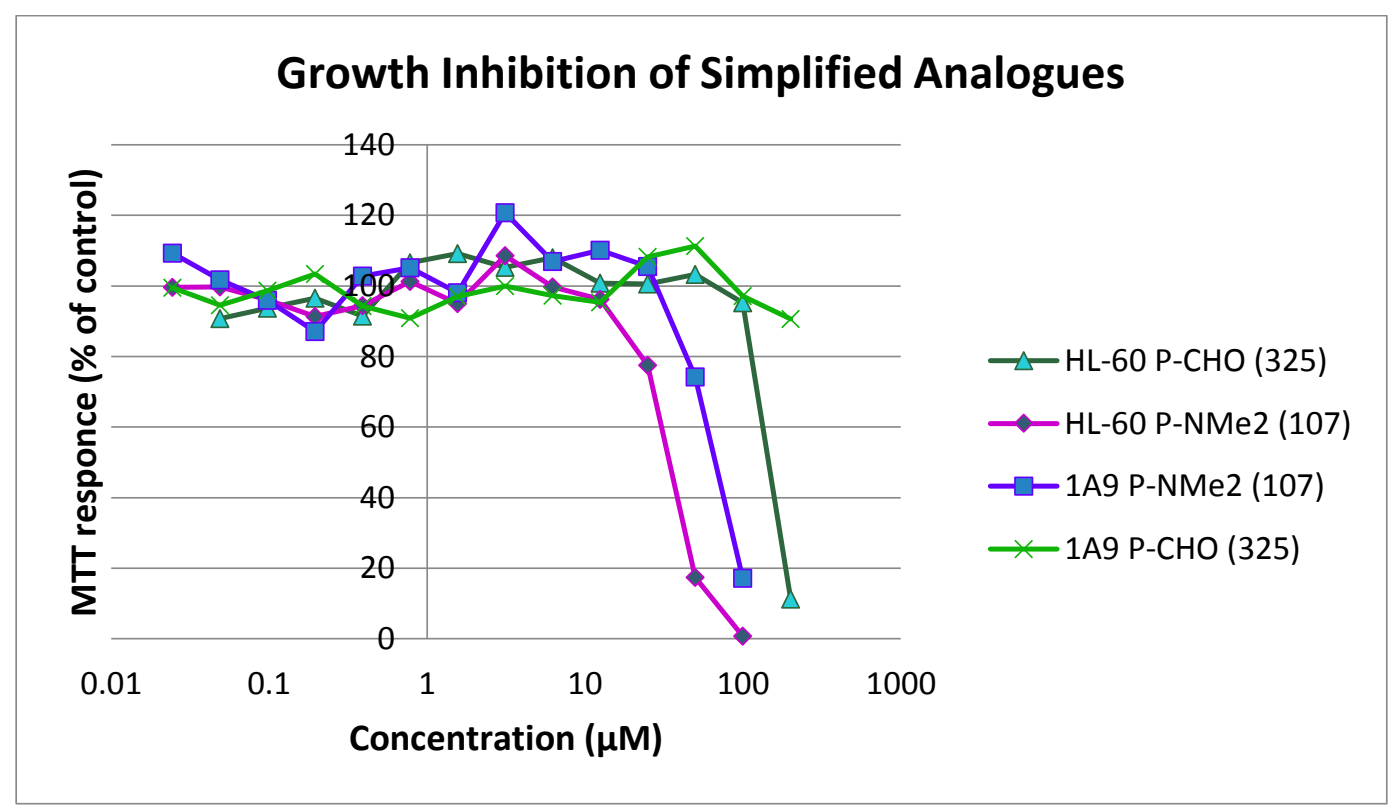

Figure 4.1 MTT assay results for pateamine analogues 325 and 107, against HL-60 and 1A9 cancer cell lines. 


\subsubsection{Searching for elF4A/Macrocycle Adducts}

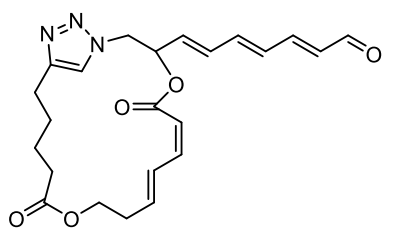

325

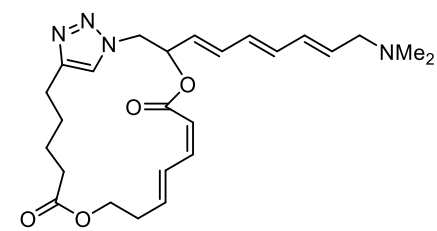

107

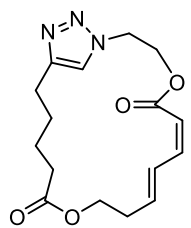

$Z, E-277$

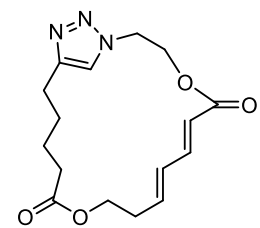

$E, E-277$

To investigate the hypothesised covalent binding of pateamine with its eIF4A protein target, Richard Little (School of Biological Sciences, Victoria University of Wellington) $)^{141}$ incubated pateamine and a range of the prepared analogues overnight in purified yeast eIF4A, along with ATP and polyU RNA. The protein was then treated with trypsin, desalted and the digested peptides analysed by Nano LC-LTQ orbitrap mass spectrometry. The resulting data was analysed using the Sequest search algorithm, searching for a mass addition of the corresponding compound on certain amino acids.

An analysis of the aldehyde 325, the pateamine analogue 107 and the two analogues lacking the sidechain, $Z, E-277$ and $E, E-277$, did not reveal any observable mass increases to any peptide sequence that could be associated with covalent modification.

When a sample of the natural compound, pateamine (14), was analysed, an increase in 560 Da was observed in the daughter ion spectra associated with C-terminal fragments of one of the tryptic digest peptides, LRNDKFTVSAIYSDLPQQER, when compared to the spectrum without pre-treatment with pateamine (compare Figure 4.2 and Figure 4.3, noting that the values are equivalent to their charge/mass ratio).

Singly charged pateamine has an exact calculated mass of 556.3 Da. Thus, the observed increase in mass of $560 \mathrm{Da}$, strongly suggests a covalent modification between pateamine and the Tif1p protein. This mass gain is equivalent to pateamine in the alkylated peptides being triply protonated, along with at least one glutamine $(\mathrm{Q})$ deamidation event. 

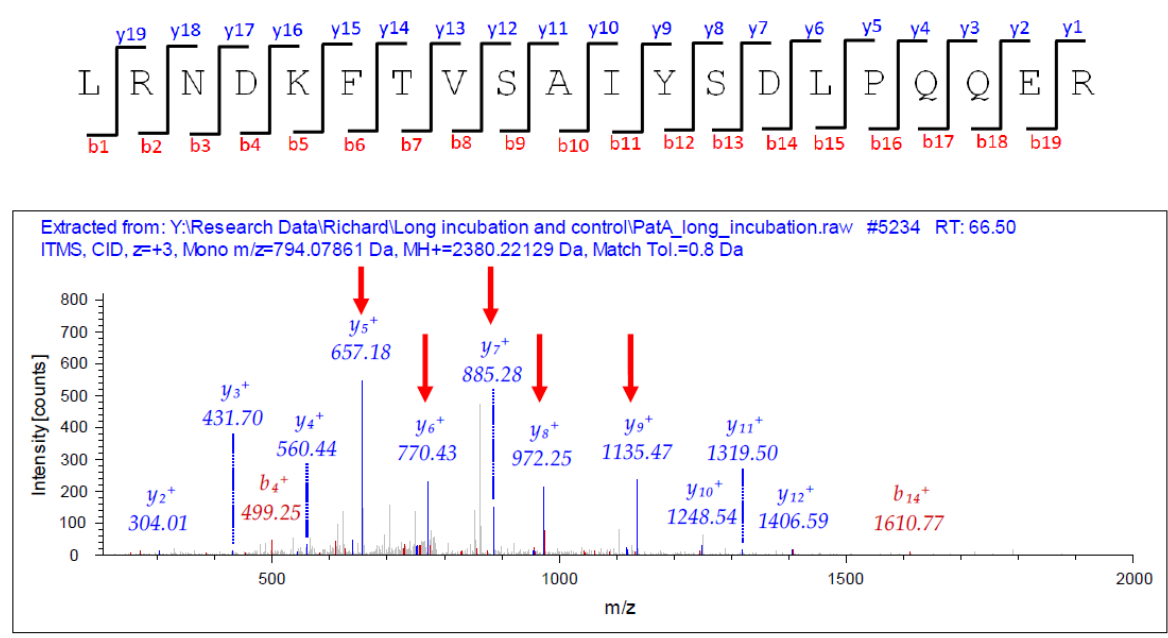

Figure 4.2 Singly charged spectrum of a section of peptide from tripsin digested Tif1p without pateamine pre-treatment.
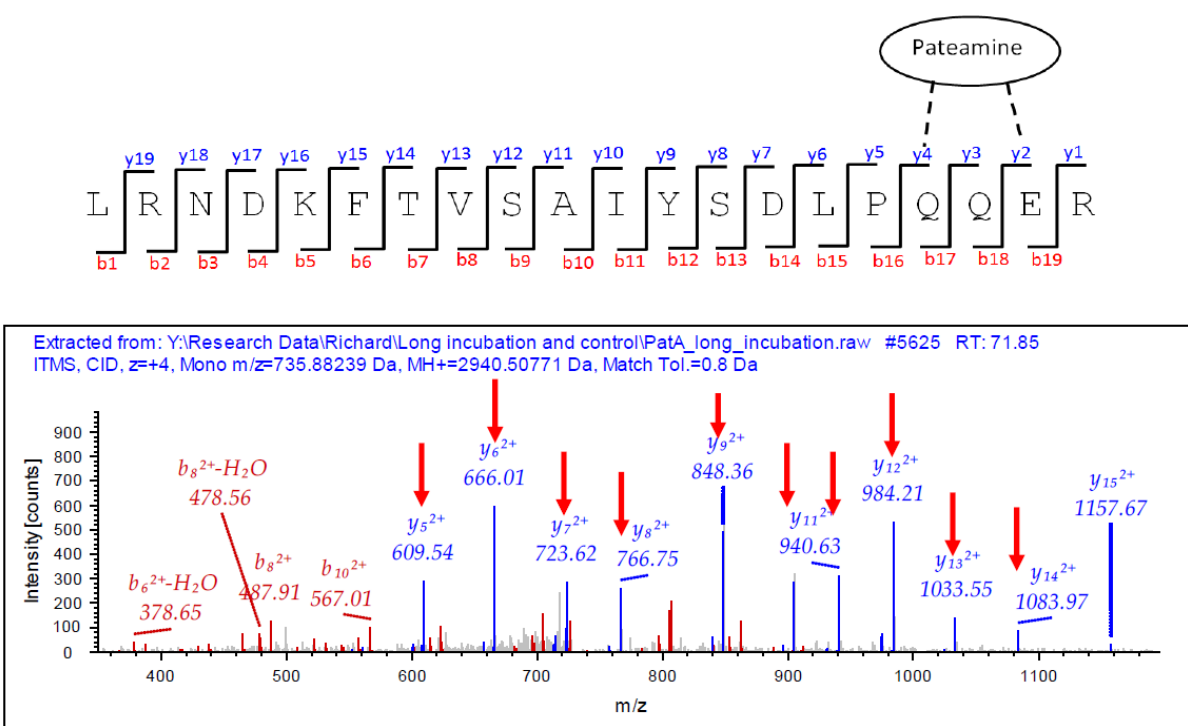

Figure 4.3 Spectrum with multi-charged fragments of a section of peptide from tripsin digested Tif1p, pretreated with pateamine. 


\subsection{Discussion}

The IC 50 of the aldehyde 325 in the MTT assay in HL-60 and 1A9 cells were measured at 100 and $>200 \mu \mathrm{M}$. Target analogue 107 had an approximately 3-fold higher potency, with an $\mathrm{IC}_{50}$ of 34.6 and $51.1 \mu \mathrm{M}$ in HL-60 and 1A9 cells respectively. Although the simplified analogues show reasonable activity, they are drastically less potent than their natural counterpart, with pateamine having an $\mathrm{IC}_{50}$ of $0.27 \mathrm{nM}$ in P388 cells. ${ }^{25}$ It is worth noting that our lead target (107) was a mixture of enantiomers, in which one enantiomer could be completely inactive. Consequently, the inhibition could be understated. Nevertheless, this still does not account for the loss in activity (relative to pateamine).

The introduction of the triazole (in place of the thiazole) is arguably the most significant modification in the simplified analogue 107 in relation to pateamine. Therefore, it would be reasonable to suggest that this modification is a cause of the observed loss in activity. It is possible that the absence of a thiazole in the simplified analogue 107 prevents an important binding interaction or results in a repulsive interaction with a protein target.

The observed modification of the LRNDKFTVSAIYSDLPQQER peptide section of Tif1p of 560 Da strongly suggested that pateamine (with a singly charged mass of $556.3 \mathrm{Da}$ ) was covalently bound. Consistent spectra were observed for the shorter peptide shown in Figure 4.4. The exact site (i.e. amino acid) of which this modification occurred along the peptide could not be unambiguously deduced from the fragment pattern, however, a glutamine residue (Gln12, based on peptide numbering in Figure 4.4) stands out as a good potential candidate, having a mildly nucleophilic residue and being highly conserved amongst the yeast and mammalian eIF4A homologues.

In order to detect a compound in the mass spectrum (in positive mode), a positively charged fragment must be produced. Figure 4.4 illustrates a possible structure for the covalently bound pateamine at $G \ln 12$ on the peptide with an overall +1 positive charge. To account for the few missing mass units and to balance the negative charges on carboxylate residues, it could be envisioned that, in addition to protonation of the primary and tertiary amino groups, the nitrogen on the thiazole could be protonated and a potential hydrolysis of Gln13 to a glutamate may occur (Figure 4.4). 


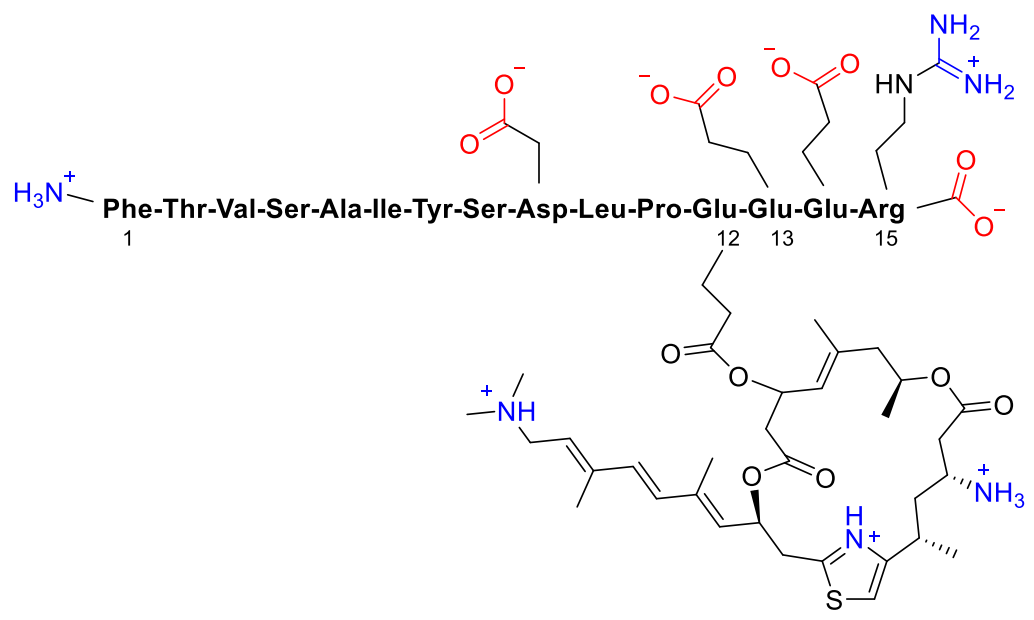

Figure 4.4 Proposed structure of the overall singly charged peptide fragment, accounting for the measured increase to $560 \mathrm{Da}$ (charge/mass). Positive charges are in blue and negative charges in red. Glutamine residues proposed by Sequest to be deaminated are shown as glutamic acid residues.

In this case, it is interesting that no covalent modifications were observed for any of the synthesised analogues, i.e. aldehyde 325, full analogue 107 and the two analogues lacking the sidechain, $Z, E-277$ and $E, E-277$. Several factors may explain this. One explanation is that the lack of easily protonatable functional groups in the analogues (i.e. lack of primary amine and lower $p \mathrm{Ka}$ of triazole relative to thiazole) when bonded to the highly electronegative peptide fragment may render the pateamine-peptide conjugate negative, and so undetectable in the mass spectrometer in the positive ion mode used. Alternatively, the introduction of the triazole might substantially reduce binding to the proposed binding site on the protein (highlighted in Figure 4.5), preventing the covalent binding. 


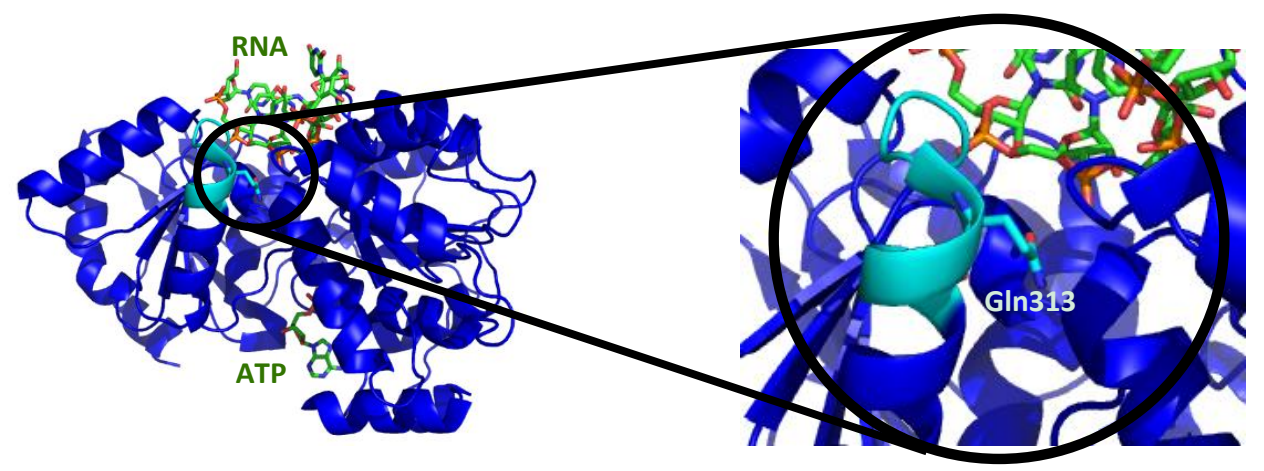

Figure 4.5 3D crystal structure of human eIF4AIII, highlighting the corresponding proposed binding residue (Gln313, corresponding to Glu12 on peptide in Figure 4.4).

(PDB 3EX7)

One interesting possibility is that, if pateamine forms a +3 ion, the protonated thiazole itself is inherent in the activity of pateamine - either as an essential component of the binding pharmacophore or as an internal catalyst for the proposed covalent bond formation. The presence of a protonated thiazole is not unreasonable, with the $\mathrm{pKa}$ of a thiazole being comparable to that of a carboxylic acid: the conjugate acid of 2,5dimethylthiazole has a $\mathrm{pKa}$ value of $3.91^{142}$ versus acetic acid with a $\mathrm{pKa}$ value of 4.76 (Figure 4.6). ${ }^{143}$ It is also noteworthy that an interaction of the thiazole with a negatively charged residue within the binding site, or indeed an intramolecular hydrogen bond within pateamine itself, would stabilise the charge, and consequently raise the $\mathrm{pKa}$ value. In contrast, the $\mathrm{pKa}$ of the conjugate acid of 1-methyltriazole is significantly lower, with a value of $1.25,{ }^{143}$ and thus, much less likely to be protonated.
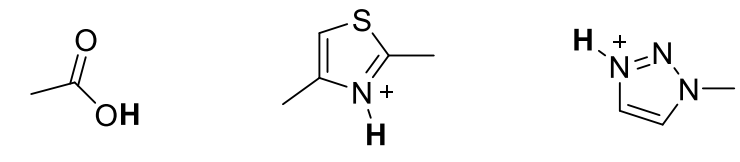

$\mathrm{pKa}=4.76$

$\mathrm{pKa}=3.91$

$\mathrm{pKa}=1.25$

Figure 4.6 Comparison of the pKa values of acetic acid ${ }^{143}$, and the conjugate acids of 2,5dimethylthiazole ${ }^{142}$ and 1-methyltriazole. ${ }^{143}$ 
Interestingly, a lowest energy conformational search ${ }^{+++++}$of DMDA-PatA 86 that is protonated on the thiazole (and the terminal amine) revealed a conformation that contained an internal interaction between the proton on the thiazole and the carbonyl oxygen at C1 (Figure 4.7a). The energy of this conformation was found to be lower, albeit only by a very slight $57 \mathrm{~J}$, to that without an internal interaction (Figure 4.7b).

(a)

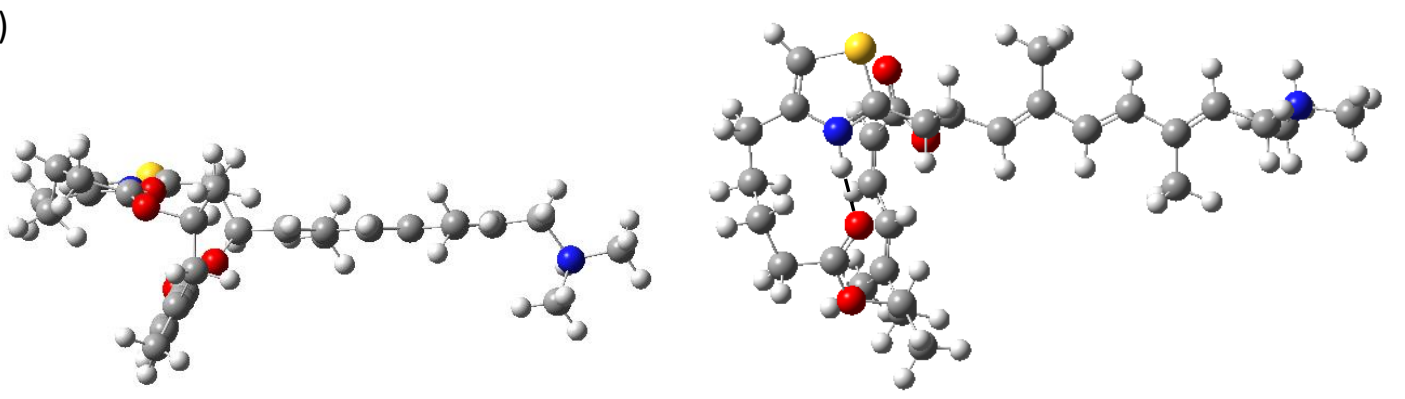

(b)

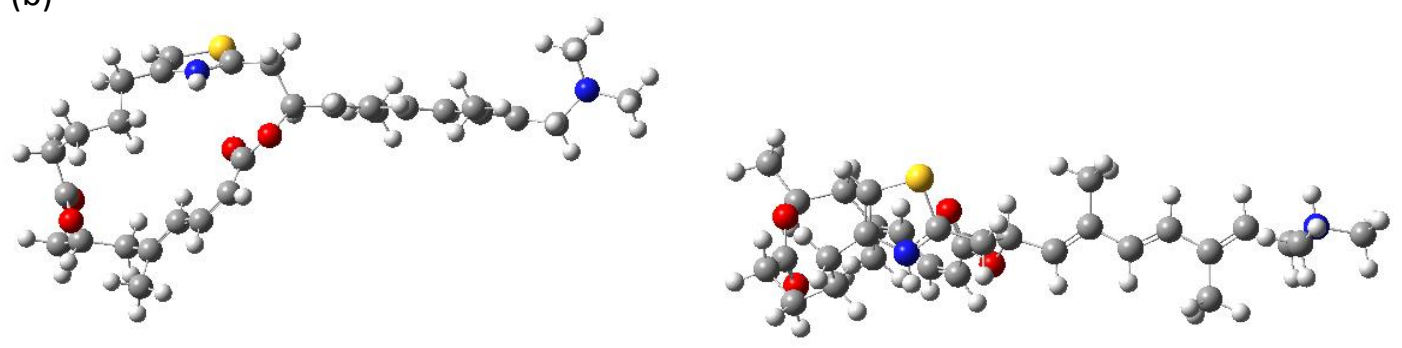

Figure 4.7 Conformations of DMDA-PatA 86 (shown from 'front' and 'top' view) that is protonated on the thiazole (and the terminal amine) both with (a) and without (b) the internal hydrogen bonding interaction.

The significance of this low energy conformation, with an internal interaction, is speculative to say the least. However, the fact that the eliminated-amino product of methyl pateamine 336 (which would not easily adopt the conformation with an internal interaction) was found to be significantly less active than the methylated product of pateamine 335, lends support for the hypothesis of an internal interaction (See appendix). ${ }^{39}$

${ }^{++t+t}$ Lowest energy conformations were calculated using Maestro 9.0.109 software (Schrödinger, LLC), using Macromodel Monte Carlo conformation search with a force field (OPLS_2005) and a continuum solvent model to simulate water as solvent. The search used 30,000 iterations. Structures were then minimised using Gaussian 09 software, performing a DFT (B3LYP) calculation with basis set 6-31G(d,p). 


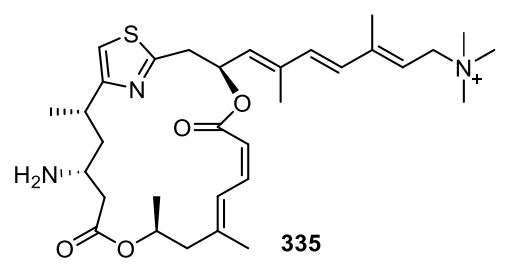

$\mathrm{IC}_{50}=141 \mathrm{nM}(\mathrm{HL}-60)$

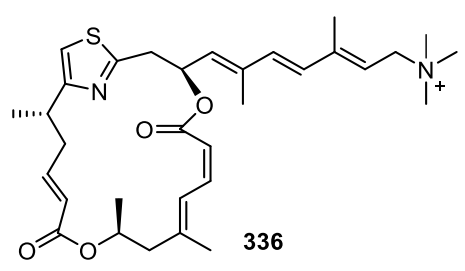

$\mathrm{IC}_{50}=290 \mathrm{nM}(\mathrm{HL}-60)$

Figure 4.8 Methylated pateamine product 335 and the eliminated-amino product 336.

These results provide further insight into the activity of pateamine on the eIF4A proteins, however, much further work is needed. Based on the results associated with Romo's pateamine analogues with oxygen-terminated side chains, it was expected that the aldehyde derivative 325 would have reduced activity and little or no interaction with eIF4A. It was hoped that the analogues lacking a side chain might retain their ability to bind to eIF4A, even if only weakly. In this regard, compounds $Z, E-277$ and $E, E-277$ were synthesised to assist determination of the binding site of pateamine on eIF4A, probe the necessity of the side chain and to determine the impact of changing the alkene geometry. Unfortunately, these analogues were not shown to bind by mass spectrometry, again suggesting that the replacement of the thiazole with triazole was strongly detrimental to binding. It must also be acknowledged that the four methyl groups 'removed' in 107 in comparison to the active simplified analogue DMDA-PatA (86), might also have a significant role in the loss of activity observed in pateamine analogue 107.

\section{Conclusion}

Pateamine was found to bind covalently to eIF4A, most likely with its thiazole in protonated form, although this may be an artefact of the mass spectral analysis. In cellular assays, triazole-containing analogues were either inactive or had dramatically reduced activity and were not found to form covalent adducts with eIF4A. It is proposed that the different electrostatic potential of the triazole or its reduced basicity, and hence reduced likelihood of protonation, may be the cause of this reduced activity. However, we cannot discount the potential loss of activity that may be caused by the removal of methyl groups. 


\section{Chapter Five: Future Work and Closing Remarks}

\subsection{Future Work}

The overall aim of this research was to develop simplified analogues of pateamine that would have selectivity between eIF4A targets. Whilst an effective synthesis of triazolecontaining analogues has been achieved, the lead compound was only weakly active.

\section{Role of Thiazole}

Future work will first focus on understanding the reason for the reduced activity, with an initial hypothesis being that the primary reason for loss of activity is the replacement of the thiazole with a triazole. To test what fraction of the loss of activity can be attributed to this replacement, reintroduction of the thiazole into the simplified pateamine framework is required. Two alternative fragments could be used and incorporated in a similar manner to the established synthesis of the triazole analogue, namely compounds $\mathbf{3 2 8}$ and 329 (Scheme 5.1). Compound 328 could be synthesised from nitrile 330, which in turn could be prepared from previously prepared chloroalkane 212. Alternatively, it is conceivable that compound 328 could be prepared from malic acid 331. Both strategies allow the incorporation of a single enantiomer from commercially available chiral starting materials. Compound fragment 329 could be prepared from easily accessible starting material, using either 1-methylcyclohexene 332 (via ozonolysis) or ع-caprolactone 333. 


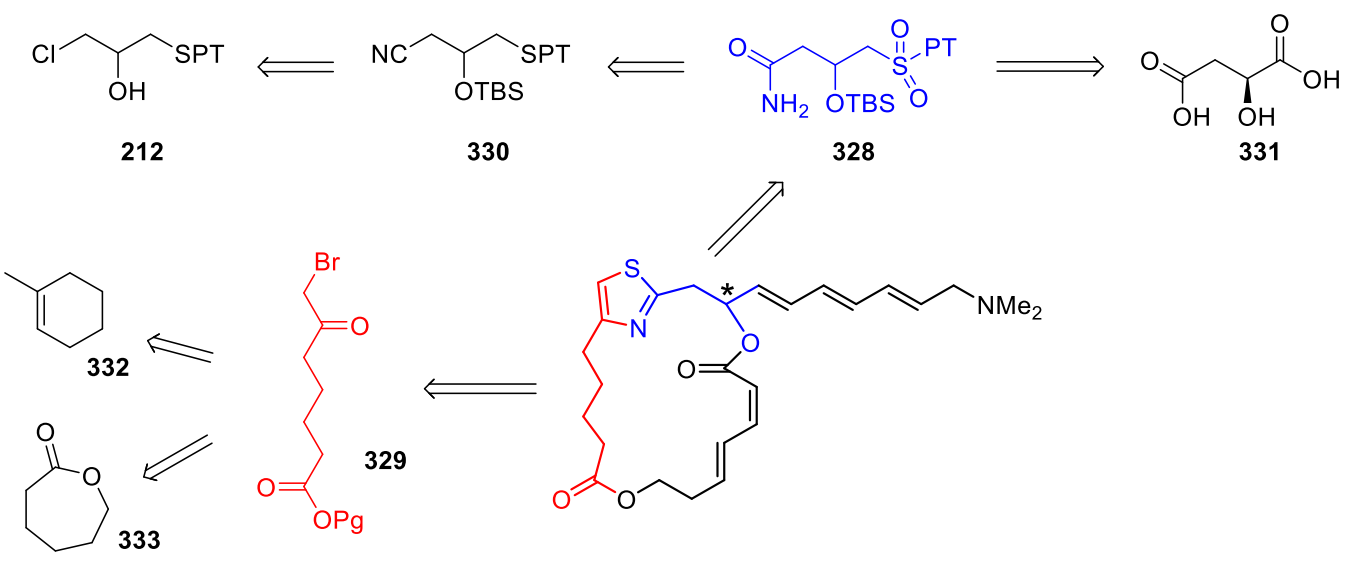

Scheme 5.1 Retrosynthetic analysis for the incorporation of a thiazole heterocycle.

If reintroduction of the thiazole does indeed result in significant regained activity, then it would raise two key questions:

1) Is the low activity against eIF4A because of a charge-based issue with the triazole (too negative an electrostatic potential), either inherently or because of a lack of protonation, leading to repulsion?

2) Is the low activity of triazole analogues because they do not protonate, and protonation is an important activation for the proposed Michael-type addition? (discussed in Section 4.3)

Preparation of analogues that either have alternative 5-membered heterocycles or extra substitution on the thiazole would be informative in addressing these questions. For example, the incorporation of either an electron withdrawing group (fluoro) or an electron donating group (methyl) on C7 would lower or raise the pKa values of the conjugate acid of the thiazole, respectively. These modifications would be conveniently introduced as depicted in Scheme 5.2, via the $\alpha$-substitution of compound 329 (from scheme above). 


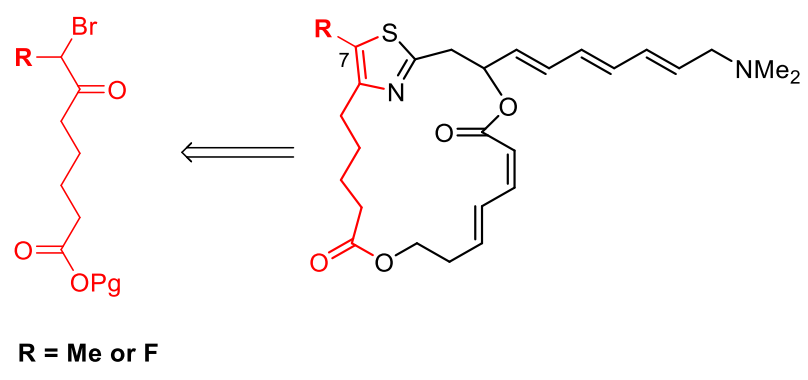

Scheme 5.2 Incorporation of methyl- or fluoro-substituted thiazole.

\section{Sidechain Methyl Groups}

An obstacle that was encountered in the synthesis of the sidechain was the unexpected degradation of polyenes that contained, most notably, an allylic oxygen (presumably via autoxidation, see section 3.4.2). Although our simplified analogue 107 was not observed to suffer this degradation, in pateamine $\mathbf{1 4}$ might provide protection to the sidechain, particularly in a cellular context. Reintroduction of one or both of the methyl groups may be advantageous.

Investigation of Covalent Modification of elF4A

Evidence from a parallel project ${ }^{141}$ looking at the potential for pateamine to bind to eIF4A through a covalent interaction, identified a putative target site in pateamine (see Section 4.3). This could be further investigated by the preparation of analogues that block or prevent Michael addition, for example the preparation of the analogue with saturation of the C19-C20 alkene or a C20 methyl group (Figure 5.1).

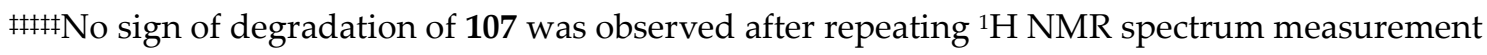
after storage, and no observed oxidation products on HR-MS. 


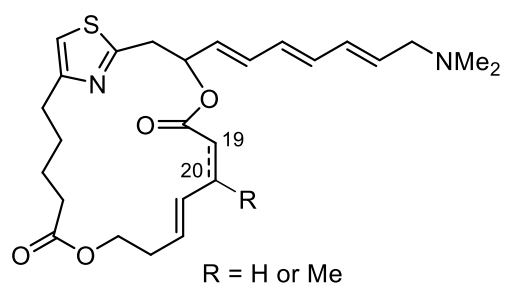

Figure 5.1 Pateamine analogues designed to block proposed Michael addition to protein target.

\section{Alternative Terminal Amines}

An analysis of the effect of alternative amines at the terminus of the sidechain would be of interest, particularly determining whether this results in differing selectivity between the eIF4A isoforms. This would be conveniently achieved in the final reductive amination step of the synthesis by using alternative alkylamines to provide a range of analogues (Scheme 5.3).
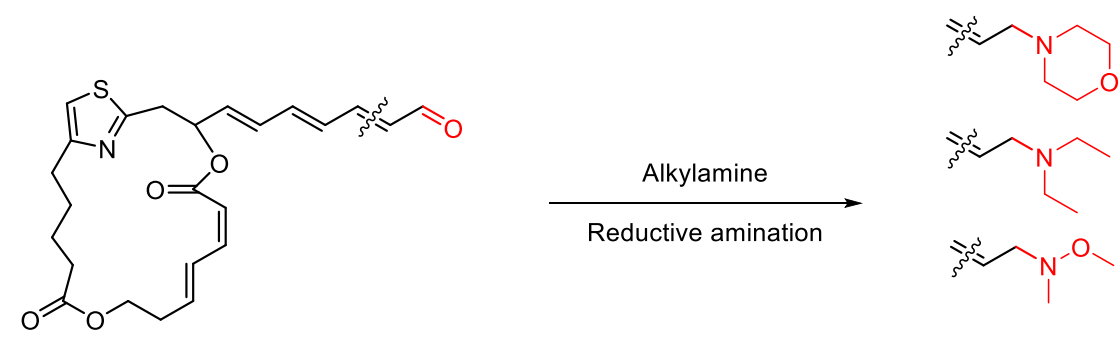<smiles>CCCN(CC)CCN(C)OC</smiles>

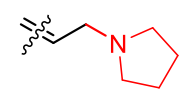

Scheme 5.3 Introduction of alternative terminal amines.

\section{Biological Testing}

All analogues prepared will be tested for their growth inhibitory activity in cancer cell lines (MTT assay). Preliminary screens to confirm that they act through eIF4A inhibition will be undertaken using selected yeast strains, including an in-house pateamine resistant eIF4A mutant ${ }^{62}$ and a strain that is deficient in the yeast homologue of eIF4B, which has been previously shown to be hypersensitive to pateamine. Compounds which show 
sufficient activity and eIF4A selectivity in these preliminary assays will be analysed for their ability to form covalent adducts with eIF4A using mass spectrometry. Selectivity between the human eIF4A isoforms will be investigated through continuation of our collaboration with Professor Jerry Pelletier, Department of Biochemistry, McGill University and his colleague Nahum Sonnenberg, who have well-established in vitro assay systems for eIF4AI/II and III, respectively. Ideally, an X-ray crystal structure of pure eIF4A protein in the presence of RNA, ATP and bound pateamine analogue would be obtained to unambiguously characterise the binding interactions.

\subsection{Closing Remarks}

Pateamine has been shown to be a potent inhibitor of the activity of eIF4A isoforms. These enzymes represent novel targets for anticancer agents (via eIF4AI/II inhibition) and treatments for certain genetically inherited diseases (via eIF4AIII inhibition).

This thesis presents the design and synthesis of simplified analogues of pateamine with some preliminary biological testing. The simplified analogue of pateamine, 107, was targeted, with the hope that this would retain significant bioactivity or inform a second generation of analogues.

\section{Synthesis Summary}

Our strategy was to make the synthesis as convergent as possible, via the separate preparation of four fragments, and combining these to provide the full analogue. Our choice of chemical methodology was strongly influenced by the desire to use cheaper and less toxic starting materials and reagents, and to limit protecting groups.

Four fragments were successfully prepared (Figure 5.2), and these were initially envisioned to be coupled together to provide the target analogue by firstly forming the macrocycle (with 167, 190 and 207 or 208), followed by the attachment of the sidechain 213. 


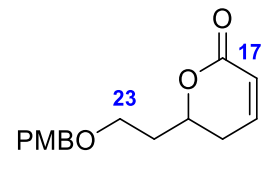

167

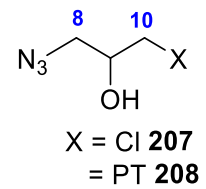

$=$ PT 208

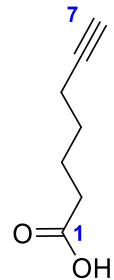

190

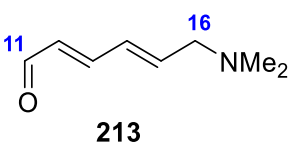

213

Figure 5.2 Four fragments prepared based on our initial synthetic strategy.

A macrocycle that lacked the sidechain (Z,E-277) was targeted (using 167, 190 and 2azidoethanol), and two synthetic sequences were explored to establish the most suitable order of connectivity, which utilised either CuAAC reaction or lactonisation (of the $Z, E-$ dienoic acid) as the macrocyclisation step (Figure 5.3). Significant isomerisation was observed in the intermolecular esterification of $Z, E$-dienoic acid, to afford the energetically favoured E,E-isomer (under Yamaguchi conditions); in contrast, no isomerisation was observed during intramolecular lactonisation. Consequently, we decided that the route using macrolactonisation forming the $Z, E$-dienoate was preferable to avoid the isomerisation.

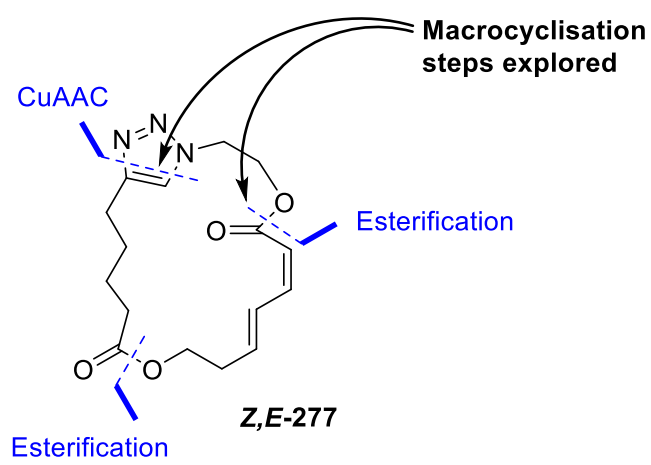

Figure 5.3 Disconnection sites for preparation of macrocycle without sidechain $(Z, E-277)$.

Using simplified acyclic models of the macrocycles prepared from the C8-C10 compounds 207 and 208, respectively, we explored the potential for the use of a Wittig or a JuliaKocienski olefination reaction, respectively, for the attachment of the sidechain. In the case of the Wittig strategy, we were unable to access the phosphonium salt $\mathbf{2 8 8}$ from 
chloroalkane 287, despite trialling a range of reaction conditions (Scheme 5.4a). Subjecting sulfone 292 to Julia-Kocienski reaction conditions resulted in $\beta$-elimination forming alkene 294 (Scheme 5.4b). The lack of success of these reactions demanded a revision of our synthetic connectivity strategy.

(a)

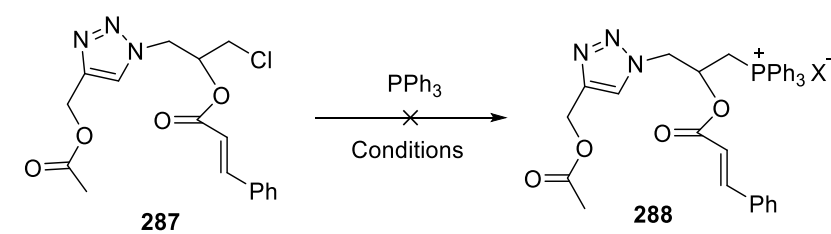

(b)

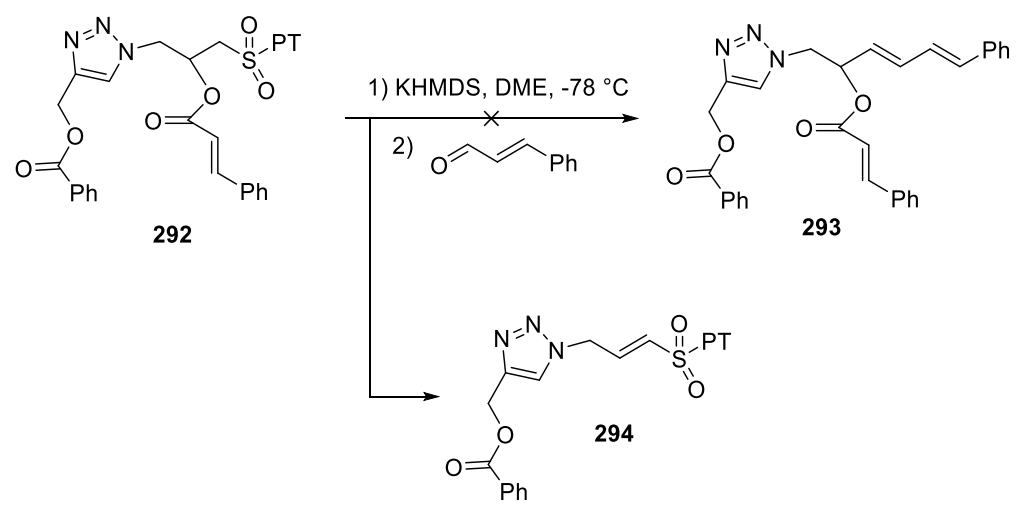

Scheme 5.4 Investigation of olefination reactions on simplified models, for the Wittig (a) and Julia-Kocienski (b) reaction strategies.

We then opted to couple the sidechain 231 with a Julia-Kocienski reaction before macrocycle formation. Unfortunately, Julia-Kocienski reaction between the TBS-protected fragment 296 and the sidechain 231 was unsuccessful (Scheme 5.5). A range of alternative conjugated aldehydes were tested that were unsuccessful in the Julia-Kocienski reaction; fortunately, the ethyl ester 301 was found to provide the triene product 300 . A distinguishing feature of the unsuccessful aldehyde substrates for the Julia-Kocienski reaction (compared with 301 and the other successful aldehyde substrate, cinnamaldehyde) was the presence of an enolisable proton. Based on this, we propose that the presence of the enolisable proton was the cause for the unsuccessful Julia-Kocienski reactions. With triene $\mathbf{3 0 0}$ in hand we were then able to access the desired amine 299 (Scheme 5.5). 


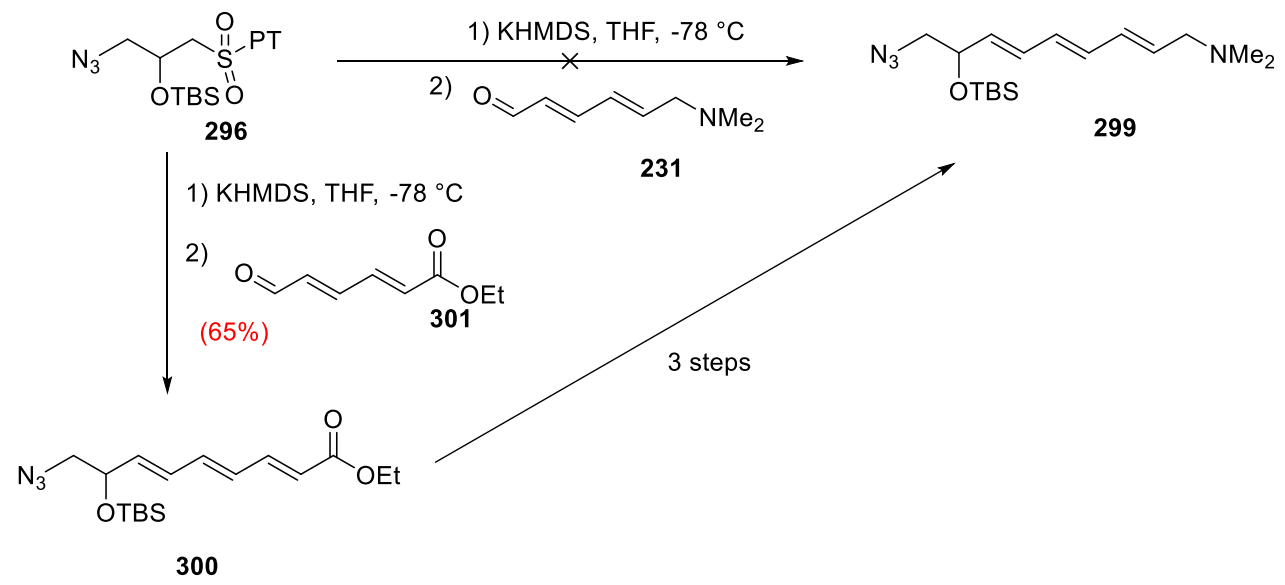

Scheme 5.5 Synthetic route using Julia-Kocienski reaction to access triene 299 .

Subjecting amine 299 to Yamaguchi reagents revealed that the amine would not be compatible with the proposed Yamaguchi macrolactonisation step, due to the reaction of the amine 299 with the acyl chloride, TCBC. We then modified our strategy to use a terminal protected alcohol instead of the amine; this could then be modified at the final stage to give our terminal amine. However, significant degradation (via oxidation) was observed in triene compounds with a terminal allylic oxygen. Fortunately, it was found that the terminal aldehyde $\mathbf{3 1 0}$ was not susceptible to oxidative degradation. Consequently, we chose to proceed with this triene (310), coupling with $Z, E$-dienoic acid 282 (via CuAAC and Yamaguchi macrolactonisation) to form the macrocycle, and subsequent reductive amination to afford the final pateamine analogue target 107 (Scheme 5.6).

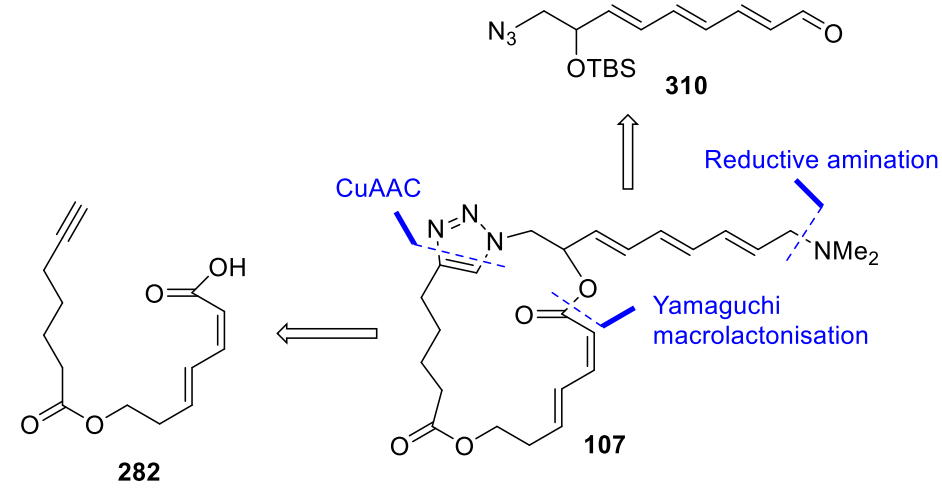

Scheme 5.6 Endgame retro-synthesis of full pateamine analogue $\mathbf{1 0 7 .}$ 


\section{Final Comments and Conclusion}

The established convergent synthetic strategy to our simplified pateamine analogue (107) has 11 longest linear steps. Our initial strategy to prepare four fragments and couple at the final stage, required multiple revisions of the connectivity strategy due to unforeseen obstacles (some of which are highlighted in the Synthesis Summary vide supra).

The $Z, E$-dienoate moiety was efficiently accessed via the ring opening of $\delta$-substituted $\alpha, \beta$-unsaturated lactones. However, incorporation of extra $\delta$-substitution (to incorporate the C21 methyl group) resulted in mixtures of the $Z, E$ - and Z,Z-stereoisomers. Efforts to improve the selectivity towards the desired $Z, E$-stereoisomer by exploring the ring opening of the gem-dimethyl lactone 155, surprisingly, resulted in reduced Z,Estereoselectivity in favour of the undesired Z,Z-stereoisomer.

The unexpected instability of triene species, which contained an allylic oxygen, was a potential barrier to the success of the synthesis. The addition of the mass of dioxygen to a triene species, as determined by HR-MS, indicated that the degradation was caused by oxidation. We propose that the mechanism of the oxidation goes via autoxidation. Fortunately, retaining the conjugated triene avoided oxidative degradation. The observed oxidation raises a possible role of the methyl groups on the sidechain of pateamine for oxidative protection.

The strength of our overall synthetic strategy, is the use of easily accessible starting materials (i.e. furan, epichlorohydrin, $\varepsilon$-caprolactone and 1,3-propanediol) and reagents, and, on the most part, the employment of easily scalable reactions.

Although the lead analogue $\mathbf{1 0 7}$ had low activity, the synthesis of it and macrocycles $Z, E$ 277 and $E, E-277$ have provided a strong indication of the role of the thiazole in pateamine's activity. This lays the platform for the design of second generation analogues of pateamine. Coupled with the efficient synthetic strategy, the groundwork is laid for new, simplified compounds to target eIF4A selectively in the treatment of cancer, cachexia $^{59}$ and Alzheimer's disease ${ }^{144}$ (via eIF4AI/II) and potentially some certain genetic diseases (via eIF4AIII). 
164 


\section{Chapter Six: Experimental}

\subsection{General Experimental}

Unless otherwise stated, the following conditions apply. All reactions were performed under an argon or nitrogen (dry and oxygen free) atmosphere, in oven- or heat gun-dried glassware, using dry solvents and standard syringe techniques. THF, DCM and toluene were either taken from solvent purification system (using Innovative Technology's PureSolv system), or freshly distilled from $\mathrm{CaH}_{2}$ (for DCM or toluene) or sodium metal and benzoquinone (for THF). DMSO, acetonitrile, diisopropylamine, triethylamine and pyridine were distilled from $\mathrm{CaH}_{2}$. Anhydrous dimethylformamide (DMF) was purchased from Aldrich Chemical Company and used without further purification. All other reagents were of commercial quality and distilled prior to use if necessary. Distilled water was used for all aqueous solutions (i.e. The reaction solvent or aqueous work-up). Organic solvents were removed by rotary evaporation with water bath temperature between 40 and $50^{\circ} \mathrm{C}$. The reaction progress was monitored using polyester-backed TLC plates pre-coated with silica UV 254 (Macherey-Nagal) and visualised by UV irradiation (254 nm), in combination with vanillin, phosphomolybdic acid (PMA) or potassium permanganate $\left(\mathrm{KMnO}_{4}\right)$ dip. ${ }^{145}$ Purification of products via silica gel flash chromatography were conducted using a column filled with silica gel 60 (220-240 mesh) eluted with the solvent systems indicated. ${ }^{1} \mathrm{H}$ NMR spectra were recorded on either a Varian Unity Inova 300 Spectrometer at $300 \mathrm{MHz}$ or a Varian Unity Inova 500 Spectrometer at $500 \mathrm{MHz}$. Data are listed as follows: chemical shift in ppm using residual $\mathrm{CHCl}_{3}$ as an internal reference (at $\delta 7.26 \mathrm{ppm})$, multiplicity $(\mathrm{s}=$ singlet, $\mathrm{d}=$ doublet, $\mathrm{t}=$ triplet, $\mathrm{q}=$ quartet, quint $=$ quintet, $\mathrm{m}=$ multiplet or overlap of non-equivalent resonances, $\mathrm{br}=$ broad), integration. ${ }^{13} \mathrm{C} \mathrm{NMR}$ spectra were recorded on a Varian Unity Inova 500 spectrometer at $125 \mathrm{MHz}$ with proton decoupled. Data are listed with chemical shift in ppm using $\mathrm{CDCl}_{3}$ as an internal reference (at $\delta 77.16 \mathrm{ppm}$ ). Infrared spectra were obtained on a Bruker Tensor 27 FTIR spectrometer (ATR). High-resolution mass spectrometry was recorded on a 6530 Accurate Mass Q-TOF LC/MS instrument (Agilent Technologies). 


\subsection{Experimental for Chapter 2}

\section{Substituted Furan}

Preparation of 1-(Triphenylphosphoranylidene)acetone, 132<smiles>CC(=O)C=[Pb]</smiles>

To a solution of triphenylphosphine (10.95 g, $41.7 \mathrm{mmol}, 1$ equiv) in THF (50 mL) was added chloroacetone $(4.7 \mathrm{~mL}, 58.4 \mathrm{mmol}, 1.4$ equiv), and the solution heated to reflux and the mixture stirred overnight. The reaction mixture was then cooled to room temperature, and the resulting white precipitate was filtered, washed with diethyl ether and air dried. The white solid phosphonium salt was then suspended in a mixture of water $(200 \mathrm{~mL})$ and methanol $(200 \mathrm{~mL})$ and the reaction stirred for 1 hour. To the resulting mixture was then added aqueous $2 \mathrm{M} \mathrm{NaOH}$ dropwise until a $\mathrm{pH}$ between 8 and 9 was reached, and stirred vigorously for a further 1 hour. The phosphorane precipitate was then filtered, washed with water and dried under reduced pressure to provide the title compound $(10.1 \mathrm{~g}, 76 \%)$ as a white solid. ${ }^{1} \mathbf{H}$ NMR (500 $\left.\mathrm{MHz}, \mathrm{CDCl}_{3}\right): \delta$ 7.77 - 7.61 (m, 6 H), 7.56 (br s, 3 H), 7.47 (br s, 6 H), 3.72 (d, J = 26.5 Hz, 1 H), 2.11 (br s, 3 H). IR (film): 3054, 1529, 1481, 1436, 1386, 1105, 716, $691 \mathrm{~cm}^{-1}$. These characterisation data match those in the literature. ${ }^{146}$

\section{Preparation of $(E)-4-(2-F u r a n y l)-3-b u t e n-2-o n e, ~ 131$}

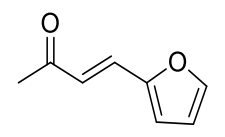

\section{Using Wittig reaction}

To a solution of 2-furfuraldehyde (526 mg, $5.4 \mathrm{mmol}, 1$ equiv) in DCM (50 mL) at room temperature was added 1-(triphenylphosphoranylidene)acetone 132 (2.05 g, 6.45 mmol, 1.2 equiv) and the mixture stirred overnight. Solvent was then removed under reduced pressure and The crude material was purified by silica gel flash chromatography (5:1 pet. ether:EtOAc) to provide the title compound (698 $\mathrm{mg}, 95 \%)$ as a yellow liquid.

\section{6}


To a solution of 2-furfuraldehyde (16.6 mL, $19.3 \mathrm{~g}$, $196 \mathrm{mmol}, 1$ equiv) and acetone (31.6 mL, $431 \mathrm{mmol}, 2.2$ equiv) in water $(150 \mathrm{~mL})$ at $10{ }^{\circ} \mathrm{C}$ (water/ice bath) was added slowly 33\% (w/w) aqueous $\mathrm{NaOH}(3.75 \mathrm{~mL})$. After stirring for 4 hours at room temperature $10 \%$ aqueous sulfuric acid was added until the reaction mixture $\mathrm{pH}$ was 5 . The organic layer was separated and the aqueous layer was extracted with diethyl ether (3 x $60 \mathrm{~mL})$. The combined organic fractions were dried $\left(\mathrm{MgSO}_{4}\right)$, filtered, then concentrated under reduced pressure. The crude material was purified by silica gel flash chromatography (5:1 pet ether:EtOAc) to provide the title compound $(22.83 \mathrm{~g}, 85 \%)$ as a yellow liquid. Rf: 0.31 (5:1 pet. ether:EtOAc). ${ }^{1} \mathbf{H}$ NMR (500 MHz, $\left.\mathrm{CDCl}_{3}\right): \delta 7.49$ (s, $\left.1 \mathrm{H}\right)$, $7.27(\mathrm{~d}, J=15.9 \mathrm{~Hz}, 1 \mathrm{H}), 6.66(\mathrm{~d}, J=2.9 \mathrm{~Hz}, 1 \mathrm{H}), 6.61(\mathrm{~d}, J=15.9 \mathrm{~Hz}, 1 \mathrm{H}), 6.49-6.46(\mathrm{~m}, 1$ H), 2.32 (s, $3 \mathrm{H}) .{ }^{13} \mathrm{C}$ NMR $\left(125 \mathrm{MHz}, \mathrm{CDCl}_{3}\right): \delta 197.8,150.9,145.0,129.4,124.3,115.6,112.5$, 27.9. These characterisation data match those in the literature. ${ }^{147}$

\section{Preparation of (E)-4-(2-Furanyl)-3-buten-2-ol, 134}

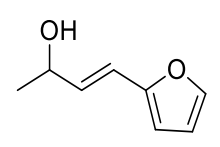

To a solution of (E)-4-(2-furanyl)-3-buten-2-one 131 (3.0 g, $22.1 \mathrm{mmol}, 1$ equiv) in methanol $(20 \mathrm{~mL})$ at $0{ }^{\circ} \mathrm{C}$ (ice bath) was added a solution of $\mathrm{NaBH}_{4}(1.4 \mathrm{~g}, 27.0 \mathrm{mmol}, 1.2$ equiv) in $2 \mathrm{M}$ aqueous sodium hydroxide $(2 \mathrm{~mL})$ and the reaction stirred for 1.5 hours. The reaction mixture was concentrated under reduced pressure, redissolved in water and extracted with diethyl ether $(3 \times 20 \mathrm{~mL})$. The combined organic fractions were dried $\left(\mathrm{MgSO}_{4}\right)$, filtered and the solvent removed under reduced pressure. The crude material was purified by silica gel flash chromatography (2:1 pet. ether: EtOAc) to provide the title compound (1.87 g, 62\%) as a colourless oil. Rf: 0.43 (2:1 pet. ether:EtOAc). ${ }^{1} \mathbf{H}$ NMR (500 $\mathrm{MHz}_{\mathrm{CDCl}}$ ): $\delta 7.35$ (s, $\left.1 \mathrm{H}\right), 6.44$ - 6.35 (m, $\left.2 \mathrm{H}\right), 6.27$ - 6.18 (m, $\left.2 \mathrm{H}\right), 4.46(\mathrm{dd}, J=5.7,9.4$ $\mathrm{Hz}, 1 \mathrm{H}), 1.70$ (s, $1 \mathrm{H}), 1.40-1.31$ (m, $3 \mathrm{H}) .{ }^{13} \mathrm{C}$ NMR (125 MHz, $\left.\mathrm{CDCl}_{3}\right)$ : $\delta$ 152.3, 141.9, 132.2, 117.7, 111.2, 108.0, 68.4, 23.4. These characterisation data match those in the literature. ${ }^{148,149,150}$ 


\section{Preparation of (E)-4-(2-Furanyl)-3-penten-2-one, 135}

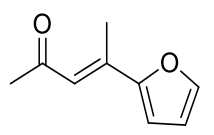

To a solution of phosphonate 137 (632 mg, $3.3 \mathrm{mmol}, 1.3$ equiv) in THF (35 mL) at room temperature was added potassium tert-butoxide (366 mg, $3.3 \mathrm{mmol}, 1.3$ equiv) and the reaction stirred for 1 hour. 2-Acetyl furan ( $276 \mathrm{mg}, 2.5 \mathrm{mmol}, 1$ equiv) was then added, heated to reflux and the mixture stirred overnight. The reaction was quenched with saturated aqueous ammonium chloride $(30 \mathrm{~mL})$, and extracted with diethyl ether $(3 \times 40$ $\mathrm{mL})$. The combined organic fractions were dried $\left(\mathrm{MgSO}_{4}\right)$, filtered and the solvent removed under reduced pressure. The crude material was purified by silica gel flash chromatography (5:1 pet. ether:EtOAc) to provide the title compound ( $45 \mathrm{mg}, 12 \%)$ as a colourless liquid. Rf: 0.46 (5:1 pet. ether:EtOAc). ${ }^{1} \mathbf{H}$ NMR (500 MHz, $\left.\mathrm{CDCl}_{3}\right): \delta 7.44$ (s, 1 H), $6.75(\mathrm{~s}, 1 \mathrm{H}), 6.69$ (d, J = 3.3 Hz, $1 \mathrm{H}), 6.48-6.43(\mathrm{~m}, 1 \mathrm{H}), 2.40(\mathrm{~s}, 3 \mathrm{H}), 2.27(\mathrm{~s}, 3 \mathrm{H}) .{ }^{13} \mathrm{C}$ NMR (125 MHz, CDCl ): $\delta$ 198.9, 154.4, 144.0, 140.2, 119.2, 112.4, 112.2, 32.3, 15.1. IR (film): 3131, 2926, 1673, 1591, 1558, 1355, 1185, 1024, 963, $744 \mathrm{~cm}^{-1}$. HRMS-ESI (m/z): $[\mathrm{M}+\mathrm{H}]^{+}$ calculated for $\mathrm{C}_{9} \mathrm{H}_{11} \mathrm{O}_{2}+1$, 151.0754; found 151.0735.

\section{Lactone Ring Opening Route}

\section{Preparation of 4,5-Dimethyl-4-hydroxyhept-1-ene, 141}

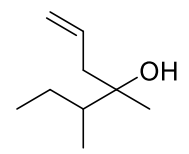

To a biphasic mixture of 3-methyl-2-pentanone (10.52 g, $105 \mathrm{mmol}, 1.1$ equiv) and allyl bromide (11.48 g, $95 \mathrm{mmol}, 1$ equiv) in THF (90 mL) and saturated aqueous ammonium chloride $(90 \mathrm{~mL})$ at $0{ }^{\circ} \mathrm{C}$ (ice bath) was added zinc powder $(6.87 \mathrm{~g}, 105 \mathrm{mmol}$, 1.1 equiv) in portions with vigorous stirring. The biphasic reaction mixture was allowed to warm to room temperature and the mixture stirred for 2 hours. The reaction mixture was filtered and the organic layer was separated. The aqueous layer was extracted with diethyl ether $(2 \times 60 \mathrm{~mL})$. The combined organic fractions were dried $\left(\mathrm{MgSO}_{4}\right)$, filtered and the solvent removed under reduced pressure. The crude material was purified by 


\section{Chapter Six: Experimental}

silica gel flash chromatography (5:1 pet. ether: EtOAc) to provide an inseparable diastereomeric mixture ( $\mathrm{dr} 1: 1)$ of title compound $(2.57 \mathrm{~g}, 22 \%)$ as a colourless liquid. Rf: 0.80 (2:1 pet. ether: EtOAc). ${ }^{1} \mathbf{H}$ NMR (500 MHz, $\left.\mathrm{CDCl}_{3}\right): \delta 5.97-5.80(\mathrm{~m}, 1 \mathrm{H}), 5.22$ - 5.06 (m, 2 H), 2.31 - $2.15(\mathrm{~m}, 2 \mathrm{H}), 1.80-1.56(\mathrm{~m}, 1 \mathrm{H}), 1.44-1.30(\mathrm{~m}, 2 \mathrm{H}), 1.09$ (d, J = 6.2 Hz, 3 H), 1.02 - 0.86 (m, 6 H). ${ }^{13} \mathrm{C}$ NMR (125 MHz, $\left.\mathrm{CDCl}_{3}\right): \delta$ 134.1, 134.0, 118.8, 118.7, 74.6, 74.5, $44.5,44.4,44.4,43.9,24.1,23.5,23.4,22.9,13.8,13.0,12.9,12.8$. These characterisation data match those in the literature. ${ }^{151}$

\section{Preparation of 4,5-Dimethyl-4-(acrolylacetate)hept-1-ene, 142}

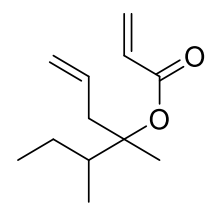

To a solution of alcohol 141 (0.92 g, $6.5 \mathrm{mmol}, 1$ equiv) in DCM (30 mL) at room temperature was added diisopropylethylamine $(2.3 \mathrm{~mL}, 1.68 \mathrm{~g}, 13.0 \mathrm{mmol}, 2$ equiv) followed by acryloyl chloride $(1.1 \mathrm{~mL}, 1.18 \mathrm{~g}, 13.0 \mathrm{mmol}, 2$ equiv). The reaction mixture was stirred at room temperature for 20 hours, then quenched with saturated aqueous ammonium chloride and organic layer was separated. The aqueous layer was extracted with DCM $(2 \times 30 \mathrm{~mL})$. The combined organic fractions were washed with water, dried $\left(\mathrm{MgSO}_{4}\right)$, filtered and the solvent removed under reduced pressure. The crude material was purified by silica gel flash chromatography (5:1 pet. ether: EtOAc) to provide an inseparable diastereomeric mixture ( $\mathrm{dr} 1: 1)$ of the title compound $(1.27 \mathrm{~g}, 30 \%)$ as a colourless liquid. Rf: 0.79 (5:1 pet. ether: EtOAc). ${ }^{1} \mathbf{H}$ NMR (500 MHz, CDCl $): \delta 6.29$ (d, J = 17.1 Hz, 1 H), $6.04(\mathrm{dd}, J=10.4,17.1 \mathrm{~Hz}, 1 \mathrm{H}), 5.85$ - 5.68 (m, 2 H), 5.13 - 5.01 (m, 2 H), 2.85 $2.66(\mathrm{~m}, 1 \mathrm{H}), 2.66$ - $2.52(\mathrm{~m}, 1 \mathrm{H}), 2.26$ - $2.02(\mathrm{~m}, 1 \mathrm{H}), 1.68$ - 1.58 (m, $0.5 \mathrm{H}), 1.58$ - 1.45 (m, $0.5 \mathrm{H}), 1.45$ - $1.28(\mathrm{~m}, 3 \mathrm{H}), 1.10$ - $0.98(\mathrm{~m}, 1 \mathrm{H}), 0.98$ - $0.79(\mathrm{~m}, 6 \mathrm{H}) .{ }^{13} \mathrm{C}$ NMR $(125 \mathrm{MHz}$, $\left.\mathrm{CDCl}_{3}\right): \delta 165.29,165.25,133.5,133.4,130.21,130.19,129.4,127.4,117.96,117.95,87.7,87.6$, 41.2, 40.10, 40.06, 31.3, 23.9, 23.6, 20.3, 20.0, 13.4, 13.2, 12.62, 12.58. IR (film): 2968, 2937, $1719,1639,1403,1297,1206,1049,916,810,734 \mathrm{~cm}^{-1}$. 


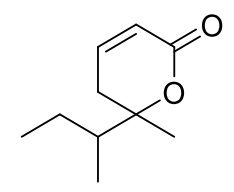

To a solution of diene 142 (374 mg, $1.9 \mathrm{mmol}, 1$ equiv) in DCM (130 mL) at room temperature was added Grubbs second generation catalyst ( $81 \mathrm{mg}, 0.10 \mathrm{mmol}, 5 \mathrm{~mol} \%$ ). The reaction mixture was stirred overnight at room temperature, and then solvent was removed under reduced pressure. The crude material was purified by silica gel flash chromatography (1:1 pet. ether:EtOAc) to provide an inseparable diastereomeric mixture (dr 1:1) of the title compound (254 mg, 80\%) as a pale brown oil. Rf: 0.62 (1:1 pet. ether: EtOAc). ${ }^{1} \mathrm{H}$ NMR (500 MHz, $\left.\mathrm{CDCl}_{3}\right): \delta 6.71-6.82(\mathrm{~m}, 1 \mathrm{H}), 6.03(\mathrm{dd}, J=9.8,1.0 \mathrm{~Hz}, 1 \mathrm{H})$, 2.46 - 2.60 (m, $1 \mathrm{H}), 2.19$ - $2.32(\mathrm{~m}, 1 \mathrm{H}), 1.76$ - 1.88 (m, $1 \mathrm{H}), 1.59$ - 1.76 (m, $2 \mathrm{H}), 1.34$ (d, $J=6.6 \mathrm{~Hz}, 3 \mathrm{H}), 0.87-1.09$ (m, $6 \mathrm{H}) .{ }^{13} \mathrm{C}$ NMR $\left(125 \mathrm{MHz}, \mathrm{CDCl}_{3}\right): \delta 163.92,163.86,143.50$, 143.46, 120.94, 120.91, 85.20, 85.19, 44.3, 44.2, 32.1, 31.3, 24.1, 23.1, 21.9, 21.0, 13.6, 13.0, 12.5, 12.4. IR (film): 2968, 2938, 2879, 1711, 1383, 1281, 1257, 1115, 1086, 996, 957, 851, $812 \mathrm{~cm}^{-1}$. HRMS-ESI (m/z): $[\mathrm{M}+\mathrm{H}]^{+}$calculated for $\mathrm{C}_{10} \mathrm{H}_{17} \mathrm{O}_{2}{ }^{+}, 169.1223$; found 169.1224. (NB: Data quoted are for the mixture of diastereomers.)

\section{General procedure for $\alpha, \beta$-unsaturated lactone opening}

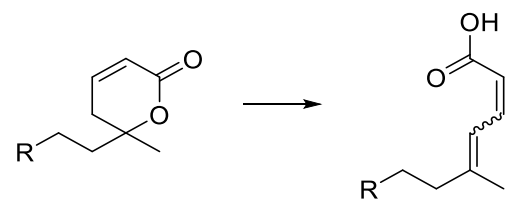

Method A (LiHMDS at $\left.-78^{\circ} \mathrm{C}\right)$

To a solution of lactone (1 equiv) in THF (volume) at $-78^{\circ} \mathrm{C}$ (dry ice/acetone bath) was added dropwise LiHMDS solution (1.3 equiv, $1 \mathrm{M}$ in THF) and the reaction stirred for 3 hours. The reaction was allowed to warm to room temperature before quenching with dilute aqueous sulfuric acid ( $2 \mathrm{vol} \%)$ and extracted with EtOAc ( 3 x volumes). The combined organic fractions were dried $\left(\mathrm{MgSO}_{4}\right)$, filtered, then concentrated under reduced pressure. The crude material was purified by flash chromatography. 
Method B (LiHMDS at room temperature)

This was performed as described in Method $A$, but the reaction was performed at room temperature instead of $-78^{\circ} \mathrm{C}$.

Method C (TBAF at room temperature)

To a solution of lactone (1 equiv) in THF (volume) at room temperature was added dropwise TBAF solution (1 M solution in THF, 4 equiv) and the reaction stirred for 3 hours. The reaction mixture was diluted with EtOAc ( $5 \times$ volumes), and washed three times with dilute aqueous sulfuric acid (2 vol\%, $5 \mathrm{x}$ volumes) to remove tetrabutylammonium salts. Organic fraction was then dried $\left(\mathrm{MgSO}_{4}\right)$, filtered, then concentrated under reduced pressure. Material was purified by flash chromatography.

\section{(2Z,4E)-5,6-Dimethyl-2,4-octadienoic acid, 143}

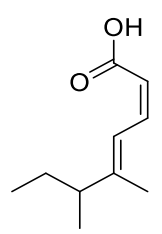

\section{Method A}

Treatment of lactone 139 (93 mg, $0.55 \mathrm{mmol}$ ) with KHMDS (used instead of LiHMDS) as outlined in Method A of General procedure for $\alpha, \beta$-unsaturated lactone opening, provided the title compound (69 $\mathrm{mg}, 74 \%)$ as a yellow liquid.

\section{Method C}

Treatment of lactone 139 (38 mg, $0.23 \mathrm{mmol})$ with TBAF as outlined in Method C of General procedure for $\alpha, \beta$-unsaturated lactone opening, provided the title compound (38 $\mathrm{mg}$, $68 \%$ ) as a yellow liquid.

Rf: 0.65 (5:1 pet. ether: EtOAc). ${ }^{1} \mathrm{H}$ NMR $\left(500 \mathrm{MHz}, \mathrm{CDCl}_{3}\right): \delta 7.20(\mathrm{~d}, J=12.0 \mathrm{~Hz}, 1 \mathrm{H}), 7.03$ $(\mathrm{t}, J=11.7 \mathrm{~Hz}, 1 \mathrm{H}), 5.60(\mathrm{~d}, J=11.5 \mathrm{~Hz}, 1 \mathrm{H}), 2.29-2.18(\mathrm{~m}, 1 \mathrm{H}), 1.80(\mathrm{~s}, 3 \mathrm{H}), 1.54-1.30$ $(\mathrm{m}, 2 \mathrm{H}), 1.06(\mathrm{~d}, J=6.8 \mathrm{~Hz}, 3 \mathrm{H}), 0.84(\mathrm{t}, J=7.4 \mathrm{~Hz}, 3 \mathrm{H}) .{ }^{13} \mathrm{C} \mathrm{NMR}\left(125 \mathrm{MHz}, \mathrm{CDCl}_{3}\right): \delta$ 172.6, 156.2, 142.9, 121.2, 114.1, 45.5, 27.8, 19.0, 13.2, 12.1. IR (film): 3046, 2962, 2932, 2876, 
1684, 1623, 1592, 1442, 1232, 1069, 920, 826, $731 \mathrm{~cm}^{-1}$. HRMS-ESI (m/z): [M - H]- calculated for $\mathrm{C}_{10} \mathrm{H}_{15} \mathrm{O}_{2}^{-}, 167.1078$; found 167.1077.

Preparation of Ethyl-3-hydroxy-3-methyl-5-hexenoate, 145

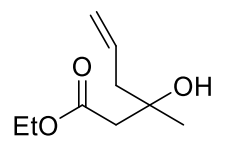

To a solution of ethyl acetoacetate $(5.15 \mathrm{~g}, 40 \mathrm{mmol}, 1$ equiv) and allyl bromide (4.84 g, $40 \mathrm{mmol}, 1$ equiv) in THF (40 mL) and saturated aqueous ammonium chloride (40 $\mathrm{mL})$ at $0{ }^{\circ} \mathrm{C}$ (ice bath) was added zinc powder $(2.88 \mathrm{~g}, 44 \mathrm{mmol}, 1.1$ equiv) in portions with vigorous stirring. The biphasic reaction mixture was allowed to warm to room temperature then stirred for 2 hours. The reaction mixture was filtered and the organic layer was separated. The aqueous layer was extracted with diethyl ether $(3 \times 40 \mathrm{~mL})$. The combined organic fractions were dried $\left(\mathrm{MgSO}_{4}\right)$, filtered and the solvent removed under reduced pressure. The crude material was purified by silica gel flash chromatography $(2: 1$ pet. ether: EtOAc) to provide the title compound (5.12 g, 76\%) as a colourless liquid. $\mathbf{R}_{\mathrm{f}}$ : 0.44 (2:1 pet. ether:EtOAc). ${ }^{1} \mathrm{H}$ NMR (500 MHz, $\left.\mathrm{CDCl}_{3}\right): \delta 5.86(\operatorname{tdd}, J=7.4,9.9,17.2 \mathrm{~Hz}, 1$ H), $5.13-5.08(\mathrm{~m}, 2 \mathrm{H}), 4.18(\mathrm{q}, J=7.0 \mathrm{~Hz}, 3 \mathrm{H}), 2.51(\mathrm{~d}, J=15.9 \mathrm{~Hz}, 1 \mathrm{H}), 2.42(\mathrm{~d}, J=15.6$ $\mathrm{Hz}, 1 \mathrm{H}), 2.30(\mathrm{~d}, J=7.3 \mathrm{~Hz}, 2 \mathrm{H}), 2.27(\mathrm{~s}, 1 \mathrm{H}), 1.28(\mathrm{t}, J=7.1 \mathrm{~Hz}, 3 \mathrm{H}), 1.25(\mathrm{~s}, 3 \mathrm{H}) .{ }^{13} \mathrm{C}$ NMR (125 MHz, $\left.\mathrm{CDCl}_{3}\right): \delta$ 173.0, 133.7, 118.5, 70.7, 60.6, 46.5, 44.3, 26.8, 14.1. These characterisation data match those in the literature. ${ }^{152}$

\section{Preparation of ( \pm )-3-Methyl-5-hexene-1,3-diol, 146}<smiles>C=CCC(C)(O)CCO</smiles>

To a solution of ethyl ester $145\left(3.81 \mathrm{~g}, 22.5 \mathrm{mmol}, 1\right.$ equiv) in THF $(50 \mathrm{~mL})$ at $0{ }^{\circ} \mathrm{C}$ (ice bath) was added slowly $\mathrm{LiAlH}_{4}(1.71 \mathrm{~g}, 45 \mathrm{mmol}, 2$ equiv), with vigorous evolution of gas observed. The reaction mixture was stirred overnight at room temperature, then quenched with saturated aqueous potassium sodium tartrate (Rochelle's salt) and the mixture stirred for one hour. The aqueous layer was extracted with EtOAc $(3 \times 40 \mathrm{~mL})$. 


\section{Chapter Six: Experimental}

The combined organic fractions were dried $\left(\mathrm{MgSO}_{4}\right)$, filtered and the solvent removed under reduced pressure. The crude material was purified by silica gel flash chromatography (3:1 to 1:1 pet. ether:EtOAc) to provide the title compound $(1.61 \mathrm{~g}, 51 \%)$ as a colourless liquid. Rf: 0.52 (1:1 pet. ether:EtOAc). ${ }^{1} \mathbf{H}$ NMR (500 MHz, $\left.\mathrm{CDCl}_{3}\right): \delta 5.86$ (tdd, $J=7.6,9.9,17.2 \mathrm{~Hz}, 1 \mathrm{H}), 5.23$ - 5.06 (m, $2 \mathrm{H}), 3.90(\mathrm{ddq}, J=4.3,6.9,11.3 \mathrm{~Hz}, 2 \mathrm{H}), 2.30$ $(\mathrm{dd}, J=1.8,6.7 \mathrm{~Hz}, 2 \mathrm{H}), 1.80(\mathrm{ddd}, J=4.4,7.7,14.5 \mathrm{~Hz}, 1 \mathrm{H}), 1.69(\mathrm{ddd}, J=4.2,6.6,14.6 \mathrm{~Hz}$, $1 \mathrm{H}), 1.25$ (s, $3 \mathrm{H}) .{ }^{13} \mathrm{C}$ NMR (125 MHz, $\left.\mathrm{CDCl}_{3}\right): \delta 133.7,118.9,67.9,59.7,47.1,26.8,25.6$. These characterisation data match those in the literature. ${ }^{153}$

Preparation of 3-Methyl-1-(tert-butyldimethylsilyloxy)hex-1-en-3-ol , 147<smiles>C=CCC(C)(O)CCOCC</smiles>

To a solution of diol 146 (1.61 g, $11.4 \mathrm{mmol}, 1$ equiv) and imidizole (1.86 g, 27.4 mmol, 2.4 equiv) in DMF (60 mL) at $0{ }^{\circ} \mathrm{C}$ (ice bath) was added TBSCl (2.06 g, $13.7 \mathrm{mmol}$, 1.2 equiv). The reaction mixture was stirred overnight at room temperature, then quenched with saturated aqueous ammonium chloride $(60 \mathrm{~mL})$. The aqueous layer was extracted with EtOAc $(3 \times 40 \mathrm{~mL})$. The combined organic fractions were dried $\left(\mathrm{MgSO}_{4}\right)$, filtered and the solvent removed under reduced pressure. The crude material was purified by silica gel flash chromatography (5:1 pet. ether:EtOAc) to provide the title compound (2.54 g, 88\%) as a colourless liquid. Rf: 0.52 (5:1 pet. ether:EtOAc). ${ }^{1} \mathbf{H}$ NMR (500 MHz, $\left.\mathrm{CDCl}_{3}\right): \delta 5.94-5.75(\mathrm{~m}, 1 \mathrm{H}), 5.09$ - $5.06(\mathrm{~m}, 2 \mathrm{H}), 3.90(\mathrm{t}, J=5.8 \mathrm{~Hz}, 2 \mathrm{H}), 2.28$ $(\mathrm{td}, J=6.5,7.4 \mathrm{~Hz}, 2 \mathrm{H}), 1.75(\mathrm{td}, J=6.1,14.6 \mathrm{~Hz}, 1 \mathrm{H}), 1.64(\mathrm{td}, J=5.4,14.5 \mathrm{~Hz}, 2 \mathrm{H}), 1.20(\mathrm{~s}$, $3 \mathrm{H}), 0.90$ (s, $9 \mathrm{H}), 0.09$ (s, $6 \mathrm{H}) .{ }^{13} \mathrm{C}$ NMR (125 MHz, $\left.\mathrm{CDCl}_{3}\right): \delta$ 134.6, 117.7, 72.4, 60.7, 47.0, 41.0, 26.5, 25.8, 18.0, -5.6. IR (film): 3509, 2930, 2858, 1255, 1086, 913, 836, 777, $734 \mathrm{~cm}^{-1}$. HRMS-ESI (m/z): [M + H] $]^{+}$calculated for $\mathrm{C}_{13} \mathrm{H}_{29} \mathrm{O}_{2} \mathrm{Si}^{+}$, 245.1931; found 245.1933. 


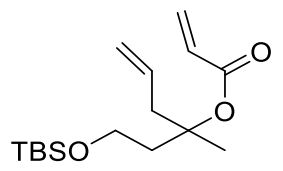

To a solution of alcohol 147 (2.54 g, $10 \mathrm{mmol}, 1$ equiv), DIPEA (4.36 mL, $3.23 \mathrm{~g}, 25$ mmol, 2.5 equiv) and DMAP (200 mg, $1.6 \mathrm{mmol}, 0.16$ equiv) in DCM (60 mL) at room temperature was added acryloyl chloride (1.62 mL, $1.81 \mathrm{~g}$, $20 \mathrm{mmol}$, 2 equiv). The reaction mixture was stirred overnight at room temperature, and then quenched with saturated aqueous ammonium chloride. The aqueous layer was extracted with DCM (3 x $30 \mathrm{~mL})$. The combined organic fractions were dried $\left(\mathrm{MgSO}_{4}\right)$, filtered and the solvent removed under reduced pressure. The crude material was purified by silica gel flash chromatography (5:1 pet. ether:EtOAc) to provide the title compound ( $808 \mathrm{mg}, 27 \%)$ as a colourless liquid. Rf: 0.63 (5:1 pet. ether:EtOAc). ${ }^{1} \mathbf{H}$ NMR (500 MHz, $\left.\mathrm{CDCl}_{3}\right): \delta 6.31$ (dd, $J=$ 1.5, 17.3 Hz, $1 \mathrm{H}), 6.04(\mathrm{dd}, J=10.3,17.3 \mathrm{~Hz}, 1 \mathrm{H}), 5.85-5.74(\mathrm{~m}, 1 \mathrm{H}), 5.75(\mathrm{dd}, J=1.4,10.4$ Hz, $1 \mathrm{H}), 5.12-5.12(\mathrm{~m}, 2 \mathrm{H}), 3.73(\mathrm{t}, J=7.0 \mathrm{~Hz}, 2 \mathrm{H}), 2.71(\mathrm{dd}, J=7.1,14.0 \mathrm{~Hz}, 1 \mathrm{H}), 2.60$ $(\mathrm{dd}, J=7.3,14.0 \mathrm{~Hz}, 1 \mathrm{H}), 2.17(\mathrm{td}, J=6.9,14.0 \mathrm{~Hz}, 1 \mathrm{H}), 2.03(\mathrm{td}, J=7.1,14.1 \mathrm{~Hz}, 1 \mathrm{H}), 1.50$ (s, $3 \mathrm{H}), 0.89$ (s, $11 \mathrm{H}), 0.05$ (s, $6 \mathrm{H}) .{ }^{13} \mathrm{C}$ NMR $\left(125 \mathrm{MHz}, \mathrm{CDCl}_{3}\right): \delta$ 165.3, 133.1, 130.0, 129.6, 118.5, 83.5, 59.0, 43.3, 40.8, 25.9, 24.1, 18.3, -5.4. IR (film): 2930, 2858, 1723, 1403, 1256, 1202, 1047, 917, 836, $776 \mathrm{~cm}^{-1}$. HRMS-ESI $(\mathrm{m} / \mathrm{z}):[\mathrm{M}+\mathrm{Na}]^{+}$calculated for $\mathrm{C}_{16} \mathrm{H}_{30} \mathrm{NaO}_{3} \mathrm{Si}^{+}$, 321.1856; found 321.1851.

\section{Preparation 6-Methyl-6-[2-(tert-butyldimethylsilyloxy)ethyl]-5,6-dihydro-2H-pyran-2- one, 144}

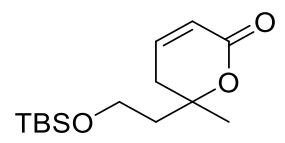

To a solution of diene 148 (375 mg, $1.26 \mathrm{mmol}, 1$ equiv) in DCM (150 mL) at room temperature was added Grubbs second generation catalyst (68 mg, $0.08 \mathrm{mmol}, 6 \mathrm{~mol} \%$ ). The reaction mixture was stirred overnight at room temperature, and then solvent was removed under reduced pressure. The crude material was purified by silica gel flash chromatography (2:1 pet. ether:EtOAc) to provide the title compound (330 $\mathrm{mg}, 97 \%)$ as a pale brown oil. Rf: 0.21 (5:1 pet. ether:EtOAc). ${ }^{1} \mathbf{H}$ NMR $\left(500 \mathrm{MHz}, \mathrm{CDCl}_{3}\right): \delta 6.82$ - $6.72(\mathrm{~m}$, 
$1 \mathrm{H}), 6.04(\mathrm{~d}, J=9.7 \mathrm{~Hz}, 1 \mathrm{H}), 3.87$ - $3.69(\mathrm{~m}, 2 \mathrm{H}), 2.73(\mathrm{~d}, J=18.5 \mathrm{~Hz}, 1 \mathrm{H}), 2.37(\mathrm{dd}, J=4.3$, $18.7 \mathrm{~Hz}, 1 \mathrm{H}), 2.06-1.91(\mathrm{~m}, 2 \mathrm{H}), 1.47$ (s, $3 \mathrm{H}), 0.89$ (s, $9 \mathrm{H}), 0.05$ (d, J = 2.6 Hz, $6 \mathrm{H}) .{ }^{13} \mathrm{C}$ NMR (125 MHz, $\left.\mathrm{CDCl}_{3}\right): \delta$ 163.8, 143.8, 120.8, 81.8, 58.6, 42.8, 34.3, 25.9, 25.8, 18.1, -5.5. These characterisation data match those in the literature. ${ }^{154}$

\section{Preparation of 4-(Methoxymethoxy)butan-2-one, 150}

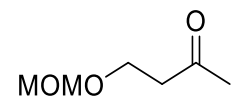

To a solution of 4-hydroxybutan-2-one (2.34 g, $26.5 \mathrm{mmol}, 1$ equiv) in DCM (25 $\mathrm{mL}$ ) at room temperature was added DIPEA (5.49 g, $4.70 \mathrm{~mL}, 42.5 \mathrm{mmol}, 1.6$ equiv) followed by $\mathrm{MOMCl}(2.99 \mathrm{~g}, 2.82 \mathrm{~mL}, 42.5 \mathrm{mmol}, 1.6$ equiv). The reaction mixture was stirred overnight, and then quenched with saturated aqueous ammonium chloride. The aqueous layer was extracted with diethyl ether $(3 \times 40 \mathrm{~mL})$. The combined organic fractions were dried $\left(\mathrm{MgSO}_{4}\right)$, filtered and the solvent removed under reduced pressure. The crude material was purified by silica gel flash chromatography (2:1 pet. ether:EtOAc) to provide the title compound $(1.93 \mathrm{~g}, 56 \%)$ as a colourless liquid. Rf: 0.42 (2:1 pet. ether:EtOAc), (Cf. Rf $=0.07$ for 4-hydroxy-2-butanone). ${ }^{1} \mathbf{H}$ NMR (500 MHz, $\left.\mathrm{CDCl}_{3}\right): \delta 4.61$ $(\mathrm{s}, 2 \mathrm{H}), 3.80(\mathrm{t}, J=6.1,2 \mathrm{H}), 3.36(\mathrm{~s}, 3 \mathrm{H}), 2.71(\mathrm{t}, J=6.1,2 \mathrm{H}), 2.20(\mathrm{~s}, 3 \mathrm{H}) .{ }^{13} \mathrm{C}$ NMR $(125$ $\left.\mathrm{MHz}_{2} \mathrm{CDCl}_{3}\right): \delta 206.9,96.6,62.7,55.3,43.6$ and 30.4. These characterisation data match those in the literature. 155

\section{Preparation of 4-Methyl-4-hydroxy-6-(methoxymethoxy)hex-1-ene, 151}

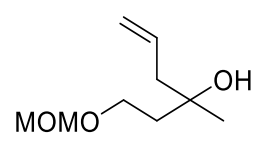

To a solution of ketone 150 (1.37 g, $10.4 \mathrm{mmol}, 1$ equiv) and allyl bromide (3.00 g, $24.9 \mathrm{mmol}, 2.4$ equiv) in THF (50 mL) and saturated aqueous ammonium chloride $(50 \mathrm{~mL})$ at $0{ }^{\circ} \mathrm{C}$ (ice bath) was added zinc powder (1.63 g, $24.9 \mathrm{mmol}, 2.4$ equiv) in portions with vigorous stirring. The biphasic reaction mixture was allowed to warm to room temperature then stirred for 2 hours. The reaction mixture was filtered and the organic layer was separated. The aqueous layer was extracted with diethyl ether $(3 \times 40 \mathrm{~mL})$. The 
combined organic fractions were dried $\left(\mathrm{MgSO}_{4}\right)$, filtered and the solvent removed under reduced pressure. The crude material was purified by silica gel flash chromatography (2:1 pet. ether:EtOAc) to provide the title compound $(754 \mathrm{mg}, 41 \%)$ as a colourless liquid. $\mathbf{R}_{\mathrm{f}}$ : 0.25 (2:1 pet. ether:EtOAc). ${ }^{1} \mathrm{H}$ NMR $\left(500 \mathrm{MHz}, \mathrm{CDCl}_{3}\right): \delta 5.85$ (tdd, $J=7.5,10.1,17.1 \mathrm{~Hz}, 1$ H), 5.14 - $5.01(\mathrm{~m}, 2 \mathrm{H}), 4.61(\mathrm{~s}, 2 \mathrm{H}), 3.75(\mathrm{t}, J=6.2 \mathrm{~Hz}, 2 \mathrm{H}), 3.36(\mathrm{~s}, 3 \mathrm{H}), 2.71$ (br s, $1 \mathrm{H}$, $\mathrm{OH}), 2.26(\mathrm{~d}, J=7.4 \mathrm{~Hz}, 2 \mathrm{H}), 1.87$ - 1.67 (m, $2 \mathrm{H}), 1.20$ (s, $3 \mathrm{H}) .{ }^{13} \mathrm{C}$ NMR (125 MHz, $\left.\mathrm{CDCl}_{3}\right)$ : $\delta$ 134.1, 118.1, 96.4, 71.8, 64.6, 55.4, 46.8, 39.6, 26.6. IR (film): 3466, 2931, 1640, 1442, 1376, 1213, 1148, 1106, 1034, 914, $818 \mathrm{~cm}^{-1}$. HRMS-ESI $(\mathrm{m} / \mathrm{z}):[\mathrm{M}+\mathrm{Na}]^{+}$calculated for $\mathrm{C}_{9} \mathrm{H}_{18} \mathrm{O}_{3} \mathrm{Na}^{+}$, 197.1148; found 197.1155.

\section{Preparation of 1-Methyl-1-(2-methoxymethoxyethyl)-3-butenyl acrylate, 152}

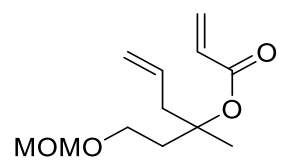

To a solution of alcohol 151 (704 mg, $4.1 \mathrm{mmol}, 1$ equiv) and DIPEA (2.12 mL, 1.57 g, $12.2 \mathrm{mmol}, 3$ equiv) in DCM (30 mL) at room temperature was added acryloyl chloride (0.82 mL, $10.1 \mathrm{mmol}, 2.5$ equiv). The reaction mixture was stirred overnight at room temperature. Starting material was still evident by TLC, so additional DIPEA $(2.1 \mathrm{~mL}, 12.2$ mmol) and acrolyl chloride $(0.82 \mathrm{~mL}, 10.1 \mathrm{mmol})$ were added, and the mixture stirred overnight. The reaction mixture was then quenched with saturated aqueous ammonium chloride and then extracted with DCM $(3 \times 30 \mathrm{~mL})$. The combined organic fractions were dried $\left(\mathrm{MgSO}_{4}\right)$, filtered and the solvent removed under reduced pressure. The crude material was purified by silica gel flash chromatography (5:1 pet. ether:EtOAc) to provide the title compound (779 mg, 89\%) as a colourless liquid. $\mathbf{R}_{\mathrm{f}}$ 0.65 (2:1 pet. ether:EtOAc). ${ }^{1} \mathbf{H}$ NMR (500 MHz, $\left.\mathrm{CDCl}_{3}\right): \delta 6.31(\mathrm{dd}, J=1.2,17.3 \mathrm{~Hz}, 1 \mathrm{H}), 6.04(\mathrm{dd}, J=10.3,17.3 \mathrm{~Hz}, 1 \mathrm{H})$, $5.82-5.74(\mathrm{~m}, 1 \mathrm{H}), 5.75(\mathrm{dd}, J=1.2,10.3 \mathrm{~Hz}, 1 \mathrm{H}), 5.16$ - $5.06(\mathrm{~m}, 2 \mathrm{H}), 4.60(\mathrm{~s}, 2 \mathrm{H}), 3.63(\mathrm{t}$, $J=7.0 \mathrm{~Hz}, 2 \mathrm{H}), 3.36(\mathrm{~s}, 3 \mathrm{H}), 2.72(\mathrm{dd}, J=7.3,14.0 \mathrm{~Hz}, 1 \mathrm{H}), 2.60(\mathrm{dd}, J=7.6,14.0 \mathrm{~Hz}, 1 \mathrm{H})$, $2.26(\mathrm{td}, J=6.9,14.1 \mathrm{~Hz}, 1 \mathrm{H}), 2.09(\mathrm{td}, J=7.2,14.3 \mathrm{~Hz}, 1 \mathrm{H}), 1.51(\mathrm{~s}, 3 \mathrm{H}) .{ }^{13} \mathrm{C}$ NMR $(125$ $\left.\mathrm{MHz}_{2} \mathrm{CDCl}_{3}\right): \delta 165.3,132.9,129.9,129.7,118.7,96.4,83.1,63.4,55.2,43.2,37.8,24.0$. IR (film): 2932, 1720, 1638, 1402, 1200, 1109, 1036, 916, $810 \mathrm{~cm}^{-1}$. HRMS-ESI (m/z): [M + Na] ${ }^{+}$ calculated for $\mathrm{C}_{12} \mathrm{H}_{20} \mathrm{O}_{4} \mathrm{Na}^{+}$, 251.1259; found 251.1254. 
Preparation of 6-Methyl-6-[2-(methoxymethoxy)ethyl]-5,6-dihydro-2H-pyran-2-one, 153<smiles>COCCC1(C)CC=CC(=O)O1</smiles>

To a solution of diene 152 (371 mg, $1.72 \mathrm{mmol}, 1$ equiv) in DCM (140 mL) at room temperature was added Grubbs second generation catalyst (134 mg, $0.16 \mathrm{mmol}, 9 \mathrm{~mol} \%$ ). The reaction mixture was stirred overnight and then solvent was removed under reduced pressure. The crude material was purified by silica gel flash chromatography (1:1 pet. ether:EtOAc) to provide the title compound (301 mg, 92\%) as a pale brown oil. Rf: 0.09 (2:1 pet. ether:EtOAc). ${ }^{1} \mathrm{H}$ NMR $\left(500 \mathrm{MHz}, \mathrm{CDCl}_{3}\right): \delta 6.78(\mathrm{td}, J=4.3,9.7 \mathrm{~Hz}, 1 \mathrm{H}), 6.04(\mathrm{td}, J=$ 1.9, $9.9 \mathrm{~Hz}, 1 \mathrm{H}), 4.60(\mathrm{~s}, 2 \mathrm{H}), 3.75-3.62(\mathrm{~m}, 2 \mathrm{H}), 3.36$ (s, $3 \mathrm{H}), 2.65(\mathrm{ddd}, J=2.3,3.6,18.7$ $\mathrm{Hz}, 1 \mathrm{H}), 2.38(\mathrm{ddd}, J=1.6,4.8,18.7 \mathrm{~Hz}, 1 \mathrm{H}), 2.06(\mathrm{dt}, J=1.7,6.5 \mathrm{~Hz}, 2 \mathrm{H}), 1.47(\mathrm{~s}, 3 \mathrm{H}) .{ }^{13} \mathrm{C}$ NMR $\left(125 \mathrm{MHz}, \mathrm{CDCl}_{3}\right): \delta 163.5,143.5,120.8,96.5,81.3,63.2,55.4,40.1,34.2,25.6$. IR (film): 2935, 1710, 1383, 1214, 1147, 1063, 953, 917, $813 \mathrm{~cm}^{-1}$. HRMS-ESI (m/z): [M + Na $]^{+}$ calculated for $\mathrm{C}_{10} \mathrm{H}_{16} \mathrm{O}_{4} \mathrm{Na}^{+}$, 223.0941; found 223.0947.

Preparation of (2Z,4E)-7-(Methoxymethoxy)-5-methyl-2,4-heptadienoic acid, Z,E-154
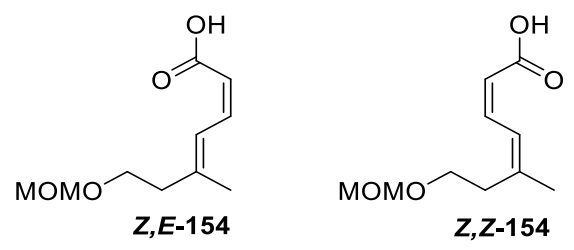

\section{Method A}

Treatment of lactone 153 to LiHMDS as outlined in Method A of General procedure for $\alpha, \beta$ unsaturated lactone opening, provided a mixture of stereoisomers Z,E-154 and Z,Z-154 in a 2.7:1 ratio, respectively, as a pale yellow oil.

\section{Method B}

Treatment of lactone 153 to LiHMDS as outlined in Method B of General procedure for $\alpha, \beta$ unsaturated lactone opening, provided a mixture of stereoisomers $Z, E-154$ and Z,Z-154 in a 1.7:1 ratio. 


\section{Method C}

Treatment of lactone 153 to TBAF as outlined in Method $C$ of General procedure for $\alpha, \beta$ unsaturated lactone opening, provided a mixture of stereoisomers Z,E-154 and Z,Z-154 in a 1.6:1 ratio, respectively.

$\mathbf{R}_{\mathrm{f}:} 0.20$ and 0.23 (2:1 pet. ether:EtOAc). Stereoisomers were not easily separable. Spectral data were measured on the mixture, with ${ }^{1} \mathrm{H}$ NMR spectra quoted as separate for clarification (determined using 2D NMR).

${ }^{1} \mathbf{H}$ NMR (500 MHz, $\left.\mathrm{CDCl}_{3}\right)$ : (Major product $\left.\mathbf{Z}, \mathbf{E - 1 5 4}\right) \delta 7.24(\mathrm{~d}, J=12.1 \mathrm{~Hz}, 1 \mathrm{H}), 7.00(\mathrm{t}, J=$ $11.7 \mathrm{~Hz}, 1 \mathrm{H}), 5.63(\mathrm{~d}, J=11.5 \mathrm{~Hz}, 1 \mathrm{H}), 4.64(\mathrm{~s}, 2 \mathrm{H}), 3.71(\mathrm{t}, J=6.6 \mathrm{~Hz}, 2 \mathrm{H}), 3.37(\mathrm{~s}, 3 \mathrm{H})$, $2.50(\mathrm{t}, J=6.6 \mathrm{~Hz}, 2 \mathrm{H}), 1.92(\mathrm{~s}, 3 \mathrm{H})$, (Minor product Z,Z-154) $7.24(\mathrm{~d}, J=12.1 \mathrm{~Hz}, 1 \mathrm{H}), 7.00$ $(\mathrm{t}, J=11.7 \mathrm{~Hz}, 1 \mathrm{H}), 5.61(\mathrm{~d}, J=11.5 \mathrm{~Hz}, 1 \mathrm{H}), 4.61(\mathrm{~s}, 2 \mathrm{H}), 3.63(\mathrm{t}, J=7.0 \mathrm{~Hz}, 2 \mathrm{H}), 3.35$ (s, 3 H), $2.58(\mathrm{t}, J=6.9 \mathrm{~Hz}, 2 \mathrm{H}), 1.98$ (s, $3 \mathrm{H}) .{ }^{13} \mathrm{C} \mathrm{NMR}\left(125 \mathrm{MHz}, \mathrm{CDCl}_{3}\right): \delta \underline{171.9}, \underline{148.0}, 142.1$, $142.0,123.8,122.9,114.8,114.7,96.4,96.3,65.84,65.77,55.3,40.5,32.6,25.1,17.0$. With three overlapping peaks, underlined.)

${ }^{1}$ H NMR (500 MHz, benzene-d): (Major product Z,E-154) $\delta 7.48(\mathrm{~d}, J=11.6 \mathrm{~Hz}, 1 \mathrm{H}), 6.57$ (t, $J=11.8 \mathrm{~Hz}, 1 \mathrm{H}), 5.56(\mathrm{~d}, J=11.6 \mathrm{~Hz}, 1 \mathrm{H}), 4.37$ (s, $2 \mathrm{H}), 3.39$ (t, J = 6.5 Hz, $2 \mathrm{H}), 3.10(\mathrm{~s}, 3 \mathrm{H})$, $2.13(\mathrm{t}, J=6.6 \mathrm{~Hz}, 2 \mathrm{H}), 1.43(\mathrm{~s}, 3 \mathrm{H})$, (Minor product Z,Z-154) 6.66 (t, J = $11.8 \mathrm{~Hz}, 1 \mathrm{H}), 5.55$ $(\mathrm{d}, J=11.5 \mathrm{~Hz}, 1 \mathrm{H}), 4.32(\mathrm{~s}, 2 \mathrm{H}), 3.28(\mathrm{t}, J=6.7 \mathrm{~Hz}, 2 \mathrm{H}), 3.05(\mathrm{~s}, 3 \mathrm{H}), 2.15(\mathrm{t}, J=6.8 \mathrm{~Hz}, 2$ $\mathrm{H}), 1.56$ (s, $3 \mathrm{H}) .{ }^{13} \mathrm{C}$ NMR (125 MHz, benzene-d): $\delta \underline{173.2}, \underline{148.9}$ 143.13, 143.11, 124.9, 124.1, 115.7, 115.6, 97.1, 97.0, 66.7, 66.6, 55.53, 55.50, 41.3, 33.3, 25.5, 17.3. With 2 overlapping peaks at the chemical shifts underlined.)

Preparation of 4-Methyl-4-(methoxymethoxy)-2 pentanone, 157<smiles>COC(C)(C)CC(C)=O</smiles>

To a solution of 4-hydroxy-4-methyl-2-pentanone (1.96 g, $16.9 \mathrm{mmol}, 1$ equiv) in DCM $(50 \mathrm{~mL})$ at room temperature was added DIPEA $(4.70 \mathrm{~mL}, 27.0 \mathrm{mmol}, 1.6$ equiv) followed by $\mathrm{MOMCl}(1.80 \mathrm{~mL}, 23.6 \mathrm{mmol}, 1.4$ equiv). The reaction mixture was stirred overnight, and then quenched with saturated aqueous ammonium chloride. The aqueous layer was extracted with diethyl ether $(3 \times 40 \mathrm{~mL})$. The combined organic fractions were 


\section{Chapter Six: Experimental}

dried $\left(\mathrm{MgSO}_{4}\right)$, filtered and the solvent removed under reduced pressure. The crude material was purified by silica gel flash chromatography (5:1 pet. ether:EtOAc) to provide the title compound $(2.70 \mathrm{~g}, 60 \%)$ as a colourless liquid. $\mathbf{R}_{\mathrm{f}} 0.40$ (3:1 pet. ether:EtOAc). ${ }^{1} \mathbf{H}$ NMR (500 MHz, CDCl $)$ ): $\delta 4.72(\mathrm{~s}, 2 \mathrm{H}), 3.35$ (s, 3H), $2.63(\mathrm{~s}, 2 \mathrm{H}), 2.18(\mathrm{~s}, 3 \mathrm{H}), 1.33(\mathrm{~s}, 6 \mathrm{H})$. ${ }^{13} \mathrm{C}$ NMR (125 MHz, $\mathrm{CDCl}_{3}$ ): $\delta$ 207.6, 91.0, 75.3, 55.23, 54.8, 32.4, 26.4. IR (film): 2976, 2934, 1706, 1360, 1212, 1145, 1041, 1015, $916 \mathrm{~cm}^{-1}$. HRMS-ESI (m/z): [M + Na $]^{+}$calculated for $\mathrm{C}_{8} \mathrm{H}_{16} \mathrm{O}_{3} \mathrm{Na}$, 183.0992; found 183.0992.

\section{Preparation of 4-Methyl-4-hydroxy-6-methyl-6-(methoxymethoxy)hept-1-ene, 158}<smiles>C=CCC(C)(O)CC(C)(C)OC</smiles>

To a solution of ketone 157 (1.62 g, $10.1 \mathrm{mmol}, 1$ equiv) in THF (40 mL) at room temperature was added allylmagnesium bromide $(1 \mathrm{M}$ solution in diethyl ether, $12.1 \mathrm{~mL}$, $12.1 \mathrm{mmol}, 1.2$ equiv). The reaction mixture was stirred overnight then quenched with saturated aqueous ammonium chloride. The aqueous layer was extracted with diethyl ether $(3 \times 40 \mathrm{~mL})$. The combined organic fractions were dried $\left(\mathrm{MgSO}_{4}\right)$, filtered and the solvent removed under reduced pressure. The crude material was purified by silica gel flash chromatography (5:1 pet. ether:EtOAc) to provide the title compound (1.54 $\mathrm{g}, 83 \%)$ as a colourless liquid. $\mathbf{R}_{\mathbf{f}} 0.44$ (3:1 pet. ether:EtOAc, $c f$. ketone starting material $R_{f}=0.40$ ). ${ }^{1} \mathrm{H}$ NMR (500 MHz, $\left.\mathrm{CDCl}_{3}\right): \delta 5.88(\mathrm{tdd}, J=7.3,10.1,17.2 \mathrm{~Hz}, 1 \mathrm{H}), 5.13-5.02(\mathrm{~m}, 2 \mathrm{H})$, 4.81 - $4.71(\mathrm{~m}, 2 \mathrm{H}), 3.38$ (s, $3 \mathrm{H}), 2.28(\mathrm{ddd}, J=7.2,13.5,20.9 \mathrm{~Hz}, 2 \mathrm{H}), 1.84(\mathrm{~d}, J=14.9 \mathrm{~Hz}, 1$ H), $1.65(\mathrm{~d}, J=14.9 \mathrm{~Hz}, 1 \mathrm{H}), 1.40(\mathrm{~s}, 3 \mathrm{H}), 1.35(\mathrm{~s}, 3 \mathrm{H}), 1.29(\mathrm{~s}, 3 \mathrm{H}) .{ }^{13} \mathrm{C}$ NMR $(125 \mathrm{MHz}$, $\mathrm{CDCl}_{3}$ ): $\delta$ 134.8, 117.7, 90.8, 78.6, 72.6, 55.6, 51.1, 49.2, 28.8, 28.5, 27.7. IR (film): 3499, 2975, 2933, 1370, 1148, 1085, 1027, 1000, $915 \mathrm{~cm}^{-1}$. HRMS-ESI (m/z): $[\mathrm{M}+\mathrm{Na}]^{+}$calculated for $\mathrm{C}_{11} \mathrm{H}_{22} \mathrm{O}_{3} \mathrm{Na}^{+}, 225.1461$; found 225.1460 . 
<smiles>C=CCC(C)(CC(C)(C)OC)OC(=O)C=C</smiles>

To a solution of alcohol $158(1.45 \mathrm{~g}, 7.2 \mathrm{mmol})$ and DIPEA (3.74 mL, $2.78 \mathrm{~g}, 21.5$ $\mathrm{mmol})$ in DCM $(50 \mathrm{~mL})$ at room temperature was added acryloyl chloride $(0.87 \mathrm{~mL}, 970$ $\mathrm{mg}, 10.2 \mathrm{mmol})$. The reaction mixture was stirred overnight then quenched with saturated aqueous ammonium chloride. The aqueous layer was extracted with DCM $(3 \times 40 \mathrm{~mL})$. The combined organic fractions were dried $\left(\mathrm{MgSO}_{4}\right)$, filtered and the solvent removed under reduced pressure. The crude material was purified by silica gel flash chromatography (5:1 pet. ether:EtOAc) to provide the title compound (513 $\mathrm{mg}, 28 \%$ ) as a colourless liquid. Rf: 0.67 (3:1 pet. ether:EtOAc). ${ }^{1} \mathbf{H}$ NMR $\left(500 \mathrm{MHz}, \mathrm{CDCl}_{3}\right): \delta 6.30$ (dd, J = 1.2, $17.3 \mathrm{~Hz}, 1 \mathrm{H}), 6.03(\mathrm{dd}, J=10.4,17.2 \mathrm{~Hz}, 1 \mathrm{H}), 5.86-5.77(\mathrm{~m}, 1 \mathrm{H}), 5.74(\mathrm{dd}, J=1.2,10.5$ $\mathrm{Hz}, 1 \mathrm{H}), 5.11$ - $5.04(\mathrm{~m}, 2 \mathrm{H}), 4.74-4.67$ (m, 2 H), 3.36 (s, $3 \mathrm{H}), 2.82(\mathrm{dd}, J=7.2,13.8 \mathrm{~Hz}, 1$ H), $2.61(\mathrm{dd}, J=7.3,13.9 \mathrm{~Hz}, 1 \mathrm{H}), 2.25(\mathrm{~d}, J=15.4 \mathrm{~Hz}, 1 \mathrm{H}), 2.06(\mathrm{~d}, J=15.6 \mathrm{~Hz}, 1 \mathrm{H}), 1.56$ (s, $3 \mathrm{H}), 1.28$ (s, $3 \mathrm{H}), 1.31$ (s, $3 \mathrm{H}) .{ }^{13} \mathrm{C}$ NMR (125 MHz, $\left.\mathrm{CDCl}_{3}\right): \delta$ 165.5, 133.5, 130.2, 129.6, 118.4, 90.8, 84.6, 76.3, 55.3, 47.6, 44.6, 27.9, 27.0, 24.9. IR (film): 2979, 1718, 1403, 1296, 1200, 1145, 1033, 985, 916, $810 \mathrm{~cm}^{-1}$. HRMS-ESI $(\mathrm{m} / \mathrm{z}):[\mathrm{M}+\mathrm{Na}]^{+}$calculated for $\mathrm{C}_{14} \mathrm{H}_{24} \mathrm{O}_{4} \mathrm{Na}^{+}$, 279.1567; found 279.1561.

Preparation of 6-Methyl-6-[2-methyl-2-(methoxymethoxy)propyl]-5,6-dihydro-2Hpyran-2-one, 155

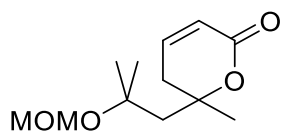

To a solution of diene 159 (400 mg, $1.56 \mathrm{mmol}, 1$ equiv) in DCM (130 mL) at room temperature was added Grubbs second generation catalyst (106 mg, $0.13 \mathrm{mmol}, 8 \mathrm{~mol} \%$ ). The reaction mixture was stirred overnight and then solvent was removed under reduced pressure. The crude material was purified by silica gel flash chromatography (1:1 pet. ether:EtOAc) to provide the title compound (336 mg, 79\%) as a pale brown oil. Rf: 0.13 (2:1 pet. ether:EtOAc). ${ }^{1} \mathrm{H}$ NMR (500 MHz, $\left.\mathrm{CDCl}_{3}\right): \delta 6.81-6.74(\mathrm{~m}, 1 \mathrm{H}), 6.02(\mathrm{~d}, J=10.0 \mathrm{~Hz}, 1$ H), $4.74-4.65(\mathrm{~m}, 2 \mathrm{H}), 3.35(\mathrm{~s}, 3 \mathrm{H}), 2.96(\mathrm{td}, J=2.8,18.8 \mathrm{~Hz}, 1 \mathrm{H}), 2.34(\mathrm{ddd}, J=1.2,4.9$, 


\section{Chapter Six: Experimental}

$18.8 \mathrm{~Hz}, 1 \mathrm{H}), 1.98$ (q, J = 15.4 Hz, $2 \mathrm{H}), 1.53(\mathrm{~s}, 3 \mathrm{H}), 1.35$ (s, $3 \mathrm{H}), 1.34(\mathrm{~s}, 3 \mathrm{H}) .{ }^{13} \mathrm{C}$ NMR (125 MHz, $\left.\mathrm{CDCl}_{3}\right): \delta$ 163.7, 144.1, 120.6, 90.9, 82.5, 76.0, 55.3, 50.8, 34.5, 27.8, 27.4, 27.2. IR (film): 2978, 1712, 1382, 1252, 1144, 1032, 998, 917, $810 \mathrm{~cm}^{-1}$. HRMS-ESI (m/z): $[\mathrm{M}+\mathrm{H}]^{+}$ calculated for $\mathrm{C}_{12} \mathrm{H}_{21} \mathrm{O}_{4}{ }^{+}, 229.1434$; found 229.1427.

Preparation of (2Z,4E)-7-(Methoxymethoxy)-5,7-dimethyl-2,4-octadienoic acid, Z,E-156
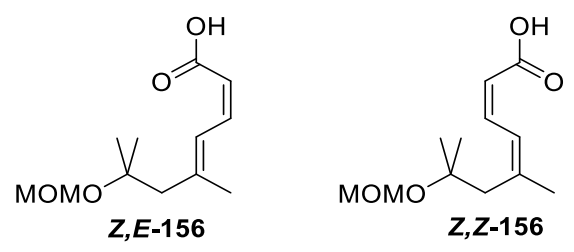

\section{Method A}

Treatment of lactone 155 with LiHMDS as outlined in Method A of General procedure for $\alpha, \beta$-unsaturated lactone opening, provided a mixture of stereoisomers $Z, E-156$ and Z,Z-156 in a 1:1.18 ratio, respectively, as a colourless oil.

\section{Method B}

Treatment of lactone 155 to LiHMDS as outlined in Method B of General procedure for $\alpha, \beta$-unsaturated lactone opening, provided a mixture of stereoisomers $Z, E-156$ and Z,Z156 in a 1:1.32 ratio, respectively.

\section{Method C}

Treatment of lactone $\mathbf{1 5 5}$ to TBAF as outlined in Method C of General procedure for $\alpha, \beta$-unsaturated lactone opening, provided a mixture of stereoisomers $Z, E-156$ and Z,Z-156 in a 1:1.35 ratio, respectively.

Rf: 0.68 (1:1 pet. ether:EtOAc). Stereoisomers were not easily separable. Spectral data were measured on the mixture, with ${ }^{1} \mathrm{H}$ NMR spectra quoted as separate for clarification (determined using 2D NMR).

${ }^{1} \mathbf{H}$ NMR (500 MHz, $\left.\mathrm{CDCl}_{3}\right)$ : (Slightly major product Z,Z-156): $\delta 7.35(\mathrm{~d}, J=12.0 \mathrm{~Hz}, 1 \mathrm{H})$, 7.03 - 6.95 (m, 1 H), 5.65 - 5.57 (m, 1 H), 4.72 (s, 2 H), 3.36 (s, 3 H), 2.50 (s, 2 H), 2.04 (s, 3 H), $1.26(\mathrm{~s}, 6 \mathrm{H})$. (Slightly minor product $Z, E-156): \delta 7.17$ (d, J = 11.7 Hz, $1 \mathrm{H}), 7.03$ - 6.95 (m, $1 \mathrm{H}), 5.65-5.57(\mathrm{~m}, 1 \mathrm{H}), 4.74(\mathrm{~s}, 2 \mathrm{H}), 3.37(\mathrm{~s}, 3 \mathrm{H}), 2.40(\mathrm{~s}, 2 \mathrm{H}), 1.97(\mathrm{~s}, 3 \mathrm{H}), 1.26(\mathrm{~m}, 6$ 
H). ${ }^{13} \mathrm{C}$ NMR $\left(125 \mathrm{MHz}, \mathrm{CDCl}_{3}\right): \delta \underline{171.4}, 148.7,148.5,143.1,142.3,125.4,125.3,125.0,114.4$, 114.0, 91.0, 90.9, 57.6, 55.2, 53.7, 52.5, 45.4, 44.4, 30.20, 30.18, 30.0, 27.6, 26.8, 26.6, 18.8. (With 1 overlapping peak at the chemical shift underlined.) IR (film): 2973, 2932, 1687, 1624, 1591, 1429, 1227, 1133, 1040, 915, $732 \mathrm{~cm}^{-1}$. HRMS-ESI (m/z): [M]- calculated for $\mathrm{C}_{12} \mathrm{H}_{19} \mathrm{O}_{4}{ }^{-}, 227.1289$; found 227.1287.

Preparation of 1-(2-Methoxymethoxy-2-methylpropyl)-1-methyl-3-butenyl acrylate, 161

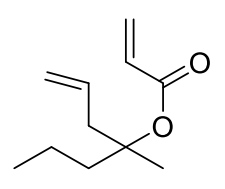

To a solution of methylpropylketone (204 mg, $2.4 \mathrm{mmol}, 1$ equiv) in THF (20 mL) at room temperature was added allylmagnesium bromide (1 M solution in diethyl ether, $3.1 \mathrm{~mL}, 3.1 \mathrm{mmol}, 1.3$ equiv) and the reaction mixture stirred overnight. Without any purification to the reaction mixture, this was added DCM $(10 \mathrm{~mL})$, triethylamine $(382 \mu \mathrm{L}$, $2.7 \mathrm{mmol}, 1.2$ equiv), acryloyl chloride ( $288 \mu \mathrm{L}, 3.6 \mathrm{mmol}, 1.5$ equiv) and DMAP (30 mg, $0.24 \mathrm{mmol}, 0.1$ equiv). The reaction mixture was stirred overnight at room temperature, then quenched with saturated aqueous ammonium chloride $(20 \mathrm{~mL})$, and extracted with EtOAc $(3 \times 20 \mathrm{~mL})$. The combined organic fractions were washed with water and brine, then dried $\left(\mathrm{MgSO}_{4}\right)$, filtered and the solvent removed under reduced pressure. The crude material was purified by silica gel flash chromatography (9:1 pet. ether:EtOAc) to provide the title compound (113 mg, 26\% over 2 steps) as a colourless oil. Rf: 0.52 (9:1 pet. ether:EtOAc). ${ }^{1} \mathbf{H}$ NMR $\left(500 \mathrm{MHz}, \mathrm{CDCl}_{3}\right) \delta 6.30(\mathrm{~d}, J=17.8 \mathrm{~Hz}, 1 \mathrm{H}), 6.04(\mathrm{dd}, J=10.4,17.2$ $\mathrm{Hz}, 1 \mathrm{H}), 5.82-5.67$ (m, 2 H), 5.16 - 4.99 (m, 2 H), 2.68 (dd, J = 6.7, 13.6 Hz, $1 \mathrm{H}), 2.54$ (dd, J = 7.3, 13.7 Hz, $1 \mathrm{H}), 1.94-1.79(\mathrm{~m}, 1 \mathrm{H}), 1.79$ - $1.66(\mathrm{~m}, 1 \mathrm{H}), 1.45(\mathrm{~s}, 3 \mathrm{H}), 1.34(\mathrm{dd}, J=7.7$, $15.7 \mathrm{~Hz}, 2 \mathrm{H}), 0.92(\mathrm{t}, J=7.2 \mathrm{~Hz}, 3 \mathrm{H}) .{ }^{13} \mathrm{C}$ NMR $\left(125 \mathrm{MHz}, \mathrm{CDCl}_{3}\right): \delta$ 165.3, 133.3, 130.1, 129.4, 118.2, 84.4, 42.8, 40.5, 23.7, 16.8, 14.4. IR (film): 2962, 2934, 1720, 1402, 1298, 1275, $1199,1135,1048,985,917 \mathrm{~cm}^{-1}$. 


\section{Preparation of 6-Methyl-6-propyl-5,6-dihydro-2H-pyran-2-one, 160}<smiles>CCCC1(C)CC=CC(=O)O1</smiles>

To a solution of diene 161 (109 mg, $0.60 \mathrm{mmol}, 1$ equiv) in DCM (50 mL) at room temperature was added Grubbs second generation catalyst ( $25 \mathrm{mg}, 0.03 \mathrm{mmol}, 5 \mathrm{~mol} \%$ ). The reaction mixture was stirred overnight and then solvent was removed under reduced pressure. The crude material was purified by silica gel flash chromatography (3:1 pet. ether:EtOAc) to provide the title compound ( $75 \mathrm{mg}, 82 \%)$ as a colourless liquid. $\mathbf{R}_{\mathrm{f}} \mathbf{0 . 1 5}$ (5:1 pet. ether:EtOAc). ${ }^{1} \mathbf{H}$ NMR $\left(500 \mathrm{MHz}, \mathrm{CDCl}_{3}\right) \delta 6.80-6.69(\mathrm{~m}, 1 \mathrm{H}), 6.01(\mathrm{~d}, J=9.5 \mathrm{~Hz}$, $1 \mathrm{H}), 2.49(\mathrm{~d}, J=18.6 \mathrm{~Hz}, 1 \mathrm{H}), 2.35-2.26(\mathrm{~m}, 1 \mathrm{H}), 1.75-1.60(\mathrm{~m}, 2 \mathrm{H}), 1.46-1.32(\mathrm{~m}, 5 \mathrm{H})$, 0.95 - 0.89 (m, 3 H). ${ }^{13} \mathrm{C}$ NMR (125 MHz, $\left.\mathrm{CDCl}_{3}\right)$ : $\delta$ 143.4, 120.9, 82.3, 43.0, 42.5, 33.8, 25.1, 17.0, 14.3. IR (film): 2959, 2924, 2853, 2361, 1712, 1458, 1383, 1246, 1113, $811 \mathrm{~cm}^{-1}$. HRMSESI (m/z): $[\mathrm{M}+\mathrm{H}]^{+}$calculated for $\mathrm{C}_{9} \mathrm{H}_{15} \mathrm{O}_{2}{ }^{+}, 155.1067$; found 155.1068.

\section{Preparation of (2Z,4E)-5-Methyl-2,4-octadienoic acid, Z,E-162}
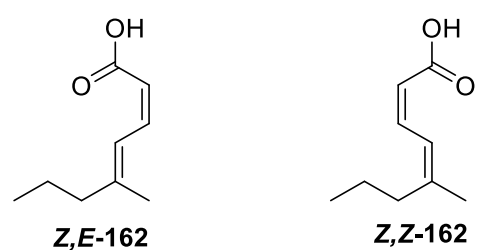

\section{Method A}

Treatment of lactone $\mathbf{1 6 0}$ to LiHMDS as outlined in Method A of General procedure for $\alpha, \beta$-unsaturated lactone opening, provided a mixture of stereoisomers $Z, E-162$ and $Z, Z$ 162 in a 1.9:1 ratio, respectively, as a yellow oil.

\section{Method C}

Treatment of lactone $\mathbf{1 6 0}$ to TBAF as outlined in Method C of General procedure for $\alpha, \beta$-unsaturated lactone opening, provided a mixture of stereoisomers Z,E-162 and Z,Z-162 in a 1.5:1 ratio, respectively. 
Rf: 0.41 (9:1 pet. ether:EtOAc). Stereoisomers were not easily separable. Spectral data were measured on the mixture, with ${ }^{1} \mathrm{H}$ NMR quoted as separate spectra quoted for clarification (determined using 2D NMR).

${ }^{1} \mathbf{H}$ NMR (500 MHz, CDCl 3 ): (Major product Z,E-162) 8.23 - $7.13(\mathrm{~m}, 1 \mathrm{H}), 7.05$ - 6.93 (m, 1 H), 5.63 - $5.51(\mathrm{~m}, 1 \mathrm{H}), 2.17(\mathrm{t}, J=7.7 \mathrm{~Hz}, 2 \mathrm{H}), 1.86(\mathrm{~s}, 3 \mathrm{H}), 1.57$ - $1.45(\mathrm{~m}, 2 \mathrm{H}), 0.92(\mathrm{t}, J=$ $7.2 \mathrm{~Hz}, 3 \mathrm{H})$, (Minor product Z,Z-162) ठ 7.23 - 7.13 (m, 1 H), 7.05 - 6.93 (m, 1 H), 5.63 - 5.51 $(\mathrm{m}, 1 \mathrm{H}), 2.25(\mathrm{t}, J=7.5 \mathrm{~Hz}, 2 \mathrm{H}), 1.93(\mathrm{~s}, 3 \mathrm{H}), 1.57-1.45(\mathrm{~m}, 2 \mathrm{H}), 0.92(\mathrm{t}, J=7.2 \mathrm{~Hz}, 3 \mathrm{H})$. ${ }^{13} \mathrm{C}$ NMR $\left(125 \mathrm{MHz}, \mathrm{CDCl}_{3}\right): \delta 172.0,152.4,152.0,142.8,142.5,122.3,121.7,113.9,113.8$, 42.8, 34.2, 24.9, 22.5, 21.7, 21.0, 16.7, 13.8. IR (film): 2961, 2931, 1704, 1625, 1593, 1291, 1228, 1162, 912, $734 \mathrm{~cm}^{-1}$. HRMS-ESI $(\mathrm{m} / \mathrm{z}):[\mathrm{M}+\mathrm{H}]^{+}$calculated for $\mathrm{C}_{9} \mathrm{H}_{15} \mathrm{O}_{2}{ }^{+}, 155.1067$; found 155.1065 .

\section{Preparation of 2-(4-Methoxyphenyl)-1,3-dioxane, 163}

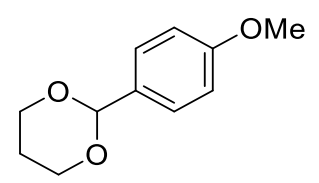

To a solution of 1,3-propanediol (6.64 g, $87.3 \mathrm{mmol}, 1.2$ equiv) and $p$-anisaldehyde (9.90 g, $72.7 \mathrm{mmol}, 1$ equiv) in toluene $(50 \mathrm{~mL})$ was treated with a catalytic amount of $p$ toluenesulfonic acid $(100 \mathrm{mg}, 0.6 \mathrm{mmol})$ and then heated at reflux in a Dean-Stark apparatus overnight. The reaction mixture was then allowed to cool to room temperature, and acid quenched with saturated aqueous sodium bicarbonate $(2 \mathrm{~mL})$ and washed excess diol with distilled water $(50 \mathrm{~mL})$. The aqueous fraction was then re-extracted with diethyl ether $(50 \mathrm{~mL})$, and the combined organic fractions were dried $\left(\mathrm{MgSO}_{4}\right)$, filtered and the solvent removed under reduced pressure to provide compound title compound (13.53 g, $96 \%)$ as a brown crystalline solid. $R_{f}: 0.73$ (1:1 pet. ether:EtOAc). Melting point: $39-42^{\circ} \mathrm{C}$ ${ }^{1} \mathrm{H}$ NMR (500 MHz, CDCl$): ~ \delta 7.42(d, J=8.6 \mathrm{~Hz}, 2 \mathrm{H}), 6.90(\mathrm{~d}, J=8.8,2 \mathrm{H}), 5.47(\mathrm{~s}, 1 \mathrm{H}), 4.26$ $(\mathrm{dd}, J=10.7$ and $4.9 \mathrm{~Hz}, 2 \mathrm{H}), 3.99(\mathrm{td}, J=12.3$ and $2.2 \mathrm{~Hz}, 2 \mathrm{H}), 3.81(\mathrm{~s}, 3 \mathrm{H}), 2.23(\mathrm{~m}, 1 \mathrm{H})$, $1.44(\mathrm{~m}, 1 \mathrm{H}) .{ }^{13} \mathrm{C}$ NMR $\left(125 \mathrm{MHz}, \mathrm{CDCl}_{3}\right): \delta$ 159.9, 131.3, 127.2, 113.6, 101.6, 67.4, 55.3, 25.7. Spectral data matched those reported in the literature. ${ }^{156}$ 


\section{Preparation of 3-[(4-Methoxyphenyl)methoxy]-1-propanol, 164}

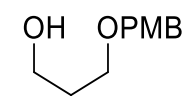

To a solution of acetal $163(2.56 \mathrm{~g}, 13.16 \mathrm{mmol})$ in DCM $(50 \mathrm{~mL})$ at $-78{ }^{\circ} \mathrm{C}$ was added a solution of DIBAL-H (4.8 mL, $4.8 \mathrm{mmol}, 1 \mathrm{M}$ solution in hexane). The reaction mixture was allowed to slowly warm to room temperature over 2 hours, then the reaction was quenched by slow addition of saturated aqueous potassium sodium tartrate (Rochelle's salt, $50 \mathrm{~mL}$ ) and the mixture stirred for 2 hours. The aqueous fraction was then extracted with EtOAc $(3 \times 30 \mathrm{~mL})$. The combined organic fractions were dried $\left(\mathrm{MgSO}_{4}\right)$, filtered, and the solvent removed under reduced pressure providing title compound (2.58 g, quant.) as a colourless oil. ${ }^{1} \mathrm{H}$ NMR indicated that the product was sufficiently pure for the subsequent steps so no further purification was undertaken. $R_{\mathrm{f}}$ : 0.36 (1:1 pet. ether:EtOAc). ${ }^{1} \mathrm{H}$ NMR (500 MHz, $\left.\mathrm{CDCl}_{3}\right) \delta 7.26(\mathrm{~d}, J=8.8 \mathrm{~Hz}, 2 \mathrm{H}), 6.89(\mathrm{~d}, J=8.5, \mathrm{~Hz}$, 2H), $4.46(\mathrm{~s}, 2 \mathrm{H}), 3.81(\mathrm{~s}, 3 \mathrm{H}), 3.78(\mathrm{q}, J=5.5 \mathrm{~Hz}, 2 \mathrm{H}), 3.65(\mathrm{t}, J=5.7 \mathrm{~Hz}, 2 \mathrm{H}), 1.86$ (quin., $J=$ $5.7 \mathrm{~Hz}, 2 \mathrm{H}) .{ }^{13} \mathrm{C}$ NMR (125 MHz, $\left.\mathrm{CDCl}_{3}\right) \delta$ 159.2, 130.1, 129.3, 113.8, 72.9, 69.2, 62.1, 55.2, 32.0. These characterisation data match those in the literature. ${ }^{156}$

\section{Preparation of 3-[(4-Methoxyphenyl)methoxy]propanal, 165}

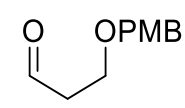

To a solution of DMSO $\left(1.16 \mathrm{~mL}, 16.4 \mathrm{mmol}, 4\right.$ equiv) in DCM $(50 \mathrm{~mL})$ at $-78{ }^{\circ} \mathrm{C}$ (dry ice/acetone bath) was added oxalyl chloride (714 mL, $8.2 \mathrm{mmol}, 2$ equiv) and the reaction stirred for 15 minutes. A solution of alcohol 164 (803 mg, $4.1 \mathrm{mmol}, 1$ equiv) in DCM (5 mL) was then added, stirred for 15 minutes then added NEt 3 (2.28 mL, 16.4 mmol, 4 equiv) and then stirred for 30 minutes at room temperature. The reaction was then quenched with saturated aqueous ammonium chloride $(40 \mathrm{~mL})$, and extracted with DCM $(3 \times 40 \mathrm{~mL})$. The combined organic fractions were washed with water and brine, then dried $\left(\mathrm{MgSO}_{4}\right)$, filtered and the solvent removed under reduced pressure. The sample was then passed through a silica plug to provide colourless oil and used in next reaction without further purification. Rf: 0.43 (1:1 pet. ether:EtOAc). ${ }^{1} \mathbf{H}$ NMR $\left(500 \mathrm{MHz}, \mathrm{CDCl}_{3}\right) \delta$ $9.81(\mathrm{~s}, 1 \mathrm{H}), 7.29(\mathrm{~d}, J=8.5,2 \mathrm{H}), 6.91(\mathrm{~d}, J=8.5,2 \mathrm{H}), 4.49(\mathrm{~s}, 2 \mathrm{H}), 3.83(\mathrm{~s}, 3 \mathrm{H}), 3.81(\mathrm{t}, J=6.1$, 
2H), $2.71(\mathrm{td}, J=6.1$ and $1.7 \mathrm{~Hz}, 2 \mathrm{H}) .{ }^{13} \mathrm{C} \mathrm{NMR}\left(125 \mathrm{MHz}, \mathrm{CDCl}_{3}\right) \delta 201.3,159.3,129.9$, $129.3,113.8,72.9,63.5,55.3,43.8$. These characterisation data match those in the literature. ${ }^{157}$

\section{Preparation of 1-\{2-[(4-methoxyphenyl)methoxy]ethyl\}-3-butenyl acrylate, 166}

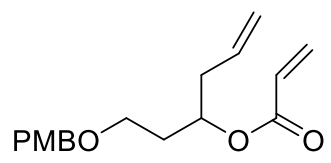

To a solution of aldehyde 165 (803 mg, $4.09 \mathrm{mmol}, 1$ equiv) in THF (25 mL) at room temperature was added allylmagnesium bromide $(1 \mathrm{M}$ solution in diethyl ether, $4.91 \mathrm{~mL}, 4.91 \mathrm{mmol}, 1.2$ equiv) and the mixture stirred overnight. Without any purification, to the reaction mixture was then added DIPEA (697 $\mu \mathrm{L}, 517 \mathrm{mg}, 4.09 \mathrm{mmol})$ and cooled to $0{ }^{\circ} \mathrm{C}$ (ice bath). Acryloyl chloride (498 $\left.\mu \mathrm{L}, 555 \mathrm{mg}, 6.14 \mathrm{mmol}\right)$ and DMAP (40 $\mathrm{mg}, 0.33 \mathrm{mmol}$ ) were added and the reaction mixture stirred overnight at room temperature. The reaction mixture was then quenched with saturated aqueous ammonium chloride $(30 \mathrm{~mL})$, and extracted with EtOAc $(3 \times 30 \mathrm{~mL})$. The combined organic fractions were washed with water and brine, then dried $\left(\mathrm{MgSO}_{4}\right)$, filtered and the solvent removed under reduced pressure. The crude material was purified by silica gel flash chromatography (5:1 pet. ether:EtOAc) to provide the title compound $(1.05 \mathrm{~g}, 88 \%$ over 3 steps) as a colourless oil. Rf: 0.64 (2:1 pet. ether:EtOAc). ${ }^{1} \mathbf{H}$ NMR (500 MHz, CDCl 3 ) $\delta 7.25(\mathrm{~d}, J=8.6,2 \mathrm{H}), 6.87(\mathrm{~d}, J=8.6,2 \mathrm{H}), 6.38(\mathrm{~d}, J=17.1,1 \mathrm{H}), 5.81(\mathrm{~m}, 1 \mathrm{H}), 5.74(\mathrm{~m}, 1 \mathrm{H})$, $5.17(\mathrm{~m}, 1 \mathrm{H}), 5.07(\mathrm{~m}, 2 \mathrm{H}), 4.41(\mathrm{~s}, 2 \mathrm{H}), 3.81(\mathrm{~s}, 3 \mathrm{H}), 3.48(\mathrm{~m}, 2 \mathrm{H}), 2.38(\mathrm{~m}, 2 \mathrm{H}), 1.91(\mathrm{~m}$, 2H). ${ }^{13} \mathrm{C}$ NMR $\left(125 \mathrm{MHz}, \mathrm{CDCl}_{3}\right) \delta 165.7,159.1,133.4,130.5,130.4,129.3,128.7,118.0$, $113.7,72.7,71.0,66.2,55.3,38.8,33.8 \mathrm{~cm}^{-1}$. These characterisation data match those in the literature. ${ }^{158}$ 


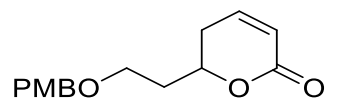

To a solution of diene 166 (504 mg, $1.74 \mathrm{mmol}, 1$ equiv) in DCM (130 mL) at room temperature was added (in two portions over 4 hours) Grubbs second generation catalyst (171 $\mathrm{mg}, 0.20 \mathrm{mmol}, 12 \mathrm{~mol} \%$ ). The reaction mixture was stirred overnight and then solvent was removed under reduced pressure. The crude material was purified by silica gel flash chromatography (1:1 pet. ether:EtOAc) to provide the title compound (179 mg, $40 \%)$ as a pale brown oil. $\mathbf{R}_{\mathbf{f}} 0.26$ (1:1 pet. ether:EtOAc). ${ }^{1} \mathbf{H}$ NMR $\left(500 \mathrm{MHz}, \mathrm{CDCl}_{3}\right) \delta 7.27$ $(\mathrm{d}, J=8.3 \mathrm{~Hz}, 2 \mathrm{H}), 6.90-6.85(\mathrm{~m}, 3 \mathrm{H}), 6.03(\mathrm{~d}, J=9.8 \mathrm{~Hz}, 1 \mathrm{H}), 4.64(\mathrm{td}, J=7.9$ and $3.3 \mathrm{~Hz}$, 1H), $4.44(\mathrm{q}, J=11.4 \mathrm{~Hz}, 2 \mathrm{H}), 3.81(\mathrm{~s}, 3 \mathrm{H}), 3.65(\mathrm{~m}, 2 \mathrm{H}), 2.37(\mathrm{~m}, 2 \mathrm{H}), 2.07(\mathrm{~m}, 1 \mathrm{H}), 1.95(\mathrm{~m}$, 1H). ${ }^{13} \mathrm{C}$ NMR $\left(125 \mathrm{MHz}, \mathrm{CDCl}_{3}\right) \delta$ 164.4, 159.2, 145.1, 130.2, 129.3, 121.4, 113.8, 75.3, 72.9, 65.3, 55.3, 35.1, 29.5. IR (film): 2932, 2863, 1716, 1612, 1512, 1244, 1174, 1087, 1031, $815 \mathrm{~cm}^{-1}$. HRMS-ESI (m/z): [M + $\left.\mathrm{NH}_{4}\right]^{+}$calculated for $\mathrm{C}_{15} \mathrm{H}_{22} \mathrm{NO}_{4}$, 280.1549; found 280.1549 .

\section{Preparation of 6-[(4-Methoxyphenyl)methoxy]-2,4-dienoic acid, 168}

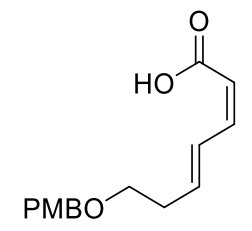

To a solution of lactone 167 (154 mg, $0.59 \mathrm{mmol}, 1$ equiv) in THF (12 mL) at room temperature was added dropwise TBAF solution $(1 \mathrm{M}$ solution in THF, $1.76 \mathrm{~mL}, 1.76$ mmol, 3 equiv) and the reaction stirred for 3 hours. The reaction mixture was diluted with EtOAc $(50 \mathrm{~mL})$, and washed three times with dilute aqueous sulfuric acid (2 vol\%, $50 \mathrm{~mL})$ to remove tetrabutylammonium salts. Organic fraction was then dried $\left(\mathrm{MgSO}_{4}\right)$, filtered and concentrated under reduced pressure. The crude material was purified by silica gel flash chromatography (2:1 pet. ether:EtOAc) to provide the title compound (134 $\mathrm{mg}, 87 \%$ ) as a colourless oil. $\mathbf{R}_{\mathbf{f}} 0.33$ (2:1 pet. ether:EtOAc). ${ }^{1} \mathbf{H}$ NMR (500 MHz, $\left.\mathrm{CDCl}_{3}\right): \delta 7.42$ (dd, J = 12.0, 14.6 Hz, $1 \mathrm{H}), 7.29$ (d, J = 8.2 Hz, $2 \mathrm{H}), 6.90$ (d, J = 8.2 Hz, $2 \mathrm{H}), 6.67$ (t, J=11.4 Hz, 1 $\mathrm{H}), 6.17(\mathrm{td}, J=7.0,14.9 \mathrm{~Hz}, 1 \mathrm{H}), 5.63(\mathrm{~d}, J=11.4 \mathrm{~Hz}, 1 \mathrm{H}), 4.47(\mathrm{~s}, 2 \mathrm{H}), 3.82(\mathrm{~s}, 3 \mathrm{H}), 3.57$ $(\mathrm{t}, J=6.5 \mathrm{~Hz}, 2 \mathrm{H}), 2.54(\mathrm{q}, J=6.5 \mathrm{~Hz}, 2 \mathrm{H}) .{ }^{13} \mathrm{C} \mathrm{NMR}\left(125 \mathrm{MHz}, \mathrm{CDCl}_{3}\right): \delta 171.2,159.2$, 
147.3, 142.9, 130.2, 129.3, 128.4, 115.1, 113.8, 72.6, 68.7, 55.3, 33.4. IR (film): 2935, 2858, 1684, 1600, 1512, 1246, 1173, 1096, 1034, $821 \mathrm{~cm}^{-1}$. HRMS-ESI (m/z): [M]- calculated for $\mathrm{C}_{15} \mathrm{H}_{18} \mathrm{O}_{4}{ }^{-}$, 262.1205; found 262.1202.

\section{Preparation of 5-[(4-Methoxyphenyl)methoxy]-1-penten-3-ol, 170}<smiles>C=CC(O)CCOCCCC</smiles>

To a solution of aldehyde $165\left(804 \mathrm{~g}, 4.1 \mathrm{mmol}, 1\right.$ equiv) in THF $(30 \mathrm{~mL})$ at $0{ }^{\circ} \mathrm{C}$ (ice bath) was added allylmagnesium bromide (1 M solution in diethyl ether, $8.3 \mathrm{~mL}, 8.3$ mmol, 2 equiv). The reaction mixture was stirred overnight at room temperature, then quenched with saturated aqueous ammonium chloride $(30 \mathrm{~mL})$. The aqueous layer was extracted with diethyl ether $(3 \times 30 \mathrm{~mL})$. The combined organic fractions were dried $\left(\mathrm{MgSO}_{4}\right)$, filtered and the solvent removed under reduced pressure. The crude material was purified by silica gel flash chromatography (2:1 pet. ether:EtOAc) to provide the title compound (950 mg, 79\%) as a colourless liquid. $\mathbf{R}_{\mathrm{f}} 0.35$ (2:1 pet. ether:EtOAc). ${ }^{1} \mathbf{H}$ NMR (500 MHz, $\left.\mathrm{CDCl}_{3}\right): \delta 7.26(\mathrm{~d}, J=8.5 \mathrm{~Hz}, 2 \mathrm{H}), 6.89$ (d, J = 8.5 Hz, $\left.2 \mathrm{H}\right), 5.87$ (ddd, J = 6.0, 10.4, $17.1 \mathrm{~Hz}, 1 \mathrm{H}), 5.27(\mathrm{~d}, J=17.3 \mathrm{~Hz}, 1 \mathrm{H}), 5.11(\mathrm{~d}, J=10.5 \mathrm{~Hz}, 1 \mathrm{H}), 4.46$ (s, $2 \mathrm{H}), 4.38$ - 4.29 $(\mathrm{m}, 1 \mathrm{H}), 3.81(\mathrm{~s}, 3 \mathrm{H}), 3.73-3.66(\mathrm{~m}, 1 \mathrm{H}), 3.66-3.59(\mathrm{~m}, 1 \mathrm{H}), 1.94-1.76(\mathrm{~m}, 2 \mathrm{H}) .{ }^{13} \mathrm{C}$ NMR (125 MHz, $\left.\mathrm{CDCl}_{3}\right)$ : $\delta$ 159.2, 140.5, 130.0, 129.3, 114.3, 113.8, 73.0, 72.0, 68.1, 55.3, 36.2. These characterisation data match those in the literature. ${ }^{159}$ 


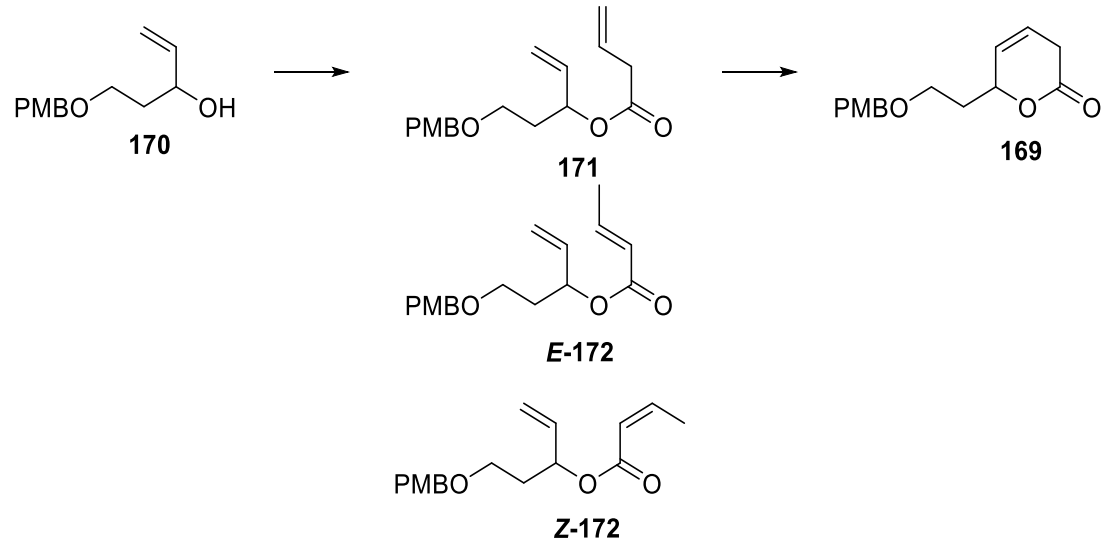

To a solution of alcohol 170 (900 mg, $4.1 \mathrm{mmol}, 1$ equiv) and vinyl acetic acid (418 $\mathrm{mg}, 4.9 \mathrm{mmol}, 1.2$ equiv) in DCM $(25 \mathrm{~mL})$ at $0{ }^{\circ} \mathrm{C}$ (ice bath) was added EDCI (932 mg, 4.9 mmol, 1.2 equiv) followed by DMAP (148 mg, $1.2 \mathrm{mmol}, 0.3$ equiv). The reaction mixture was then stirred overnight at room temperature and quenched with saturated aqueous ammonium chloride $(20 \mathrm{~mL})$. The aqueous layer was extracted with DCM $(3 \times 20 \mathrm{~mL})$. The combined organic fractions were dried $\left(\mathrm{MgSO}_{4}\right)$, filtered and the solvent removed under reduced pressure. The crude material was purified by silica gel flash chromatography (2:1 pet. ether:EtOAc) to provide a mixture of diene products $\mathbf{1 7 1}, \mathbf{E}-\mathbf{1 7 2}$ and Z-172. The diene mixture was then dissolved in DCM (150 mL) and Grubbs second generation catalyst $(34 \mathrm{mg}, 0.04 \mathrm{mmol}$ ) added. The reaction mixture was stirred overnight at room temperature, and then solvent was removed under reduced pressure. The crude material was purified by silica gel flash chromatography (1:1 pet. ether:EtOAc) to provide the title compound (169) (171 mg, 16\% over 2 steps) as a pale brown oil. Rf: 0.38 (1:1 pet. ether:EtOAc). ${ }^{1} \mathbf{H}$ NMR (500 MHz, $\left.\mathrm{CDCl}_{3}\right): \delta 7.26(\mathrm{~d}, J=8.7 \mathrm{~Hz}, 2 \mathrm{H}), 6.89$ (d, J = 8.4 Hz, 2 H), 5.90 - $5.81(\mathrm{~m}, 2 \mathrm{H}), 5.21-5.10(\mathrm{~m}, 1 \mathrm{H}), 4.45(\mathrm{dd}, J=11.4,24.4 \mathrm{~Hz}, 2 \mathrm{H}), 3.81(\mathrm{~s}, 3 \mathrm{H})$, $3.69(\mathrm{dt}, J=5.6,8.6 \mathrm{~Hz}, 1 \mathrm{H}), 3.64-3.55(\mathrm{~m}, 1 \mathrm{H}), 3.06($ br s, $2 \mathrm{H}), 2.09-1.85(\mathrm{~m}, 3 \mathrm{H}) .{ }^{13} \mathrm{C}$ NMR (125 MHz, $\left.\mathrm{CDCl}_{3}\right)$ : $\delta$ 169.0, 159.2, 130.2, 129.4, 126.7, 121.5, 113.87, 113.82, 72.9, 65.3, 55.3, 36.0, 29.9. IR (film): 2866, 2361, 1733, 1612, 1512, 1245, 1085, 1031, $816 \mathrm{~cm}^{-1}$. 
Preparation of Ethyl 7-[(4-methoxyphenyl)methoxy]-5-hydroxy-3-oxoheptanoate, 180

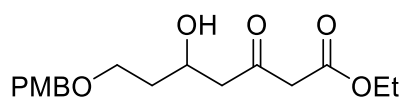

To a solution of $\mathrm{NaH}$ (as a $60 \%$ oil suspension, $72 \mathrm{mg}, 1.78 \mathrm{mmol}, 2.4$ equiv) in THF (15 mL) at $0{ }^{\circ} \mathrm{C}$ (ice bath) was added ethyl acetoacetate (188 $\mu \mathrm{L}, 1.48 \mathrm{mmol}, 2$ equiv) dropwise, then stirred for 10 minutes. The reaction mixture was then added $n$-BuLi $(2 \mathrm{M}$ solution in hexane, $815 \mu \mathrm{L}, 1.63 \mathrm{mmol}, 2.2$ equiv) dropwise, and then stirred for 10 minutes. The reaction mixture was then added a solution of aldehyde 165 (144 mg, 0.74 mmol, 1 equiv, dissolved in $5 \mathrm{~mL}$ of THF) dropwise, and stirred for a further 30 minutes. The reaction was then quenched with saturated aqueous ammonium chloride $(15 \mathrm{~mL})$. The aqueous layer was then extracted with diethyl ether $(3 \times 15 \mathrm{~mL})$. The combined organic fractions were dried $\left(\mathrm{MgSO}_{4}\right)$, filtered and the solvent removed under reduced pressure. The crude material was purified by silica gel flash chromatography (2:1 pet. ether:EtOAc) to provide the title compound ( $240 \mathrm{mg}, 66 \%)$ as a pale brown oil. $\mathbf{R}_{\mathrm{f}}$ : 0.41 (2:1 pet. ether:EtOAc). ${ }^{1} \mathrm{H}$ NMR (500 MHz, $\left.\mathrm{CDCl}_{3}\right): \delta 7.25(\mathrm{~d}, J=8.6 \mathrm{~Hz}, 3 \mathrm{H}), 6.88(\mathrm{~d}, J=8.4$ $\mathrm{Hz}, 2 \mathrm{H}), 4.50-4.41(\mathrm{~m}, 2 \mathrm{H}), 4.28(\mathrm{dt}, J=3.8,7.6 \mathrm{~Hz}, 1 \mathrm{H}), 4.20(\mathrm{q}, J=7.3 \mathrm{~Hz}, 2 \mathrm{H}), 3.81(\mathrm{~s}, 3$ H), 3.71 - $3.59(\mathrm{~m}, 2 \mathrm{H}), 3.48(\mathrm{~s}, 2 \mathrm{H}), 2.75-2.68(\mathrm{~m}, 2 \mathrm{H}), 1.84-1.71(\mathrm{~m}, 2 \mathrm{H}), 1.28(\mathrm{t}, J=7.3$ $\mathrm{Hz}, 3 \mathrm{H})$.

Preparation of Methyl 7-[(4-methoxyphenyl)methoxy]-5-hydroxy-3-oxoheptanoate, 187

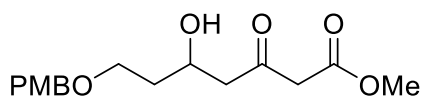

To a solution of $\mathrm{NaH}$ (as a $60 \%$ oil suspension, $162 \mathrm{mg}, 4.1 \mathrm{mmol}, 1.5$ equiv) in THF (30 mL) at $0{ }^{\circ} \mathrm{C}$ (ice bath) was added methyl acetoacetate (584 $\mu \mathrm{L}, 5.4 \mathrm{mmol}, 2$ equiv) dropwise, and stirred for 10 minutes. The reaction mixture was then added $n$-BuLi (of a 2 M solution in hexane, $3.1 \mathrm{~mL}, 6.2 \mathrm{mmol}, 2.3$ equiv) dropwise, and stirred for 10 minutes. The reaction mixture was then added a solution of aldehyde 165 (525 mg, $2.7 \mathrm{mmol}, 1$ equiv, dissolved in $10 \mathrm{~mL}$ of THF) dropwise, and stirred for a further 30 minutes. The reaction was then quenched with saturated aqueous ammonium chloride $(30 \mathrm{~mL})$. The aqueous layer was extracted with diethyl ether $(3 \times 30 \mathrm{~mL})$. The combined organic fractions were dried $\left(\mathrm{MgSO}_{4}\right)$, filtered and the solvent removed under reduced pressure. 


\section{Chapter Six: Experimental}

The crude material was purified by silica gel flash chromatography (2:1 pet. ether:EtOAc) to provide the title compound $(149 \mathrm{mg}, 56 \%)$ as a pale brown oil. Rf: 0.73 (1:1 pet. ether:EtOAc). ${ }^{1} \mathbf{H}$ NMR (500 MHz, $\left.\mathrm{CDCl}_{3}\right): \delta 7.25(\mathrm{~d}, J=8.5 \mathrm{~Hz}, 2 \mathrm{H}), 6.88(\mathrm{~d}, J=8.5 \mathrm{~Hz}, 2$ H), $4.48-4.41(\mathrm{~m}, 2 \mathrm{H}), 4.28(\mathrm{dt}, J=3.9,7.6 \mathrm{~Hz}, 1 \mathrm{H}), 3.81(\mathrm{~s}, 3 \mathrm{H}), 3.74(\mathrm{~s}, 3 \mathrm{H}), 3.70-3.58$ (m, 2 H), 3.50 (s, 2 H), 2.77 - 2.65 (m, 2 H), 1.85 - 1.69 (m, 2 H). ${ }^{13} \mathrm{C}$ NMR (125 MHz, $\left.\mathrm{CDCl}_{3}\right)$ : ठ 202.8, 167.4, 159.3, 129.9, 129.4, 113.8, 73.0, 67.8, 67.0, 55.3, 52.4, 49.8, 49.7, 35.9 .

\section{Acid-Yne Fragment}

Preparation of Methyl-6-hydroxyhexanoate, 202<smiles>COC(=O)CCCCCO</smiles>

To a solution of $\varepsilon$-caprolactone $(0.96 \mathrm{mg}$, $8.4 \mathrm{mmol})$ in methanol $(20 \mathrm{~mL})$ was added a catalytic amount of $\mathrm{H}_{2} \mathrm{SO}_{4}(0.5 \mathrm{~mL})$ and refluxed $\left(65^{\circ} \mathrm{C}\right)$ overnight. The reaction mixture was then allowed to cool to room temperature, quenched with saturated $\mathrm{NaHCO}_{3}$ $(3 \mathrm{~mL})$ and methanol removed under reduced pressure. The mixture was then redissolved in water and extracted with diethyl ether $(3 \times 30 \mathrm{~mL})$. The combined organic fractions were then dried $\left(\mathrm{MgSO}_{4}\right)$, filtered and the solvent removed under reduced pressure providing title compound $(1.06 \mathrm{~g}, 85 \%)$ as a colourless oil. ${ }^{1} \mathrm{H}$ NMR indicated that the product was sufficiently pure for the subsequent steps so no further purification was necessary. Rf: 0.12 (2:1 pet. ether:EtOAc). ${ }^{1} \mathbf{H}$ NMR (500 MHz, $\left.\mathrm{CDCl}_{3}\right): \delta 3.66$ (s, 3H), 3.64 $(\mathrm{t}, J=6.5 \mathrm{~Hz}, 2 \mathrm{H}), 2.33(\mathrm{t}, 7.5 \mathrm{~Hz}, 2 \mathrm{H}), 1.81(\mathrm{~s}, \mathrm{br}, 1 \mathrm{H},-\mathrm{OH}), 1.66$ (quin, $J=7.6 \mathrm{~Hz}, 2 \mathrm{H}$ ), 1.58 $(\mathrm{m}, 2 \mathrm{H}), 1.40(\mathrm{~m}, 2 \mathrm{H}) .{ }^{13} \mathrm{C}$ NMR $\left(125 \mathrm{MHz}, \mathrm{CDCl}_{3}\right): \delta 174.2,62.6,51.5,33.9,32.2,25.2$ and 24.6. These characterisation data match those in the literature. ${ }^{160}$

\section{Preparation of Methyl 5-formylvalerate, 203}<smiles>COC(=O)CCCCC=O</smiles>

To a solution of alcohol 202 (1.06 g, $7.2 \mathrm{mmol}, 1$ equiv) in DCM (20 mL) at room temperature was added TEMPO (113 mg, $0.72 \mathrm{mmol}, 0.1$ equiv) followed by BAIB ( $2.54 \mathrm{~g}$, 
$7.9 \mathrm{mmol}, 1.1$ equiv). The reaction mixture was stirred for 2 hours then quenched with aqueous saturated sodium thiosulfate $(5 \mathrm{~mL})$. The aqueous layer was basified with aqueous $\mathrm{NaOH}(1 \mathrm{M}$, to approximately $\mathrm{pH} 8)$, then extracted with DCM $(2 \times 20 \mathrm{~mL})$. The combined organic fractions were then dried $\left(\mathrm{MgSO}_{4}\right)$, filtered and the solvent removed under reduced pressure. The crude material was purified by silica gel flash chromatography (2:1 pet. ether:EtOAc) to provide the title compound ( $892 \mathrm{mg}, 85 \%$ ) as a colourless liquid. $\mathbf{R}_{\mathrm{f}} 0.38$ (2:1 pet. ether:EtOAc). ${ }^{1} \mathbf{H}$ NMR $\left(500 \mathrm{MHz}, \mathrm{CDCl}_{3}\right): \delta 9.77$ (s, 1 $\mathrm{H}), 3.67(\mathrm{~s}, 3 \mathrm{H}), 2.47(\mathrm{t}, J=5.5 \mathrm{~Hz}, 2 \mathrm{H}), 2.34(\mathrm{t}, J=6.5 \mathrm{~Hz}, 2 \mathrm{H}), 1.73-1.61(\mathrm{~m}, 4 \mathrm{H}) .{ }^{13} \mathrm{C}$ NMR (125 MHz, $\left.\mathrm{CDCl}_{3}\right): \delta 202.1,173.7,51.6,43.5,33.7,24.3,21.5$. These characterisation data match those in the literature. ${ }^{160}$

\section{Preparation of Methyl 7,7-dibromo-6-heptenoate, 204}

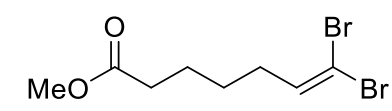

To a solution of aldehyde 203 (3.56 g, $24.4 \mathrm{mmol}, 1$ equiv) in DCM (110 mL) was added $\mathrm{CBr}_{4}(32.3 \mathrm{~g}$, $97.5 \mathrm{mmol}, 4$ equiv) and zinc dust (6.38 g, $97.5 \mathrm{mmol}, 4$ equiv) followed by triphenylphosphine (25.6 g, $97.5 \mathrm{mmol}, 4$ equiv) added in portions so as to not exceed $25^{\circ} \mathrm{C}$. The reaction mixture was stirred at room temperature for 1.5 hours, pet. ether was added and mixture was passed through a plug of silica (washing silica plug with diethyl ether). Solvent was removed under reduced pressure and the crude material was purified with silica gel flash chromatography (5:1 pet. ether:EtOAc) to provide the title compound $(4.15 \mathrm{~g}, 57 \%)$ as a colourless liquid. $\mathbf{R}_{\mathbf{f}}: 0.72$ (2:1 pet. ether:EtOAc). ${ }^{1} \mathbf{H}$ NMR (500 MHz, CDCl $): \delta 6.39(\mathrm{t}, J=7.3 \mathrm{~Hz}, 1 \mathrm{H}), 3.68(\mathrm{~s}, 3 \mathrm{H}), 2.34(\mathrm{t}, J=7.4 \mathrm{~Hz}, 2 \mathrm{H})$, $2.13\left(\mathrm{q}, J=7.4 \mathrm{~Hz}, 2 \mathrm{H}\right.$ ), 1.67 (quin, $J=7.6 \mathrm{~Hz}, 2 \mathrm{H}$ ), 1.47 (quin, $J=7.6 \mathrm{~Hz}, 2 \mathrm{H}$ ). ${ }^{13} \mathrm{C}$ NMR (125 MHz, $\left.\mathrm{CDCl}_{3}\right): \delta 173.8,138.1,89.1,51.5,33.7,32.6,27.2,24.2$. IR (CDCl3 film): 2949, 2862, 1736, 1436, 1363, 1174, $783 \mathrm{~cm}^{-1}$. HRMS (ESI): calculated for $\mathrm{C}_{8} \mathrm{H}_{12} \mathrm{O}_{2} 79 \mathrm{Br}_{2}[\mathrm{M}+\mathrm{Na}]^{+}$ 320.9102 , found 320.9113 . 


\section{Preparation of 7,7-Dibromo-6-heptenoic acid, 205}<smiles>O=C(O)CCCCC=C(Br)Br</smiles>

To a solution of methyl ester 204 (200 mg, $0.67 \mathrm{mmol}, 1$ equiv) in $\mathrm{MeOH}(15 \mathrm{~mL})$ at room temperature was added $\mathrm{LiOH}_{2} \mathrm{H}_{2} \mathrm{O}(141 \mathrm{mg}$, $3.35 \mathrm{mmol}, 5$ equiv $)$ and the mixture stirred overnight. Solvent was then removed under reduced pressure, redissolved in 0.5 $\mathrm{M}$ aqueous $\mathrm{NaOH}(20 \mathrm{~mL})$ and washed with diethyl ether $(20 \mathrm{~mL})$. The aqueous fraction was then acidified with $10 \% \mathrm{H}_{2} \mathrm{SO}_{4}$ to $\mathrm{pH} 2$ and extracted with EtOAc $(3 \times 20 \mathrm{~mL})$. The combined organic fractions were then dried $\left(\mathrm{MgSO}_{4}\right)$, filtered and the solvent removed under reduced pressure to provide the title compound (126 mg,66\%) as a colourless liquid. Rf: 0.51 (2:1 pet. ether:EtOAc). ${ }^{1} \mathbf{H}$ NMR (500 MHz, $\left.\mathrm{CDCl}_{3}\right): \delta 6.39$ (t, J=7.3 Hz, 1H), $2.39(\mathrm{t}, J=7.4 \mathrm{~Hz}, 2 \mathrm{H}), 2.14(\mathrm{q}, J=7.4,2 \mathrm{H}), 1.68(\mathrm{dt}, J=15.4$ and $7.5,2 \mathrm{H}), 1.50$ (quin, $J=7.6$ $\mathrm{Hz}, 2 \mathrm{H}) .{ }^{13} \mathrm{C}$ NMR $\left(125 \mathrm{MHz}, \mathrm{CDCl}_{3}\right): \delta 179.5,138.0,89.2,33.6,32.6,27.1,23.9$.

\section{Preparation of 6-Heptynoic acid, 190 (via Corey-Fuchs)}<smiles>C#CCCCCC(=O)O</smiles>

To a solution of gem-dibromo alkene 205 (723 mg, $2.5 \mathrm{mmol}, 1$ equiv) in THF (30 $\mathrm{mL})$ at $-78{ }^{\circ} \mathrm{C}$ (dry ice/acetone bath) was added $n$-BuLi (2 M solution in hexane, $4.17 \mathrm{~mL}$, $8.3 \mathrm{mmol}, 3.3$ equiv) and the reaction stirred for 1 hour, then warmed to room temperature and the reaction stirred for another 1 hour. The reaction mixture was quenched with $0.5 \mathrm{M}$ aqueous $\mathrm{NaOH}(20 \mathrm{~mL})$ and washed with diethyl ether $(20 \mathrm{~mL})$. The aqueous fraction was then acidified with $10 \% \mathrm{H}_{2} \mathrm{SO}_{4}$ to $\mathrm{pH} 2$ and extracted with EtOAc (3 x $20 \mathrm{~mL})$. The combined organic fractions were then dried $\left(\mathrm{MgSO}_{4}\right)$, filtered and the solvent removed under reduced pressure to provide the title compound (297 $\mathrm{mg}, 93 \%$ ) as a colourless liquid. Rf: 0.53 (1:1 pet. ether:EtOAc). ${ }^{1} \mathbf{H}$ NMR $\left(500 \mathrm{MHz}, \mathrm{CDCl}_{3}\right): \delta 2.40$ (t, J = $7.4 \mathrm{~Hz}, 2 \mathrm{H}$ ), $2.23(\mathrm{dt}, J=2.6,7.0 \mathrm{~Hz}, 2 \mathrm{H}$ ), $1.97(\mathrm{t}, J=2.6 \mathrm{~Hz}, 1 \mathrm{H}$ ), 1.77 (quin, $J=7.6 \mathrm{~Hz}, 2$ H), 1.60 (td, $J=7.2,15.0 \mathrm{~Hz}, 2 \mathrm{H}) .{ }^{13} \mathrm{C}$ NMR $\left(125 \mathrm{MHz}, \mathrm{CDCl}_{3}\right): \delta$ 179.4, 83.8, 68.7, 33.4, 27.7, 23.7, 18.1. These characterisation data match those in the literature. ${ }^{161}$ 


\section{Preparation of $p$-Toluenesulfonylazide}<smiles>Cc1ccc(S(N)(=O)=O)cc1</smiles>

To a solution of tosyl chloride (5.52 g, $29.0 \mathrm{mmol}, 1$ equiv) in ethanol (100 mL) at room temperature was added sodium azide ( $4.70 \mathrm{~g}$, $72.4 \mathrm{mmol}, 2.5$ equiv) and the reaction stirred for 10 minutes. Ethanol was then removed under reduced pressure, redissolved in distilled water $(50 \mathrm{~mL})$, and extracted with DCM $(3 \times 50 \mathrm{~mL})$. The combined organic fractions were dried $\left(\mathrm{MgSO}_{4}\right)$, filtered and the solvent removed under reduced pressure to provide the title compound $(5.34 \mathrm{~g}, 94 \%)$ as a colourless oil. Rf: 0.87 (EtOAc). ${ }^{1} \mathbf{H}$ NMR (500 MHz, CDCl 3 ): $\delta 7.94-7.79(\mathrm{~m}, J=8.3 \mathrm{~Hz}, 2 \mathrm{H}), 7.48-7.35$ (m, J = 8.1 Hz, $2 \mathrm{H}), 2.49$ (s, 3 H). ${ }^{13} \mathrm{C}$ NMR $\left(125 \mathrm{MHz}, \mathrm{CDCl}_{3}\right): \delta$ 146.2, 135.4, 130.2, 127.5, 21.7. IR (film): 2122 (-N3 stretch), 1367, 1162, 1085, 813, 743, $657 \mathrm{~cm}^{-1}$. These characterisation data match those in the literature. ${ }^{162,163}$

\section{Preparation of Diethyl (2-oxopropyl)phosphonate, 137}
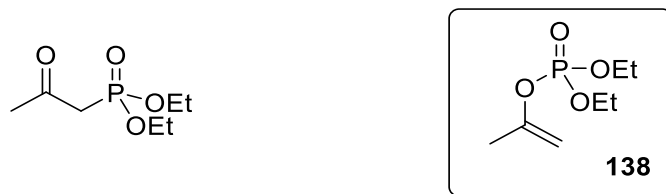

To a solution of chloroacetone (11 mL, $132 \mathrm{mmol}, 1$ equiv) in acetone (50 mL) and acetonitrile $(50 \mathrm{~mL})$ at $0{ }^{\circ} \mathrm{C}$ (ice bath) was added $\mathrm{KI}(24.4 \mathrm{~g}$, $147 \mathrm{mmol}, 1.1$ equiv) and the reaction stirred for 20 minutes. The reaction mixture was then added triethylphosphite (22.6 mL, $132 \mathrm{mmol}, 1$ equiv) at $0{ }^{\circ} \mathrm{C}$ (ice bath) and then stirred overnight at room temperature. The reaction slurry was then added water $(100 \mathrm{~mL})$ and the organic solvents were carefully removed under reduced pressure. $\mathrm{LiCO}_{3}$ was then added to the aqueous mixture until a $\mathrm{pH}$ of 10 was reached, and washed with 19:1 pet. ether:DCM (50 mL) to separate phosphate product 138 (2.34 g, 9\%). The aqueous layer was then extracted with $\operatorname{DCM}(2 \times 50 \mathrm{~mL})$. The combined organic fractions were dried $\left(\mathrm{MgSO}_{4}\right)$, filtered and the solvent removed under reduced pressure to provide the title compound ( $22.4 \mathrm{~g}, 88 \%)$ as a colourless oil. Rf: 0.36 (EtOAc). ${ }^{1} \mathbf{H}$ NMR (500 MHz, CDCl 3 ): $\delta 4.19$ - 4.09 (m, 4 H), 3.08 (d, J $=22.9 \mathrm{~Hz}, 2 \mathrm{H}), 2.32(\mathrm{~s}, 3 \mathrm{H}), 1.33(\mathrm{t}, J=7.1 \mathrm{~Hz}, 6 \mathrm{H}) .{ }^{13} \mathrm{C} \mathbf{N M R}\left(125 \mathrm{MHz}, \mathrm{CDCl}_{3}\right): \delta$ 200.0, 
62.6, 62.5, $43.4(\mathrm{~d}, J=127 \mathrm{~Hz}, \mathrm{C}-\mathrm{P}), 31.3,16.3,16.2$. These characterisation data match those in the literature. ${ }^{76}$

Preparation of Diethyl 1-diazo-2-oxopropylphosphonate, 195<smiles>CCOP(=O)(OCC)C(=N)C(C)=O</smiles>

To a solution of phosphonate 137 (4.0 g, $20.6 \mathrm{mmol}, 1$ equiv) in $\mathrm{MeCN}$ (100 mL) at $0{ }^{\circ} \mathrm{C}$ (ice bath) at room temperature was added $\mathrm{K}_{2} \mathrm{CO}_{3}(3.14 \mathrm{~g}, 22.7 \mathrm{mmol}, 1.1$ equiv) then tosyl azide (4.47 g, $22.7 \mathrm{mmol}, 1.1$ equiv) and the reaction stirred for 3 hours. Solvent was then removed under reduced pressure, redissolved in distilled water (50 mL), and extracted with EtOAc $(3 \times 50 \mathrm{~mL})$. The combined organic fractions were dried $\left(\mathrm{MgSO}_{4}\right)$, filtered and the solvent removed under reduced pressure. The crude material was purified by silica gel flash chromatography (1:1 pet. ether:EtOAc) to provide the title compound (2.86 g, 63\%) as a colourless oil. Rf: 0.51 (EtOAc). ${ }^{1} \mathbf{H}$ NMR (500 MHz, $\left.\mathrm{CDCl}_{3}\right): \delta$ 4.29 - $4.13(\mathrm{~m}, 4 \mathrm{H}), 2.28(\mathrm{~s}, 3 \mathrm{H}), 1.39(\mathrm{t}, J=7.1 \mathrm{~Hz}, 6 \mathrm{H})$. Characterisation data match those in the literature. ${ }^{164}$

Preparation of Methyl 6-heptynoate, 206 (via Ohira-Bestmann reagent)

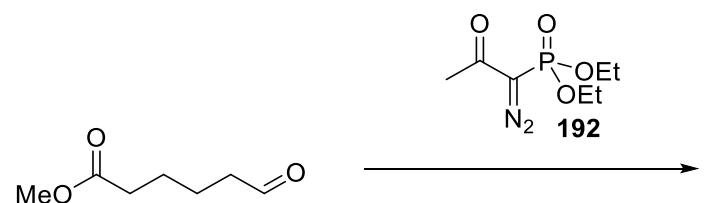

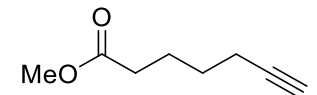

$(51 \%)$

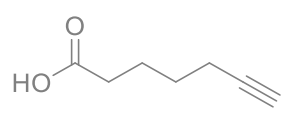

$(40 \%)$

To a solution of aldehyde 203 (1.44 g, $10.0 \mathrm{mmol}, 1$ equiv) in dry methanol (40 mL) at room temperature was added $\mathrm{K}_{2} \mathrm{CO}_{3}(2.76 \mathrm{~g}$, $20.0 \mathrm{mmol}$, 2 equiv) followed by a solution of Ohira-Bestmann reagent 192 (2.86 g, $13.0 \mathrm{mmol}, 1.3$ equiv). The reaction mixture was stirred overnight at room temperature then quenched with saturated aqueous ammonium chloride $(30 \mathrm{~mL})$, some of the methanol was carefully removed under reduced pressure, and then extracted with EtOAc $(3 \times 40 \mathrm{~mL})$. The combined organic fractions were dried $\left(\mathrm{MgSO}_{4}\right)$, filtered and the solvent removed under reduced pressure. The crude material 
was purified by silica gel flash chromatography (5:1 pet. ether:EtOAc) to provide the title compound (716 mg, 51\%) as a colourless liquid. Rf: 0.61 (2:1 pet. ether:EtOAc). ${ }^{1} \mathbf{H}$ NMR (500 MHz, $\left.\mathrm{CDCl}_{3}\right): \delta 3.68(\mathrm{~s}, 3 \mathrm{H}), 2.35(\mathrm{t}, J=7.3 \mathrm{~Hz}, 2 \mathrm{H}), 2.22(\mathrm{dt}, J=2.5,7.0 \mathrm{~Hz}, 2 \mathrm{H}), 1.96$ (t, $J=2.6 \mathrm{~Hz}, 1 \mathrm{H}$ ), 1.76 (quin, $J=7.5 \mathrm{~Hz}, 2 \mathrm{H}), 1.57$ (td, $J=7.3,14.7 \mathrm{~Hz}, 2 \mathrm{H}) .{ }^{13} \mathrm{C}$ NMR (125 $\left.\mathrm{MHz}_{2} \mathrm{CDCl}_{3}\right): \delta 173.8,83.9,68.6,51.5,33.5,27.8,24.0,18.1$. These characterisation data match those in the literature. ${ }^{165}$

The aqueous layer of the above reaction was acidified with $10 \% \mathrm{H}_{2} \mathrm{SO}_{4}$ to $\mathrm{pH} 2$ and extracted with EtOAc $(3 \times 20 \mathrm{~mL})$. The combined organic fractions were dried $\left(\mathrm{MgSO}_{4}\right)$, filtered and the solvent removed under reduced pressure to reveal the saponified product $203(510 \mathrm{mg}, 40 \%)$ as a pale yellow oil. Characterisation data match previous preparation and literature.

\section{Preparation of 6-Heptynoic acid, 190 (via Ohira-Bestmann reagent)}

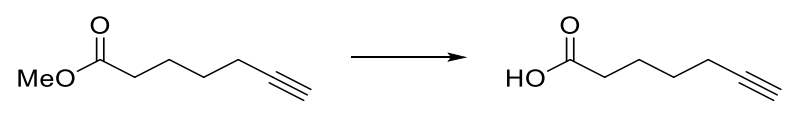

To a solution of methyl ester 206 (716 mg, $5.1 \mathrm{mmol}, 1$ equiv) in $\mathrm{MeOH}$ (35 mL) and water $(25 \mathrm{~mL})$ at room temperature was added $\mathrm{LiOH}_{2} \mathrm{H}_{2} \mathrm{O}(1.07 \mathrm{~g}, 25.5 \mathrm{mmol}, 5$ equiv) and the mixture stirred overnight. Solvent was then removed under reduced pressure, redissolved in $0.5 \mathrm{M}$ aqueous $\mathrm{NaOH}(40 \mathrm{~mL})$ and washed with diethyl ether $(30 \mathrm{~mL})$. The aqueous fraction was then acidified with $10 \% \mathrm{H}_{2} \mathrm{SO}_{4}$ to $\mathrm{pH} 2$ and extracted with EtOAc (3 x $30 \mathrm{~mL})$. The combined organic fractions were then dried $\left(\mathrm{MgSO}_{4}\right)$, filtered and the solvent removed under reduced pressure to provide the title compound ( $515 \mathrm{mg}, 80 \%$ ) as a colourless liquid. Characterisation data match previous preparation and literature.

Combining the products over the two steps gave $203(1.03 \mathrm{~g})$ in 81\% yield from 200 . 


\section{Preparation of 1-Azido-3-chloro-2-propanol, 207}<smiles>[NH3+]CC(O)CCl</smiles>

To a solution of epichlorohydrin (590 mg, $6.4 \mathrm{mmol}, 1$ equiv) in 4:1 acetonitrile:water $(30 \mathrm{~mL})$ at room temperature was added sodium azide $(705 \mathrm{mg}, 10.8$ mmol, 1.7 equiv) followed by $\mathrm{CeCl}_{3} . \mathrm{H}_{2} \mathrm{O}(713 \mathrm{mg}$, $1.2 \mathrm{mmol}, 0.3$ equiv) and the reaction stirred overnight. The reaction mixture was then filtered through celite to remove insoluable solid, and extracted with EtOAc $(3 \times 30 \mathrm{~mL})$. The combined organic fractions were then dried $\left(\mathrm{MgSO}_{4}\right)$, filtered and the solvent removed under reduced pressure to provide the title compound (509 $\mathrm{mg}, 59 \%$ ) as a colourless liquid. $\mathbf{R}_{\mathbf{f}}$ : 0.43 (5:1 pet. ether:EtOAc). ${ }^{1} \mathrm{H}$ NMR (500 MHz, $\mathrm{CDCl}_{3}$ ): $\delta 4.01$ (quin, J = $5.4 \mathrm{~Hz}, 1 \mathrm{H}$ ), 3.67 - 3.57 (m, 2 H), 3.49 (d, J = 5.1 Hz, 2 H), 2.49 (br s, $1 \mathrm{H},-\mathrm{OH}) .{ }^{13} \mathrm{C}$ NMR $\left(125 \mathrm{MHz}, \mathrm{CDCl}_{3}\right): \delta 70.3,53.5$, 46.4. IR (film): 3393, 2926, 2099, 1442, 1279, 1099, 1067, 930, $752 \mathrm{~cm}^{-1}$. HRMS-ESI (m/z): [M $+\mathrm{Na}]^{+}$calculated for $\mathrm{C}_{3} \mathrm{H}_{6} \mathrm{~N}_{3} \mathrm{ONa}^{+}, 158.0092$; found 158.0097. These characterisation data match those in the literature. ${ }^{166}$

\section{Preparation of 3-Chloro-1-(1-phenyl-1H-1,2,3,4-tetrazol-5-ylthio)-2-propanol, 212}

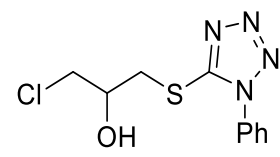

Phenyl tetrazolethiol $(1.53 \mathrm{~g}$, $8.6 \mathrm{mmol})$ was dissolved in epichlorohydrin $(8 \mathrm{~mL})$ and the mixture stirred overnight at room temperature. Solvent (epichlorohydrin, boiling point $=118^{\circ} \mathrm{C}$ at $1 \mathrm{~atm}$ ) was then removed under reduced pressure. To assist in the removal of the final traces of epichlorohydrin, material was redissolved in toluene $(3 \mathrm{~mL})$ and removed under reduced pressure and this was repeated twice. This provided the title compound (2.33 g, quant) as a colourless oil. $\mathbf{R}_{\mathbf{f}} 0.26$ (2:1 pet. ether:EtOAc). ${ }^{\mathbf{1}} \mathbf{H}$ NMR (500 $\mathrm{MHz}^{\mathrm{CDCl}}$ ): $\delta 7.59$ (s, $\left.5 \mathrm{H}\right), 4.39-4.31(\mathrm{~m}, 1 \mathrm{H}), 3.91(\mathrm{~d}, J=5.0 \mathrm{~Hz}, 1 \mathrm{H},-\mathrm{OH}), 3.73$ (t, J = $4.7 \mathrm{~Hz}, 2 \mathrm{H}), 3.69(\mathrm{dd}, J=3.6,14.6 \mathrm{~Hz}, 1 \mathrm{H}), 3.60-3.51(\mathrm{~m}, 2 \mathrm{H}) .{ }^{13} \mathrm{C}$ NMR $(125 \mathrm{MHz}$, $\mathrm{CDCl}_{3}$ ): $\delta$ 154.4, 133.3, 130.4, 129.9, 123.8, 70.4, 47.4, 36.9. IR (film): Approx. 3400 (broad), 
1596, 1499, 1411, 1387, 1243, 1074, 1042, 1015759, 729, $685 \mathrm{~cm}^{-1}$. HRMS-ESI (m/z): [M + H] $]^{+}$ calculated for $\mathrm{C}_{10} \mathrm{H}_{12} \mathrm{ClN}_{4} \mathrm{OS}^{+}, 271.0415$; found 271.0403 .

Preparation of 3-Azido-1-(1-phenyl-1H-1,2,3,4-tetrazol-5-ylthio)-2-propanol, 208<smiles>NCC(O)CSc1nnnn1-c1ccccc1</smiles>

To a solution of alkyl chloride $212(1.80 \mathrm{~g}$, $6.65 \mathrm{mmol}, 1$ equiv) in DMF (40 mL) at room temperature was added sodium azide ( $864 \mathrm{mg}, 13.30 \mathrm{mmol}, 2$ equiv) and TBAI (491 $\mathrm{mg}, 1.33 \mathrm{mmol}, 0.2$ equiv) and the reaction mixture heated $\left(80^{\circ} \mathrm{C}\right)$ overnight. The mixture was carefully concentrated to a few mL's under reduced pressure (potentially explosive) then diluted with water $(30 \mathrm{~mL})$ and extracted with DCM $(3 \times 30 \mathrm{~mL})$. The combined organic fractions were dried $\left(\mathrm{MgSO}_{4}\right)$, filtered, and solvent removed under reduced pressure providing title compound $\left(1.708 \mathrm{~g}\right.$, 93\%) as a slightly yellow oil. ${ }^{1} \mathrm{H}$ NMR indicated that the product was sufficiently pure for the subsequent steps so no further purification was necessary. $\mathbf{R}_{\mathbf{f}} 0.26$ (2:1 pet. ether:EtOAc). ${ }^{1} \mathbf{H}$ NMR (500 MHz, $\left.\mathrm{CDCl}_{3}\right): \delta$ 7.59 (s, 5H), $4.28(\mathrm{~m}, 1 \mathrm{H}), 3.60-3.47(\mathrm{~m}, 4 \mathrm{H}) .{ }^{13} \mathrm{C} \mathrm{NMR}\left(125 \mathrm{MHz}, \mathrm{CDCl}_{3}\right): \delta 154.3,133.3$, 130.4, 129.9, 123.8, 69.6, 55.0, 37.0. IR (film): 3400 (broad), 2099, 1662, 1596, 1499, 1411, 1387, 1279, 1243, 1091, 1075, 981, 731, $686 \mathrm{~cm}^{-1}$. HRMS-ESI (m/z): [M + H $]^{+}$calculated for C10H12N7OS+, 278.0819; found 278.0806.

\section{Sidechain Fragment}

\section{Preparation of 4,7-Dihydro-2-(4-methoxyphenyl)-1,3-dioxepin, 217}

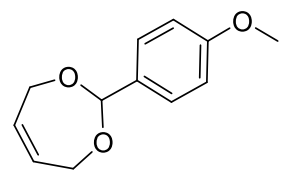

To a solution of cis-2-butene-1,4-diol (1.70 g, $19.3 \mathrm{mmol}, 1$ equiv) in toluene (50 $\mathrm{mL}$ ) was added $p$-anisaldehyde ( $2.63 \mathrm{~g}, 19.3 \mathrm{mmol}, 1$ equiv) followed by $p$-toluene sulfonic acid (100 mg) and heated in a Dean-Stark apparatus overnight. The reaction was 


\section{Chapter Six: Experimental}

quenched with saturated aqueous $\mathrm{NaHCO}_{3}(1 \mathrm{~mL})$ and solvent reduced to about $5 \mathrm{~mL}$ under reduced pressure. To simplify the purification of the product from the unreacted $p$ anisaldehyde, the crude solution was redissolved in $\mathrm{MeOH}(50 \mathrm{~mL})$ and $\mathrm{NaBH}_{4}(730 \mathrm{mg}$, $19.3 \mathrm{mmol}$ ) added at $0{ }^{\circ} \mathrm{C}$ (ice bath) which reduced the $p$-anisaldehyde to the

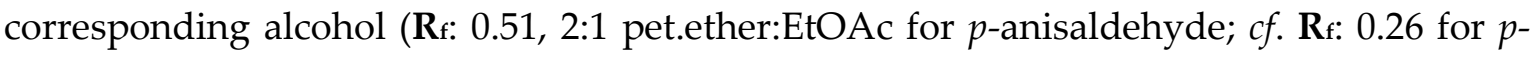
anisalcohol). After removal of solvent under reduced pressure the crude mixture was purified by silica gel flash chromatography (9:1 pet. ether:EtOAc) to provide the title compound (2.43 g, 61\%) as a colourless liquid. Rf: 0.63 (2:1 pet. ether:EtOAc). ${ }^{1} \mathbf{H}$ NMR (500 MHz, $\left.\mathrm{CDCl}_{3}\right): \delta 7.51-7.41(\mathrm{~m}, J=8.7 \mathrm{~Hz}, 2 \mathrm{H}), 6.94-6.87(\mathrm{~m}, J=8.7 \mathrm{~Hz}, 2 \mathrm{H}), 5.84(\mathrm{~s}, 1$ H), $5.78(\mathrm{~s}, 2 \mathrm{H}), 4.39$ (d, J = 15.5 Hz, $2 \mathrm{H}), 4.26$ (d, J = 14.9 Hz, $2 \mathrm{H}), 3.82(\mathrm{~s}, 3 \mathrm{H}) .{ }^{13} \mathrm{C}$ NMR (125 MHz, $\left.\mathrm{CDCl}_{3}\right): \delta 159.7,131.2,130.0,127.7,113.5,102.0,64.4$, 55.3. These characterisation data match those in the literature. ${ }^{167}$

\section{Preparation of cis-4-[(4-Methoxyphenyl)methoxy]-2-buten-1-ol, 216}

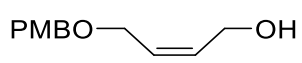

To a solution of acetal $217\left(2.43 \mathrm{~g}, 11.8 \mathrm{mmol}, 1\right.$ equiv) in DCM $(50 \mathrm{~mL})$ at $-78{ }^{\circ} \mathrm{C}$ (dry ice/acetone bath) was added a solution of DIBAL-H (1 M solution in hexane, $24 \mathrm{~mL}$, $24 \mathrm{mmol}, 2$ equiv). The reaction mixture was allowed to slowly warm to room temperature over 2 hours, then the reaction was quenched by slow addition of saturated aqueous potassium sodium tartrate (Rochelle's salt, $50 \mathrm{~mL}$ ) and the mixture stirred for 1 hour. The aqueous fraction was then extracted with EtOAc $(3 \times 30 \mathrm{~mL})$, organic fractions dried $\left(\mathrm{MgSO}_{4}\right)$, filtered, then solvent removed under reduced pressure providing title compound (2.36 g, 96\%) as a colourless oil. $\mathbf{R}_{\mathbf{f}}$ : 0.38 (2:1 pet. ether:EtOAc). ${ }^{1} \mathrm{H}$ NMR indicated that the product was sufficiently pure for the subsequent steps so no further purification was necessary. ${ }^{1} \mathrm{H}$ NMR $\left(500 \mathrm{MHz}, \mathrm{CDCl}_{3}\right): \delta 7.32-7.23(\mathrm{~d}, J=8.3 \mathrm{~Hz}, 2 \mathrm{H})$, $6.94-6.85(\mathrm{~d}, J=8.3 \mathrm{~Hz}, 2 \mathrm{H}), 5.87$ - $5.78(\mathrm{~m}, 1 \mathrm{H}), 5.78$ - $5.68(\mathrm{~m}, 1 \mathrm{H}), 4.46(\mathrm{~s}, 2 \mathrm{H}), 4.16(\mathrm{~d}$, $J=5.9 \mathrm{~Hz}, 2 \mathrm{H}), 4.07$ (d, J = 6.3 Hz, $2 \mathrm{H}), 3.81$ (s, $3 \mathrm{H}) .{ }^{13} \mathrm{C}$ NMR $\left(125 \mathrm{MHz}, \mathrm{CDCl}_{3}\right): \delta$ 159.3, $132.4,129.9,129.5,128.3,113.9,72.2,65.4,58.7,55.3$. These characterisation data match those in the literature. ${ }^{168}$ 


\section{Preparation of (2E)- 4-[(4-Methoxyphenyl)methoxy]-2-butenal, 218}

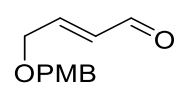

To a solution of alcohol 216 (1.34 g, $6.4 \mathrm{mmol}, 1$ equiv) in DCM $(40 \mathrm{~mL})$ at room temperature was added DMSO $(10 \mathrm{~mL})$ and triethylamine $(4.5 \mathrm{~mL}, 32.2 \mathrm{mmol}, 5$ equiv). The reaction mixture was cooled to $0{ }^{\circ} \mathrm{C}$ (ice bath), $\mathrm{SO}_{3} . \mathrm{Pyr}(3.6 \mathrm{~g}, 22.5 \mathrm{mmol}, 3.5$ equiv) added and the reaction stirred for 1 hour before warming to room temperature and stirring for another 1 hour. The reaction was quenched with saturated aqueous $\mathrm{NH}_{4} \mathrm{Cl}$ (30 $\mathrm{mL})$ and extracted with DCM $(3 \times 40 \mathrm{~mL})$. The combined organic fractions were washed with distilled $\mathrm{H}_{2} \mathrm{O}$ then brine, dried $\left(\mathrm{MgSO}_{4}\right)$, filtered and concentrated under reduced pressure. The crude material was purified by silica gel flash chromatography (5:1 pet. ether:EtOAc) to provide the title compound $\left(1.08 \mathrm{~g}\right.$, $81 \%$ ) as a pale yellow liquid. $\mathbf{R}_{\mathrm{f}} 0.60$ (2:1 pet. ether:EtOAc). ${ }^{1} \mathrm{H}$ NMR $\left(500 \mathrm{MHz}, \mathrm{CDCl}_{3}\right): \delta 9.58(\mathrm{~d}, J=7.9 \mathrm{~Hz}, 1 \mathrm{H}), 7.33-7.21$ $(\mathrm{m}, J=8.7 \mathrm{~Hz}, 2 \mathrm{H}), 6.92-6.88(\mathrm{~m}, J=8.5 \mathrm{~Hz}, 2 \mathrm{H}), 6.85(\mathrm{td}, J=4.0,15.7 \mathrm{~Hz}, 1 \mathrm{H}), 6.39(\mathrm{dd}, J$ = 7.9, $15.7 \mathrm{~Hz}, 1 \mathrm{H}), 4.53(\mathrm{~s}, 2 \mathrm{H}), 4.31-4.22$ (m, $2 \mathrm{H}), 3.82(\mathrm{~s}, 3 \mathrm{H}) .{ }^{13} \mathrm{C}$ NMR $(125 \mathrm{MHz}$, $\left.\mathrm{CDCl}_{3}\right): \delta 193.3,159.4,153.2,131.8,129.4,129.4,113.9,72.7,68.2$, 55.3. These characterisation data match those in the literature. ${ }^{168}$

\section{Preparation of Ethyl (2E,4E)-6-[(4-methoxyphenyl)methoxy]-2,4-hexadienoate, 220}

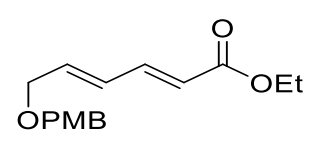

To a solution of aldehyde 218 (1.07 g, $5.2 \mathrm{mmol}, 1$ equiv) in DCM (40 mL) at room temperature was added triphenylphosphorane ylide 219 ( $2.17 \mathrm{~g}, 6.2 \mathrm{mmol}, 1.2$ equiv) and the mixture stirred overnight. Solvent was then removed under reduced pressure and the crude material was purified by silica gel flash chromatography (5:1 pet. ether:EtOAc) to provide the title compound $(1.43 \mathrm{~g}, 82 \%)$ as a colourless liquid. $\mathbf{R}_{\mathrm{f}}$ : 0.77 (1:1 pet. ether:EtOAc). ${ }^{1} \mathbf{H}$ NMR (500 MHz, $\left.\mathrm{CDCl}_{3}\right): \delta 7.28(\mathrm{t}, J=8.1 \mathrm{~Hz}, 2 \mathrm{H}), 7.28(\mathrm{~d}, J=8.3 \mathrm{~Hz}, 2$ H), $6.90(\mathrm{~d}, J=8.4 \mathrm{~Hz}, 2 \mathrm{H}), 6.41(\mathrm{dd}, J=11.2,15.3 \mathrm{~Hz}, 1 \mathrm{H}), 6.18(\mathrm{td}, J=5.3,15.3 \mathrm{~Hz}, 1 \mathrm{H})$, $5.88(\mathrm{~d}, J=15.3 \mathrm{~Hz}, 1 \mathrm{H}), 4.48(\mathrm{~s}, 2 \mathrm{H}), 4.21(\mathrm{q}, J=7.2 \mathrm{~Hz}, 2 \mathrm{H}), 4.11(\mathrm{~d}, J=5.2 \mathrm{~Hz}, 2 \mathrm{H}), 3.82$ (s, $3 \mathrm{H}), 1.30(\mathrm{t}, J=7.1 \mathrm{~Hz}, 3 \mathrm{H}) .{ }^{13} \mathrm{C}$ NMR $\left(125 \mathrm{MHz}, \mathrm{CDCl}_{3}\right): \delta 167.0,159.3,143.7,138.7$, 
$129.9,129.4,129.1,121.4,113.8,72.3,69.3,60.3,55.3,14.3$. These characterisation data match those in the literature. ${ }^{169}$

Preparation of (2E,4E)-6-[(4-Methoxyphenyl)methoxy]-2,4-hexadien-1-ol, 221

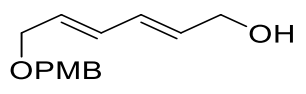

To a solution of ethyl ester $220(1.17 \mathrm{~g}, 4.2 \mathrm{mmol})$ in THF $(40 \mathrm{~mL})$ at $-78{ }^{\circ} \mathrm{C}$ (dry ice/acetone bath) was added a solution of DIBAL-H $(8.5 \mathrm{~mL}, 8.5 \mathrm{mmol}, 2$ equiv, $1 \mathrm{M}$ in hexane). The reaction mixture was allowed to slowly warm to room temperature over 2 hours, before the reaction was quenched by slow addition of saturated potassium sodium tartrate (Rochelle's salt, $30 \mathrm{~mL}$ ) and the mixture stirred for 1 hour. The aqueous fraction was then extracted with EtOAc $(3 \times 30 \mathrm{~mL})$, and the combined organic fractions were dried $\left(\mathrm{MgSO}_{4}\right)$, filtered, then concentrated under reduced pressure providing title compound (956 mg, 99\%) as a colourless oil. ${ }^{1} \mathrm{H}$ NMR indicated that the product was sufficiently pure for the subsequent steps so no further purification was necessary. $\mathbf{R}_{\mathrm{f}} \mathbf{0 . 2 0}$ (2:1 pet. ether:EtOAc). ${ }^{1} \mathrm{H}$ NMR (500 MHz, $\left.\mathrm{CDCl}_{3}\right): \delta 7.28(\mathrm{~d}, J=7.3 \mathrm{~Hz}, 2 \mathrm{H}), 6.89(\mathrm{~d}, J=$ $8.5 \mathrm{~Hz}, 2 \mathrm{H}), 6.31$ - 6.25 (m, $2 \mathrm{H}), 5.83$ (tdd, J = 6.0, 14.4, $19.9 \mathrm{~Hz}, 2 \mathrm{H}), 4.45$ (s, $2 \mathrm{H}), 4.20$ (d, J $=5.4 \mathrm{~Hz}, 2 \mathrm{H}), 4.04(\mathrm{~d}, J=5.9 \mathrm{~Hz}, 2 \mathrm{H}), 3.81$ (s, $3 \mathrm{H}) .{ }^{13} \mathrm{C} \mathrm{NMR}\left(125 \mathrm{MHz}, \mathrm{CDCl}_{3}\right): \delta$ 159.2, 132.3, 131.8, 130.6, 130.2, 130.1, 129.4, 113.8, 71.8, 69.9, 63.2, 55.3. These characterisation data match those in the literature. ${ }^{169}$

\section{Preparation of [(Ethoxycarbonyl)methylene]triphenylphosphorane, 219}<smiles>CCOC(=O)C=Cc1ccccc1</smiles>

A solution of triphenylphosphine (9.61 g, $36.6 \mathrm{mmol}, 1.05$ equiv) and ethyl bromoacetate (5.81 g, $34.8 \mathrm{mmol}, 1$ equiv) in toluene $(50 \mathrm{~mL})$ was stirred overnight at room temperature. The resulting white precipitate was then filtered, washed with diethyl ether and air dried. The white solid phosphonium salt was then dissolved in $2 \mathrm{M} \mathrm{NaOH}$ $(40 \mathrm{~mL})$ and the reaction stirred for 30 minutes. The reaction mixture was then extracted with DCM (3 x $40 \mathrm{~mL})$. The combined organic fractions were dried $\left(\mathrm{MgSO}_{4}\right)$, filtered, then concentrated under reduced pressure to provide the title compound $(10.5 \mathrm{~g}, 87 \%)$ as a 
white solid. ${ }^{1} \mathrm{H}$ NMR (500 MHz, $\mathrm{CDCl}_{3}$ ): $\delta 7.75$ - 7.61 (m, $\left.6 \mathrm{H}\right), 7.55$ (br s, $\left.4 \mathrm{H}\right), 7.46$ (br s, 6 H), 3.97 (br s, 2 H), 2.88 (br s, 1 H), 1.06 (br s, 3 H). IR (film): 3058, 2976, 1613, 1437, 1370, 1330, 1103, 889, 717, $691 \mathrm{~cm}^{-1}$. These characterisation data match those in the literature. ${ }^{170,171}$

\section{Preparation of (Z)-4-(tert-Butyldimethylsilyloxy)-2-buten-1-ol, 226}

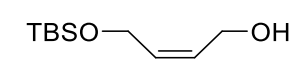

To a solution of cis-2-butene-1,4-diol (10.0 g, $110 \mathrm{mmol}, 3$ equiv) and triethylamine (7.7 mL, $55 \mathrm{mmol}, 1.5$ equiv) in 5:1 DCM:DMF $(60 \mathrm{~mL})$ at $0{ }^{\circ} \mathrm{C}$ (ice bath) was added TBSCl (5.7 g, $38 \mathrm{mmol}, 1$ equiv). The reaction mixture was allowed to warm to room temperature and the mixture stirred overnight. The reaction was quenched with saturated aqueous ammonium chloride $(50 \mathrm{~mL})$. The aqueous solution was then extracted with DCM $(3 \times 40$ $\mathrm{mL}$ ). The combined organic fractions were then washed with water and brine, dried $\left(\mathrm{MgSO}_{4}\right)$, filtered and concentrated under reduced pressure. The crude material was purified by silica gel flash chromatography (2:1 pet. ether:EtOAc) to provide the title compound (8.03 g, 99\%) as a colourless liquid. $\mathbf{R}_{\mathbf{f}}$ 0.32 (2:1 pet. ether:EtOAc). ${ }^{1} \mathbf{H}$ NMR $\left(500 \mathrm{MHz}, \mathrm{CDCl}_{3}\right): \delta 5.77-5.62(\mathrm{~m}, 2 \mathrm{H}), 4.26(\mathrm{~d}, J=5.4,2 \mathrm{H}), 4.20(\mathrm{~d}, J=5.9,2 \mathrm{H}), 0.91(\mathrm{~s}, 9$ $\mathrm{H}), 0.10(\mathrm{~s}, 6 \mathrm{H}) .{ }^{13} \mathrm{C}$ NMR (125 MHz, $\left.\mathrm{CDCl}_{3}\right): \delta 131.3,130.0,59.6,58.9,25.9,18.3,-5.3$. These characterisation data match those in the literature. ${ }^{172,168}$

\section{Preparation of (E)-4-(tert-Butyldimethylsilyloxy)but-2-enal, 227}

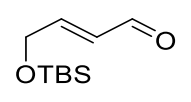

To a solution of alcohol 226 (609 mg, 3.0 mmol, 1 equiv) in DCM:DMSO (4:1, 40 $\mathrm{mL})$ was added triethylamine $(2.1 \mathrm{~mL}, 15.0 \mathrm{mmol}, 5$ equiv). The reaction mixture was then cooled to $0^{\circ} \mathrm{C}$ (ice bath) and added portionwise $\mathrm{SO}_{3} . \mathrm{Pyr}(1.68 \mathrm{~g}, 10.5 \mathrm{mmol}, 3.5$ equiv). The reaction was allowed to gradually warm to room temperature (over approximately 20 minutes), then stirred for a further 2 hours, quenched with saturated aqueous ammonium chloride and extracted with DCM $(3 \times 30 \mathrm{~mL})$. The combined organic fractions were then washed with distilled $\mathrm{H}_{2} \mathrm{O}$ then brine, dried $\left(\mathrm{MgSO}_{4}\right)$ and concentrated under reduced 


\section{Chapter Six: Experimental}

pressure. The crude material was purified by silica gel flash chromatography (5:1 pet. ether:EtOAc) to provide the title compound (508 $\mathrm{mg}$, $84 \%$ ) as a colourless liquid. $\mathbf{R}_{\mathbf{f}} 0.46$ (5:1 pet. ether:EtOAc). ${ }^{1} \mathrm{H}$ NMR $\left(500 \mathrm{MHz}, \mathrm{CDCl}_{3}\right): \delta 9.61(\mathrm{~d}, J=8.0 \mathrm{~Hz}, 1 \mathrm{H}), 6.89(\mathrm{~d}, J=$ $15.5 \mathrm{~Hz}, 1 \mathrm{H}), 6.41(\mathrm{~m}, 1 \mathrm{H}), 4.45(\mathrm{~m}, 2 \mathrm{H}), 0.92(\mathrm{~s}, 9 \mathrm{H}), 0.09$ (s, $6 \mathrm{H}) .{ }^{13} \mathrm{C}$ NMR $(125 \mathrm{MHz}$, $\left.\mathrm{CDCl}_{3}\right): \delta 193.3,156.5,130.5,62.2,25.8,18.3,-5.5$. These characterisation data match those in the literature. ${ }^{168}$

\section{Preparation of Ethyl (2E,4E)-6-(tert-butyldimethylsilyloxy)-2,4-hexadienoate, 228}

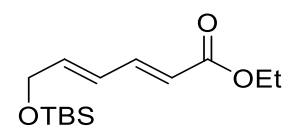

To a solution of aldehyde 227 (1.16 g, $5.79 \mathrm{mmol}, 1$ equiv) in DCM (45 mL) at room temperature was added ylide 219 (2.42 g, $6.95 \mathrm{mmol}, 1.2$ equiv) and the mixture stirred overnight. Solvent was then removed under reduced pressure and The crude material was purified by silica gel flash chromatography (5:1 pet. ether:EtOAc) to provide the title compound (1.41 g, 90\%) as a colourless liquid. Rf: 0.77 (2:1 pet. ether:EtOAc). ${ }^{1} \mathbf{H}$ NMR (500 MHz, $\left.\mathrm{CDCl}_{3}\right): \delta 7.20$ - $7.14(\mathrm{~m}, 1 \mathrm{H}), 6.31$ (d, J = 11.7 Hz, $\left.1 \mathrm{H}\right), 6.09$ (td, J = 4.2, $15.1 \mathrm{~Hz}$, $1 \mathrm{H}), 5.79(\mathrm{~d}, J=15.4 \mathrm{~Hz}, 1 \mathrm{H}), 4.27-4.17(\mathrm{~m}, 2 \mathrm{H}), 4.12(\mathrm{q}, J=7.1 \mathrm{~Hz}, 2 \mathrm{H}), 1.21(\mathrm{t}, J=7.1$ $\mathrm{Hz}, 4 \mathrm{H}), 0.84$ (s, $12 \mathrm{H}), 0.00$ (s, $6 \mathrm{H}) .{ }^{13} \mathrm{C}$ NMR (125 MHz, $\left.\mathrm{CDCl}_{3}\right): \delta$ 167.1, 144.0, 141.8, $126.8,120.7,62.9,60.3,25.9,18.4,14.3,-5.3$. These characterisation data match those in the literature. ${ }^{173}$

\section{Preparation of (2E,4E)-6-(tert-Butyldimethylsilyloxy)-2,4-hexadien-1-ol, 225}

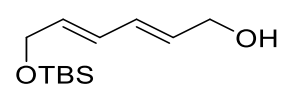

To a solution of ethyl ester $228(641 \mathrm{mg}, 2.37 \mathrm{mmol})$ in THF $(30 \mathrm{~mL})$ at $-78{ }^{\circ} \mathrm{C}($ dry ice/acetone bath) was added a solution of DIBAL-H $(4.8 \mathrm{~mL}, 4.8 \mathrm{mmol}, 1 \mathrm{M}$ solution in hexane). The reaction mixture was allowed to slowly warm to room temperature over 2 hours, then the reaction was quenched by slow addition of saturated potassium sodium tartrate (Rochelle's salt, $30 \mathrm{~mL}$ ) and the reaction stirred for 2 hours. The aqueous fraction was then extracted with EtOAc $(3 \times 30 \mathrm{~mL})$, The combined organic fractions were dried 
$\left(\mathrm{MgSO}_{4}\right)$, filtered, then concentrated under reduced pressure providing title compound (491 mg, 93\%) as a colourless oil. ${ }^{1} \mathrm{H}$ NMR indicated that the product was sufficiently pure for the subsequent steps so no further purification was necessary. $\mathbf{R}_{\mathbf{f}}$ : 0.51 (2:1 pet. ether:EtOAc). ${ }^{1} \mathbf{H}$ NMR (500 MHz, $\left.\mathrm{CDCl}_{3}\right): \delta 6.26(\mathrm{~m}, 2 \mathrm{H}), 5.80(\mathrm{~m}, 2 \mathrm{H}), 4.21(\mathrm{~m}, 4 \mathrm{H}), 0.92$ (s, 9), 0.08 (s, 6H). ${ }^{13} \mathrm{C}$ NMR (125 MHz, $\left.\mathrm{CDCl}_{3}\right): \delta 133.1,131.4,130.9,128.9,63.3,63.2,25.9$, $18.4,-5.3$. These characterisation data match those in the literature. ${ }^{174}$

\section{Preparation of (2E,4E)-1-Bromo-6-(tert-Butyldimethylsilyloxy)-2,4-hexadiene, 229}

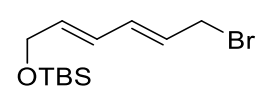

To a solution of alcohol 225 (50 mg, $0.22 \mathrm{mmol}, 1$ equiv) in DCM (5 mL) at $-30{ }^{\circ} \mathrm{C}$ (dry ice/acetone bath) was added triphenylphosphine (69 mg, $0.26 \mathrm{mmol}, 1.2$ equiv) followed by $\mathrm{N}$-bromosuccinimide ( $46 \mathrm{mg}, 0.26 \mathrm{mmol}, 1.2$ equiv). The reaction mixture was stirred for 20 minutes then quenched with saturated aqueous ammonium chloride (10 $\mathrm{mL})$ and extracted with DCM $(3 \times 10 \mathrm{~mL})$. The combined organic fractions were then dried $\left(\mathrm{MgSO}_{4}\right)$ and concentrated under reduced pressure. Crude material was passed through a plug of silica and concentrated to give title compound (57 $\mathrm{mg}, 89 \%$ ) as a colourless oil and used immediately in the next step due to instability. $\mathbf{R}_{\mathrm{f}} 0.82$ (2:1 pet. ether:EtOAc). ${ }^{1} \mathbf{H}$ NMR (500 MHz, $\left.\mathrm{CDCl}_{3}\right): \delta 6.36$ - 6.17 (m, $\left.2 \mathrm{H}\right), 5.96$ - 5.68 (m, $\left.2 \mathrm{H}\right), 4.23$ $(\mathrm{d}, J=5.3 \mathrm{~Hz}, 2 \mathrm{H}), 4.03(\mathrm{~d}, J=7.9 \mathrm{~Hz}, 2 \mathrm{H}), 0.91(\mathrm{~s}, 9 \mathrm{H}), 0.07$ (s, $6 \mathrm{H}) .{ }^{1} \mathrm{H}$ NMR matches literature. ${ }^{175}$

\section{Preparation of $N, N$-Dimethyl[(2E,4E)-6-(tert-butyldimethylsilyloxy)-2,4-} hexadienyl]amine, 231 (via alkyl bromide 229)

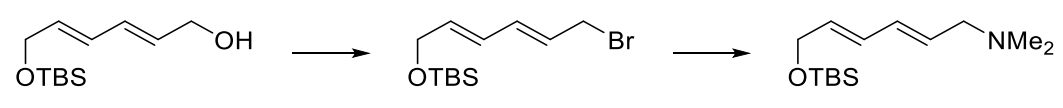

To a solution of alcohol 225 (172 mg, $0.75 \mathrm{mmol}, 1$ equiv) in DCM $(15 \mathrm{~mL})$ at $-30^{\circ} \mathrm{C}$ (dry ice/acetone bath) was added triphenylphosphine (237 mg, $0.90 \mathrm{mmol}, 1.2$ equiv) followed by $N$-bromosuccinimide $(160 \mathrm{mg}$, $0.90 \mathrm{mmol}, 1.2$ equiv). The reaction mixture was stirred for 20 minutes then quenched with saturated aqueous ammonium chloride (15 $\mathrm{mL})$ and extracted with DCM $(3 \times 15 \mathrm{~mL})$. The combined organic fractions were then 


\section{Chapter Six: Experimental}

dried $\left(\mathrm{MgSO}_{4}\right)$, concentrated under reduced pressure, passed through a plug of silica and concentrated again to give alkyl bromide 229. This was then immediately redissolved in DCM (10 mL), cooled to $0{ }^{\circ} \mathrm{C}$ (ice bath), and $\mathrm{HCl} . \mathrm{HNMe} 2(918 \mathrm{mg}, 11.3 \mathrm{mmol}, 15$ equiv) added, followed by DIPEA (2.6 mL, $15.0 \mathrm{mmol}, 20$ equiv). This was stirred overnight on ice, in a closed system (to prevent loss of dimethylamine; bp $=7-9{ }^{\circ} \mathrm{C}$ ). The reaction mixture was then quenched with $0.5 \mathrm{M}$ aqueous $\mathrm{NaOH}(15 \mathrm{~mL})$, and extracted with DCM (3 $\times 15 \mathrm{~mL})$. The combined organic fractions were dried $\left(\mathrm{MgSO}_{4}\right)$, filtered, then concentrated under reduced pressure. The crude material was purified by silica gel flash chromatography (90:10:1 DCM:MeOH:saturated aqueous $\mathrm{NH}_{4} \mathrm{OH}$ ) to provide the title compound (57 mg, 30\%) as a colourless oil. Rf: 0.42 (90:10:1 DCM:MeOH:saturated aqueous $\left.\mathrm{NH}_{4} \mathrm{OH}\right) .{ }^{1} \mathrm{H}$ NMR (500 MHz, $\left.\mathrm{CDCl}_{3}\right)$ : $\delta 6.30$ - $6.10(\mathrm{~m}, 2 \mathrm{H}), 5.78$ - $5.63(\mathrm{~m}, 2 \mathrm{H})$, $4.22(\mathrm{~d}, J=4.9 \mathrm{~Hz}, 2 \mathrm{H}), 2.94(\mathrm{~d}, J=6.7 \mathrm{~Hz}, 2 \mathrm{H}), 2.22(\mathrm{~s}, 6 \mathrm{H}), 0.92(\mathrm{~s}, 9 \mathrm{H}), 0.08(\mathrm{~s}, 6 \mathrm{H}) .{ }^{13} \mathrm{C}$ NMR $\left(125 \mathrm{MHz}, \mathrm{CDCl}_{3}\right): \delta$ 132.4, 132.1, 130.5, 129.4, 63.5, 61.7, 45.2, 25.9, 18.4, -5.2. IR (film): 2930, 2857, 2772, 1462, 1254, 1083, 990, 835, 775, $733 \mathrm{~cm}^{-1}$. HRMS-ESI (m/z): [M+ H] $]^{+}$ calculated for $\mathrm{C}_{14} \mathrm{H}_{30} \mathrm{NOSi}^{+}$, 256.2091; found 256.2087.

\section{Characterisation data for pseudo-dimerisation product, 232}

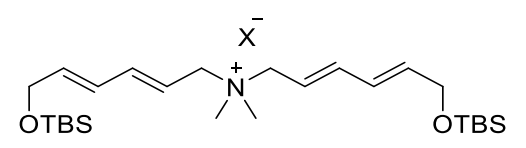

In addition desired product 231, the preceeding reaction generated pseudo-dimer 232 .

Rf: 0.53 (90:10:1 DCM : $\mathrm{MeOH}$ : saturated aqueous $\left.\mathrm{NH}_{4} \mathrm{OH}\right) .{ }^{1} \mathbf{H}$ NMR (500 MHz, $\mathrm{CDCl}_{3}$ ): $\delta 6.70$ - $6.59(\mathrm{~m}, 2 \mathrm{H}), 6.32-6.19(\mathrm{~m}, 2 \mathrm{H}), 5.95(\mathrm{~d}, J=15.2 \mathrm{~Hz}, 2 \mathrm{H}), 5.61-5.50(\mathrm{~m}, 2 \mathrm{H}), 4.23$ $(\mathrm{d}, J=7.9 \mathrm{~Hz}, 4 \mathrm{H}), 4.18$ (br s, $4 \mathrm{H}), 3.14(\mathrm{~s}, 6 \mathrm{H}), 0.84(\mathrm{~s}, 18 \mathrm{H}), 0.02$ (s, 12H). ${ }^{13} \mathrm{C}$ NMR (125 $\left.\mathrm{MHz}, \mathrm{CDCl}_{3}\right): \delta$ 143.9, 139.0, 126.4, 115.8, 65.3, 62.7, 49.0, 25.9, 18.4, -5.4. IR (film): 3394, 2955, 2930, 2857, 2360, 2341, 1472, 1254, 1112, 1004, 835, $776 \mathrm{~cm}^{-1}$. HRMS-ESI (m/z): [M]+ calculated for $\mathrm{C}_{26} \mathrm{H}_{52} \mathrm{NO}_{2} \mathrm{Si}_{2}{ }^{+}$, 466.3531; found 466.3542 . 


\section{Preparation of (2E,4E)-6-(tert-Butyldimethylsilyloxy)-2,4-hexadienal, 233}

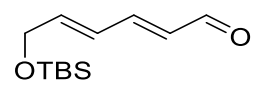

To a solution of alcohol 225 (155 mg, 0.68 mmol, 1 equiv) in DCM:DMSO (4:1, 5 $\mathrm{mL})$ was added triethylamine $(474 \mu \mathrm{L}, 3.4 \mathrm{mmol}, 5$ equiv). The reaction mixture was then cooled to $0{ }^{\circ} \mathrm{C}$ (ice bath) and added $\mathrm{SO}_{3} . \mathrm{Pyr}(324 \mathrm{mg}, 2.04 \mathrm{mmol}, 3$ equiv). The reaction was allowed to gradually warm to room temperature stirred for 2 hours, quenched with saturated aqueous ammonium chloride $(5 \mathrm{~mL})$ and extracted with DCM $(3 \times 10 \mathrm{~mL})$. The combined organic fractions were then washed with water then brine, dried $\left(\mathrm{MgSO}_{4}\right)$ and solvent removed under reduced pressure. The crude material was purified by silica gel flash chromatography (5:1 pet. ether:EtOAc) to provide the title compound (106 $\mathrm{mg}, 69 \%)$ as a yellow liquid. Rf: 0.73 (2:1 pet. ether:EtOAc) 0.94 (90:10:1 DCM:MeOH:saturated aqueous $\left.\mathrm{NH}_{4} \mathrm{OH}\right) .{ }^{1} \mathrm{H}$ NMR $\left(500 \mathrm{MHz}, \mathrm{CDCl}_{3}\right): \delta 9.56(\mathrm{~d}, J=7.9 \mathrm{~Hz}, 1 \mathrm{H}), 7.13(\mathrm{dd}, J=11.1$, $15.2 \mathrm{~Hz}, 1 \mathrm{H}), 6.55(\mathrm{t}, J=13.1 \mathrm{~Hz}, 1 \mathrm{H}), 6.32(\mathrm{td}, J=3.8,15.1 \mathrm{~Hz}, 1 \mathrm{H}), 6.14(\mathrm{dd}, J=7.8,15.2$ $\mathrm{Hz}, 1 \mathrm{H}), 4.34$ (br s, $2 \mathrm{H}), 0.92$ (s, $9 \mathrm{H}), 0.08$ (s, $6 \mathrm{H}) .{ }^{13} \mathrm{C}$ NMR (125 MHz, $\left.\mathrm{CDCl}_{3}\right): \delta$ 193.8, $151.6,144.3,131.2,126.7,62.8,25.8,18.4,-5.4$. These characterisation data match those in the literature. ${ }^{174}$

\section{Preparation of $N, N$-Dimethyl[(2E,4E)-6-(tert-butyldimethylsilyloxy)-2,4-} hexadienyl]amine, 231 (via reductive amination)

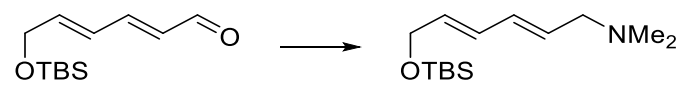

To a solution of aldehyde 233 (151 mg, $0.67 \mathrm{mmol}, 1$ equiv) in THF $(20 \mathrm{~mL})$ at $0{ }^{\circ} \mathrm{C}$ (ice bath) was added HCl.HNMe $(163 \mathrm{mg}, 2.0 \mathrm{mmol}, 3$ equiv) followed by $\mathrm{NMe}_{4} \mathrm{BH}(\mathrm{OAc}) 3$ (263 mg, $1.0 \mathrm{mmol}, 1.5$ equiv). This was stirred overnight on ice, in a closed system (to prevent loss of dimethylamine with a $b p=7-9{ }^{\circ} \mathrm{C}$ ). The reaction mixture was then quenched with $0.5 \mathrm{M}$ aqueous $\mathrm{NaOH}(20 \mathrm{~mL})$, and extracted with EtOAc $(3 \times 20$ $\mathrm{mL})$. The combined organic fractions were dried $\left(\mathrm{MgSO}_{4}\right)$, filtered, then concentrated under reduced pressure. The crude material was purified by silica gel flash chromatography (90:10:1 DCM:MeOH:saturated aqueous $\mathrm{NH}_{4} \mathrm{OH}$ ) to provide the title compound (108 mg, 64\%) as a pale orange oil. Rf: 0.41 (90:10:1 DCM:MeOH:saturated aqueous $\left.\mathrm{NH}_{4} \mathrm{OH}\right) .{ }^{1} \mathrm{H}$ NMR (500 MHz, $\left.\mathrm{CDCl}_{3}\right)$ : $\delta 6.28$ - $6.09(\mathrm{~m}, 2 \mathrm{H}), 5.77$ - $5.60(\mathrm{~m}, 2 \mathrm{H})$, 
$4.20(\mathrm{~d}, J=5.0 \mathrm{~Hz}, 2 \mathrm{H}), 2.93(\mathrm{~d}, J=6.6 \mathrm{~Hz}, 2 \mathrm{H}), 2.21(\mathrm{~s}, 6 \mathrm{H}), 0.90(\mathrm{~s}, 9 \mathrm{H}), 0.08(\mathrm{~s}, 6 \mathrm{H}) .{ }^{13} \mathrm{C}$

NMR (125 MHz, $\left.\mathrm{CDCl}_{3}\right): \delta 132.4,132.1,130.4,129.4,63.5,61.6,45.2,25.9,18.4,-5.2$. Characterisation data matched those of compound 231 from previous synthesis (vide supra).

Preparation of (2E,4E)-6-(Dimethylamino)-2,4-hexadien-1-ol, 236

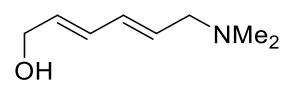

To a solution of silyl ether $231(17 \mathrm{mg}, 0.067 \mathrm{mmol})$ in methanol $(2 \mathrm{~mL})$ at room temperature was added concentrated $\mathrm{HCl}$ (2 drops). The reaction mixture was allowed to stir for 20 minutes, after which TLC had indicated full conversion of starting material. A few drops of saturated aqueous $\mathrm{NaHCO}_{3}$ were added to neutralise the acid, then methanol was removed under reduced pressure, and the the product partitioned between EtOAc $(5 \mathrm{~mL})$ and $1 \mathrm{M}$ aqueous $\mathrm{NaOH}(5 \mathrm{~mL})$. The aqueous layer was extracted with EtOAc $(2 \times 10 \mathrm{~mL})$, dried $\left(\mathrm{MgSO}_{4}\right)$, filtered, and solvent removed under reduced pressure to provide the title compound $(9.0 \mathrm{mg}, 96 \%)$ as a colourless liquid. Rf: 0.22 (90:10:1 DCM:MeOH:saturated aqueous $\left.\mathrm{NH}_{4} \mathrm{OH}\right) .{ }^{1} \mathbf{H}$ NMR (500 MHz, $\left.\mathrm{CDCl}_{3}\right): \delta 6.32$ - 6.23 (m, 1 H), 6.22 - $6.13(\mathrm{~m}, 1 \mathrm{H}), 5.81(\mathrm{td}, J=5.4,14.6 \mathrm{~Hz}, 1 \mathrm{H}), 5.73(\mathrm{td}, J=6.6,15.1 \mathrm{~Hz}, 1 \mathrm{H}), 4.18(\mathrm{~d}$, $J=5.1 \mathrm{~Hz}, 2 \mathrm{H}), 2.97(\mathrm{~d}, J=6.9 \mathrm{~Hz}, 2 \mathrm{H}), 2.22$ (s, $6 \mathrm{H}) .{ }^{13} \mathrm{C} \mathrm{NMR}\left(125 \mathrm{MHz}, \mathrm{CDCl}_{3}\right): \delta$ 132.5, 132.1, 130.6, 130.5, 63.0, 61.4, 44.9. IR (film): 3163, 2946, 2820, 2779, 1627, 1459, 1355, 990, 912, $731 \mathrm{~cm}^{-1}$. HRMS-ESI (m/z): $[\mathrm{M}+\mathrm{H}]^{+}$calculated for $\mathrm{C}_{8} \mathrm{H}_{16} \mathrm{NO}^{+}, 142.1226$; found 142.1237.

Preparation of (2E,4E)-6-(Dimethylamino)-2,4-hexadienal, 213

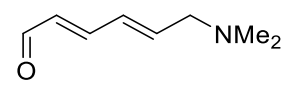

To a solution of alcohol 236 (49 mg, $0.35 \mathrm{mmol}$, 1 equiv) in DCM (4 mL) was added DMSO (0.50 mL, $7.0 \mathrm{mmol}, 20$ equiv) and triethylamine (0.24 mL, $1.75 \mathrm{mmol}, 5$ equiv). The reaction mixture was then cooled to $0^{\circ} \mathrm{C}$ (ice bath), then added $\mathrm{SO}_{3} . \mathrm{Pyr}(166 \mathrm{mg}, 1.04$ mmol, 3 equiv) and the reaction stirred at this temperature for 30 minutes and for a further 60 minutes at room temperature. The reaction was then quenched with water (5 
$\mathrm{mL})$, and extracted with DCM $(3 \times 10 \mathrm{~mL})$. The combined organic fractions were dried $\left(\mathrm{MgSO}_{4}\right)$, filtered and the solvent removed under reduced pressure. The crude material was purified by silica gel flash chromatography (90:10:1 DCM:MeOH:saturated aqueous $\mathrm{NH}_{4} \mathrm{OH}$ ) provided the title compound $(42 \mathrm{mg}, 86 \%)$ as a pale yellow liquid. $\mathbf{R}_{\mathrm{f}}: 0.49$ (90:10:1 DCM:MeOH:saturated aqueous $\left.\mathrm{NH}_{4} \mathrm{OH}\right) .{ }^{1} \mathbf{H}$ NMR (500 MHz, $\left.\mathrm{CDCl}_{3}\right): \delta 9.57$ (d, J $=7.9 \mathrm{~Hz}, 1 \mathrm{H}), 7.12(\mathrm{dd}, J=10.8,15.4 \mathrm{~Hz}, 1 \mathrm{H}), 6.45(\mathrm{dd}, J=11.0,15.2 \mathrm{~Hz}, 1 \mathrm{H}), 6.27(\mathrm{td}, J=$ 6.5, $15.2 \mathrm{~Hz}, 1 \mathrm{H}), 6.13(\mathrm{dd}, J=7.9,15.4 \mathrm{~Hz}, 1 \mathrm{H}), 3.07(\mathrm{~d}, J=6.3 \mathrm{~Hz}, 2 \mathrm{H}), 2.25(\mathrm{~s}, 6 \mathrm{H}) .{ }^{13} \mathrm{C}$ NMR (125 MHz, CDCl $)$ ): $\delta$ 193.8, 151.6, 142.9, 131.3, 130.3, 61.4, 45.5. IR (film): 3385, 2946, 2820, 2778, 2360, 1727, 1681, 1016, 992, 918, $731 \mathrm{~cm}^{-1}$. HRMS-ESI $(\mathrm{m} / \mathrm{z}):[\mathrm{M}+\mathrm{H}]^{+}$calculated for $\mathrm{C}_{8} \mathrm{H}_{14} \mathrm{NO}^{+}, 140.1070$; found 140.1080 .

Preparation of N-(2,4-Dinitrophenyl)pyridinium chloride, 238

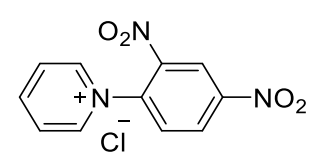

To a solution of 1-chloro-2,4-dinitrobenzene 239 (4.47 g, $22.0 \mathrm{mmol}, 1$ equiv) in acetone $(20 \mathrm{~mL})$ was added pyridine $(1.8 \mathrm{~mL}, 22.0 \mathrm{mmol}, 1$ equiv) and refluxed overnight. After this time, a white precipitate had formed. The reaction mixture was allowed to cool to room temperature, pet. ether added and the precipitate filtered. The solid was then washed with pet. ether and air dried to provide the title compound $(3.71 \mathrm{~g}, 60 \%)$ as a white solid. ${ }^{1}$ H NMR (500 MHz, methanol): $\delta 9.41$ - 9.20 (m, 3 H), 9.02 - 8.87 (m, 2 H), 8.41 $(\mathrm{t}, J=6.8 \mathrm{~Hz}, 2 \mathrm{H}), 8.36-8.27(\mathrm{~m}, 1 \mathrm{H}) .{ }^{13} \mathrm{C} \mathrm{NMR}\left(125 \mathrm{MHz}, \mathrm{CDCl}_{3}\right): \delta 151.3,150.3,147.4$, $144.7,140.3,132.8,131.3,129.7,123.3$. These characterisation data match those in the literature. ${ }^{176}$

\section{Preparation of (2E,4E)-5-N,N-Dimethylamino-2,4-pentadien-1-al, 237}

$$
\text { o }>\mathrm{NMe}_{2}
$$

To a solution of pyridinium salt 238 (980 mg, $3.5 \mathrm{mmol}, 1$ equiv) in ethanol (20 mL) was added a solution of $\mathrm{HNMe} . \mathrm{HCl}(709 \mathrm{mg}, 8.7 \mathrm{mmol}, 2.5$ equiv) in water ( $3 \mathrm{~mL})$ and refluxed for 1 hour. Solvent was then removed under reduced pressure, the product 


\section{Chapter Six: Experimental}

redissolved in $4 \mathrm{M}$ aqueous $\mathrm{NaOH}(50 \mathrm{~mL})$ and the reaction stirred for 5 minutes. The aqueous mixture was then extracted with DCM $(3 \times 50 \mathrm{~mL})$. The combined organic fractions were concentrated under reduced pressure. The crude material was purified by silica gel flash chromatography (2:1 pet. ether:EtOAc) to provide the title compound (359 mg, 83\%) as a brown solid. ${ }^{1} \mathrm{H}$ NMR $\left(500 \mathrm{MHz}, \mathrm{CDCl}_{3}\right): \delta 9.29(\mathrm{~d}, J=8.5 \mathrm{~Hz}, 1 \mathrm{H}), 7.10$ (dd, $J=11.6,14.3 \mathrm{~Hz}, 1 \mathrm{H}), 6.78(\mathrm{~d}, J=12.5 \mathrm{~Hz}, 1 \mathrm{H}), 5.85(\mathrm{dd}, J=8.4,14.3 \mathrm{~Hz}, 1 \mathrm{H}), 5.28(\mathrm{t}, J=$ $12.1 \mathrm{~Hz}, 1 \mathrm{H}), 2.96$ (br s, $6 \mathrm{H}) .{ }^{13} \mathrm{C}$ NMR $\left(125 \mathrm{MHz}, \mathrm{CDCl}_{3}\right): \delta$ 192.3, 156.5, 152.4, 119.9, 97.3, 38.0. These characterisation data match those in the literature. ${ }^{177}$

\subsection{Experimental for Chapter 3}

\section{Macrocycle Formation}

\section{Preparation of 6-(2-Hydroxyethyl)-5,6-dihydropyran-2-one, 283}

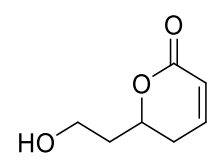

To a solution of lactone 167 (160 mg, $0.61 \mathrm{mmol}, 1$ equiv) in 9:1 DCM: $\mathrm{H}_{2} \mathrm{O}(15 \mathrm{~mL})$ at room temperature was added 2,3-dichloro-5,6-dicyano-1,4-benzoquinone (152 mg, 0.67 mmol, 1.1 equiv) and the mixture stirred overnight. The reaction mixture was then filtered (filter paper) and extracted with DCM $(3 \times 15 \mathrm{~mL})$. The crude material was purified by silica gel flash chromatography (1:1 pet.ether:EtOAc to EtOAc) to provide the title compound (73 mg, 84\%) as a colourless oil. $\mathbf{R}_{\mathbf{f}} 0.26$ (EtOAc). ${ }^{1} \mathbf{H}$ NMR (500 MHz, $\mathrm{CDCl}_{3}$ ):

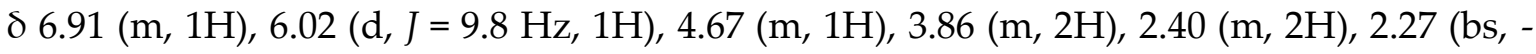
$\mathrm{OH}, 1 \mathrm{H}), 2.03(\mathrm{~m}, 1 \mathrm{H}), 1.91(\mathrm{~m}, 1 \mathrm{H}) .{ }^{13} \mathrm{C}$ NMR $\left(125 \mathrm{MHz}, \mathrm{CDCl}_{3}\right): \delta$ 164.4, 145.4, 121.2, 75.6, $58.3,37.3,29.5$. These characterisation data match those in the literature. ${ }^{178}$ 


\section{Preparation of 2-(6-Oxo-2,3-dihydropyran-2-yl)ethyl 6-heptynoate, 284}

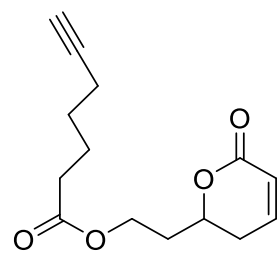

To a solution of alcohol 283 (64 mg, $0.45 \mathrm{mmol}, 1$ equiv) and acid 190 (68 mg, 0.54 mmol, 1.2 equiv) in DCM (10 mL) at $0{ }^{\circ} \mathrm{C}$ (ice bath) was added EDCI (121 mg, $0.63 \mathrm{mmol}$, 1.4 equiv) followed by DMAP (17 $\mathrm{mg}, 0.14 \mathrm{mmol}, 0.3$ equiv). The reaction mixture was stirred overnight at room temperature, then quenched with saturated aqueous ammonium chloride. The aqueous layer was extracted with DCM ( $3 \times 15 \mathrm{~mL})$. The combined organic fractions were dried $\left(\mathrm{MgSO}_{4}\right)$, filtered and concentrated under reduced pressure. The crude material was purified by silica gel flash chromatography (1:1 pet. ether:EtOAc) to provide the title compound (113 $\mathrm{mg}, 71 \%)$ as a colourless liquid. $\mathbf{R}_{\mathrm{f}:} 0.33$ (1:1 pet. ether:EtOAc). ${ }^{1} \mathrm{H}$ NMR (500 MHz, $\left.\mathrm{CDCl}_{3}\right): \delta 8.20$ (br s, $\left.1 \mathrm{H}\right), 6.89$ (dd, J = 3.7, 8.9 $\mathrm{Hz}, 2 \mathrm{H}), 6.47(\mathrm{~d}, J=4.8 \mathrm{~Hz}, 1 \mathrm{H}), 6.02(\mathrm{~d}, J=9.7 \mathrm{~Hz}, 2 \mathrm{H}), 4.61-4.50(\mathrm{~m}, 2 \mathrm{H}), 4.34-4.17$ (m, 5 H), $2.98(\mathrm{~s}, 3 \mathrm{H}), 2.40$ - $2.35(\mathrm{~m}, 4 \mathrm{H}), 2.32(\mathrm{t}, J=7.3 \mathrm{~Hz}, 5 \mathrm{H}), 2.23$ - $2.16(\mathrm{~m}, 5 \mathrm{H}), 2.15$ $2.06(\mathrm{~m}, 3 \mathrm{H}), 2.04-1.96(\mathrm{~m}, 3 \mathrm{H}), 1.94(\mathrm{br} \mathrm{s}, 2 \mathrm{H}), 1.73$ (quin, $J=7.6 \mathrm{~Hz}, 5 \mathrm{H}), 1.54$ (quin, $J=$ $7.3 \mathrm{~Hz}, 5 \mathrm{H}) .{ }^{13} \mathrm{C}$ NMR $\left(125 \mathrm{MHz}, \mathrm{CDCl}_{3}\right): \delta$ 173.1, 163.8, 144.8, 121.3, 83.8, 74.6, 68.6, 59.8, 33.9, 33.5, 29.3, 27.7, 23.8, 18.0. IR (film): 3277, 2935, 1771, 1720, 1389, 1246, 1170, 1037, 818, $638 \mathrm{~cm}^{-1}$. HRMS-ESI (m/z): [M + H] $]^{+}$calculated for $\mathrm{C}_{14} \mathrm{H}_{19} \mathrm{O}_{4}{ }^{+}, 251.1278$; found 251.1282.

\section{Preparation of (2Z,4E)-7-(5-Hexynylcarbonyloxy)-2,4-heptadienoic acid, 282}

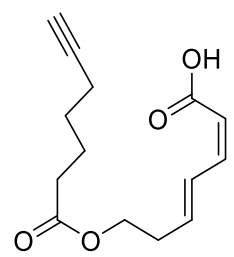

To a solution of lactone 284 (55 mg, $0.22 \mathrm{mmol}, 1$ equiv) in THF (4 mL) at room temperature was added dropwise TBAF solution $(1 \mathrm{M}$ solution in THF, $0.88 \mathrm{~mL}, 0.88$ mmol, 4 equiv) and the reaction stirred for 2 hours. The reaction mixture was diluted with EtOAc $(20 \mathrm{~mL})$, and washed with dilute aqueous sulfuric acid (2 vol\%, $3 \times 20 \mathrm{~mL})$ to remove tetrabutylammonium salts. The organic fraction was then dried $\left(\mathrm{MgSO}_{4}\right)$, filtered, 


\section{Chapter Six: Experimental}

and concentrated under reduced pressure to provide the title compound ( $52 \mathrm{mg}, 95 \%$ ) as a colourless oil. Rf: 0.54 (1:1 pet. ether:EtOAc). ${ }^{1} \mathbf{H}$ NMR (500 MHz, $\left.\mathrm{CDCl}_{3}\right): \delta 7.42(\mathrm{dd}, J=$ 11.8, 14.8 Hz, $1 \mathrm{H}), 6.66(\mathrm{t}, J=11.3 \mathrm{~Hz}, 1 \mathrm{H}), 6.08(\mathrm{td}, J=7.2,15.1 \mathrm{~Hz}, 1 \mathrm{H}), 5.65(\mathrm{~d}, J=11.5$ $\mathrm{Hz}, 1 \mathrm{H}), 4.19(\mathrm{t}, J=6.5 \mathrm{~Hz}, 2 \mathrm{H}), 2.56(\mathrm{q}, J=6.6 \mathrm{~Hz}, 2 \mathrm{H}), 2.35(\mathrm{t}, J=7.4 \mathrm{~Hz}, 2 \mathrm{H}), 2.21(\mathrm{dt}, J$ = 2.4, $6.9 \mathrm{~Hz}, 3 \mathrm{H}$ ), $1.96(\mathrm{t}, J=2.3 \mathrm{~Hz}, 1 \mathrm{H}$ ), 1.75 (quin, $J=7.6 \mathrm{~Hz}, 2 \mathrm{H}$ ), 1.56 (quin, $J=7.4 \mathrm{~Hz}$, $2 \mathrm{H}) .{ }^{13} \mathrm{C}$ NMR (125 MHz, $\left.\mathrm{CDCl}_{3}\right): \delta 173.3,171.2,146.7,140.9,129.1,115.8,83.9,68.6,62.8$, 33.7, 32.4, 27.8, 24.0, 18.1. IR (film): 3302, 2951, 2867, 1728, 1687, 1441, 1227, 1172, 1146, 910, 731, $646 \mathrm{~cm}^{-1}$. HRMS-ESI (m/z): [M - H] calculated for $\mathrm{C}_{14} \mathrm{H}_{17} \mathrm{O}_{4}^{-}, 249.1132$; found 249.1137.

\section{Preparation of 2-Azidoethanol}

$$
\mathrm{HO}^{\sim} \mathrm{N}_{3}
$$

To a solution of 2-chloroethanol (2.93 g, $36.4 \mathrm{mmol}$, 1 equiv) in $\mathrm{H}_{2} \mathrm{O}(60 \mathrm{~mL})$ was added sodium iodide (540 mg, $3.6 \mathrm{mmol}, 0.1$ equiv) and sodium azide ( $4.73 \mathrm{~g}, 72.7 \mathrm{mmol}$, 2 equiv) then heated to reflux for 6 hours. After cooling to room temperature The reaction mixture was extracted with EtOAc $(3 \times 40 \mathrm{~mL})$, dried $\left(\mathrm{MgSO}_{4}\right)$, filtered and concentrated under reduced pressure to provide the title compound $(2.20 \mathrm{~g}, 69 \%)$ as a colourless liquid. ${ }^{1} \mathbf{H}$ NMR (500 MHz, CDCl $)$ : $\delta 3.79$ (q, J = 5.1 Hz, $\left.2 \mathrm{H}\right), 3.46$ (t, J = 4.9 Hz, $\left.2 \mathrm{H}\right), 1.94$ (t, J = 5.7 $\mathrm{Hz}, 1 \mathrm{H},-\mathrm{OH}) .{ }^{13} \mathrm{C}$ NMR $\left(125 \mathrm{MHz}, \mathrm{CDCl}_{3}\right): \delta 61.5,53.5$. These characterisation data match those in the literature. ${ }^{132}$

\section{Preparation of 2-Azidoethyl 5-hexynyl (2Z,4E)-2,4-octadienedioate, Z,E-285}

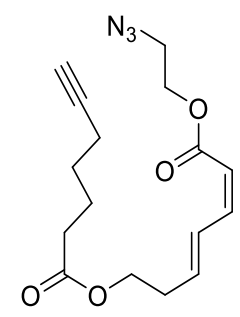

$Z, E-285$ Major<smiles>C#CCCCCC(=O)OCC/C=C/C=C/C(=O)OCCN</smiles>

$E, E-285$

Minor

To a solution of acid $282(22 \mathrm{mg}, 0.09 \mathrm{mmol}, 1$ equiv) in THF (1.5 mL) at room temperature was added triethylamine $(18 \mu \mathrm{L}, 0.13 \mathrm{mmol}, 1.5$ equiv) followed by 2,4,6trichlorobenzoyl chloride $(18 \mu \mathrm{L}, 0.11 \mathrm{mmol}, 1.3$ equiv). After stirring for 2 hours, The 
reaction mixture was diluted with DCM $(8 \mathrm{~mL})$, cooled to $-78{ }^{\circ} \mathrm{C}$ (dry ice/acetone bath) and 2-azidoethanol (12 mg, $0.13 \mathrm{mmol}, 1.5$ equiv) followed by DMAP ( $3 \mathrm{mg}, 0.03 \mathrm{mmol}$, 0.3 equiv) were added. The reaction mixture was stirred overnight, whereby dry ice/acetone cold bath gradually warmed to room temperature. The reaction was then quenched with saturated aqueous ammonium chloride $(3 \mathrm{~mL})$. The aqueous layer was extracted with EtOAc $(3 \times 10 \mathrm{~mL})$. The combined organic fractions were dried $\left(\mathrm{MgSO}_{4}\right)$, filtered and concentrated under reduced pressure. The crude material was purified by silica gel flash chromatography (5:1 pet. ether:EtOAc) to provide the title compound and the $\boldsymbol{E}, \boldsymbol{E}-285$ isomerised product in a 2:1 ratio (14 $\mathrm{mg}, 50 \%)$ as a colourless oil. $\mathbf{R}_{\mathrm{f}} 0.49$ (2:1 pet. ether:EtOAc). Only NMR spectra for major $Z, E-285$ are quoted, refer to next reaction for NMR spectra data for minor isomer: ${ }^{1} \mathrm{H}$ NMR $\left(500 \mathrm{MHz}, \mathrm{CDCl}_{3}\right): \delta 7.43(\mathrm{dd}, J=12.1$, 14.6 Hz, 1 H), 6.60 (t, J = 11.3 Hz, 1 H), 6.09 - 6.00 (m, 1 H), 5.66 (d, J = 11.2 Hz, 1 H), 4.32 $4.27(\mathrm{~m}, 2 \mathrm{H}), 4.17(\mathrm{q}, J=5.8 \mathrm{~Hz}, 2 \mathrm{H}), 3.51(\mathrm{q}, J=4.5 \mathrm{~Hz}, 2 \mathrm{H}), 2.58-2.48(\mathrm{~m}, 2 \mathrm{H}), 2.34(\mathrm{t}, J$ = $7.3 \mathrm{~Hz}, 2 \mathrm{H}), 2.24$ - $2.16(\mathrm{~m}, 2 \mathrm{H}), 1.99-1.90$ (m, $1 \mathrm{H}), 1.74(\mathrm{td}, J=7.6,15.2 \mathrm{~Hz}, 2 \mathrm{H}), 1.61$ 1.50 (m, $2 \mathrm{H}) .{ }^{13} \mathrm{C}$ NMR (125 MHz, $\left.\mathrm{CDCl}_{3}\right): \delta 173.3,165.8,145.6,140.5,129.0,115.7,83.9$, 68.6, 62.8, 62.4, 49.8, 33.7, 32.4, 27.8, 24.0, 18.1. IR (film): 3300, 2956, 2107, 1718, 1643, 1602, 1426, 1258, 1172, 1138, 1000, 914, 734, $629 \mathrm{~cm}^{-1}$. HRMS-ESI (m/z): [M + NH4 $]^{+}$calculated for $\mathrm{C}_{16} \mathrm{H}_{25} \mathrm{~N}_{4} \mathrm{O}_{4}{ }^{+}$, 337.1870; found 337.1869.

\section{Preparation of 2-Azidoethyl 5-hexynyl (2E,4E)-2,4-octadienedioate, E,E-285}

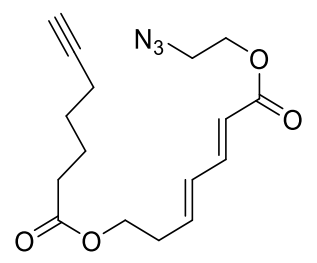

To a solution of acid $282(27 \mathrm{mg}, 0.11 \mathrm{mmol}, 1$ equiv) in THF (1.5 mL) at room temperature was added triethylamine $(23 \mu \mathrm{L}, 0.16 \mathrm{mmol}, 1.5$ equiv) followed by 2,4,6trichlorobenzoyl chloride ( $22 \mu \mathrm{L}, 0.16 \mathrm{mmol}, 1.5$ equiv). After stirring for 2 hours, The reaction mixture was diluted with DCM $(8 \mathrm{~mL})$, and added 2-azidoethanol (14 mg, 0.16 mmol, 1.5 equiv) followed by DMAP (67 mg, $0.55 \mathrm{mmol}, 5$ equiv). The reaction was stirred overnight then quenched with saturated aqueous ammonium chloride $(5 \mathrm{~mL})$. The aqueous layer was extracted with EtOAc $(3 \times 5 \mathrm{~mL})$. The combined organic fractions were 


\section{Chapter Six: Experimental}

dried $\left(\mathrm{MgSO}_{4}\right)$, filtered and concentrated under reduced pressure. The crude material was purified by silica gel flash chromatography (pet. ether:EtOAc) to provide a 10:1 mixture of the isomerised $E, E-285$ compound and the non-isomerised Z,E-285product ( $35 \mathrm{mg}, 71 \%$ ) as a colourless oil. ${ }^{1} \mathrm{H}$ NMR $\left(500 \mathrm{MHz}, \mathrm{CDCl}_{3}\right): \delta 7.29(\mathrm{dd}, J=10.5,15.0 \mathrm{~Hz}, 1 \mathrm{H}), 6.25(\mathrm{dd}, J$ = 11.0, 15.1 Hz, $1 \mathrm{H}), 6.14-6.05(\mathrm{~m}, 1 \mathrm{H}), 5.85(\mathrm{~d}, J=15.2 \mathrm{~Hz}, 1 \mathrm{H}), 4.31(\mathrm{t}, J=5.1 \mathrm{~Hz}, 2 \mathrm{H})$, $4.16(\mathrm{t}, J=6.4 \mathrm{~Hz}, 2 \mathrm{H}), 3.50(\mathrm{t}, J=4.8 \mathrm{~Hz}, 3 \mathrm{H}), 2.51(\mathrm{q}, J=6.6 \mathrm{~Hz}, 2 \mathrm{H}), 2.32(\mathrm{t}, J=7.3 \mathrm{~Hz}, 2$ H), 2.26 - $2.14(\mathrm{~m}, 3 \mathrm{H}), 1.95(\mathrm{br} \mathrm{s}, 1 \mathrm{H}), 1.73(\mathrm{td}, J=7.6,15.2 \mathrm{~Hz}, 3 \mathrm{H}), 1.62$ - $1.48(\mathrm{~m}, 3 \mathrm{H})$. ${ }^{13} \mathrm{C}$ NMR $\left(125 \mathrm{MHz}, \mathrm{CDCl}_{3}\right)$ : $\delta$ 173.2, 166.5, 145.3, 139.6, 130.5, 119.4, 83.9, 68.6, 62.9, 62.7, 49.8, 33.6, 32.3, 27.8, 23.9, 18.1. IR (film): 3301, 2931, 2868, 2361, 2104, 1715, 1644, 1246, 1173, 1135, 1000, 732, $639 \mathrm{~cm}^{-1}$. HRMS-ESI (m/z): $[\mathrm{M}+\mathrm{Na}]^{+}$calculated for $\mathrm{C}_{16} \mathrm{H}_{21} \mathrm{~N}_{3} \mathrm{O}_{4} \mathrm{Na}^{+}$, 342.1424; found 342.1421.

(6E,8E)--4.12-Dioxa-1.19.20-triazabicyclo[16.2.1]henicosa-6,8,18(21),19-tetraene-5,13dione, $E, E-277$

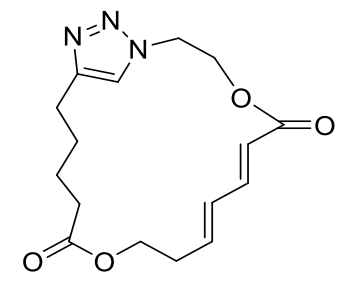

To an aqueous solution (10 mL) of $\mathrm{CuSO}_{4} .5 \mathrm{H}_{2} \mathrm{O}(2 \mathrm{mg}, 0.008 \mathrm{mmol}, 0.1$ equiv) and ascorbic acid ( $7 \mathrm{mg}, 0.014 \mathrm{mmol}, 0.5$ equiv), that was neutralised to approximately $\mathrm{pH} 7$ with aqueous $\mathrm{NaOH}$, was added a solution of alkyne/azide $\boldsymbol{E}, \boldsymbol{E}-285$ (24 mg, $0.075 \mathrm{mmol}, 1$ equiv) in THF $(10 \mathrm{~mL})$. The yellow reaction mixture was stirred overnight at room temperature which gradually turned yellow/green. The aqueous layer was extracted with EtOAc $(3 \times 10 \mathrm{~mL})$ and the combined organic fractions were dried $\left(\mathrm{MgSO}_{4}\right)$, filtered and concentrated under reduced pressure. The crude material was purified by silica gel flash chromatography (EtOAc) to provide the title compound (11 mg, 46\%) as a white solid. Melting point: $140-143^{\circ} \mathrm{C}$ (decomposition). Rf: 0.34 (EtOAc). ${ }^{1} \mathbf{H}$ NMR (500 MHz, CDCl $)$ : $\delta 7.25(\mathrm{~s}, 1 \mathrm{H}), 6.98(\mathrm{dd}, J=11.0,15.5 \mathrm{~Hz}, 1 \mathrm{H}), 6.19(\mathrm{dd}, J=11.2,14.9 \mathrm{~Hz}, 1 \mathrm{H}), 5.99$ (td, J = 7.3, 15.0 Hz, $1 \mathrm{H}), 5.70(\mathrm{~d}, J=15.5 \mathrm{~Hz}, 1 \mathrm{H}), 4.61(\mathrm{t}, J=4.8 \mathrm{~Hz}, 3 \mathrm{H}), 4.52(\mathrm{t}, J=4.9 \mathrm{~Hz}, 2 \mathrm{H})$, $4.31(\mathrm{t}, J=5.6 \mathrm{~Hz}, 2 \mathrm{H}), 2.79-2.71(\mathrm{~m}, 2 \mathrm{H}), 2.50(\mathrm{q}, J=6.0 \mathrm{~Hz}, 3 \mathrm{H}), 2.37(\mathrm{t}, J=6.2 \mathrm{~Hz}, 2 \mathrm{H})$, 1.79 - $1.63(\mathrm{~m}, 3 \mathrm{H}), 1.60$ - 1.48 (m, $3 \mathrm{H}) .{ }^{13} \mathrm{C}$ NMR (125 MHz, $\left.\mathrm{CDCl}_{3}\right)$ : $\delta$ 173.3, 165.4, 148.0, 
145.5, 140.9, 130.3, 122.1, 119.1, 62.0, 61.9, 48.7, 34.0, 32.6, 29.1, 26.1, 25.0. IR (film): 2958, 2862, 1747, 1716, 1435, 1367, 1218, 1075, 1038, 922, 797, $733 \mathrm{~cm}^{-1}$. HRMS-ESI (m/z): [M + H] $]^{+}$ calculated for $\mathrm{C}_{16} \mathrm{H}_{22} \mathrm{~N}_{3} \mathrm{O}_{4}^{+}$, 320.1605; found 320.1623 .

\section{Preparation of (2Z,4E)-7-\{4-[1-(2-Hydroxyethyl)-1H-1,2,3-triazol-4-yl]valeroxy\}-2,4-} heptadienoic acid, 286

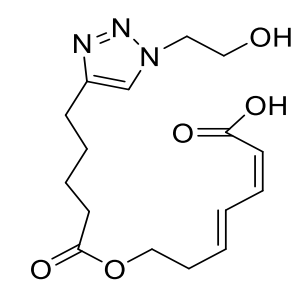

To an aqueous solution (2 mL) of $\mathrm{CuSO}_{4} .5 \mathrm{H}_{2} \mathrm{O}(3 \mathrm{mg}, 0.01 \mathrm{mmol}, 0.1$ equiv) and ascorbic acid (11 mg, $0.06 \mathrm{mmol}, 0.5$ equiv), that was neutralised to $\mathrm{pH} 7$ with aqueous $\mathrm{NaOH}$, was added a solution of alkyne $282(28 \mathrm{mg}, 0.11 \mathrm{mmol}, 1$ equiv) and 2azidoethanol (15 mg, $0.17 \mathrm{mmol}, 1.5$ equiv) in THF $(2 \mathrm{~mL})$. The yellow reaction mixture was stirred overnight at room temperature which gradually turned yellow/green.Reaction was acidified with $10 \%$ aqueous $\mathrm{H}_{2} \mathrm{SO}_{4}(1 \mathrm{~mL})$ extracted with EtOAc $(3 \times 10 \mathrm{~mL})$ and the combined organic fractions were dried $\left(\mathrm{MgSO}_{4}\right)$, filtered and concentrated under reduced pressure. The crude material was purified by silica gel flash chromatography (1:1 pet. ether:EtOAc to EtOAc) to provide the title compound (23 mg, 62\%) as a slightly pale yellow oil. ${ }^{1} \mathrm{H}$ NMR (500 MHz, $\left.\mathrm{CDCl}_{3}\right)$ : $\delta 7.47$ (s, $\left.1 \mathrm{H}\right), 7.38(\mathrm{dd}, J=11.7,14.8 \mathrm{~Hz}, 1 \mathrm{H}), 6.59$ (t, $J=11.3 \mathrm{~Hz}, 1 \mathrm{H}), 6.09$ - $5.95(\mathrm{~m}, 1 \mathrm{H}), 5.62(\mathrm{~d}, J=11.3 \mathrm{~Hz}, 1 \mathrm{H}), 4.49$ - 4.38 (m, $2 \mathrm{H}), 4.26$ 4.15 (m, 2 H), 4.08 - 3.95 (m, 2 H), 2.68 (br s, 2 H), 2.52 (q, J = 6.0 Hz, 2 H), 2.42 - 2.29 (m, 2 H), 1.66 (br s, 4 H). ${ }^{13} \mathrm{C}$ NMR (125 MHz, $\left.\mathrm{CDCl}_{3}\right)$ : $\delta$ 173.6, 169.4, 147.3, 145.1, 139.8, 129.1, 122.3, 116.9, 62.5, 61.0, 52.6, 34.0, 32.0, 28.6, 24.9, 24.3. IR (film): 3145, 2936, 1720, 1640, 1602, 1433, 1177, 1053, 1001, 966, 911, $729 \mathrm{~cm}^{-1}$. HRMS-ESI $(\mathrm{m} / \mathrm{z}):[\mathrm{M}+\mathrm{H}]^{+}$calculated for $\mathrm{C}_{16} \mathrm{H}_{24} \mathrm{~N}_{3} \mathrm{O}_{5}^{+}, 338.1710$; found 338.1714. 


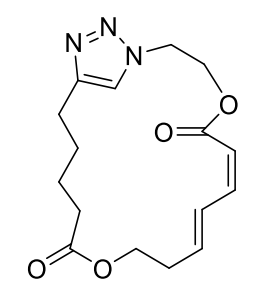

To a solution of seco acid 286 (19 mg, $0.056 \mathrm{mmol}, 1$ equiv) in THF ( $3 \mathrm{~mL}$ ) at room temperature was added triethylamine $(12 \mu \mathrm{L}, 0.084 \mathrm{mmol}, 2$ equiv) followed by 2,4,6trichlorobenzoyl chloride (13 $\mu \mathrm{L}, 0.084 \mathrm{mmol}, 1.5 \mathrm{equv})$. After stirring for 2 hours, The reaction mixture was diluted with $\mathrm{DCM}(16 \mathrm{~mL})$, cooled to $-78{ }^{\circ} \mathrm{C}$ (dry ice/acetone bath) and DMAP (2 mg, $0.02 \mathrm{mmol}, 0.3$ equiv) added. The reaction mixture was allowed to gradually warm to room temperature (over a few hours) and the mixture stirred overnight. The reaction was then quenched with saturated aqueous ammonium chloride $(3 \mathrm{~mL})$. The aqueous layer was extracted with EtOAc $(3 \times 10 \mathrm{~mL})$ and the combined organic fractions were dried $\left(\mathrm{MgSO}_{4}\right)$, filtered and concentrated under reduced pressure. The crude material was purified by silica gel flash chromatography (pet. ether:EtOAc) to provide the title compound $(4 \mathrm{mg}, 22 \%)$ as a pale yellow oil. Rf: 0.22 (EtOAc). ${ }^{1} \mathbf{H}$ NMR $\left(600 \mathrm{MHz}, \mathrm{CDCl}_{3}\right): \delta 7.26(\mathrm{~s}, 1 \mathrm{H}), 7.07(\mathrm{dd}, J=12.0,14.6 \mathrm{~Hz}, 1 \mathrm{H}), 6.56(\mathrm{t}, J=11.3 \mathrm{~Hz}, 1 \mathrm{H})$, $6.00(\mathrm{td}, J=7.2,15.2 \mathrm{~Hz}, 1 \mathrm{H}), 5.54(\mathrm{~d}, J=11.2 \mathrm{~Hz}, 1 \mathrm{H}), 4.62(\mathrm{~s}, 4 \mathrm{H}), 4.28-4.20(\mathrm{~m}, 2 \mathrm{H})$, $2.71(\mathrm{t}, J=7.1 \mathrm{~Hz}, 2 \mathrm{H}), 2.53-2.44(\mathrm{~m}, 2 \mathrm{H}), 2.29(\mathrm{t}, J=7.6 \mathrm{~Hz}, 2 \mathrm{H}), 1.68(\mathrm{dd}, J=7.4,15.0 \mathrm{~Hz}$, $2 \mathrm{H}), 1.56(\mathrm{td}, J=7.6,15.4 \mathrm{~Hz}, 2 \mathrm{H}) .{ }^{13} \mathrm{C}$ NMR (151 MHz, $\left.\mathrm{CDCl}_{3}\right): \delta$ 173.3, 165.1, 145.8, 142.1, 128.5, 128.2, 121.2, 115.0, 61.9, 61.0, 49.6, 34.7, 33.0, 28.4, 25.1, 24.2. IR (film): 2928, 2858, 1723, 1640, 1459, 1370, 1270, 1220, 1161, 1126, 1046, $731 \mathrm{~cm}^{-1}$. HRMS-ESI (m/z): [M + H] $]^{+}$ calculated for $\mathrm{C}_{16} \mathrm{H}_{22} \mathrm{~N}_{3} \mathrm{O}_{4}{ }^{+}, 320.1605$; found 320.1611 . 


\section{Preparation of 1-(Azidomethyl)-2-chloroethyl (E)-cinnamate, 278}<smiles>NCC(CCl)OC(=O)/C=C/c1ccccc1</smiles>

To a solution of alcohol 207 (902 mg, $7.3 \mathrm{mmol}, 1$ equiv) and (E)-cinnamic acid (1.30 g, $8.8 \mathrm{mmol}, 1.2$ equiv) in DCM $(50 \mathrm{~mL})$ at $0{ }^{\circ} \mathrm{C}$ (ice bath) was added EDCI (1.68 g, $8.8 \mathrm{mmol}, 1.2$ equiv) followed by DMAP (268 mg, $2.2 \mathrm{mmol}, 0.3$ equiv). The reaction mixture was stirred overnight at room temperature, then quenched with saturated aqueous ammonium chloride. The aqueous layer was extracted with DCM $(3 \times 30 \mathrm{~mL})$ and the combined organic fractions were dried $\left(\mathrm{MgSO}_{4}\right)$, filtered and concentrated under reduced pressure. The crude material was purified by silica gel flash chromatography (9:1 pet. ether:EtOAc) to provide the title compound $(1.50 \mathrm{~g}, 80 \%)$ as a colourless liquid. $\mathbf{R}_{\mathrm{f}}$ : 0.54 (2:1 pet. ether:EtOAc). ${ }^{1} \mathbf{H}$ NMR (500 MHz, $\left.\mathrm{CDCl}_{3}\right): \delta 7.77(\mathrm{~d}, J=16.1 \mathrm{~Hz}, 1 \mathrm{H}), 7.61$ $7.51(\mathrm{~m}, 2 \mathrm{H}), 7.46-7.35(\mathrm{~m}, 3 \mathrm{H}), 6.48(\mathrm{~d}, J=16.1 \mathrm{~Hz}, 1 \mathrm{H}), 5.26$ (quin, $J=5.1 \mathrm{~Hz}, 1 \mathrm{H}$ ), 3.81 - 3.72 (m, 2 H), 3.71 - 3.63 (m, 2 H). $\left.{ }^{13} \mathrm{C} \mathrm{NMR} \mathrm{(125} \mathrm{MHz,} \mathrm{CDCl}_{3}\right)$ : $\delta$ 165.7, 146.6, 134.0, 130.7, 128.9, 128.3, 116.7, 71.4, 50.9, 42.3. IR (film): 3029, 2968, 2104, 1714, 1635, 1282, 1202, 1154, $979,766,683 \mathrm{~cm}^{-1}$.

Preparation of 2-[4-(Acetoxymethyl)-1H-1,2,3-triazol-1-yl]-1-(chloromethyl)ethyl (E)cinnamate, 287

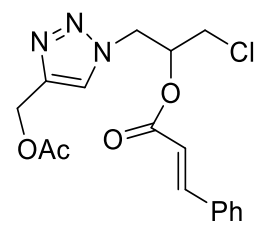

To an aqueous solution (30 mL) of $\mathrm{CuSO}_{4} .5 \mathrm{H}_{2} \mathrm{O}(142 \mathrm{mg}, 0.6 \mathrm{mmol}, 0.1$ equiv) and ascorbic acid (497 mg, $2.8 \mathrm{mmol}, 0.5$ equiv), that was neutralised to approximately $\mathrm{pH} 7$ with $1 \mathrm{M}$ aqueous $\mathrm{NaOH}$, was added a solution of propargyl acetate $(831 \mathrm{mg}$, $8.5 \mathrm{mmol}$, 1.5 equiv) and azide 278 (1.50 g, $5.7 \mathrm{mmol}, 1$ equiv) in THF (30 mL). The yellow reaction mixture was stirred overnight at room temperature which gradually turned yellow/green. 


\section{Chapter Six: Experimental}

The aqueous layer was extracted with EtOAc $(3 \times 30 \mathrm{~mL})$ and the combined organic fractions were dried $\left(\mathrm{MgSO}_{4}\right)$, filtered and concentrated under reduced pressure. The crude material was purified by silica gel flash chromatography (5:1 pet. ether:EtOAc) to provide the title compound $(1.57 \mathrm{~g}, 76 \%)$ as a slightly orange oil. Rf: 0.76 (2:1 pet. ether:EtOAc). ${ }^{1} \mathbf{H}$ NMR (500 MHz, $\left.\mathrm{CDCl}_{3}\right): \delta 7.73(\mathrm{~d}, J=16.0 \mathrm{~Hz}, 0 \mathrm{H}), 7.72(\mathrm{~s}, 1 \mathrm{H}), 7.60$ $7.52(\mathrm{~m}, 2 \mathrm{H}), 7.45$ - $7.38(\mathrm{~m}, 3 \mathrm{H}), 6.43(\mathrm{~d}, J=16.0 \mathrm{~Hz}, 1 \mathrm{H}), 5.53$ - $5.43(\mathrm{~m}, 1 \mathrm{H}), 5.26$ - 5.16 $(\mathrm{m}, 2 \mathrm{H}), 4.78(\mathrm{~d}, J=5.5 \mathrm{~Hz}, 3 \mathrm{H}), 3.75(\mathrm{dd}, J=5.5,12.3 \mathrm{~Hz}, 1 \mathrm{H}), 3.68(\mathrm{dd}, J=4.3,11.7 \mathrm{~Hz}, 1$ H), 2.03 (s, $2 \mathrm{H}) .{ }^{13} \mathrm{C}$ NMR (125 MHz, $\left.\mathrm{CDCl}_{3}\right): \delta$ 170.9, 165.5, 147.0, 133.8, 130.9, 129.0, 128.3, 125.0, 116.2, 116.2, 70.8, 57.4, 50.1, 42.4, 20.8. IR (film): 2961, 1715, 1634, 1230, 1203, 1154, 1037, 980, 911, 767, 730, $684 \mathrm{~cm}^{-1}$. HRMS-ESI (m/z): $[\mathrm{M}+\mathrm{H}]^{+}$calculated for $\mathrm{C}_{17} \mathrm{H}_{19} \mathrm{ClN}_{3} \mathrm{O}_{4}{ }^{+}$, 364.1059; found 364.1066.

\section{Preparation of 1-(Azidomethyl)-2-(1-phenyl-1H-1,2,3,4-tetrazol-5-ylthio)ethyl} (E) cinnamate, 289

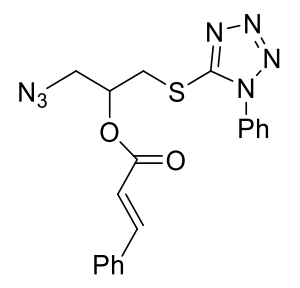

To a solution of alcohol 208 (413 mg, $1.5 \mathrm{mmol}, 1$ equiv) and cinnamic acid (265 $\mathrm{mg}, 1.8 \mathrm{mmol}, 1.2$ equiv) in DCM (20 mL) at $0{ }^{\circ} \mathrm{C}$ (ice bath) was added EDCI (343 mg, 1.8 mmol, 1.2 equiv) followed by DMAP ( $55 \mathrm{mg}, 0.45 \mathrm{mmol}, 0.3$ equiv). The reaction mixture was stirred overnight at room temperature, then quenched with saturated aqueous ammonium chloride. The aqueous layer was extracted with DCM $(3 \times 10 \mathrm{~mL})$ and the combined organic fractions were dried $\left(\mathrm{MgSO}_{4}\right)$, filtered and concentrated under reduced pressure. The crude material was purified by silica gel flash chromatography (pet. ether:EtOAc) to provide the title compound $(603 \mathrm{mg}, 99 \%)$ as a pale yellow oil. $\mathbf{R}_{\mathbf{f}} 0.33$ (2:1 pet. ether:EtOAc). ${ }^{1} \mathbf{H}$ NMR $\left(500 \mathrm{MHz}, \mathrm{CDCl}_{3}\right): \delta 7.69(\mathrm{~d}, J=16.0 \mathrm{~Hz}, 1 \mathrm{H}), 7.59-7.47$ $(\mathrm{m}, 8 \mathrm{H}), 7.41(\mathrm{br} \mathrm{s}, 3 \mathrm{H}), 6.41(\mathrm{~d}, J=16.0 \mathrm{~Hz}, 1 \mathrm{H}), 5.51(\mathrm{qd}, J=4.0,8.0 \mathrm{~Hz}, 1 \mathrm{H}), 3.84(\mathrm{dd}, J$ $=4.3,14.2 \mathrm{~Hz}, 1 \mathrm{H}), 3.74(\mathrm{dd}, J=3.5,13.4 \mathrm{~Hz}, 1 \mathrm{H}), 3.70$ - $3.59(\mathrm{~m}, 2 \mathrm{H}) .{ }^{13} \mathrm{C}$ NMR $(125 \mathrm{MHz}$, $\left.\mathrm{CDCl}_{3}\right): \delta 165.7,153.4,146.5,133.9,133.3,130.7,130.3,129.8,128.9,128.2,123.9,116.6,70.5$, 52.6, 34.2. IR (film): 2931, 2250, 2102, 1687, 1637, 1597, 1499, 1451, 1386, 1280, 1244, 1090, 
907, 760, $726 \mathrm{~cm}^{-1}$. HRMS-ESI (m/z): $[\mathrm{M}+\mathrm{H}]^{+}$calculated for $\mathrm{C}_{19} \mathrm{H}_{18} \mathrm{~N}_{7} \mathrm{O}_{2} \mathrm{~S}^{+}, 408.1237$; found 408.1224 .

\section{Preparation of 2-[4-(Benzoyloxymethyl)-1H-1,2,3-triazol-1-yl]-1-[(1-phenyl-1H-1,2,3,4-} tetrazol-5-ylthio)methyl]ethyl (E)cinnamate, 291

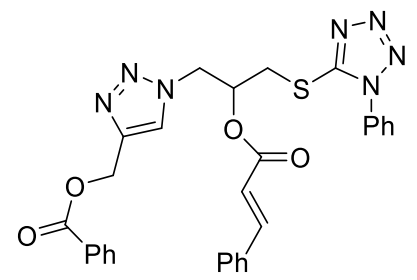

To an aqueous solution $(5 \mathrm{~mL})$ of $\mathrm{CuSO}_{4} .5 \mathrm{H}_{2} \mathrm{O}(16 \mathrm{mg}, 0.06 \mathrm{mmol}, 0.2)$ and ascorbic acid ( $26 \mathrm{mg}, 0.15 \mathrm{mmol}, 0.5$ equiv), that was neutralised to $\mathrm{pH} 7$ with aqueous $\mathrm{NaOH}$, was added a solution of propargyl benzoate (146 mg, $0.9 \mathrm{mmol}, 3$ equiv) and azide 289 (124 mg, $0.3 \mathrm{mmol}, 1$ equiv) in THF ( $5 \mathrm{~mL})$. The yellow reaction mixture was stirred overnight at room temperature which gradually turned yellow/green. The aqueous layer was extracted with EtOAc $(3 \times 5 \mathrm{~mL})$ and the combined organic fractions were dried $\left(\mathrm{MgSO}_{4}\right)$, filtered and concentrated under reduced pressure. The crude material was purified by silica gel flash chromatography (pet. ether:EtOAc) to provide the title compound (135 mg, 79\%) as a colourless oil. Rf: 0.33 (1:1 pet. ether:EtOAc). ${ }^{1} \mathbf{H}$ NMR (500 $\left.\mathrm{MHz}, \mathrm{CDCl}_{3}\right): \delta 8.00(\mathrm{~d}, J=7.7 \mathrm{~Hz}, 2 \mathrm{H}), 7.80(\mathrm{~s}, 1 \mathrm{H}), 7.63(\mathrm{~d}, J=16.0 \mathrm{~Hz}, 1 \mathrm{H}), 7.59-7.47$ $(\mathrm{m}, 8 \mathrm{H}), 7.47$ - $7.32(\mathrm{~m}, 5 \mathrm{H}), 6.34(\mathrm{~d}, J=15.7 \mathrm{~Hz}, 1 \mathrm{H}), 5.78-5.69(\mathrm{~m}, 1 \mathrm{H}), 5.53$ - $5.43(\mathrm{~m}, 2$ H), $4.91-4.78(\mathrm{~m}, 2 \mathrm{H}), 3.82(\mathrm{dd}, J=4.6,14.6 \mathrm{~Hz}, 1 \mathrm{H}), 3.53(\mathrm{dd}, J=7.4,14.4 \mathrm{~Hz}, 1 \mathrm{H}) .{ }^{13} \mathrm{C}$ NMR (125 MHz, $\left.\mathrm{CDCl}_{3}\right): \delta$ 166.4, 165.5, 153.3, 147.0, 143.4, 133.8, 133.3, 133.2, 130.9, 130.4, 129.9, 129.7, 129.0, 128.4, 125.3, 123.8, 116.1, 69.9, 58.0, 51.5, 34.0, 14.2. IR (film): 3063, 1714, 1634, 1499, 1270, 1154, 1107, 765, 712, $685 \mathrm{~cm}^{-1}$. HRMS-ESI (m/z): $[\mathrm{M}+\mathrm{H}]^{+}$calculated for $\mathrm{C}_{29} \mathrm{H}_{26} \mathrm{~N}_{7} \mathrm{O}_{4} \mathrm{~S}^{+}, 568.1761$; found 568.1742 . 


\section{Chapter Six: Experimental}

\section{Preparation of 2-[4-(Benzoyloxymethyl)-1H-1,2,3-triazol-1-yl]-1-[(1-phenyl-1H-1,2,3,4-}

tetrazol-5-ylsulfonyl)methyl]ethyl (E)cinnamate, 292

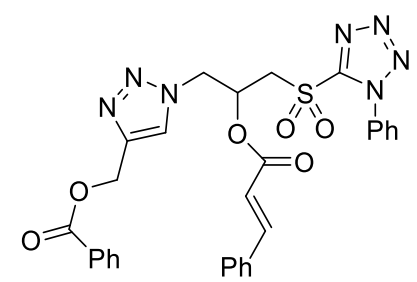

To a solution of thioether 291 (38 $\mathrm{mg}, 0.064 \mathrm{mmol}, 1$ equiv) and ammonium heptamolybdate (18 mg, $0.014 \mathrm{mmol}, 0.2$ equiv) in ethanol $(4 \mathrm{~mL})$ at $0{ }^{\circ} \mathrm{C}$ (ice bath) was added aqueous $\mathrm{H}_{2} \mathrm{O}_{2}$ (30 wt $\%, 100 \mu \mathrm{L}$, approximately 12 equiv) and the mixture stirred overnight at room temperature. The reaction was quenched with saturated aqueous sodium bisulfite $(0.1 \mathrm{~mL})$ and ethanol removed under reduced pressure. The crude mixture was then redissolved in water $(5 \mathrm{~mL})$ and extracted with EtOAc $(2 \times 10 \mathrm{~mL})$. The combined organic fractions were dried $\left(\mathrm{MgSO}_{4}\right)$, filtered and concentrated under reduced pressure. The crude material was purified by silica gel flash chromatography (2: 1 pet. ether:EtOAc) to provide the title compound (34 $\mathrm{mg}, 85 \%)$ as a oil. $\mathbf{R}_{\mathbf{f}}: 0.40$ (1:1 pet. ether:EtOAc). ${ }^{1} \mathbf{H}$ NMR (500 MHz, CDCl $): \delta 8.01$ (d, J = $\left.7.8 \mathrm{~Hz}, 2 \mathrm{H}\right), 7.79$ (s, $\left.1 \mathrm{H}\right), 7.69$ $7.48(\mathrm{~m}, 9 \mathrm{H}), 7.46-7.33(\mathrm{~m}, 5 \mathrm{H}), 6.25(\mathrm{~d}, J=16.1 \mathrm{~Hz}, 1 \mathrm{H}), 5.94(\mathrm{dd}, J=3.8,7.7 \mathrm{~Hz}, 1 \mathrm{H})$, 5.55 - $5.45(\mathrm{~m}, 3 \mathrm{H}), 4.90(\mathrm{dd}, J=4.2,14.6 \mathrm{~Hz}, 1 \mathrm{H}), 4.82(\mathrm{dd}, J=4.6,14.6 \mathrm{~Hz}, 1 \mathrm{H}), 4.30(\mathrm{dd}, J$ $=3.2,15.6 \mathrm{~Hz}, 1 \mathrm{H}), 4.01(\mathrm{dd}, J=7.9,15.6 \mathrm{~Hz}, 1 \mathrm{H}) .{ }^{13} \mathrm{C}$ NMR $\left(125 \mathrm{MHz}, \mathrm{CDCl}_{3}\right): \delta$ 166.4, 165.0, 153.4, 147.7, 143.6, 133.6, 133.2, 132.7, 131.6, 131.1, 130.1, 129.8, 129.7, 129.6, 129.0, 128.5, 128.4, 125.5, 125.1, 115.4, 65.9, 57.9, 56.8, 51.6. IR (film): 3317, 1715, 1634, 1352, 1270 , 1148, 909, 764, 730, 711, $687 \mathrm{~cm}^{-1}$. HRMS-ESI (m/z): $[\mathrm{M}+\mathrm{H}]^{+}$calculated for $\mathrm{C}_{29} \mathrm{H}_{26} \mathrm{~N}_{7} \mathrm{O}_{6} \mathrm{~S}^{+}$, 600.1660; found 600.1653. 


\section{Preparation of 5-(3-Chloro-2-(tert-butyldimethylsilyloxy)propylthio)-1-phenyl-1H-}

\section{1,2,3,4-tetrazole, 297}

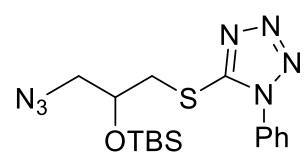

To a solution of alcohol 208 (435 mg, $1.57 \mathrm{mmol}, 1$ equiv) in DCM (16 mL) at $0{ }^{\circ} \mathrm{C}$ (ice bath) was added imidizole (214 mg, $3.14 \mathrm{mmol}, 2$ equiv) followed by TBSOTf (432 $\mu \mathrm{L}$, $498 \mathrm{mg}$, $1.88 \mathrm{mmol}, 1.2$ equiv). The reaction mixture was allowed to warm to room temperature, stirred overnight and quenched with saturated aqueous ammonium chloride $(16 \mathrm{~mL})$. The aqueous layer was extracted with DCM $(3 \times 10 \mathrm{~mL})$. The combined organic fractions were dried $\left(\mathrm{MgSO}_{4}\right)$, filtered and the solvent removed under reduced pressure. Silica gel flash chromatography (5:1 to 2:1 pet. ether:EtOAc) provided the title compound $(475 \mathrm{~g}, 70 \%)$ as a colourless oil that slowly crystallised during storage in freezer. Rf: 0.74 in 2:1 (pet. ether:EtOAc). ${ }^{1} \mathbf{H}$ NMR (500 MHz, $\left.\mathrm{CDCl}_{3}\right): \delta 7.58$ (m, 5H), 4.32 $(\mathrm{m}, 1 \mathrm{H}), 3.55(\mathrm{~d}, J=5.7 \mathrm{~Hz}, 2 \mathrm{H}), 3.49(\mathrm{dd}, J=12.7$ and $4.4 \mathrm{~Hz}, 1 \mathrm{H}), 3.34(\mathrm{dd}, J=12.7$ and 4.4 $\mathrm{Hz}, 1 \mathrm{H}), 0.90$ (s, 9H), 0.14 (s, 3H), 0.12 (s, 3H). $\left.{ }^{13} \mathrm{C} \mathrm{NMR} \mathrm{(125} \mathrm{MHz,} \mathrm{CDCl}_{3}\right): \delta$ 154.0, 133.5, 130.2, 130.0, 123.7, 70.0, 55.3, 36.9, 25.7, 25.6, 17.9, -3.6, -4.7. Melting point: $49-51{ }^{\circ} \mathrm{C}$. IR (film): 2930, 2857, 2360, 2101, 1597, 1500, 1463, 1388, 1281, 1252, 1106, 961, 837, 807, 778, 760, $694 \mathrm{~cm}^{-1}$. HRMS-ESI (m/z): $[\mathrm{M}+\mathrm{H}]^{+}$calculated for $\mathrm{C}_{16} \mathrm{H}_{25} \mathrm{~N}_{7} \mathrm{OSSi}^{+}, 392.1683$; found 392.1678.

Preparation of 5-(3-Chloro-2-(tert-butyldimethylsilyloxy)propylsulfonyl)-1-phenyl-1H1,2,3,4-tetrazole, 296

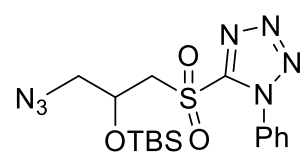

To a solution of thioether 297 ( $8.09 \mathrm{~g}, 20.0 \mathrm{mmol}, 1$ equiv) in absolute ethanol (100 $\mathrm{mL}$ ) was added ammonium heptamolybdate ( $3.8 \mathrm{~g}, 3.1 \mathrm{mmol}, 0.15$ equiv). The reaction mixture was then cooled to $0{ }^{\circ} \mathrm{C}$ (ice bath) and added $\mathrm{H}_{2} \mathrm{O}_{2}(30 \%$ aqueous solution, $1.3 \mathrm{~mL}$, 
approximately $12.0 \mathrm{mmol}$ ) and the reaction stirred at room temperature. The reaction progress was followed by TLC, observing formation of slower running spot of sulfoxide ( $\mathbf{R}_{\mathrm{f}:} 0.53$ in 2:1 (pet. ether:EtOAc)) and then observing the loss of this spot as it converts to the desired sulfone. Sulfide and sulfone, with same $\mathrm{R}_{\mathrm{f}}$, were distinguishable as the sulfone was UV active and reacted instantly with $\mathrm{KMnO}_{4}$ dip, and sulfide was only UV active. The reaction mixture was stirred overnight or until reaction was complete by TLC, adding extra $\mathrm{H}_{2} \mathrm{O}_{2}$ solution if needed. When complete, peroxides were quenched with saturated aqueous $\mathrm{Na}_{2} \mathrm{SO}_{3}(15 \mathrm{~mL})$, ethanol removed under reduced pressure and diluted with distilled water $(80 \mathrm{~mL})$ and extracted with DCM $(3 \times 60 \mathrm{~mL})$. The combined organic fractions were dried $\left(\mathrm{MgSO}_{4}\right)$, filtered and the solvent removed under reduced pressure to provide the title compound $(7.6 \mathrm{~g}, 90 \%)$ as a colourless oil that slowly crystallised during storage in freezer. $\mathbf{R}_{\mathbf{f}}$ 0.74 in 2:1 (pet. ether:EtOAc). ${ }^{1} \mathbf{H}$ NMR $\left(500 \mathrm{MHz}, \mathrm{CDCl}_{3}\right)$ : $\delta$ 7.69$7.62(\mathrm{~m}, 5 \mathrm{H}), 4.60(\mathrm{~m}, 1 \mathrm{H}), 4.04(\mathrm{dd}, J=15.2$ and 5.6, 2H), $3.57(\mathrm{dd}, J=12.8$ and $3.8,1 \mathrm{H})$, $3.41\left(\mathrm{dd}, J=12.8\right.$ and 3.8, 1H), $0.89(\mathrm{~s}, 9 \mathrm{H}), 0.15(\mathrm{~s}, 3 \mathrm{H}), 0.12(\mathrm{~s}, 3 \mathrm{H}) .{ }^{13} \mathrm{C}$ NMR $(125 \mathrm{MHz}$, $\left.\mathrm{CDCl}_{3}\right): \delta 153.8,132.9,131.6,129.8,125.0,66.4,60.0,55.8,25.6,17.8,-4.7,-5.0$. Melting point: $40-42^{\circ} \mathrm{C}$. IR (film): 2931, 2858, 2105, 1498, 1463, 1343, 1287, 1255, 1154, 1104, 961, $839,808,780,762,731,688 \mathrm{~cm}^{-1}$. HRMS-ESI (m/z): $[\mathrm{M}+\mathrm{H}]^{+}$calculated for $\mathrm{C}_{16} \mathrm{H}_{26} \mathrm{~N}_{7} \mathrm{O}_{3} \mathrm{SSi}^{+}$, 424.1582; found 424.1571.

\section{Preparation of (1E,3E)-6-Azido-5-(tert-butyldimethylsilyloxy)-1-phenyl-1,3-hexadiene}

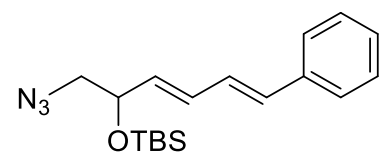

To a solution of sulfone 296 (33 mg, $0.08 \mathrm{mmol}, 1$ equiv) in THF $(5 \mathrm{~mL})$ at $-78{ }^{\circ} \mathrm{C}$ (dry ice/acetone bath) was added KHMDS (of a 0.5 M solution in toluene, $200 \mu \mathrm{L}, 0.10$ mmol, 1.3 equiv) and the reaction stirred for 15 minutes. Cinnamaldehyde (13 mg, 0.10 mmol, 1.3 equiv) in THF ( $1 \mathrm{~mL})$ was then added dropwise to the cold reaction mixture and stirred for two hours. The reaction was allowed to warm to room temperature before quenching with saturated aqueous ammonium chloride and extracting with DCM $(3 \times 5$ $\mathrm{mL})$. The combined organic fractions was washed with water then brine, dried $\left(\mathrm{MgSO}_{4}\right)$, filtered and concentrated under reduced pressure. The crude material was purified by silica gel flash chromatography (9:1 pet. ether:EtOAc) to provide the title compound (17 
mg, 65\%) as a yellow oil. $\mathbf{R}_{\mathbf{f}} 0.76$ (5:1 pet. ether:EtOAc). ${ }^{1} \mathbf{H}$ NMR (500 MHz, $\left.\mathrm{CDCl}_{3}\right): \delta 7.41$ $(\mathrm{d}, J=7.7 \mathrm{~Hz}, 2 \mathrm{H}), 7.33(\mathrm{t}, J=7.4 \mathrm{~Hz}, 2 \mathrm{H}), 7.28-7.20(\mathrm{~m}, 1 \mathrm{H}), 6.77(\mathrm{dd}, J=10.5,15.8 \mathrm{~Hz}, 1$ H), $6.57(\mathrm{~d}, J=15.8 \mathrm{~Hz}, 1 \mathrm{H}), 6.43(\mathrm{dd}, J=10.7,15.0 \mathrm{~Hz}, 1 \mathrm{H}), 5.77(\mathrm{dd}, J=6.2,15.2 \mathrm{~Hz}, 1 \mathrm{H})$, $4.39(\mathrm{~d}, J=5.6 \mathrm{~Hz}, 1 \mathrm{H}), 3.22(\mathrm{~d}, J=4.3 \mathrm{~Hz}, 2 \mathrm{H}), 0.95(\mathrm{~s}, 10 \mathrm{H}), 0.15(\mathrm{~s}, 3 \mathrm{H}), 0.10(\mathrm{~s}, 3 \mathrm{H}) .{ }^{13} \mathrm{C}$ NMR (125 MHz, $\left.\mathrm{CDCl}_{3}\right)$ : $\delta$ 137.0, 133.2, 131.8, 128.7, 128.6, 127.9, 127.7, 126.4, 73.0, 57.3, 25.8, 18.2, -4.5, -4.9. IR (film): 2955, 2929, 2857, 2099, 1284, 1256, 1105, 989, 957, 909, 837, $810,778,734,691 \mathrm{~cm}^{-1}$.

\section{Preparation of Ethyl (2E,4E)-6-hydroxy-2,4-hexadienoate, 302}

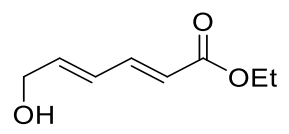

To a solution of silyl ether $228(795 \mathrm{mg}, 2.94 \mathrm{mmol})$ in $\mathrm{EtOH}(40 \mathrm{~mL})$ at room temperature was added concentrated aqueous $\mathrm{HCl}(0.5 \mathrm{~mL})$ and the reaction stirred for 1 hour. The reaction was then quenched with aqueous saturated $\mathrm{NaHCO}_{3}(1 \mathrm{~mL})$ and solvent was removed under reduced pressure. The crude material was purified by silica gel flash chromatography (5:1 pet. ether:EtOAc) to provide the title compound (427 mg, 93\%) as a colourless oil. $\mathbf{R}_{\mathrm{f}:} 0.37$ (2:1 pet. ether:EtOAc). ${ }^{1} \mathbf{H} \mathbf{N M R}\left(500 \mathrm{MHz}, \mathrm{CDCl}_{3}\right): \delta 7.29$ $(\mathrm{dd}, J=10.9,15.1 \mathrm{~Hz}, 1 \mathrm{H}), 6.41(\mathrm{dd}, J=11.1,15.3 \mathrm{~Hz}, 1 \mathrm{H}), 6.22(\mathrm{td}, J=4.8,15.3 \mathrm{~Hz}, 1 \mathrm{H})$, $5.89(\mathrm{~d}, J=15.5 \mathrm{~Hz}, 1 \mathrm{H}), 4.29(\mathrm{~d}, J=4.0 \mathrm{~Hz}, 2 \mathrm{H}), 4.20(\mathrm{q}, J=7.2 \mathrm{~Hz}, 3 \mathrm{H}), 1.30(\mathrm{t}, J=7.1 \mathrm{~Hz}$, $4 \mathrm{H}) .{ }^{13} \mathrm{C}$ NMR $\left(125 \mathrm{MHz}, \mathrm{CDCl}_{3}\right): \delta 167.0,143.7,141.0,127.7,121.4,62.6,60.4,14.3$. These characterisation data match those in the literature. ${ }^{137}$

\section{Preparation of Ethyl (2E,4E)-6-hydroxy-2,4-hexadienoate, 301 (via $1^{\text {st }}$ route)}<smiles>CCOC(=O)/C=C/C=C/C=O</smiles>

To a solution of alcohol $302(130 \mathrm{mg}, 0.83 \mathrm{~mol}, 1$ equiv) in DCM (10 mL) at room temperature was added DMSO (590 $\mu \mathrm{L}, 8.3 \mathrm{mmol}, 10$ equiv) and triethylamine (580 $\mu \mathrm{L}$, $4.2 \mathrm{mmol}, 5$ equiv). The reaction mixture was cooled to $0{ }^{\circ} \mathrm{C}$ (ice bath) and added $\mathrm{SO}_{3} . \mathrm{Pyr}$ (397 mg, $2.5 \mathrm{mmol}, 3$ equiv) and the reaction stirred for 1 hour before warming to room temperature and stirring for another 1 hour. The reaction was quenched with saturated 


\section{Chapter Six: Experimental}

aqueous ammonium chloride and extracted with DCM $(3 \times 10 \mathrm{~mL})$. The combined organic fractions were washed with water then brine, dried $\left(\mathrm{MgSO}_{4}\right)$, filtered and concentrated under reduced pressure. The crude material was purified by silica gel flash chromatography (5:1 pet. ether:EtOAc) to provide the title compound (98 $\mathrm{mg}, 77 \%$ ) as a pale yellow liquid. $\mathbf{R}_{\mathrm{f}} 0.61$ (2:1 pet. ether:EtOAc). ${ }^{1} \mathbf{H}$ NMR $\left(500 \mathrm{MHz}, \mathrm{CDCl}_{3}\right): \delta 9.67(\mathrm{~d}, J=$ $7.9 \mathrm{~Hz}, 1 \mathrm{H}), 7.42(\mathrm{dd}, J=11.3,15.3 \mathrm{~Hz}, 1 \mathrm{H}), 7.17(\mathrm{dd}, J=11.3,15.3 \mathrm{~Hz}, 1 \mathrm{H}), 6.42(\mathrm{dd}, J=$ 7.8, 15.4 Hz, $1 \mathrm{H}), 6.31(\mathrm{~d}, J=15.5 \mathrm{~Hz}, 1 \mathrm{H}), 4.26(\mathrm{q}, J=7.1 \mathrm{~Hz}, 2 \mathrm{H}), 1.32(\mathrm{t}, J=7.2 \mathrm{~Hz}, 3 \mathrm{H})$. ${ }^{13} \mathrm{C}$ NMR (125 MHz, $\left.\mathrm{CDCl}_{3}\right): \delta 192.9,165.4,147.2,140.3,136.9,129.9,61.1,14.2$. These characterisation data match those in the literature. ${ }^{137}$

\section{Scalable Route to Aldehyde Compound 301}

Preparation of fumaraldehyde bis(dimethyl acetal), 306

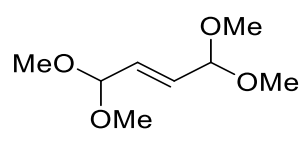

To a solution of furan $(9.40 \mathrm{~mL}, 129 \mathrm{mmol}, 1$ equiv) in dry methanol $(60 \mathrm{~mL})$ at -45 ${ }^{\circ} \mathrm{C}$ (dry ice/acetone bath) was added dropwise a solution of bromine $(6.8 \mathrm{~mL}, 133 \mathrm{mmol}$, 1.03 equiv) in dry methanol $(60 \mathrm{~mL})$. The reaction was then stirred for 3 hours maintaining the temperature between -40 and $-10{ }^{\circ} \mathrm{C}$ (dry ice/acetone bath). Anhydrous sodium carbonate (40 g) was then added portionwise over about 30 minutes and then stirred overnight at room temperature. The mixture was filtered and the solvent was removed under reduced pressure. Crude material was then purified by distillation in a kugelrohr apparatus $\left(65-70{ }^{\circ} \mathrm{C}, 1 \mathrm{mmHg}\right)$, providing title compound $(18.02 \mathrm{~g}, 79 \%)$ as a colourless liquid. $\mathbf{R}_{\mathbf{f}} 0.52$ (2:1 pet. ether:EtOAc). ${ }^{1} \mathbf{H}$ NMR (500 MHz, $\mathrm{CDCl}_{3}$ ): $\delta 5.81$ (s, 2 $\mathrm{H}), 4.82(\mathrm{~s}, 2 \mathrm{H}), 3.31$ (s, $12 \mathrm{H}) .{ }^{13} \mathrm{C}$ NMR (125 MHz, $\left.\mathrm{CDCl}_{3}\right): \delta$ 130.9, 101.9, 52.7. These characterisation data match those in the literature. ${ }^{179}$ 


\section{Preparation of $(E)$-4,4-dimethoxybut-2-enal, 307}<smiles>COC(C=CC=O)OC</smiles>

To a solution of fumaraldehyde bis(dimethyl acetal) 306 (3.81 g, $21.6 \mathrm{mmol}, 1$ equiv) in acetone at room temperature was added Amberlyst-15 (800 mg) and water (1.2 $\mathrm{mL}, 64.9 \mathrm{mmol}, 3$ equiv). The reaction was stirred vigorously for 10 minutes before removal of acidic resin by filtration. Solvent was removed under reduced pressure and purified by silica gel flash chromatography (4:1 pet. ether:diethyl ether) to provide the title compound (1.95g, 69\%) as a colourless oil. $\mathbf{R}_{\mathbf{f}} 0.48$ (2:1 pet. ether:EtOAc). ${ }^{1} \mathbf{H}$ NMR $\left(500 \mathrm{MHz}, \mathrm{CDCl}_{3}\right): \delta 9.62(\mathrm{~d}, J=7.9 \mathrm{~Hz}, 1 \mathrm{H}), 6.63(\mathrm{dd}, J=3.9,16.0 \mathrm{~Hz}, 1 \mathrm{H}), 6.37(\mathrm{dd}, J=$ 7.8, $15.9 \mathrm{~Hz}, 1 \mathrm{H}), 6.07(\mathrm{~d}, J=5.6 \mathrm{~Hz}, 1 \mathrm{H}), 3.36$ (s, $6 \mathrm{H}) .{ }^{13} \mathrm{C}$ NMR $\left(125 \mathrm{MHz}, \mathrm{CDCl}_{3}\right): \delta$ 193.2, 150.4, 134.2, 100.4, 53.0. These characterisation data match those in the literature. ${ }^{180}$

\section{Preparation of Ethyl (2E,4E)-6,6-dimethoxy-2,4-hexadienoate, 308}<smiles>CCOC(=O)/C=C/C=C/C(OC)O[C@@H](O)CC</smiles>

To a solution of aldehyde 307 (2.27 g, $17.5 \mathrm{mmol}, 1$ equiv) in DCM (40 mL) at room temperature was added triphenylphosphorane ylide 219 (6.0 g, $17.5 \mathrm{mmol}, 1$ equiv) and the mixture stirred overnight. Solvent was then removed under reduced pressure and the crude material was passed through a short silica plug (5:1 pet. ether:EtOAc) to remove triphenylphosphine oxide by-product. Solvent was then removed under reduced pressure and the residue was used in the subsequent reaction. A small sample was purified by silica gel flash chromatography (5:1 pet. ether:EtOAc) to provide a sample for NMR spectra. Rf: 0.61 (2:1 pet. ether:EtOAc). ${ }^{1} \mathbf{H}$ NMR (500 MHz, $\left.\mathrm{CDCl}_{3}\right): \delta 7.28(\mathrm{dd}, J=11.0$, $15.5 \mathrm{~Hz}, 1 \mathrm{H}), 6.49(\mathrm{dd}, J=11.3,15.5 \mathrm{~Hz}, 1 \mathrm{H}), 6.00(\mathrm{dd}, J=4.1,15.6 \mathrm{~Hz}, 1 \mathrm{H}), 5.95(\mathrm{~d}, J=$ $15.6 \mathrm{~Hz}, 1 \mathrm{H}), 4.92(\mathrm{~d}, J=4.1 \mathrm{~Hz}, 1 \mathrm{H}), 4.22(\mathrm{q}, J=7.1 \mathrm{~Hz}, 2 \mathrm{H}), 3.33(\mathrm{~s}, 6 \mathrm{H}), 1.30(\mathrm{t}, J=7.1$ $\mathrm{Hz}, 3 \mathrm{H}) .{ }^{13} \mathrm{C}$ NMR $\left(125 \mathrm{MHz}, \mathrm{CDCl}_{3}\right): \delta$ 166.7, 142.9, 137.3, 131.1, 123.2, 101.3, 60.5, 52.7, 14.3. These characterisation data match those in the literature. ${ }^{181}$ 


\section{Preparation of Ethyl (2E,4E)-6-oxo-2,4-hexadienoate, 301}

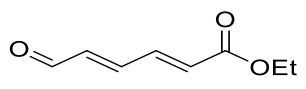

To a solution of dimethyl acetal 308 (from previous reaction) in acetone at room temperature was added Amberlyst $15(1 \mathrm{~g})$ and water $(1.3 \mathrm{~mL}, 70 \mathrm{mmol}, 4$ equiv). The reaction was stirred vigorously for 60 minutes at $60^{\circ} \mathrm{C}$. After removal of acidic resin by filtration, solvent was removed under reduced pressure and purified by silica gel flash chromatography (4:1 pet. ether : diethyl ether) to provide the title compound $(1.95 \mathrm{~g}, 69 \%)$ as a colourless oil. $\mathbf{R}_{\mathbf{f}} 0.61$ (2:1 pet. ether:EtOAc). Proton and carbon NMR data matched those of previous synthesis and literature. ${ }^{137}$

\section{Preparation of Ethyl (2E,4E,6E)-9-azido-8-(tert-butyldimethylsilyloxy)-2,4,6-} nonatrienoate, 300

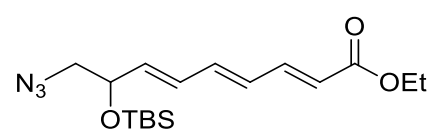

To a solution of sulfone 296 (5.69 g, $13.4 \mathrm{mmol}, 1$ equiv) in THF $(80 \mathrm{~mL})$ at $-78{ }^{\circ} \mathrm{C}$ (dry ice/acetone bath) was added KHMDS (of a $0.5 \mathrm{M}$ solution in toluene, $40.2 \mathrm{~mL}, 20.1$ mmol, 1.5 equiv) and the reaction stirred for 15 minutes. Aldehyde 301 (2.28 g, $14.8 \mathrm{mmol}$, 1.1 equiv) in THF (30 mL) was then added slowly to the reaction mixture and allowed to stir for 1 hour at $-78^{\circ} \mathrm{C}$, before slowly warming to room temperature over 30 minutes. The reaction was then quenched with saturated aqueous ammonium chloride $(80 \mathrm{~mL})$ and extracted three times with EtOAc $(3 \times 50 \mathrm{~mL})$. The combined organic fractions were then washed with water then brine, dried $\left(\mathrm{MgSO}_{4}\right)$, filtered and the solvent removed under reduced pressure. Silica gel flash chromatography (5:1 pet. ether:EtOAc) provided the title compound (3.05 g, 65\%) as a colourless oil. $\mathbf{R}_{\mathrm{f}} 0.77$ (2:1 pet. ether:EtOAc). ${ }^{1} \mathbf{H}$ NMR (500 MHz, $\left.\mathrm{CDCl}_{3}\right): \delta 7.34$ - $7.25(\mathrm{~m}, 1 \mathrm{H}), 6.54(\mathrm{dd}, J=11.0,14.8 \mathrm{~Hz}, 1 \mathrm{H}), 6.36(\mathrm{dd}, J=11.2$, $21.2 \mathrm{~Hz}, 2 \mathrm{H}), 6.33(\mathrm{dd}, J=11.1,20.9 \mathrm{~Hz}, 1 \mathrm{H}), 5.90(\mathrm{~d}, J=15.6 \mathrm{~Hz}, 1 \mathrm{H}), 5.88-5.83(\mathrm{~m}, 1 \mathrm{H})$, $4.38(\mathrm{~d}, J=5.2 \mathrm{~Hz}, 1 \mathrm{H}), 4.21(\mathrm{q}, J=7.0 \mathrm{~Hz}, 2 \mathrm{H}), 3.24-3.15(\mathrm{~m}, 2 \mathrm{H}), 1.30(\mathrm{t}, J=7.1 \mathrm{~Hz}, 3 \mathrm{H})$, 0.93 (s, 9 H), 0.12 (s, 4 H), 0.07 (s, 3 H). $\left.{ }^{13} \mathrm{C} \mathrm{NMR} \mathrm{(125} \mathrm{MHz,} \mathrm{CDCl}\right): \delta 166.9,144.0,139.2$, 137.2, 130.6, 130.6, 121.5, 72.6, 60.3, 56.9, 25.7, 18.1, 14.3, -4.6, -5.0. IR (film): 2955, 2930, 
2858, 2099, 1708, 1620, 1254, 1112, 1005, 956, 836, $778 \mathrm{~cm}^{-1}$. HRMS-ESI $(\mathrm{m} / \mathrm{z}):[\mathrm{M}+\mathrm{H}]^{+}$ calculated for $\mathrm{C}_{17} \mathrm{H}_{30} \mathrm{~N}_{3} \mathrm{O}_{3} \mathrm{Si}^{+}$, 352.2051; found 352.2044.

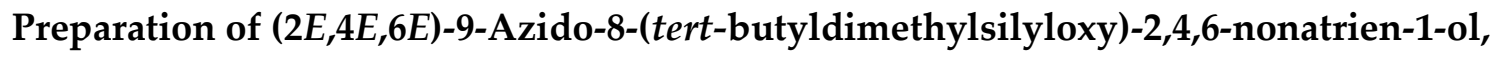
309

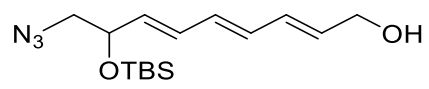

To a solution of ethyl ester 300 (701 mg, $2.0 \mathrm{mmol}, 1$ equiv) in THF (30 mL) at $78^{\circ} \mathrm{C}$ (dry ice/acetone bath) was added a solution of DIBAL-H $(4.0 \mathrm{~mL}, 4.0 \mathrm{mmol}, 2$ equiv, $1 \mathrm{M}$ in hexane). The reaction mixture was allowed to slowly warm to room temperature over 2 hours, before the reaction was quenched by slow addition of saturated potassium sodium tartrate (Rochelle's salt, $30 \mathrm{~mL}$ ) and the mixture stirred for 2 hours. The aqueous fraction was then extracted with EtOAc $(3 \times 30 \mathrm{~mL})$, and the combined organic fractions were dried $\left(\mathrm{MgSO}_{4}\right)$, filtered, then concentrated under reduced pressure. ${ }^{1} \mathrm{H}$ NMR indicated material was sufficiently pure, thus no further purification was necessary, providing title compound (595 $\mathrm{mg}, 96 \%$ ) as a pale yellow oil. R: 0.45 (2:1 pet. ether:EtOAc). ${ }^{1} \mathbf{H}$ NMR (500 MHz, $\left.\mathrm{CDCl}_{3}\right)$ : $\delta 6.35$ - 6.18 (m, $\left.4 \mathrm{H}\right), 5.89$ (d, J = $\left.14.5 \mathrm{~Hz}, 1 \mathrm{H}\right)$, $5.67(\mathrm{dd}, J=6.3,14.8 \mathrm{~Hz}, 1 \mathrm{H}), 4.34(\mathrm{~d}, J=5.7 \mathrm{~Hz}, 1 \mathrm{H}), 4.22(\mathrm{~d}, J=5.7 \mathrm{~Hz}, 2 \mathrm{H}), 3.18(\mathrm{~d}, J=$ $5.3 \mathrm{~Hz}, 2 \mathrm{H}), 0.93$ (s, $9 \mathrm{H}), 0.12$ (s, $3 \mathrm{H}), 0.07$ (s, $3 \mathrm{H}) .{ }^{13} \mathrm{C}$ NMR (125 MHz, $\left.\mathrm{CDCl}_{3}\right): \delta$ 133.2, 132.8, 132.6, 131.9, 131.4, 131.1, 73.0, 63.3, 57.2, 25.8, 18.1, -4.5, -5.0. IR (film): 3413, 2930, 2858, 2100, 1707, 1620, 1256, 1101, 999, 837, $779 \mathrm{~cm}^{-1}$. HRMS-ESI $(\mathrm{m} / \mathrm{z}):[\mathrm{M}+\mathrm{Na}]^{+}$ calculated for $\mathrm{C}_{15} \mathrm{H}_{27} \mathrm{~N}_{3} \mathrm{NaO}_{2} \mathrm{Si}^{+}, 332.1765$; found 332.1763 .

Preparation of (2E,4E,6E)-9-azido-8-(tert-butyldimethylsilyloxy)-2,4,6-nonatrienal, 310

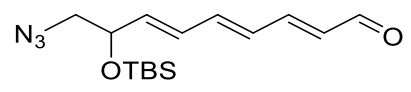

To a solution of alcohol 309 (89 mg, $0.29 \mathrm{mmol}, 1$ equiv) in DCM (10 mL) at room temperature was added DMSO $(206 \mu \mathrm{L}, 2.9 \mathrm{mmol}, 10$ equiv) and triethylamine $(202 \mu \mathrm{L}$, $1.45 \mathrm{mmol}, 5$ equiv). The reaction mixture was cooled to $0{ }^{\circ} \mathrm{C}$ (ice bath), SO $3 . P y r(137 \mathrm{mg}$, $0.86 \mathrm{mmol}, 3$ equiv) added and the reaction stirred for 1 hour before warming to room temperature and stirring for another 1 hour. The reaction was quenched with saturated 
aqueous ammonium chloride $(10 \mathrm{~mL})$ and extracted with DCM $(3 \times 10 \mathrm{~mL})$. The combined organic fractions were washed with distilled $\mathrm{H}_{2} \mathrm{O}$ then brine, dried $\left(\mathrm{MgSO}_{4}\right)$, filtered and concentrated under reduced pressure. The crude material was purified by silica gel flash chromatography (5:1 pet. ether:EtOAc) to provide the title compound (73 $\mathrm{mg}, 82 \%$ ) as a pale yellow oil. Rf: 0.79 (2:1 pet. ether:EtOAc). ${ }^{1} \mathbf{H}$ NMR $\left(500 \mathrm{MHz}, \mathrm{CDCl}_{3}\right): \delta 9.58$ (d, J= 7.8 $\mathrm{Hz}, 1 \mathrm{H}), 7.13(\mathrm{dd}, J=11.2,15.3 \mathrm{~Hz}, 1 \mathrm{H}), 6.67(\mathrm{dd}, J=11.1,14.6 \mathrm{~Hz}, 1 \mathrm{H}), 6.50-6.39$ (m, 2 H), $6.19(\mathrm{dd}, J=7.7,15.4 \mathrm{~Hz}, 1 \mathrm{H}), 5.97(\mathrm{dd}, J=5.8,15.2 \mathrm{~Hz}, 1 \mathrm{H}), 4.41(\mathrm{~d}, J=5.1 \mathrm{~Hz}, 1 \mathrm{H})$, 3.27 - 3.17 (m, $2 \mathrm{H}), 0.94$ (s, $9 \mathrm{H}), 0.14$ (s, $3 \mathrm{H}), 0.08$ (s, $3 \mathrm{H}) .{ }^{13} \mathrm{C}$ NMR $\left(125 \mathrm{MHz}, \mathrm{CDCl}_{3}\right)$ : $\delta$ 193.5, 151.4, 141.1, 139.1, 131.8, 130.5, 130.3, 72.5, 56.9, 25.7, 18.1, -4.6, -5.0. IR (film): 2930, 2858, 2101, 1679, 1616, 1346, 1254, 1157, 1109, 993, 835, 778, $688 \mathrm{~cm}^{-1}$. HRMS-ESI (m/z): [M $+\mathrm{H}]^{+}$calculated for $\mathrm{C}_{15} \mathrm{H}_{26} \mathrm{~N}_{3} \mathrm{O}_{2} \mathrm{Si}^{+}, 308.1789$; found 308.1769 .

\section{Preparation of $N, N$-Dimethyl[(2E,4E,6E)-9-azido-8-(tert-butyldimethylsilyloxy)-2,4,6- nonatrienyl]amine, 299}

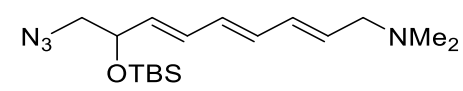

To a solution of aldehyde 310 (141 mg, $0.46 \mathrm{mmol}, 1$ equiv) in THF $(10 \mathrm{~mL})$ at $0{ }^{\circ} \mathrm{C}$ (ice bath) was added HCl.HNMe $(113 \mathrm{mg}, 1.38 \mathrm{mmol}, 3$ equiv) followed by $\mathrm{NMe}_{4} \mathrm{BH}(\mathrm{OAc})_{3}(181 \mathrm{mg}, 0.69 \mathrm{mmol}, 1.5$ equiv). This was stirred overnight on ice, in a closed system (to prevent loss of dimethylamine with a $b p=7-9{ }^{\circ} \mathrm{C}$ ). The reaction mixture was then quenched with $0.5 \mathrm{M}$ aqueous $\mathrm{NaOH}(10 \mathrm{~mL})$, and extracted with EtOAc $(3 \times 15$ $\mathrm{mL})$. The combined organic fractions were dried $\left(\mathrm{MgSO}_{4}\right)$, filtered, then concentrated under reduced pressure. The crude material was purified by silica gel flash chromatography (90:10:1 DCM:MeOH:saturated aqueous $\mathrm{NH}_{4} \mathrm{OH}$ ) to provide the title compound (94 $\mathrm{mg}, 59 \%$ or $85 \%$ BRSM) as a pale orange oil. $\mathbf{R}_{\mathbf{f}}: 0.0$ (90:10:1 DCM:MeOH:saturated aqueous $\mathrm{NH}_{4} \mathrm{OH}$ ); cf. aldehyde 310 with Rf: $0.15 .{ }^{1} \mathbf{H}$ NMR (500 $\left.\mathrm{MHz}_{2} \mathrm{CDCl}_{3}\right): \delta 6.33$ - $6.13(\mathrm{~m}, 4 \mathrm{H}), 5.81-5.72(\mathrm{~m}, 1 \mathrm{H}), 5.65(\mathrm{dd}, J=6.5,15.1 \mathrm{~Hz}, 1 \mathrm{H}), 4.33$ $(\mathrm{d}, J=5.6 \mathrm{~Hz}, 1 \mathrm{H}), 3.20$ - $3.13(\mathrm{~m}, 2 \mathrm{H}), 2.99(\mathrm{~d}, J=6.8 \mathrm{~Hz}, 2 \mathrm{H}), 2.24(\mathrm{~s}, 8 \mathrm{H}), 0.92(\mathrm{~s}, 13 \mathrm{H})$, $0.12(\mathrm{~s}, 4 \mathrm{H}), 0.06$ (s, $4 \mathrm{H}) .{ }^{13} \mathrm{C}$ NMR (125 MHz, $\left.\mathrm{CDCl}_{3}\right): \delta 133.0,132.9,132.7,131.7,131.5$, 131.2, 73.0, 61.6, 57.2, 45.2, 45.1, 25.8, 18.1, -4.5, -5.0. IR (film): 2930, 2857, 2774, 2098, 1471, 1362, 1255, 1083, 998, 836, 778, $734 \mathrm{~cm}^{-1}$. HRMS-ESI $(\mathrm{m} / \mathrm{z}):[\mathrm{M}+\mathrm{H}]^{+}$calculated for $\mathrm{C}_{17} \mathrm{H}_{33} \mathrm{~N}_{4} \mathrm{OSi}^{+}$, 337.2418; found 337.2422. 


\section{Preparation of (2E,4E,6E)-9-Azido-8-(tert-butyldimethylsilyloxy)-1-(trityloxy)-2,4,6-}

nonatriene, 312

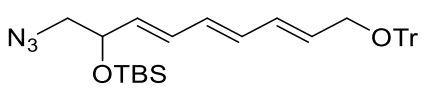

To a solution of alcohol 309 (340 mg, $1.1 \mathrm{mmol}, 1$ equiv) in DCM (15 mL) at room temperature was added pyridine $(8 \mathrm{~mL})$ followed by trityl chloride $(617 \mathrm{mg}, 2.2 \mathrm{mmol}, 2$ equiv) and the mixture stirred overnight. The reaction mixture was then quenched with $2 \%$ aqueous $\mathrm{H}_{2} \mathrm{SO}_{4}(20 \mathrm{~mL})$ and extracted with DCM $(3 \times 20 \mathrm{~mL})$. The combined organic fractions were washed with water then brine, dried $\left(\mathrm{MgSO}_{4}\right)$, filtered and concentrated under reduced pressure. The crude material was purified by silica gel flash chromatography (9:1 pet. ether:EtOAc) to provide the title compound (552 $\mathrm{mg}, 90 \%$ ) as a colourless oil. Rf: 0.88 (2:1 pet. ether:EtOAc). ${ }^{1} \mathbf{H}$ NMR (500 MHz, $\left.\mathrm{CDCl}_{3}\right): \delta 7.47$ (d, J = 7.6 $\mathrm{Hz}, 6 \mathrm{H}), 7.35$ - $7.22(\mathrm{~m}, 9 \mathrm{H}), 6.48$ - $6.37(\mathrm{~m}, 1 \mathrm{H}), 6.33$ - 6.17 (m, $3 \mathrm{H}), 5.84(\mathrm{~d}, J=15.1 \mathrm{~Hz}, 1$ H), $5.67(\mathrm{dd}, J=6.3,14.8 \mathrm{~Hz}, 1 \mathrm{H}), 4.35(\mathrm{~d}, J=5.0 \mathrm{~Hz}, 1 \mathrm{H}), 3.68(\mathrm{~d}, J=5.0 \mathrm{~Hz}, 2 \mathrm{H}), 3.21$ 3.17 (m, $2 \mathrm{H}), 0.94$ (s, $9 \mathrm{H}), 0.13$ (s, $3 \mathrm{H}), 0.08$ (s, $3 \mathrm{H}) .{ }^{13} \mathrm{C}$ NMR (125 MHz, CDCl $)$ : $\delta$ 144.1, $133.2,132.8,131.6,131.2,131.2,130.6,128.6,127.9,127.8,86.9,73.1,64.4,57.2,25.8,14.2$, $4.5,-4.9$.

\section{Coupling Fragments}

Preparation of 2-(6-Oxo-2,3-dihydropyran-2-yl)ethyl 5-\{1-[(3E,5E,7E)-9-(trityloxy)-2-(tertbutyldimethylsilyloxy)-3,5,7-nonatrienyl]-1H-1,2,3-triazol-4-yl\}valerate, 314

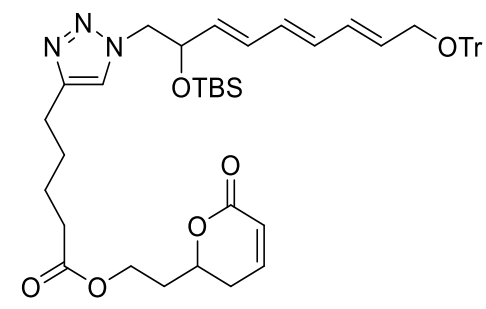

To an aqueous solution ( $5 \mathrm{~mL})$ of $\mathrm{CuSO}_{4} .5 \mathrm{H}_{2} \mathrm{O}(10 \mathrm{mg}$, $0.04 \mathrm{mmol}, 0.1$ equiv) and ascorbic acid ( $35 \mathrm{mg}, 0.2 \mathrm{mmol}, 0.5$ equiv), that was neutralised to $\mathrm{pH} 7$ with aqueous $\mathrm{NaOH}$, was added a solution of alkyne $284(99 \mathrm{mg}, 0.40 \mathrm{mmol}, 1$ equiv) and azide 312 (221 $\mathrm{mg}$, $0.40 \mathrm{mmol}, 1$ equiv) in THF (5 mL). The reaction mixture was stirred overnight at 


\section{Chapter Six: Experimental}

room temperature which gradually turned yellow/green. The mixture was then extracted with EtOAc $(3 \times 10 \mathrm{~mL})$ and the combined organic fractions were dried $\left(\mathrm{MgSO}_{4}\right)$, filtered and concentrated under reduced pressure. The crude material was purified by silica gel flash chromatography (1:1 pet. ether:EtOAc) to provide the title compound (196 $\mathrm{mg}, 62 \%)$ as a pale yellow oil. Rf: 0.66 (EtOAc). ${ }^{1} \mathbf{H}$ NMR (500 MHz, $\left.\mathrm{CDCl}_{3}\right): \delta 7.46(\mathrm{~d}, J=7.4 \mathrm{~Hz}, 6$ $\mathrm{H}), 7.31(\mathrm{t}, J=8.1 \mathrm{~Hz}, 6 \mathrm{H}), 7.28-7.21(\mathrm{~m}, 3 \mathrm{H}), 6.92-6.86(\mathrm{~m}, 1 \mathrm{H}), 6.40(\mathrm{~d}, J=15.1 \mathrm{~Hz}, 1$ H), $6.33-6.16(\mathrm{~m}, 3 \mathrm{H}), 6.04(\mathrm{~d}, J=9.8 \mathrm{~Hz}, 1 \mathrm{H}), 5.84(\mathrm{td}, J=5.2,15.2 \mathrm{~Hz}, 1 \mathrm{H}), 5.63(\mathrm{dd}, J=$ 6.6, 14.7 Hz, $1 \mathrm{H}), 4.62$ - $4.51(\mathrm{~m}, 2 \mathrm{H}), 4.41(\mathrm{dd}, J=3.3,13.7 \mathrm{~Hz}, 1 \mathrm{H}), 4.34-4.17(\mathrm{~m}, 3 \mathrm{H})$, $3.68(\mathrm{~d}, J=5.0 \mathrm{~Hz}, 2 \mathrm{H}), 2.73(\mathrm{br} \mathrm{s}, 2 \mathrm{H}), 2.42-2.29(\mathrm{~m}, 4 \mathrm{H}), 2.11(\mathrm{dd}, J=5.7,8.4 \mathrm{~Hz}, 2 \mathrm{H})$, 2.04 - 1.93 (m, 2 H), 1.71 (br s, 4 H), 0.83 (s, 9 H), - -0.06 (s, 3 H), - -0.19 (s, 3 H). ${ }^{13}$ C NMR (125 $\left.\mathrm{MHz}_{2} \mathrm{CDCl}_{3}\right): \delta 173.3,163.9,147.0,144.8,144.1,133.7,132.4,131.6,130.9,130.4,128.7,128.6$, $127.8,127.0,122.5,121.5,86.9,74.7,72.6,64.3,60.4,59.8,56.3,34.0,33.9,29.4,28.9,25.7$, 25.2, 24.4, 18.0, 14.2, -4.6, -5.4. HRMS-ESI (m/z): $[\mathrm{M}+\mathrm{H}]^{+}$calculated for $\mathrm{C}_{48} \mathrm{H}_{60} \mathrm{~N}_{3} \mathrm{O}_{6} \mathrm{Si}^{+}$, 802.4246; found 802.4240.

\section{Preparation of 2-(6-Oxo-2,3-dihydropyran-2-yl)ethyl 5-\{1-[(3E,5E,7E)-9-oxo-2-(tert-} butyldimethylsilyloxy)-3,5,7-nonatrienyl]-1H-1,2,3-triazol-4-yl\}valerate, 323

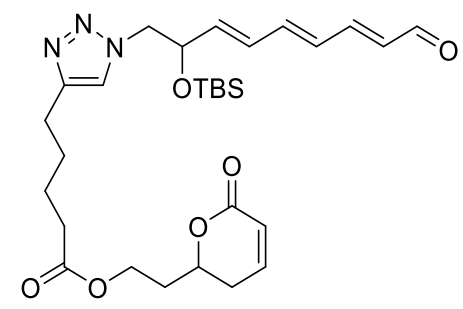

To an aqueous solution ( $2 \mathrm{~mL})$ of $\mathrm{CuSO}_{4} .5 \mathrm{H}_{2} \mathrm{O}(3 \mathrm{mg}, 0.01 \mathrm{mmol}, 0.1$ equiv) and ascorbic acid (11 mg, $0.06 \mathrm{mmol}, 0.5$ equiv), that was neutralised to $\mathrm{pH} 7$ with aqueous $\mathrm{NaOH}$, was added a solution of alkyne $284(31 \mathrm{mg}, 0.12 \mathrm{mmol}, 1$ equiv) and azide 310 (35 $\mathrm{mg}, 0.12 \mathrm{mmol}, 1$ equiv) in THF (2 mL). The reaction mixture was stirred overnight at room temperature which gradually turned yellow/green. The mixture was then extracted with EtOAc $(3 \times 5 \mathrm{~mL})$ and the combined organic fractions were dried $\left(\mathrm{MgSO}_{4}\right)$, filtered and concentrated under reduced pressure. The crude material was purified by silica gel flash chromatography (1:1 pet. ether:EtOAc) to provide the title compound (36 $\mathrm{mg}, 54 \%)$ as a pale yellow oil. Rf: 0.14 (1:1 pet. ether:EtOAc). ${ }^{1} \mathbf{H}$ NMR (500 MHz, $\left.\mathrm{CDCl}_{3}\right)$ : $\delta 9.57$ (d, J $=8.0 \mathrm{~Hz}, 1 \mathrm{H}), 7.36-7.29(\mathrm{~m}, 1 \mathrm{H}), 7.11(\mathrm{dd}, J=11.2,15.1 \mathrm{~Hz}, 1 \mathrm{H}), 6.96-6.85(\mathrm{~m}, 1 \mathrm{H}), 6.69$ 
- $6.57(\mathrm{~m}, 1 \mathrm{H}), 6.51-6.33(\mathrm{~m}, 2 \mathrm{H}), 6.18(\mathrm{dd}, J=8.0,15.3 \mathrm{~Hz}, 1 \mathrm{H}), 6.03(\mathrm{~d}, J=9.8 \mathrm{~Hz}, 1 \mathrm{H})$, $5.91(\mathrm{dd}, J=6.0,15.1 \mathrm{~Hz}, 1 \mathrm{H}), 4.65(\mathrm{br} \mathrm{s}, 1 \mathrm{H}), 4.56$ (br s, $1 \mathrm{H}), 4.34-4.16(\mathrm{~m}, 4 \mathrm{H}), 2.72$ (br $\mathrm{s}, 2 \mathrm{H}), 2.43-2.37(\mathrm{~m}, 2 \mathrm{H}), 2.37-2.29(\mathrm{~m}, 2 \mathrm{H}), 2.15-2.05(\mathrm{~m}, 2 \mathrm{H}), 2.04-1.94(\mathrm{~m}, 2 \mathrm{H})$, 1.69 (br s, $4 \mathrm{H}), 0.84$ (s, $9 \mathrm{H}),-0.06$ (s, $3 \mathrm{H}),-0.18$ (s, $3 \mathrm{H}) .{ }^{13} \mathrm{C}$ NMR $\left(125 \mathrm{MHz}, \mathrm{CDCl}_{3}\right): \delta$ 193.5, 173.2, 163.9, 151.1, 147.1, 144.8, 140.7, 137.7, 132.0, 130.9, 122.6, 121.4, 74.7, 72.0, 59.8, 55.9, 34.0, 33.8, 29.4, 28.8, 25.7, 25.6, 25.1, 24.3, 18.0, -4.7, -5.4. IR (film): 2952, 2930, 2857, 1724, 1674, 1614, 1250, 1160, 1110, 1043, 1014, 940, 836, 779, 730 $\mathrm{cm}^{-1}$. HRMS-ESI (m/z): [M + $\mathrm{H}]^{+}$calculated for $\mathrm{C}_{29} \mathrm{H}_{44} \mathrm{~N}_{3} \mathrm{O}_{6} \mathrm{Si}^{+}$, 558.2994; found 558.3014.

Preparation of (2E,4E,6E)-9-Azido-8-(tert-butyldimethylsilyloxy)-2,4,6-nonatrienal, 324<smiles>NCC(O)C=CC=CC=CC=O</smiles>

To a solution of compound $310(412 \mathrm{mg}, 1.34 \mathrm{mmol})$ in $\mathrm{MeOH}(20 \mathrm{~mL})$ at room temperature was added a few drops of conc. $\mathrm{HCl}$. The reaction mixture was stirred for two hours, then quenched with saturated aqueous sodium carbonate $(1 \mathrm{~mL})$ and solvent removed under reduced pressure. (Reaction was not complete based on TLC, however we believed that the product would be susceptible to elimination.) The crude material was purified by silica gel flash chromatography (2:1 pet. ether:EtOAc) to provide the title compound (57 mg, $22 \%$ or $84 \%$ BRSM) as a yellow oil. $\mathbf{R}_{\text {f: }} 0.21$ (2:1 pet. ether:EtOAc). ${ }^{1} \mathbf{H}$ NMR (500 MHz, $\left.\mathrm{CDCl}_{3}\right): \delta 9.57(\mathrm{~d}, J=7.9 \mathrm{~Hz}, 1 \mathrm{H}), 7.18-7.06(\mathrm{~m}, 1 \mathrm{H}), 6.73-6.58(\mathrm{~m}, 1 \mathrm{H})$, 6.58 - 6.36 (m, $2 \mathrm{H}), 6.18(\mathrm{dd}, J=7.9,15.0 \mathrm{~Hz}, 1 \mathrm{H}), 5.97$ (dd, J=4.9, $15.1 \mathrm{~Hz}, 1 \mathrm{H}), 4.45$ (br s, $1 \mathrm{H}), 3.43(\mathrm{~d}, J=11.8 \mathrm{~Hz}, 1 \mathrm{H}), 3.39$ - 3.25 (m, $1 \mathrm{H}) .{ }^{13} \mathrm{C}$ NMR (125 MHz, $\left.\mathrm{CDCl}_{3}\right): \delta$ 193.5, 151.2, 140.8, 137.3, 132.0, 131.1, 131.0, 71.1, 56.3. IR (film): 3381, 2924, 2834, 2097, 1667, 1610, 1283, 1159, 1115, 912, 843, $730 \mathrm{~cm}^{-1}$. HRMS-ESI $(\mathrm{m} / \mathrm{z}):[\mathrm{M}+\mathrm{H}]^{+}$calculated for $\mathrm{C}_{9} \mathrm{H}_{12} \mathrm{~N}_{3} \mathrm{O}_{2}{ }^{+}, 194.0924$; found 194.0899. 


\section{Preparation of $(2 Z, 4 E)-7-(4-\{1-[(3 E, 5 E, 7 E)-9-O x o-2-h y d r o x y-3,5,7-n o n a t r i e n y l]-1 H-1,2,3-$} triazol-4-yl\}valeroxy)-2,4-heptadienoic acid, 322

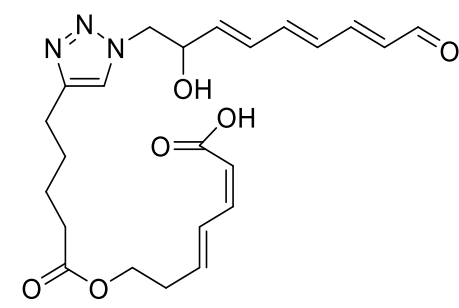

To an aqueous solution ( $5 \mathrm{~mL})$ of $\mathrm{CuSO}_{4} .5 \mathrm{H}_{2} \mathrm{O}(10 \mathrm{mg}, 0.04 \mathrm{mmol}, 0.1$ equiv) and ascorbic acid (30 mg, $0.17 \mathrm{mmol}, 0.5$ equiv), that was neutralised to $\mathrm{pH} 7$ with aqueous $\mathrm{NaOH}$, was added a solution of alkyne $282(103 \mathrm{mg}, 0.41 \mathrm{mmol}, 1.2$ equiv) and azide 324 (66 mg, $0.34 \mathrm{mmol}, 1$ equiv) in THF ( $5 \mathrm{~mL}$ ). The reaction mixture was stirred overnight at room temperature which gradually turned yellow/green. The mixture was then extracted with EtOAc $(3 \times 15 \mathrm{~mL})$ and the combined organic fractions were dried $\left(\mathrm{MgSO}_{4}\right)$, filtered and concentrated under reduced pressure. The crude material was purified by silica gel flash chromatography (EtOAc) to provide the title compound (114 $\mathrm{mg}, 75 \%)$ as a pale yellow oil. Rf: 0.40 (4:1 DCM:MeOH). ${ }^{1} \mathbf{H}$ NMR (500 MHz, CDCl $)$ : $\delta 9.56$ (d, J = $7.8 \mathrm{~Hz}, 1$ H), $7.46(\mathrm{~s}, 1 \mathrm{H}), 7.38(\mathrm{dd}, J=11.8,14.9 \mathrm{~Hz}, 1 \mathrm{H}), 7.12(\mathrm{dd}, J=11.2,15.1 \mathrm{~Hz}, 1 \mathrm{H}), 6.67$ - 6.56 $(\mathrm{m}, 2 \mathrm{H}), 6.56-6.41(\mathrm{~m}, 2 \mathrm{H}), 6.17(\mathrm{dd}, J=7.8,15.2 \mathrm{~Hz}, 1 \mathrm{H}), 6.09-5.95(\mathrm{~m}, 2 \mathrm{H}), 5.62(\mathrm{~d}, J=$ $11.3 \mathrm{~Hz}, 1 \mathrm{H}), 4.75(\mathrm{br} \mathrm{s}, 1 \mathrm{H}), 4.50(\mathrm{dd}, J=2.9,13.8 \mathrm{~Hz}, 1 \mathrm{H}), 4.28(\mathrm{dd}, J=8.0,13.8 \mathrm{~Hz}, 1 \mathrm{H})$, $4.19(\mathrm{t}, J=5.8 \mathrm{~Hz}, 2 \mathrm{H}), 2.68(\mathrm{br} \mathrm{s}, 2 \mathrm{H}), 2.52(\mathrm{q}, J=5.8 \mathrm{~Hz}, 2 \mathrm{H}), 2.32(\mathrm{br} \mathrm{s}, 2 \mathrm{H}), 1.65$ (br s, 4

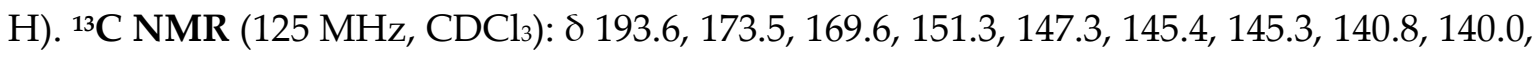
136.7, 132.0, 131.2, 131.1, 129.1, 116.6, 70.5, 62.5, 55.4, 33.9, 32.0, 28.6, 24.9, 24.2. IR (film): 2947, 2865, 1722, 1673, 1640, 1609, 1429, 1365, 1161, 1117, 1014, 997, 967, 913, 822, $730 \mathrm{~cm}^{-1}$. HRMS-ESI (m/z): $[\mathrm{M}+\mathrm{H}]^{+}$calculated for $\mathrm{C}_{23} \mathrm{H}_{30} \mathrm{~N}_{3} \mathrm{O}_{6}{ }^{+}$, 444.2129; found 444.2106. 


\section{Preparation of (2E,4E,6E)-7-\{(6Z,8E)-5,13-Dioxo-4.12-dioxa-1.19.20-}

\section{triazabicyclo[16.2.1]henicosa-6,8,18(21),19-tetraen-3-yl\}-2,4,6-heptatrienal, 325}

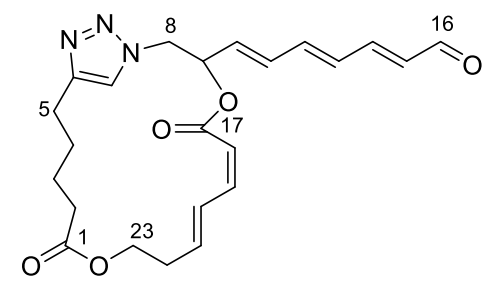

To a solution of seco acid 322 (60 mg, $0.14 \mathrm{mmol}, 1$ equiv) in THF ( $3 \mathrm{~mL})$ at room temperature was added triethylamine $(39 \mu \mathrm{L}, 0.28 \mathrm{mmol}, 2$ equiv) followed by 2,4,6trichlorobenzoyl chloride ( $33 \mu \mathrm{L}, 0.21 \mathrm{mmol}, 1.5 \mathrm{equv})$. After stirring for 2 hours, the reaction mixture was diluted with $\mathrm{DCM}(40 \mathrm{~mL})$, cooled to $-78^{\circ} \mathrm{C}$ (dry ice/acetone bath) and DMAP (5 mg, $0.042 \mathrm{mmol}, 0.3$ equiv) added. The reaction mixture was allowed to gradually warm to room temperature (over a few hours) and the mixture stirred overnight. The reaction was then quenched with saturated aqueous ammonium chloride $(10 \mathrm{~mL})$. The aqueous layer was extracted with EtOAc $(3 \times 15 \mathrm{~mL})$ and the combined organic fractions were dried $\left(\mathrm{MgSO}_{4}\right)$, filtered and concentrated under reduced pressure. The crude material was purified by silica gel flash chromatography (9:1 DCM:MeOH) to provide the title compound $(8 \mathrm{mg}, 13 \%)$ as a yellow oil. $\mathbf{R}_{\mathbf{f}}$ $0.66(4: 1 \mathrm{DCM}: \mathrm{MeOH}) .{ }^{1} \mathbf{H}$

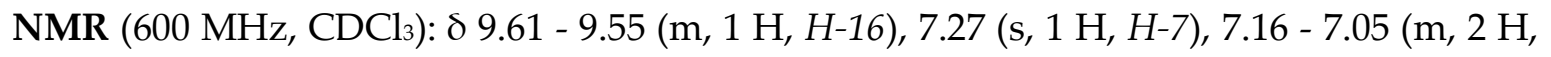
H-14 and 20), $6.66(\mathrm{dd}, J=10.8,14.9 \mathrm{~Hz}, 1 \mathrm{H}, H-10), 6.59$ (t, $J=11.4 \mathrm{~Hz}, 1 \mathrm{H}, H-19), 6.56$ $6.49(\mathrm{~m}, 2 \mathrm{H}, \mathrm{H}-12$ and 13), $6.21(\mathrm{dd}, J=7.9,15.2 \mathrm{~Hz}, 1 \mathrm{H}, H-15), 6.07-5.91$ (m, $3 \mathrm{H}, H-9,11$ and 21), $5.56(\mathrm{~d}, J=11.4 \mathrm{~Hz}, 1 \mathrm{H}, H-18), 4.71-4.65(\mathrm{~m}, 1 \mathrm{H}, \mathrm{H}-8 a), 4.41(\mathrm{dd}, J=10.1,14.1 \mathrm{~Hz}$, $1 \mathrm{H}, H-8 b), 4.37-4.30$ (m, $1 \mathrm{H}, H-23 a), 4.19$ (ddd, J = 3.5, 5.0, $11.1 \mathrm{~Hz}, 1 \mathrm{H}, H-23 b), 2.76$ (td, J = 7.0, 14.6 Hz, $1 \mathrm{H}, H-5 a), 2.64(\mathrm{td}, J=7.4,14.8 \mathrm{~Hz}, 1 \mathrm{H}, H-5 b), 2.59$ - 2.49 (m, $1 \mathrm{H}, \mathrm{H}-22 a)$, 2.49 - 2.41 (m, 1 H, H-22b), 2.38 - 2.21 (m, 2 H, H-2), 1.77 - 1.63 (m, 2 H, H-4), 1.60 - 1.51 (m, $2 \mathrm{H}, \mathrm{H}-3) .{ }^{13} \mathrm{C}$ NMR $\left(151 \mathrm{MHz}, \mathrm{CDCl}_{3}\right): \delta 193.4,173.3,164.0,150.6,146.8,142.5,139.9,132.9$, 132.6, 132.2, 131.6, 128.2, 128.0, 121.4, 114.3, 70.5, 61.8, 53.3, 34.7, 33.2, 28.6, 25.1, 24.3. IR (film): 2929, 2856, 2361, 1721, 1673, 1615, 1222, 1157, 1116, 996, $731 \mathrm{~cm}^{-1}$. HRMS-ESI (m/z): $[\mathrm{M}+\mathrm{H}]^{+}$calculated for $\mathrm{C}_{23} \mathrm{H}_{28} \mathrm{~N}_{3} \mathrm{O}_{5}{ }^{+}, 426.2023$; found 426.2010. 


\section{Preparation of $(6 Z, 8 E)-3-[(1 E, 3 E, 5 E)-7-(D i m e t h y l a m i n o)-1,3,5-h e p t a t r i e n y l]-4.12-d i o x a-$} 1.19.20-triazabicyclo[16.2.1]henicosa-6,8,18(21),19-tetraene-5,13-dione, 107

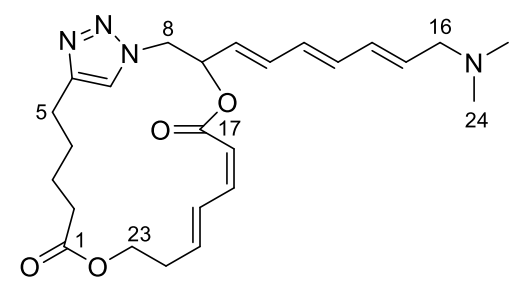

To a solution of aldehyde $325\left(8 \mathrm{mg}, 0.02 \mathrm{mmol}, 1\right.$ equiv) in THF $(2 \mathrm{~mL})$ at $0{ }^{\circ} \mathrm{C}$ (ice bath) was added $\mathrm{HCl} . \mathrm{HNMe} 2\left(6 \mathrm{mg}, 0.08 \mathrm{mmol}, 4\right.$ equiv) followed by $\mathrm{NMe}_{4} \mathrm{BH}(\mathrm{OAc})_{3}(10$ $\mathrm{mg}, 0.04 \mathrm{mmol}, 2$ equiv) and Triethylamine $(11 \mu \mathrm{L}, 0.08 \mathrm{mmol}, 4$ equiv). This was stirred overnight on ice, in a closed system (to prevent loss of dimethylamine with a bp $=7-9{ }^{\circ} \mathrm{C}$ ). The reaction mixture was then quenched with $0.5 \mathrm{M}$ aqueous $\mathrm{NaOH}(2 \mathrm{~mL})$, and extracted with EtOAc $(3 \times 5 \mathrm{~mL})$. The combined organic fractions were dried $\left(\mathrm{MgSO}_{4}\right)$, filtered, then concentrated under reduced pressure. The crude material was purified by silica gel flash chromatography (90:10:1 DCM:MeOH:saturated aqueous $\mathrm{NH}_{4} \mathrm{OH}$ ) to provide the title compound (5 mg, 56\%) as a pale orange oil. $\mathbf{R}_{\mathbf{f}} 0.67$ (80:20:1 magic base). ${ }^{1} \mathbf{H}$ NMR (500 $\mathrm{MHz}_{\mathrm{CDCl}}$ ): $\delta 7.24$ (s, $\left.1 \mathrm{H}, H-7\right), 7.18-7.09$ (m, $\left.1 \mathrm{H}, \mathrm{H}-20\right), 6.56$ (t, J = 11.3 Hz, $\left.1 \mathrm{H}, \mathrm{H}-19\right)$, $6.47-6.40(\mathrm{~m}, 1 \mathrm{H}, \mathrm{H}-11), 6.34-6.18(\mathrm{~m}, 3 \mathrm{H}, \mathrm{H}-12,13$ and 14), $6.01(\mathrm{~d}, J=9.2 \mathrm{~Hz}, 1 \mathrm{H}, H-$ 21), $5.92-5.84(\mathrm{~m}, 2 \mathrm{H}, \mathrm{H}-2$ and 4), $5.74(\mathrm{dd}, J=6.9,15.2 \mathrm{~Hz}, 1 \mathrm{H}, \mathrm{H}-10), 5.55(\mathrm{~d}, J=11.4 \mathrm{~Hz}$, $1 \mathrm{H}, H-18), 4.62(\mathrm{~d}, J=14.3 \mathrm{~Hz}, 1 \mathrm{H}, H-8 a), 4.39(\mathrm{dd}, J=10.2,14.2 \mathrm{~Hz}, 1 \mathrm{H}, H-8 b), 4.32$ (d, J = $9.7 \mathrm{~Hz}, 1 \mathrm{H}, H-23 a), 4.23$ - 4.16 (m, $1 \mathrm{H}, \mathrm{H}-23 b), 3.26$ (br s, $2 \mathrm{H}, \mathrm{H}-16), 2.78$ - 2.67 (m, $1 \mathrm{H}, \mathrm{H}-$ 5a), 2.67 - $2.56(\mathrm{~m}, 1 \mathrm{H}, \mathrm{H}-5 b), 2.56-2.46$ (m, $2 \mathrm{H}, \mathrm{H}-22), 2.39$ - 2.23 (m, $2 \mathrm{H}, \mathrm{H}-2), 2.17$ (s, 6 $\mathrm{H}, \mathrm{H}-24), 1.76$ - 1.61 (m, $2 \mathrm{H}, \mathrm{H}-4), 1.60$ - 1.50 (m, $2 \mathrm{H}, \mathrm{H}-3) .{ }^{13} \mathrm{C}$ NMR (151 MHz, CDCl$): ~ \delta$ 173.3, 164.1, 146.5, 142.2, 139.8, 134.6, 133.3, 132.2, 130.4, 128.9, 128.3, 121.2, 120.7, 114.5, 70.7, 61.8, 53.3, 45.7, 41.9, 34.7, 33.2, 28.6, 25.2, 24.4. IR (film): 2934, 2862, 1717, 1640, 1612, 1513, 1246, 1159, 1090, 1033, 1001, 912, 817, $728 \mathrm{~cm}^{-1}$. HRMS-ESI (m/z): [M + H] $]^{+}$calculated for $\mathrm{C}_{25} \mathrm{H}_{35} \mathrm{~N}_{4} \mathrm{O}_{4}{ }^{+}, 455.2653$; found 455.2650 . 


\section{4}




\section{NMR Spectra of Novel Compounds}

Chapter Two Spectra

${ }^{1} \mathrm{H}-\mathrm{NMR}\left(500 \mathrm{MHz}, \mathrm{CDCl}_{3}\right)$<smiles>CC(=O)/C=C(\C)c1ccco1</smiles>

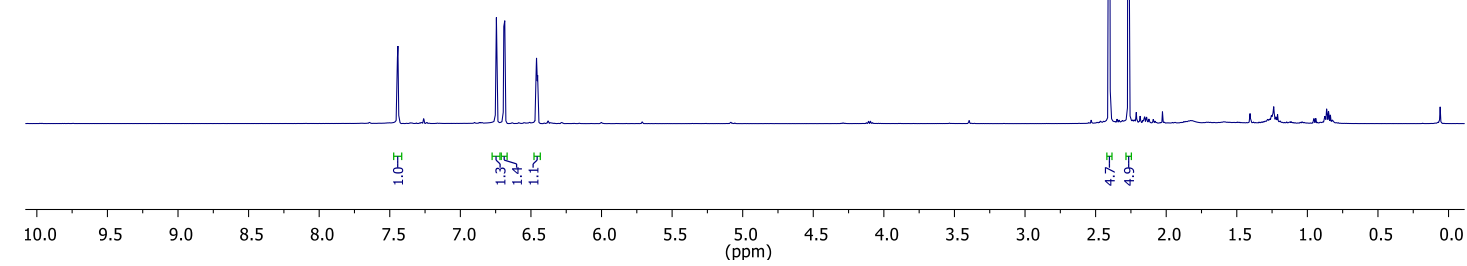

${ }^{13} \mathrm{C}-\mathrm{NMR}$

(125 MHz, $\mathrm{CDCl}_{3}$ )

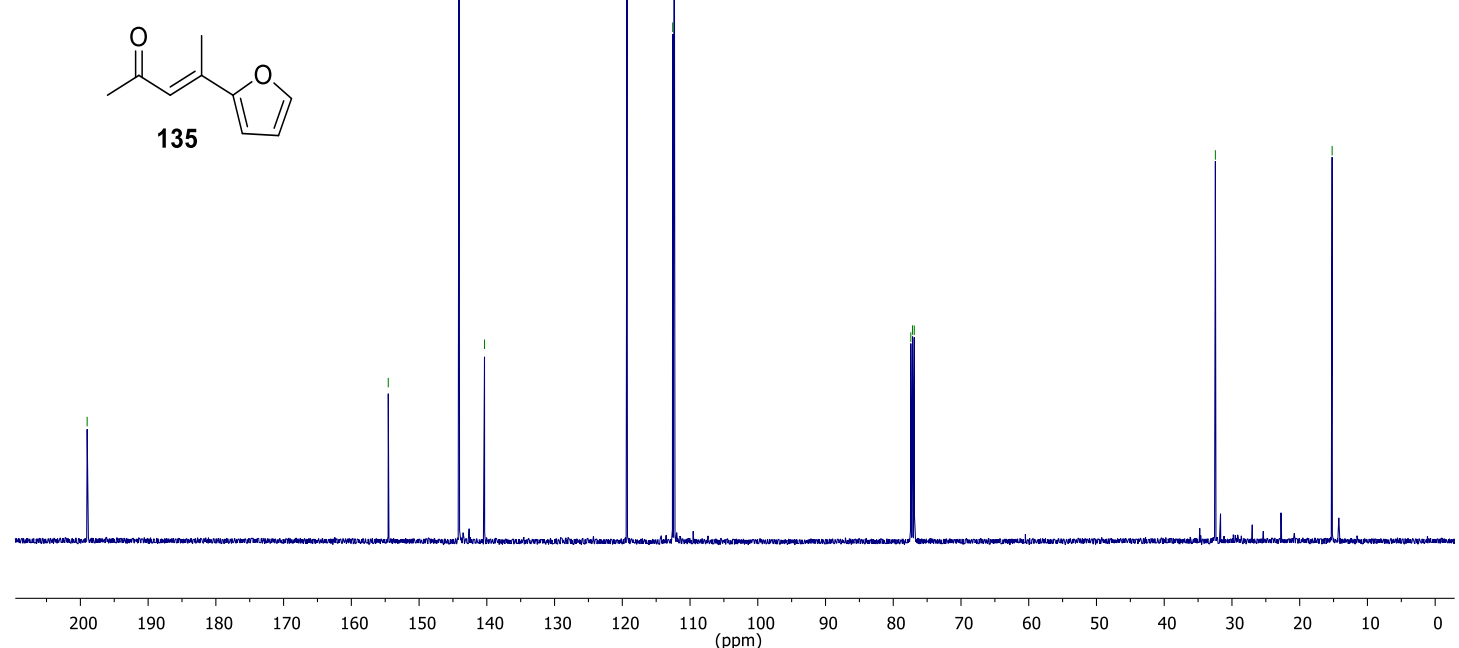


${ }^{1} \mathrm{H}-\mathrm{NMR}\left(500 \mathrm{MHz}, \mathrm{CDCl}_{3}\right.$ )

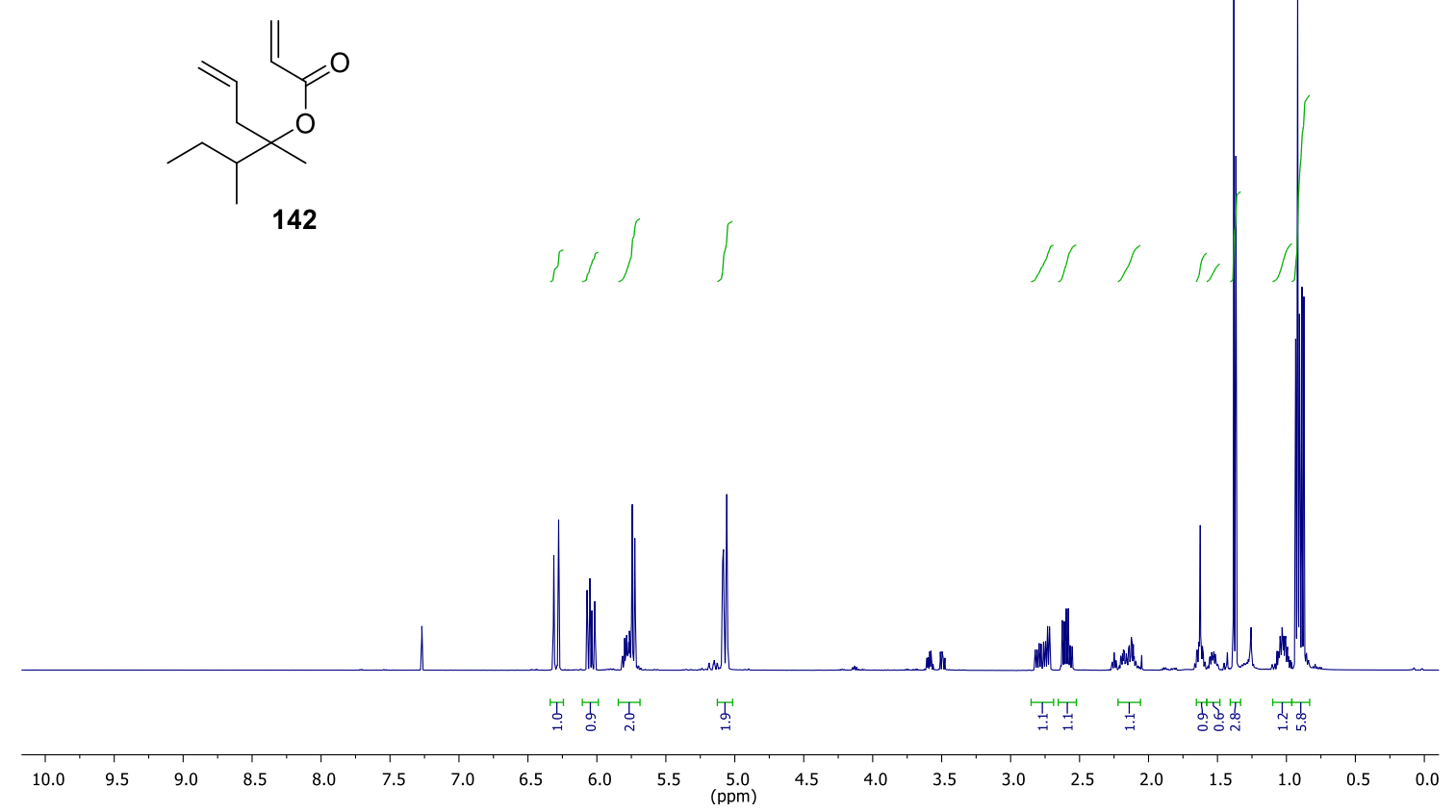

${ }^{13} \mathrm{C}-\mathrm{NMR}\left(125 \mathrm{MHz}, \mathrm{CDCl}_{3}\right.$ )<smiles>C=CCC(C)(OC(=O)C=C)C(C)CC</smiles>

\section{-}

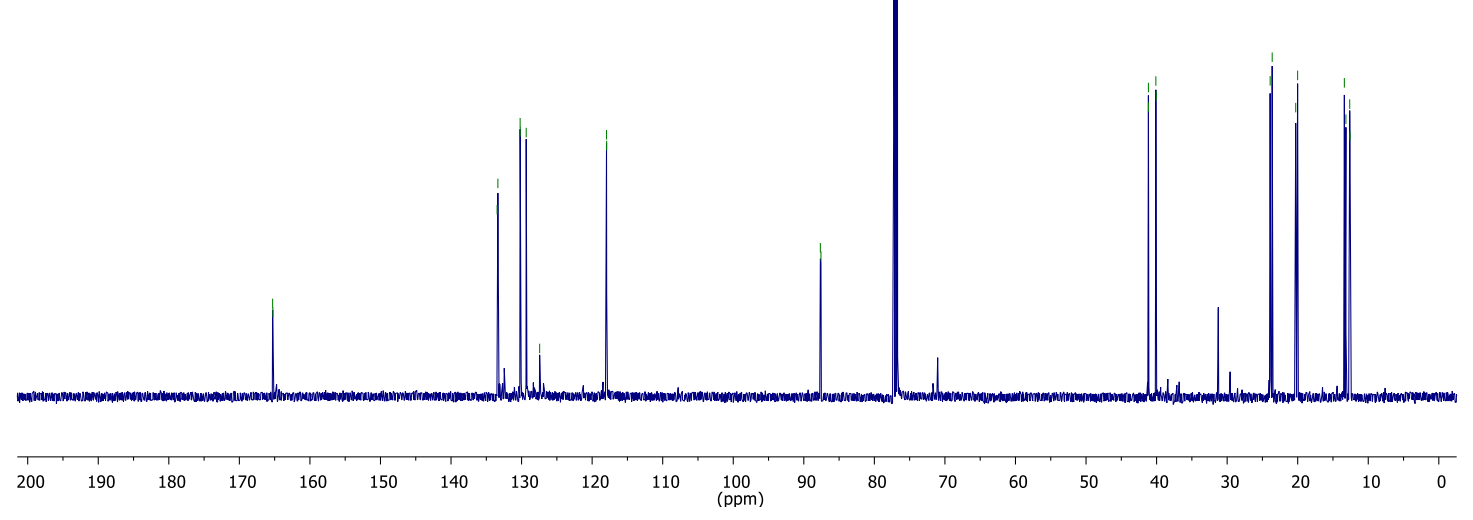


${ }^{1} \mathrm{H}-\mathrm{NMR}\left(500 \mathrm{MHz}, \mathrm{CDCl}_{3}\right)$

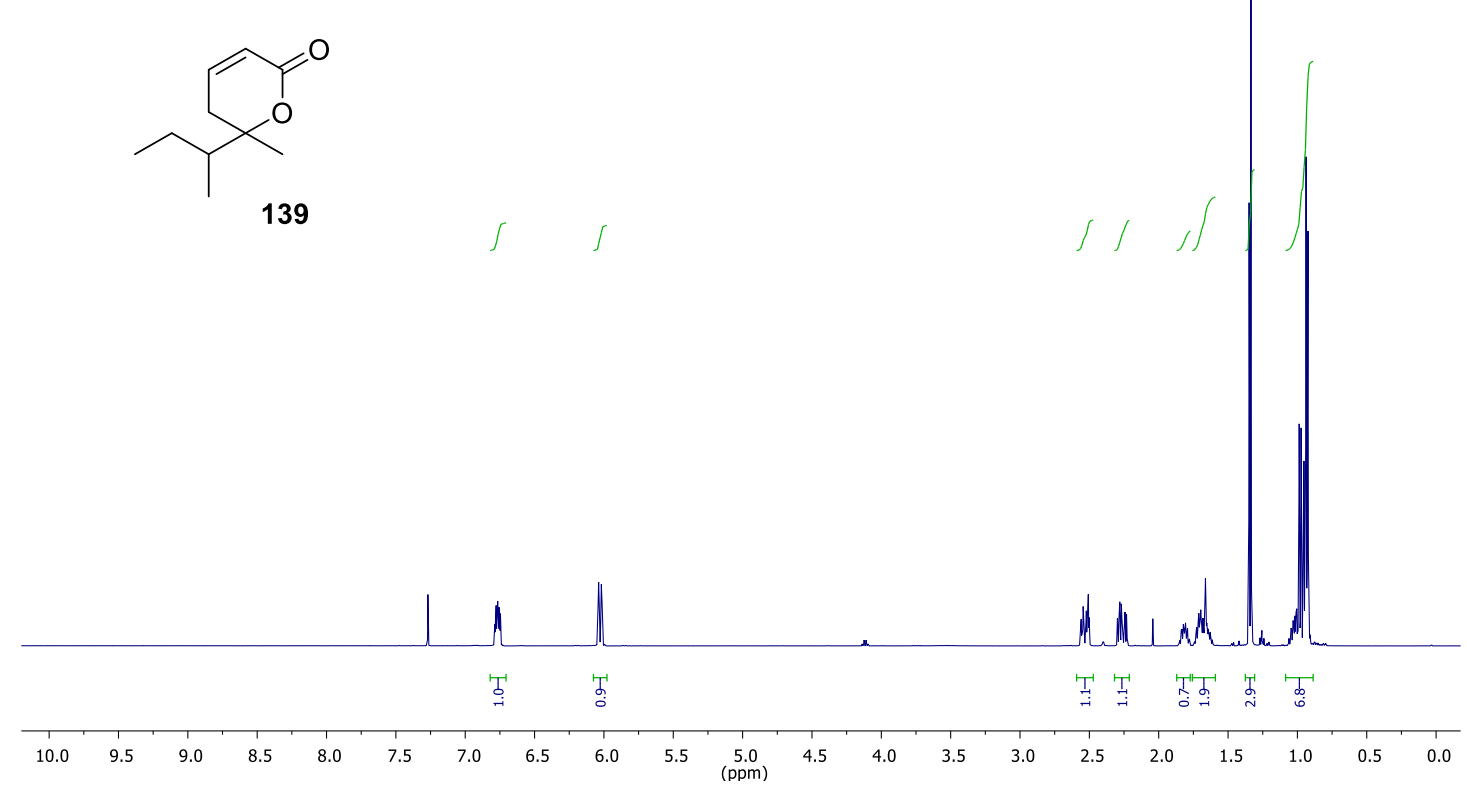

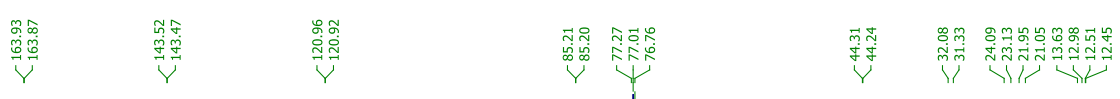

${ }^{13} \mathrm{C}-\mathrm{NMR}\left(125 \mathrm{MHz}, \mathrm{CDCl}_{3}\right.$ )

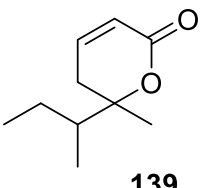

139

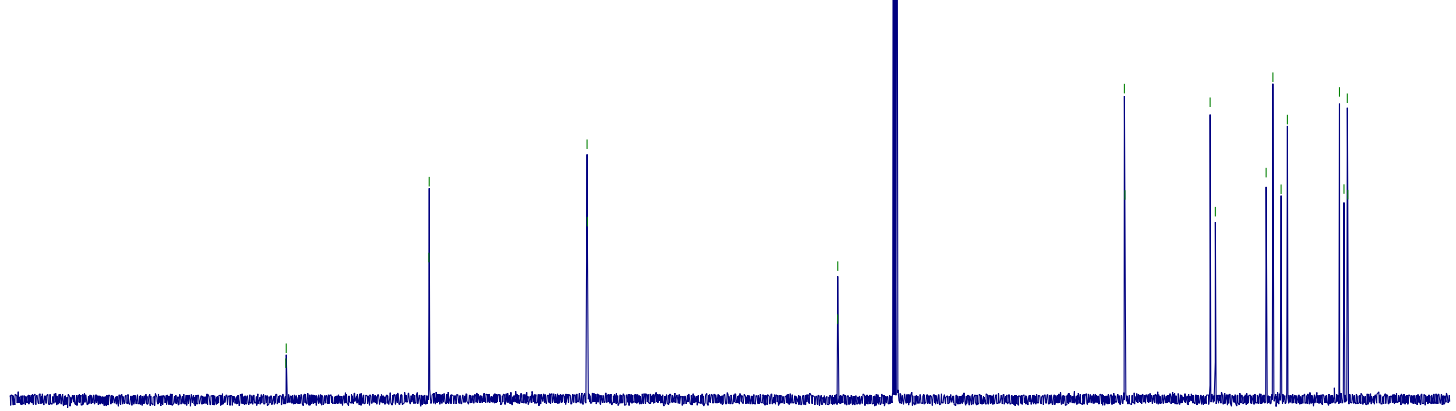

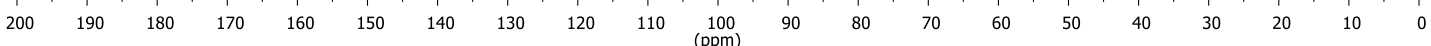


${ }^{1} \mathrm{H}-\mathrm{NMR}\left(500 \mathrm{MHz}, \mathrm{CDCl}_{3}\right)$

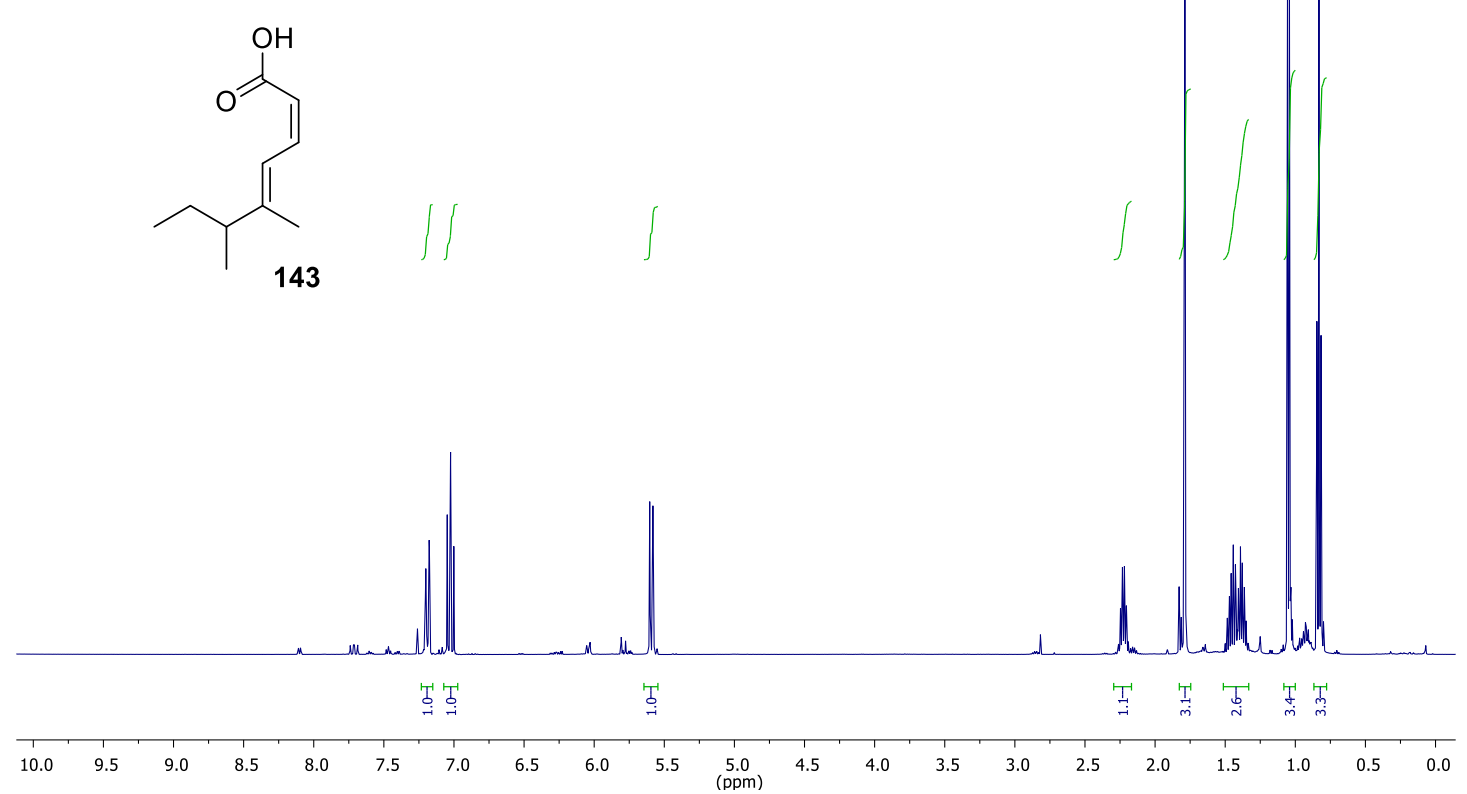

${ }^{13} \mathrm{C}-\mathrm{NMR}\left(125 \mathrm{MHz}, \mathrm{CDCl}_{3}\right.$ )
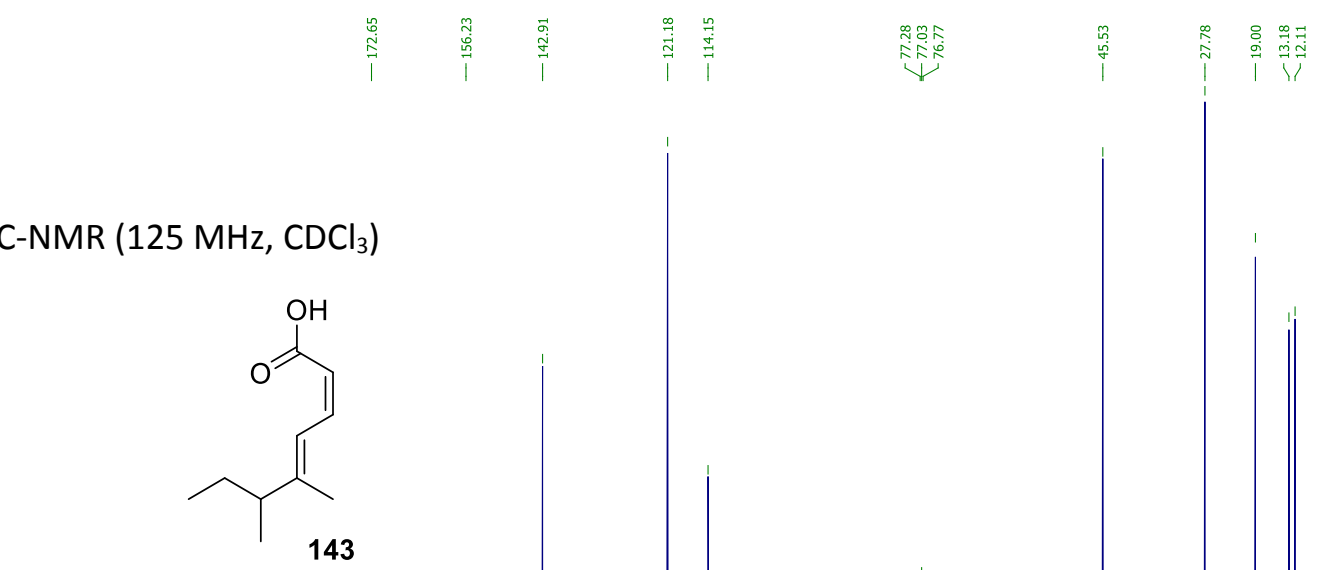

143

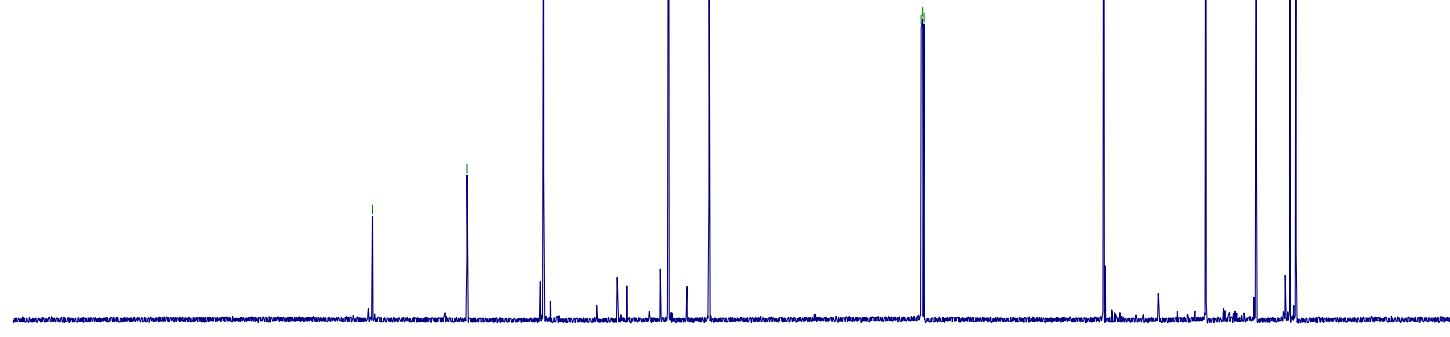

$\begin{array}{llllllllllllllllllllllllllllllll}230 & 220 & 210 & 200 & 190 & 180 & 170 & 160 & 150 & 140 & 130 & 120 & 110 & 100 & 90 & 80 & 70 & 60 & 50 & 40 & 30 & 20 & 10 & 0 & -10\end{array}$ 
${ }^{1} \mathrm{H}-\mathrm{NMR}\left(500 \mathrm{MHz}, \mathrm{CDCl}_{3}\right)$

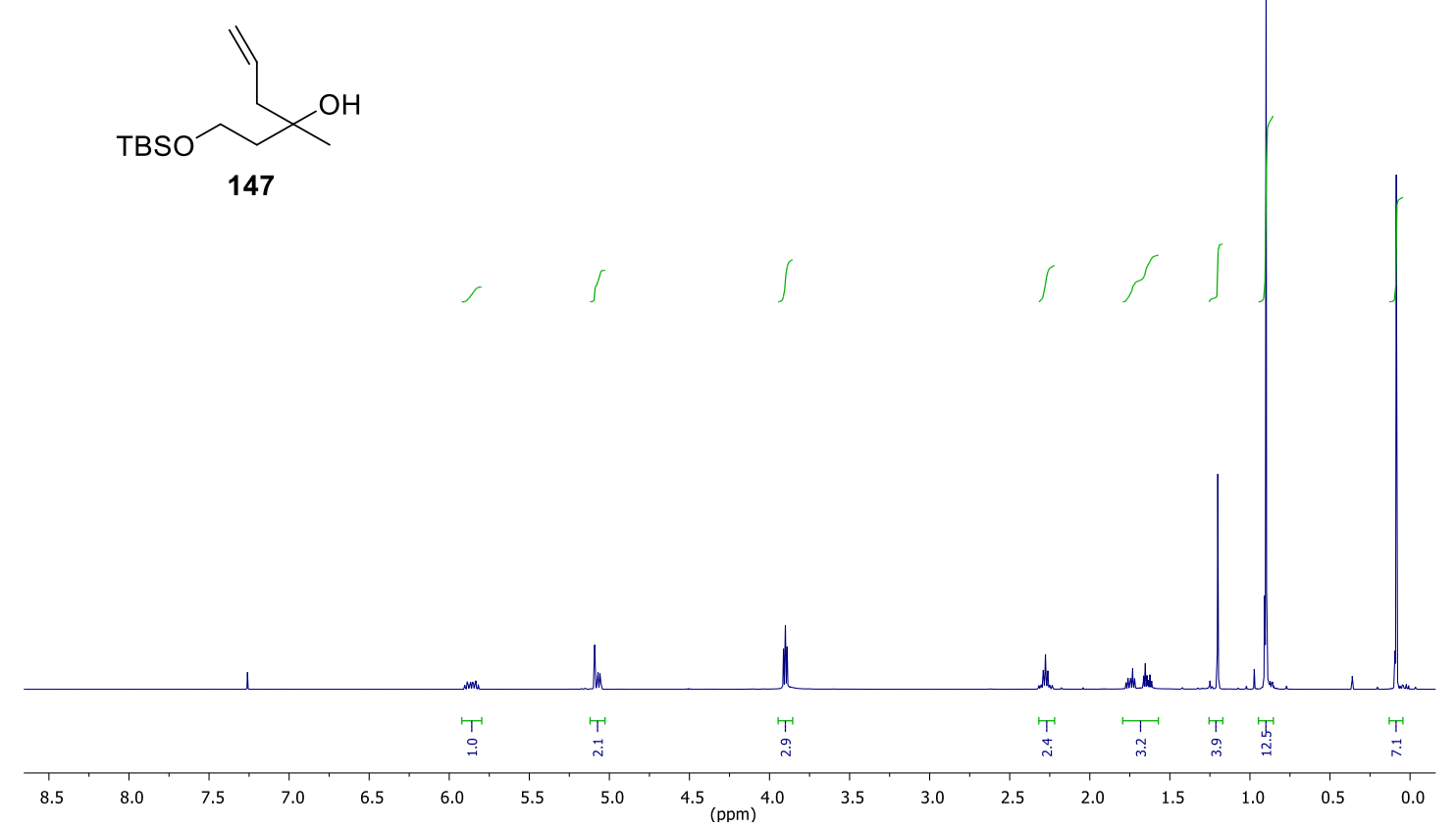

${ }^{13} \mathrm{C}-\mathrm{NMR}\left(125 \mathrm{MHz}, \mathrm{CDCl}_{3}\right)$

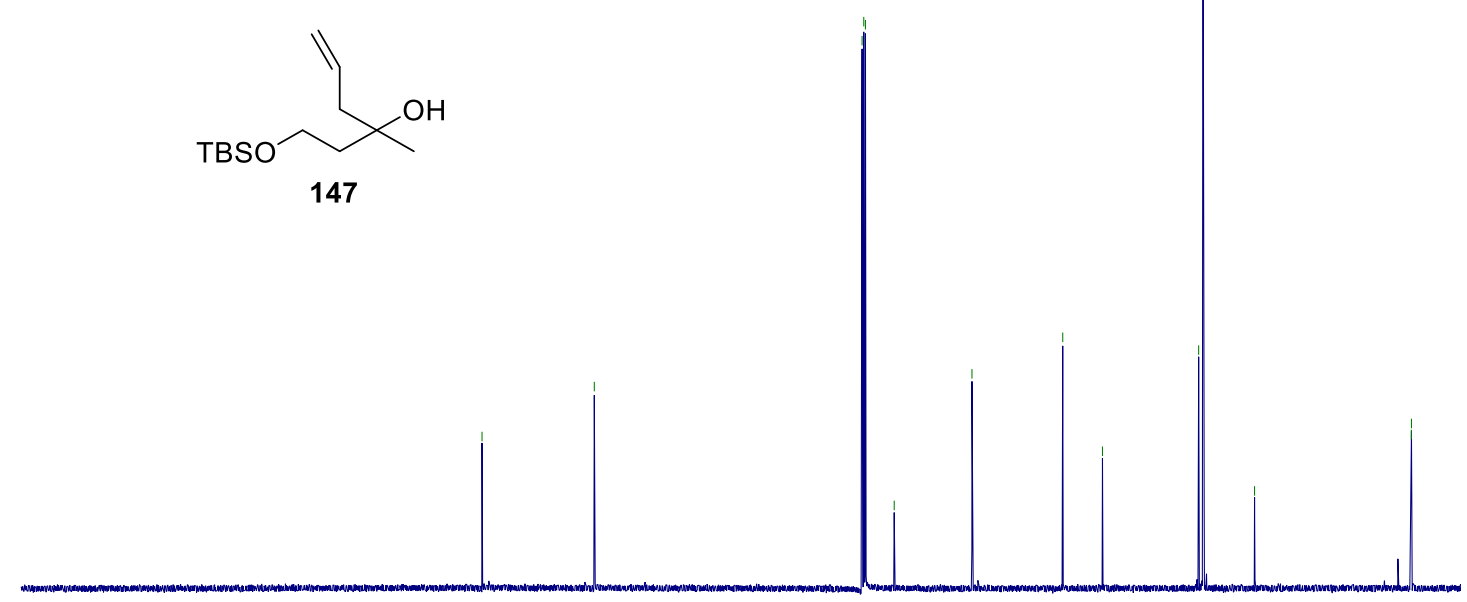

$\begin{array}{llllllllllllllllllllllll}200 & 190 & 180 & 170 & 160 & 150 & 140 & 130 & 120 & 110 & 100 & 90 & 80 & 70 & 60 & 50 & 40 & 30 & 20 & 10 & 0 & -10\end{array}$ 
${ }^{1} \mathrm{H}-\mathrm{NMR}\left(500 \mathrm{MHz}, \mathrm{CDCl}_{3}\right.$ )
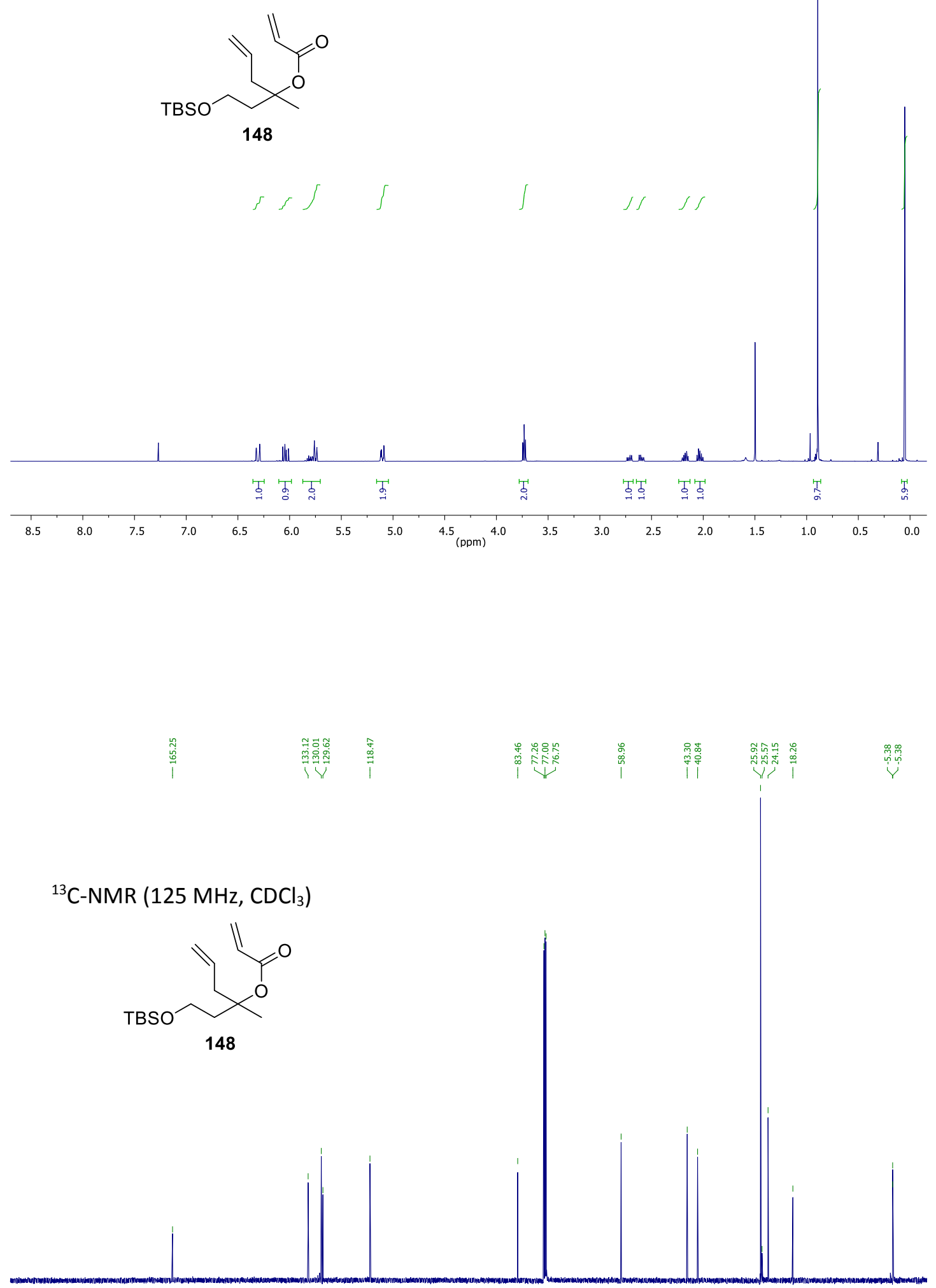

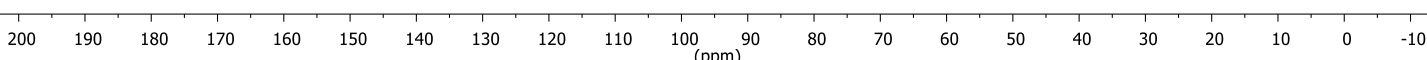


${ }^{1} \mathrm{H}-\mathrm{NMR}\left(500 \mathrm{MHz}, \mathrm{CDCl}_{3}\right)$

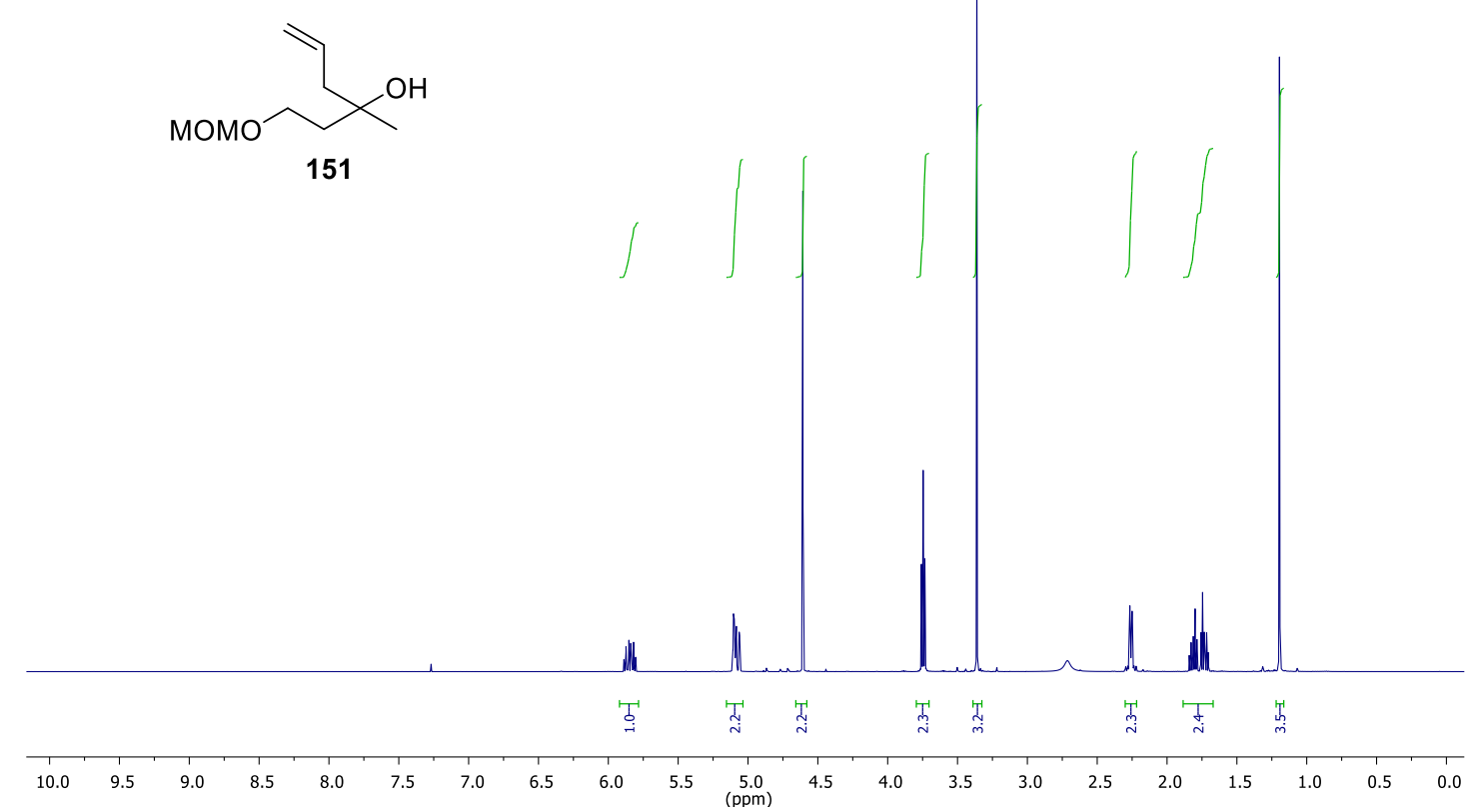

${ }^{13} \mathrm{C}-\mathrm{NMR}\left(125 \mathrm{MHz}, \mathrm{CDCl}_{3}\right.$ )

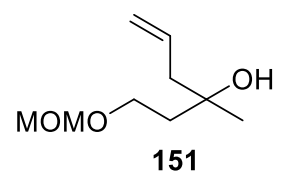

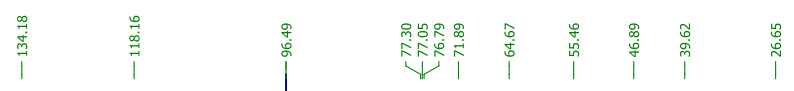

$\begin{array}{llllllllllllllllllllllll} & 1 \\ 200 & 190 & 180 & 170 & 160 & 150 & 140 & 130 & 120 & 110 & \begin{array}{c}100 \\ (\mathrm{ppm})\end{array} & 90 & 80 & 70 & 60 & 50 & 40 & 30 & 20 & 10 & 0\end{array}$ 
${ }^{1} \mathrm{H}-\mathrm{NMR}\left(500 \mathrm{MHz}, \mathrm{CDCl}_{3}\right)$
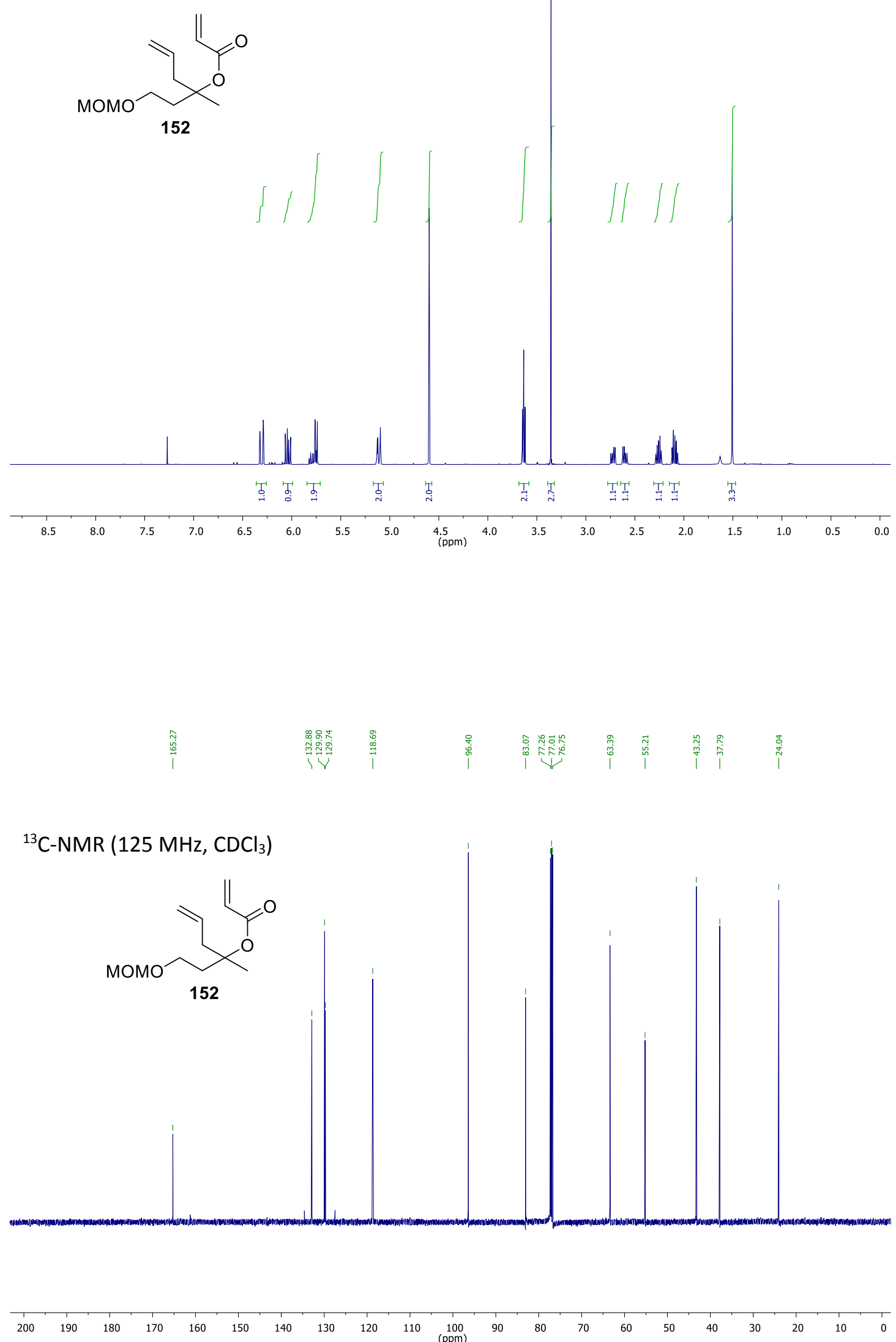
${ }^{1} \mathrm{H}-\mathrm{NMR}\left(500 \mathrm{MHz}, \mathrm{CDCl}_{3}\right.$ )

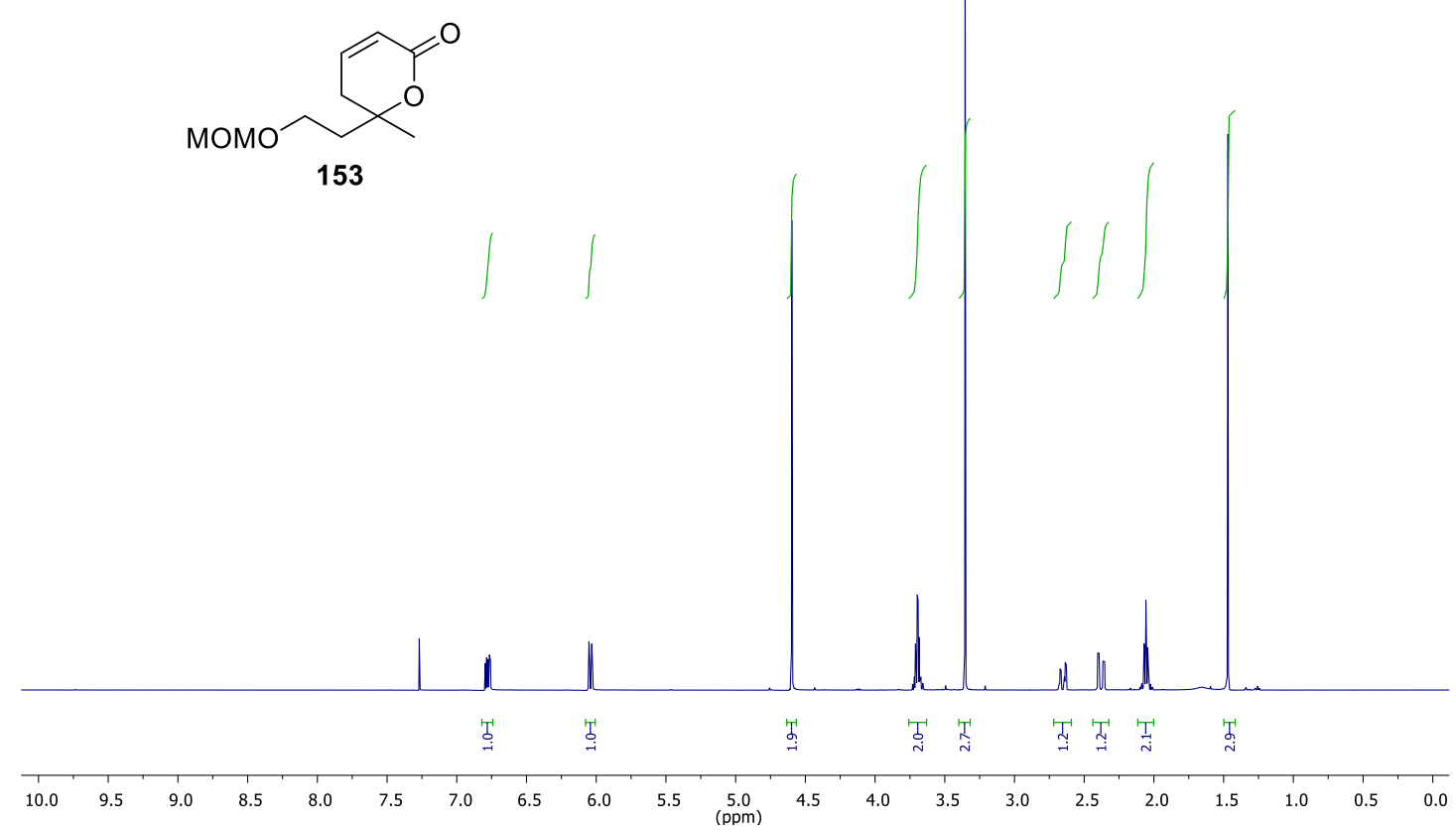

${ }^{13} \mathrm{C}-\mathrm{NMR}\left(125 \mathrm{MHz}, \mathrm{CDCl}_{3}\right)$

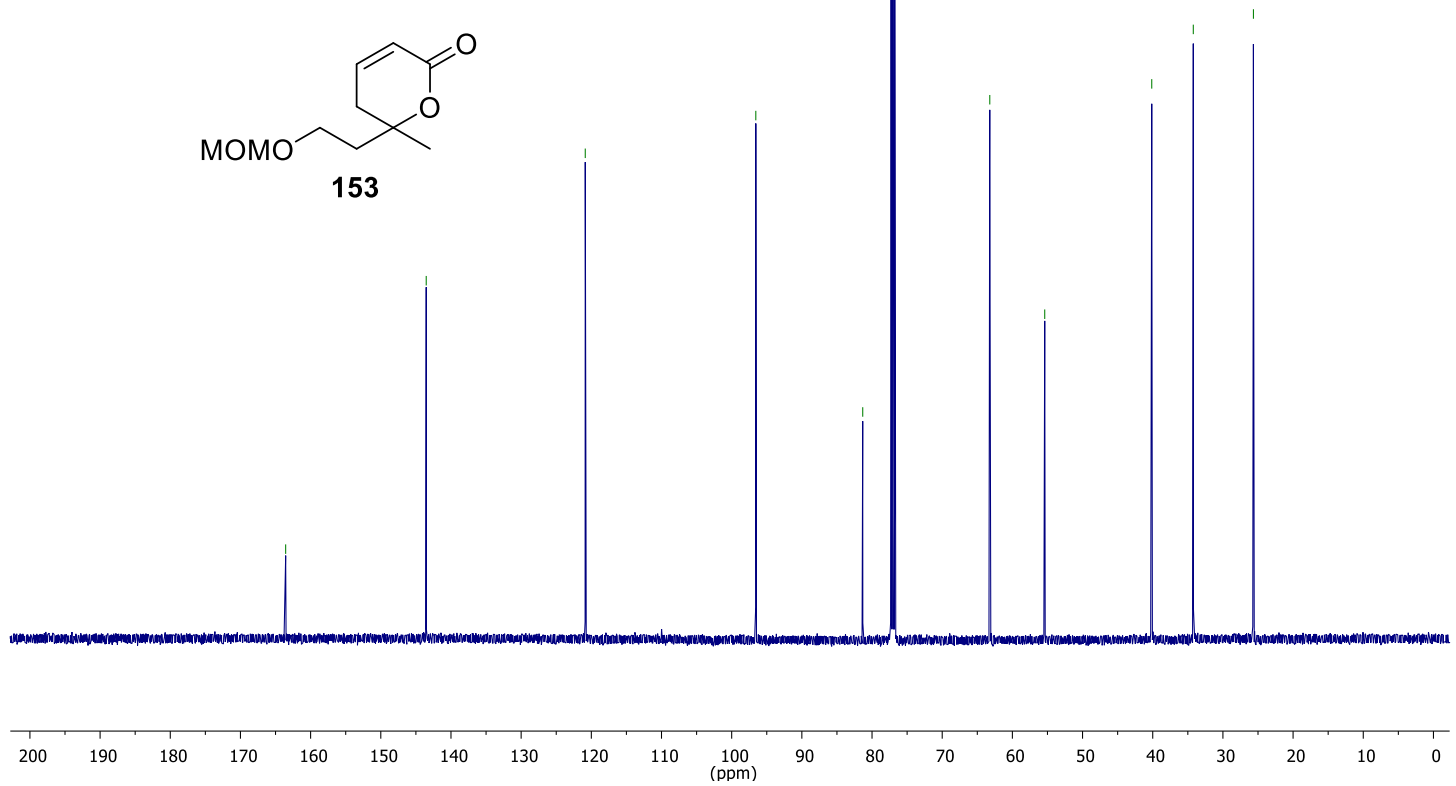


${ }^{1} \mathrm{H}-\mathrm{NMR}\left(500 \mathrm{MHz}, \mathrm{CDCl}_{3}\right)$

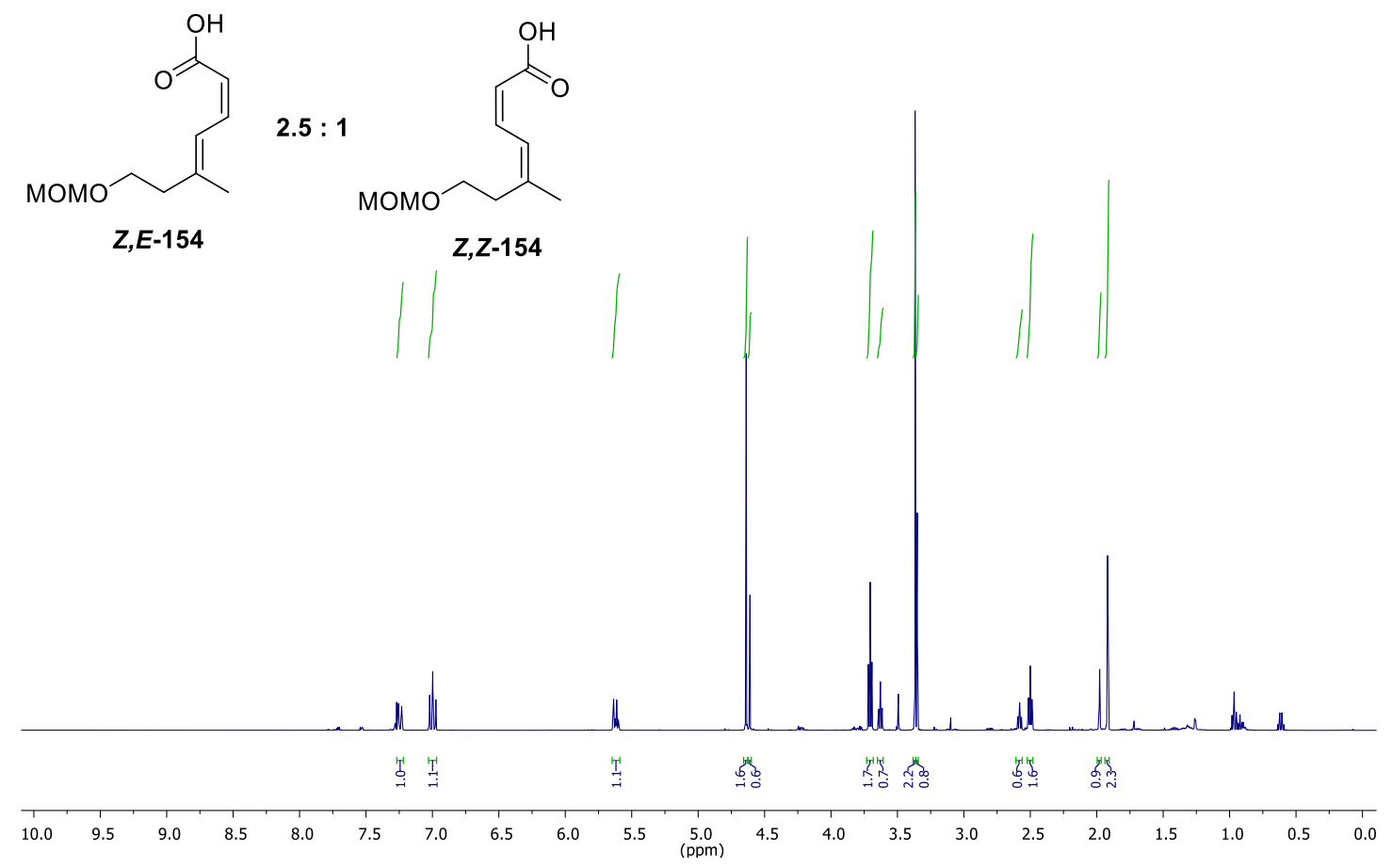

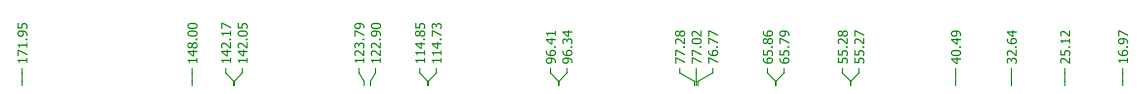

${ }^{13} \mathrm{C}-\mathrm{NMR}\left(125 \mathrm{MHz}, \mathrm{CDCl}_{3}\right)$

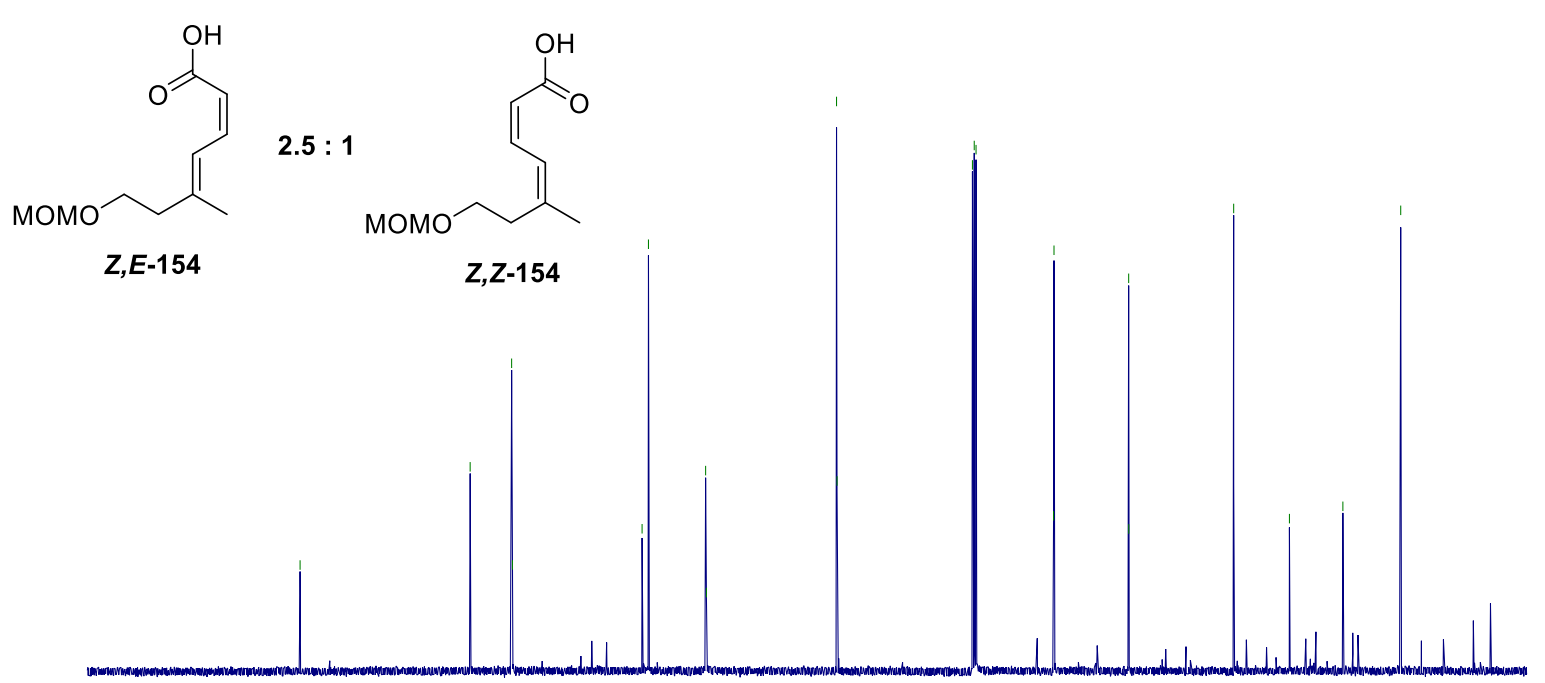

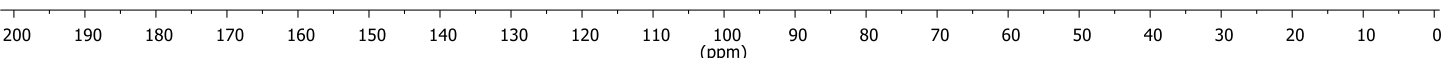


${ }^{1} \mathrm{H}-\mathrm{NMR}\left(500 \mathrm{MHz}, \mathrm{C}_{6} \mathrm{D}_{6}\right)$

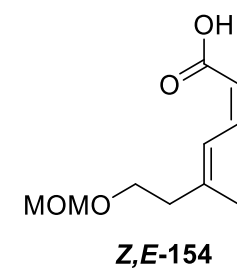

$2.5: 1$

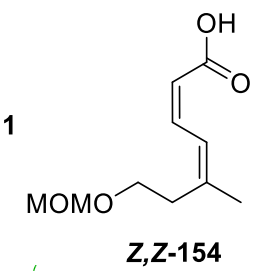
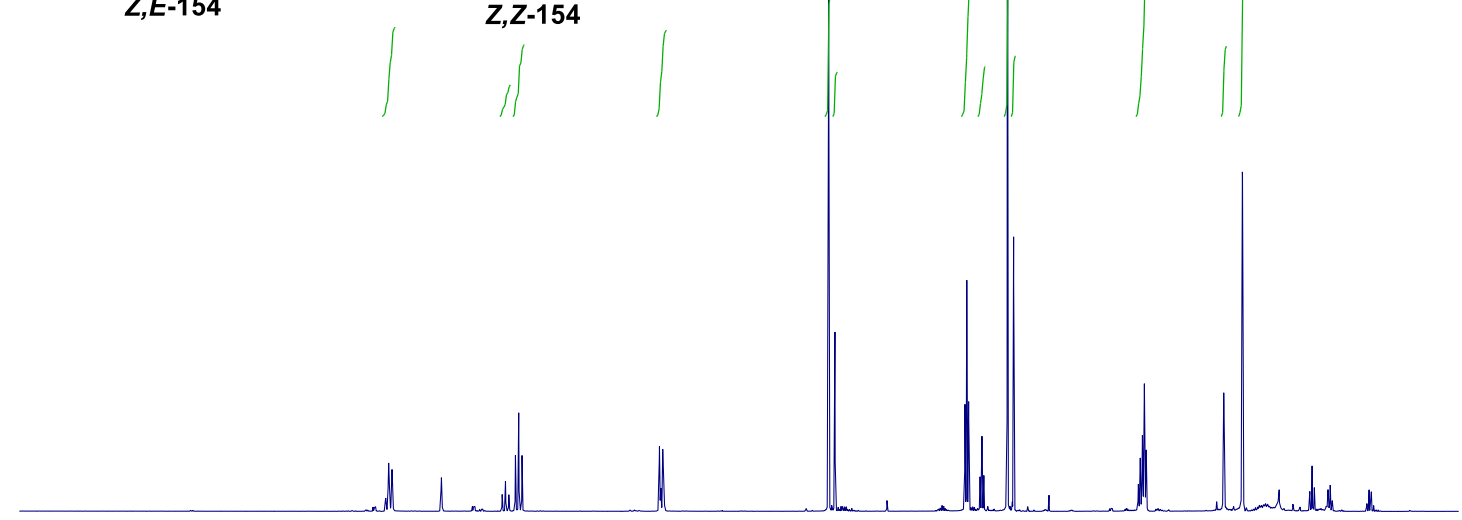

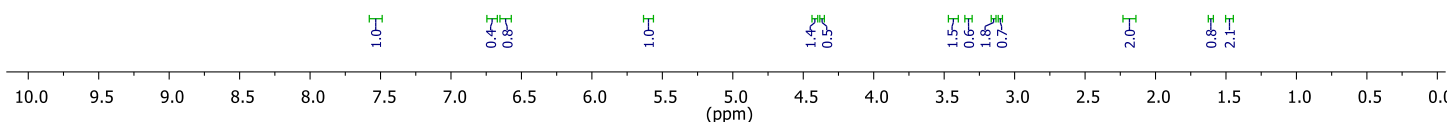

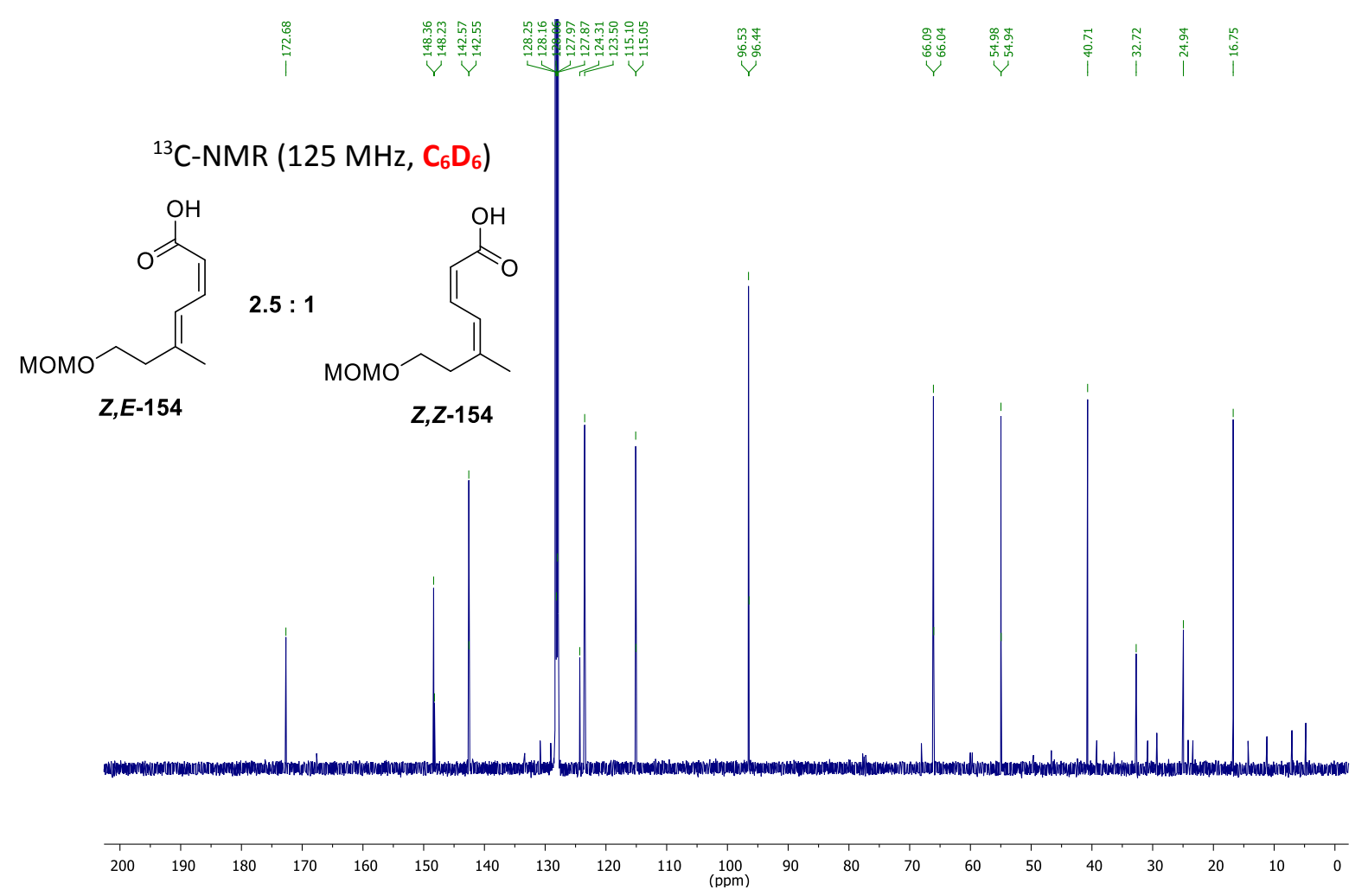


${ }^{1} \mathrm{H}-\mathrm{NMR}\left(500 \mathrm{MHz}, \mathrm{CDCl}_{3}\right.$ )
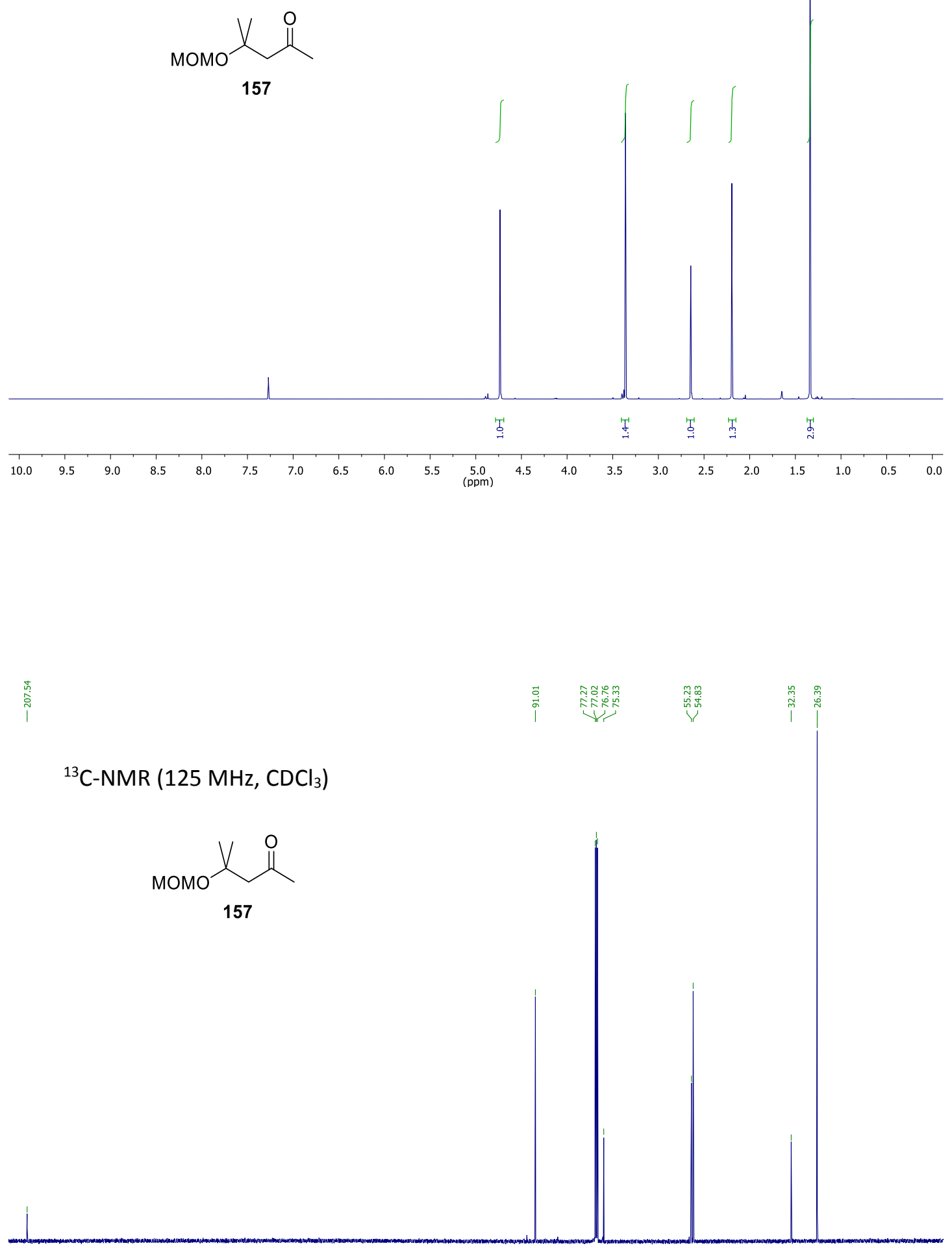

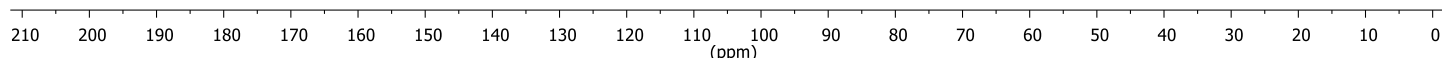


${ }^{1} \mathrm{H}-\mathrm{NMR}\left(500 \mathrm{MHz}, \mathrm{CDCl}_{3}\right.$ )

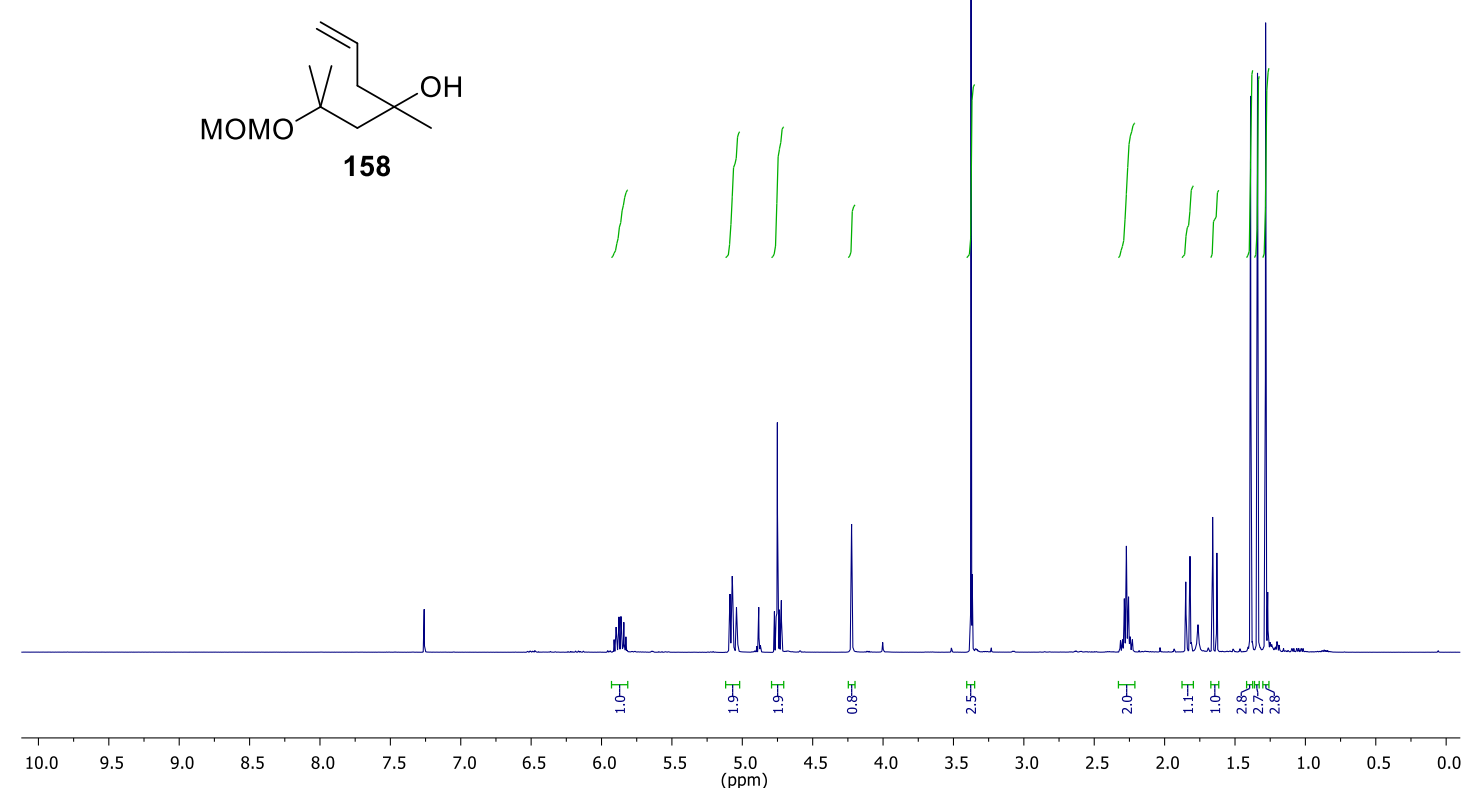

${ }^{13} \mathrm{C}-\mathrm{NMR}\left(125 \mathrm{MHz}, \mathrm{CDCl}_{3}\right)$

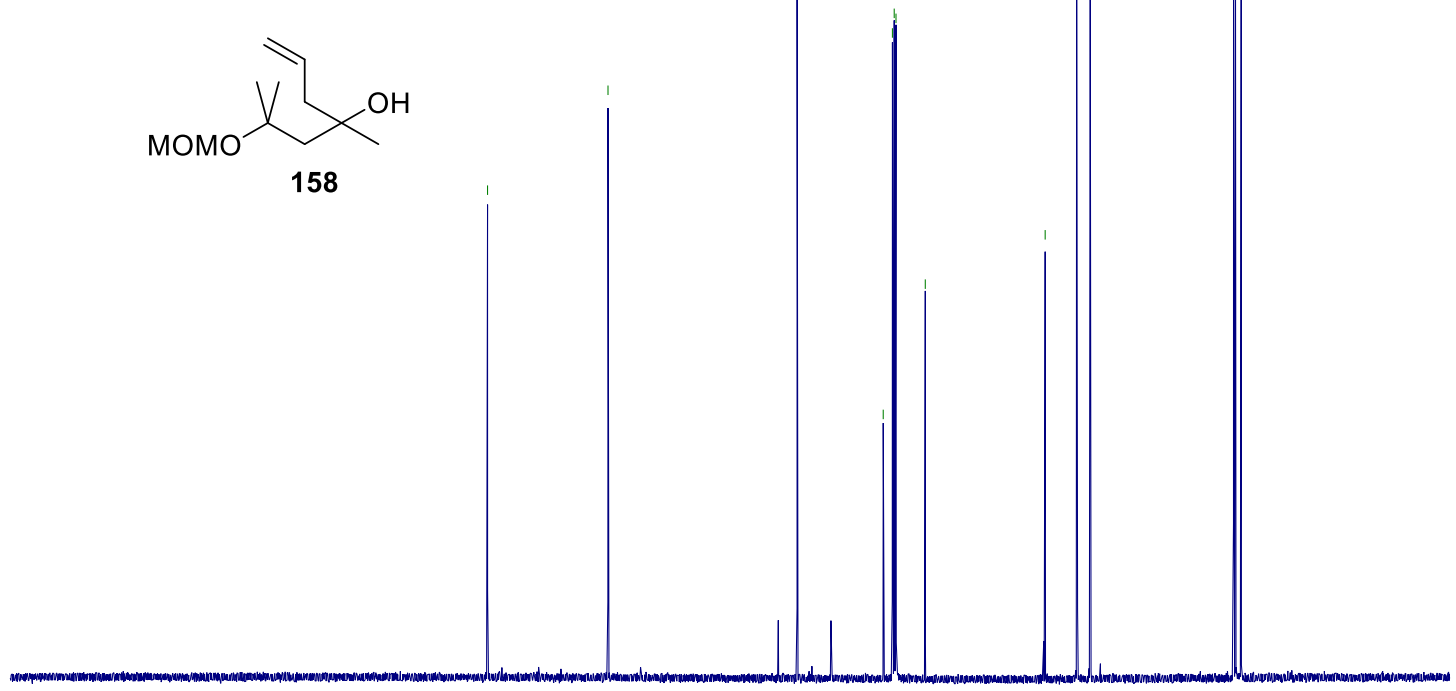

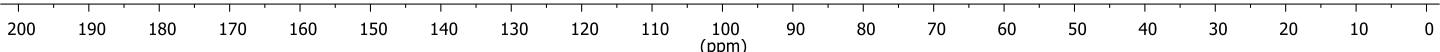


${ }^{1} \mathrm{H}-\mathrm{NMR}\left(500 \mathrm{MHz}, \mathrm{CDCl}_{3}\right)$

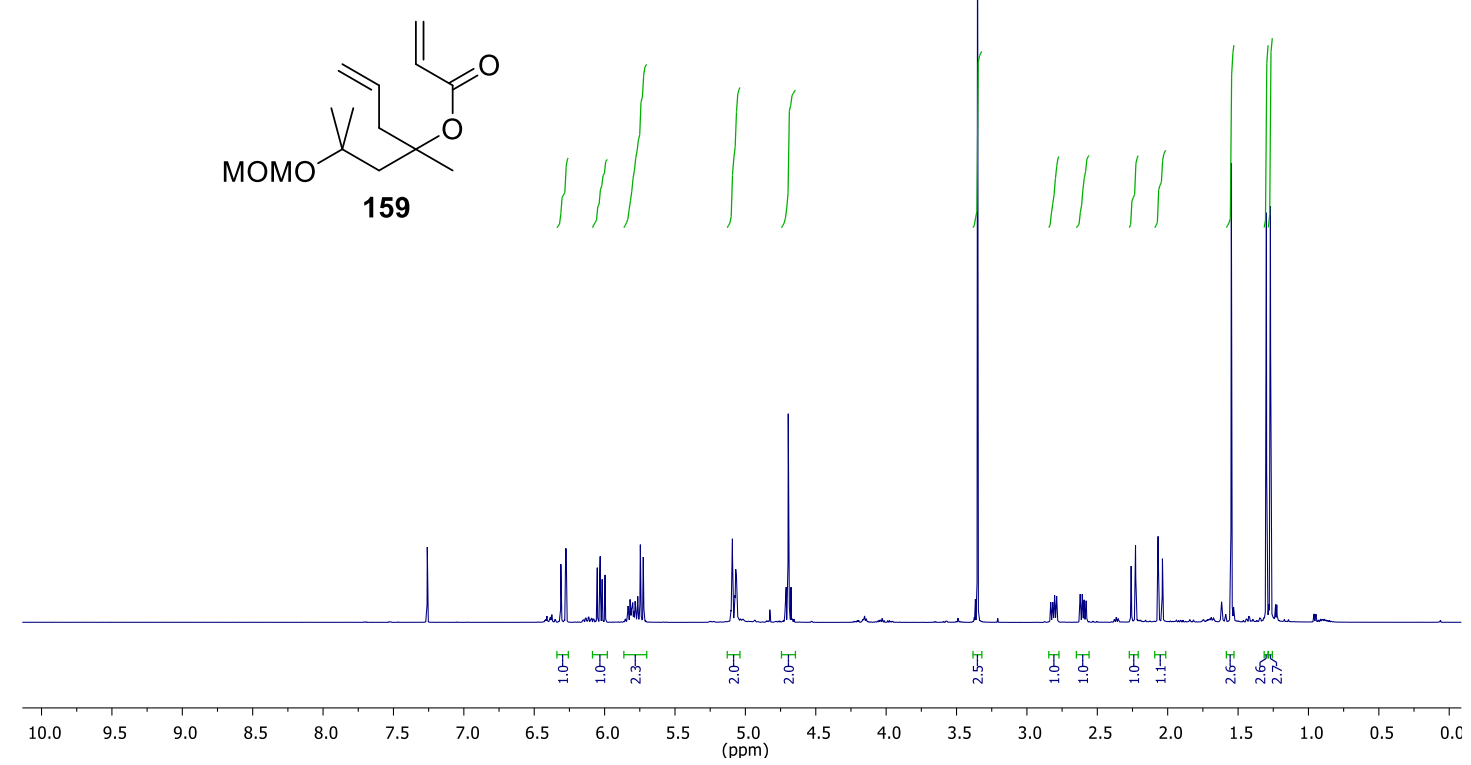

${ }^{13} \mathrm{C}-\mathrm{NMR}\left(125 \mathrm{MHz}, \mathrm{CDCl}_{3}\right)$

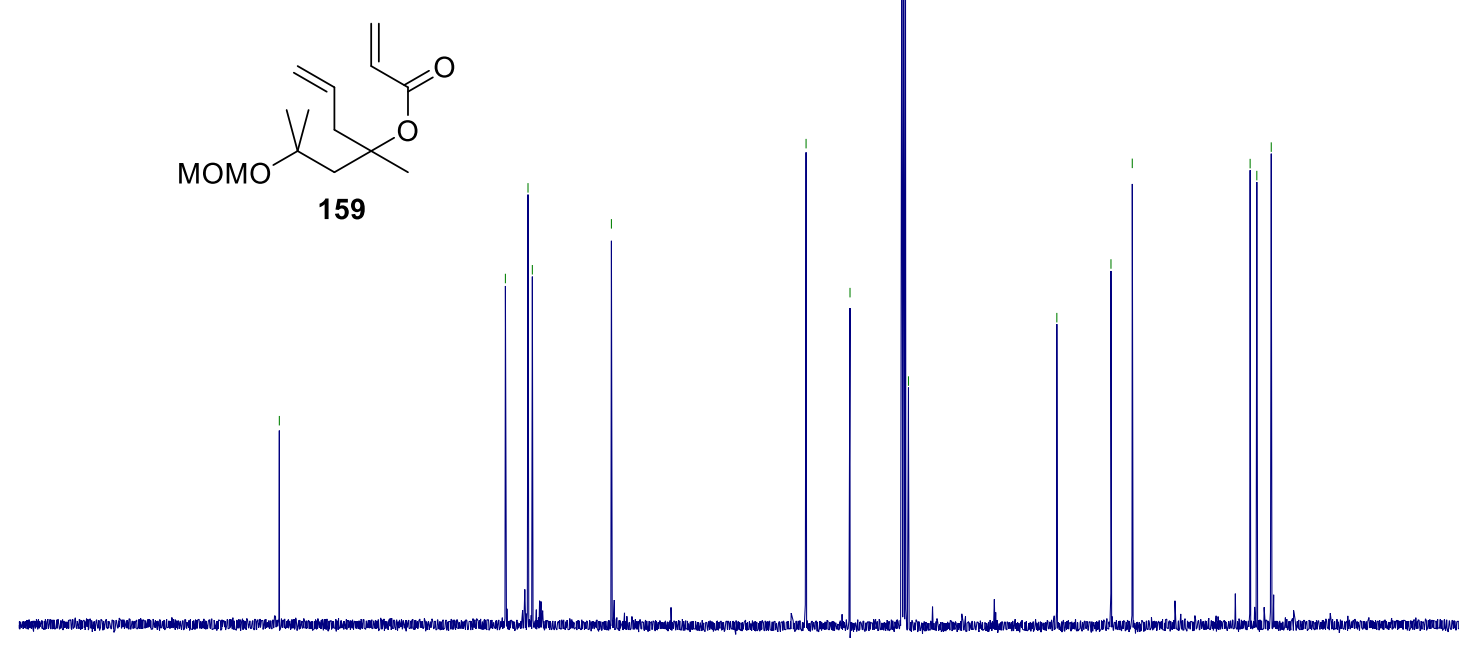

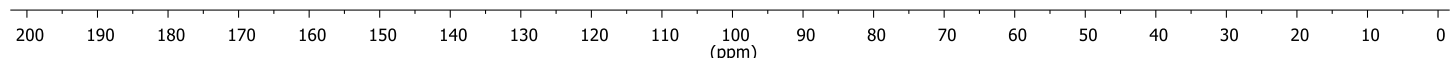


${ }^{1} \mathrm{H}-\mathrm{NMR}\left(500 \mathrm{MHz}, \mathrm{CDCl}_{3}\right.$ )

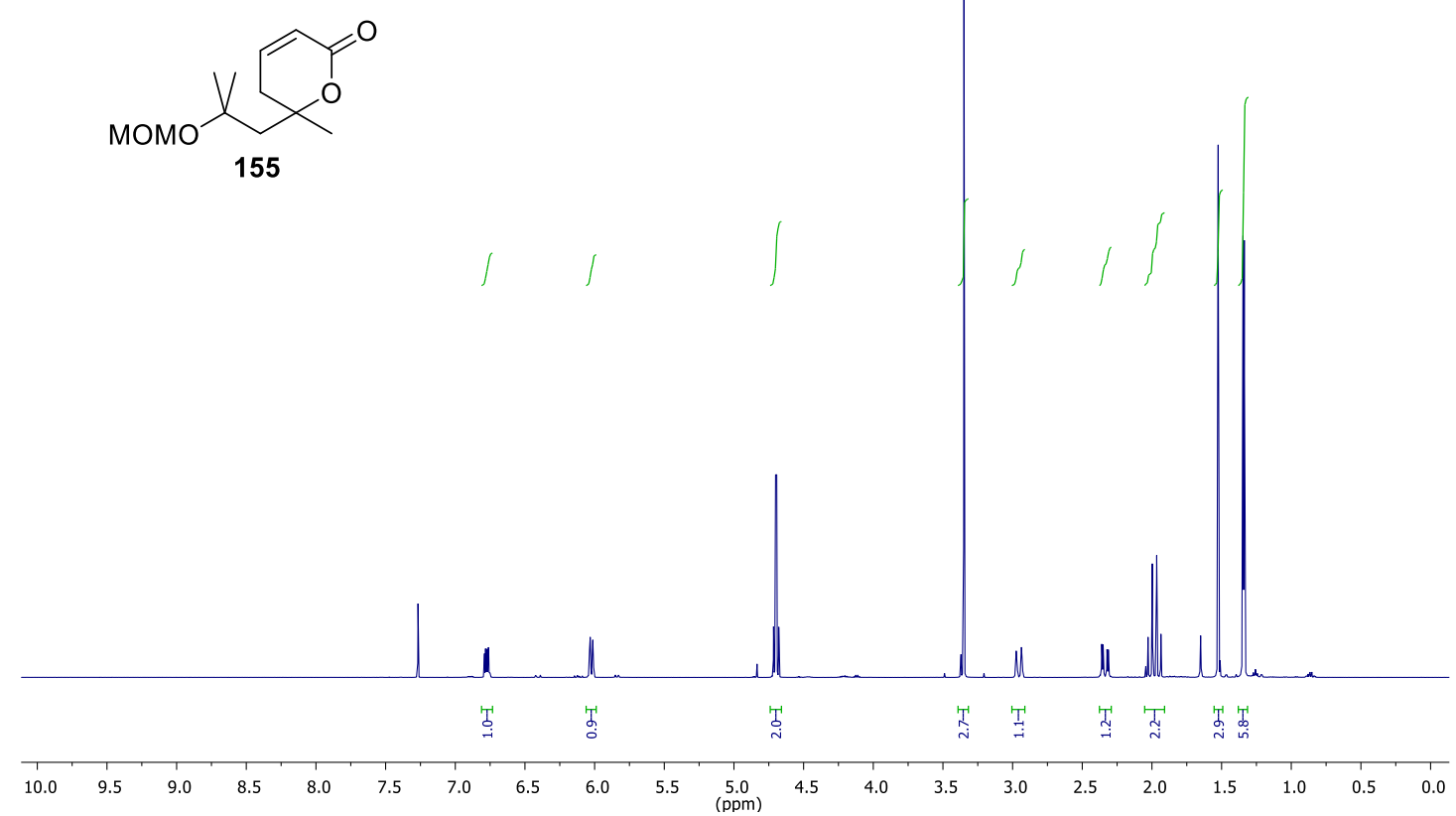

${ }^{13} \mathrm{C}-\mathrm{NMR}\left(125 \mathrm{MHz}, \mathrm{CDCl}_{3}\right)$<smiles>COC(C)(C)CC1(C)CC=CC(=O)O1</smiles>

155 
${ }^{1} \mathrm{H}-\mathrm{NMR}\left(500 \mathrm{MHz}, \mathrm{CDCl}_{3}\right.$ )

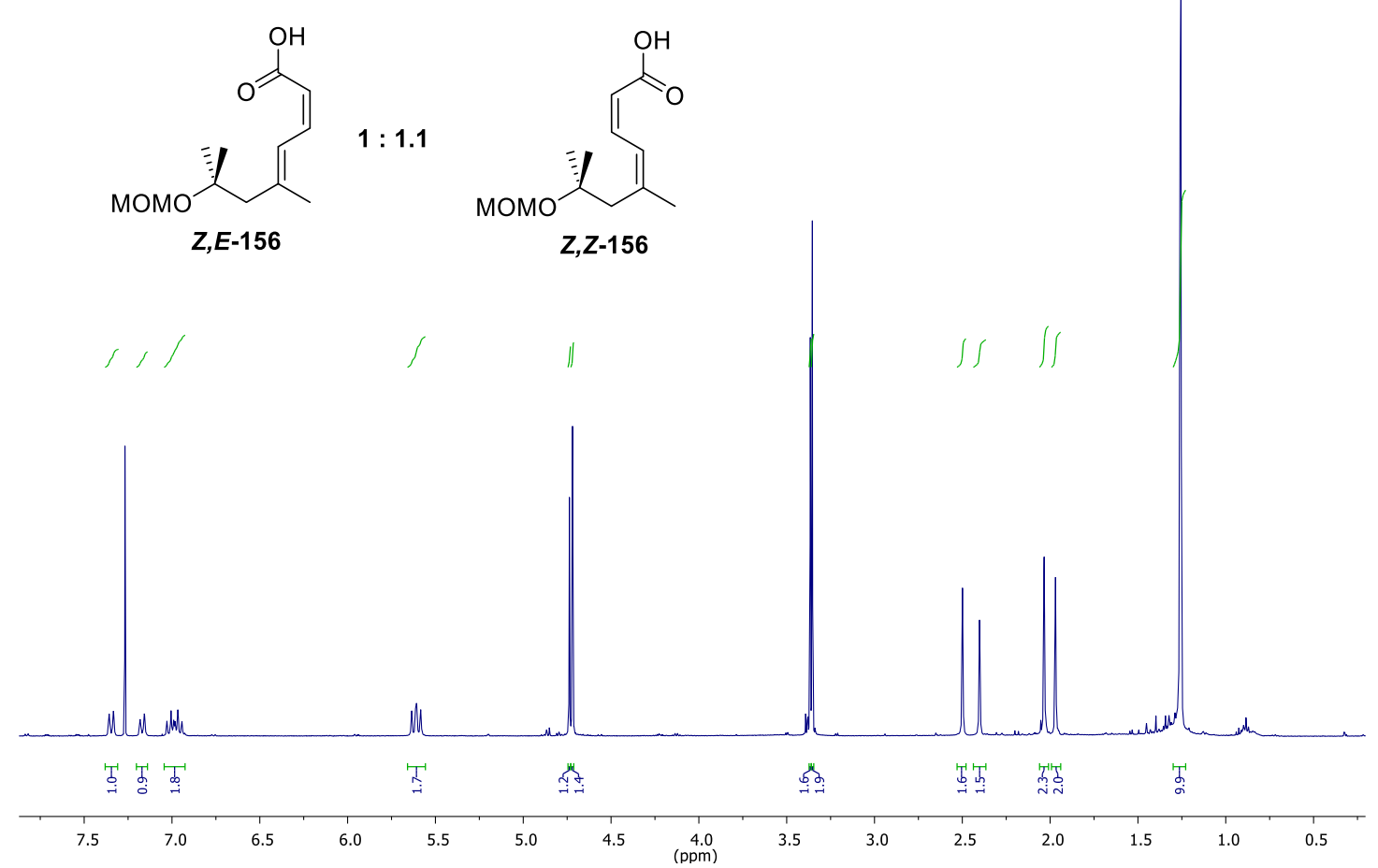

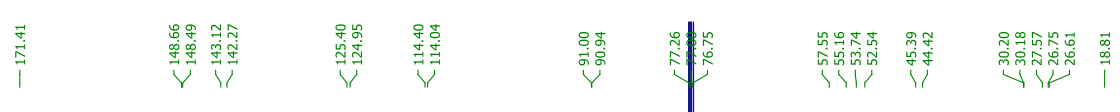

${ }^{13} \mathrm{C}-\mathrm{NMR}\left(125 \mathrm{MHz}, \mathrm{CDCl}_{3}\right)$<smiles>CO[C@H](C)C/C(C)=C\C=C/C(=O)O</smiles>

Z,E-156
$1: 1.1$<smiles>[Z16]OC(C)C(C)=CC=CC(=O)O</smiles>

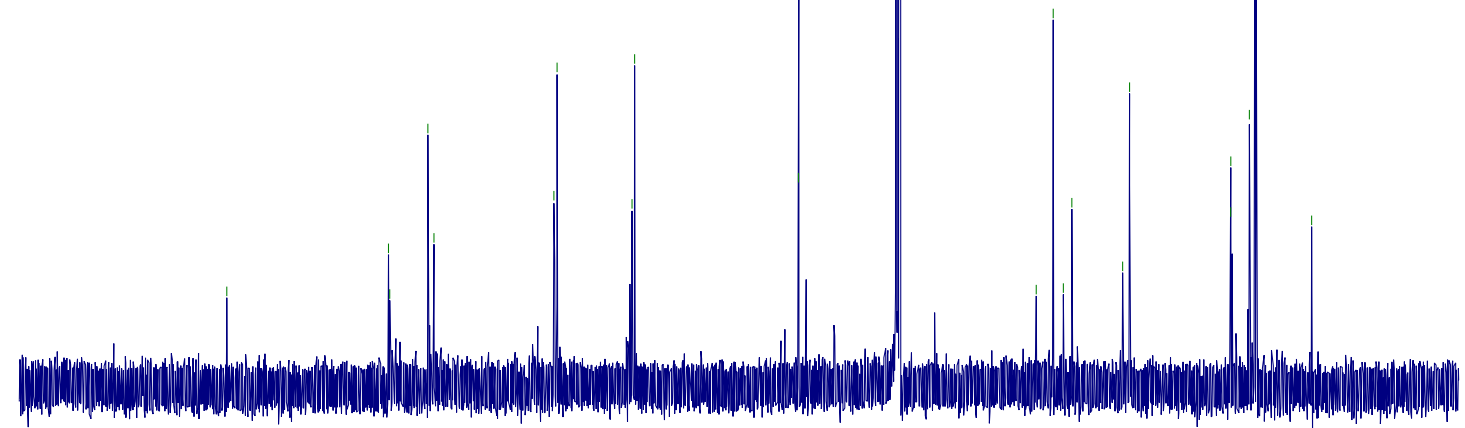

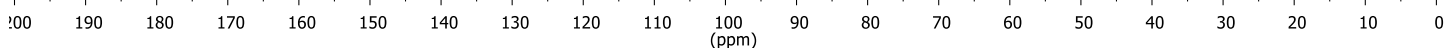


${ }^{1} \mathrm{H}-\mathrm{NMR}\left(500 \mathrm{MHz}, \mathrm{CDCl}_{3}\right)$

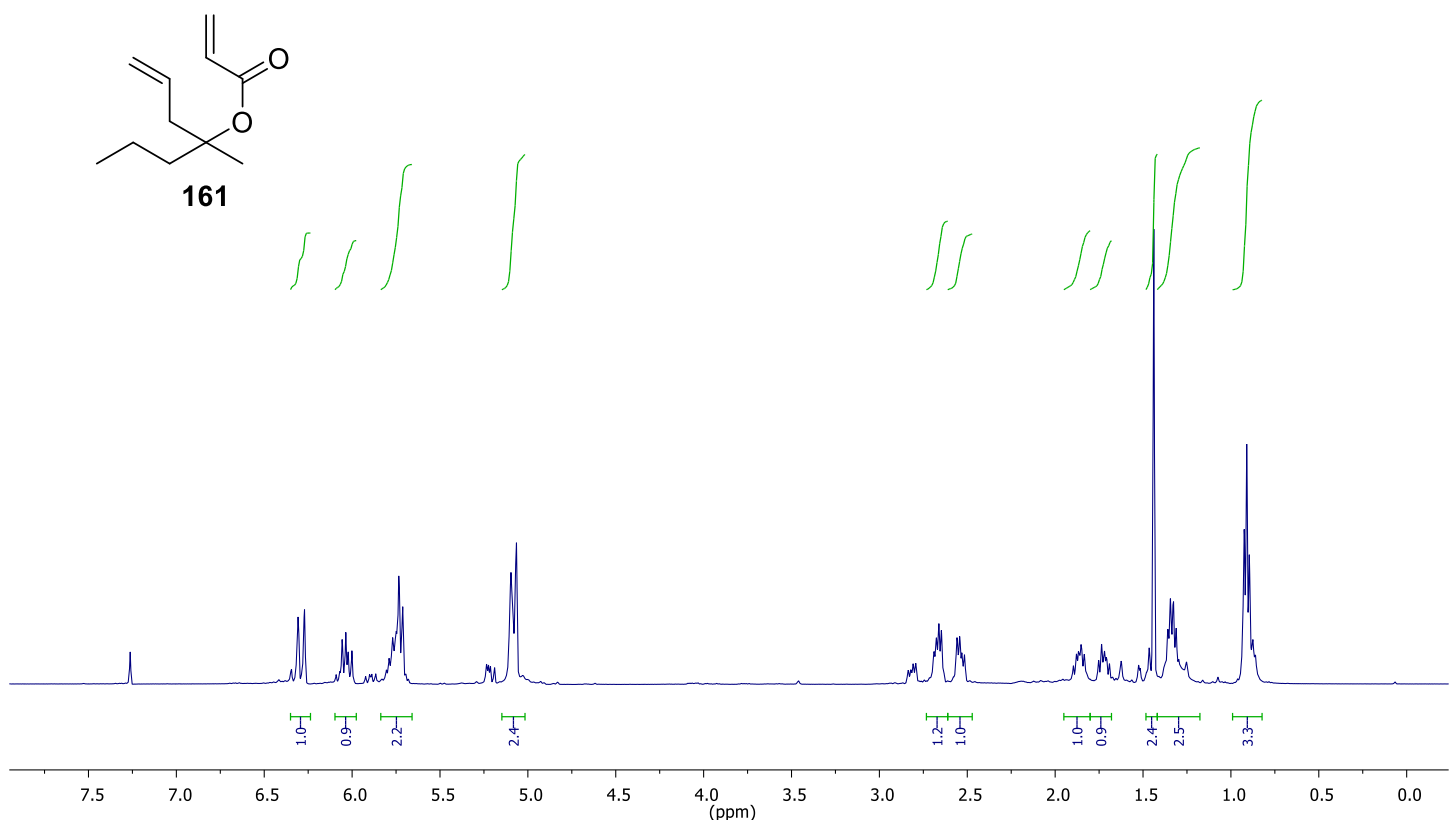

${ }^{13} \mathrm{C}-\mathrm{NMR}\left(125 \mathrm{MHz}, \mathrm{CDCl}_{3}\right.$ )

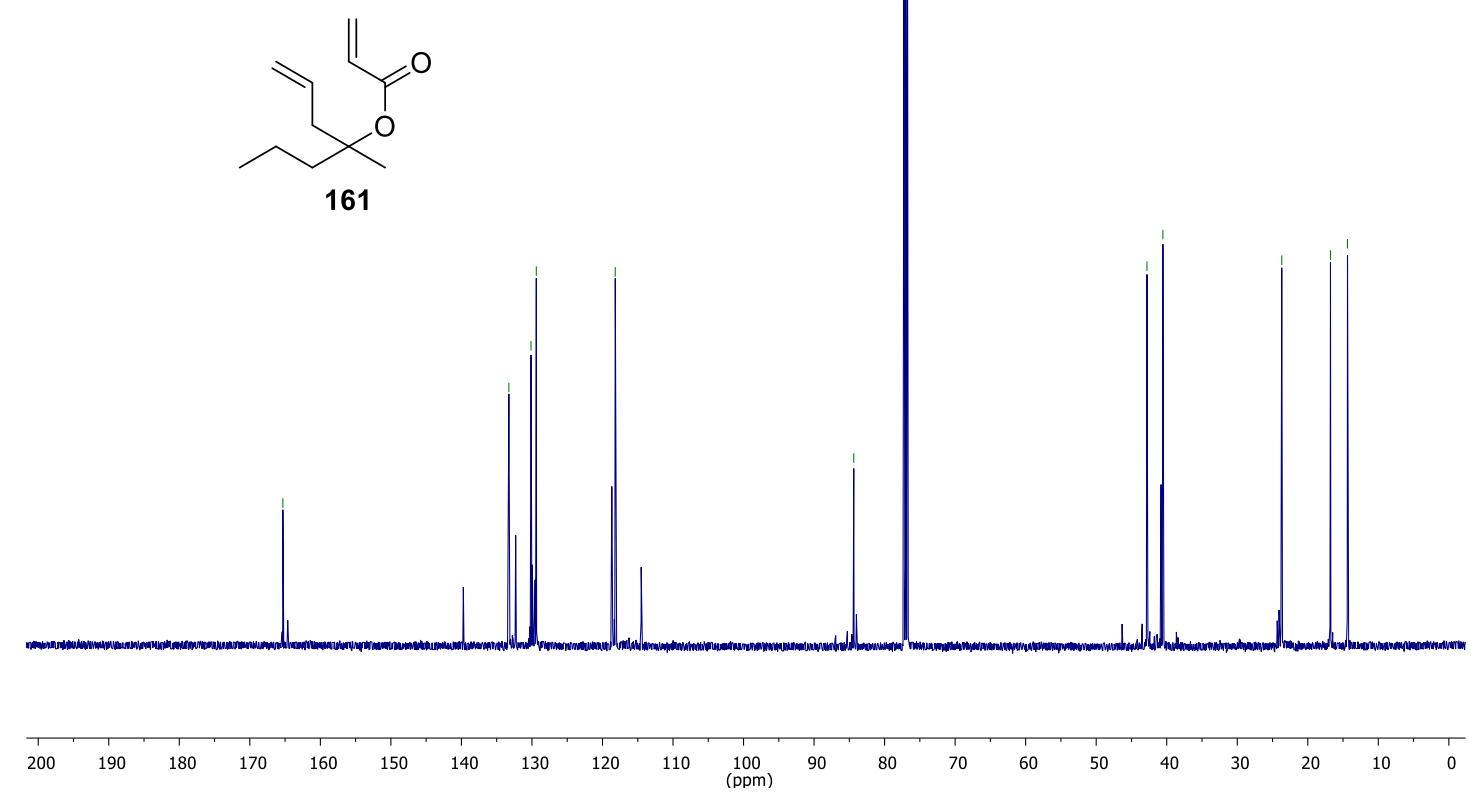


${ }^{1} \mathrm{H}-\mathrm{NMR}\left(500 \mathrm{MHz}, \mathrm{CDCl}_{3}\right.$ )
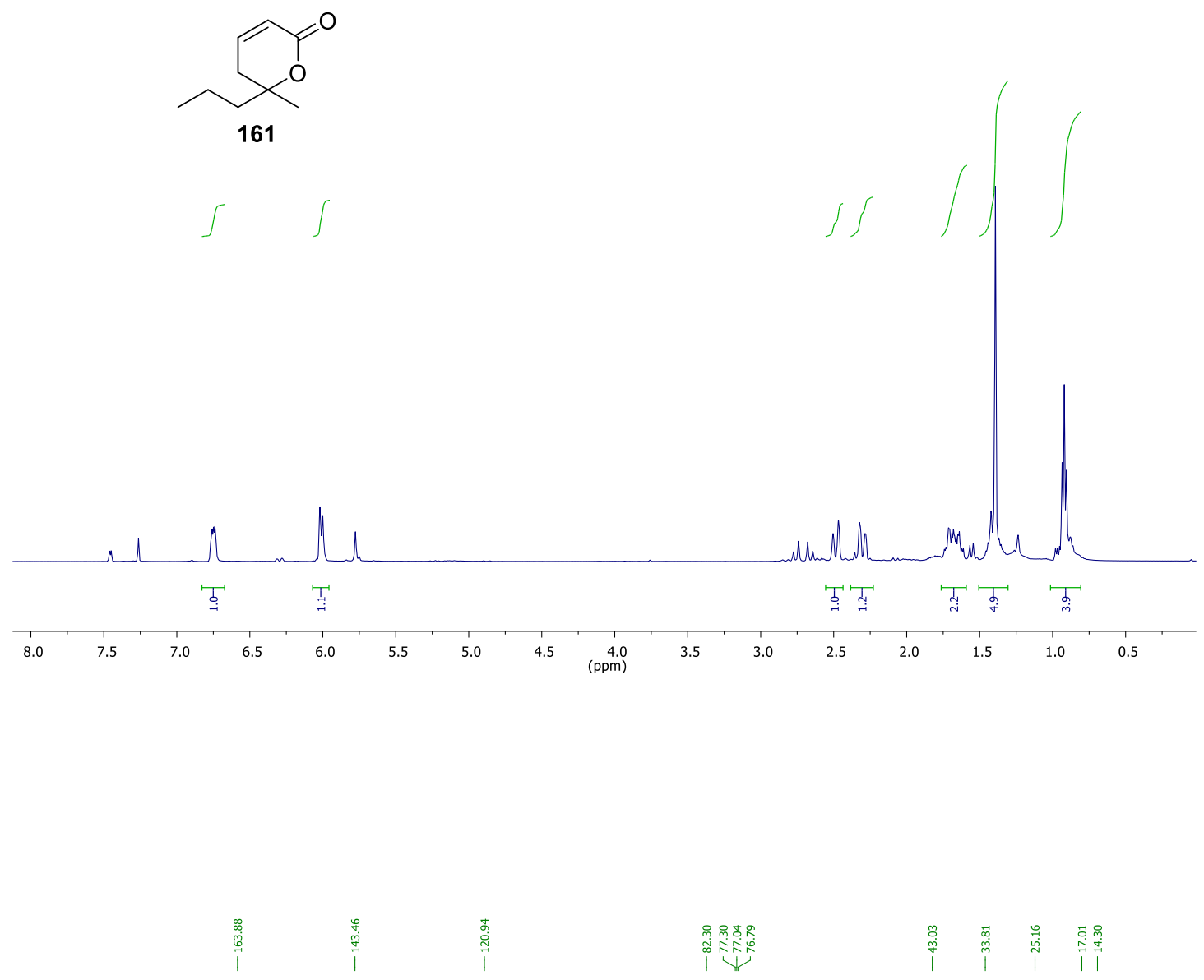

${ }^{13} \mathrm{C}-\mathrm{NMR}\left(125 \mathrm{MHz}, \mathrm{CDCl}_{3}\right.$ )<smiles>CCCC1(C)CC=CC(=O)O1</smiles>

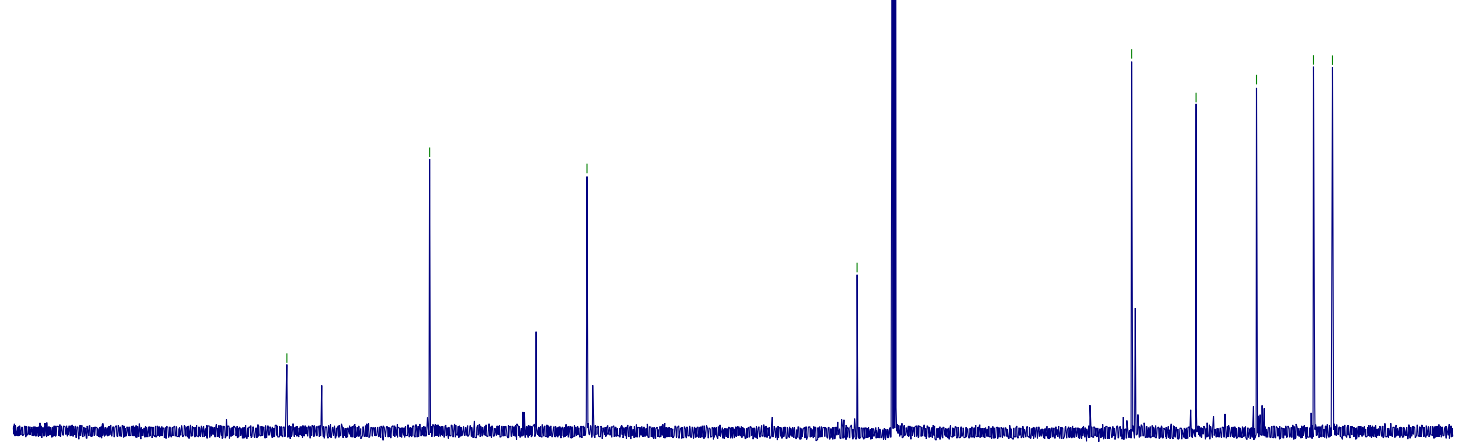

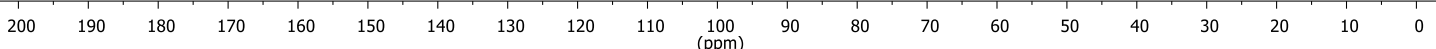


${ }^{1} \mathrm{H}-\mathrm{NMR}\left(500 \mathrm{MHz}, \mathrm{CDCl}_{3}\right)$

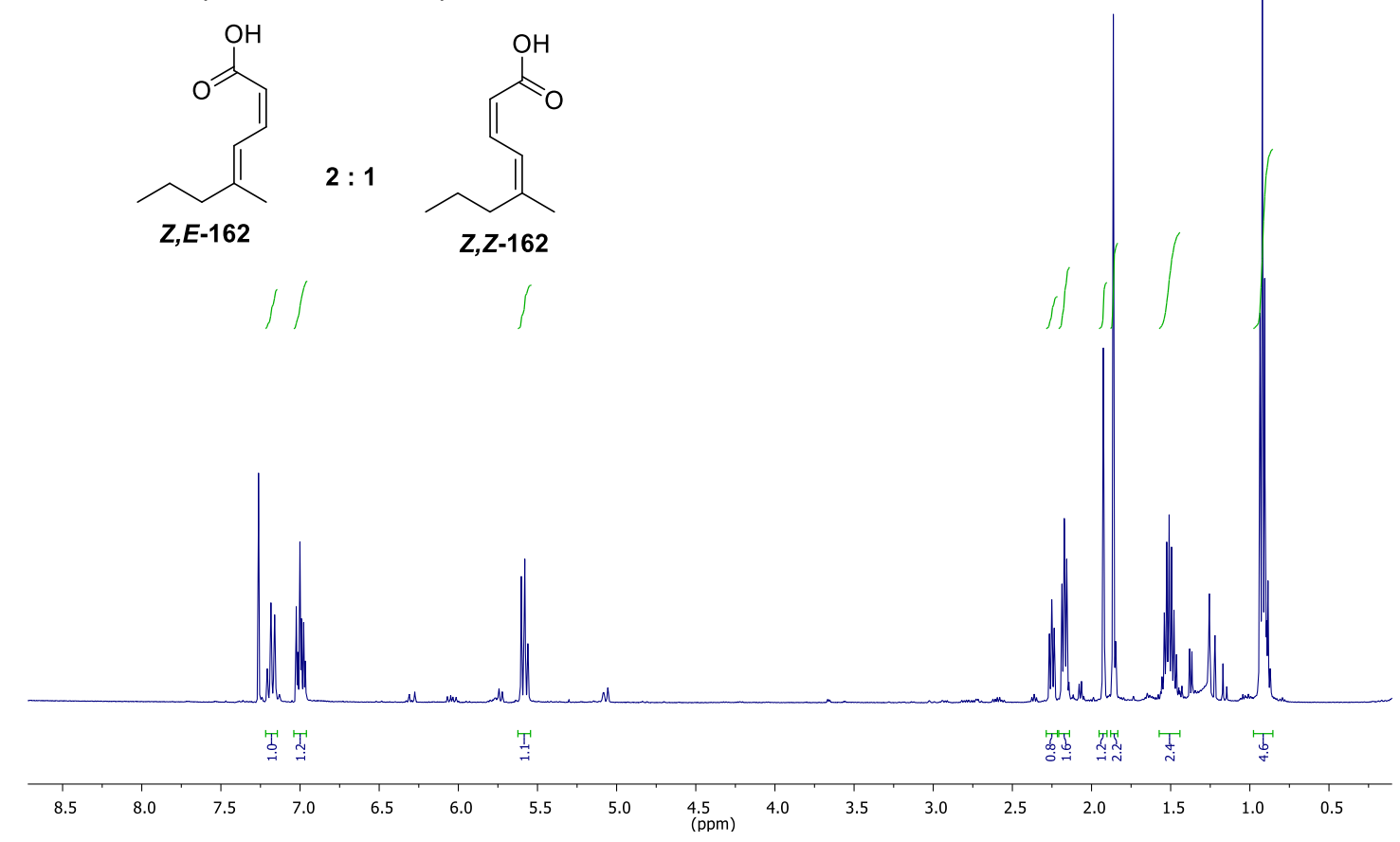

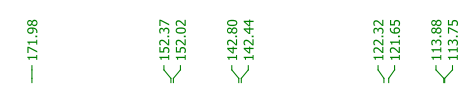

${ }^{13} \mathrm{C}-\mathrm{NMR}\left(125 \mathrm{MHz}, \mathrm{CDCl}_{3}\right.$ )

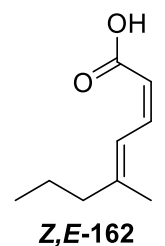

$2: 1$<smiles>[Z20]C/C=C(C)\C=C/C(=O)O</smiles>

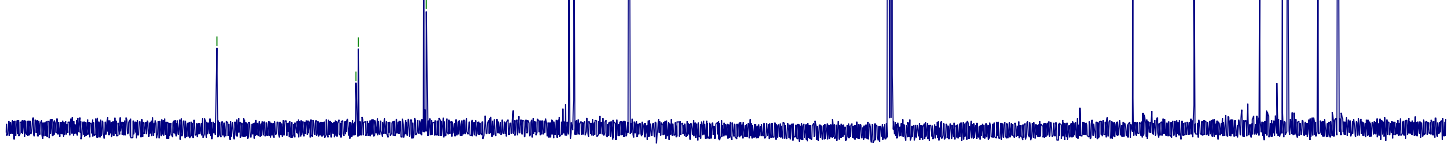


${ }^{1} \mathrm{H}-\mathrm{NMR}\left(500 \mathrm{MHz}, \mathrm{CDCl}_{3}\right)$

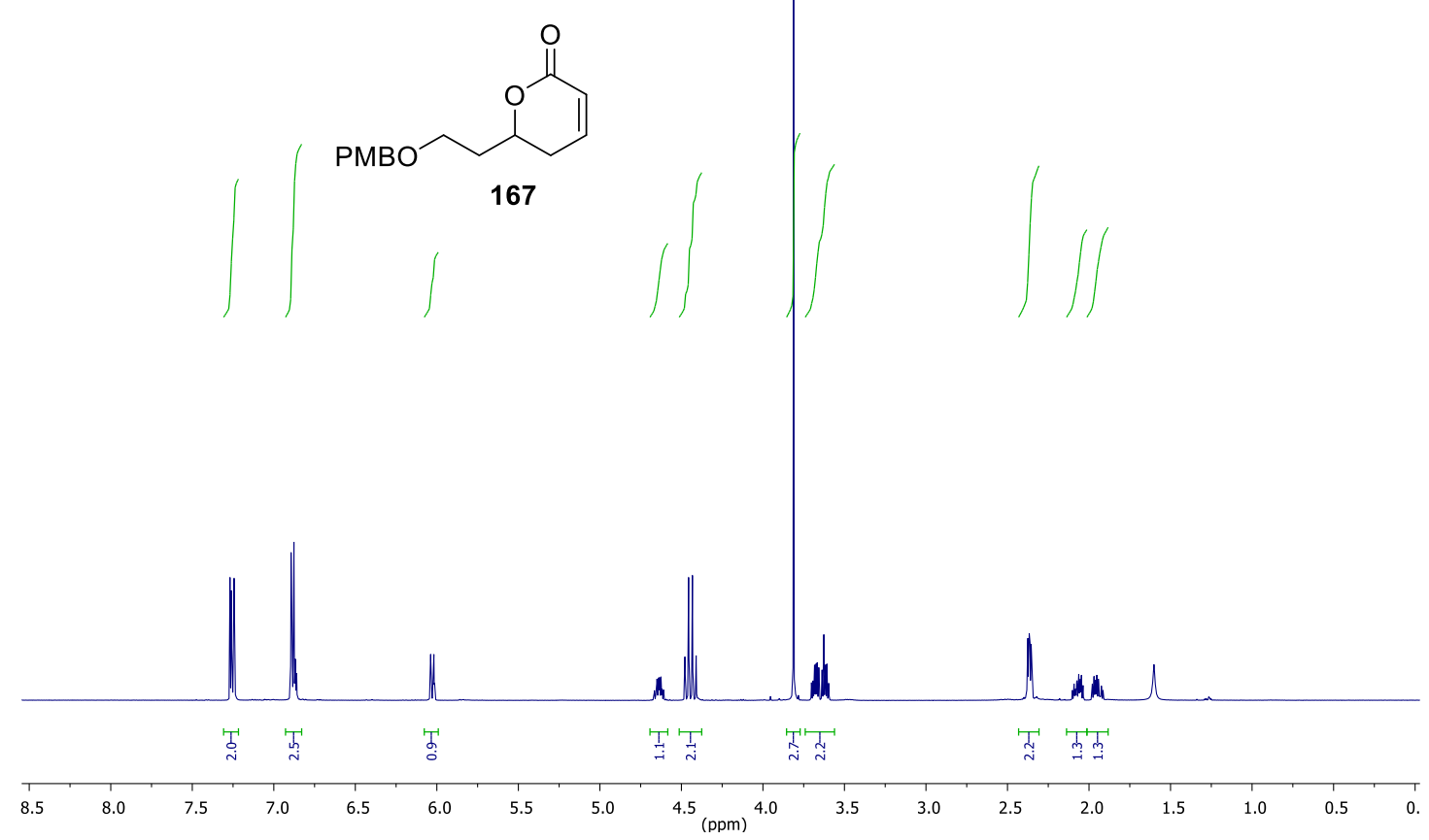

${ }^{13} \mathrm{C}-\mathrm{NMR}\left(125 \mathrm{MHz}, \mathrm{CDCl}_{3}\right)$

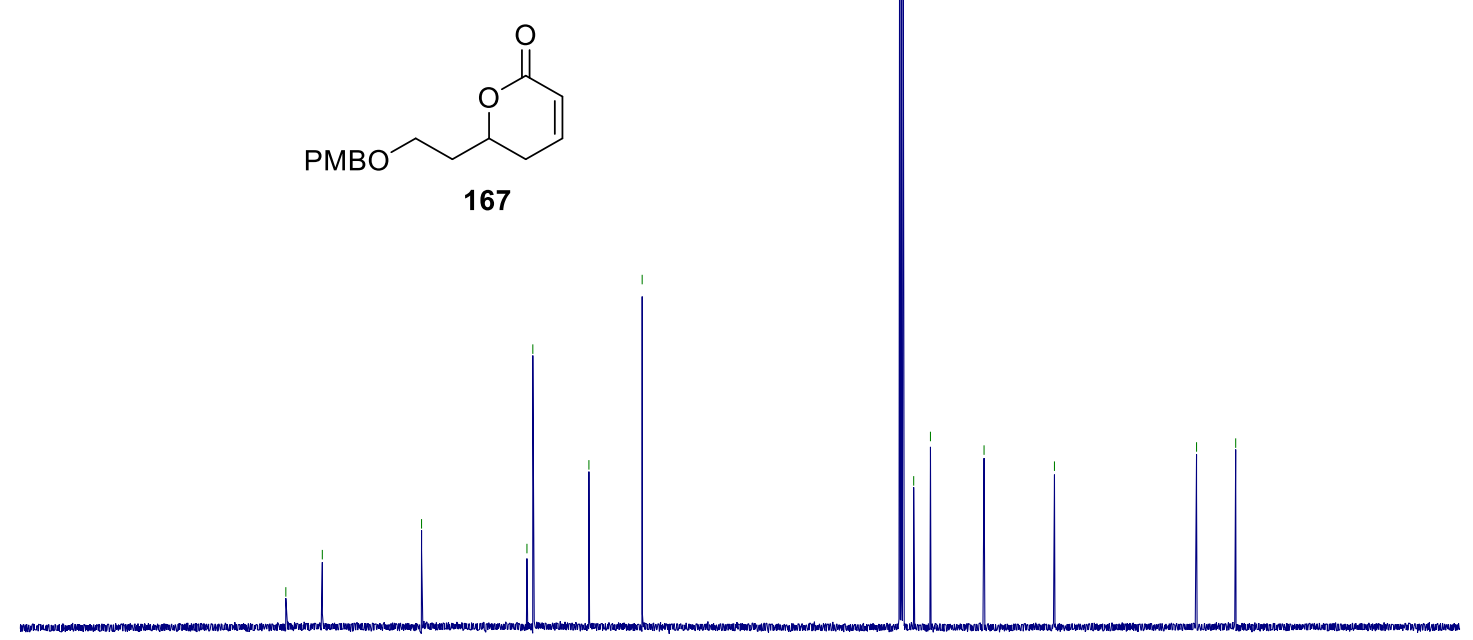

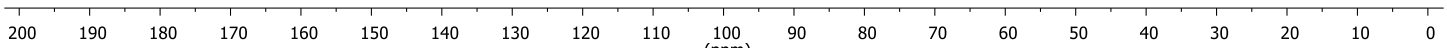


${ }^{1} \mathrm{H}-\mathrm{NMR}\left(500 \mathrm{MHz}, \mathrm{CDCl}_{3}\right)$

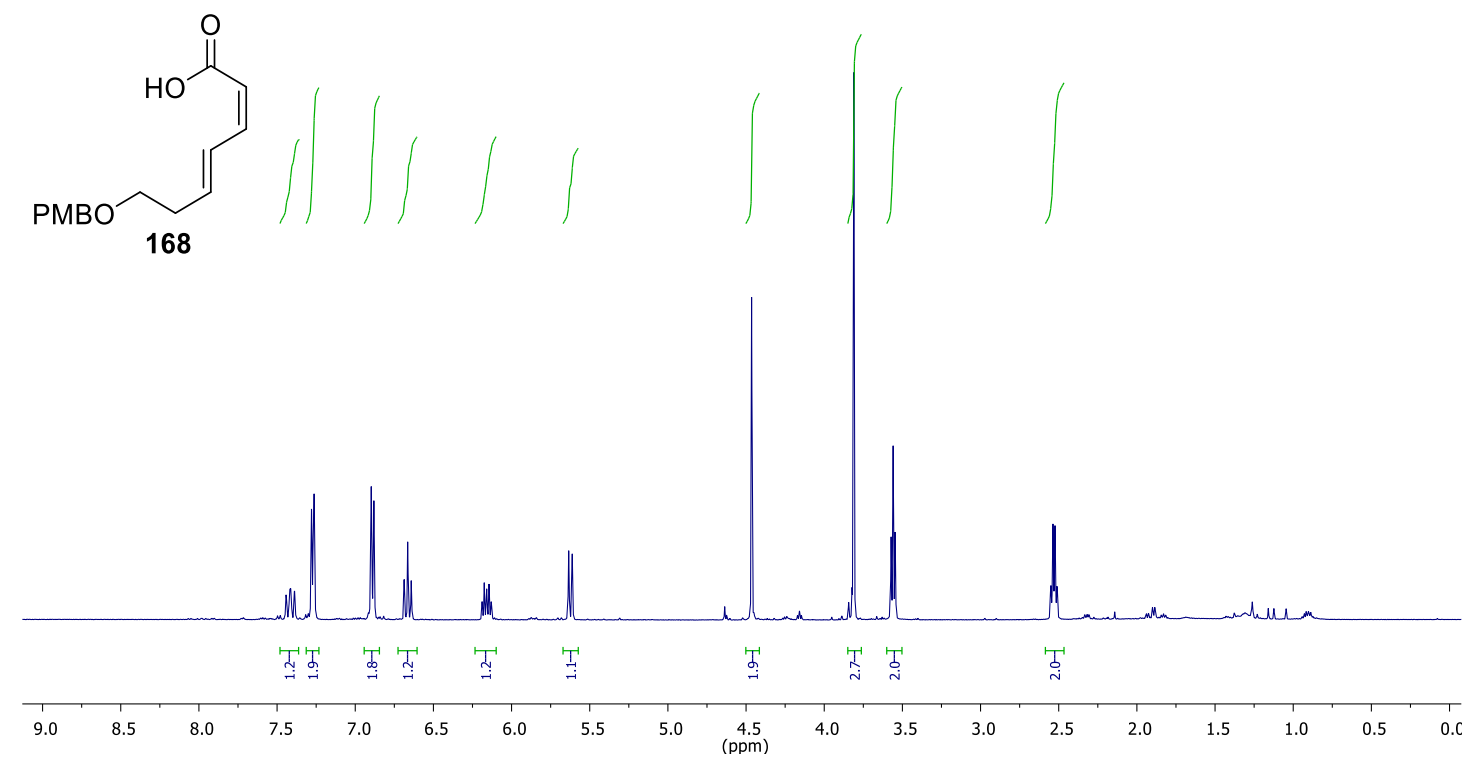

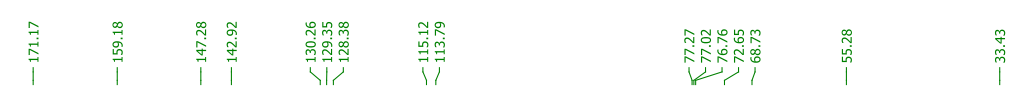

${ }^{13} \mathrm{C}-\mathrm{NMR}\left(125 \mathrm{MHz}, \mathrm{CDCl}_{3}\right.$ )
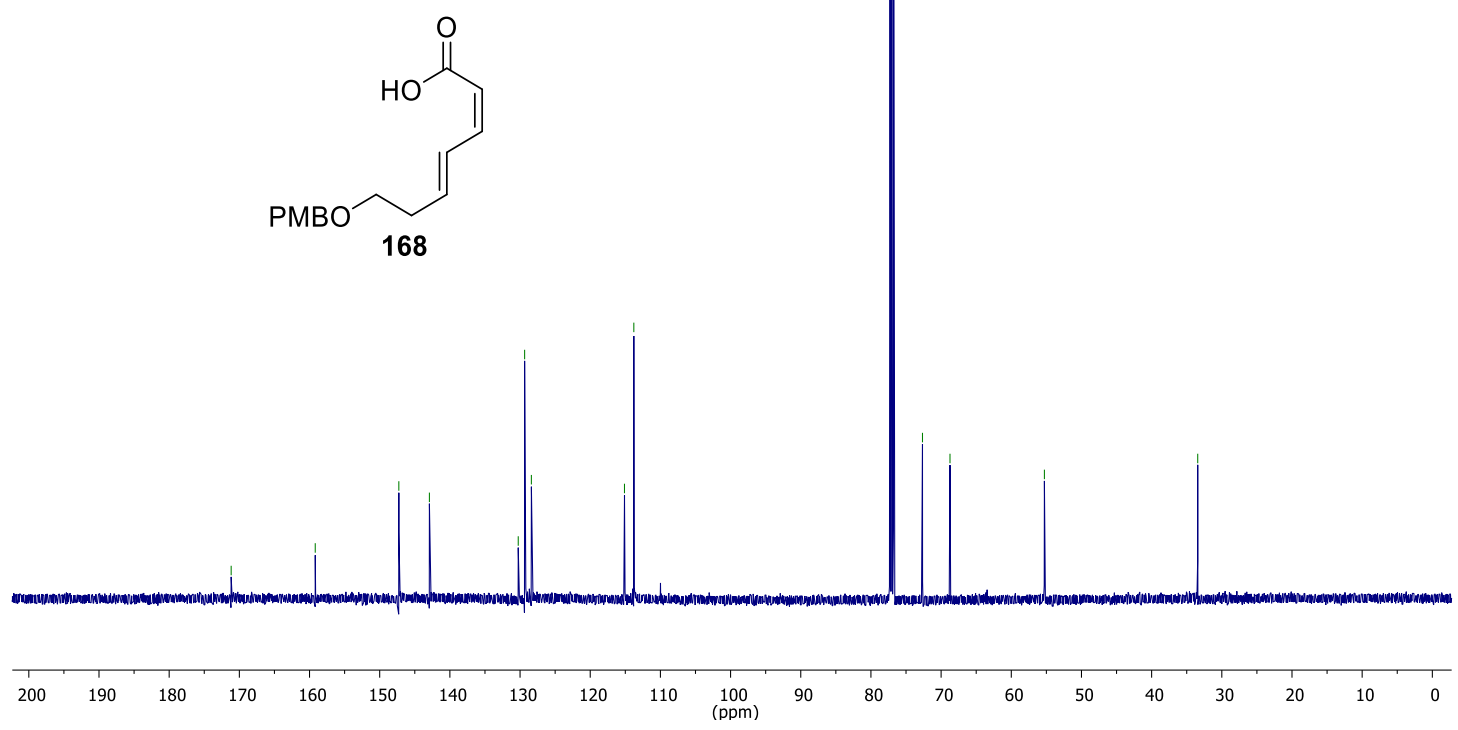
${ }^{1} \mathrm{H}-\mathrm{NMR}\left(500 \mathrm{MHz}, \mathrm{CDCl}_{3}\right.$ )

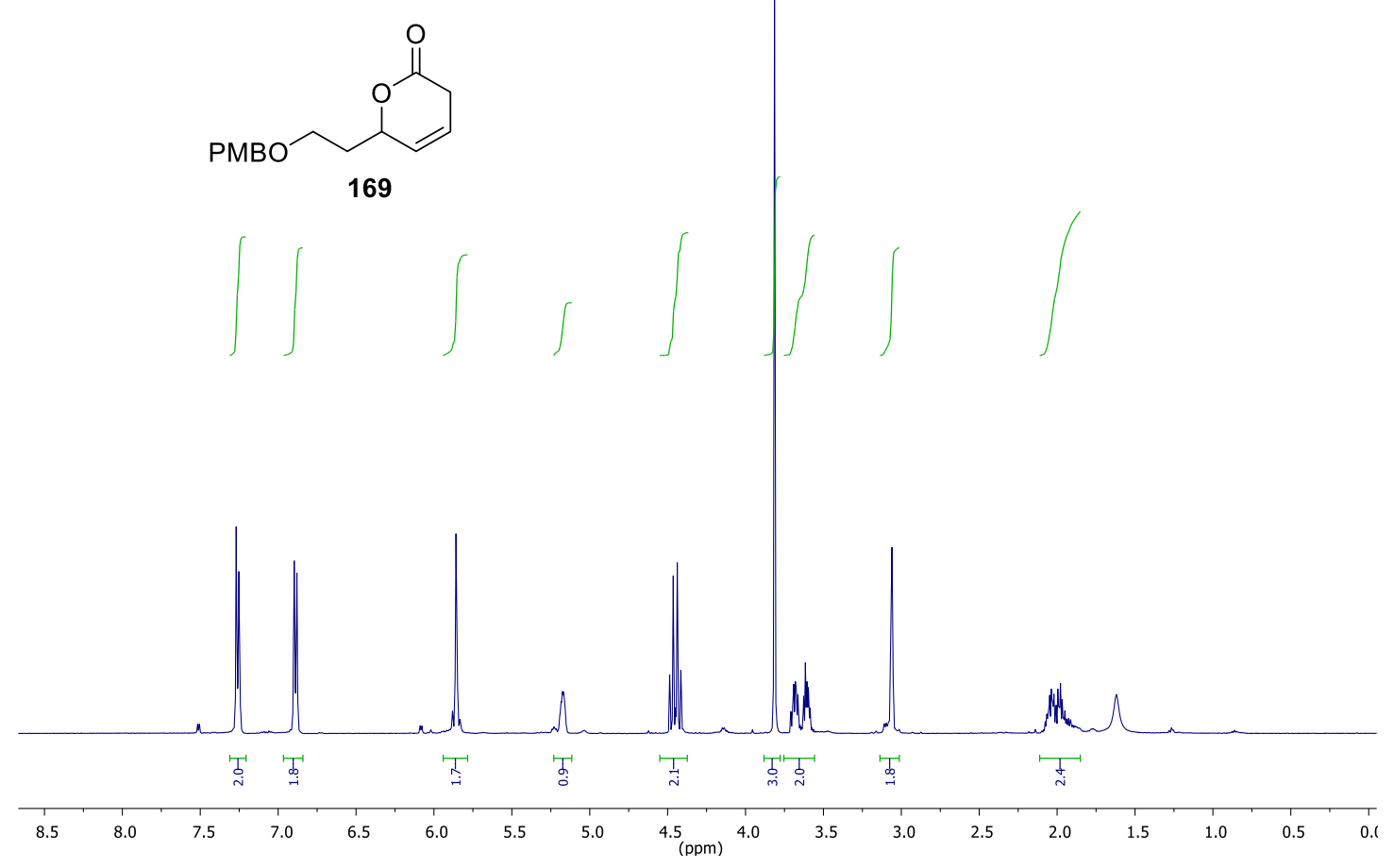

${ }^{13}$ C-NMR (125 MHz, CDCl 3 )
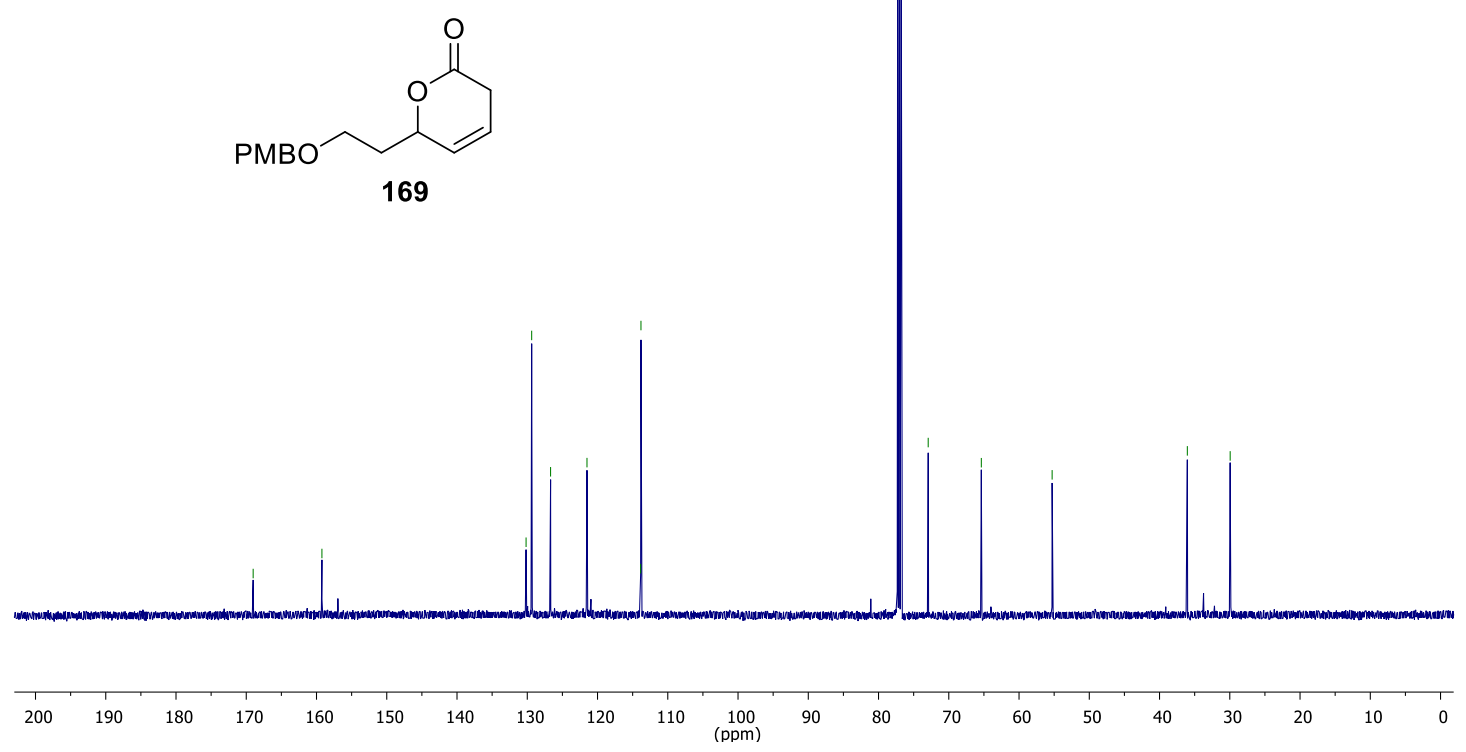
${ }^{1} \mathrm{H}-\mathrm{NMR}\left(500 \mathrm{MHz}, \mathrm{CDCl}_{3}\right.$ )

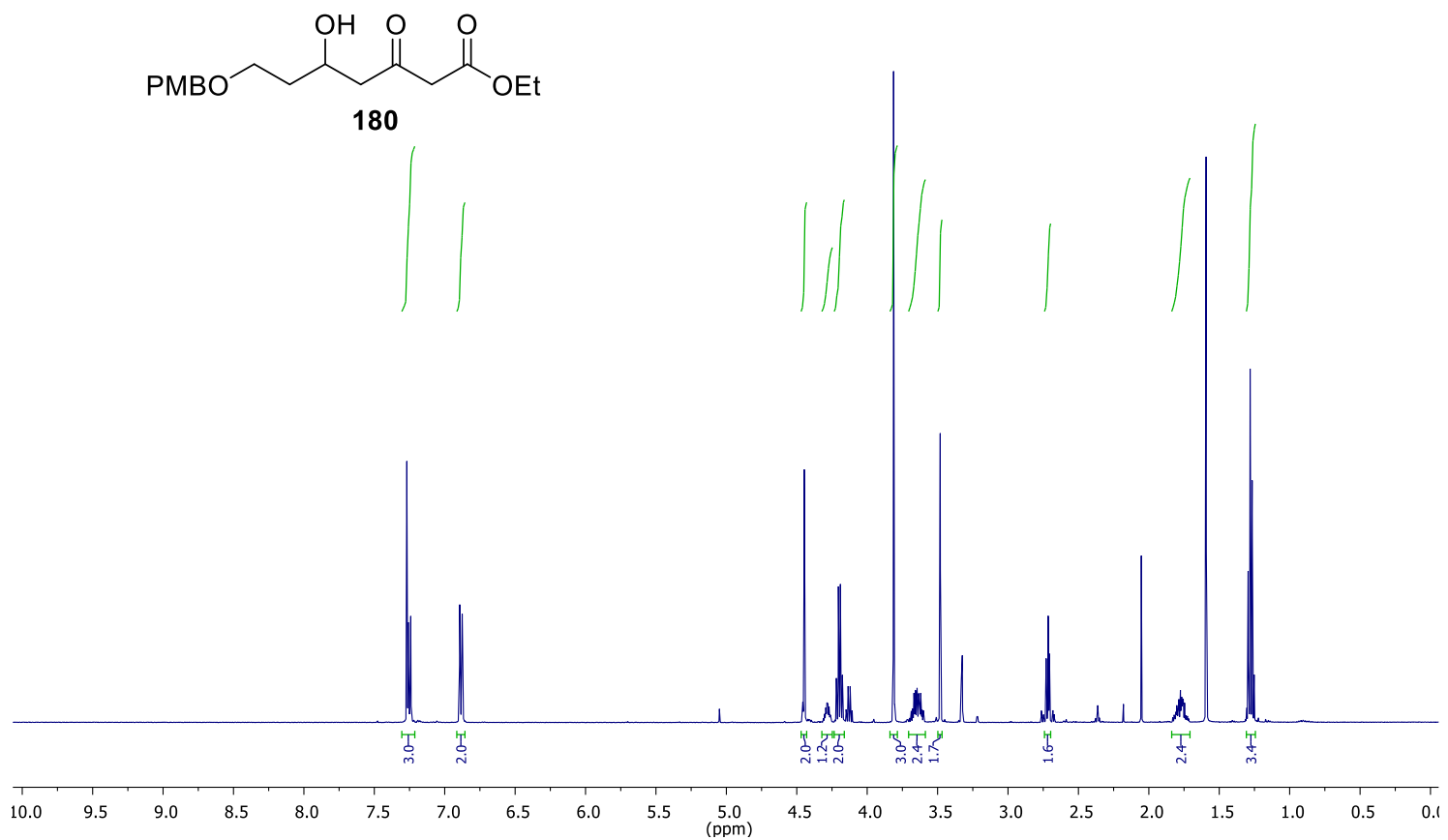

${ }^{1} \mathrm{H}-\mathrm{NMR}\left(500 \mathrm{MHz}, \mathrm{CDCl}_{3}\right)$

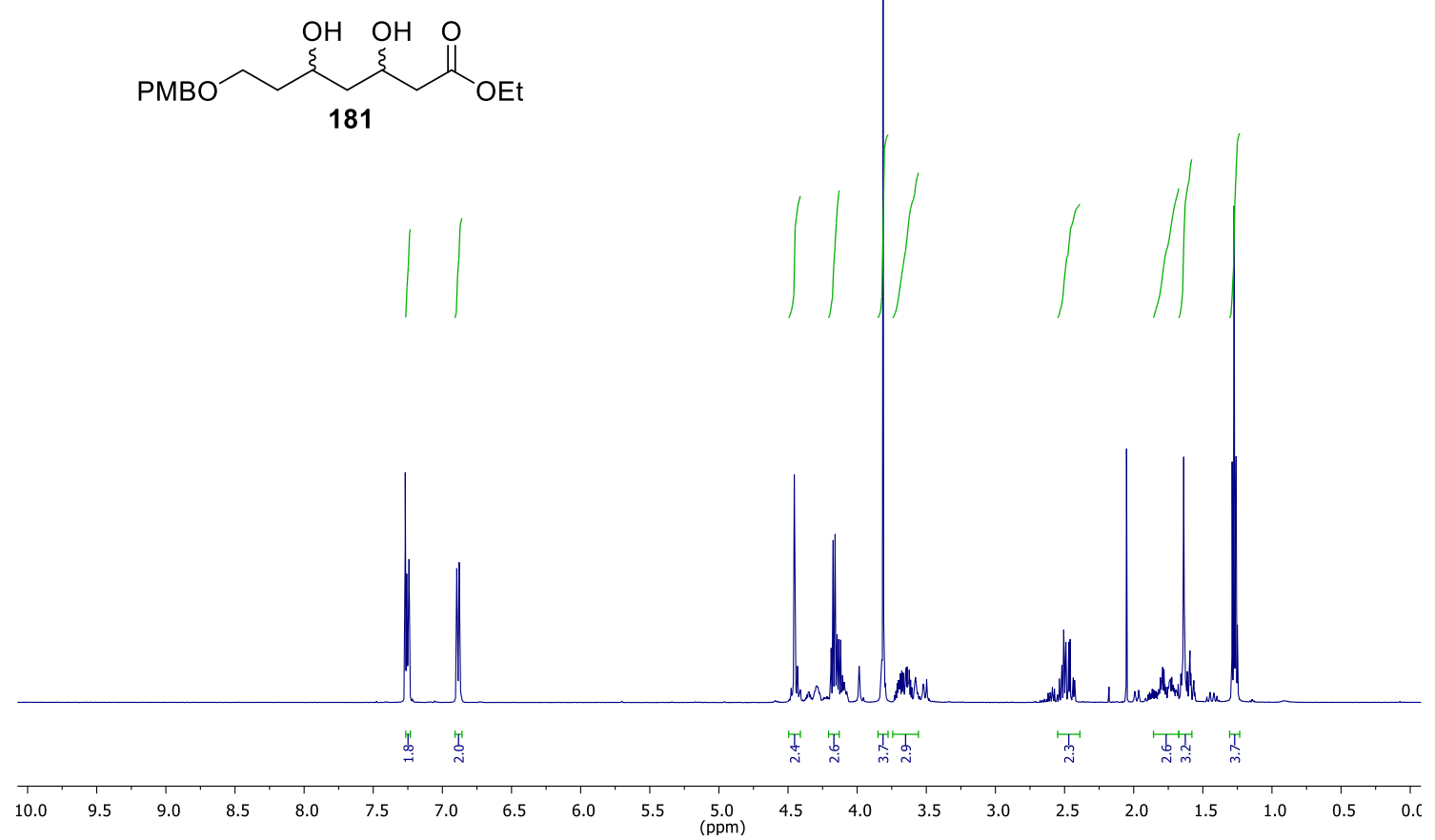


${ }^{1} \mathrm{H}-\mathrm{NMR}\left(500 \mathrm{MHz}, \mathrm{CDCl}_{3}\right.$ )

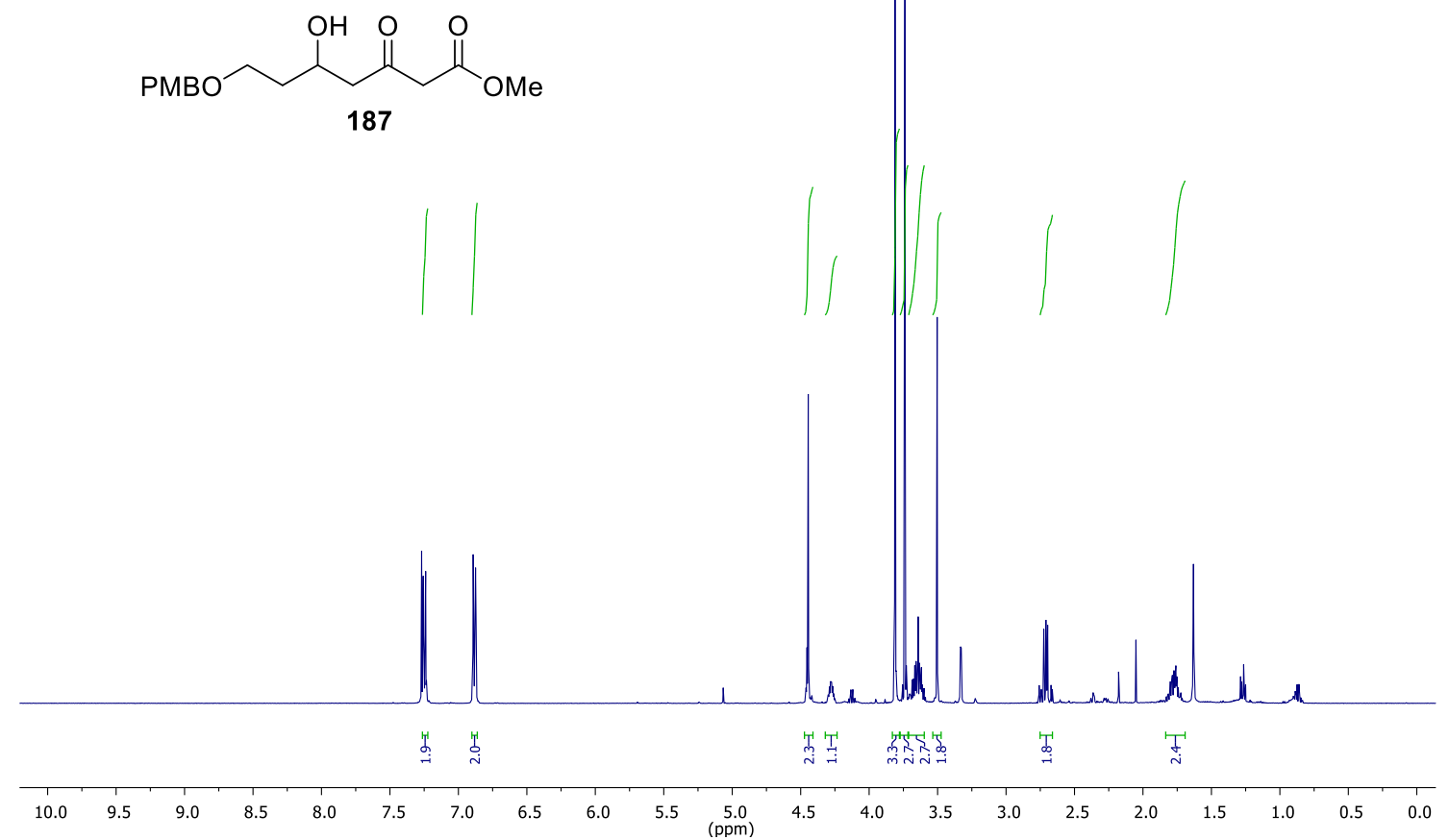

${ }^{13} \mathrm{C}-\mathrm{NMR}\left(125 \mathrm{MHz}, \mathrm{CDCl}_{3}\right)$
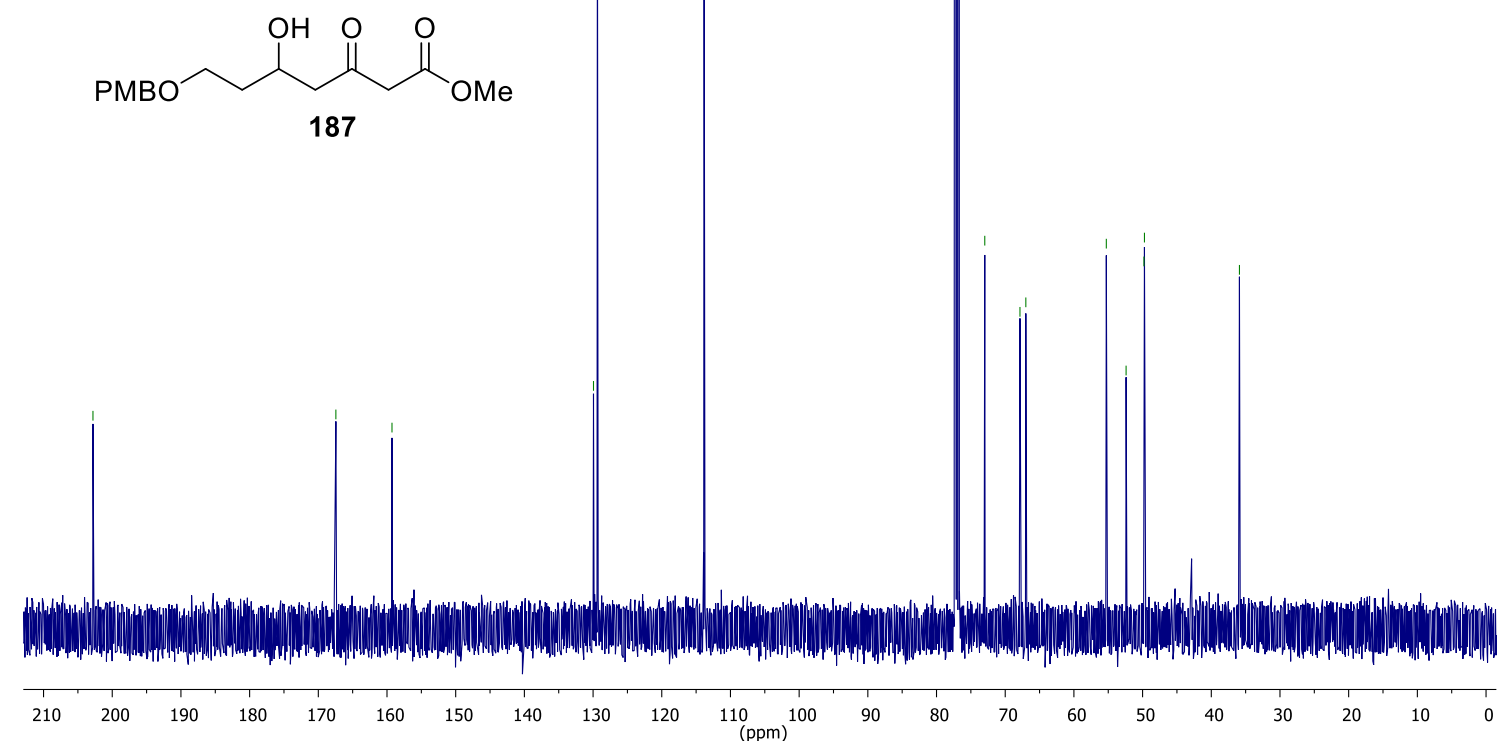
${ }^{1} \mathrm{H}-\mathrm{NMR}\left(500 \mathrm{MHz}, \mathrm{CDCl}_{3}\right)$

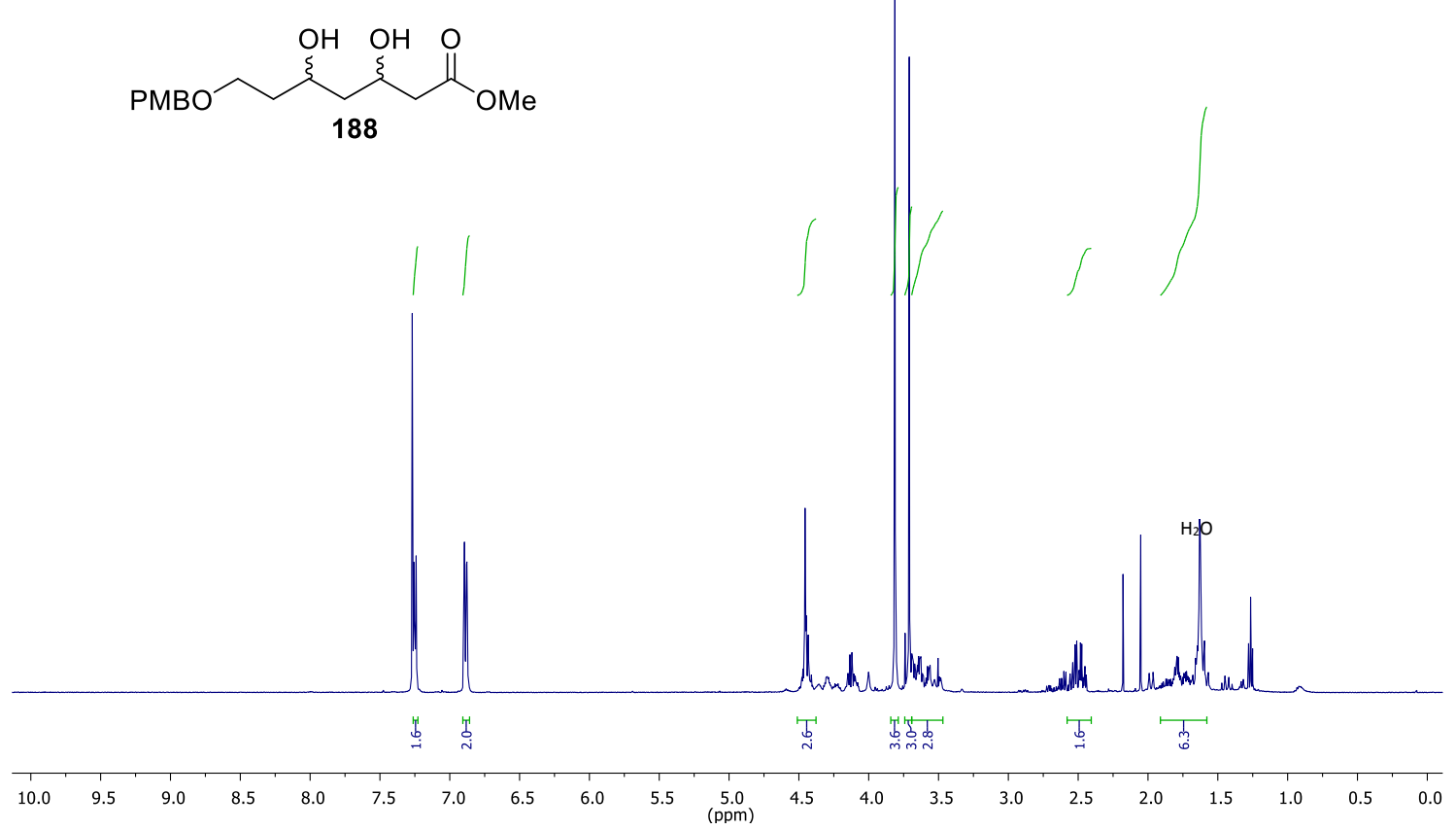


${ }^{1} \mathrm{H}-\mathrm{NMR}\left(500 \mathrm{MHz}, \mathrm{CDCl}_{3}\right)$

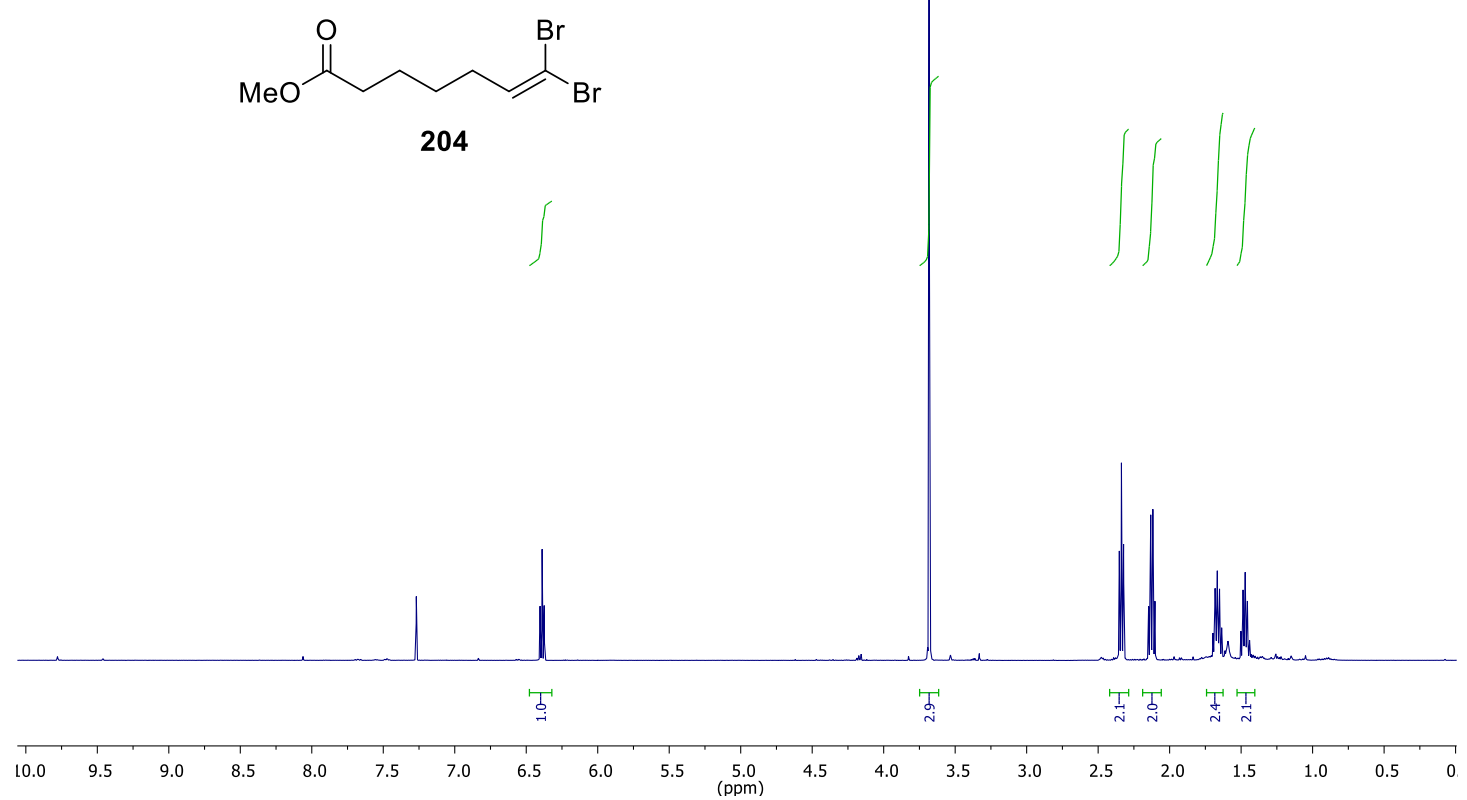

${ }^{13} \mathrm{C}$-NMR (125 MHz, $\left.\mathrm{CDCl}_{3}\right)$
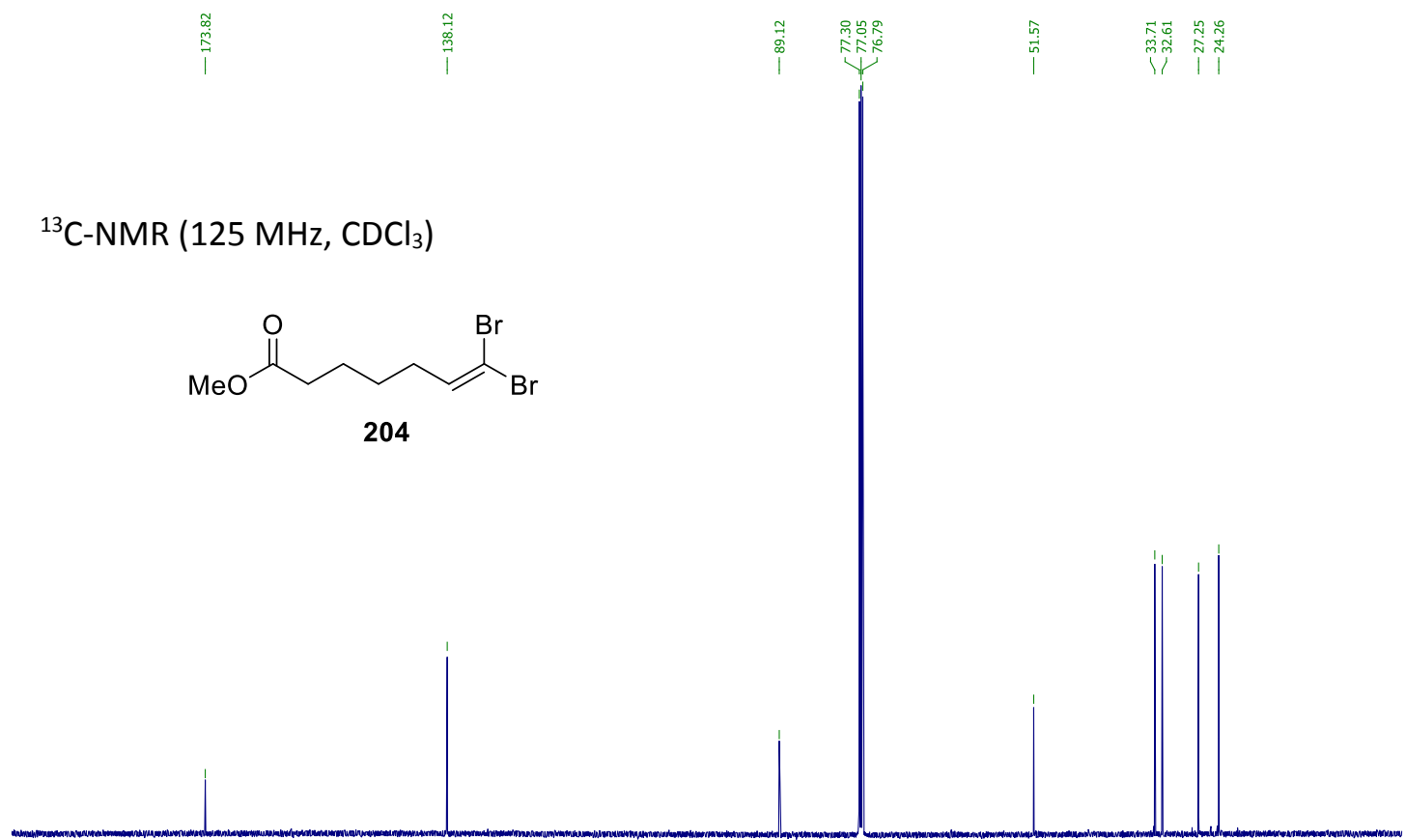

200 190
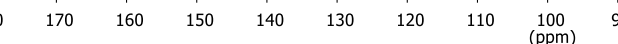

100
$(\mathrm{ppm})$
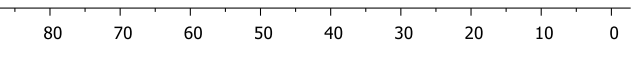
${ }^{1} \mathrm{H}-\mathrm{NMR}\left(500 \mathrm{MHz}, \mathrm{CDCl}_{3}\right.$ )

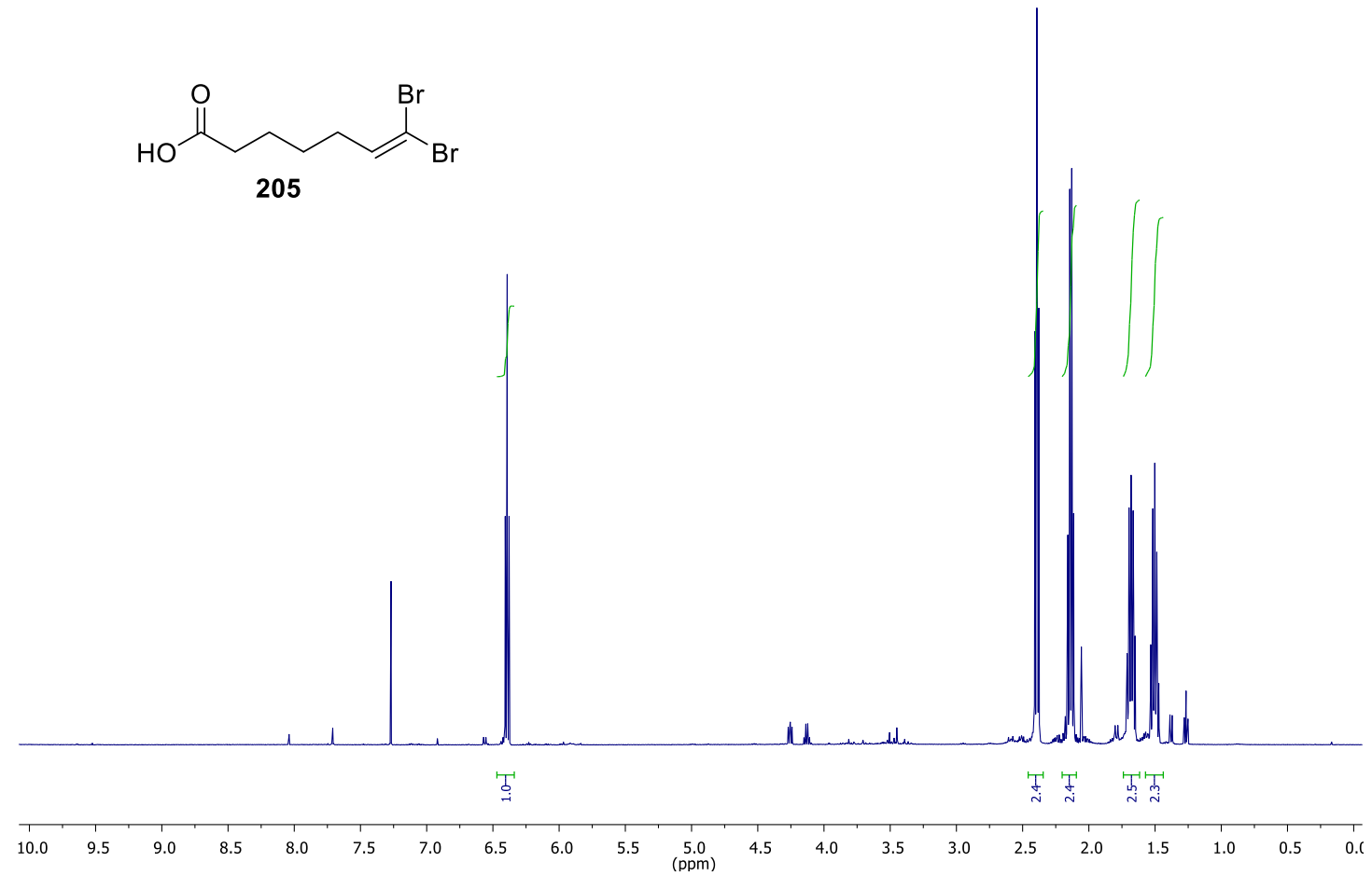

${ }^{13} \mathrm{C}-\mathrm{NMR}\left(125 \mathrm{MHz}, \mathrm{CDCl}_{3}\right)$

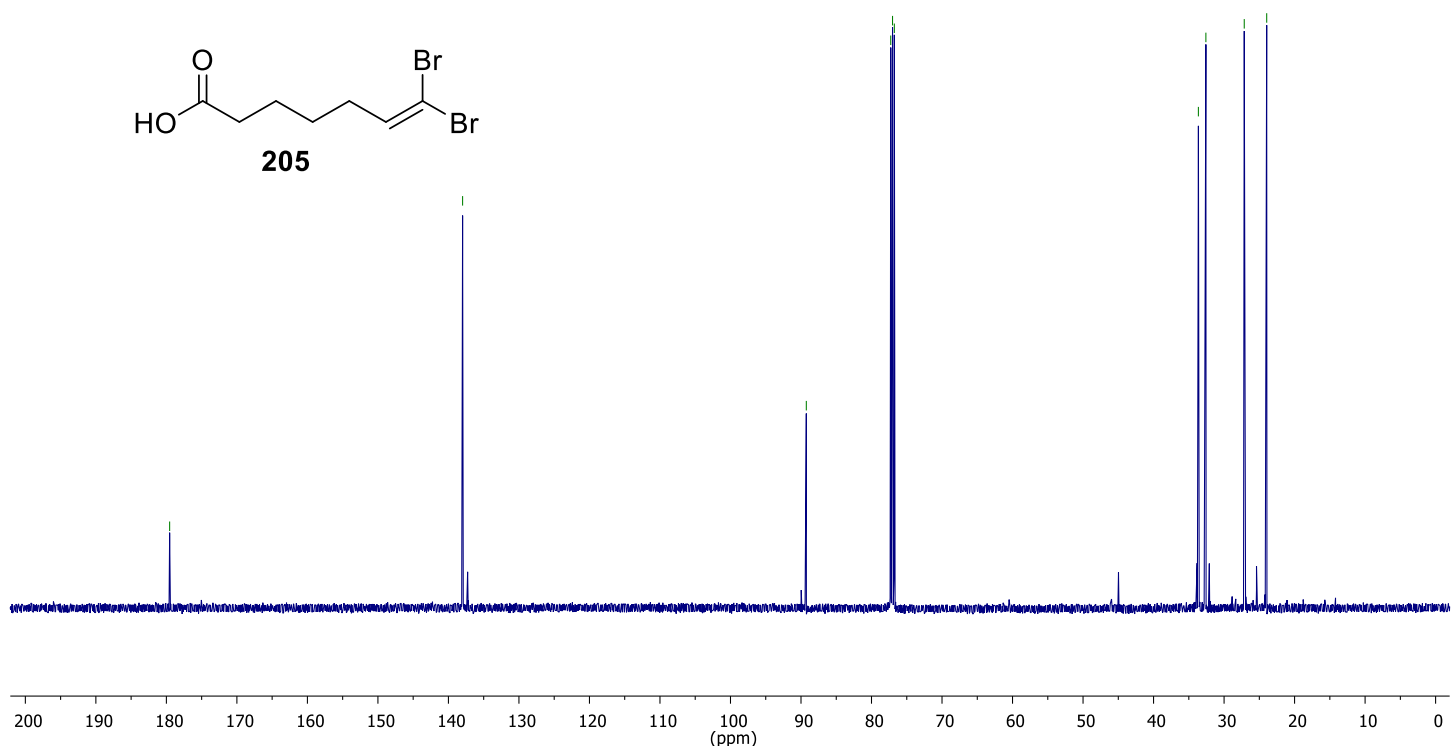


${ }^{1} \mathrm{H}-\mathrm{NMR}\left(500 \mathrm{MHz}, \mathrm{CDCl}_{3}\right)$

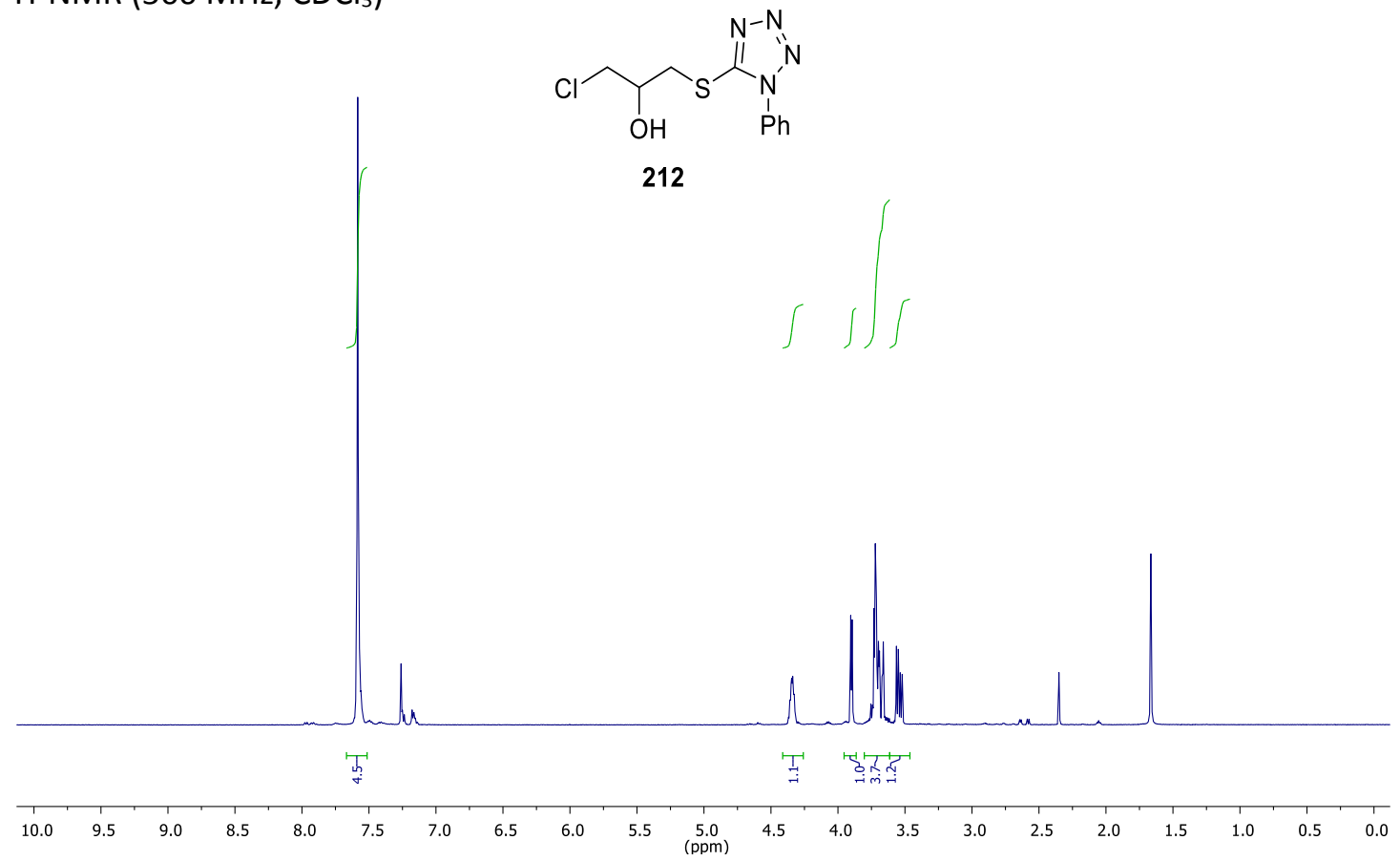

${ }^{13} \mathrm{C}-\mathrm{NMR}\left(125 \mathrm{MHz}, \mathrm{CDCl}_{3}\right)$

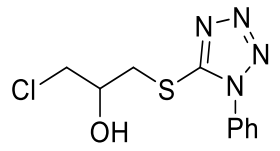

212

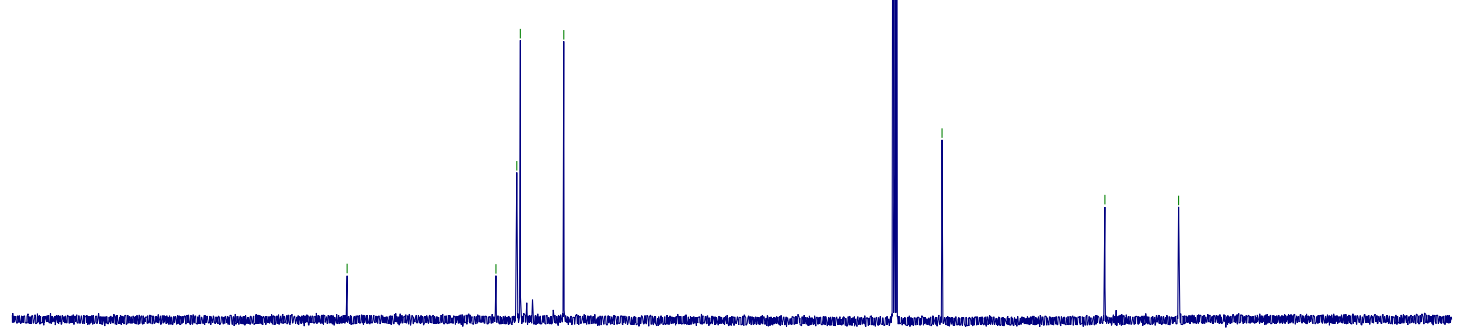

$\begin{array}{llllllllllllllllllllll} & 1 & 190 & 180 & 170 & 160 & 150 & 140 & 130 & 120 & 110 & 100 & 90 & 80 & 70 & 60 & 50 & 40 & 30 & 20 & 10 & 0\end{array}$ 
${ }^{1} \mathrm{H}-\mathrm{NMR}\left(500 \mathrm{MHz}, \mathrm{CDCl}_{3}\right)$

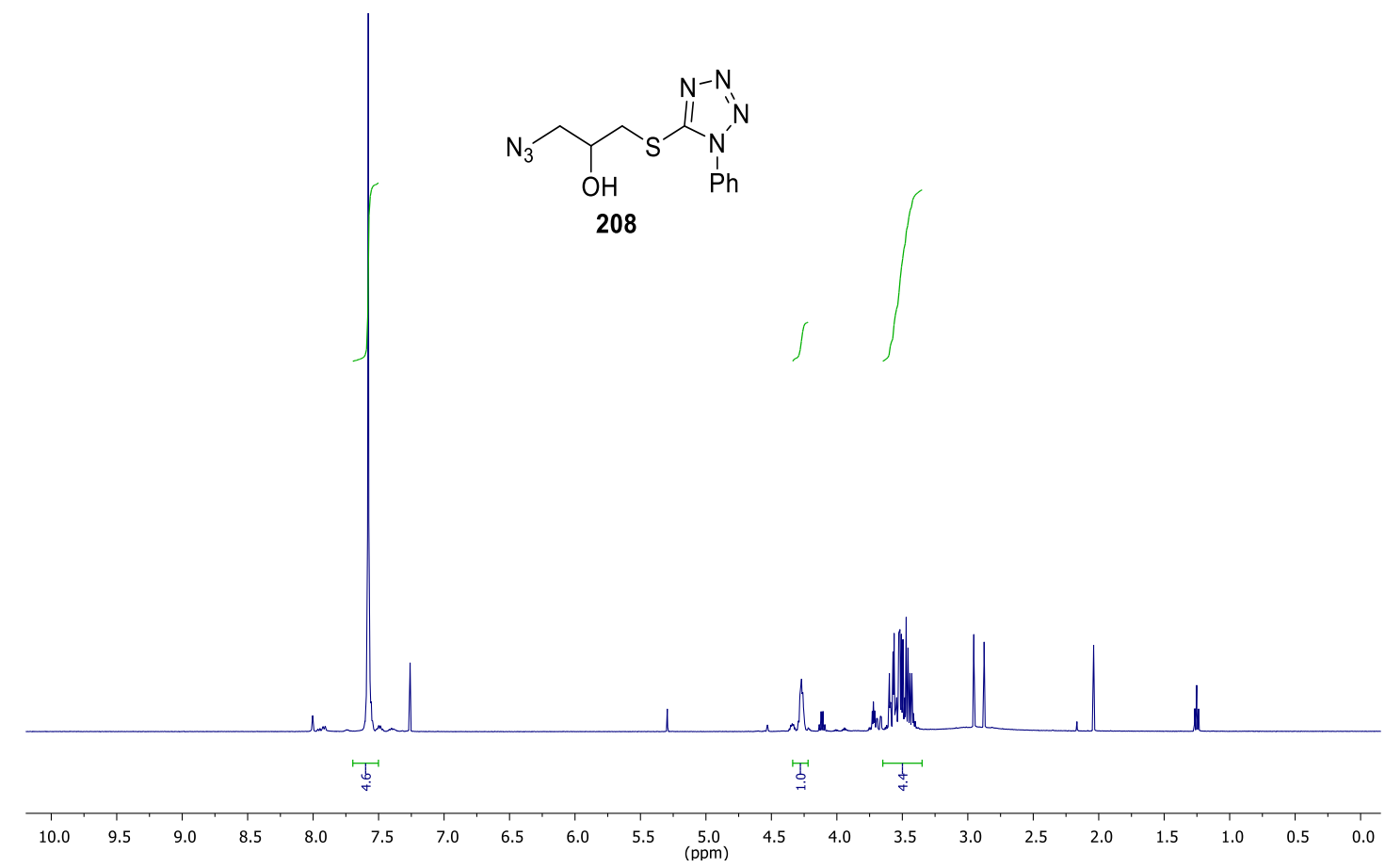

${ }^{13} \mathrm{C}-\mathrm{NMR}\left(125 \mathrm{MHz}, \mathrm{CDCl}_{3}\right)$

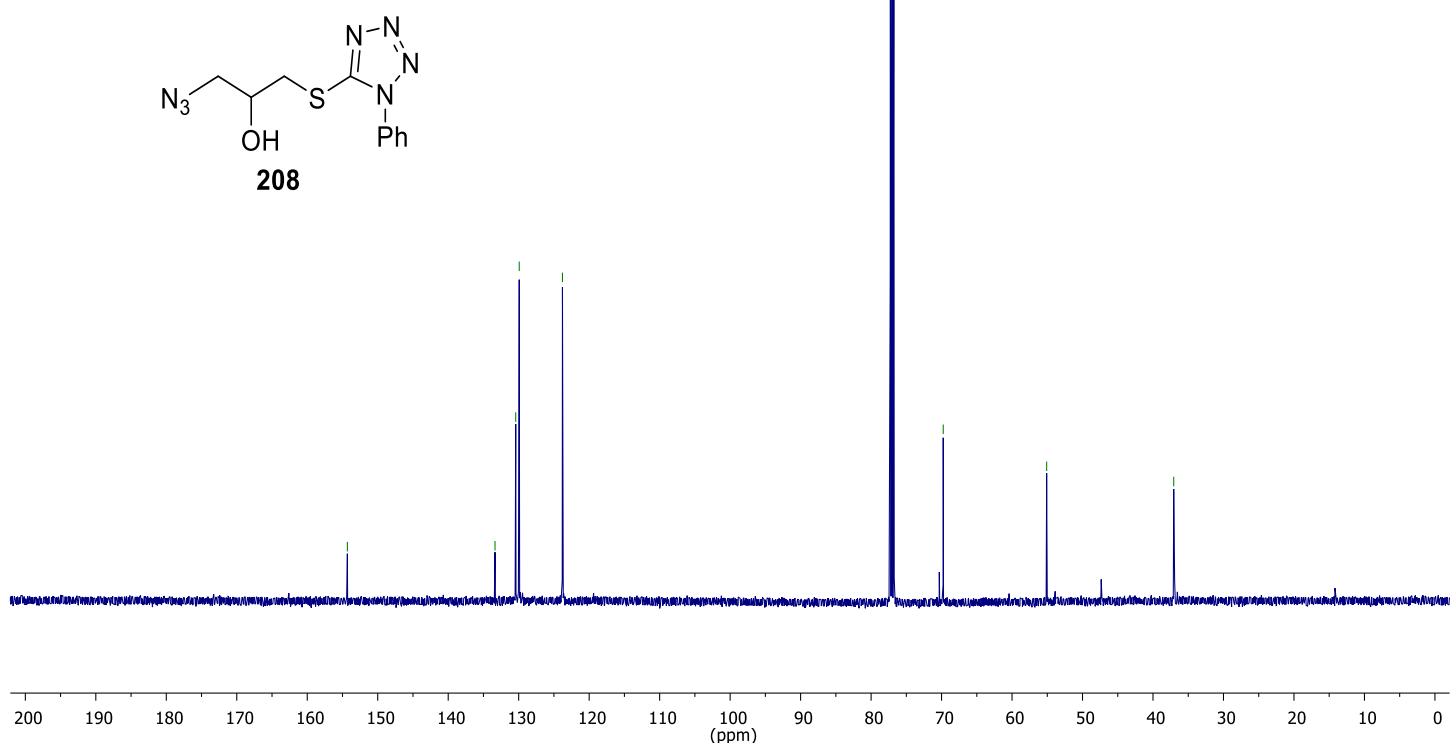

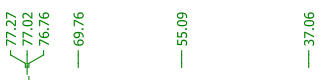


${ }^{1} \mathrm{H}-\mathrm{NMR}\left(500 \mathrm{MHz}, \mathrm{CDCl}_{3}\right)$

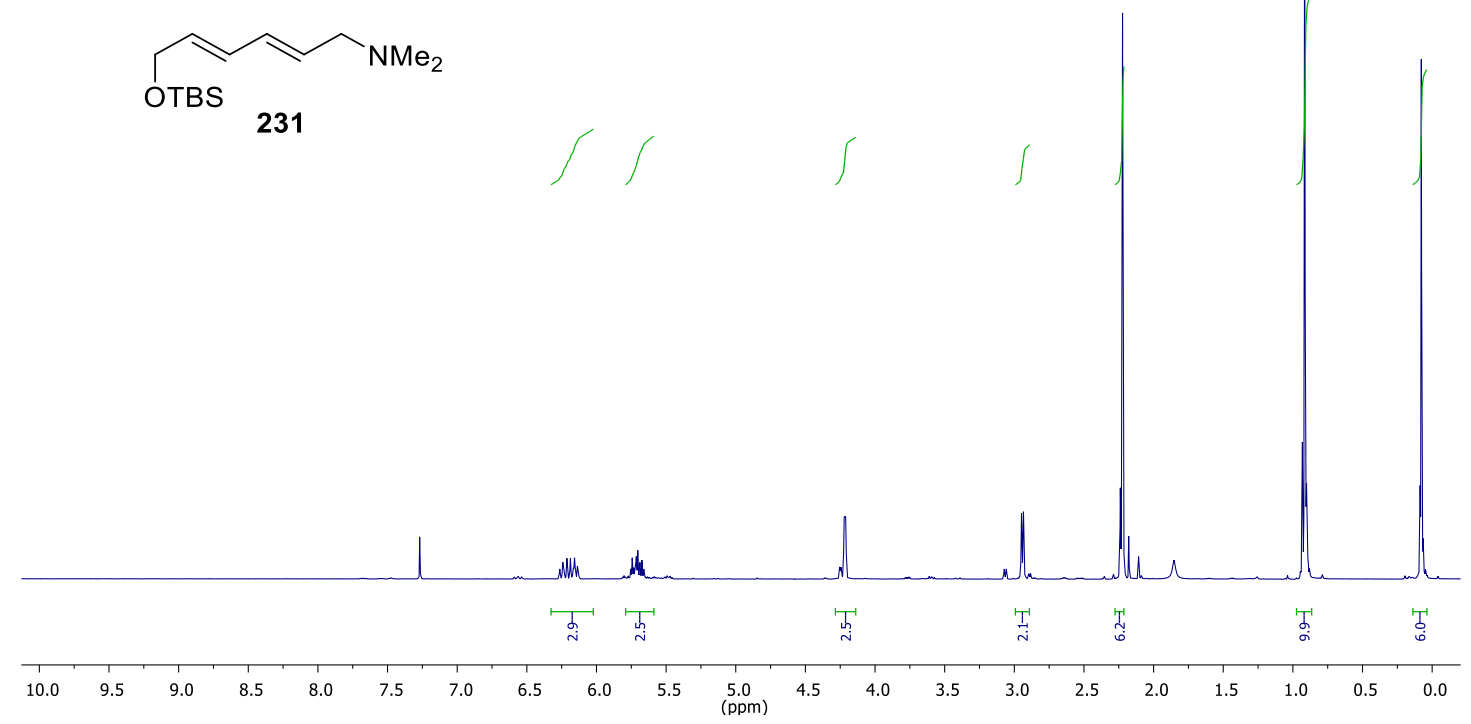

${ }^{13} \mathrm{C}-\mathrm{NMR}\left(125 \mathrm{MHz}, \mathrm{CDCl}_{3}\right)$

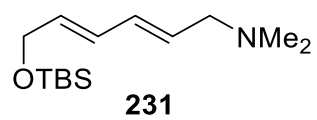

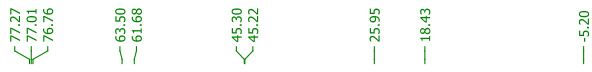

象穿

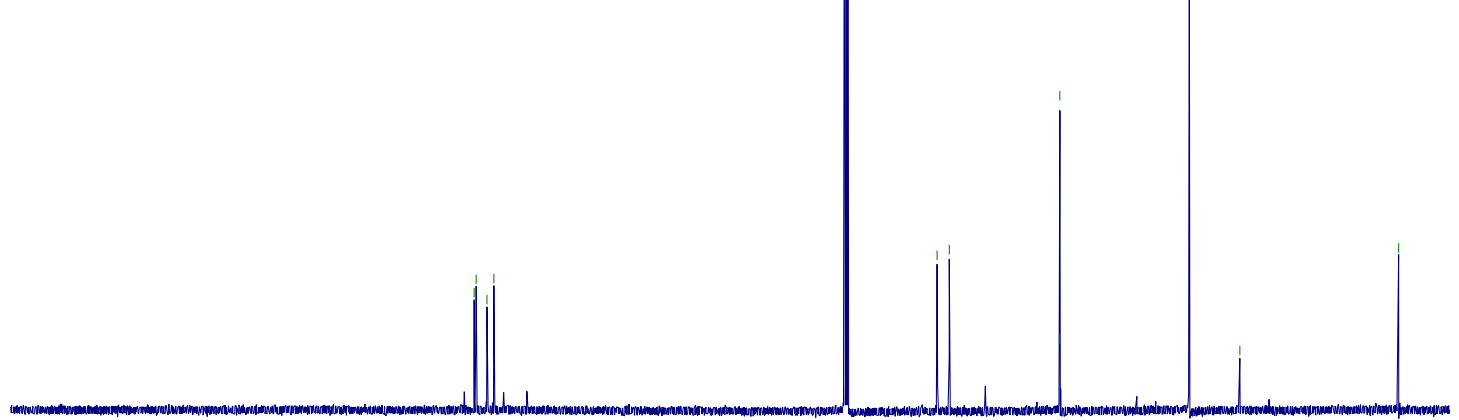

$\begin{array}{llllllllllllllllllllll}200 & 190 & 180 & 170 & 160 & 150 & 140 & 130 & 120 & 110 & 100 & 90 & 80 & 70 & 60 & 50 & 40 & 30 & 20 & 10 & 0 & -10\end{array}$ 
${ }^{1} \mathrm{H}-\mathrm{NMR}\left(500 \mathrm{MHz}, \mathrm{CDCl}_{3}\right)$

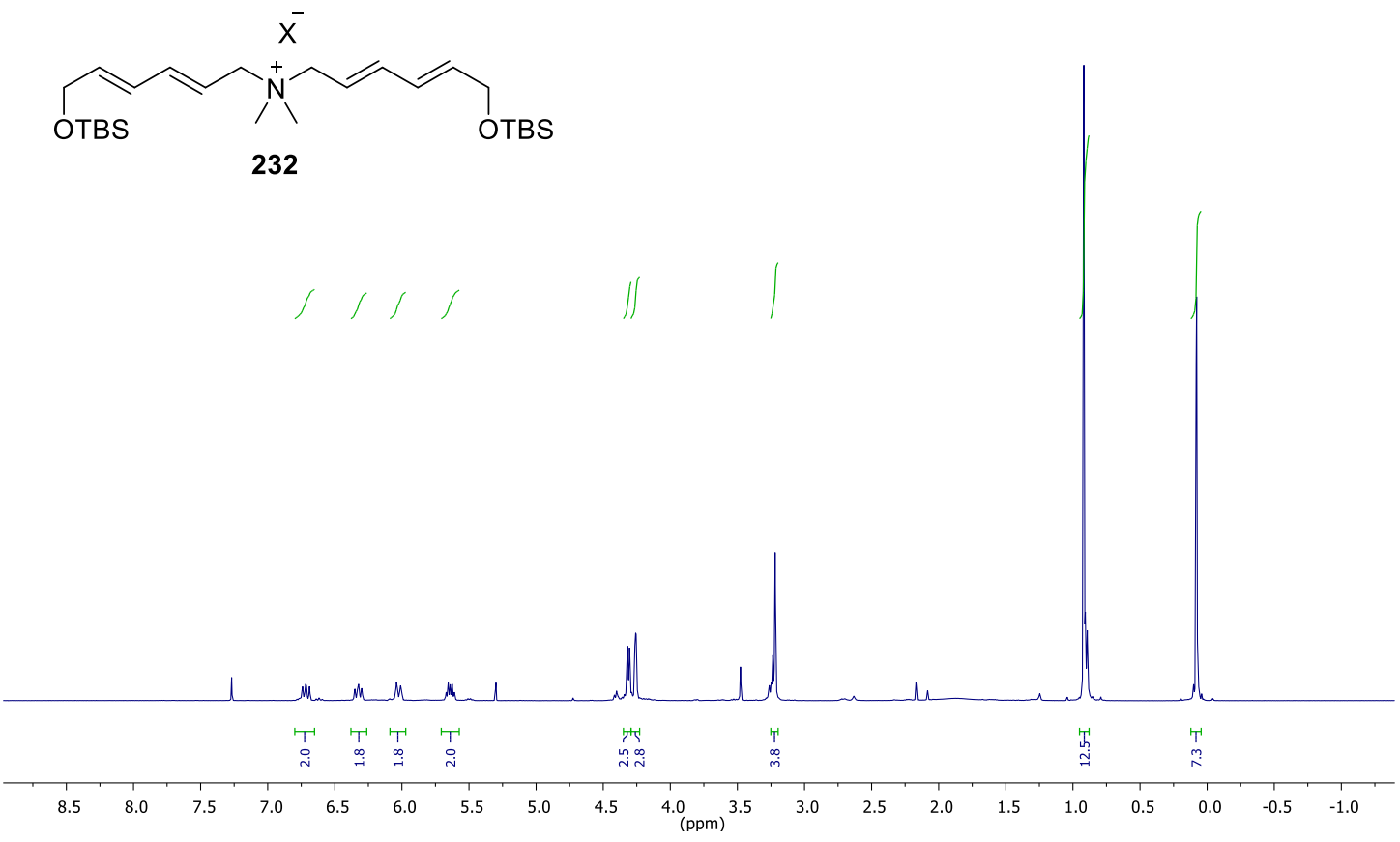

\section{|c}

至

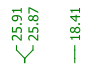

${ }^{13} \mathrm{C}-\mathrm{NMR}\left(125 \mathrm{MHz}, \mathrm{CDCl}_{3}\right)$

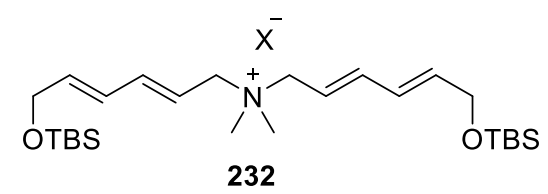

200

$\begin{array}{lllllllllll}190 & 180 & 170 & 160 & 150 & 140 & 130 & 120 & 110 & 100 \\ (\mathrm{ppm})\end{array}$ 
${ }^{1} \mathrm{H}-\mathrm{NMR}\left(500 \mathrm{MHz}, \mathrm{CDCl}_{3}\right.$ )

$\overbrace{236} \mathrm{NMe}_{2}$

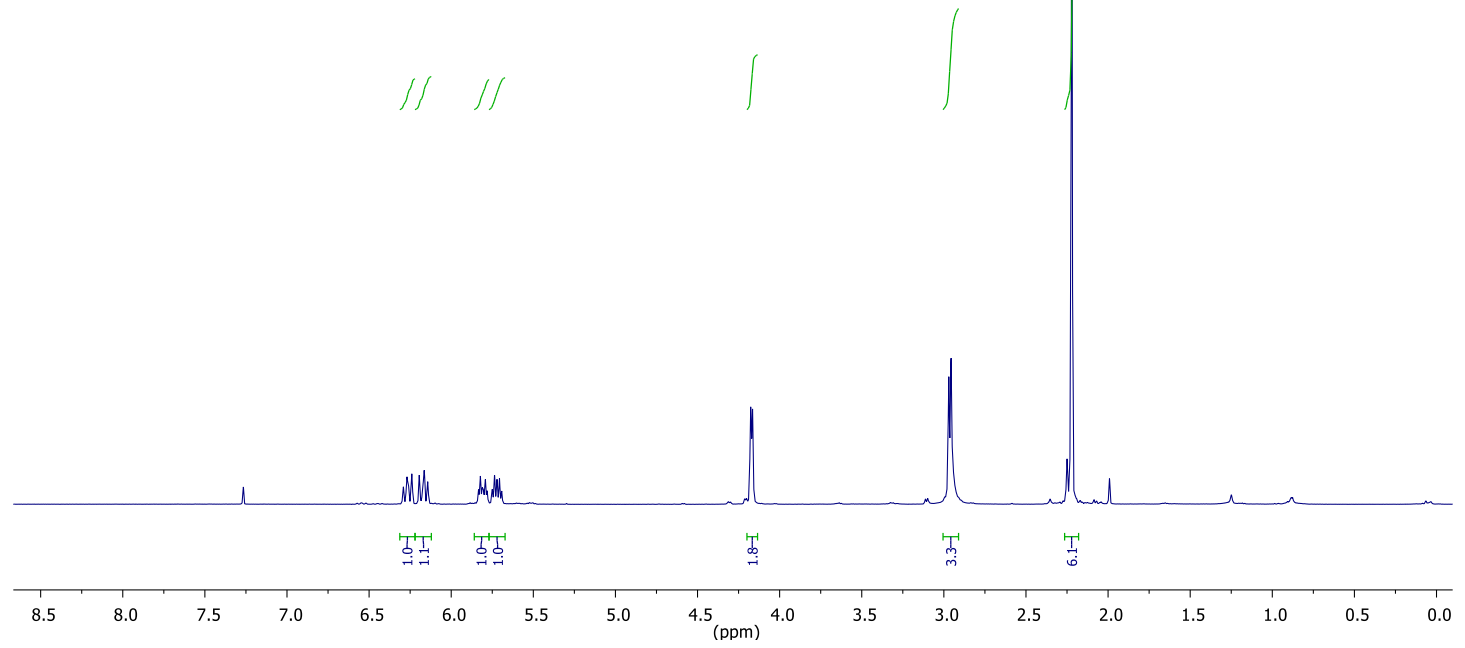

${ }^{13} \mathrm{C}-\mathrm{NMR}\left(125 \mathrm{MHz}, \mathrm{CDCl}_{3}\right.$ )
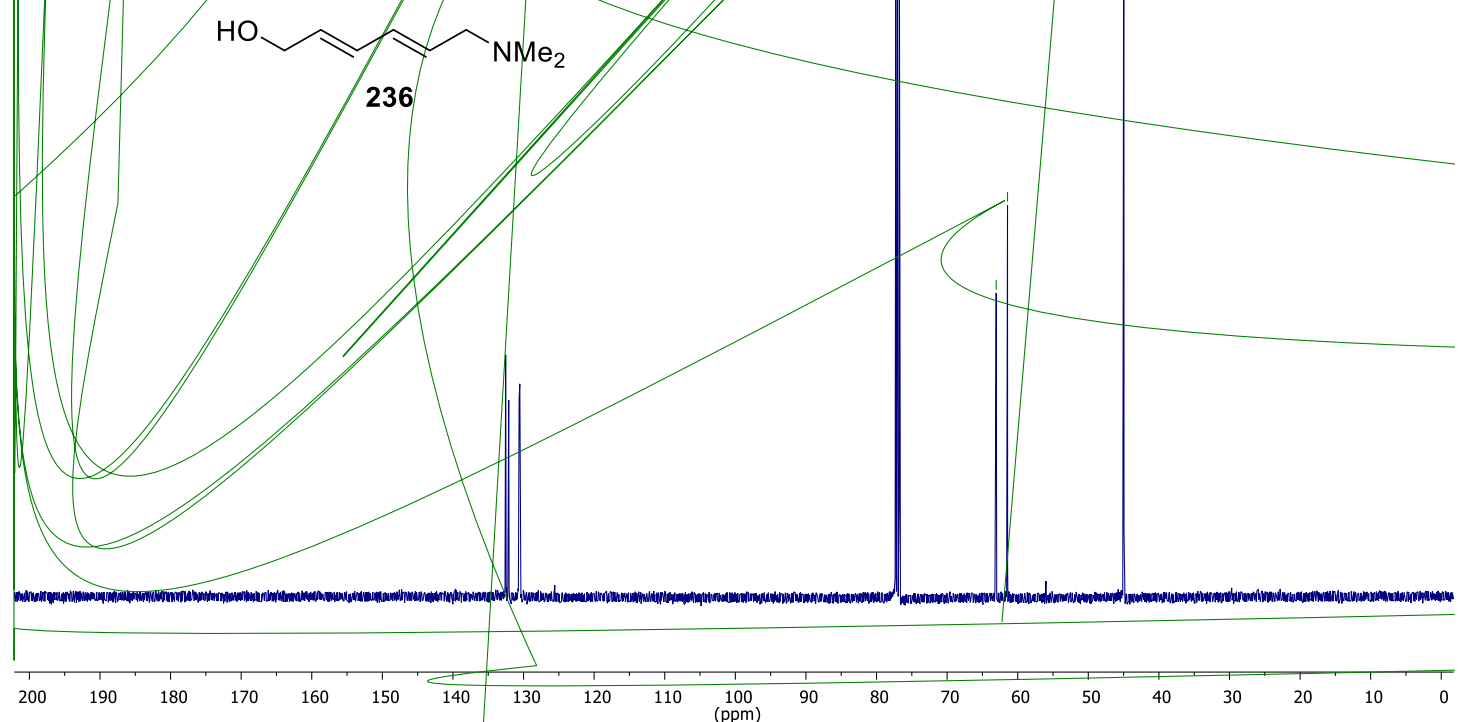
${ }^{1} \mathrm{H}-\mathrm{NMR}\left(500 \mathrm{MHz}, \mathrm{CDCl}_{3}\right.$ )

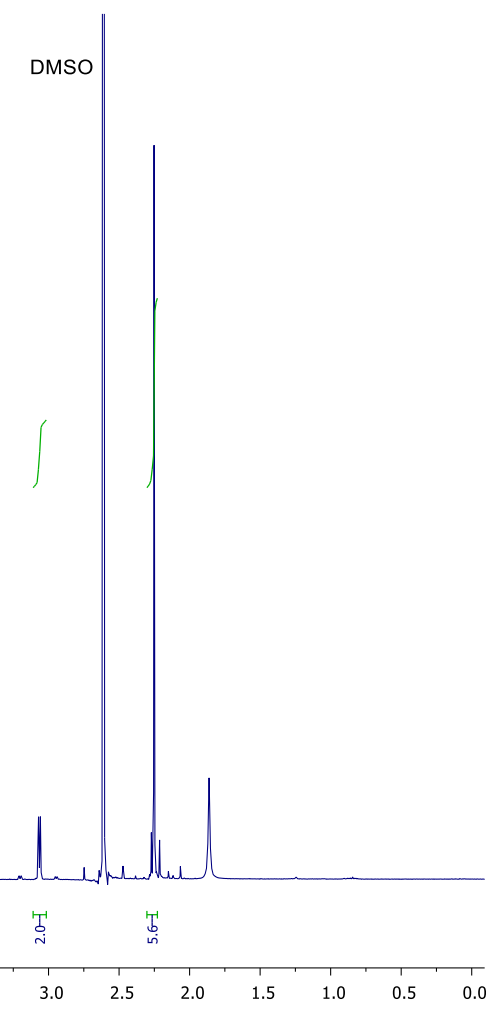

i

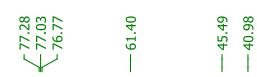

${ }^{13} \mathrm{C}-\mathrm{NMR}\left(125 \mathrm{MHz}, \mathrm{CDCl}_{3}\right)$<smiles>CN(C)C/C=C/C=C/C=O</smiles>

213

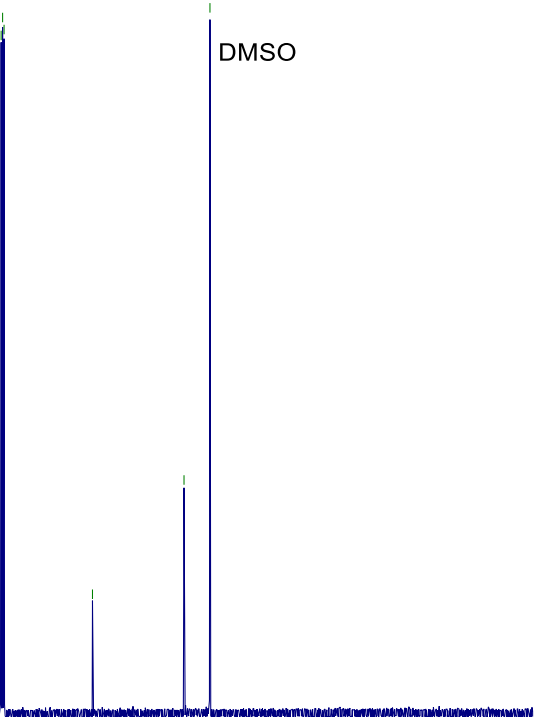

$\begin{array}{lllllllllllllllllllllllllllllllll}230 & 220 & 210 & 200 & 190 & 180 & 170 & 160 & 150 & 140 & 130 & 120 & \begin{array}{c}110 \\ (\mathrm{ppm})\end{array} & 100 & 90 & 80 & 70 & 60 & 50 & 40 & 30 & 20 & 10 & 0 & -10\end{array}$ 
Chapter Three Spectra

${ }^{1} \mathrm{H}-\mathrm{NMR}\left(500 \mathrm{MHz}, \mathrm{CDCl}_{3}\right.$ )<smiles>C#CCCCCC(=O)OCCC1CC=CC(=O)O1</smiles>

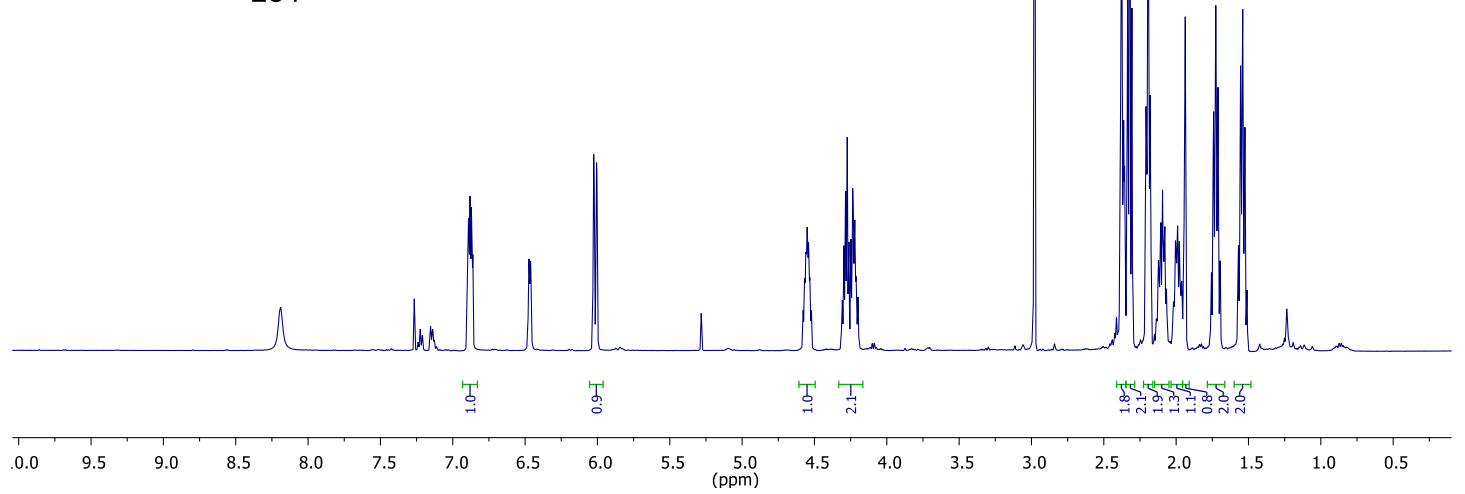

${ }^{13} \mathrm{C}-\mathrm{NMR}\left(125 \mathrm{MHz}, \mathrm{CDCl}_{3}\right.$ )

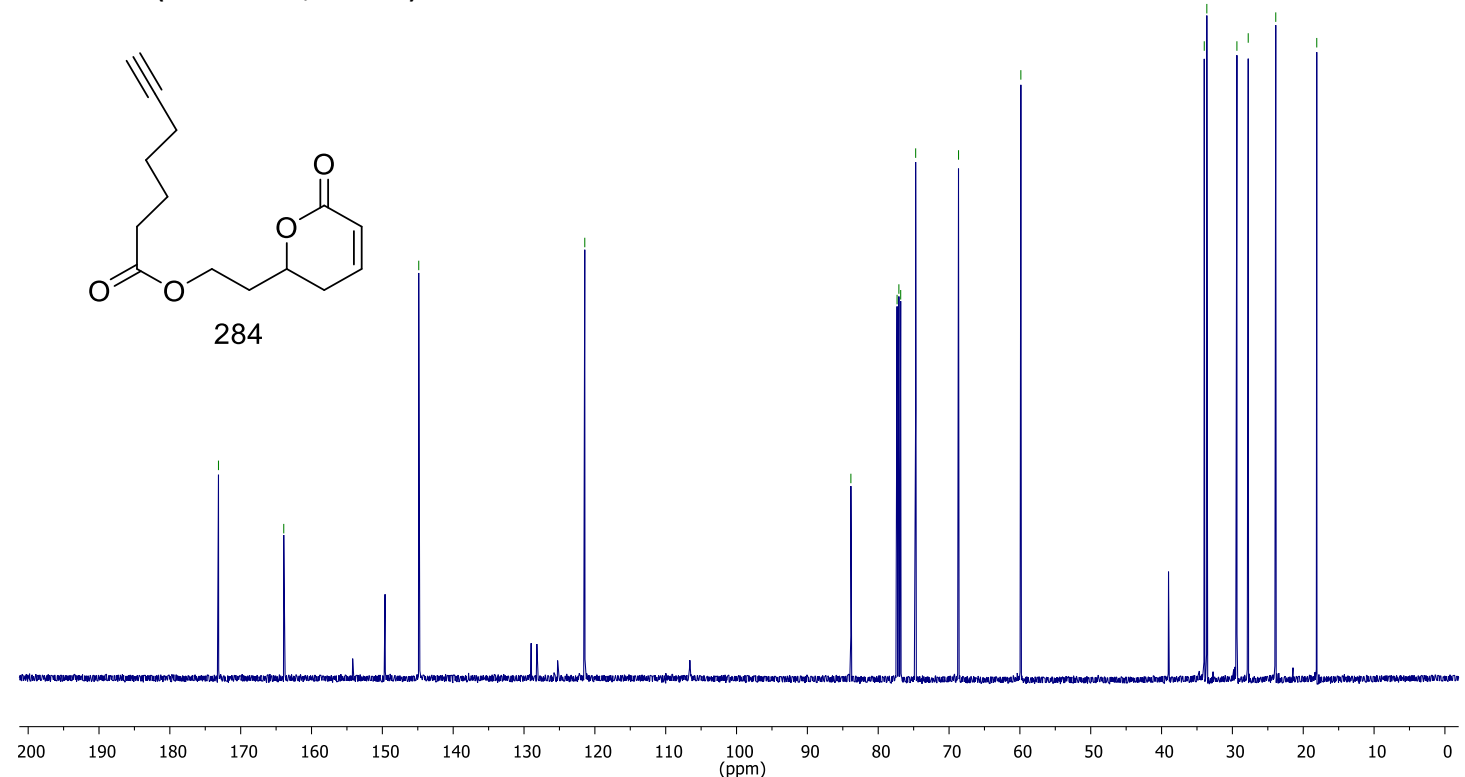


${ }^{1} \mathrm{H}-\mathrm{NMR}\left(500 \mathrm{MHz}, \mathrm{CDCl}_{3}\right.$ )

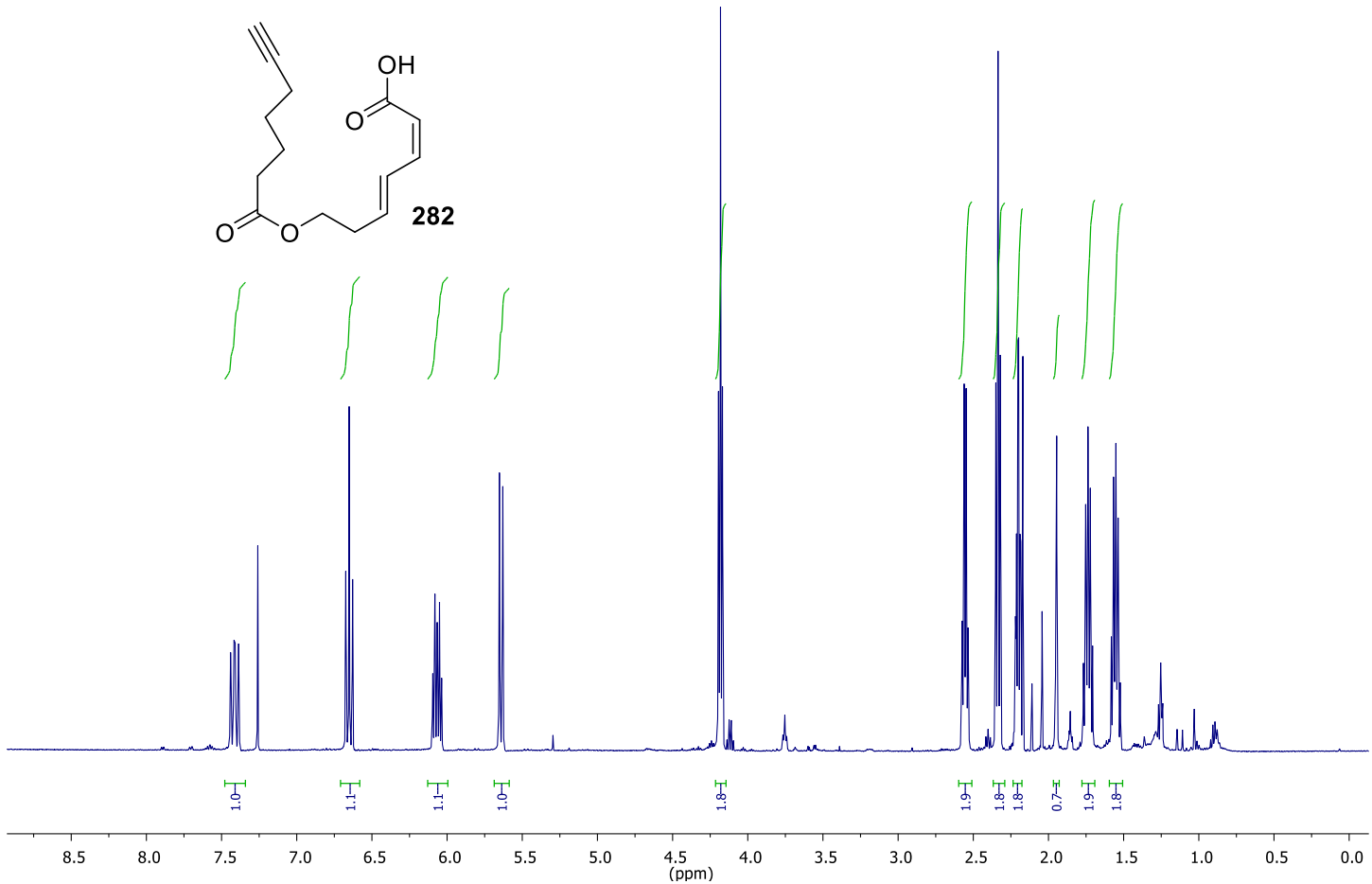

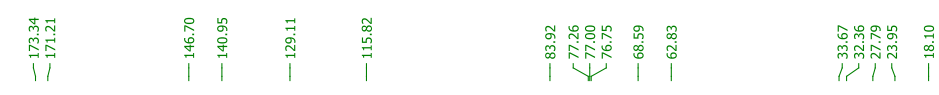

${ }^{13} \mathrm{C}-\mathrm{NMR}\left(125 \mathrm{MHz}, \mathrm{CDCl}_{3}\right.$ )

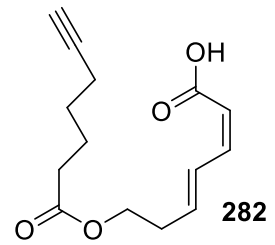

82

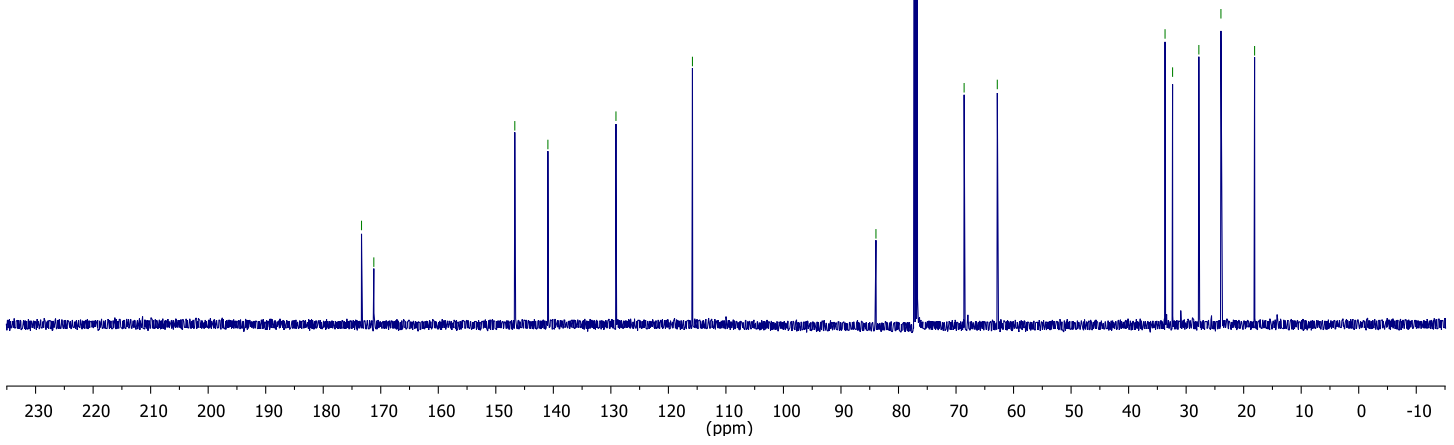


${ }^{1} \mathrm{H}-\mathrm{NMR}\left(500 \mathrm{MHz}, \mathrm{CDCl}_{3}\right.$ )

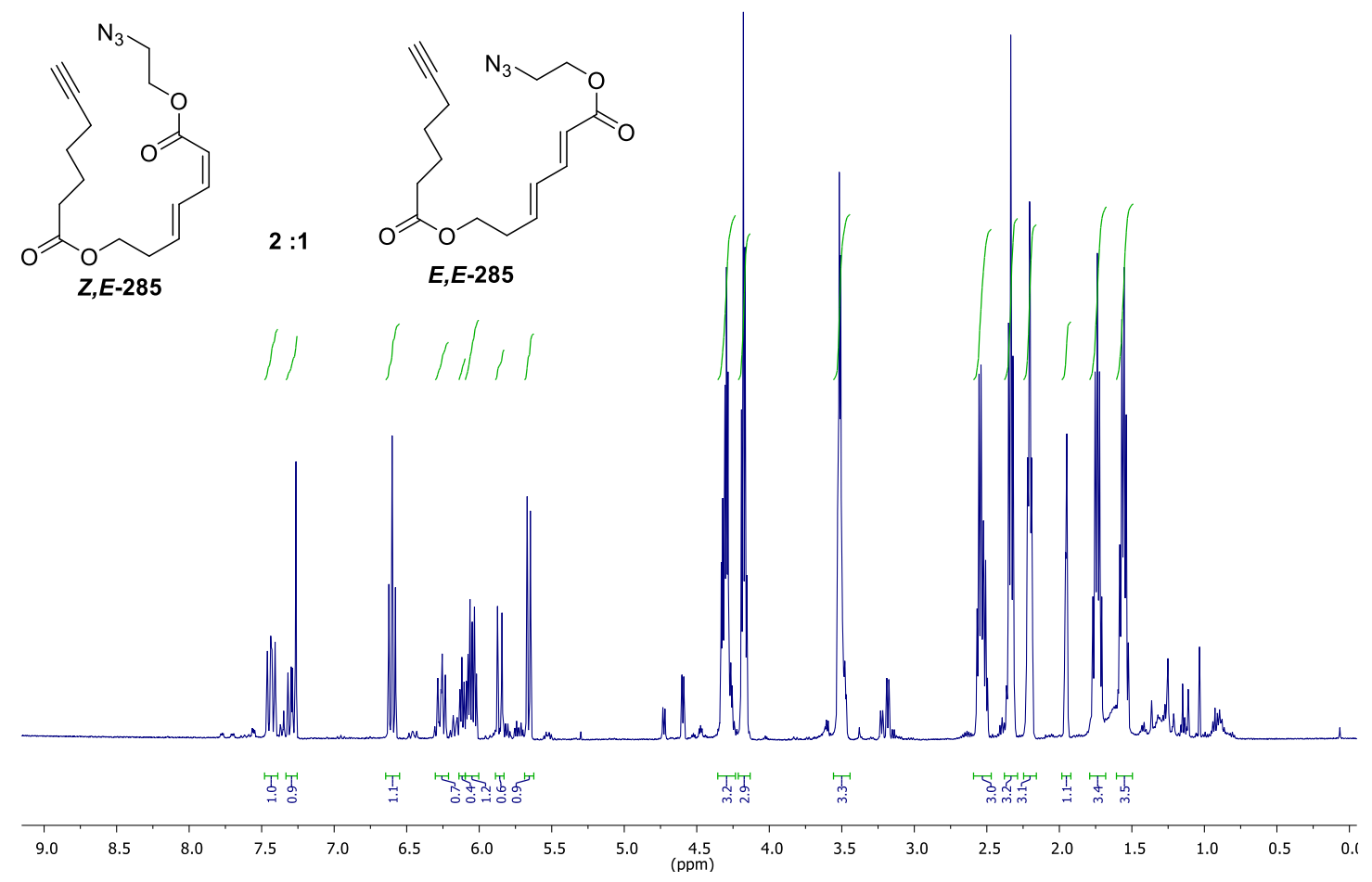

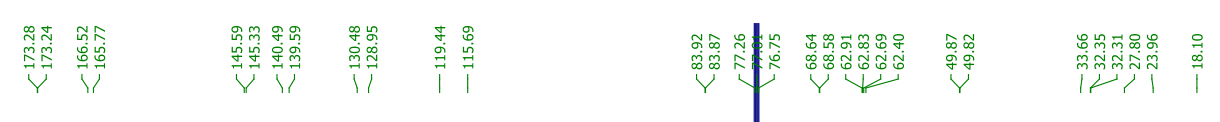

${ }^{13} \mathrm{C}-\mathrm{NMR}\left(125 \mathrm{MHz}, \mathrm{CDCl}_{3}\right)$<smiles>C#CCCCCC(=O)OCC/C=C\C=C/C(=O)OCCN</smiles>

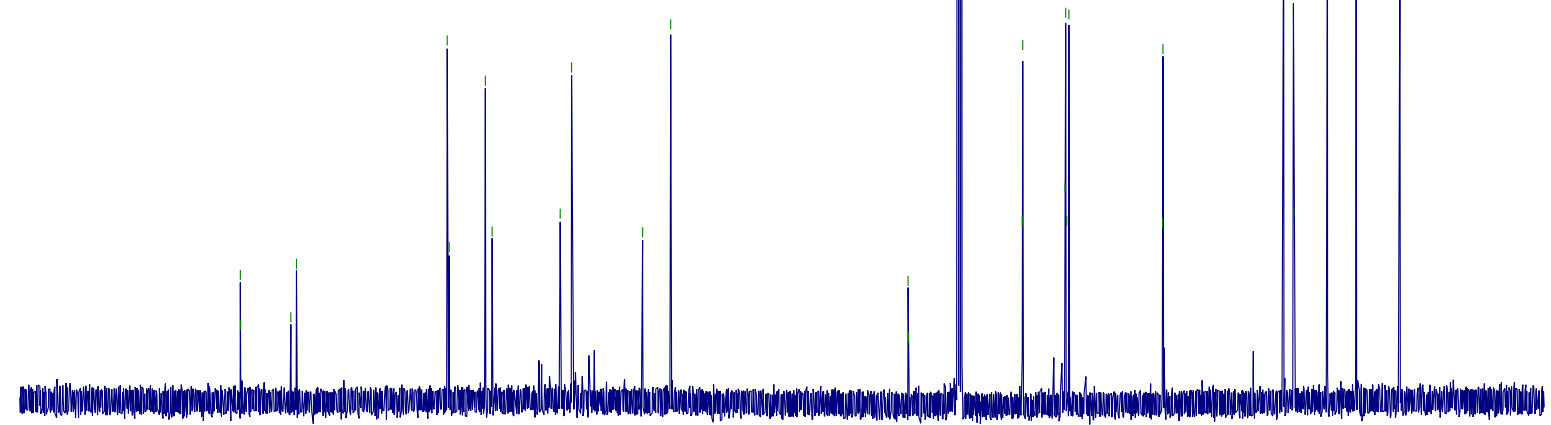

$\begin{array}{lllllllllllllllllllll} & 1 \\ 200 & 190 & 180 & 170 & 160 & 150 & 140 & 130 & 120 & 110 & \begin{array}{l}100 \\ (\mathrm{ppm})\end{array} & 90 & 80 & 70 & 60 & 50 & 40 & 30 & 20 & 10 & 0\end{array}$ 
${ }^{1} \mathrm{H}-\mathrm{NMR}\left(500 \mathrm{MHz}, \mathrm{CDCl}_{3}\right)$

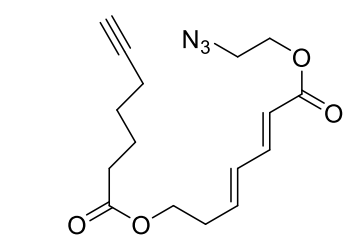

$E, E-285$

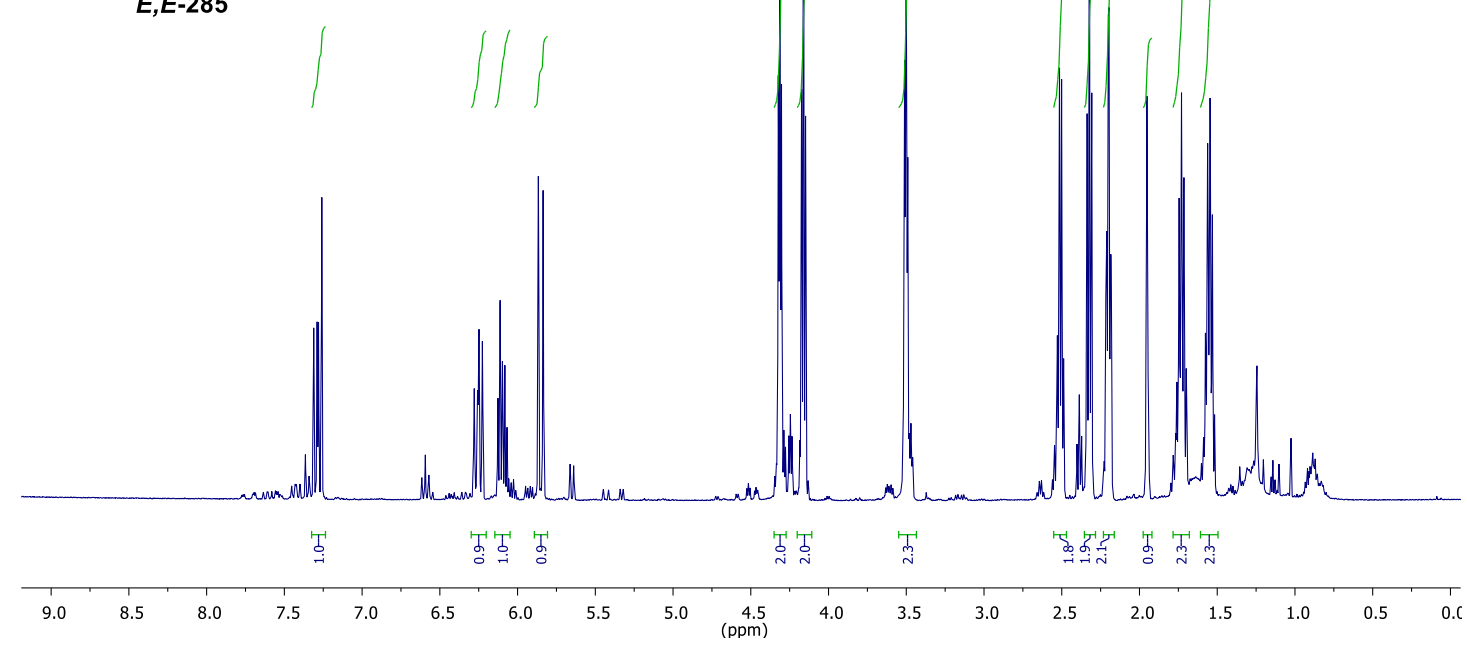

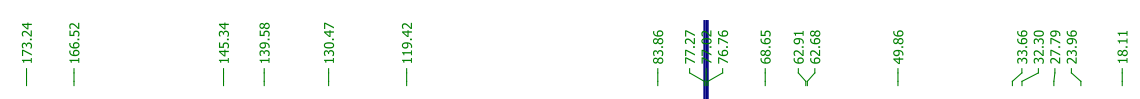

${ }^{13} \mathrm{C}-\mathrm{NMR}\left(125 \mathrm{MHz}, \mathrm{CDCl}_{3}\right)$

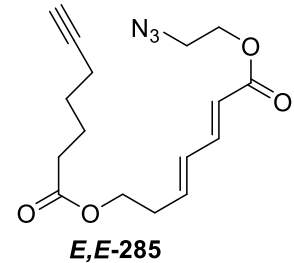

$\begin{array}{lllllllllllllllllllll}200 & 190 & 180 & 170 & 160 & 150 & 140 & 130 & 120 & 110 & 100 & 90 & 80 & 70 & 60 & 50 & 40 & 30 & 20 & 10 & 0\end{array}$ 


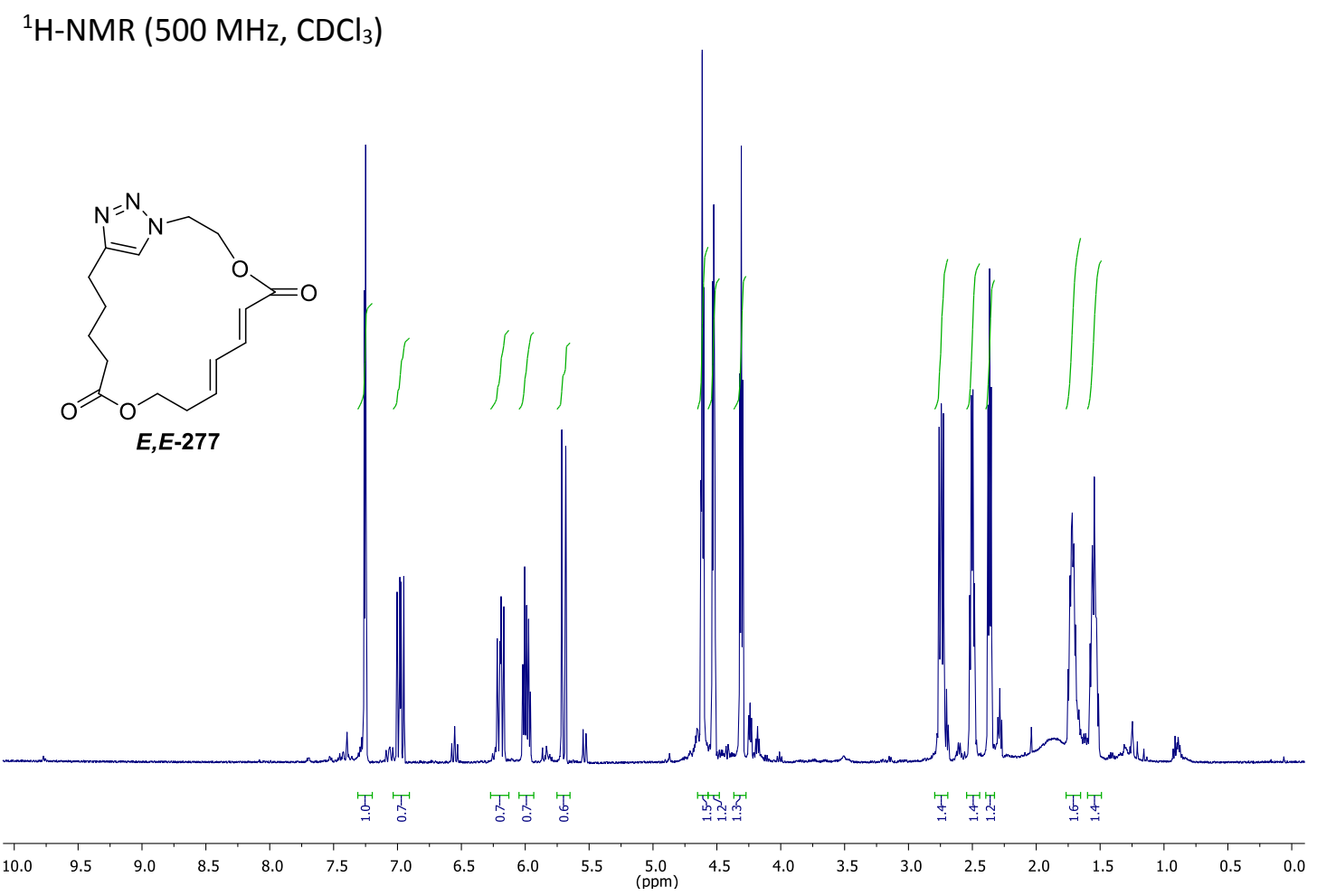

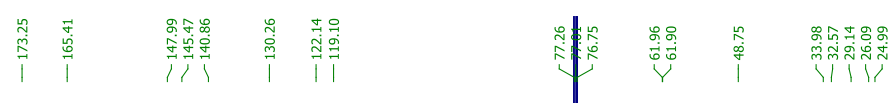

${ }^{13} \mathrm{C}-\mathrm{NMR}\left(125 \mathrm{MHz}, \mathrm{CDCl}_{3}\right)$
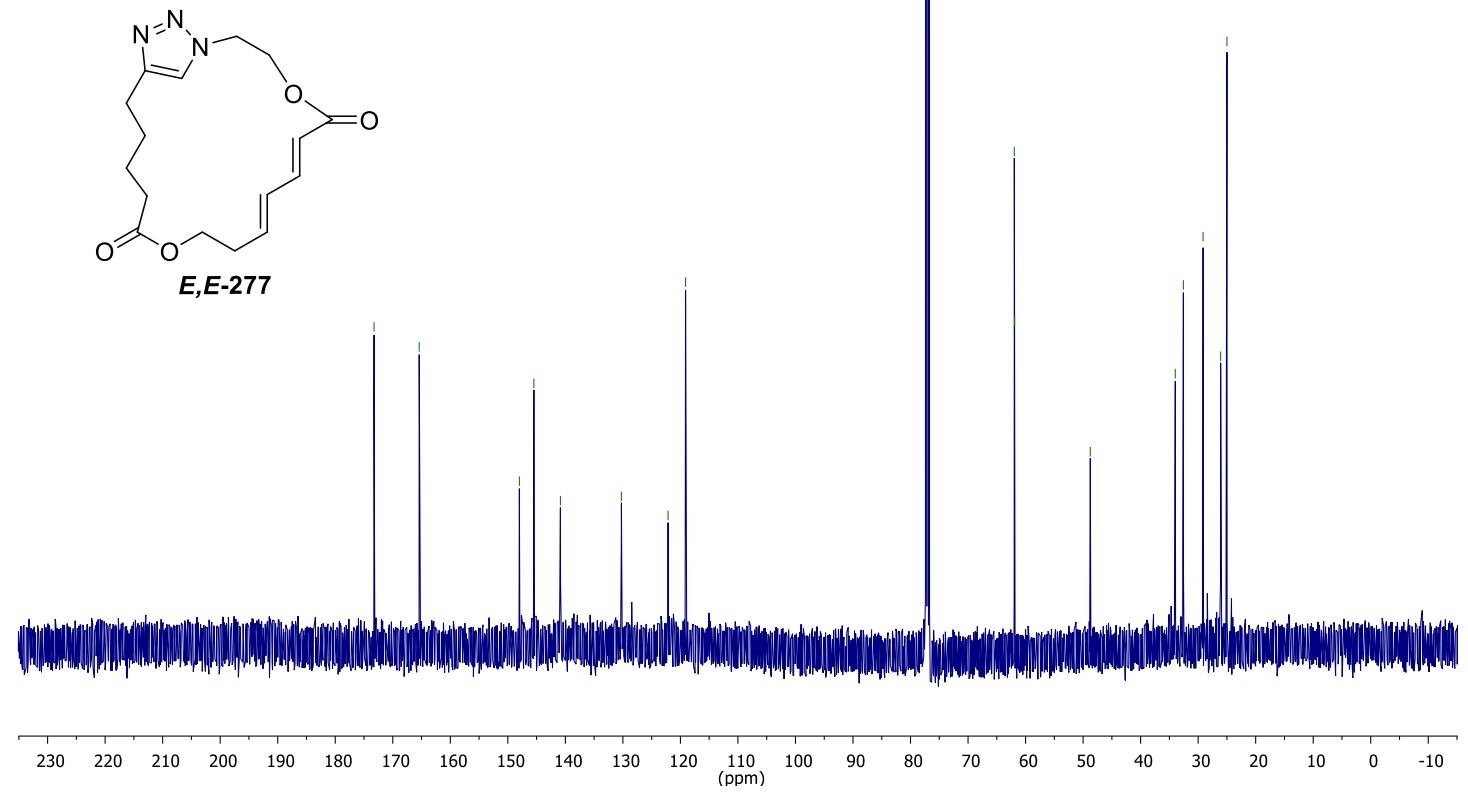
${ }^{1} \mathrm{H}-\mathrm{NMR}\left(500 \mathrm{MHz}, \mathrm{CDCl}_{3}\right)$

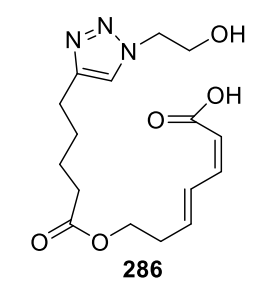

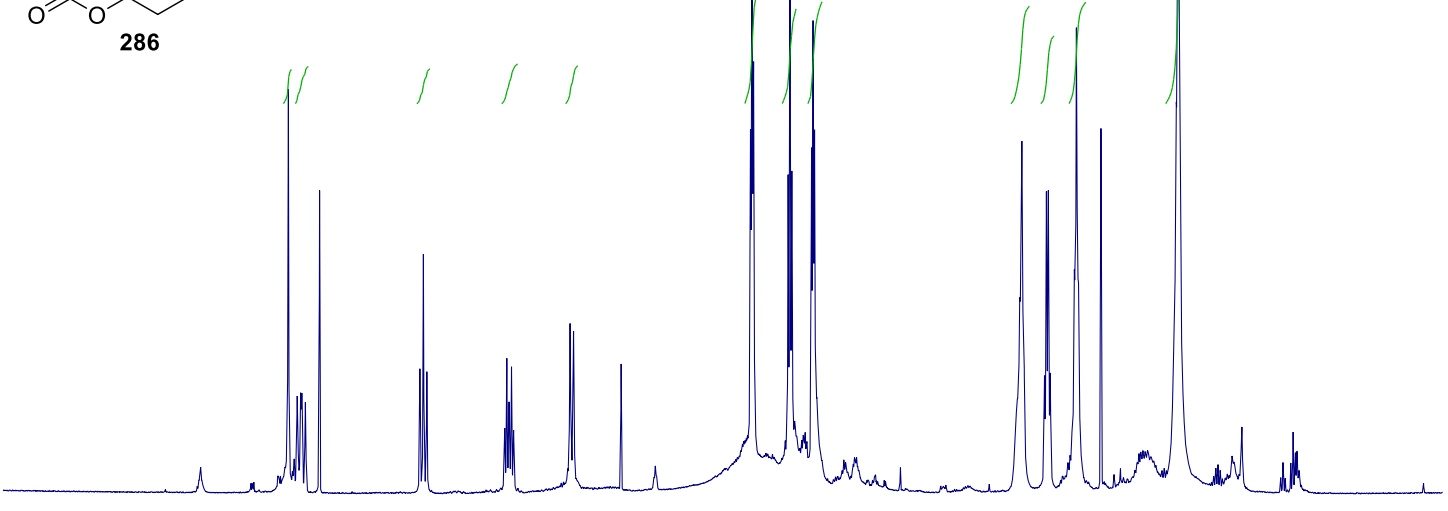

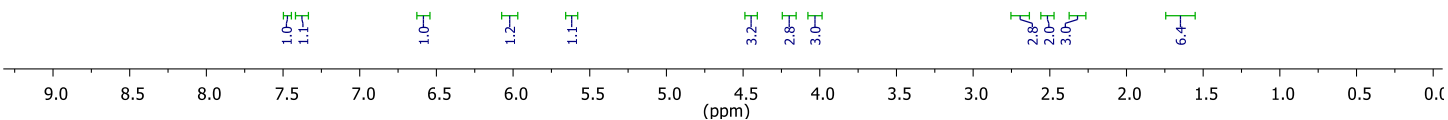

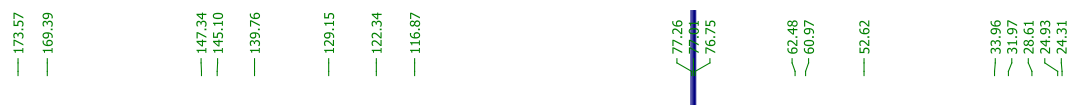

${ }^{13} \mathrm{C}-\mathrm{NMR}\left(125 \mathrm{MHz}, \mathrm{CDCl}_{3}\right.$ )<smiles>O=C(O)/C=C\C=C/CCOC(=O)CCCCc1cn(CCO)nn1</smiles>
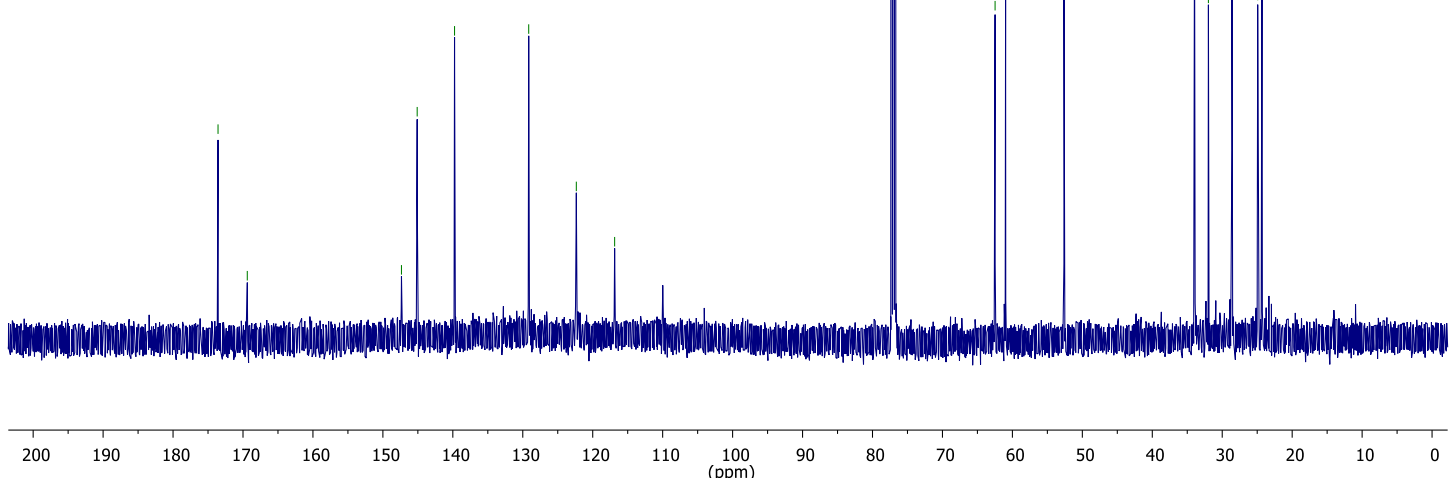
${ }^{1} \mathrm{H}-\mathrm{NMR}\left(600 \mathrm{MHz}, \mathrm{CDCl}_{3}\right)$

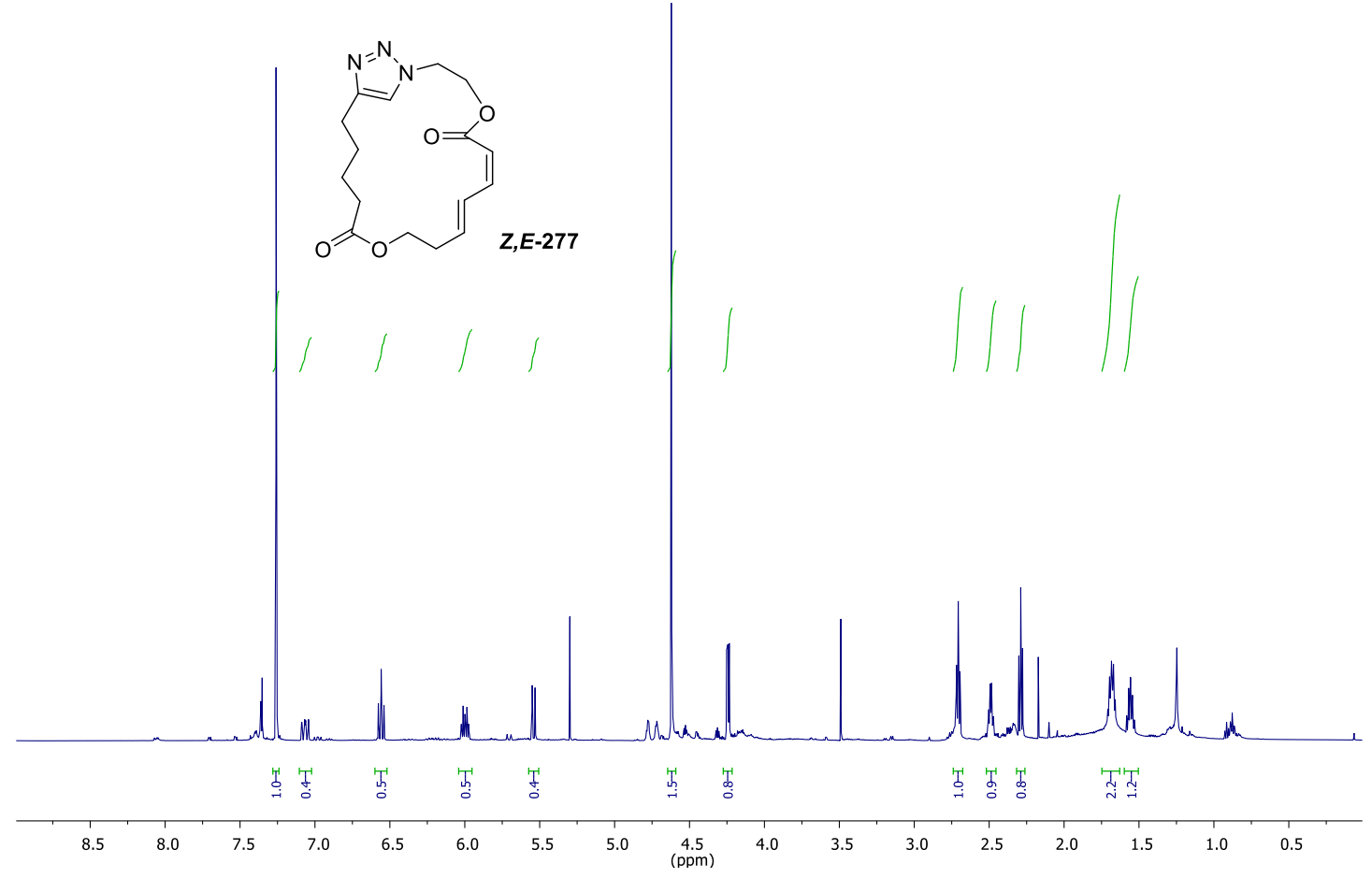

H n

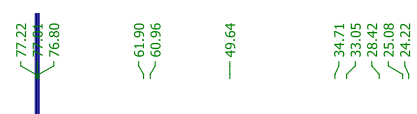

${ }^{13} \mathrm{C}-\mathrm{NMR}\left(151 \mathrm{MHz}, \mathrm{CDCl}_{3}\right)$
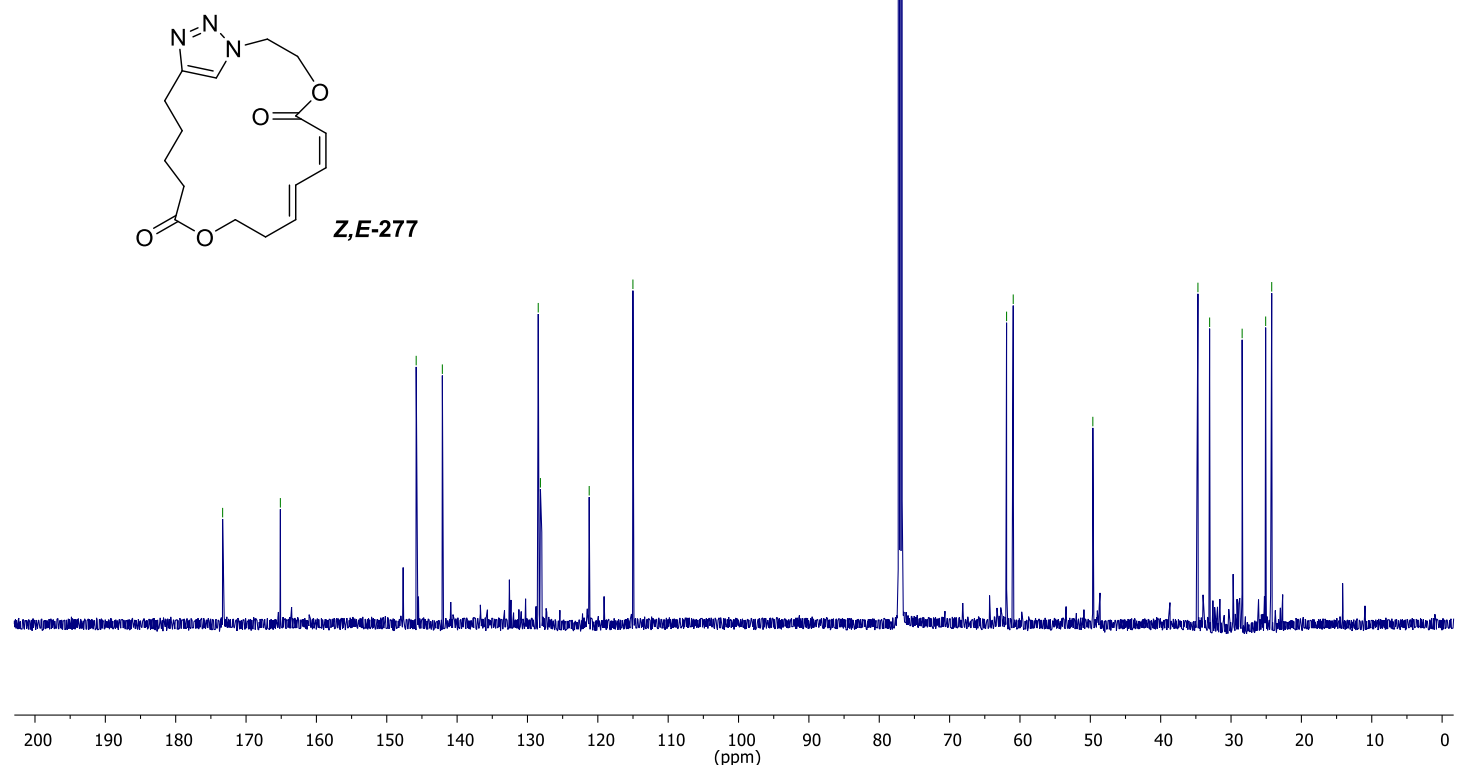
${ }^{1} \mathrm{H}-\mathrm{NMR}\left(500 \mathrm{MHz}, \mathrm{CDCl}_{3}\right)$

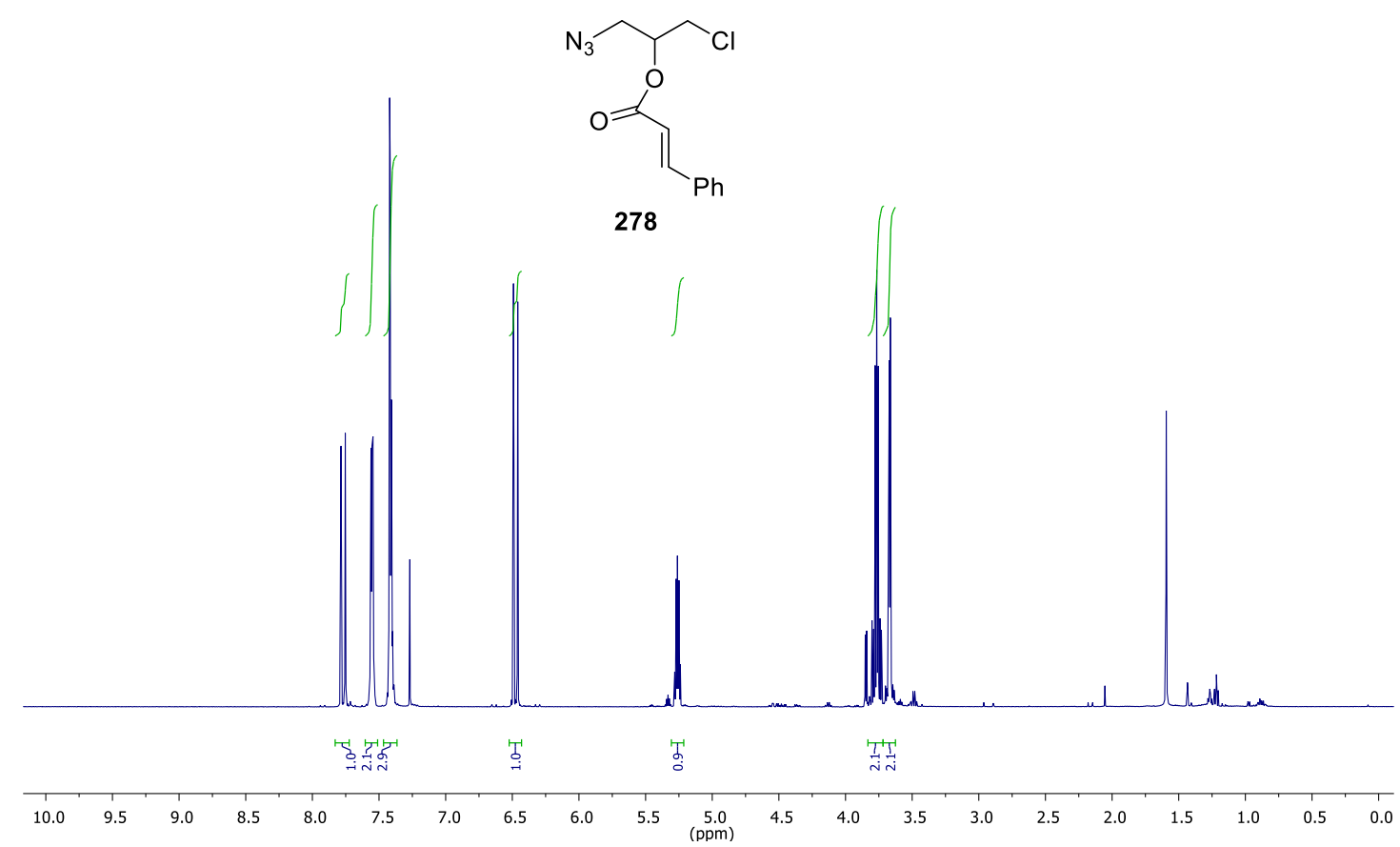

${ }^{13} \mathrm{C}-\mathrm{NMR}\left(125 \mathrm{MHz}, \mathrm{CDCl}_{3}\right.$ )

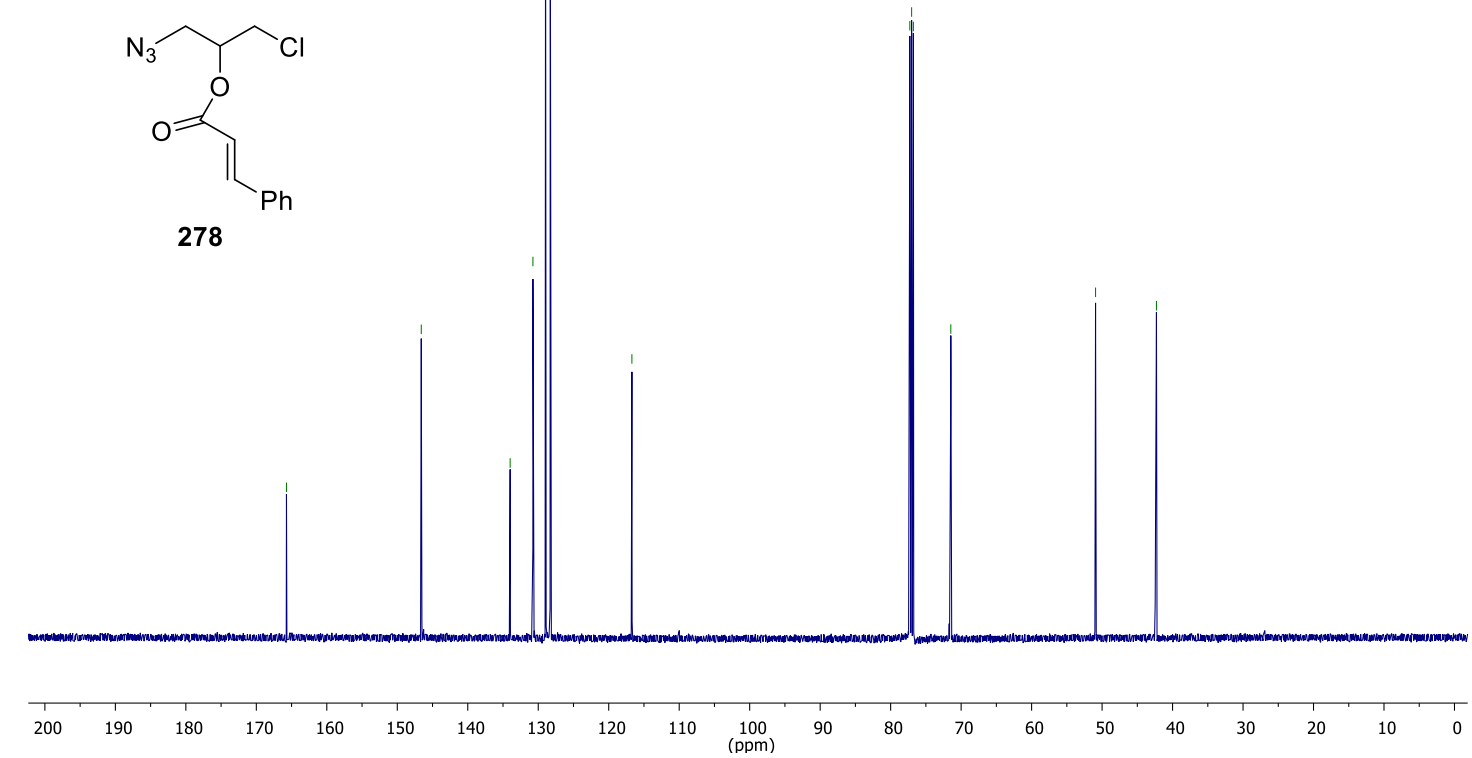

278

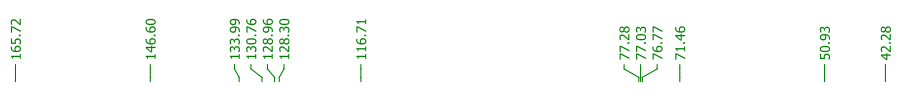


${ }^{1} \mathrm{H}-\mathrm{NMR}\left(500 \mathrm{MHz}, \mathrm{CDCl}_{3}\right)$
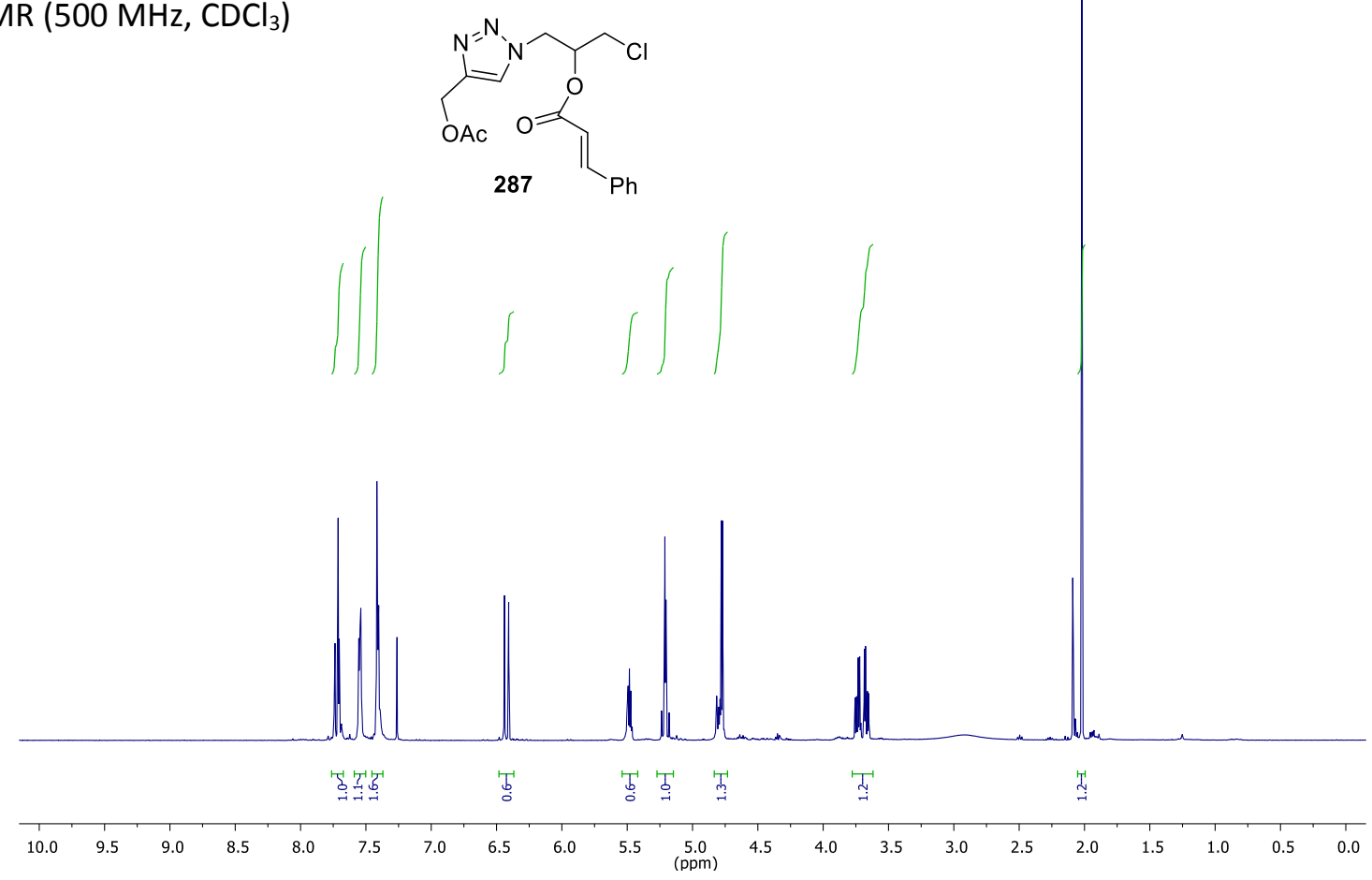

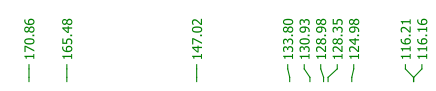

${ }^{13} \mathrm{C}-\mathrm{NMR}\left(125 \mathrm{MHz}, \mathrm{CDCl}_{3}\right.$ )

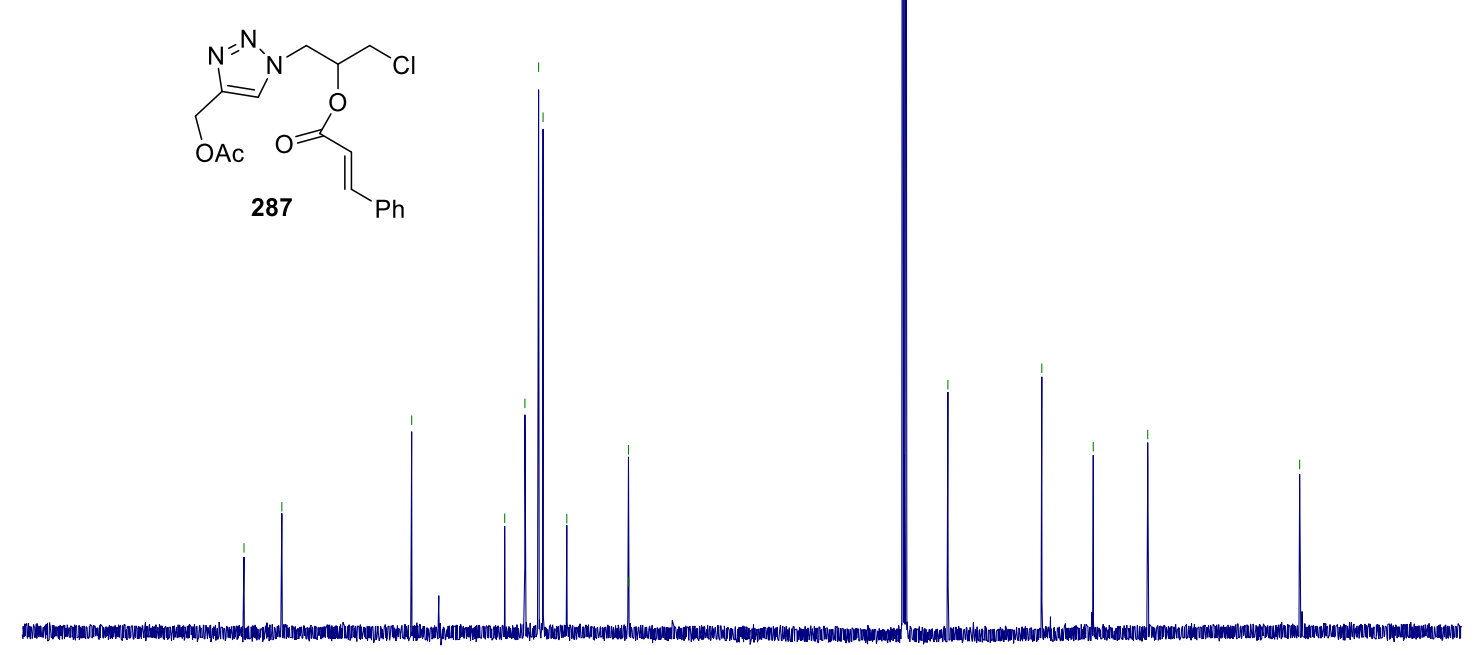

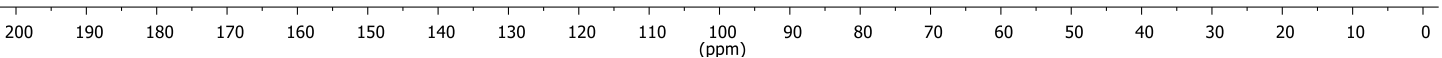




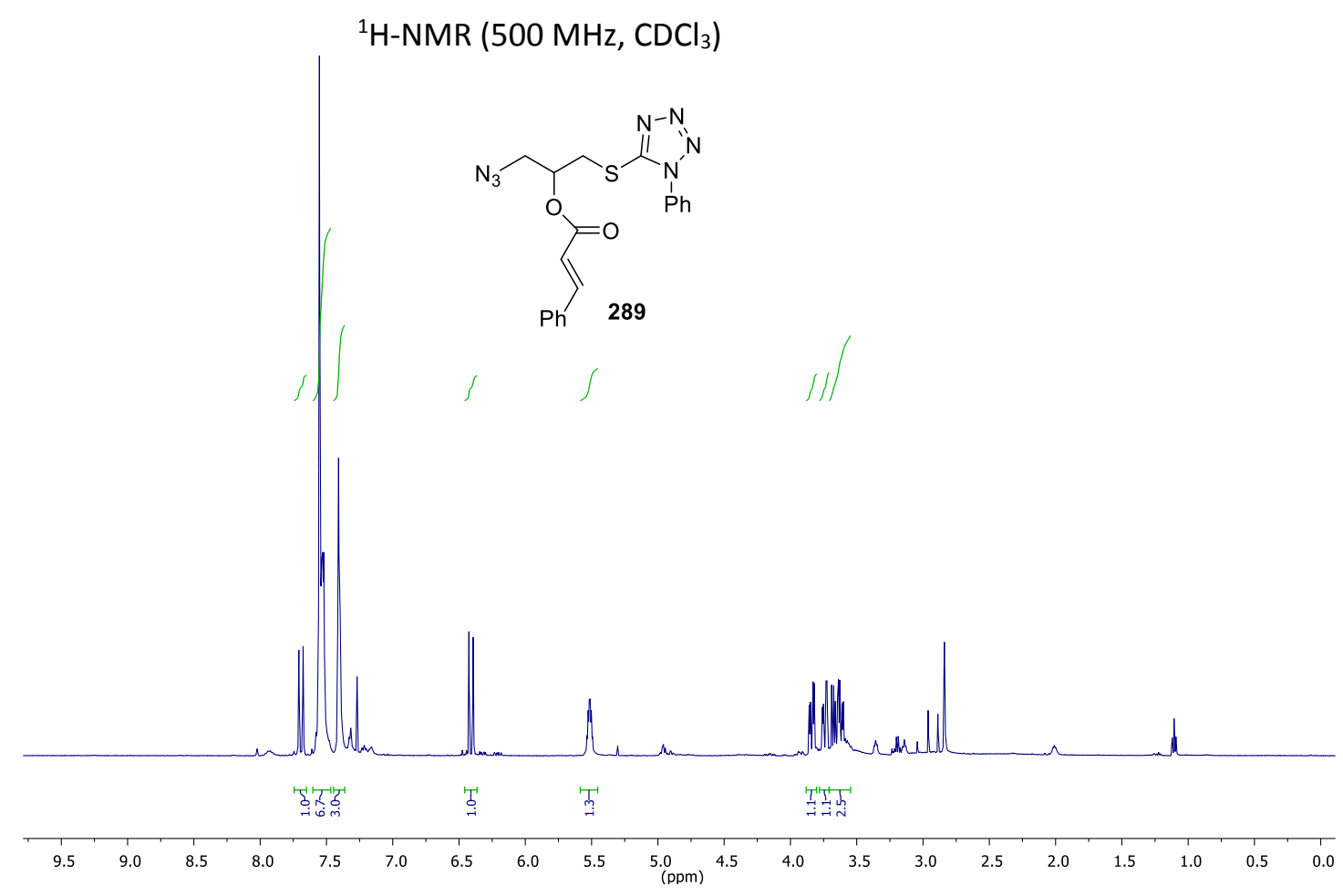

\section{| l|}

许|

${ }^{13} \mathrm{C}-\mathrm{NMR}\left(125 \mathrm{MHz}, \mathrm{CDCl}_{3}\right)$

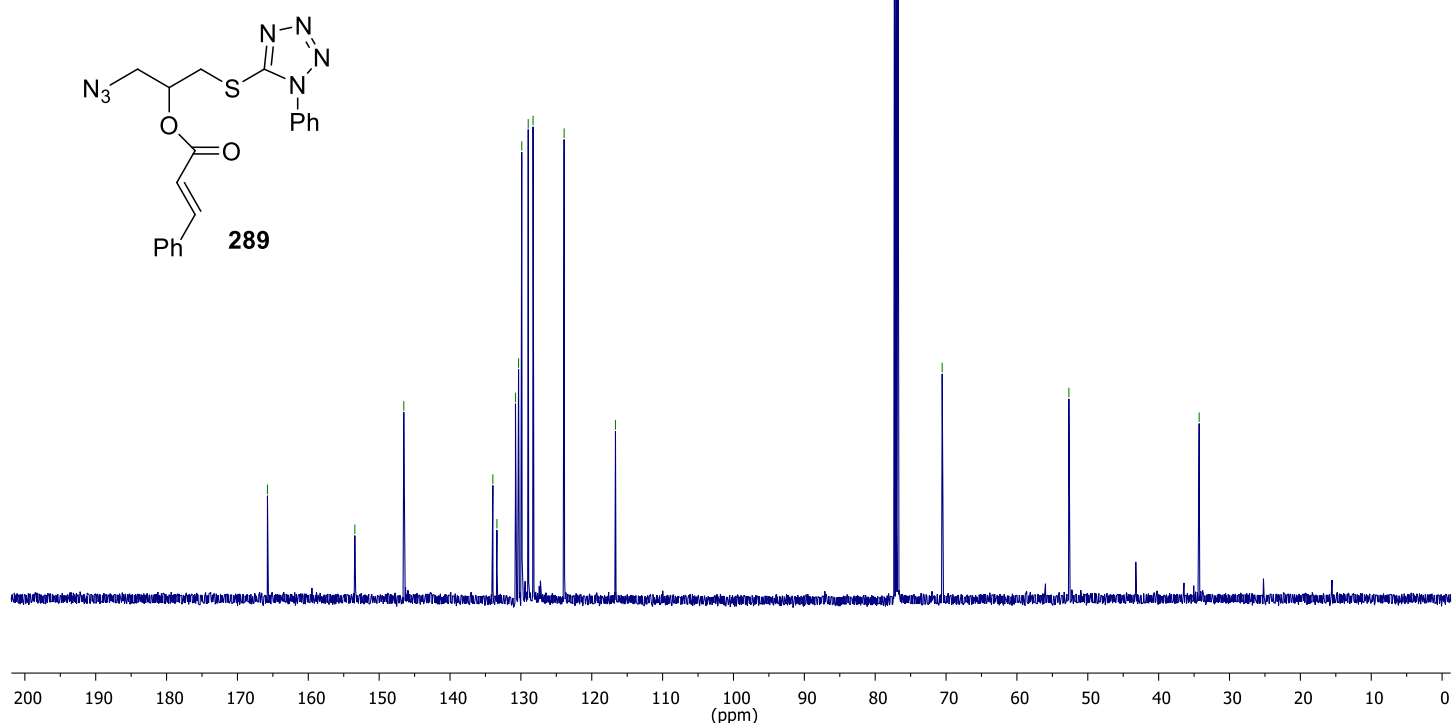


${ }^{1} \mathrm{H}-\mathrm{NMR}\left(500 \mathrm{MHz}, \mathrm{CDCl}_{3}\right)$

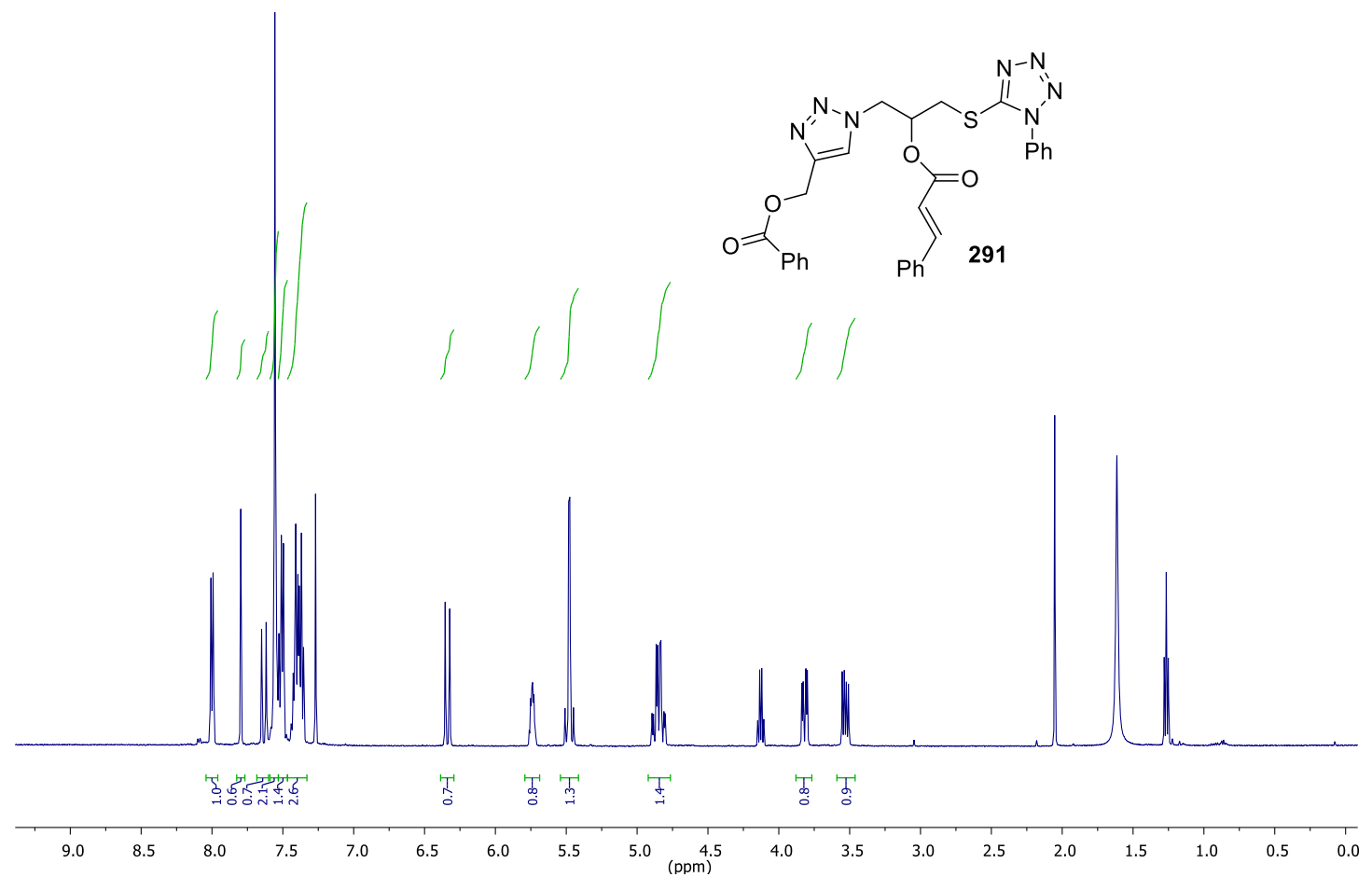

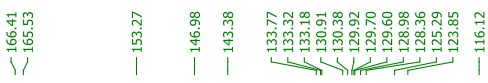

${ }^{13} \mathrm{C}-\mathrm{NMR}\left(125 \mathrm{MHz}, \mathrm{CDCl}_{3}\right)$
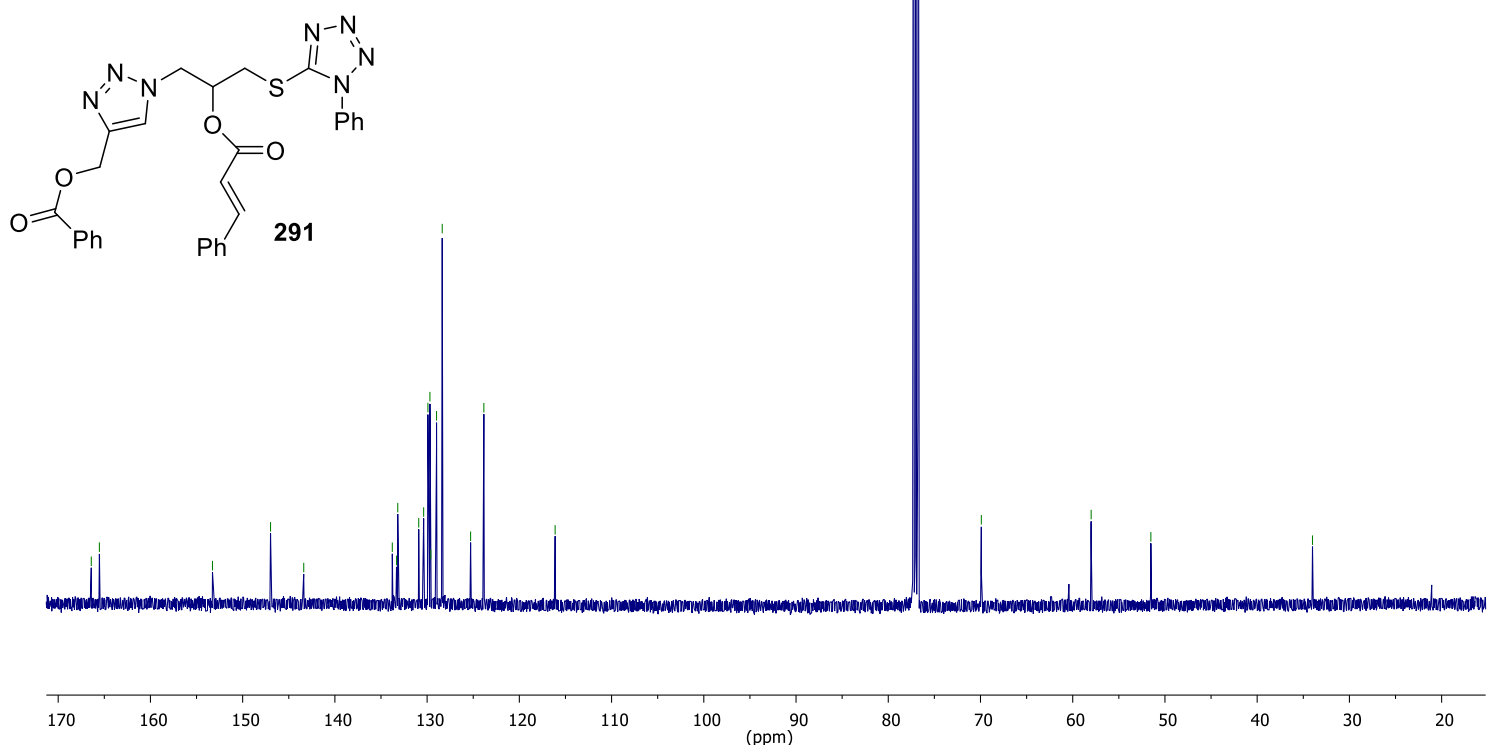
${ }^{1} \mathrm{H}-\mathrm{NMR}\left(500 \mathrm{MHz}, \mathrm{CDCl}_{3}\right.$ )

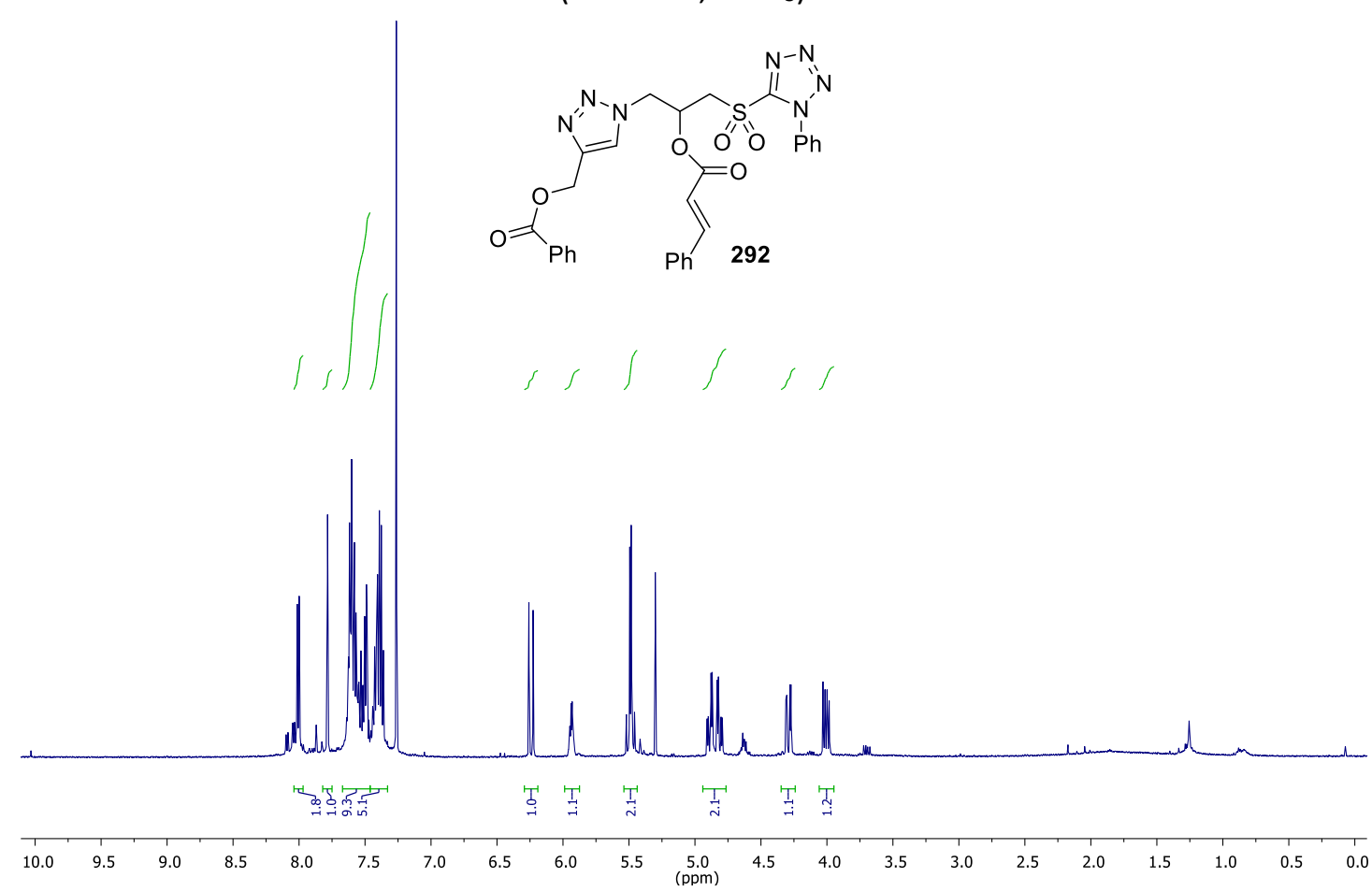

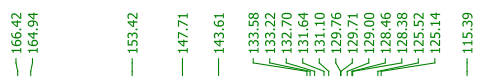

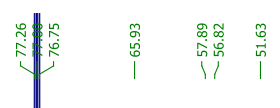

${ }^{13} \mathrm{C}-\mathrm{NMR}\left(125 \mathrm{MHz}, \mathrm{CDCl}_{3}\right.$ )
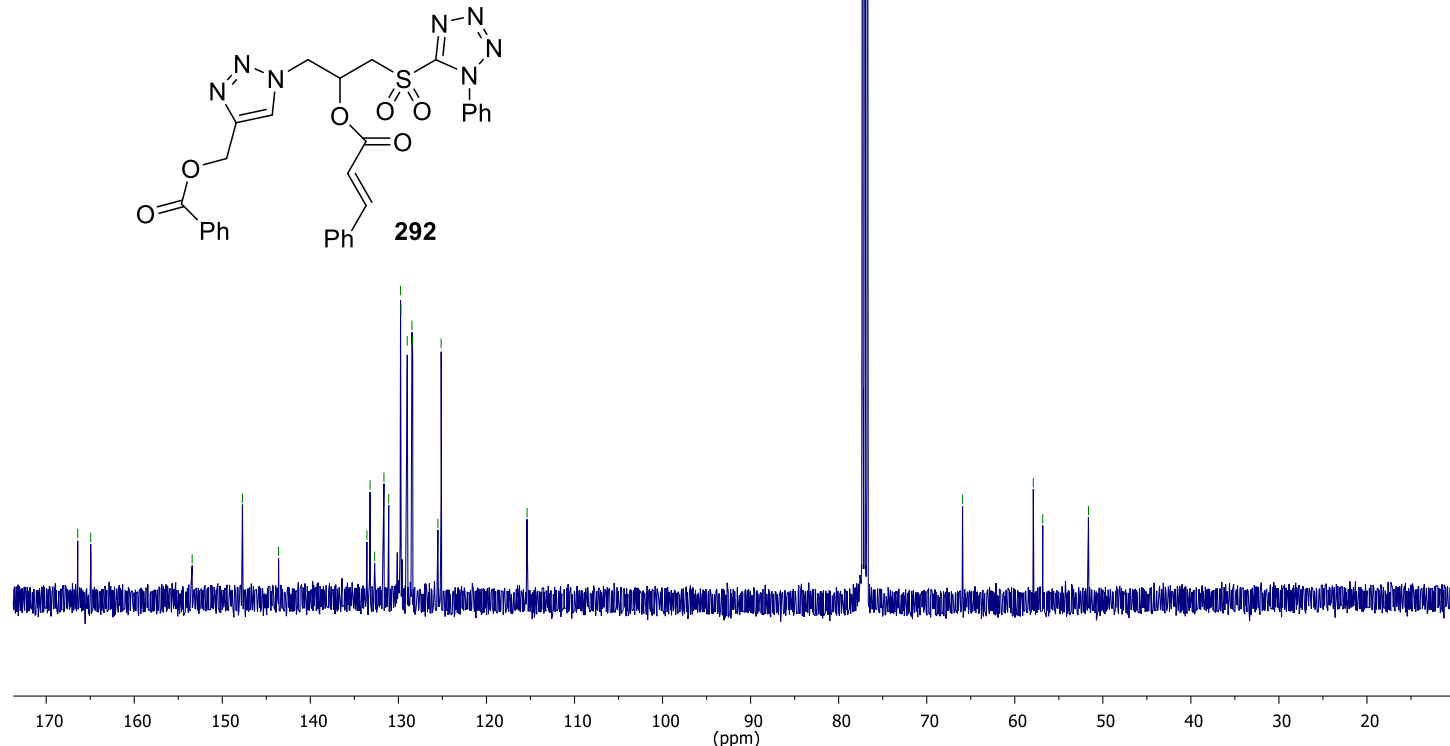
${ }^{1} \mathrm{H}-\mathrm{NMR}\left(500 \mathrm{MHz}, \mathrm{CDCl}_{3}\right)$

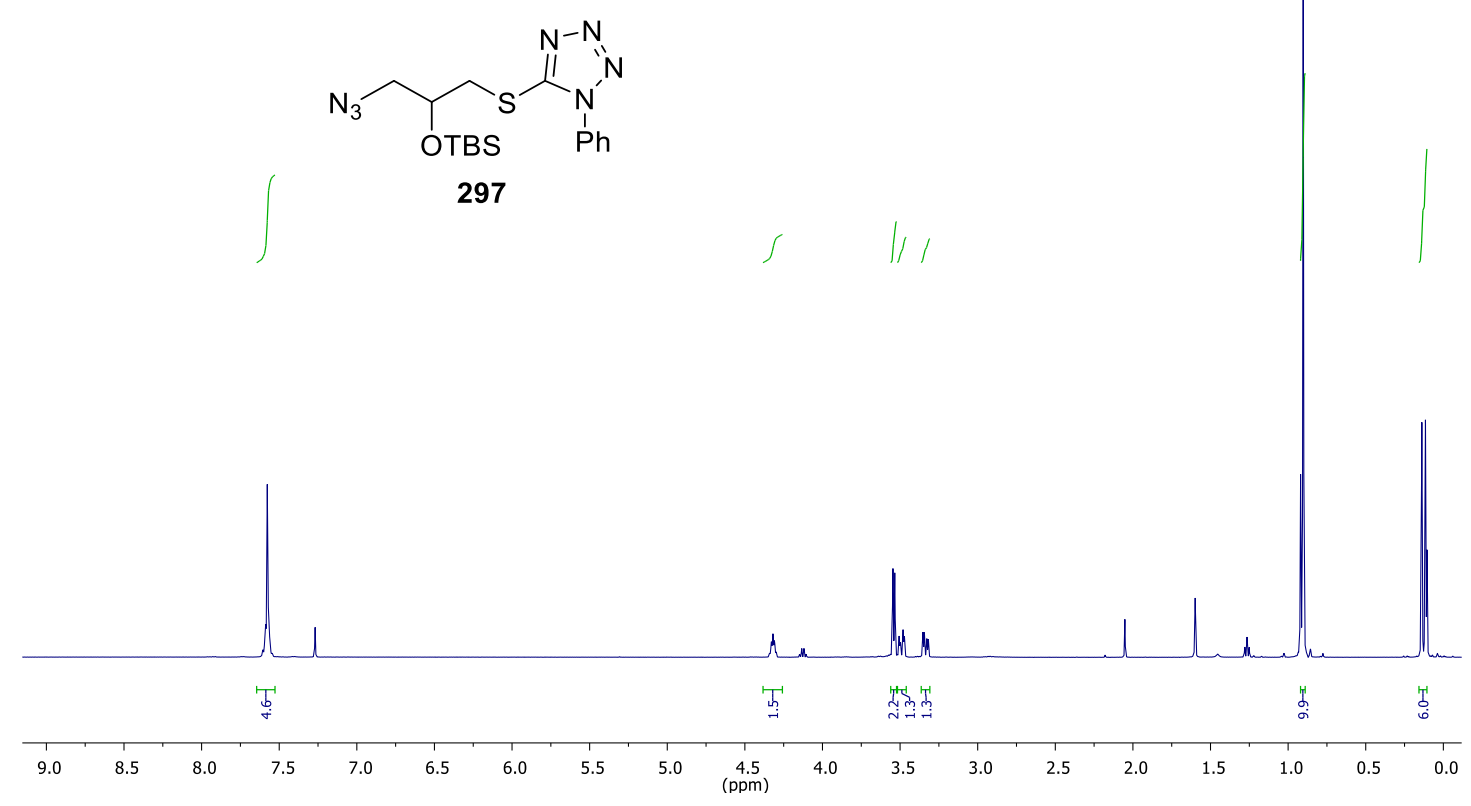

${ }^{13} \mathrm{C}-\mathrm{NMR}\left(125 \mathrm{MHz}, \mathrm{CDCl}_{3}\right)$
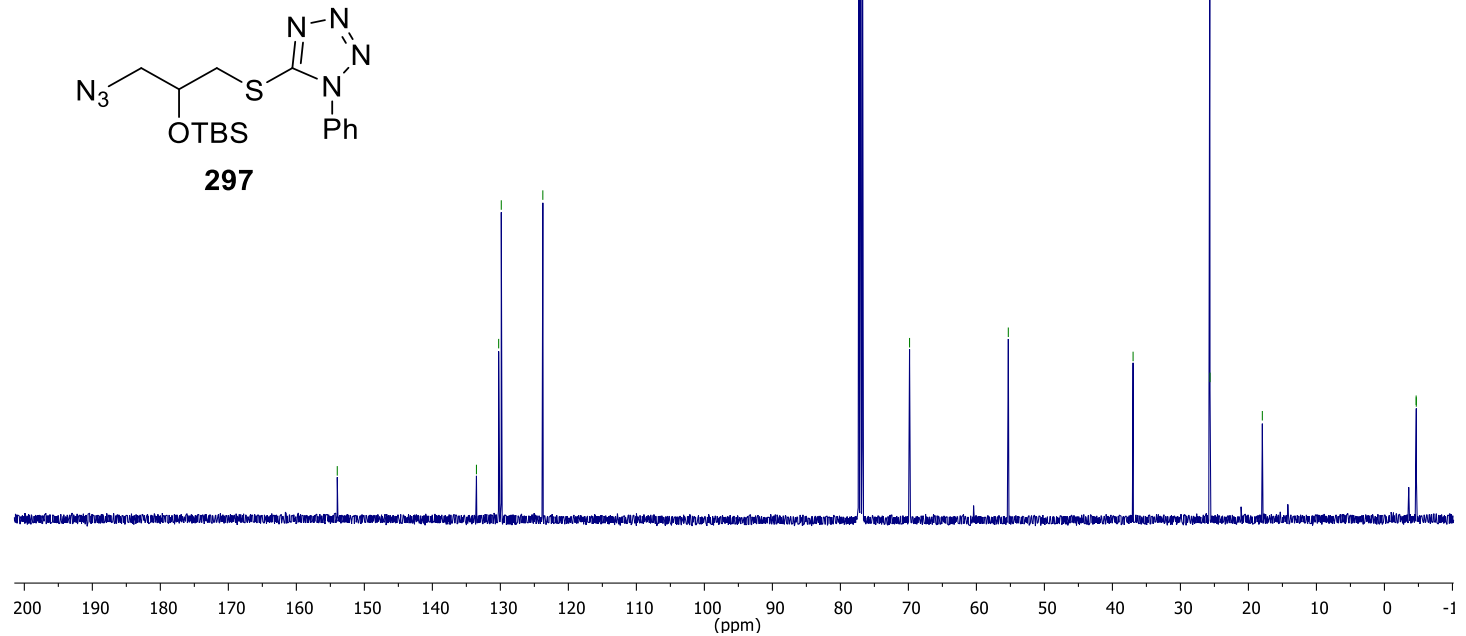
${ }^{1} \mathrm{H}-\mathrm{NMR}\left(500 \mathrm{MHz}, \mathrm{CDCl}_{3}\right.$ )

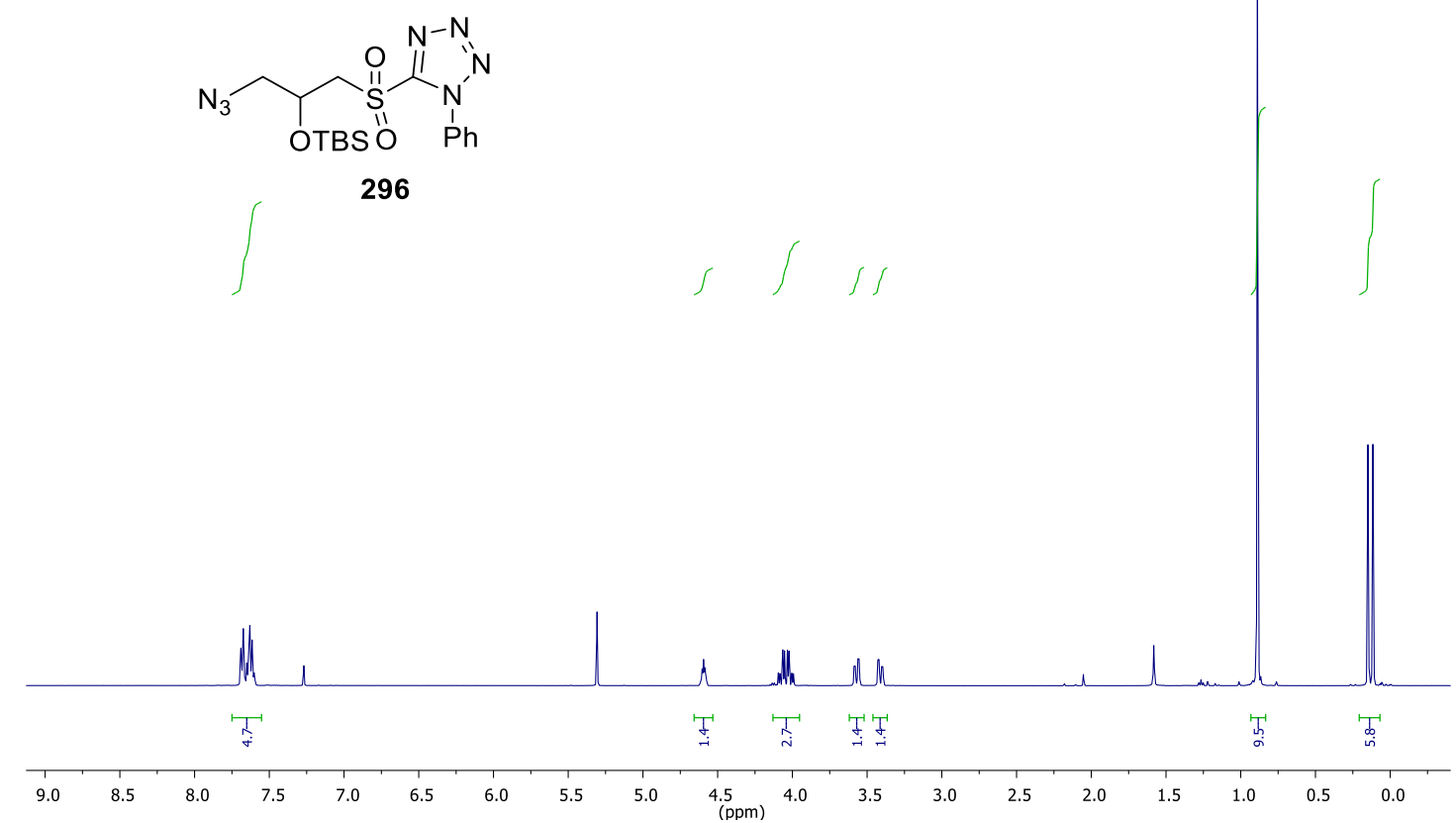

${ }^{13} \mathrm{C}-\mathrm{NMR}\left(125 \mathrm{MHz}, \mathrm{CDCl}_{3}\right)$

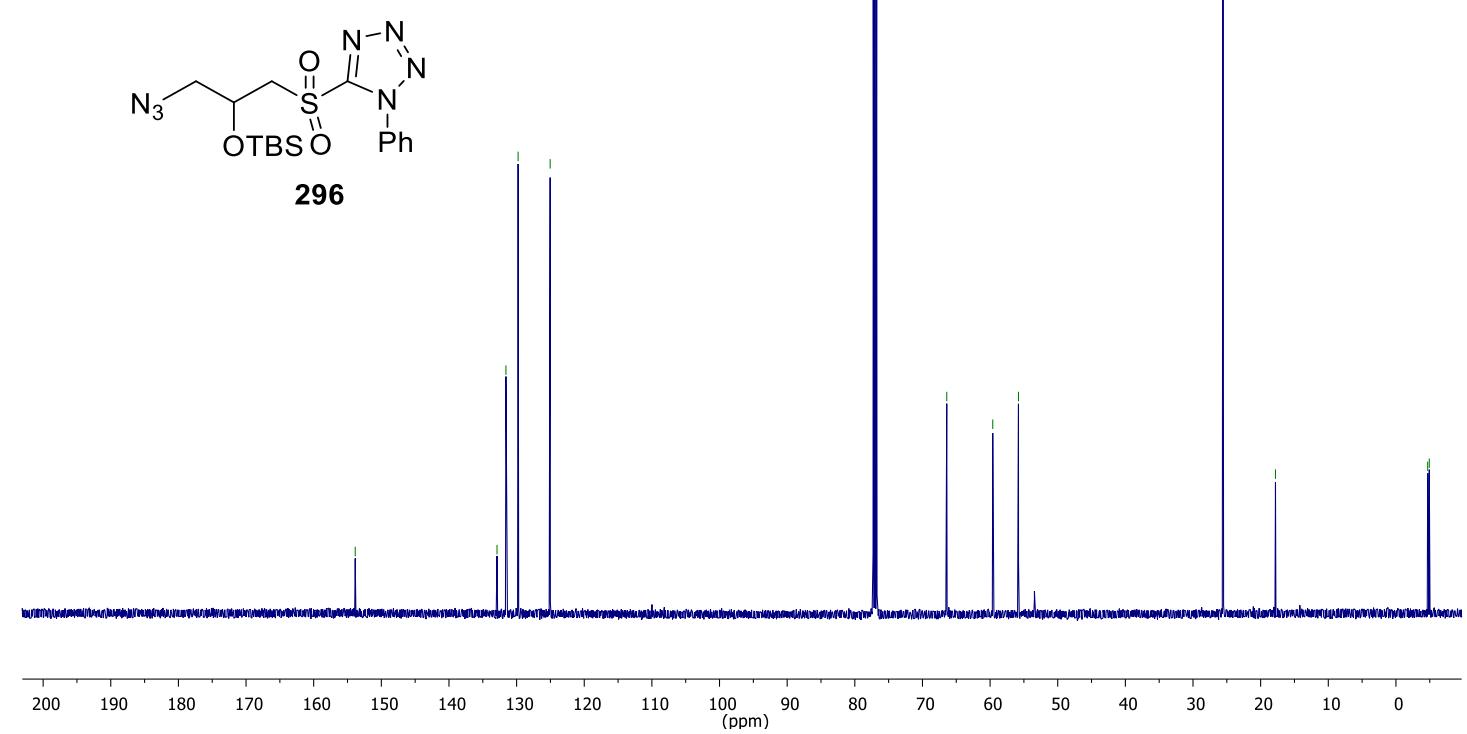


${ }^{1} \mathrm{H}-\mathrm{NMR}\left(500 \mathrm{MHz}, \mathrm{CDCl}_{3}\right)$

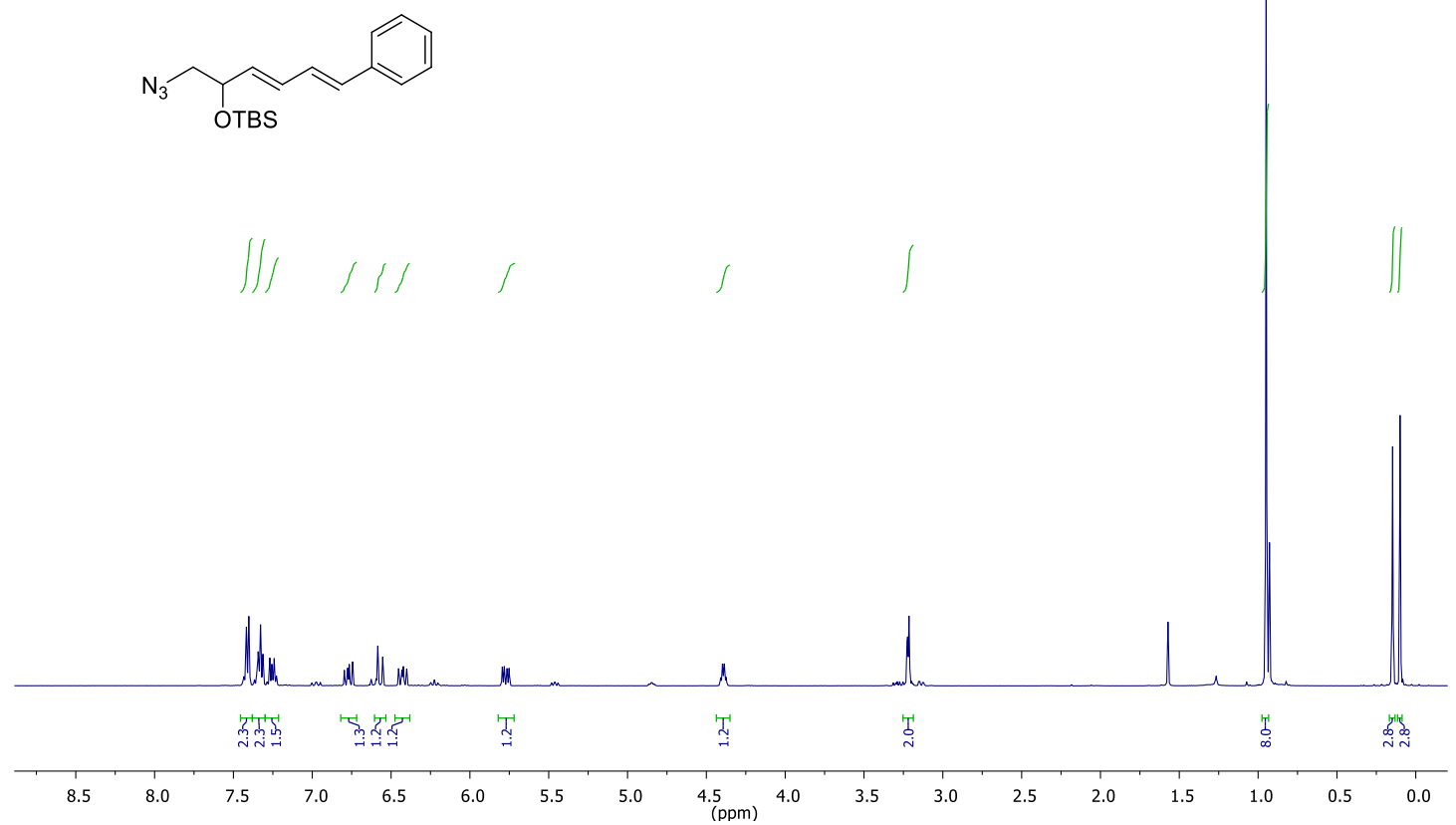

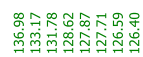

选过

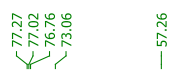

变索

${ }^{13} \mathrm{C}-\mathrm{NMR}\left(125 \mathrm{MHz}, \mathrm{CDCl}_{3}\right)$

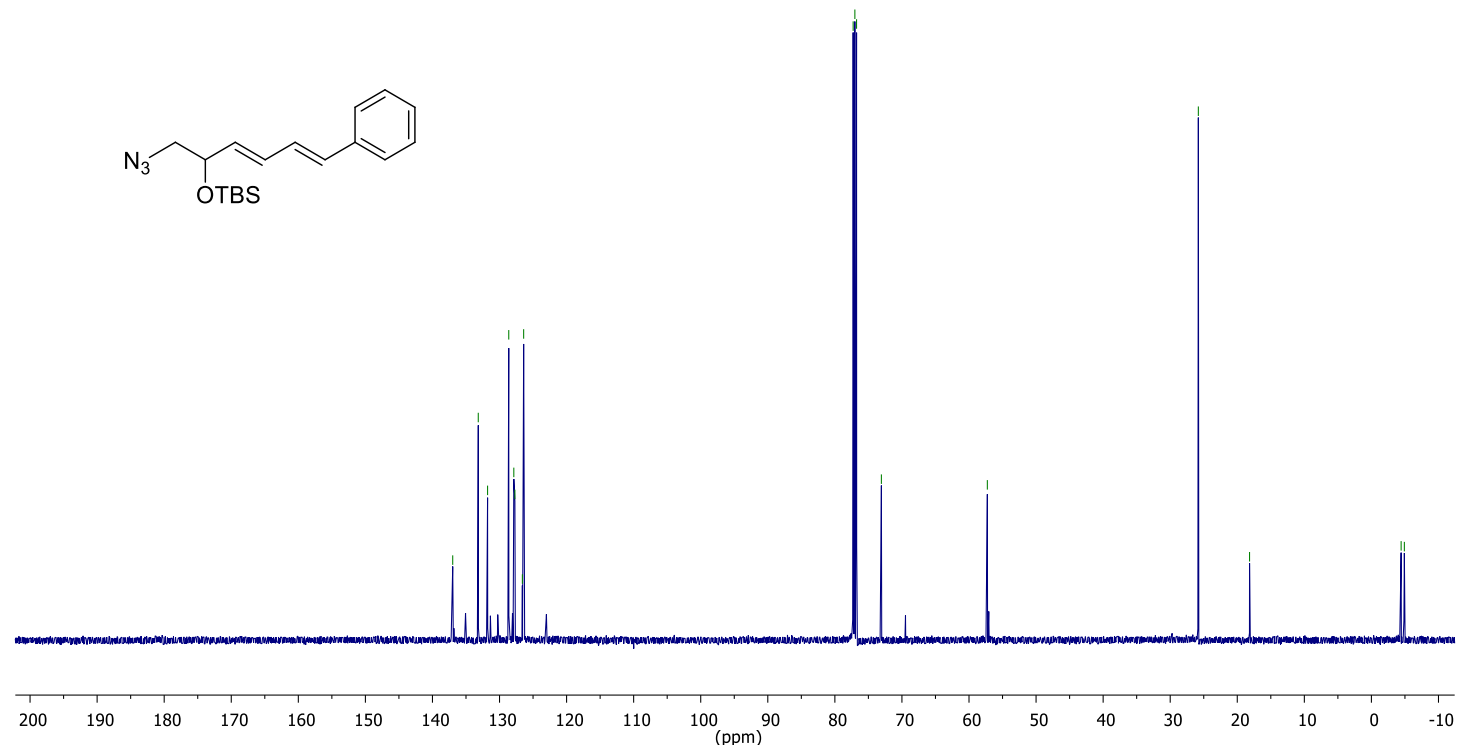


${ }^{1} \mathrm{H}-\mathrm{NMR}\left(500 \mathrm{MHz}, \mathrm{CDCl}_{3}\right.$ )

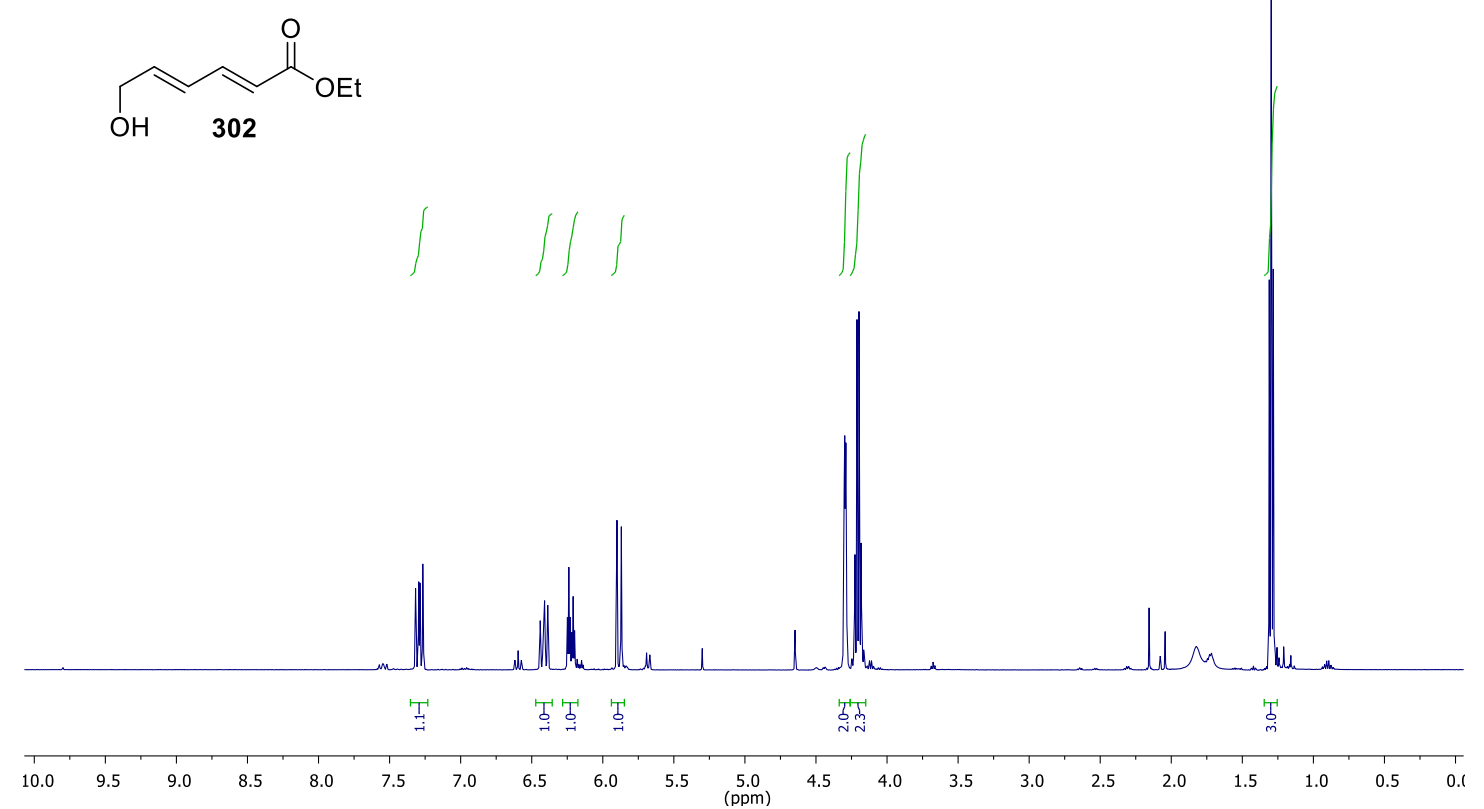

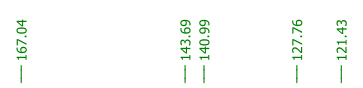

${ }^{13} \mathrm{C}-\mathrm{NMR}\left(125 \mathrm{MHz}, \mathrm{CDCl}_{3}\right)$
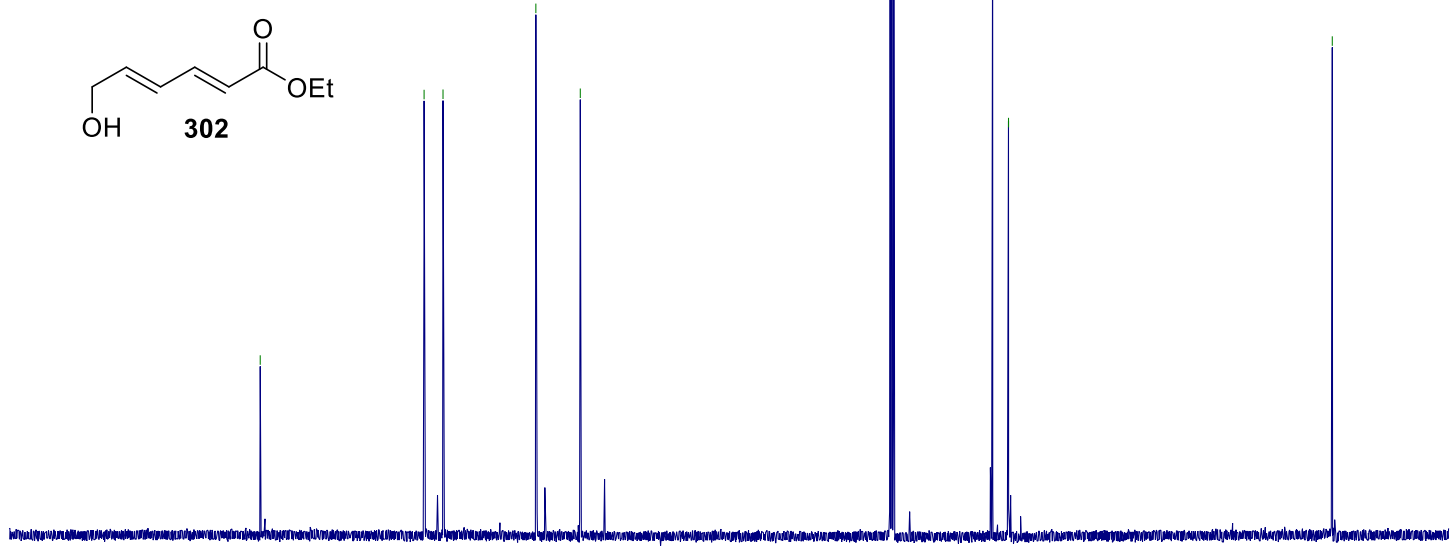

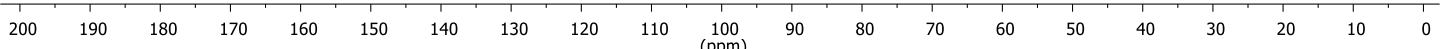


${ }^{1} \mathrm{H}-\mathrm{NMR}\left(500 \mathrm{MHz}, \mathrm{CDCl}_{3}\right)$

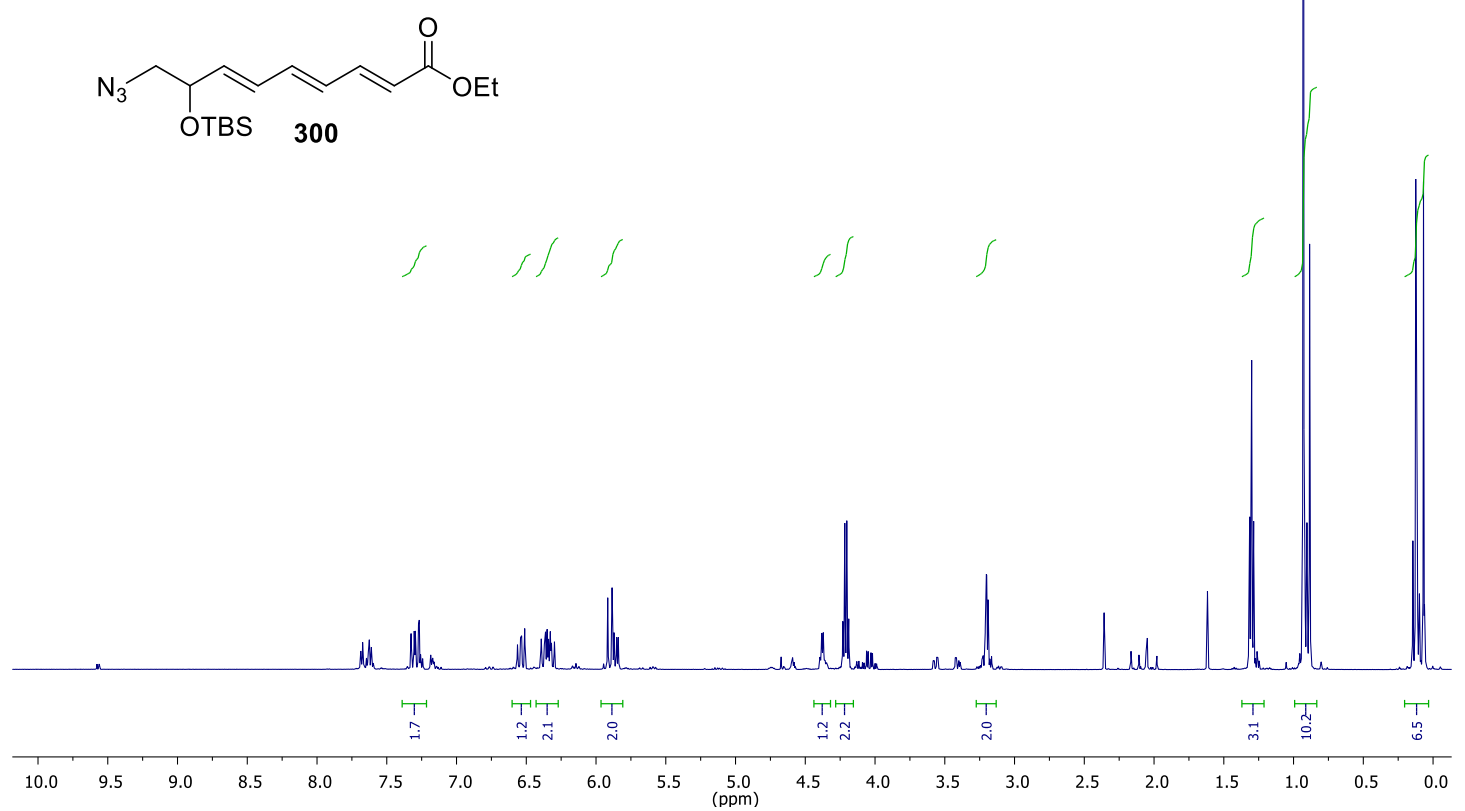

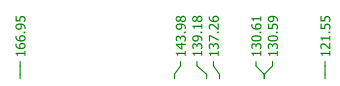

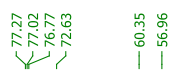

$\stackrel{\substack{0 \\ \infty}}{\substack{0 \\ i}}$

辝

${ }^{13} \mathrm{C}-\mathrm{NMR}\left(125 \mathrm{MHz}, \mathrm{CDCl}_{3}\right.$ )<smiles>CCOC(=O)/C=C/C=C/C=C/C([18OH])CN</smiles>

$\begin{array}{lllllllllll}200 & 190 & 180 & 170 & 160 & 150 & 140 & 130 & 120 & 110 & 100(\mathrm{ppm})\end{array}$ 
${ }^{1} \mathrm{H}-\mathrm{NMR}\left(500 \mathrm{MHz}, \mathrm{CDCl}_{3}\right)$

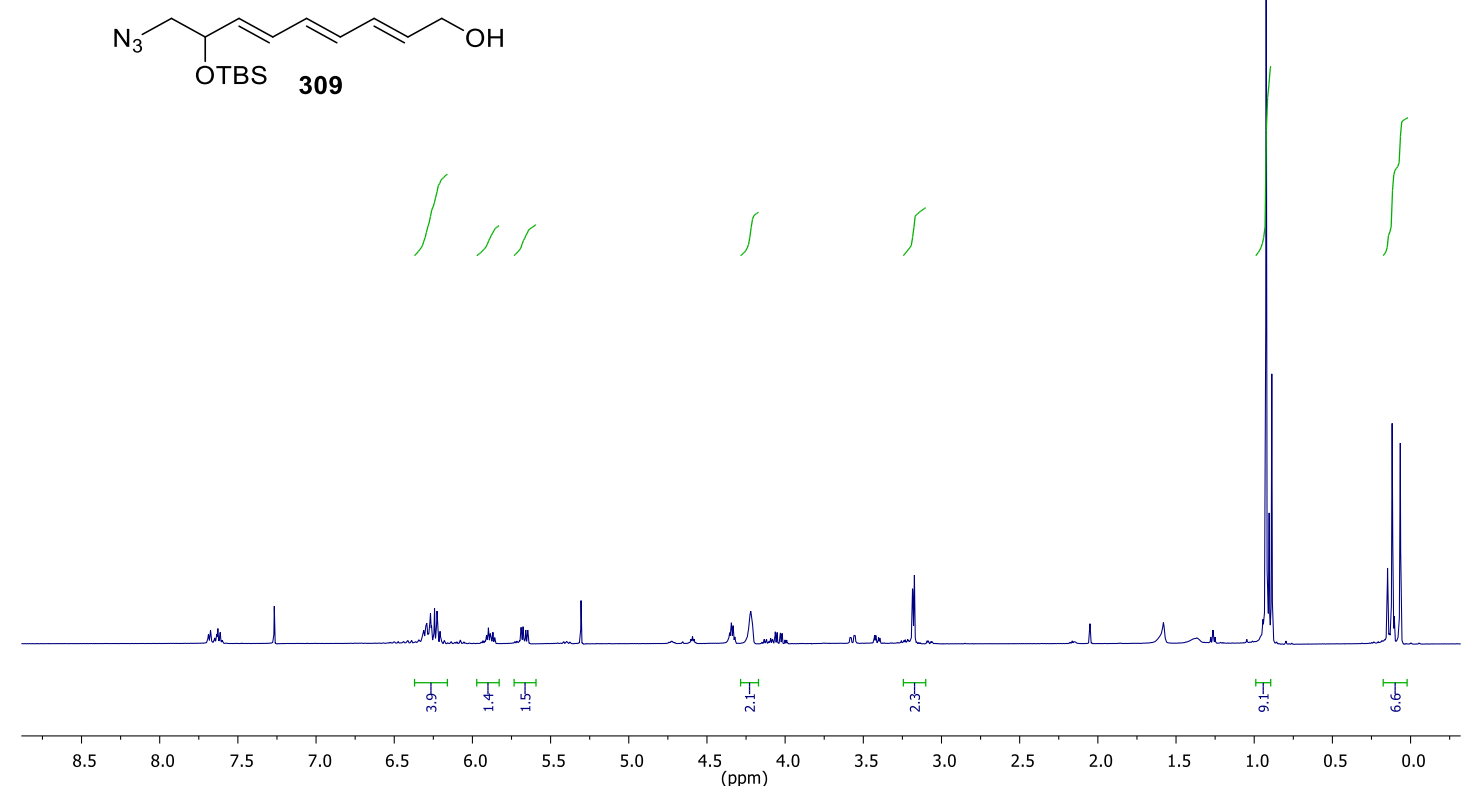

${ }^{13} \mathrm{C}-\mathrm{NMR}\left(125 \mathrm{MHz}, \mathrm{CDCl}_{3}\right)$
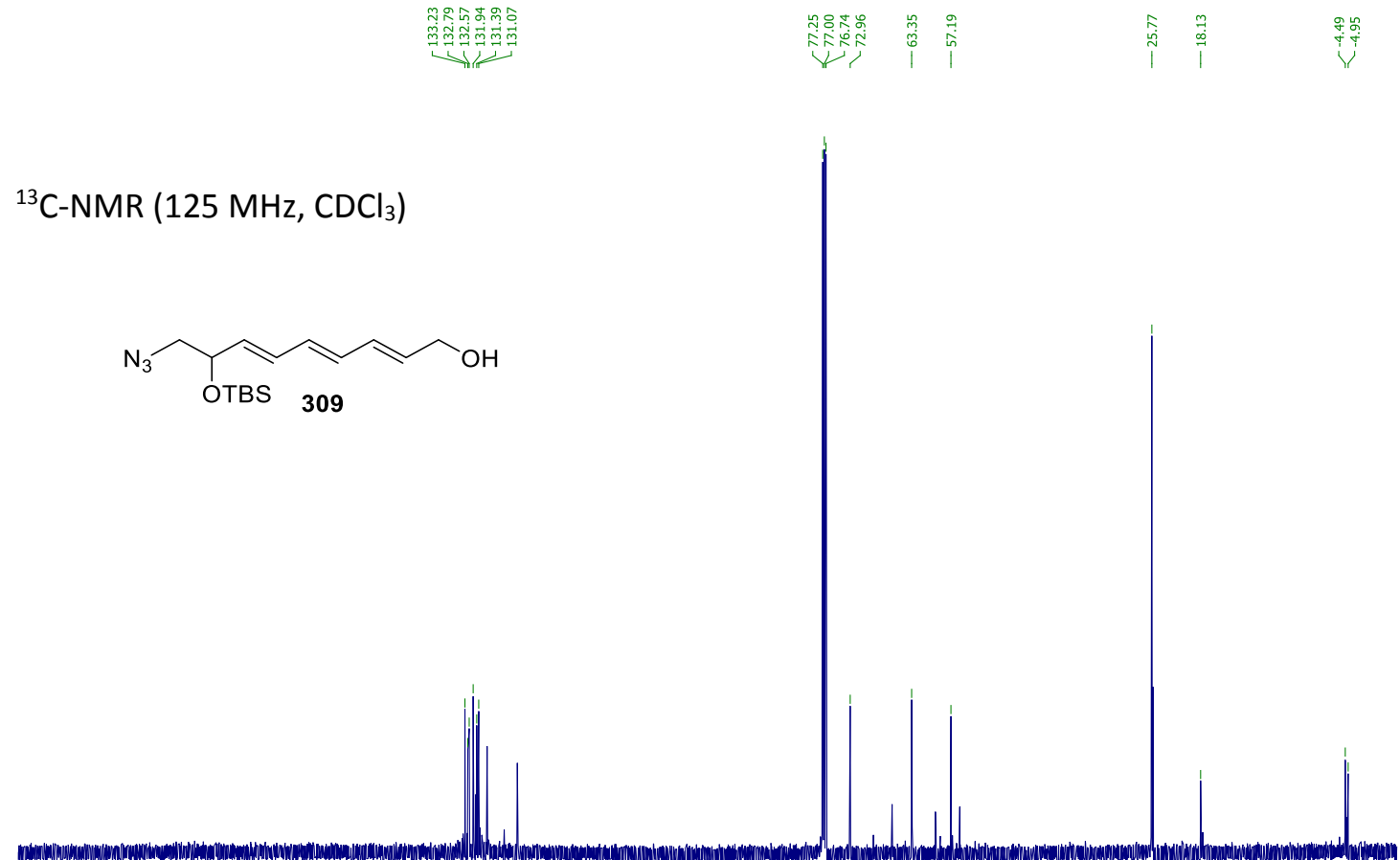

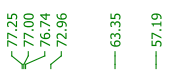

200

$\begin{array}{llllllllll}180 & 170 & 160 & 150 & 140 & 130 & 120 & 110 & 100(\mathrm{ppm})\end{array}$

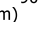


${ }^{1} \mathrm{H}-\mathrm{NMR}\left(500 \mathrm{MHz}, \mathrm{CDCl}_{3}\right)$

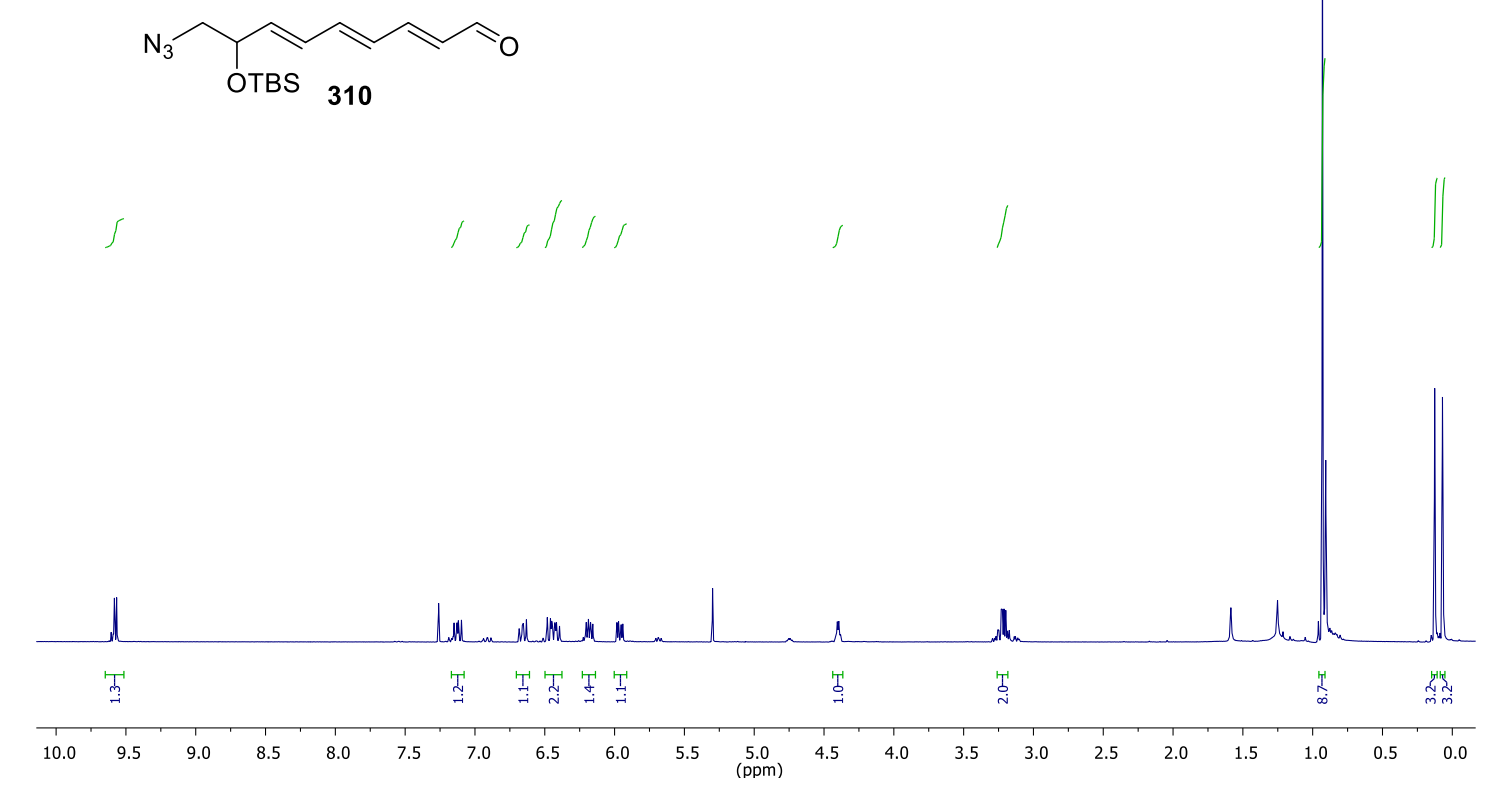

${ }^{13} \mathrm{C}-\mathrm{NMR}\left(125 \mathrm{MHz}, \mathrm{CDCl}_{3}\right)$

$\overbrace{\text { OTBS }} \overbrace{0}$

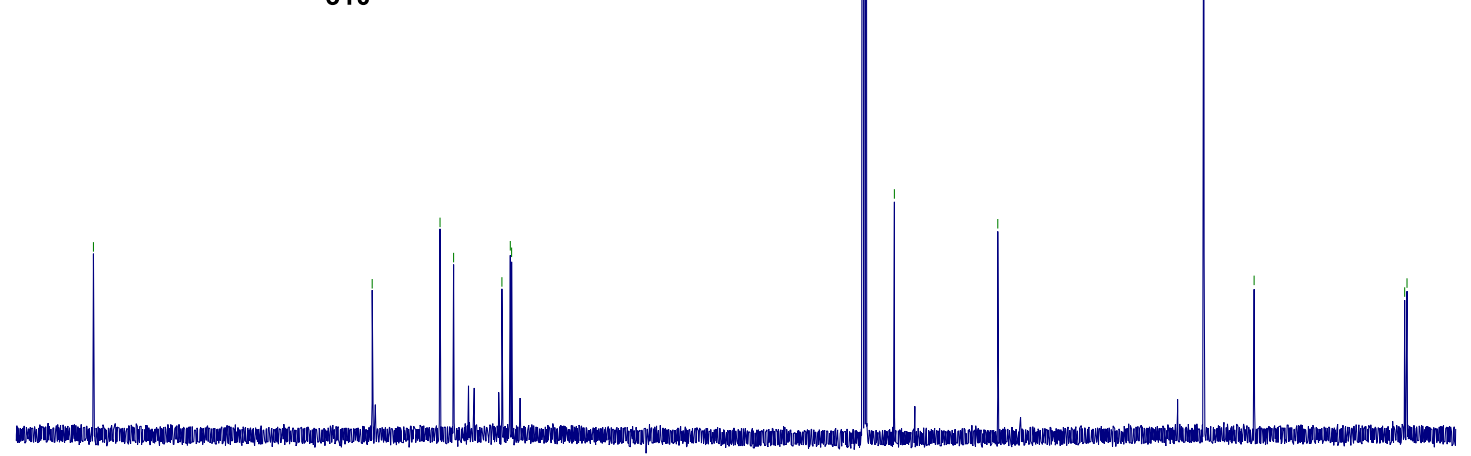

$\begin{array}{llllllllllllllllllllll} & 200 & 190 & 180 & 170 & 160 & 150 & 140 & 130 & 120 & 110 & \underset{(\mathrm{ppm})}{100} & 90 & 70 & 60 & 50 & 40 & 30 & 20 & 10 & 0 & -10\end{array}$ 
${ }^{1} \mathrm{H}-\mathrm{NMR}\left(500 \mathrm{MHz}, \mathrm{CDCl}_{3}\right)$

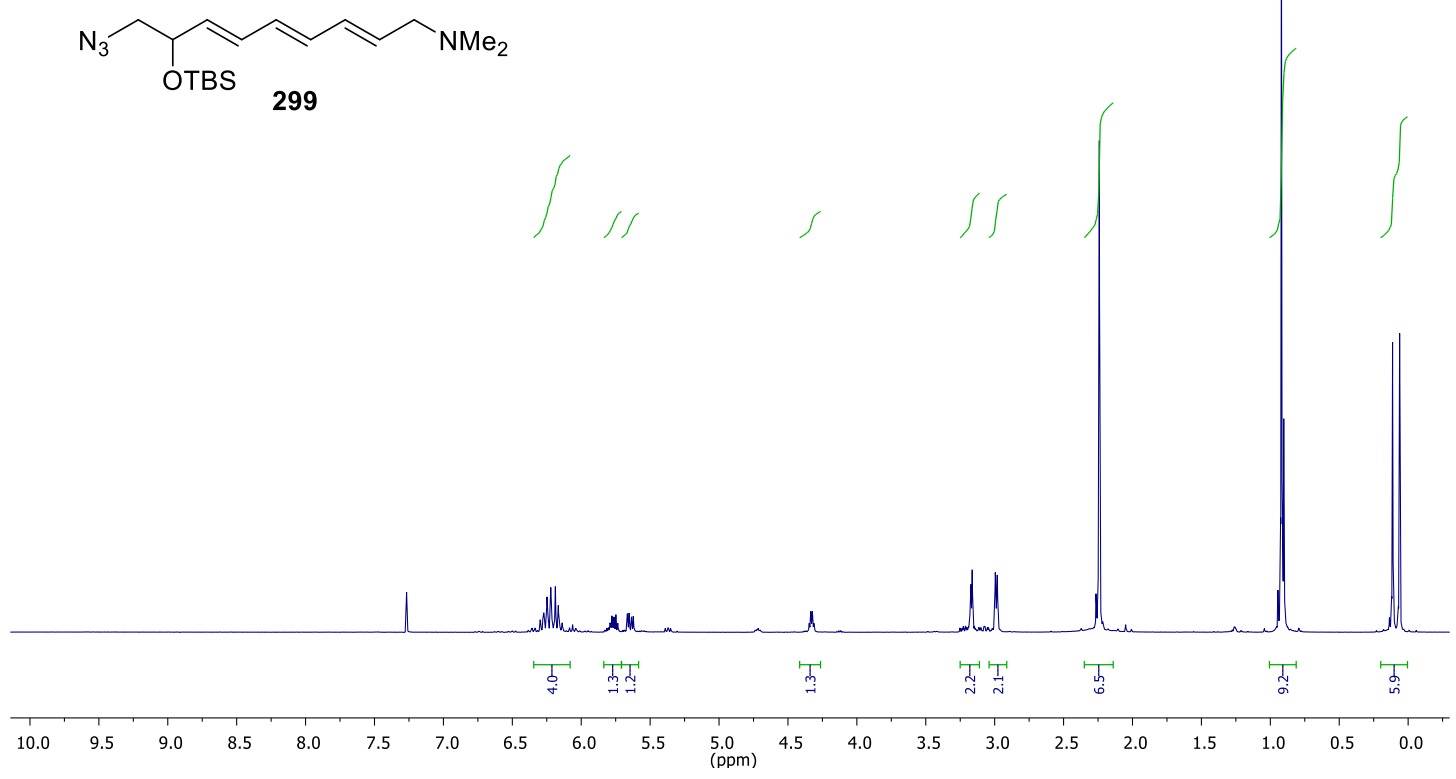

${ }^{13} \mathrm{C}-\mathrm{NMR}\left(125 \mathrm{MHz}, \mathrm{CDCl}_{3}\right.$ )<smiles>CNCC=CC=CC=CC([18OH])CN</smiles>

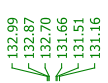

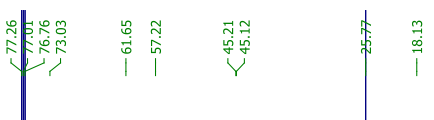


${ }^{1} \mathrm{H}-\mathrm{NMR}\left(500 \mathrm{MHz}, \mathrm{CDCl}_{3}\right.$ )

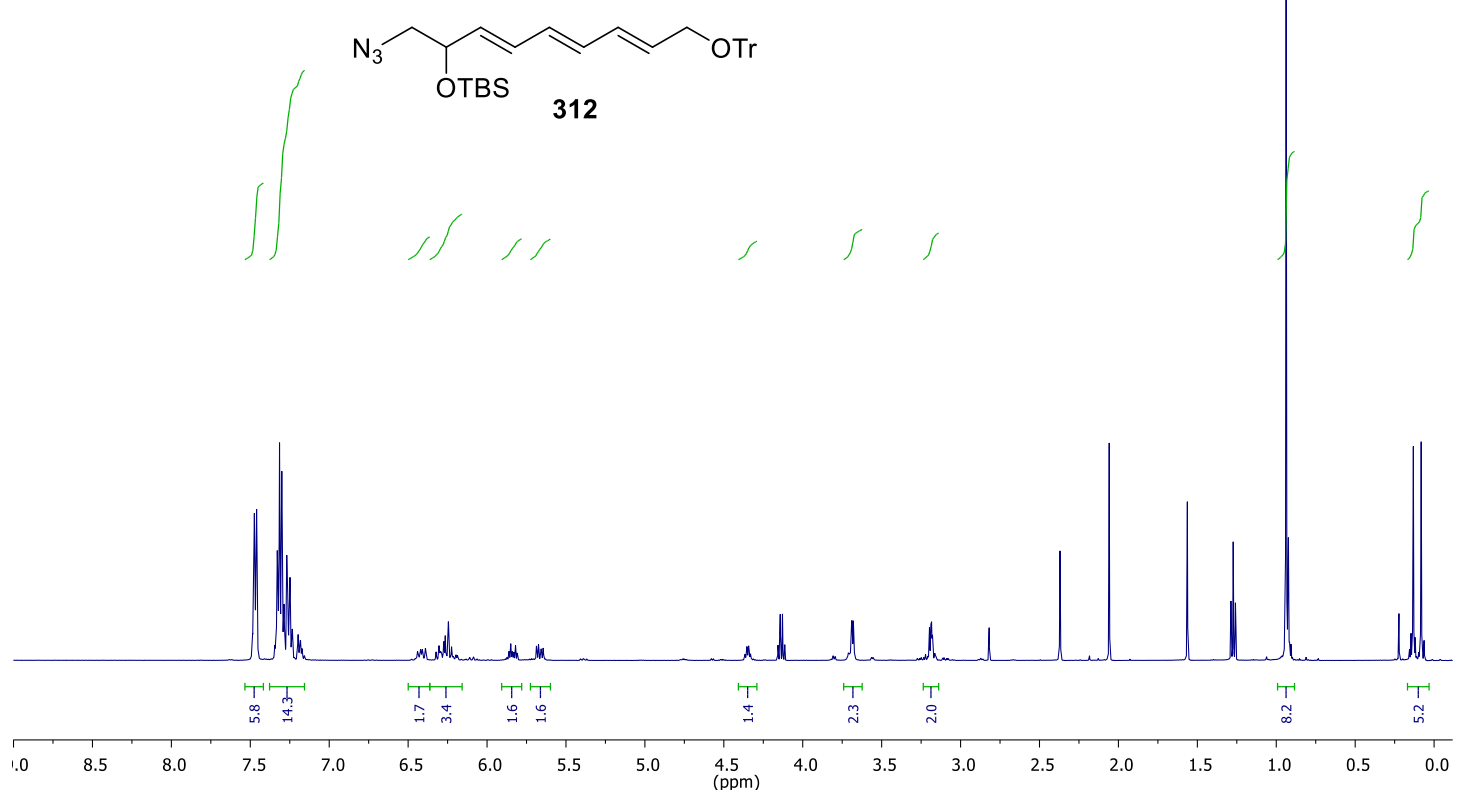

${ }^{13} \mathrm{C}-\mathrm{NMR}\left(125 \mathrm{MHz}, \mathrm{CDCl}_{3}\right)$

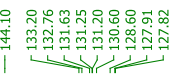

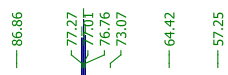

i

OTBS 312

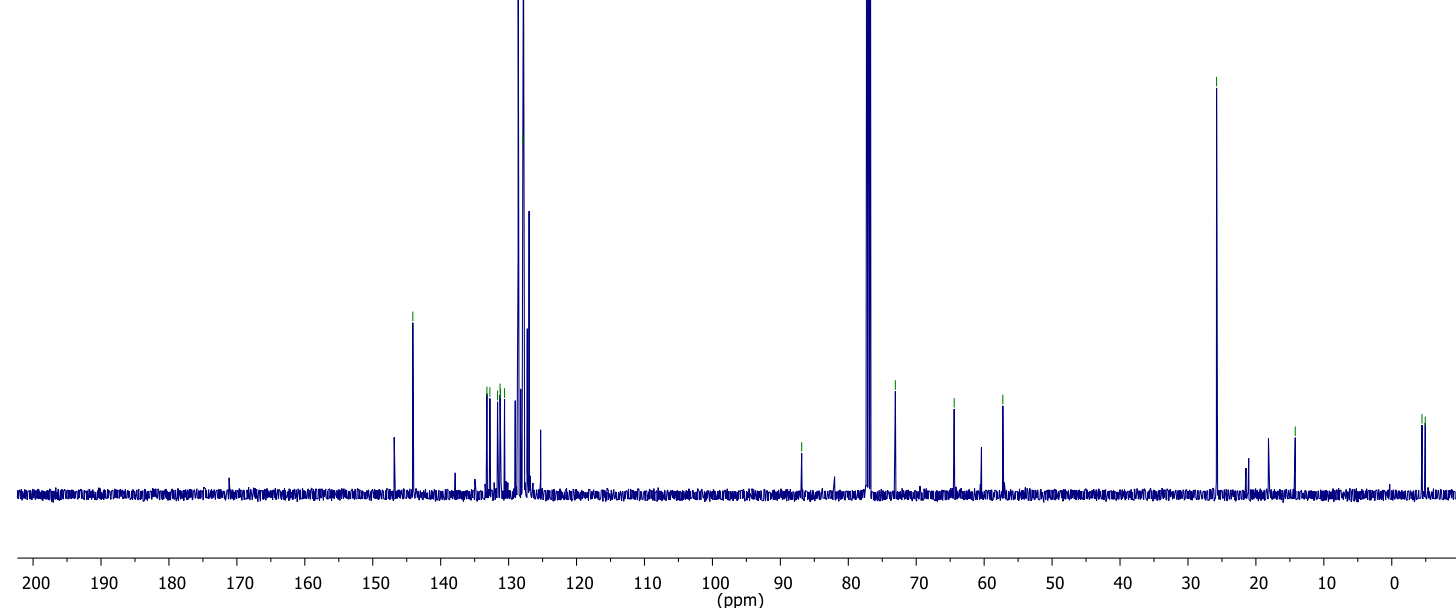


${ }^{1} \mathrm{H}-\mathrm{NMR}\left(500 \mathrm{MHz}, \mathrm{CDCl}_{3}\right)$
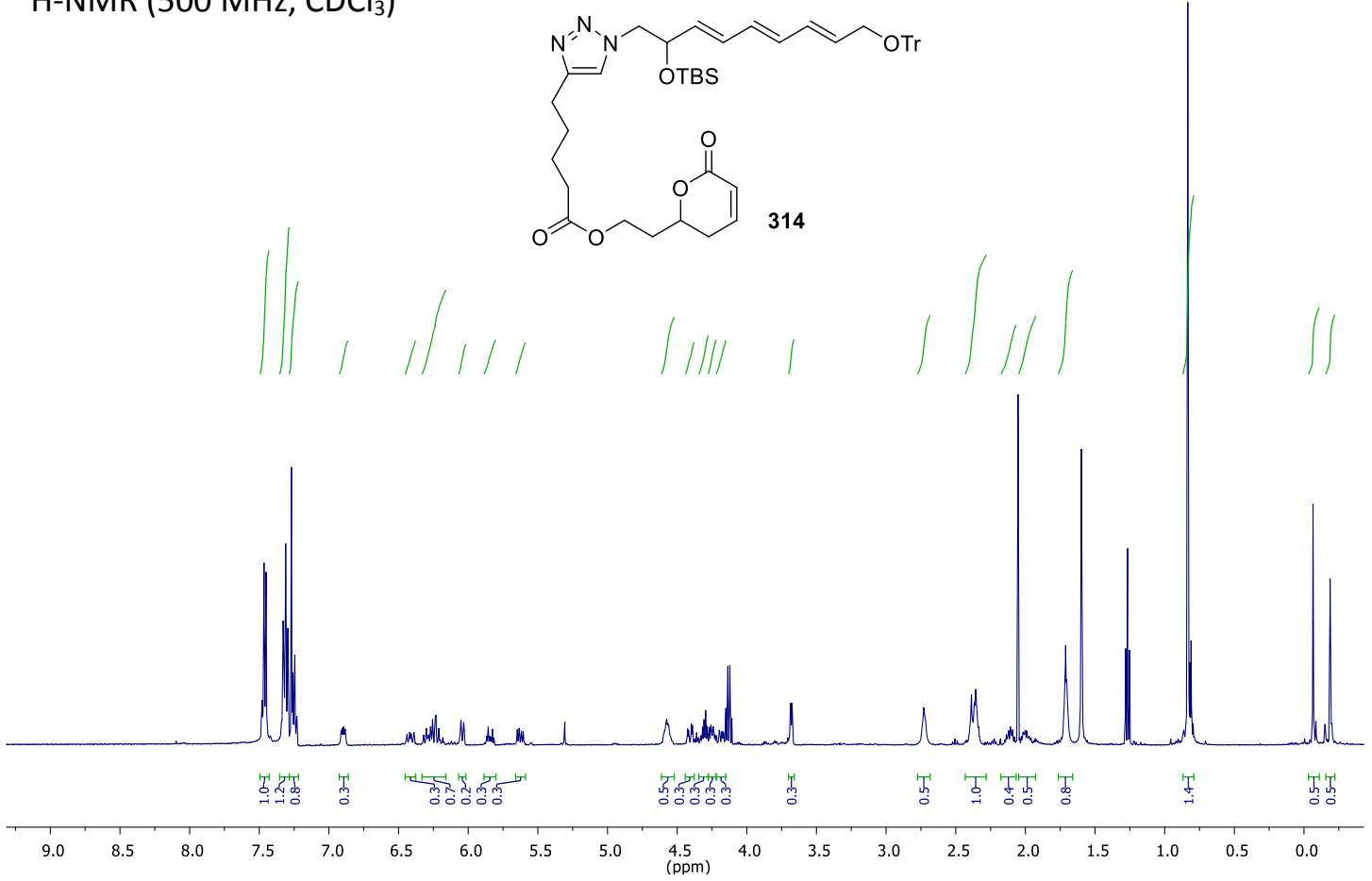

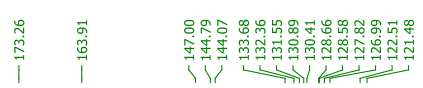

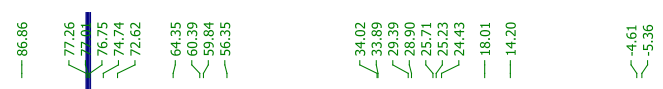

${ }^{13} \mathrm{C}-\mathrm{NMR}\left(125 \mathrm{MHz}, \mathrm{CDCl}_{3}\right.$ )

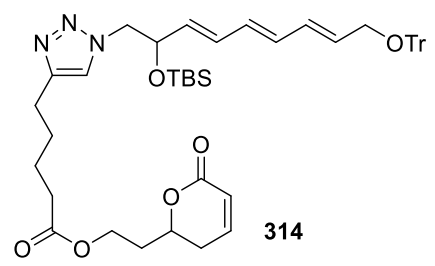

$\begin{array}{lllllllllll}200 & 190 & 180 & 170 & 160 & 150 & 140 & 130 & 120 & 110 & 100 \\ (\mathrm{ppm}) & 90\end{array}$ 
${ }^{1} \mathrm{H}-\mathrm{NMR}\left(500 \mathrm{MHz}, \mathrm{CDCl}_{3}\right)$
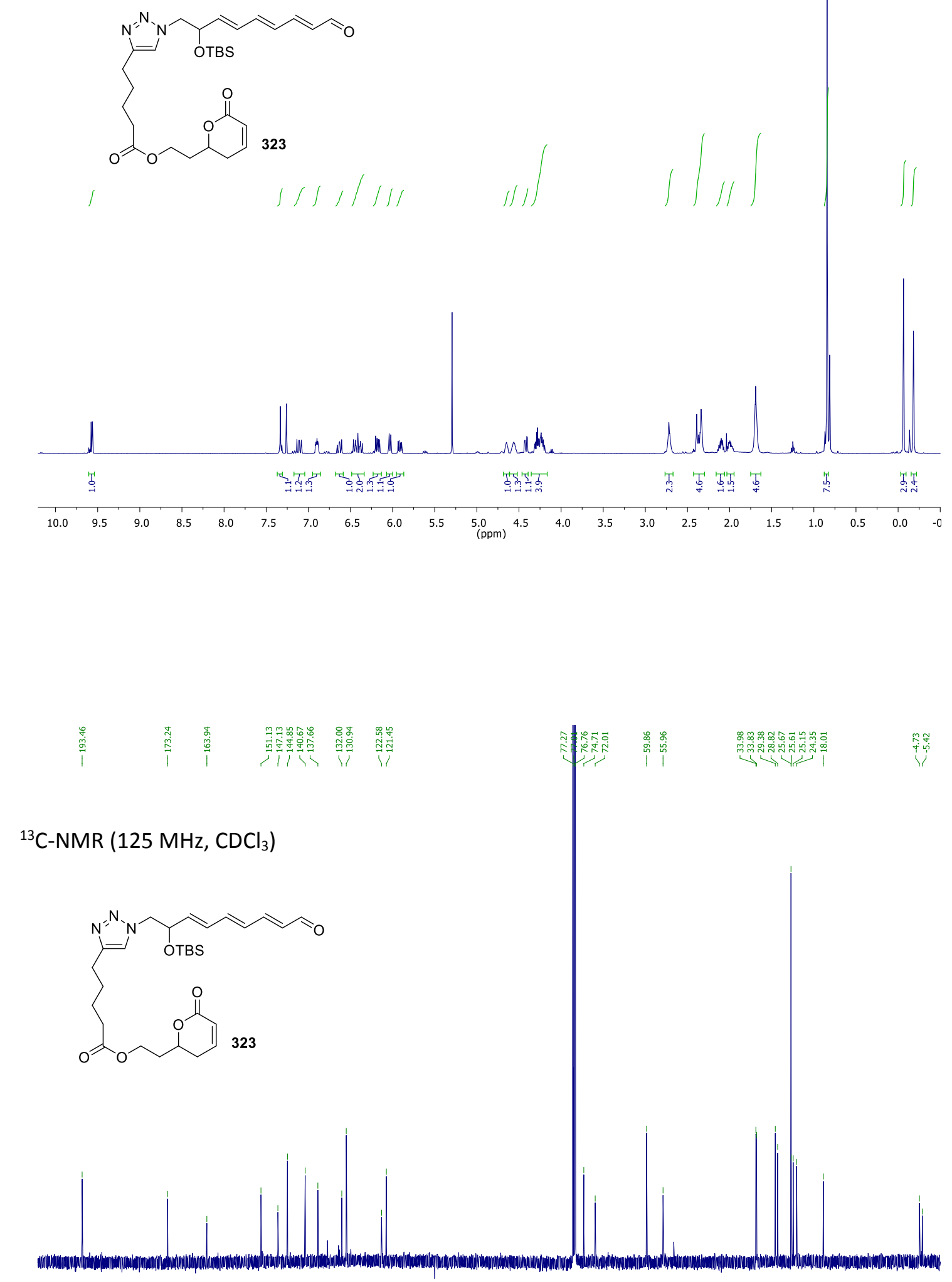

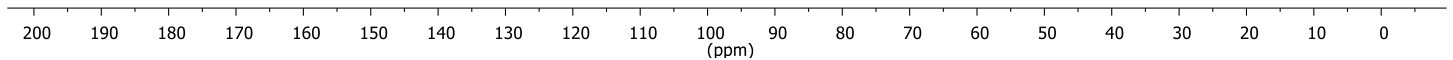


${ }^{1} \mathrm{H}-\mathrm{NMR}\left(500 \mathrm{MHz}, \mathrm{CDCl}_{3}\right)$<smiles>NCC(O)C=CC=CC=CC=O</smiles>
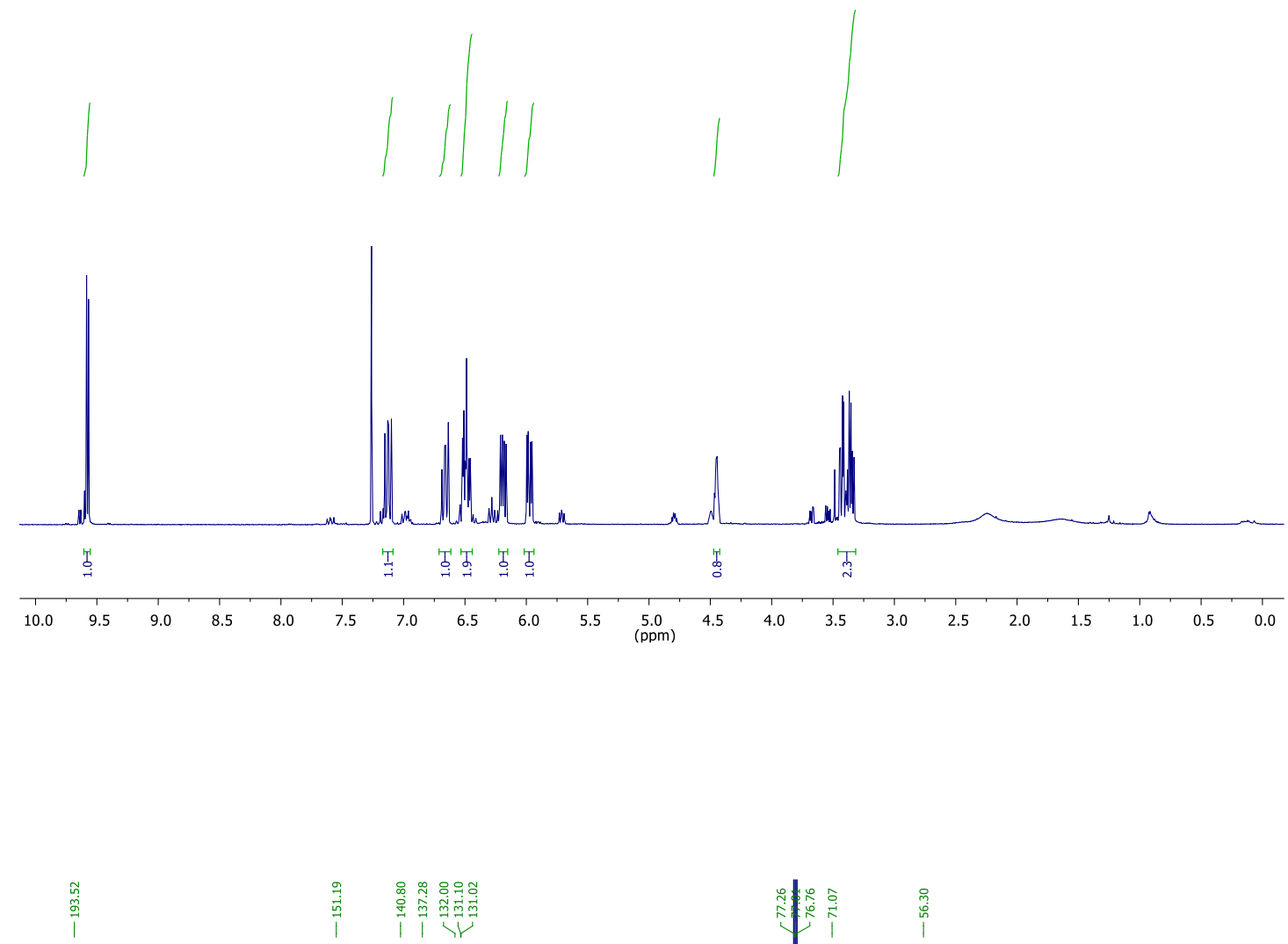

${ }^{13} \mathrm{C}-\mathrm{NMR}\left(125 \mathrm{MHz}, \mathrm{CDCl}_{3}\right)$<smiles>[N]CC(O)/C=C/C=C/C=C/C=O</smiles>

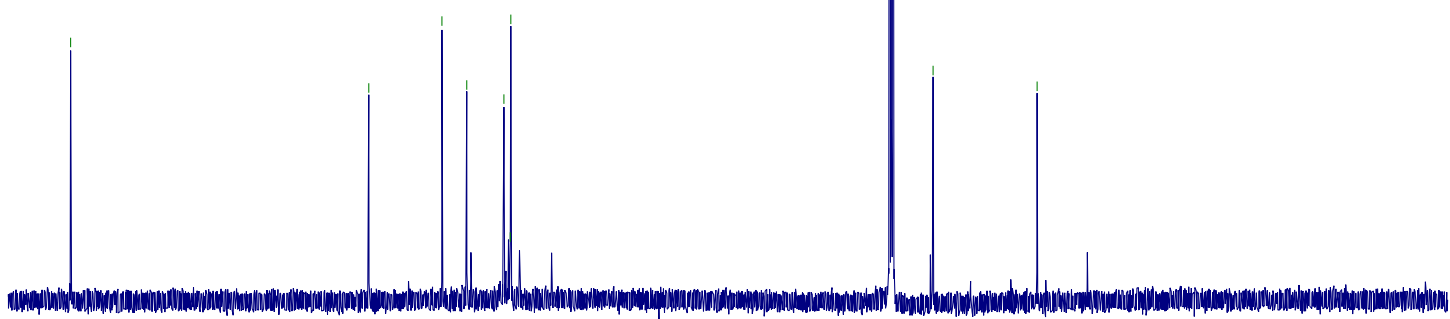

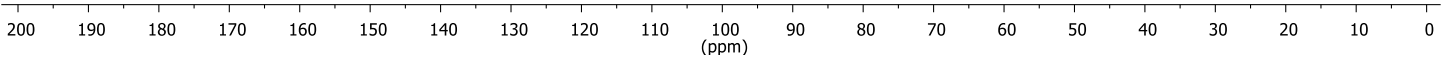


${ }^{1} \mathrm{H}-\mathrm{NMR}\left(500 \mathrm{MHz}, \mathrm{CDCl}_{3}\right)$

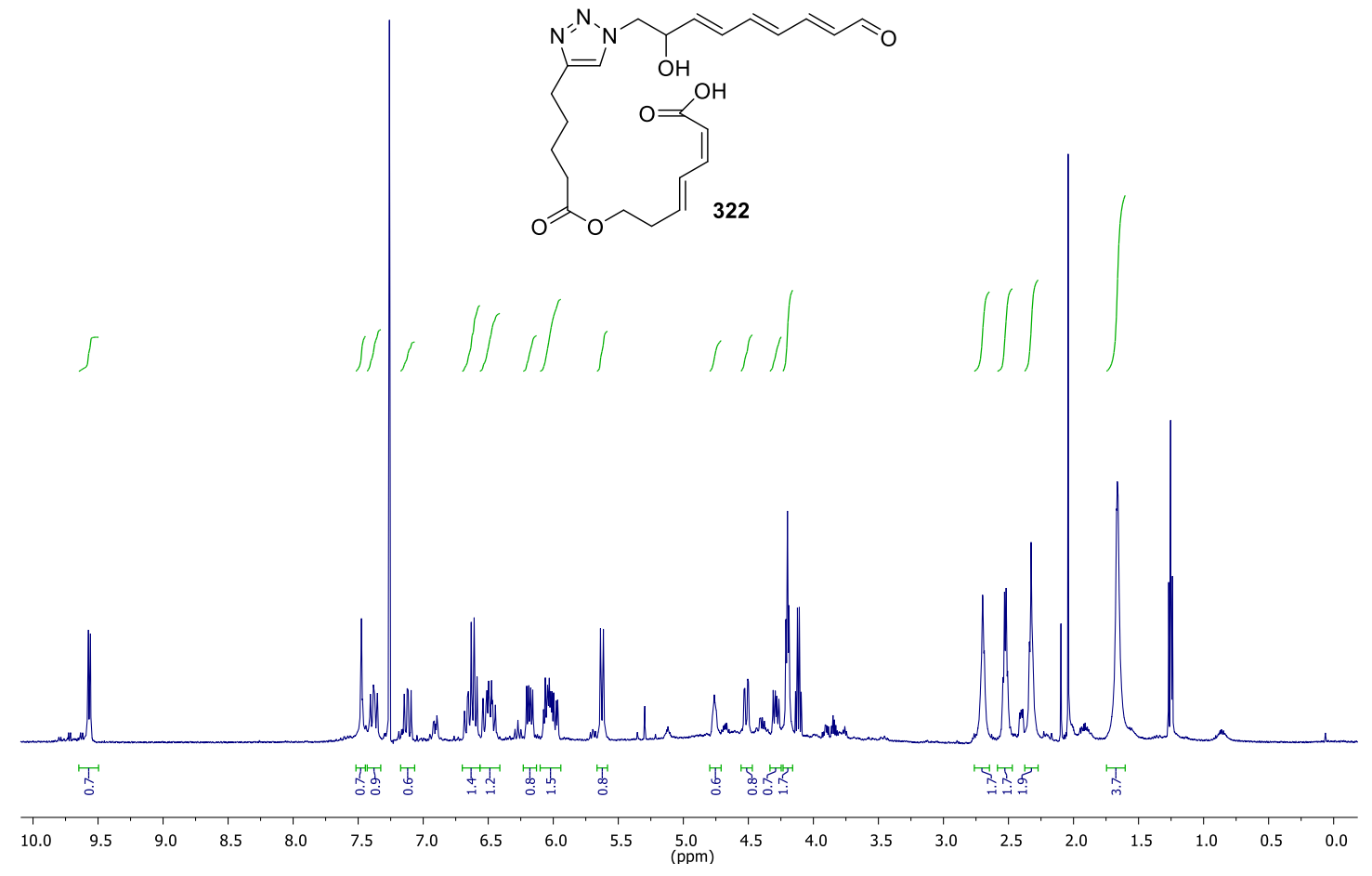

${ }^{13} \mathrm{C}-\mathrm{NMR}\left(125 \mathrm{MHz}, \mathrm{CDCl}_{3}\right.$ )

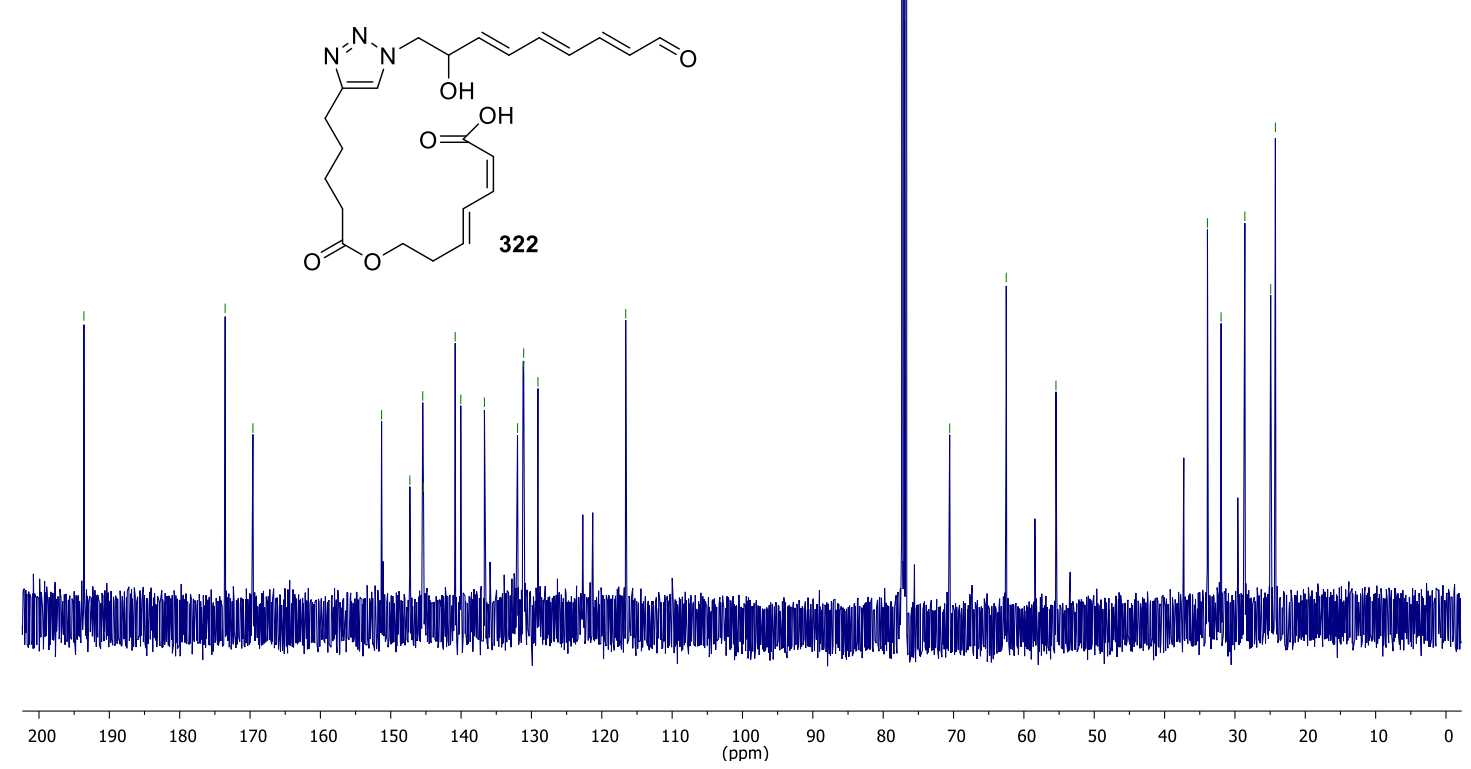



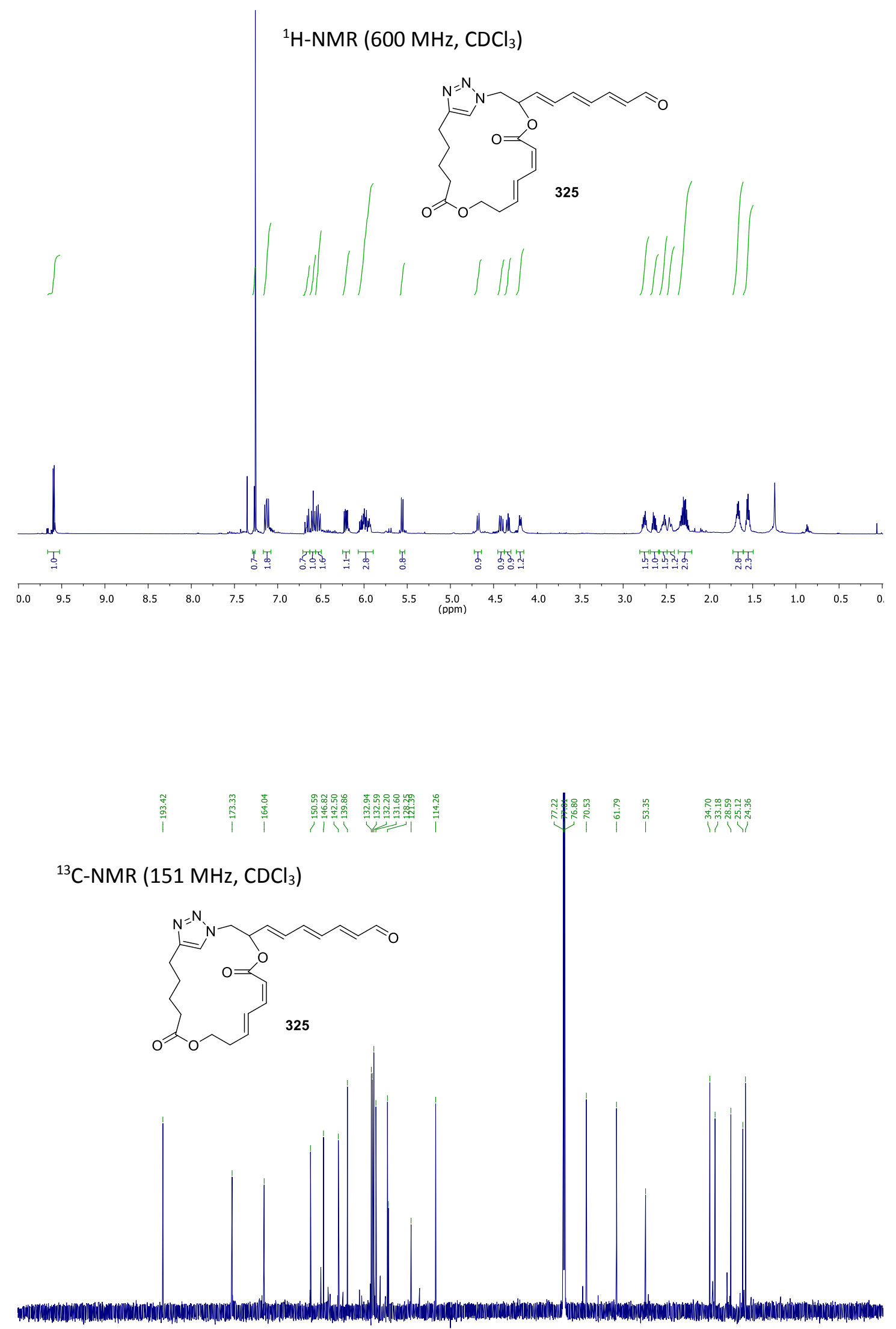

$\begin{array}{llllllllllllllllllllllllllll}230 & 220 & 210 & 200 & 190 & 180 & 170 & 160 & 150 & 140 & 130 & 120 & \begin{array}{l}110 \\ (\mathrm{ppm})\end{array} & 100 & 90 & 80 & 70 & 60 & 50 & 40 & 30 & 20 & 10 & 0 & -10\end{array}$ 
${ }^{1} \mathrm{H}-\mathrm{NMR}\left(500 \mathrm{MHz}, \mathrm{CDCl}_{3}\right)$
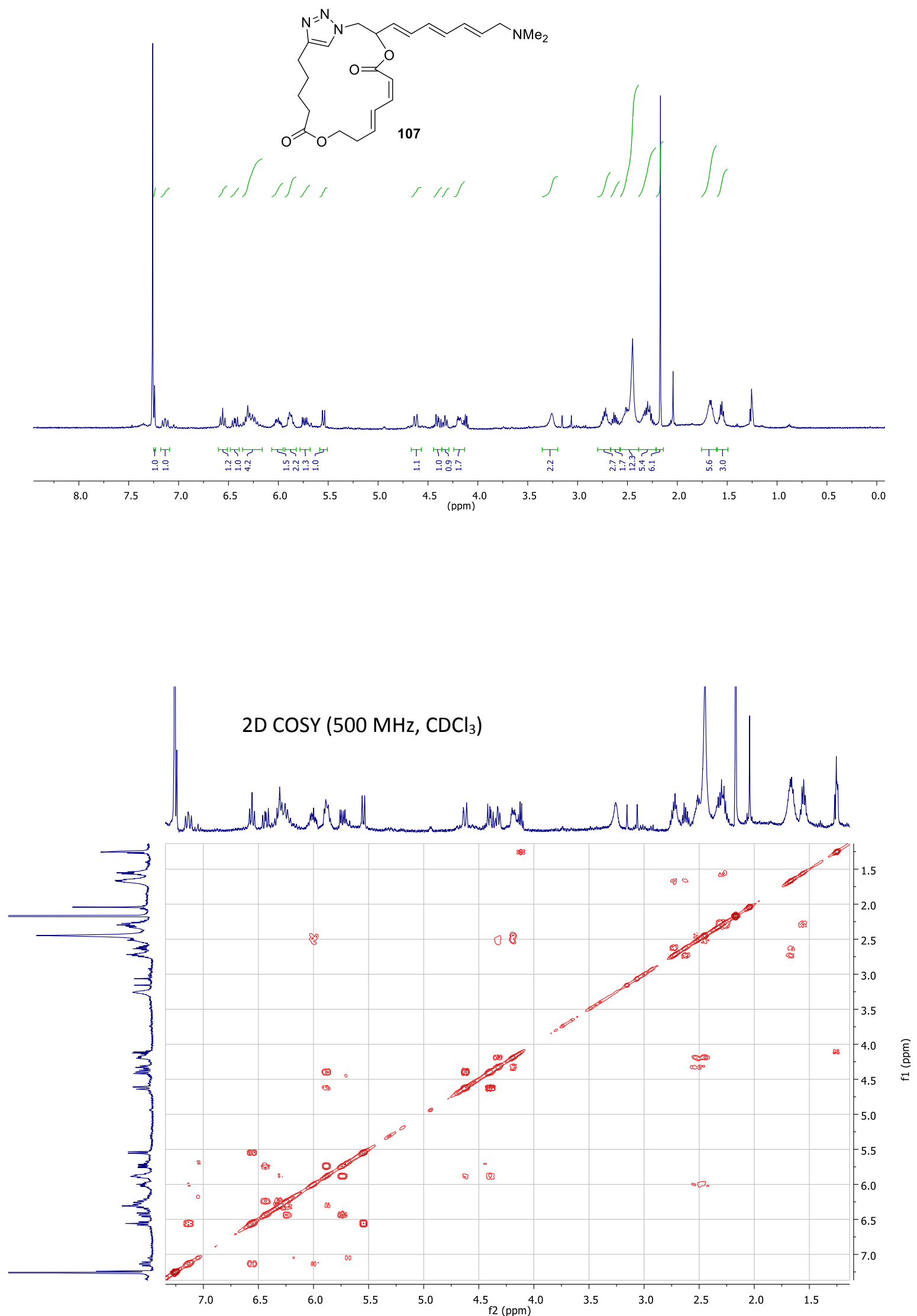


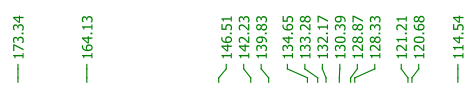

${ }^{13} \mathrm{C}-\mathrm{NMR}\left(151 \mathrm{MHz}, \mathrm{CDCl}_{3}\right.$ )
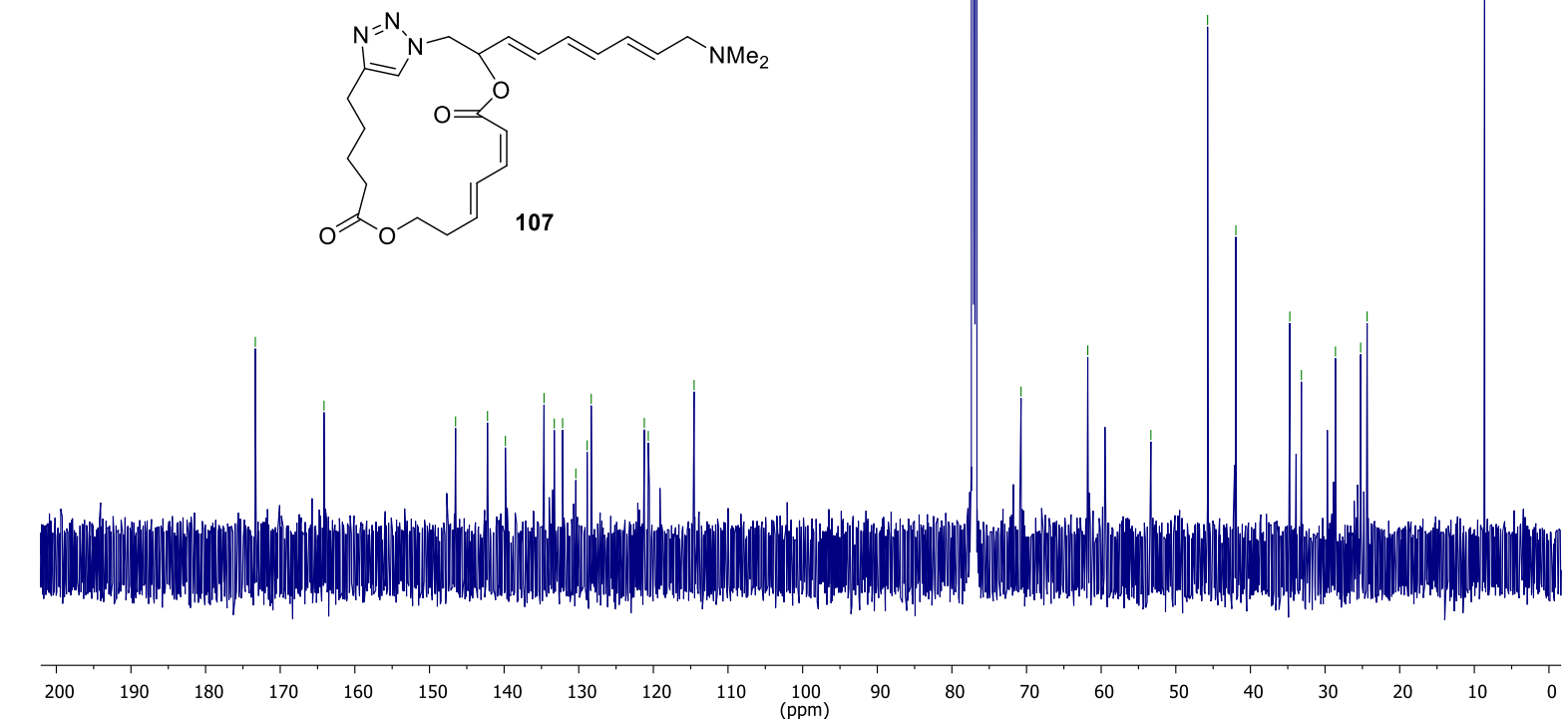

$\begin{array}{lllllllllll}200 & 190 & 180 & 170 & 160 & 150 & 140 & 130 & 120 & 110 & \left.\begin{array}{l}100 \\ (\mathrm{ppm})\end{array}\right)\end{array}$

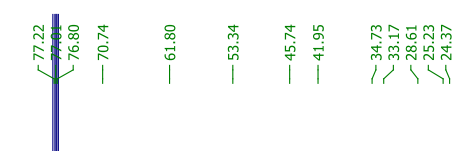


296 


\section{Appendix}

Biological data for derivatisation products of pateamine A

From Joanna's honours report 2002 ${ }^{39}$
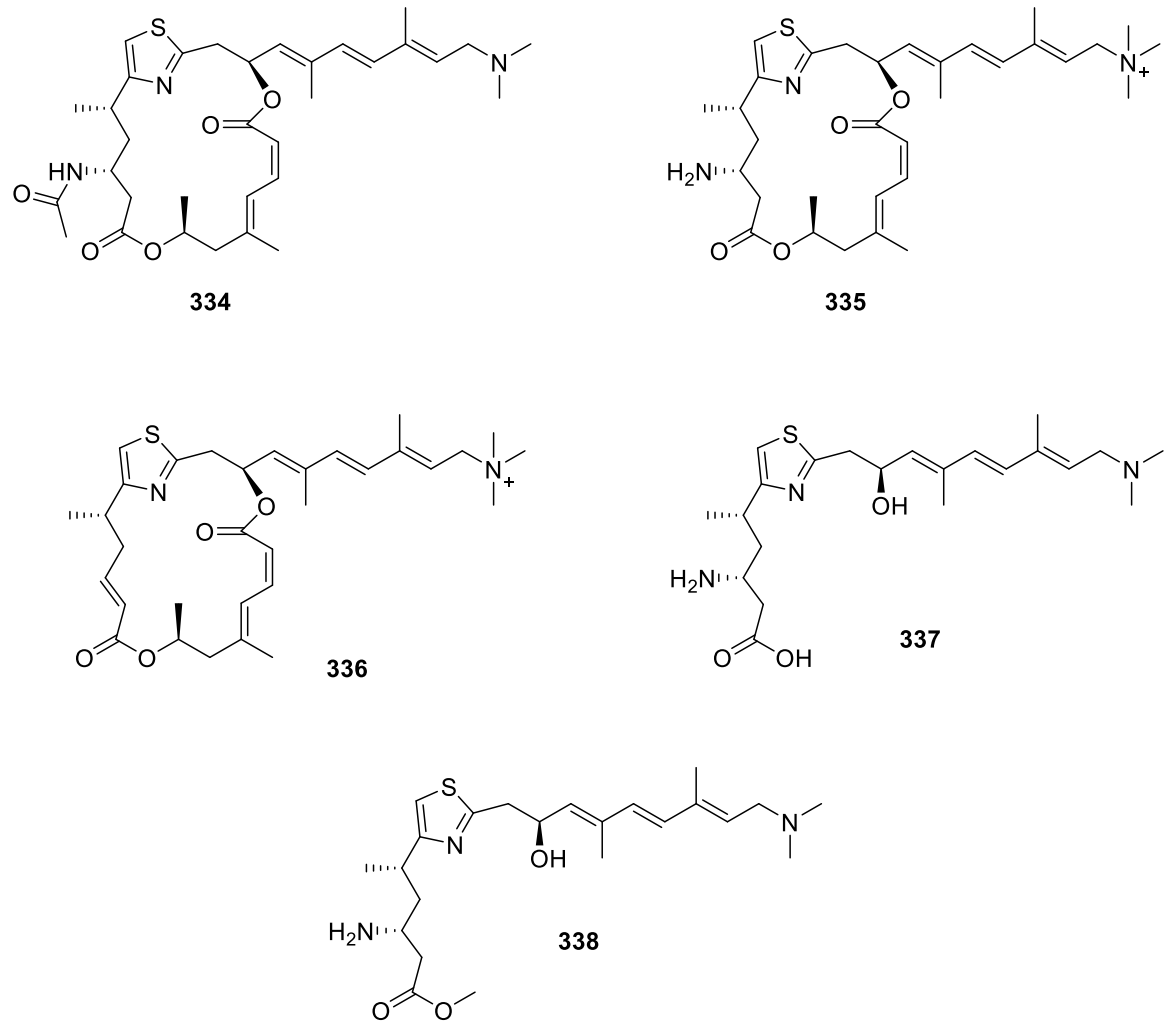

\begin{tabular}{lc}
\hline \multicolumn{1}{c}{ Entry } & IC \\
\hline Pateamine A & $<0.17$ \\
\hline Acetyl- Pateamine (334) & 0.17 \\
\hline Methyl- Pateamine (335) & 141 \\
\hline Eliminated Methyl- Pateamine (336) & 290 \\
\hline Large hydrolysis product (337) & 3332 \\
\hline Methylated hydrolysis product (338) & 307 \\
\hline \multicolumn{2}{c}{ Summary of IC S5 $_{50}$ values for MTT assay in HL-60 cell line. }
\end{tabular}




\section{Bibliography}

1. Singh, S.; Pelaez, F., Biodiversity, chemical diversity and drug discovery. In Natural Compounds as Drugs Volume I, Petersen, F.; Amstutz, R., Eds. Birkhäuser Basel: 2008; Vol. 65, pp 141-174.

2. Newman, D. J.; Cragg, G. M., Natural products as sources of new drugs over the 30 years from 1981 to 2010. J. Nat. Prod. 2012, 75 (3), 311-335.

3. Ortholand, J.-Y.; Ganesan, A., Natural products and combinatorial chemistry: back to the future. Curr. Opin. Chem. Biol. 2004, 8 (3), 271-280.

4. Reymond, J.-L.; van Deursen, R.; Blum, L. C.; Ruddigkeit, L., Chemical space as a source for new drugs. MedChem Comm 2010, 1 (1), 30-38.

5. Lipinski, C. A.; Lombardo, F.; Dominy, B. W.; Feeney, P. J., Experimental and computational approaches to estimate solubility and permeability in drug discovery and development settings. Adv. Drug Deliv. Rev. 1997, 23 (1-3), 3-25.

6. Zhu, F.; Ma, X. H.; Qin, C.; Tao, L.; Liu, X.; Shi, Z.; Zhang, C. L.; Tan, C. Y.; Chen, Y. Z.; Jiang, Y. Y., Drug discovery prospect from untapped species: Indications from approved natural product drugs. PLoS ONE 2012, 7 (7), e39782.

7. Danishefsky, S., On the potential of natural products in the discovery of pharma leads: A case for reassessment. Nat. Prod. Rep. 2010, 27 (8), 1114-1116.

8. Su, D.-S.; Balog, A.; Meng, D.; Bertinato, P.; Danishefsky, S. J.; Zheng, Y.-H.; Chou, T.-C.; He, L.; Horwitz, S. B., Structure-Activity Relationship of the Epothilones and the First In Vivo Comparison with Paclitaxel. Angewandte Chemie International Edition in English 1997, 36 (19), 2093-2096.

9. Nakajima, H.; Sato, B.; Fujita, T.; Takase, S.; Terano, H.; Okuhara, M., New antitumor substances, FR901463, FR901464 and FR901465: I. Taxonomy, fermentation, isolation, physico-chemical properties and biological activities. J. Antibiot. 1996, 49 (12), 1196-1203.

10. Albert, B. J.; Sivaramakrishnan, A.; Naka, T.; Czaicki, N. L.; Koide, K., Total syntheses, fragmentation studies, and antitumor/antiproliferative activities of FR901464 and its low picomolar analogue. J. Am. Chem. Soc. 2007, 129 (9), 26482659 .

11. Wender, P. A.; Verma, V. A.; Paxton, T. J.; Pillow, T. H., Function-oriented synthesis, step economy, and drug design. Acc. Chem. Res. 2008, 41 (1), 40-49.

12. Wall, N. R.; Mohammad, R. M.; Al-Katib, A. M., Bax:Bcl-2 ratio modulation by bryostatin 1 and novel antitubulin agents is important for susceptibility to drug 
induced apoptosis in the human early pre-B acute lymphoblastic leukemia cell line, Reh. Leukemia Res. 1999, 23 (10), 881-888.

13. Spitaler, M.; Utz, I.; Hilbe, W.; Hofmann, J.; Grunicke, H., PKC-independent modulation of multidrug resistance in cells with mutant (V185) but not wild-type (G185) p-glycoprotein by bryostatin 1. Biochem. Pharm. 1998, 56 (7), 861-869.

14. Oz, H. S.; Hughes, W. T.; Rehg, J. E.; Thomas, E. K., Effect of CD40 ligand and other immunomodulators on Pneumocystis carinii infection in rat model. Microb. Pathogenesis 2000, 29 (3), 187-190.

15. Kuzirian, A. M.; Epstein, H. T.; Gagliardi, C. J.; Nelson, T. J.; et al., Bryostatin enhancement of memory in hermissenda. Biol. Bull. 2006, 210 (3), 201-14.

16. Keck, G. E.; Poudel, Y. B.; Cummins, T. J.; Rudra, A.; Covel, J. A., Total synthesis of bryostatin 1. J. Am. Chem. Soc. 2010, 133 (4), 744-747.

17. Wender, P. A.; Baryza, J. L.; Brenner, S. E.; DeChristopher, B. A.; Loy, B. A.; Schrier, A. J.; Verma, V. A., Design, synthesis, and evaluation of potent bryostatin analogs that modulate PKC translocation selectivity. Proc. Natl. Acad. Sci. USA 2011, 108 (17), 6721-6726.

18. Wender, P. A.; Baryza, J. L.; Bennett, C. E.; Bi, F. C.; Brenner, S. E.; Clarke, M. O.; Horan, J. C.; Kan, C.; LacÃ'te, E.; Lippa, B.; Nell, P. G.; Turner, T. M., The practical synthesis of a novel and highly potent analogue of bryostatin. J. Am. Chem. Soc. 2002, 124 (46), 13648-13649.

19. Wender, P. A.; Hilinski, M. K.; Soldermann, N.; Mooberry, S. L., Total synthesis and biological evaluation of 11-desmethyllaulimalide, a highly potent simplified laulimalide analogue. Org. Lett. 2006, 8 (7), 1507-1510.

20. Mori, K.; Kuwahara, S., Synthesis of optically active forms of (E)-6-isopropyl-3,9dimethyl-5,8-decadienyl acetate, the pheromone of the yellow scale. Tetrahedron 1982, $38(4), 521-525$.

21. Wender, P. A.; Hegde, S. G.; Hubbard, R. D.; Zhang, L., Total synthesis of (-)laulimalide. J. Am. Chem. Soc. 2002, 124 (18), 4956-4957.

22. Copeland, R. A., Enzyme reaction mechanisms. In Evaluation of Enzyme Inhibitors in Drug Discovery, John Wiley \& Sons, Inc.: 2013; pp 25-55.

23. Shin, Y.; Fournier, J. H.; Balachandran, R.; Madiraju, C.; Raccor, B. S.; Zhu, G.; Edler, M. C.; Hamel, E.; Day, B. W.; Curran, D. P., Synthesis and biological evaluation of (-)-16-normethyldictyostatin: A potent analogue of (-)-dictyostatin. Org. Lett. 2005, 7 (14), 2873-2876.

24. Smith III, A. B.; Freeze, B. S., (+)-Discodermolide: total synthesis, construction of novel analogues, and biological evaluation. Tetrahedron 2008, 64 (2), 261-298. 
25. Northcote, P. T.; Blunt, J. W.; Munro, M. H. G., Pateamine: A potent cytotoxin from the New Zealand marine sponge, Mycale sp. Tetrahedron Lett. 1991, 32 (44), 6411-6414.

26. Page, M.; West, L.; Northcote, P.; Battershill, C.; Kelly, M., Spatial and temporal variability of cytotoxic metabolites in populations of the New Zealand sponge Mycale hentscheli. J. Chem. Ecol. 2005, 31 (5), 1161-1174.

27. Northcote, P. T., Personal communication. Victoria University of Wellington. 2012.

28. Hood, K. A.; West, L. M.; Northcote, P. T.; Berridge, M. V.; Miller, J. H., Induction of apoptosis by the marine sponge (Mycale) metabolites, mycalamide $\mathrm{A}$ and pateamine. Apoptosis 2001, 6 (3), 207-219.

29. Clardy, J., Stopping trouble before it starts. ACS Chem. Biol. 2006, 1 (1), 17-19.

30. Romo, D.; Rzasa, R. M.; Shea, H. A.; Park, K.; Langenhan, J. M.; Sun, L.; Akhiezer, A.; Liu, J. O., Total synthesis and immunosuppressive activity of (-)-pateamine A and related compounds: Implementation of a $\beta$-lactam-based macrocyclization. J. Am. Chem. Soc. 1998, 120 (47), 12237-12254.

31. Rzasa, R. M.; Romo, D.; Stirling, D. J.; Blunt, J. W.; Munro, M. H. G., Structural and synthetic studies of the pateamines: Synthesis and absolute configuration of the hydroxydienoate fragment. Tetrahedron Lett. 1995, 36 (30), 5307-5310.

32. Aguilar, E.; Meyers, A. I., Reinvestigation of a modified Hantzsch thiazole synthesis. Tetrahedron Lett. 1994, 35 (16), 2473-2476.

33. Nicolas, E.; Russell, K. C.; Knollenberg, J.; Hruby, V. J., Efficient method for the total asymmetric-synthesis of the isomers of beta-methyltyrosine. J. Org. Chem. 1993, 58 (26), 7565-7571.

34. Aksela, R.; Oehlschlager, A. C., Stannylmetallation of conjugated enynes. Tetrahedron 1991, 47 (7), 1163-1176.

35. Palomo, C.; Aizpurua, J. M.; Cuevas, C.; Mielgo, A.; Galarza, R., A mild method for the alcoholysis of beta-lactams. Tetrahedron Lett. 1995, 36 (49), 9027-9030.

36. Critcher, D. J.; Pattenden, G., Synthetic studies towards pateamine, a novel thiazole-based 19-membered bis-lactone from Mycale sp. Tetrahedron Lett. 1996, 37 (50), 9107-9110.

37. Remuinan, M. J.; Pattenden, G., Total synthesis of (-)-pateamine, a novel polyene bis-macrolide with immunosuppressive activity from the sponge Mycale sp. Tetrahedron Lett. 2000, 41 (38), 7367-7371. 
38. Romo, D.; Choi, N. S.; Li, S.; Buchler, I.; Shi, Z.; Liu, J. O., Evidence for separate binding and scaffolding domains in the immunosuppressive and antitumor marine natural product, pateamine A: Design, synthesis, and activity studies leading to a potent simplified derivative. J. Am. Chem. Soc. 2004, 126 (34), 1058210588 .

39. Wojnar, J. M. A study of the structure-activity relationship of pateamine. Honours report, Victoria University of Wellington, 2002.

40. Bordeleau, M. E.; Matthews, J.; Wojnar, J. M.; Lindqvist, L.; Novac, O.; Jankowsky, E.; Sonenberg, N.; Northcote, P.; Teesdale-Spittle, P.; Pelletier, J., Stimulation of mammalian translation initiation factor eIF4A activity by a small molecule inhibitor of eukaryotic translation. Proc. Natl. Acad. Sci. USA 2005, 102 (30), 10460-10465.

41. Low, W. K.; Dang, Y.; Schneider-Poetsch, T.; Shi, Z.; Choi, N. S.; Merrick, W. C.; Romo, D.; Liu, J. O., Inhibition of eukaryotic translation initiation by the marine natural product pateamine A. Mol. Cell 2005, 20 (5), 709-722.

42. Rogers, G. W.; Komar, A. A.; Merrick, W. C., elF4A: The godfather of the DEAD box helicases. Prog. Nucleic Acid Res. Mol. Biol. 2002, 72, 307-331.

43. Yoderhill, J.; Pause, A.; Sonenberg, N.; Merrick, W. C., The p46 subunit of eukaryotic initiation-factor (eIF)-4F exchanges with eIF-4A. J. Biol. Chem. 1993, 268 (8), 5566-5573.

44. Li, Q. Y.; Imataka, H.; Morino, S.; Rogers, G. W.; Richter-Cook, N. J.; Merrick, W. C.; Sonenberg, N., Eukaryotic translation initiation factor 4AIII (eIF4AIII) is functionally distinct from eIF4AI and eIF4AII. Mol. Cell. Biol. 1999, 19 (11), 73367346.

45. Caruthers, J. M.; Johnson, E. R.; McKay, D. B., Crystal structure of yeast initiation factor 4A, a DEAD-box RNA helicase. Proc. Natl. Acad. Sci. USA 2000, 97 (24), 13080-13085.

46. Nielsen, K. H.; Chamieh, H.; Andersen, C. B. F.; Fredslund, F.; Hamborg, K.; Le Hir, H.; Andersen, G. R., Mechanism of ATP turnover inhibition in the EJC. RNA 2009, 15 (1), 67-75.

47. Gingras, A. C.; Raught, B.; Sonenberg, N., eIF4 initiation factors: Effectors of mRNA recruitment to ribosomes and regulators of translation. Annu. Rev. Biochem. 1999, 68, 913-963.

48. Korneeva, N. L.; First, E. A.; Benoit, C. A.; Rhoads, R. E., Interaction between the $\mathrm{NH}_{2}$-terminal domain of eIF4A and the central domain of eIF4G modulates RNAstimulated ATPase activity. J. Biol. Chem. 2005, 280 (3), 1872-1881. 
49. Bordeleau, M. E.; Cencic, R.; Lindqvist, L.; Oberer, M.; Northcote, P.; Wagner, G.; Pelletier, J., RNA-mediated sequestration of the RNA helicase eIF4A by pateamine A inhibits translation initiation. Chem. Biol. 2006, 13 (12), 1287-1295.

50. Chang, Y.-F.; Imam, J. S.; Wilkinson, M. F., The nonsense-mediated decay RNA surveillance pathway. Annu. Rev. Biochem. 2007, 76 (1), 51-74.

51. Le Hir, H.; Izaurralde, E.; Maquat, L. E.; Moore, M. J., The spliceosome deposits multiple proteins 20-24 nucleotides upstream of mRNA exon-exon junctions. EMBO J 2000, 19 (24), 6860-6869.

52. Le Hir, H.; Gatfield, D.; Izaurralde, E.; Moore, M. J., The exon-exon junction complex provides a binding platform for factors involved in mRNA export and nonsense-mediated mRNA decay. EMBO J 2001, 20 (17), 4987-4997.

53. Maquat, L. E., Nonsense-mediated mRNA decay: splicing, translation and mRNP dynamics. Nat. Rev. Mol. Cell Biol. 2004, 5 (2), 89-99.

54. Ishigaki, Y.; Li, X.; Serin, G.; Maquat, L. E., Evidence for a pioneer round of mRNA translation: mRNAs subject to nonsense-mediated decay in mammalian cells are bound by CBP80 and CBP20. Cell 2001, 106 (5), 607-617.

55. Behm-Ansmant, I.; Kashima, I.; Rehwinkel, J.; Sauliere, J.; Wittkopp, N.; Izaurralde, E., mRNA quality control: An ancient machinery recognizes and degrades mRNAs with nonsense codons. FEBS Lett. 2007, 581, 2845-2853.

56. Longman, D.; Plasterk, R. H. A.; Johnstone, I. L.; Caceres, J. F., Mechanistic insights and identification of two novel factors in the C. elegans NMD pathway. Genes Dev. 2007, 21 (9), 1075-1085.

57. Bono, F.; Ebert, J.; Lorentzen, E.; Conti, E., The crystal structure of the exon junction complex reveals how it maintains a stable grip on mRNA. Cell 2006, 126 (4), 713-725.

58. Dang, Y. J.; Kedersha, N.; Low, W. K.; Romo, D.; Gorospe, M.; Kaufman, R.; Anderson, P.; Liu, J. O., Eukaryotic initiation factor 2 alpha-independent pathway of stress granule induction by the natural product pateamine A. J. Biol. Chem. 2006, 281 (43), 32870-32878.

59. Di Marco, S.; Cammas, A.; Lian, X. J.; Kovacs, E. N.; Ma, J. F.; Hall, D. T.; Mazroui, R.; Richardson, J.; Pelletier, J.; Gallouzi, I. E., The translation inhibitor pateamine A prevents cachexia-induced muscle wasting in mice. Nat Commun 2012, 3, 896.

60. Kuznetsov, G.; Xu, Q.; Rudolph-Owen, L.; TenDyke, K.; Liu, J.; Towle, M.; Zhao, N.; Marsh, J.; Agoulnik, S.; Twine, N.; Parent, L.; Chen, Z.; Shie, J. L.; Jiang, Y.; Zhang, H.; Du, H.; Boivin, R.; Wang, Y.; Romo, D.; Littlefield, B. A., Potent in vitro and in vivo anticancer activities of des-methyl, des-amino pateamine $\mathrm{A}, \mathrm{a}$ 
synthetic analogue of marine natural product pateamine A. Mol. Cancer Ther. 2009, 8 (5), 1250-1260.

61. Dang, Y.; Low, W. K.; Xu, J.; Gehring, N. H.; Dietz, H. C.; Romo, D.; Liu, J. O., Inhibition of nonsense-mediated mRNA decay by the natural product pateamine a through eukaryotic initiation factor 4AIII. J. Biol. Chem. 2009, 284 (35), 2361323621.

62. Matthews, J. The molecular pharmacology of pateamine A. PhD Thesis, Victoria University of Wellington, 2010.

63. Li, X.; Tu, Z.; Li, H.; Liu, C.; Li, Z.; Sun, Q.; Yao, Y.; Liu, J.; Jiang, S., Biological evaluation of new largazole analogues: Alteration of macrocyclic scaffold with click chemistry. ACS Med. Chem. Lett. 2012, 4 (1), 132-136.

64. Smith III, A. B.; Ott, G. R., Total syntheses of (-)-macrolactin A, (+)-macrolactin E, and (-)-macrolactinic acid: An exercise in Stille cross-coupling chemistry. J. Am. Chem. Soc. 1998, 120 (16), 3935-3948.

65. Allred, G. D.; Liebeskind, L. S., Copper-mediated cross-coupling of organostannanes with organic iodides at or below room temperature. J. Am. Chem. Soc. 1996, 118 (11), 2748-2749.

66. Paterson, I.; Gardner, N. M.; Guzman, E.; Wright, A. E., Total synthesis and biological evaluation of potent analogues of dictyostatin: Modification of the C2C6 dienoate region. Bioorg. Med. Chem. Lett. 2008, 18 (23), 6268-6272.

67. Yadav, J. S.; Rajender, V., Studies directed towards the total synthesis of (-)dictyostatin. Eur. J. Org. Chem. 2010, 2010 (11), 2148-2156.

68. Still, W. C.; Gennari, C., Direct synthesis of Z-unsaturated esters. A useful modification of the Horner-Emmons olefination. Tetrahedron Lett. 1983, 24 (41), 4405-4408.

69. Sabitha, G.; Padmaja, P.; Reddy, P. N.; Jadav, S. S.; Yadav, J. S., Stereoselective total synthesis of stagonolide E. Tetrahedron Lett. 2010, 51 (47), 6166-6168.

70. Eisner, U.; Elvidge, J. A.; Linstead, R. P., 281. Polyene acids. Part VI. A new (cistrans-)isomer of sorbic acid and its relation to hexenolactones. J. Chem. Soc. (Resumed) 1953, 0 (0), 1372-1379.

71. Cardillo, G.; Orena, M.; Sandri, S., Synthesis of compounds containing the isoprene unit : A stereospecific synthesis of dehydronerol isovalerate, a component of anthemis Montana L. Tetrahedron 1976, 32 (1), 107-108.

72. Gammill, R. B.; Bell, L. T.; Mizsak, S. A., Mechanistic aspects of a novel, 1,2-3,4 reductive-fragmentation that establishes four stereocenters in a single step. Tetrahedron Lett. 1990, 31 (37), 5301-5302. 
73. Baldwin, J. E.; Reddy, V. P., Primary deuterium kinetic isotope effects for the thermal [1,7] sigmatropic rearrangement of 7-methylocta-1,3(Z),5(Z)-triene. J. Am. Chem. Soc. 1988, 110 (24), 8223-8228.

74. Isaacs, N. S.; El-Din, G. N., The application of high pressure to some difficult Wittig reactions. Tetrahedron Lett. 1987, 28 (19), 2191-2192.

75. Smith III, A. B.; Walsh, S. P.; Frohn, M.; Duffey, M. O., Diversity-oriented synthesis of polyketide natural products via iterative chemo- and stereoselective functionalization of polyenoates: Development of a unified approach for the C(1-19) segments of lituarines A-C. Org. Lett. 2004, 7 (1), 139-142.

76. Lengkeek, N. A.; Greenwood, P. F.; Nguyen, B.; Koutsantonis, G. A.; Piggott, M. J., Making mixtures to solve structures: Structural elucidation via combinatorial synthesis. ACS Comb. Sci. 2010, 12 (1), 141-150.

77. Schmidt, B.; Kunz, O., One-flask tethered ring closing metathesis-electrocyclic ring opening for the highly stereoselective synthesis of conjugated $Z / E$-dienes. Eur. J. Org. Chem. 2012, 2012 (5), 1008-1018.

78. Carey, F.; Sundberg, R., Polar addition and elimination reactions. In Advanced Organic Chemistry, Springer US: 2007; pp 473-577.

79. Jung, M. E.; Piizzi, G., gem-Gisubstituent effect: Theoretical basis and synthetic applications. Chem. Rev. 2005, 105 (5), 1735-1766.

80. Ciabatti, R.; Maffioli, S. I.; Panzone, G.; Canavesi, A.; Michelucci, E.; Tiseni, P. S.; Marzorati, E.; Checchia, A.; Giannone, M.; Jabes, D.; Romano, G.; Brunati, C.; Candiani, G.; Castiglione, F., Synthesis and preliminary biological characterization of new semisynthetic derivatives of ramoplanin. J. Med. Chem. 2007, 50 (13), 3077-3085.

81. Huckin, S. N.; Weiler, L., Alkylation of dianions of beta-keto esters. J. Am. Chem. Soc. 1974, 96 (4), 1082-1087.

82. Wuts, P. G. M.; Greene, T. W., Protection for the hydroxyl group, including 1,2and 1,3-diols. In Greene's Protective Groups in Organic Synthesis, John Wiley \& Sons, Inc.: 2006; pp 16-366.

83. Sarel, S.; Tsai, L.; Newman, M. S., Rates of alkaline hydrolysis of a series of primary and secondary alkyl acetates. J. Am. Chem. Soc. 1956, 78 (20), 5420-5423.

84. Trost, B. M.; Weiss, A. H., An alkyne strategy for the asymmetric synthesis of natural products: Application to (+)-spirolaxine methyl ether. Angew. Chem. Int. Ed. 2007, 46 (40), 7664-7666.

85. Lozano-Vila, A. M.; Monsaert, S.; Bajek, A.; Verpoort, F., Ruthenium-based olefin metathesis catalysts derived from alkynes. Chem. Rev. 2010, 110 (8), 4865-4909. 
86. Chinchilla, R.; Nájera, C., The sonogashira reaction: A booming methodology in synthetic organic chemistry. Chem. Rev. 2007, 107 (3), 874-922.

87. (a) Hashmi, A. S., Homogeneous gold catalysts and alkynes: A successful liaison. Gold Bull. 2003, 36 (1), 3-9; (b) Ma, S.; Yu, S.; Gu, Z., Gold-catalyzed cyclization of enynes. Angew. Chem. Int. Ed. 2006, 45 (2), 200-203.

88. Eymery, F.; Iorga, B.; Savignac, P., The usefulness of phosphorus compounds in alkyne synthesis. Synthesis 2000, (02), 185-213.

89. Gilbert, J. C.; Weerasooriya, U., Diazoethenes: their attempted synthesis from aldehydes and aromatic ketones by way of the Horner-Emmons modification of the Wittig reaction. A facile synthesis of alkynes. J. Org. Chem. 1982, 47 (10), 18371845 .

90. Habrant, D.; Rauhala, V.; Koskinen, A. M. P., Conversion of carbonyl compounds to alkynes: general overview and recent developments. Chem. Soc. Rev. 2010, 39 (6), 2007-2017.

91. Fringuelli, F.; Piermatti, O.; Pizzo, F.; Vaccaro, L., Ring opening of epoxides with sodium azide in water. A regioselective $\mathrm{pH}$-controlled reaction. J. Org. Chem. 1999, 64 (16), 6094-6096.

92. Iranpoor, N.; Kazemi, F., Regioselective azidolysis of epoxides catalyzed with Ce(IV). Synth. Commun. 1999, 29 (4), 561-566.

93. Kazemi, F.; Kiasat, A. R.; Ebrahimi, S., Regioselective azidolysis of epoxides catalyzed with $\mathrm{LiBF}_{4}$. Synth. Commun. 2003, 33 (6), 999.

94. Boruwa, J.; Borah, J. C.; Kalita, B.; Barua, N. C., Highly regioselective ring opening of epoxides using $\mathrm{NaN}_{3}$ : a short and efficient synthesis of (-)-cytoxazone. Tetrahedron Lett. 2004, 45 (39), 7355-7358.

95. Sabitha, G.; Babu, R. S.; Reddy, M. S. K.; Yadav, J. S., Ring opening of epoxides and aziridines with sodium azide using Oxone ${ }^{\circledR}$ in aqueous acetonitrile: A highly regioselective azidolysis reaction. Synthesis 2002, 2002 (15), 2254-2258.

96. Procopio, A.; Costanzo, P.; Dalpozzo, R.; Maiuolo, L.; Nardi, M.; Oliverio, M., Efficient ring opening of epoxides with trimethylsilyl azide and cyanide catalyzed by erbium(III) triflate. Tetrahedron Lett. 2010, 51 (39), 5150-5153.

97. Yadollahi, B.; Danafar, H., A facile synthesis of 1,2-azidoalcohols by (TBA) $4 \mathrm{PFeW}_{11} \mathrm{O}_{39} \cdot 3 \mathrm{H}_{2} \mathrm{O}$-catalyzed azidolysis of epoxides with $\mathrm{NaN}_{3}$. Catal. Lett. 2007, 113 (3-4), 120-123.

98. Sukata, K., Efficient synthesis of silyl azides using sodium azide impregnated on amberlite XAD resin. J. Org. Chem. 1988, 53 (20), 4867-4869. 
99. Sabitha, G.; Babu, R. S.; Rajkumar, M.; Yadav, J. S., Cerium(III) chloride promoted highly regioselective ring opening of epoxides and aziridines using $\mathrm{NaN}_{3}$ in acetonitrile: A facile synthesis of 1,2-azidoalcohols and 1,2-azidoamines. Org. Lett. 2002, 4 (3), 343-345.

100. Lieber, E.; Ramachandran, J., Isomeric 5-(substituted)aminothiatriazole and 1substituted-tetrazolinethiones. Can. J. Chem. 1959, 37 (1), 101-109.

101. Oremus, V.; Heimgartner, H., Reaction of 1,4-dihydro-1-phenyltetrazole-5-thione with epoxides ; formation of 1-[(1-phenyl-1H-tetrazol-5-yl)thio]-alkan-2-ols. J. Chem. Res., Synop. 1991, (10), 296.

102. Bandgar, B. P.; Joshi, N. S.; Kamble, V. T.; Sawant, S. S., Cyanuric chloride: An efficient catalyst for ring opening of epoxides with thiols under solvent-free conditions. Aus. J. Chem. 2008, 61 (3), 231-234.

103. Movassagh, B.; Soleiman-Beigi, M., Stereo- and regioselective thiolysis of 1,2epoxides in water. Synth. Commun. 2007, 37 (18), 3239-3244.

104. Thirsk, C.; Whiting, A., Polyene natural products. J. Chem. Soc., Perkin Trans. 1 2002, (8), 999-1023.

105. Kovacic, P.; Cooksy, A., Novel, unifying mechanism for amphotericin B and other polyene drugs: electron affinity, radicals, electron transfer, autoxidation, toxicity, and antifungal action. MedChem Comm 2012, 3 (3), 274-280.

106. Mills, J. E.; Maryanoff, C. A.; McComsey, D. F.; Stanzione, R. C.; Scott, L., Reaction of amines with methylene chloride. Evidence for rapid aminal formation from $\mathrm{N}$-methylenepyrrolidinium chloride and pyrrolidine. J. Org. Chem. 1987, 52 (9), 1857-1859.

107. Zincke, T.; Heuser, G.; Möller, W., I. Ueber dinitrophenylpyridiniumchlorid und dessen umwandlungsproducte. Liebigs Ann. 1904, 333 (2-3), 296-345.

108. Wang, Q.; Deredas, D.; Huynh, C.; Schlosser, M., Sequestered alkyllithiums: Why phenyllithium alone is suitable for betaine-ylide generation. Chem. Eur. J. 2003, 9 (2), 570-574.

109. Schlosser, M.; Tuong, H. B.; Schaub, B., The betaine-ylid route to trans-alkenols. Tetrahedron Lett. 1985, 26 (3), 311-314.

110. Maryanoff, B. E.; Reitz, A. B., The Wittig olefination reaction and modifications involving phosphoryl-stabilized carbanions. Stereochemistry, mechanism, and selected synthetic aspects. Chem. Rev. 1989, 89 (4), 863-927. 
111. Tamura, R.; Saegusa, K.; Kakihana, M.; Oda, D., Stereoselective $E$ and Z olefin formation by Wittig olefination of aldehydes with allylic phosphorus ylides. Stereochemistry. J. Org. Chem. 1988, 53 (12), 2723-2728.

112. Murakami, N.; Sugimoto, M.; Kobayashi, M., Participation of the $\beta$ hydroxyketone part for potent cytotoxicity of callystatin A, a spongean polyketide. Bioorg. Med. Chem. 2001, 9 (1), 57-67.

113. Blakemore, P. R., The modified Julia olefination: Alkene synthesis via the condensation of metallated heteroarylalkylsulfones with carbonyl compounds. J. Chem. Soc., Perkin Trans. 1 2002, (23), 2563-2585.

114. Kocienski, P., Recent sulphone-based olefination reactions. Phosphorus Sulfur Relat. Elem. 1985, 24 (1-2), 97.

115. Blakemore, P. R.; Cole, W. J.; Kocieński, P. J.; Morley, A., A stereoselective synthesis of trans-1,2-disubstituted alkenes based on the condensation of aldehydes with metallated 1-phenyl-1H-tetrazol-5-yl sulfones. Synlett 1998, 1998 (01), 26-28.

116. Mallinson, J.; Collins, I., Macrocycles in new drug discovery. Future Med. Chem. 2012, 4 (11), 1409-1438.

117. Illuminati, G.; Mandolini, L., Ring closure reactions of bifunctional chain molecules. Acc. Chem. Res. 1981, 14 (4), 95-102.

118. Parenty, A.; Moreau, X.; Niel, G.; Campagne, J. M., Update 1 of: Macrolactonizations in the total synthesis of natural products. Chem. Rev. 2013, 113 (1), PR1-PR40.

119. Roush, W. R.; Blizzard, T. A., Synthesis of epoxytrichothecenes: verrucarin J and verrucarin J isomers. J. Org. Chem. 1984, 49 (10), 1772-1783.

120. Shiina, I.; Kubota, M.; Oshiumi, H.; Hashizume, M., An effective use of benzoic anhydride and its derivatives for the synthesis of carboxylic esters and lactones: A powerful and convenient mixed anhydride method promoted by basic catalysts. J. Org. Chem. 2004, 69 (6), 1822-1830.

121. Zhu, W.; Jimenez, M.; Jung, W. H.; Camarco, D. P.; Balachandran, R.; Vogt, A.; Day, B. W.; Curran, D. P., Streamlined syntheses of (-)-dictyostatin, 16-desmethyl25,26- dihydrodictyostatin, and 6- epi-16-desmethyl-25,26-dihydrodictyostatin. J. Am. Chem. Soc. 2010, 132 (26), 9175-9187.

122. Devos, A.; Remion, J.; Frisque-Hesbain, A.-M.; Colens, A.; Ghosez, L., Synthesis of acyl halides under very mild conditions. J. Chem. Soc., Chem. Comm. 1979, 0 (24), 1180-1181. 
123. Zanato, C.; Pignataro, L.; Ambrosi, A.; Hao, Z.; Trigili, C.; Diaz, J. F.; Barasoain, I.; Gennari, C., Highly stereoselective total synthesis of (+)-9-epi-dictyostatin and ()-12,13-bis-epi-dictyostatin. Eur. J. Org. Chem. 2011, (14), 2643-2661.

124. Michael, A., Ueber die einwirkung von diazobenzolimid auf acetylendicarbonsäuremethylester. J. Prakt. Chem. 1893, 48 (1), 94-95.

125. Huisgen, R., Proceedings of the chemical society. October 1961. Proc. Chem. Soc. 1961, (October), 357-396.

126. Kolb, H. C.; Finn, M. G.; Sharpless, K. B., Click chemistry: Diverse chemical function from a few good reactions. Angew. Chem. Int. Ed. 2001, 40 (11), 2004-2021.

127. Thirumurugan, P.; Matosiuk, D.; Jozwiak, K., Click chemistry for drug development and diverse chemical-biology applications. Chem. Rev. 2013.

128. Iha, R. K.; Wooley, K. L.; Nyström, A. M.; Burke, D. J.; Kade, M. J.; Hawker, C. J., Applications of orthogonal "click" chemistries in the synthesis of functional soft materials. Chem. Rev. 2009, 109 (11), 5620-5686.

129. Binder, W. H.; Kluger, C., Azide/alkyne-"click" reactions: Applications in material science and organic synthesis. In Curr. Opin. Chem. Biol., Bentham Science Publishers Ltd.: 2006; Vol. 10, pp 1791-1815.

130. Meldal, M.; Tornoe, C. W., Cu-catalyzed azide - Alkyne cycloaddition. Chem. Rev. 2008, 108 (8), 2952-3015.

131. Allam, A.; Dupont, L.; Behr, J.-B.; Plantier-Royon, R., Convenient Synthesis of a Galacturonic Acid Based Macrocycle with Potential Copper-Complexation Ability. Eur. J. Org. Chem. 2012, 2012 (4), 817-823.

132. Wu, J.-C.; Wang, D.-X.; Huang, Z.-T.; Wang, M.-X., Synthesis of diverse N,Obridged calix[1]arene[4]pyridine-C60 dyads and triads and formation of intramolecular self-inclusion complexes. J. Org. Chem. 2010, 75 (24), 8604-8614.

133. Smith III, A. B.; Wan, Z., Total synthesis of the ansamycin antibiotic (+)thiazinotrienomycin E. J. Org. Chem. 2000, 65 (12), 3738-3753.

134. Kocienski, P. J.; Ruston, S., Scope and stereochemistry of an olefin synthesis from $\beta$-hydroxy-sulphones. J. Chem. Soc., Perkin Trans. 1 1978, 829.

135. Smith III, A. B.; Brandt, B. M., Total synthesis of (-)-callystatin A. Org. Lett. 2001, 3 (11), 1685-1688.

136. Grée, R.; Tourbah, H.; Carrié, R., Fumaraldehyde monodimethyl acetal: An easily accessible and versatile intermediate. Tetrahedron Lett. 1986, 27 (41), 4983-4986. 
137. Kashin, D.; Meyer, A.; Wittenberg, R.; Schoning, K.-U.; Kamlage, S.; Kirschning, A., A practical synthesis of the ansa chain of benzenic ansamycin antibiotics: Total synthesis of an ansatrienol derivative. Synthesis 2007, 2007 (02), 304-319.

138. Yao, G.; Steliou, K., Synthetic studies toward bioactive cyclic peroxides from the marine sponge Plakortis angulospiculatus. Org. Lett. 2002, 4 (4), 485-488.

139. Finkelshtein, E. I.; Krasnokutskaya, I. S., Reactivity of retinoids and carotenoids in autoxidation. J. Phys. Org. Chem. 1996, 9 (6), 411-418.

140. Krasnokutskaya, I. S.; Leontieva, S. V.; Finkelshtein, E. I.; Khodonov, A. A.; Shvets, V. I., Effect of carbonyls on the reactivity of polyenes in autoxidation. J. Phys. Org. Chem. 2003, 16 (4), 226-231.

141. Little, R. Characterization of the interaction between pateamine and eIF4A. Honours project report, Victoria University of Wellington, 2013.

142. Katritzky, A. R.; Ogretir, C.; Tarhan, H. O.; Dou, H. M.; Metzger, J. V., The kinetics and mechanism of the electrophilic substitution of heteroaromatic compounds. Part XLII. The nitration of thiazoles and thiazolones. J. Chem. Soc., Perkin Trans. 2 1975, (14), 1614-1620.

143. Jencks, W. P.; Regenstein, J., Ionization constants of acids and bases. In Handbook of Biochemistry and Molecular Biology, Fourth Edition, CRC Press: 2010; pp 595-635.

144. Bottley, A.; Phillips, N. M.; Webb, T. E.; Willis, A. E.; Spriggs, K. A., eIF4A inhibition allows translational regulation of mRNAs encoding proteins involved in Alzheimer's disease. PLoS ONE 2010, 5 (9), e13030.

145. Pirrung, M. C., Appendix 3: Recipes for TLC stains. In The Synthetic Organic Chemist's Companion, John Wiley \& Sons, Inc.: 2006; pp 171-172.

146. Kuroda, H.; Hanaki, E.; Izawa, H.; Kano, M.; Itahashi, H., A convenient method for the preparation of $\alpha$-vinylfurans by phosphine-initiated reactions of various substituted enynes bearing a carbonyl group with aldehydes. Tetrahedron 2004, 60 (8), 1913-1920.

147. Bellassoued, M.; Aatar, J.; Bouzid, M.; Damak, M., A convenient route to (E)- $\alpha$, $\beta$ unsaturated methyl ketones. Phosphorus Sulfur Relat. Elem. 2010, 185 (9), 18861895.

148. Li, X.; Li, L.; Tang, Y.; Zhong, L.; Cun, L.; Zhu, J.; Liao, J.; Deng, J., Chemoselective conjugate reduction of $\alpha, \beta$-unsaturated ketones catalyzed by rhodium amido complexes in aqueous media. J. Org. Chem. 2010, 75 (9), 29812988. 
149. Nakano, T.; Ishii, Y.; Ogawa, M., Selective oxidation of alcohol function in allylic alcohols to $\alpha, \beta$-unsaturated carbonyl compounds catalyzed by zirconocene complexes. J. Org. Chem. 1987, 52 (22), 4855-4859.

150. Mamaghani, M.; Mahmoodi, N. O.; Moghisseh, A. A.; Pourmohamad, L., Synthesis and kinetic resolution of furyl substituted secondary carbinols by porcine pancreatic lipase under solvent free conditions. J. Iran. Chem. Soc. 2008, 5 (2), 238-243.

151. Galletti, P.; Moretti, F.; Samorì, C.; Tagliavini, E., Chemoselective allylation of ketones in ionic liquids containing sulfonate anions. ChemSusChem 2009, 2 (11), 1045-1050.

152. Nowrouzi, F.; Thadani, A. N.; Batey, R. A., Allylation and crotylation of ketones and aldehydes using potassium organotrifluoroborate salts under lewis acid and montmorillonite K10 catalyzed conditions. Org. Lett. 2009, 11 (12), 2631-2634.

153. Ferraboschi, P.; Canevotti, R.; Grisenti, P.; Santaniello, E., A new flexible synthesis of (R,S)-mevalonolactone. J. Chem. Soc., Perkin Trans. 1 1987, 0 (0), 23012303.

154. Moreau, X.; Bazán-Tejeda, B.; Campagne, J.-M., Catalytic and asymmetric vinylogous Mukaiyama reactions on aliphatic ketones: Formal asymmetric synthesis of taurospongin A. J. Am. Chem. Soc. 2005, 127 (20), 7288-7289.

155. Tanaka, N.; Masaki, Y., Deprotection of acetals and silyl ethers using a polymersupported $\pi$-acid catalyst: Chemoselectivity and polymer effect. Synlett 1999, (12), 1960-1962.

156. Arikan, F.; Li, J.; Menche, D., Diastereodivergent aldol reactions of beta-alkoxy ethyl ketones: Modular access to $(1,4)$-syn and -anti polypropionates. Org. Lett. 2008, 10 (16), 3521-3524.

157. Zampella, A.; Sepe, V.; D'Orsi, R.; Bifulco, G.; Bassarello, C.; D'Auria, M. V., Stereochemical assignment of the C23-C35 portion of sphinxolide/reidispongiolide class of natural products by asymmetric synthesis. Tetrahedron: Asymm. 2003, 14 (13), 1787-1798.

158. Mohapatra, D. K.; Das, P. P.; Sai Reddy, D.; Yadav, J. S., First total syntheses and absolute configuration of rugulactone and 6(R)-(4'-oxopent-2'-enyl)-5,6-dihydro2H-pyran-2-one. Tetrahedron Lett. 2009, 50 (43), 5941-5944.

159. Batt, F.; Fache, F., Towards the synthesis of the 4,19-diol derivative of (-)mycothiazole: synthesis of a potential key intermediate. Eur. J. Org. Chem. 2011, 2011 (30), 6039-6055. 
160. Choi, S. E.; Pflum, M. K. H., The structural requirements of histone deacetylase inhibitors: Suberoylanilide hydroxamic acid analogs modified at the C6 position. Bioorg. Med. Chem. Lett. 2012, 22 (23), 7084-7086.

161. Rousseau, G.; Strzalko, T.; Roux, M.-C., Preparation of large ring acetylenic lactones by iodo lactonisation. Tetrahedron Lett. 2004, 45 (23), 4503-4505.

162. Jaschinski, T.; Hiersemann, M., $\{1,6\}$-Transannular catalytic asymmetric GosteliClaisen rearrangement. Org. Lett. 2012, 14 (16), 4114-4117.

163. Jafarpour, M.; Rezaeifard, A.; Golshani, T., A green, catalyst-free method for the synthesis of sulfonamides and sulfonylazides. Phosphorus Sulfur Relat. Elem. 2010, 186 (1), 140-148.

164. Schnaars, C.; Hansen, T., Halodiazophosphonates, a new class of diazo compounds for the diastereoselective intermolecular Rh(II) catalyzed cyclopropanation. Org. Lett. 2012, 14 (11), 2794-2797.

165. Kang, S. H.; Jung, B. M.; Kim, W. J.; Chang, J. Y., Embedding nanofibers in a polymer matrix by polymerization of organogels comprising heterobifunctional organogelators and monomeric solvents. Chem. Mat. 2008, 20 (17), 5532-5540.

166. Kishore Kumar, G. D.; Baskaran, S., A facile, catalytic, and environmentally benign method for selective deprotection of tert-butyldimethylsilyl ether mediated by phosphomolybdic acid supported on silica gel. J. Org. Chem. 2005, 70 (11), 4520-4523.

167. Williams, R. M.; Rollins, S. B.; Judd, T. C., Synthesis of the first photo-triggered pro-mitosene based on FR-900482. Tetrahedron 2000, 56 (4), 521-532.

168. Könning, D.; Hiller, W.; Christmann, M., One-pot oxidation/isomerization of Zallylic alcohols with oxygen as stoichiometric oxidant. Org. Lett. 2012, 14 (20), 5258-5261.

169. Olofsson, B.; Somfai, P., A regio- and stereodivergent route to all isomers of vicamino alcohols. J. Org. Chem. 2002, 67 (24), 8574-8583.

170. Werkhoven, T. M.; van Nispen, R.; Lugtenburg, J., Specific isotope enrichment of methyl methacrylate. Eur. J. Org. Chem. 1999, 1999 (11), 2909-2914.

171. Mao, Y.; Mathey, F., A phosphinine 2-carboxaldehyde. Org. Lett. 2012, 14 (4), 1162-1163.

172. Kondo, Y.; Suzuki, N.; Takahashi, M.; Kumamoto, T.; Masu, H.; Ishikawa, T., Enantioselective construction of a polyhydroxylated pyrrolidine skeleton from 3vinylaziridine-2-carboxylates: Synthesis of (+)-DMDP and a potential common intermediate for (+)-hyacinthacine a1 and (+)-1-epi-australine. J. Org. Chem. 2012, 77 (18), 7988-7999. 
173. Amans, D.; Bellosta, V.; Dacquet, C.; Ktorza, A.; Hennuyer, N.; Staels, B.;

Caignard, D.-H.; Cossy, J., Synthesis and evaluation of new polyenic compounds as potential PPARs modulators. Org. Biomol. Chem. 2012, 10 (30), 6169-6185.

174. Han, S. B.; Hassan, A.; Kim, I. S.; Krische, M. J., Total synthesis of (+)-roxaticin via $\mathrm{C}-\mathrm{C}$ bond forming transfer hydrogenation: A departure from stoichiometric chiral reagents, auxiliaries, and premetalated nucleophiles in polyketide construction. J. Am. Chem. Soc. 2010, 132 (44), 15559-15561.

175. Huang, Y.; Fananas-Mastral, M.; Minnaard, A. J.; Feringa, B. L., A novel catalytic asymmetric route towards skipped dienes with a methyl-substituted central stereogenic carbon. Chem. Comm. 2013, 49 (32), 3309-3311.

176. Shi, L.; Lundberg, D.; Musaev, D. G.; Menger, F. M., [12]Annulene gemini surfactants: Structure and self-assembly. Angew. Chem. Int. Ed. 2007, 46 (31), 58895891.

177. (a) Michels, T. D.; Rhee, J. U.; Vanderwal, C. D., Synthesis of $\delta$-tributylstannyl$\alpha, \beta, \gamma, \delta$-unsaturated aldehydes from pyridines. Org. Lett. 2008, 10 (21), 4787-4790; (b) Nguyen, T. M.; Peixoto, S.; Ouairy, C.; Nguyen, T. D.; Bénéchie, M.; Marazano, C.; Michel, P., Simple and convenient method for the synthesis of 2substituted glutaconaldehyde salts and 2-substituted glutaconaldehyde derivatives. Synthesis 2010, 2010 (01), 103-109.

178. Bose, D.; Fernandez, E.; Pietruszka, J., Stereoselective synthesis of both enantiomers of rugulactone. J. Org. Chem. 2011, 76 (9), 3463-3469.

179. Frederico, D.; Donate, P. M.; Constantino, M. G.; Bronze, E. S.; Sairre, M. I., A short and efficient synthesis of crocetin-dimethylester and crocetindial. J. Org. Chem. 2003, 68 (23), 9126-9128.

180. Trost, B. M.; Bartlett, M. J., Transition-metal-catalyzed synthesis of aspergillide B: An alkyne addition strategy. Org. Lett. 2012, 14 (5), 1322-1325.

181. Schoning, K.-U.; Wittenberg, R.; Kirschning, A., Stereocontrolled preparation of the C1-C14 polyene fragment of benzenic ansamycin antibiotics ansatrienin A and B. Synlett 1999, 1999 (10), 1624-1626. 